$w 1125.7$

R299

\#282

Report 282

\title{
CHEMICAL AND PHYSICAL \\ CHARACTERISTICS OF WATER IN ESTUARIES OF TEXAS \\ OCTOBER 1976-SEPTEMBER 1978
}

Government Publications

DEC 51983

Dallas Public Library

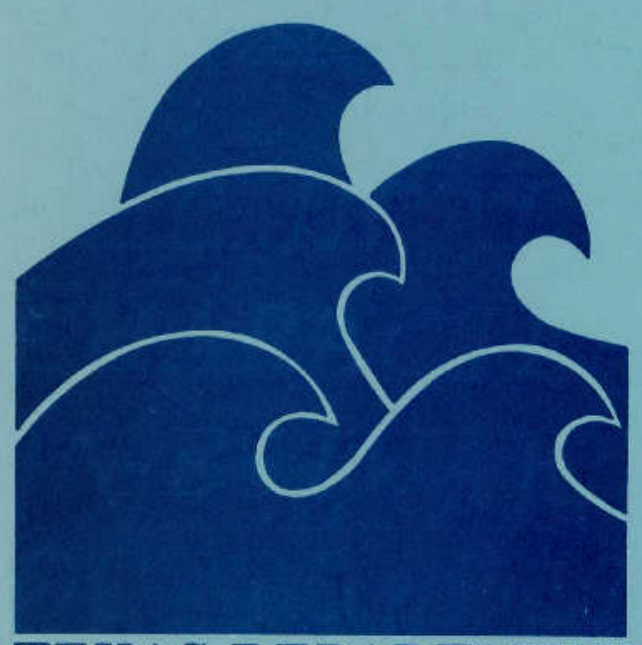





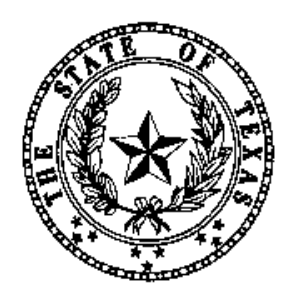

TEXAS DEPARTMENT OF WATER RESOURCES

\author{
REPORT 282
}

\title{
CHEMICAL AND PHYSICAL CHARACTERISTICS OF WATER IN ESTUARIES OF TEXAS OCTOBER 1976-SEPTEMBER 1978
}

\author{
By \\ J. C. Fisher \\ U.S. Geological Survey \\ This report was prepared by the U.S. Geological Survey \\ under cooperative agreement with the \\ Texas Department of Water Resources
}




\section{TEXAS DEPARTMENT OF WATER RESOURCES}

Charles E. Nemir, Executive Director

\section{TEXAS WATER DEVELOPMENT BOARD}

Louis A. Beecherl Jr., Chairman

Glen E. Roney

George W. McCleskey, Vice Chairmąn

w. O. Bankston Lonnie A. "Bo" Pilgrim

Louie Welch

\section{TEXAS WATER COMMISSION}

Felix McDonald, Chairman

Lee B. M. Biggart, Commissioner

John D. Stover, Commissioner

Authorization for use or reproduction of any original material contained in this publication, ie., not obtained from other sources, is freely granted. The Department would appreciate acknowledgement.

Published and distributed

by' the

Texas Department of Water Resources

Post Office Box 13087

Austin, Texas 78711 


\begin{abstract}
This report presents basic data on the chemical and physical characteristics of water in the estuaries of Texas for October 1976-September 1978. The properties or constituents that were measured in the field are dissolved oxygen (DO), specific conductance, temperature, $\mathrm{pH}$, and transparency by Secchi disk. Analyses conducted in the laboratory include the principal inorganic ions, biochemical oxygen demand (BOD), total organic carbon (TOC), ammonium, nitrite, nitrate, and total phosphate.
\end{abstract}




\section{TABLE OF CONTENTS}

Page

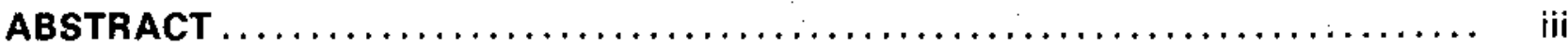

INTRODUCTION $\ldots \ldots, \ldots \ldots \ldots, \ldots, \ldots, \ldots, \ldots, \ldots, \ldots, \ldots \ldots \ldots \ldots, 1$

Purpose and Scope of the Investigation $\ldots \ldots \ldots \ldots \ldots \ldots \ldots \ldots \ldots \ldots \ldots \ldots, 1$

Status of the Project $\ldots \ldots \ldots \ldots \ldots \ldots \ldots \ldots \ldots \ldots \ldots \ldots \ldots \ldots \ldots \ldots \ldots, 3$

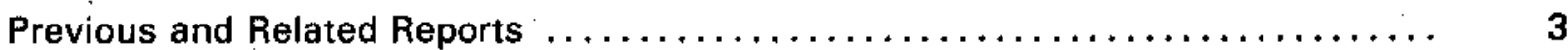

Metric Conversions $\ldots \ldots \ldots \ldots \ldots \ldots \ldots \ldots \ldots \ldots \ldots \ldots \ldots \ldots \ldots \ldots, \quad 3$

Acknowledgements $\ldots \ldots \ldots \ldots \ldots \ldots \ldots \ldots \ldots \ldots \ldots \ldots \ldots \ldots \ldots, \quad 4$

DATA-COLLECTION METHODS $. \ldots \ldots \ldots \ldots \ldots \ldots \ldots \ldots \ldots \ldots \ldots \ldots \ldots \ldots \ldots, 4$

Field Instruments $\ldots \ldots \ldots \ldots \ldots \ldots \ldots \ldots \ldots \ldots \ldots \ldots \ldots \ldots \ldots \ldots, \quad 4$

Treatment of Samples $\ldots \ldots \ldots \ldots \ldots \ldots \ldots \ldots \ldots \ldots \ldots \ldots \ldots \ldots \ldots \ldots \ldots, \quad 5$

QUALITY OF WATER IN THE ESTUARIES $\ldots \ldots \ldots \ldots \ldots \ldots \ldots \ldots \ldots \ldots, \quad 7$

Sabine-Neches Estuary $\ldots \ldots \ldots \ldots \ldots \ldots \ldots \ldots \ldots \ldots \ldots \ldots \ldots \ldots \ldots \ldots \ldots, 7$

Trinity-San Jacinto Estuary $\ldots \ldots \ldots \ldots \ldots \ldots \ldots \ldots \ldots \ldots \ldots \ldots \ldots \ldots \ldots \ldots \ldots \ldots \ldots, \quad 33$

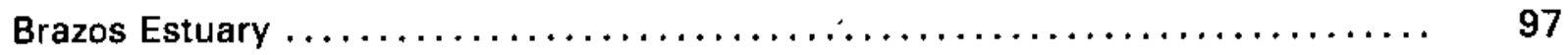

East Matagorda Estuary $\ldots \ldots \ldots \ldots \ldots \ldots \ldots \ldots \ldots \ldots \ldots \ldots \ldots \ldots \ldots, 103$

Colorado Estuary $\ldots \ldots \ldots \ldots \ldots \ldots \ldots \ldots \ldots \ldots \ldots \ldots \ldots \ldots \ldots \ldots, 111$

Lavaca-Tres Palacios Estuary $\ldots \ldots \ldots \ldots \ldots \ldots \ldots \ldots \ldots \ldots \ldots \ldots \ldots, 123$

Guadalupe Estuary $\ldots \ldots \ldots \ldots \ldots \ldots \ldots \ldots \ldots \ldots \ldots \ldots \ldots \ldots \ldots \ldots, 153$

Mission-Aransas Estuary $\ldots \ldots \ldots \ldots \ldots \ldots \ldots \ldots \ldots \ldots \ldots \ldots \ldots \ldots \ldots, 169$

Nueces Estuary $\ldots \ldots \ldots \ldots \ldots \ldots \ldots \ldots \ldots \ldots \ldots \ldots \ldots \ldots \ldots \ldots \ldots \ldots \ldots \ldots \ldots, 179$ 
Laguna Madre Estuary $\ldots \ldots \ldots \ldots \ldots \ldots \ldots \ldots \ldots \ldots \ldots \ldots \ldots \ldots, 193$

SELECTED HYDROLOGIC RECORDS $\ldots \ldots \ldots \ldots \ldots \ldots \ldots \ldots \ldots \ldots \ldots \ldots, 211$

Climatological Records $\ldots \ldots \ldots \ldots \ldots \ldots \ldots \ldots \ldots \ldots \ldots \ldots \ldots \ldots \ldots, 211$

Streamflow and Water-Quality Records $\ldots \ldots \ldots \ldots \ldots \ldots \ldots \ldots \ldots \ldots \ldots, 212$

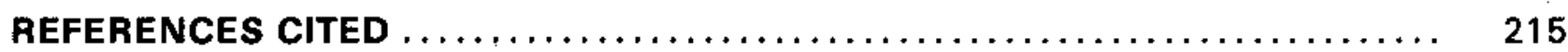

\section{TABLES}

1. Quality of Water in the Sabine-Neches Estuary, Water Years 1977-78
A. Field Determinations
B. Nutrient Analyses
C. Chemical Analyses

2. Quality of Water in the Trinity-San Jacinto Estuary, Water Years 1977-78
A. Field Determinations
B. Nutrient Analyses
C. Chemical Analyses

3. Quality of Water in the Brazos Estuary, Water Years 1977-78
A. Field Determinations
B. Nutrient Analyses
C. Chemical Analyses

101

102

4. Quality of Water in the East Matagorda Estuary, Water Years 1977-78
A. Field Determinations
B. Nutrient Analyses

104 


\section{TABLE OF CONTENTS-Continued}

Page

C. Chemical Analyses $\ldots \ldots \ldots \ldots \ldots \ldots \ldots \ldots \ldots \ldots \ldots \ldots \ldots \ldots, 110$

5. Quality of Water in the Colorado Estuary, Water Years 1977-78

A. Field Determinations $\ldots \ldots \ldots \ldots \ldots \ldots \ldots \ldots \ldots \ldots \ldots \ldots \ldots \ldots, 113$

B. Nutrient Analyses $\ldots \ldots \ldots \ldots \ldots \ldots \ldots \ldots \ldots \ldots \ldots \ldots \ldots \ldots \ldots, 119$

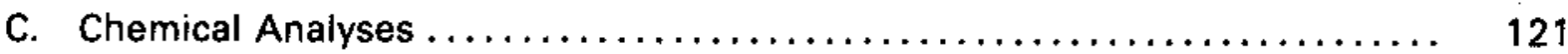

6. Quality of Water in the Lavaca-Tres Palacios Estuary, Water Years 1977-78

A. Field Determinations $\ldots \ldots \ldots \ldots \ldots \ldots \ldots \ldots \ldots \ldots \ldots \ldots \ldots \ldots \ldots, 124$

B. Nutrient Analyses $\ldots \ldots \ldots \ldots \ldots \ldots \ldots \ldots \ldots \ldots \ldots \ldots \ldots \ldots, 140$

C. Chemical Analyses $\ldots \ldots \ldots \ldots \ldots \ldots \ldots \ldots \ldots \ldots \ldots \ldots \ldots \ldots, 148$

7. Quality of Water in the Guadalupe Estuary, Water Years 1977-78

A. Field Determinations $\ldots \ldots \ldots \ldots \ldots \ldots \ldots \ldots \ldots \ldots \ldots \ldots \ldots \ldots \ldots, 154$

B. Nutrient Analyses $\ldots \ldots \ldots \ldots \ldots \ldots \ldots \ldots \ldots \ldots \ldots \ldots \ldots \ldots, 162$

C. Chemical Analyses $\ldots \ldots \ldots \ldots \ldots \ldots \ldots \ldots \ldots \ldots \ldots \ldots \ldots \ldots, 166$

8. Quality of Water in the Mission-Aransas Estuary, Water Years 1977-78
A. Field Determinations
B. Nutrient Analyses
176
C. Chemical Analyses

9. Quality of Water in the Nueces Estuary, Water Years 1977-78
A. Field Determinations
B. Nutrient Analyses . 186
C. Chemical Analyses 


\section{TABLE OF CONTENTS-Continued}

Page

10. Quality of Water in the Laguna Madre Estuary, Water Years 1977-78
A. Field Determinations
B. Nutrient Analyses
C. Chemical Analyses

\section{FIGURES}

1-14. Maps Showing:

1. Locations of the Estuaries $\ldots \ldots \ldots \ldots \ldots \ldots \ldots \ldots \ldots \ldots \ldots \ldots \ldots \ldots \ldots \ldots \ldots$

2. Data-Collection Sites in the Sabine-Neches Estuary $\ldots \ldots \ldots \ldots \ldots \ldots \ldots \ldots \ldots .8$

3. Data-Collection Sites in the Trinity-San Jacinto Estuary $\ldots \ldots \ldots \ldots \ldots \ldots \ldots \ldots . \quad 35$

4. Data-Collection Sites in the Brazos Estuary $\ldots \ldots \ldots \ldots \ldots \ldots \ldots \ldots \ldots \ldots \ldots . . \ldots 8$

5. Data-Collection Sites in the East Matagorda Estuary $\ldots \ldots \ldots \ldots \ldots \ldots \ldots \ldots \ldots .103$

6. Data-Collection Sites in the Colorado Estuary .................... 112

7. Data-Collection Sites in the Lavaca-Tres Palacios Estuary $\ldots \ldots \ldots \ldots \ldots \ldots \ldots \ldots$

8. Data-Collection Sites in the Guadalupe Estuary $\ldots \ldots \ldots \ldots \ldots \ldots \ldots \ldots \ldots \ldots \ldots$

9. Data-Collection Sites in the Mission-Aransas Estuary . . . . . . . . . . ... 169

10. Data-Collection Sites in the Nueces Estuary ................... 179

11. Data-Coliection Sites in the Laguna-Madre Estuary ................... 194

12. Locations of Selected Climatological Stations $\ldots \ldots \ldots \ldots \ldots \ldots \ldots \ldots \ldots \ldots \ldots . \ldots \ldots$

13. Location of Streamflow-Measuring Sites and Daily Water-Quality

14. Location of Selected Water-Quality and Streamflow Data-Collection Sites ..... 214 
. 


\title{
CHEMICAL AND PHYSICAL CHARACTERISTICS \\ OF WATER IN ESTUARIES OF TEXAS \\ OCTOBER 1976-SEPTEMBER 1978
}

\author{
By \\ J. C. Fisher \\ U.S. Geological Survey
}

INTRODUCTION

\section{Purpose and Scope of the Investigation}

The Texas Water Plan (Texas Water Development Board, 1968) proposes development and utilization of water resources in Texas and includes provision for the use and preservation of water in the estuaries of the State. Management of estuarine waters requires knowledge of the hydrodynamics and of the continuing changes in the chemical and physical characteristics of water in the estuaries.

In September 1967, the U.S. Geological Survey and the Texas Water Development Board (now Texas Department of Water Resources) began a cooperative water-resources investigation of the principal estuaries along the Texas Coast (Figure 1) except the Rio Grande estuary, which is under the jurisdiction of the International Boundary and Water Commission, United States and Mexico.

The objectives of the investigation are to define: (1) the occurrence, source, and distribution of nutrients; (2) the physical, organic, and inorganic water-quality constituents and their areal distribution and time variations; (3) the chemical and physical characteristics of gulf water that enters the estuaries; (4) the occurrence, quality, quantity, and dispersion of drainage entering the estuarine systems; and (5) the current patterns, directions, and rates of water movement.

The coastal waters of Texas are not classical estuaries, but are similar to them in ecosystems and mixing phenomena. A description of various types of estuaries is presented in "Estuaries" edited by Lauff (1967, p. 3-11). The term estuary as used in this report, refers to concomitant water bodies in which streamflow mixes with seawater. 


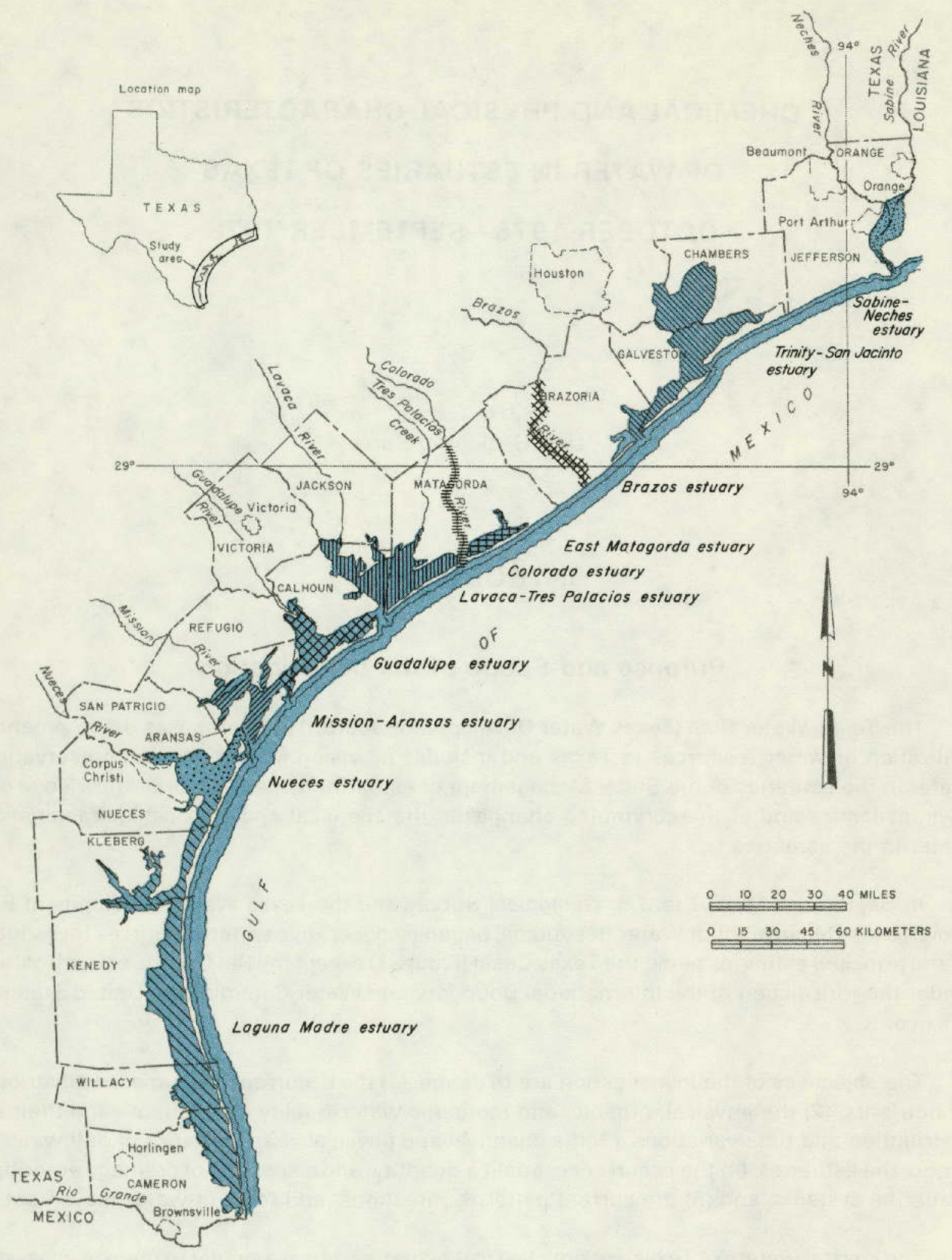

Figure 1

Locations of the Estuaries

Base from Official Stafe Highway Map of Texas, 1971 


\section{Status of the Project}

The first three objectives of the project are being met by a three-phased water-quality data-collection program of: (1) reconnaissance for establishment of an optimum data-collection network; (2) repetitive surveys throughout this network to determine the general chemical and physical characteristics of the estuarine systems; and (3) continued data collection at a reduced number of sites and at a reduced frequency to maintain definition of the chemical and physical characteristics of each estuarine system and of the relationship between systems. The first two phases have been completed and the third phase began in September 1973.

The fourth objective of the project is being met by data collection at six continuous streamflow-measuring stations and 11 stations at which monthly data on streamflow and water quality are obtained. The dispersion of water entering an estuary is being documented under the data-collection activities to meet the first three objectives.

The fifth objective of the project is being met by short-duration, intensive studies of inflow. Two such studies will be completed for each estuary. The studies on the Guadalupe estuary were completed in November 1970 and August 1973; the studies on the Lavaca-Tres Palacios estuary were completed in March 1971 and October 1972; the studies on the Mission-Aransas and Nueces estuaries were completed in November 1971 and May-June 1974; the studies on the Sabine-Neches estuary were completed in September 1974 and July 1975; one study on the Trinity-San Jacinto estuary was completed in July 1976; and two studies on the Colorado estuary were completed in May and July 1977. These studies are providing data on inflow and exchange of water through the passes. Three short-duration studies of water quality and water exchange were done on the Trinity River tidal marsh in November and December 1976 and July and August 1977.

\section{Previous and Related Reports}

This report, which presents data collected during water years $1977-78$, is one of a series of basic-data reports that have been prepared since 1970 (Hahl and Ratzlaff, 1970,1972, 1973, and 1975; Ratzlaff, 1976; Lind and Ratzlaff, 1979; and Lind, 1980 and 1983). A report by Grozier and others (1968, p. 47-61) includes data collected during flooding caused by Hurricane Beulah.

\section{Metric Conversions}

Metric equivalents of inch-pound units of measurements are given in parentheses in the text. The inch-pound units used in this report may be converted to metric units by using the following conversion factors:

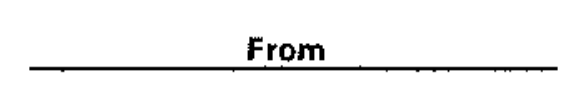

acre-foot

cubic foot per second $\left(\mathrm{ft}^{3} / \mathrm{s}\right)$

toot
Multiply by

1,233

.02832

.3048
To obtain

cubic meter $\left(\mathrm{m}^{3}\right)$

cubic meter per second $\left(\mathrm{m}^{3} / \mathrm{s}\right)$

meter (m) 


\section{Acknowledgements}

The U.S. Army Corps of Engineers (Galveston District), the Texas Parks and Wildlife Department, and the Texas Department of Water Resources provided data and field assistance. Many private citizens and commercial fishermen furnished information on historical changes and existing conditions in the estuaries.

\section{DATA-COLLECTION METHODS}

Approximately 234 data-collection sites were visited during the 1977.78 water years. About 50 percent of these sites are located adjacent to or between navigation aids, bridge piers, power poles, survey platforms, well structures, or other landmarks and can be reoccupied exactly. About 19 percent of the sites are close to shore features or reefs and are located by onboard radar or by compass heading and distance from the feature and water depth at the site; these sites can be reoccupied within 100 feet $(30.5 \mathrm{~m})$. About 31 percent of the sites are remote to any reference. They are reached by traveling from a known landmark at a known speed on a predetermined compass course. Verification of site location is made by checking the alignment of one or more distant landmarks by visual observation or by onboard radar. These sites can be reoccupied within approximately 0.25 mile $(0.4 \mathrm{~km})$.

At each data-collection site, field data are collected from several points along a vertical. Samples for laboratory analyses are collected from a predetermined number of data-collection sites and at other sites in the network when significant changes in field data indicate a need for additional samples.

The properties or constituents that are measured in the field are dissolved oxygen (DO), specific conductance, temperature, $\mathrm{pH}$, and transparency by Secchi disk. Analyses conducted in the laboratory include the principal inorganic ions, biochemical oxygen demand (BOD), total organic carbon (TOC), ammonium, nitrite, nitrate, and total phosphate.

\section{Field Instruments}

The field instruments used in this investigation are given in the following table. The mention of the names of the manufacturers of the instruments is for identification purposes only and does not constitute an endorsement by the Geological Survey.

Parameter measured

$\mathrm{pH}$, dissolved oxygen, temperature, specific conductance

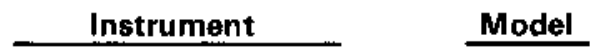

6

Surveyor
Manufacturer
Hydrolab Corporation 
The instruments used for $\mathrm{pH}$ measurements were calibrated daily during each water-quality

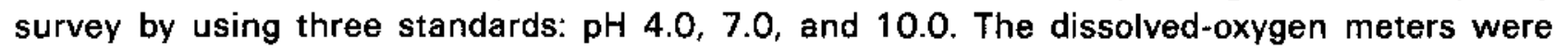
calibrated at least twice daily by using the oxygen-saturation data compiled by the American Public Health Association and others (1971, p. 480). The Surveyor was calibrated using the procedure provided by the manufacturer. In addition, instrument calibrations were rechecked at the end of each day.

\section{Treatment of Samples}

All water samples, except those for TOC, were collected in plastic throw-away bottles. The BOD, TOC, and nutrient samples were chilled and stored in crushed ice and shipped to the laboratory as soon as possible.

Water samples for the principal dissolved constituents were filtered through 0.45 micrometer membrane filters. Water samples for dissolved inorganic cations, heavy metals, and other selected trace constituents were treated with $1: 1$ nitric acid until the $\mathrm{pH}$ of the sample was less than 2.0 . 



\section{QUALITY OF WATER IN THE ESTUARIES}

\section{Sabine-Neches Estuary}

The Sabine-Neches estuary, which has an area of about 100 square miles $\left(260 \mathrm{~km}^{2}\right)$, consists of the tidal parts of the Sabine and Neches Rivers and other tributaries, Sabine Lake, the Sabine-Neches Canal, the Port Arthur Canal, parts of the Intracoastal Waterway, and Sabine Pass (Figure 2). Water depth at mean low water is greater than 40 feet $(12.2 \mathrm{~m})$ in dredged parts of the rivers, canals, and pass; about 15 feet $(4.6 \mathrm{~m})$ in the Intracoastal Waterway; and generally about 10 feet $(3.0 \mathrm{~m})$ in Sabine Lake.

Water-quality data (Table 1) were collected during October-December 1976; January, February, June, August, and September 1977; and February, May, and June 1978. 


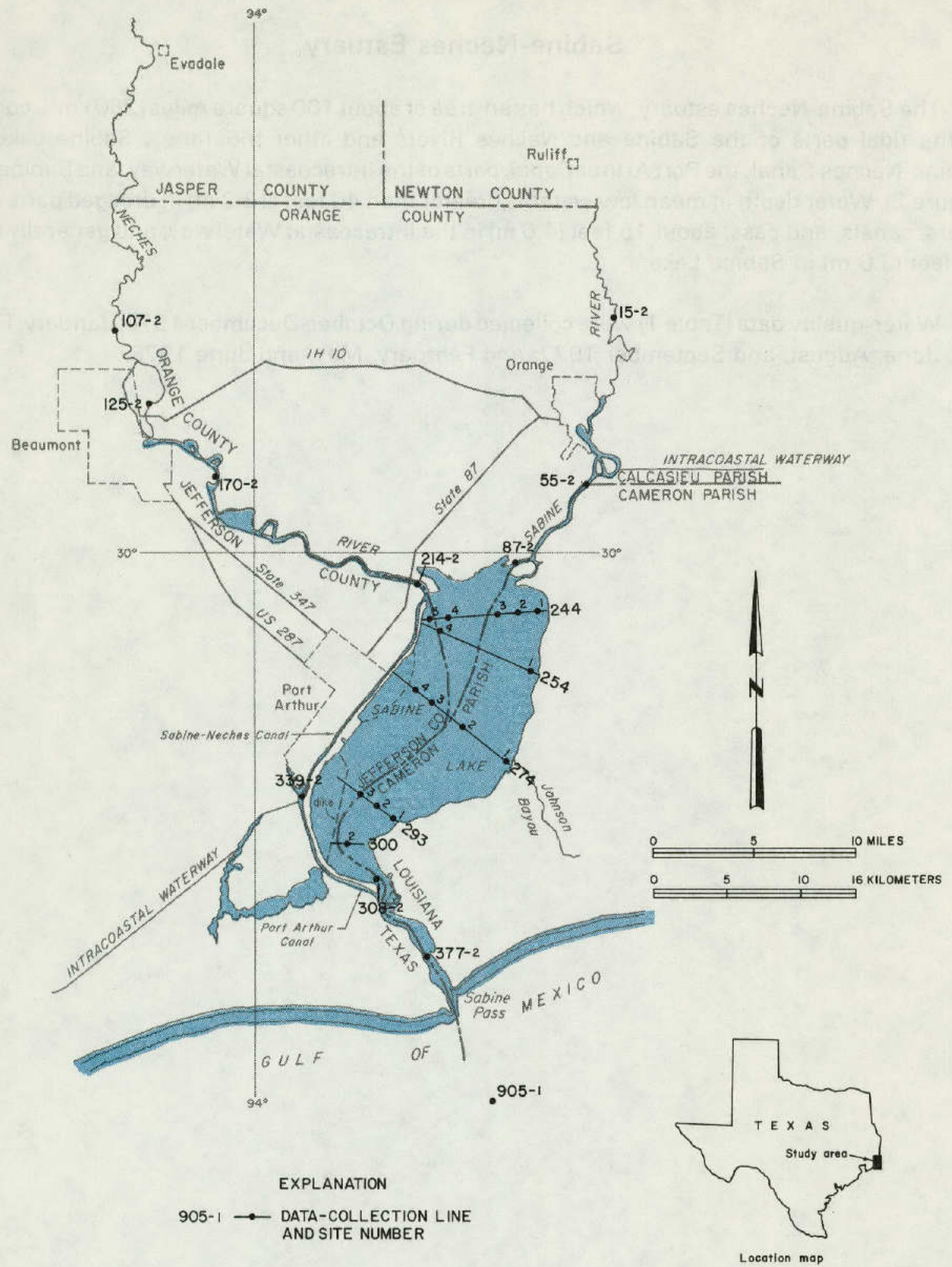

Figure 2

Data-Collection Sites in the Sabine-Neches Estuary 
(FT a feet; MICROAHOS - micronhos per centimeter at $25^{\circ}$ Celsius; OEG $\mathrm{C}=$ degrees Celsius; M = meters; $M G / L=$ milligraus per liter)

300953093420600 iINE 015 SITE 02

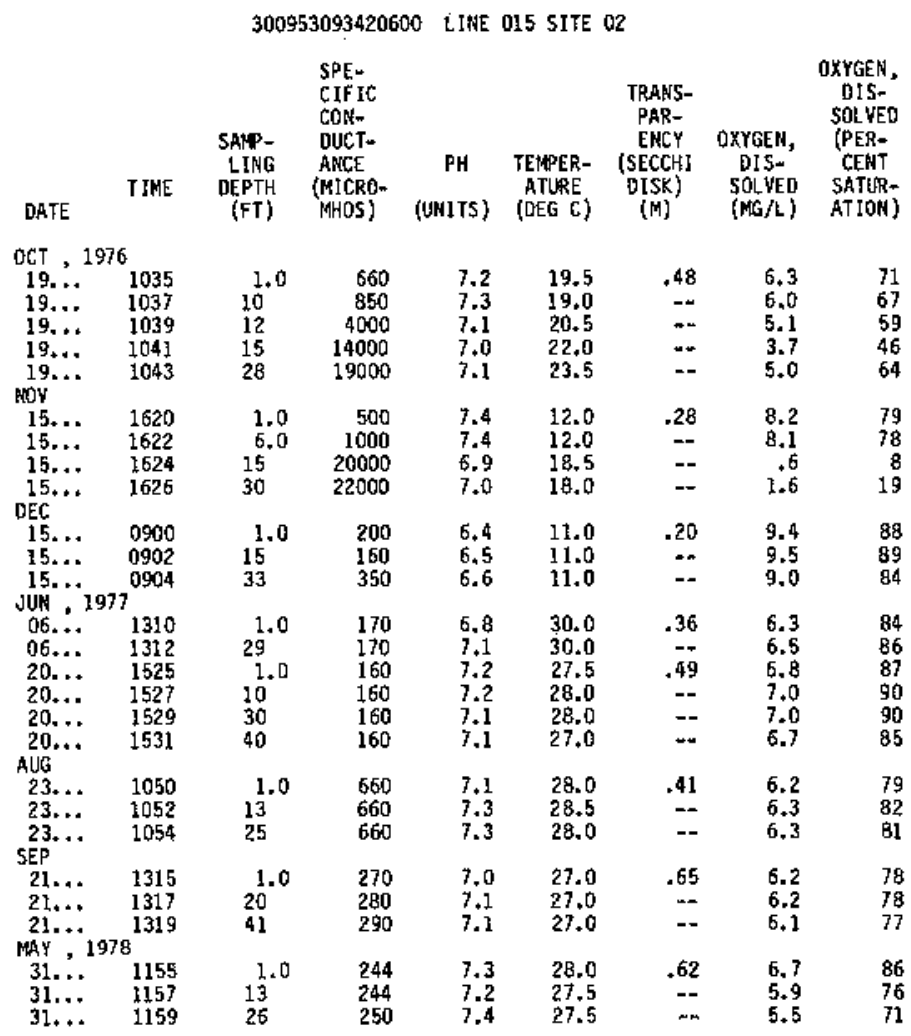

300252093433000 LINE 055 S1TE 02

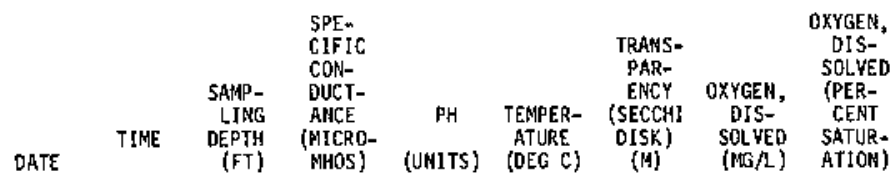

OCT , 1976

$19 . . \quad 1155$

$19 . . . \quad 115$

19... 1201

$15 \ldots .1705$

$\begin{array}{ll}15 . . . & 1705\end{array}$

15... 1709

DEC

15... 0930

5... 0932

$\begin{array}{ll}15 \ldots . & 0934 \\ 15 & 0936\end{array}$

JUN, 1977

$06 . .21415$

$06 . . . \quad 1419$

20... 1610

20... 1612

20... 1614

AUG

$\begin{array}{ll}23 . \ldots . & 1120 \\ 23 . . . & 1122\end{array}$

23... 1124

SEP $\quad 1400$

$\begin{array}{ll}21 \ldots & 1400 \\ 21 \ldots & 1402\end{array}$

21... 1404

21... 1406

21....

07... 1320

$07 . . .1320$

07... 1324

MAY

$\begin{array}{ll}1.0 & 14000 \\ 10 & 20000 \\ 15 & 23000 \\ 30 & 28000\end{array}$

7.6
7.8
7.9
8.0

20.5
20.5
21.0
21.0

$\begin{array}{ccc}.54 & 6.3 & 76 \\ -- & 6.1 & 75 \\ -- & 6.0 & 75\end{array}$

$\begin{array}{ll}1.0 & 15000 \\ 15 & 25000\end{array}$

$7.7 \quad 12.5$

$\begin{array}{ll}15 & 25000 \\ 33 & 34000\end{array}$

8.1

14.0

$\begin{array}{rr}1.0 & 2500 \\ 8.0 & 3000 \\ 15 & 13000\end{array}$

$6.8 \quad 11.5$

$33 \quad 25000$

7.

\begin{tabular}{lr}
1.0 & 5000 \\
15 & 5000 \\
25 & 24000 \\
\hline & 1500
\end{tabular}

$1.0 \quad 1500$

151600

7.4
7.
7.
7.

11.0
12.0

12.0
12.0

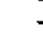

$.61 \quad 8.0$

-- 7.4

.27

27
--

$1.0 \quad 1300$

$\begin{array}{ll}14 & 1200 \\ 29 & 1400\end{array}$

200

$\begin{array}{rr}1.0 & 1200 \\ 5.0 & 1500 \\ 10 & 3000 \\ 20 & 13000\end{array}$

$\begin{array}{ll}10 & 3000 \\ 20 & 13000 \\ 33 & 18000\end{array}$

$\begin{array}{ll}7.4 & 30.0 \\ 7.2 & 29.5\end{array}$

$7.2 \quad 29.5$

$7.2 \quad 28.5$

.55
--

- 3.1

$\begin{array}{lllll}7.2 & 29.0 & .64 & 6.2 & 82 \\ 7.3 & 29.0 & -- & 5.9 & 78\end{array}$

--

6.7

$\begin{array}{rr}31 \ldots . & 1215 \\ 31 \ldots . & 1216\end{array}$

$\begin{array}{cc}1.0 & 240 \\ 16 & 880\end{array}$

$\begin{array}{lr}1.0 & 5000 \\ 19 & 11500 \\ 38 & 20000\end{array}$

7.0
7.0
7.0
7.1

$\begin{array}{ll}7.0 & 28.5 \\ 7.0 & 27.5 \\ 7.0 & 28.0 \\ 7.1 & 28.5 \\ 7.1 & 28.5\end{array}$

6.8
7.1

6.5
6.5
6.5

$\begin{array}{ll}7.5 & 29.5 \\ 6.9 & 29.5 \\ 6.9 & 29 .\end{array}$ 
Table JA,--Duality of water in the Sabine-Neches estuary, water years 1977-78--Continued Field Determinations--Cont inued

295938093465000 LINE 087 SITE D2

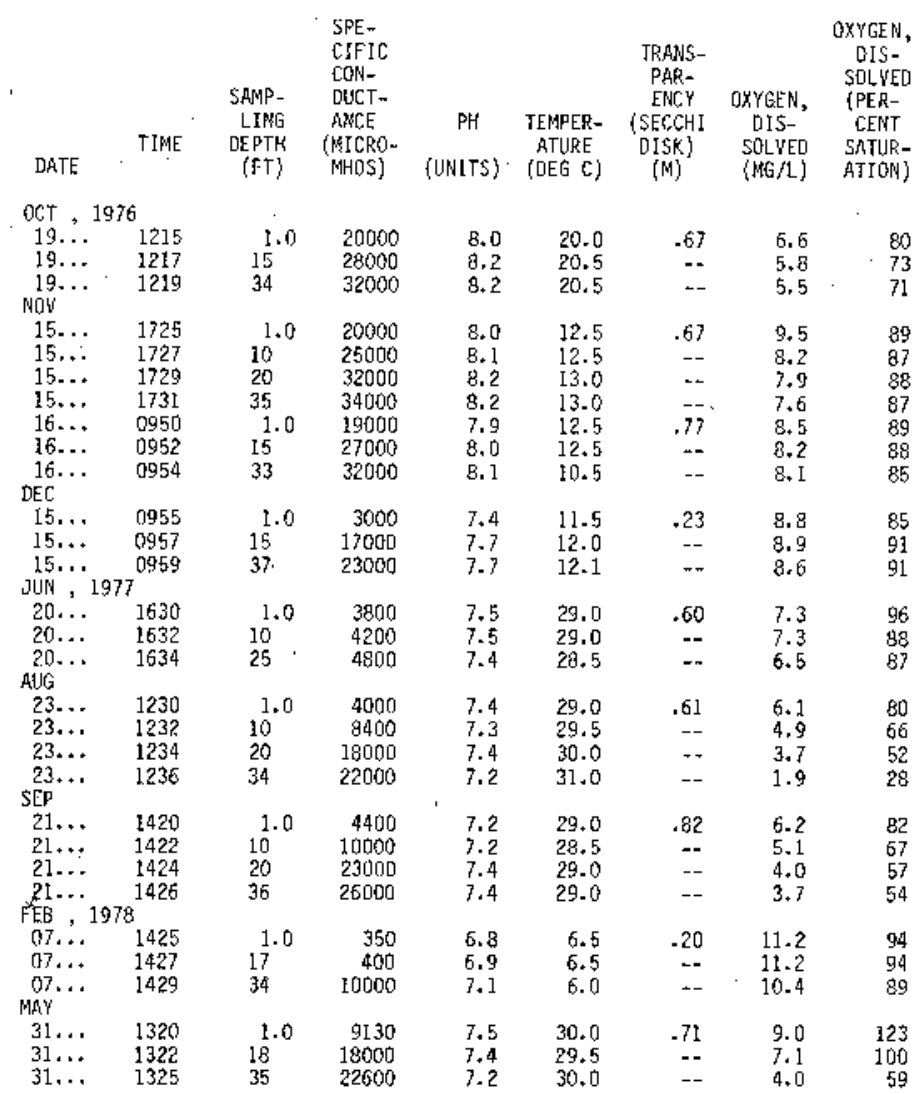

300922094064900 LINE 107 SITE 02

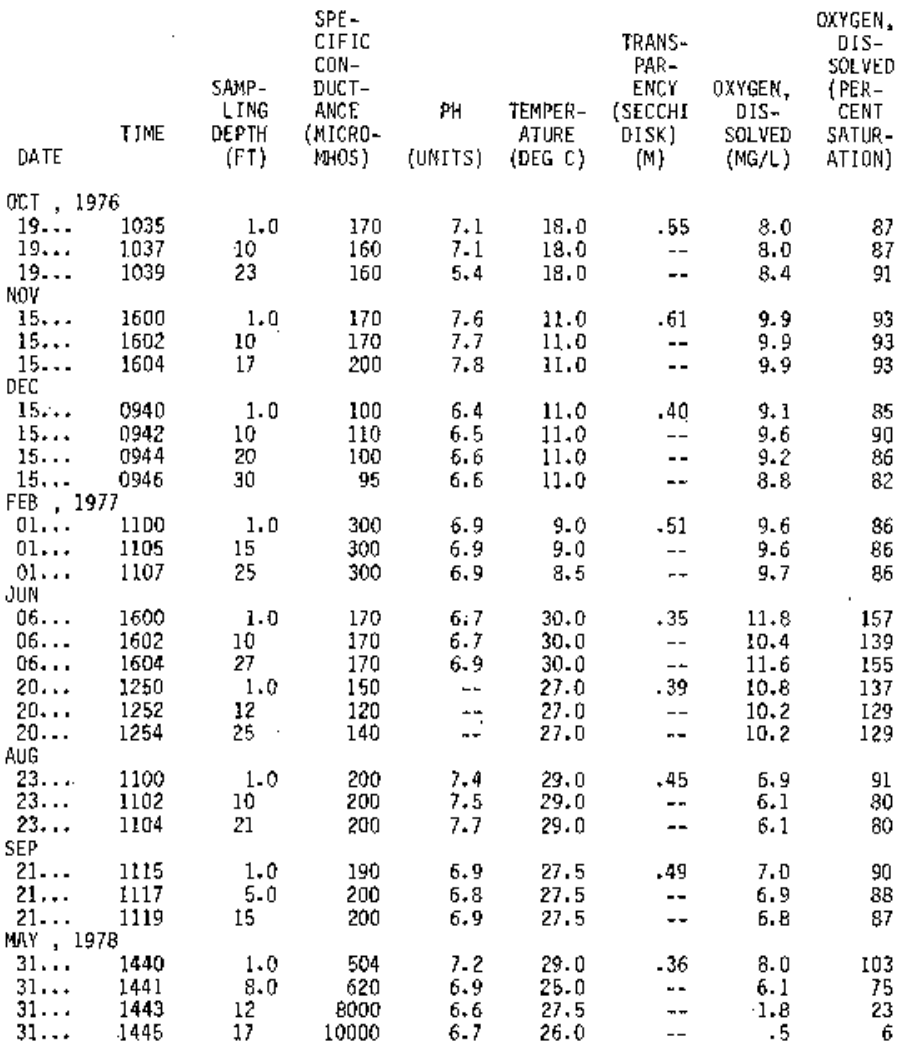


Table 1A...Quality of water in the Sabine-Neches estuary, water years 1977-78--Continued Field Determinations--Cont inued

300618094051300 LIHE 125 SITE 02

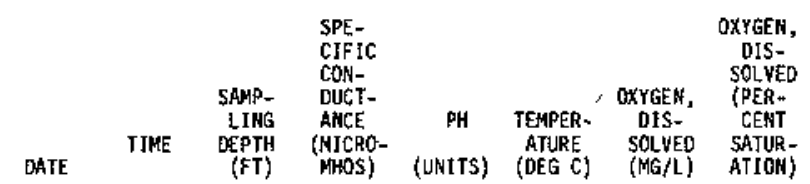

$\begin{array}{cccccccr}\text { MAY }, 1978 & & & & & & \\ 31 \ldots & 1510 & 1.0 & 2000 & 6.9 & 28.5 & 6.6 & 85 \\ 31 \ldots & 1511 & 5.0 & 2000 & 6.8 & 27.0 & 6.0 & 77 \\ 31 \ldots & 1512 & 10 & 9000 & 6.6 & 26.5 & .7 & 9 \\ 31 \ldots & 1513 & 15 & 11000 & 6.6 & 26.5 & .3 & 4 \\ 31 \ldots & 1514 & 25 & 14000 & 6.6 & 26.5 & .3 & 4 \\ 31 \ldots . & 1515 & 35 & 14000 & 6.6 & 27.0 & .3 & 4 \\ 31 \ldots & 1516 & 45 & 14000 & 6.5 & 27.0 & .5 & 6\end{array}$

300319054014600 LINE 170 SITE 02

\begin{tabular}{|c|c|c|c|c|c|c|c|c|}
\hline DATE & TINE & $\begin{array}{c}\text { SAMP- } \\
\text { LIHG } \\
\text { DEPTH } \\
\text { (FT) } \\
(00003)\end{array}$ & $\begin{array}{l}\text { SPE- } \\
\text { CIFIC } \\
\text { CON- } \\
\text { DUCT- } \\
\text { ANCE } \\
\text { (MICRO- } \\
\text { MHOS) } \\
(00095)\end{array}$ & $\begin{array}{c}\text { PH } \\
(\text { INITS }) \\
(00400)\end{array}$ & $\begin{array}{l}\text { TEMPER- } \\
\text { ATURE } \\
\text { (DEG C) } \\
\text { (00010) }\end{array}$ & $\begin{array}{c}\text { TRAPSS- } \\
\text { PAR- } \\
\text { ENCY } \\
\text { (SECCHI } \\
\text { DISK) } \\
(M) \\
(00078)\end{array}$ & $\begin{array}{c}\text { OXYGEN, } \\
\text { DIS- } \\
\text { SOLWED } \\
(N G G / L) \\
(00300)\end{array}$ & $\begin{array}{c}\text { OXYGEN, } \\
\text { DIS- } \\
\text { SOLVED } \\
\text { (PER- } \\
\text { CENT } \\
\text { SATUR- } \\
\text { AT ION) } \\
\text { (OO3O1) }\end{array}$ \\
\hline $\begin{array}{l}\text { OCT , } 1 \\
19 . . . \\
19 \ldots \\
19 . . . \\
19 . . \\
\text { NOY }\end{array}$ & $\begin{array}{l}6 \\
1115 \\
1117 \\
1119 \\
1121\end{array}$ & $\begin{array}{l}1.0 \\
10 \\
20 \\
40\end{array}$ & $\begin{array}{r}3000 \\
12000 \\
22000 \\
27000\end{array}$ & $\begin{array}{l}7.5 \\
7.5 \\
7.6 \\
7.8\end{array}$ & $\begin{array}{l}21.5 \\
22.0 \\
23.0 \\
23.0\end{array}$ & $\begin{array}{c}.43 \\
-. \\
-- \\
--\end{array}$ & $\begin{array}{l}6.4 \\
4.8 \\
3.8 \\
4.6\end{array}$ & $\begin{array}{l}75 \\
59 \\
49 \\
60\end{array}$ \\
\hline $\begin{array}{l}15 \ldots \\
15 \ldots \\
15 \ldots \\
15 \ldots \\
\text { DEC }\end{array}$ & $\begin{array}{l}1630 \\
1632 \\
1634 \\
1636\end{array}$ & $\begin{array}{l}1.0 \\
10 \\
20 \\
40\end{array}$ & $\begin{array}{l}7000 \\
14000 \\
27000 \\
34000\end{array}$ & $\begin{array}{l}7.3 \\
7.3 \\
7.4 \\
7.8\end{array}$ & $\begin{array}{l}12.5 \\
16.0 \\
16.0 \\
15.0\end{array}$ & $\begin{array}{c}.64 \\
-- \\
--\end{array}$ & $\begin{array}{l}8.2 \\
6.4 \\
4.3 \\
6.9\end{array}$ & $\begin{array}{l}82 \\
70 \\
50 \\
81\end{array}$ \\
\hline $\begin{array}{l}15 \ldots \\
15 \ldots \\
15 \ldots \\
15 \ldots \\
15 \ldots \\
15 \ldots \\
\text { FEB... }\end{array}$ & $\begin{array}{l}0835 \\
0937 \\
0839 \\
0841 \\
0843 \\
0845\end{array}$ & $\begin{array}{l}1.0 \\
10 \\
20 \\
25 \\
30 \\
43\end{array}$ & $\begin{array}{r}2000 \\
2000 \\
6000 \\
15000 \\
23000 \\
28000\end{array}$ & $\begin{array}{l}6.8 \\
6.9 \\
7.0 \\
7.4 \\
7.6 \\
7.8\end{array}$ & $\begin{array}{l}11.5 \\
11.5 \\
12.5 \\
12.5 \\
13.0 \\
12.0\end{array}$ & $\begin{array}{c}49 \\
-- \\
=- \\
=- \\
= \\
=\end{array}$ & $\begin{array}{l}8.0 \\
7.8 \\
7.5 \\
6.3 \\
5.7 \\
5.7\end{array}$ & $\begin{array}{l}76 \\
74 \\
74 \\
64 \\
61 \\
61\end{array}$ \\
\hline $\begin{array}{l}01 \ldots . \\
01 \ldots \\
01 \ldots \\
01 \ldots \\
01 \ldots \\
01 \ldots \\
\text { Ju…. }\end{array}$ & $\begin{array}{l}1130 \\
1132 \\
1134 \\
1136 \\
1138 \\
1140\end{array}$ & $\begin{array}{l}1.0 \\
10 \\
20 \\
25 \\
30 \\
47\end{array}$ & $\begin{array}{r}2900 \\
3200 \\
13000 \\
21000 \\
25000 \\
3 \$ 000\end{array}$ & $\begin{array}{l}7.1 \\
7.2 \\
7.4 \\
7.6 \\
7.8 \\
7.9\end{array}$ & $\begin{array}{r}10.0 \\
9.5 \\
9.0 \\
9.0 \\
9.5 \\
9.5\end{array}$ & $\begin{array}{l}.51 \\
-- \\
-- \\
-- \\
-- \\
--\end{array}$ & $\begin{array}{l}9.4 \\
9.3 \\
9.8 \\
8.4 \\
8.0 \\
7.7\end{array}$ & $\begin{array}{l}87 \\
85 \\
82 \\
82 \\
80 \\
79\end{array}$ \\
\hline $\begin{array}{l}06 \ldots \\
06 \ldots \\
06 \ldots \\
20 \ldots \\
20 \ldots \\
20 \ldots \\
20 \ldots \\
20 \ldots \\
\text { AUG }\end{array}$ & $\begin{array}{l}1515 \\
1517 \\
1519 \\
1225 \\
1227 \\
1229 \\
1231 \\
1233\end{array}$ & $\begin{array}{l}1.0 \\
20 \\
45 \\
1.0 \\
10 \\
20 \\
35 \\
47\end{array}$ & $\begin{array}{r}5000 \\
22000 \\
30000 \\
900 \\
1000 \\
1300 \\
9000 \\
30000\end{array}$ & $\begin{array}{l}7.4 \\
7.1 \\
7.2 \\
-- \\
-. \\
- \\
- \\
-\end{array}$ & $\begin{array}{l}32.5 \\
28.0 \\
28.5 \\
29.0 \\
27.5 \\
27.5 \\
27.5 \\
28.5\end{array}$ & $\begin{array}{l}.48 \\
-- \\
.- \\
.48 \\
- \\
- \\
-+ \\
--\end{array}$ & $\begin{array}{r}10.1 \\
3.7 \\
3.1 \\
10.8 \\
9.6 \\
7.8 \\
6.3 \\
5.6\end{array}$ & $\begin{array}{r}142 \\
53 \\
45 \\
142 \\
123 \\
100 \\
83 \\
81\end{array}$ \\
\hline $\begin{array}{l}23 \ldots \\
23 \ldots \\
23 \ldots \\
23 \ldots \\
\text { SEP }\end{array}$ & $\begin{array}{l}1135 \\
1137 \\
1139 \\
1141\end{array}$ & $\begin{array}{l}1.0 \\
10 \\
20 \\
42\end{array}$ & $\begin{array}{r}3500 \\
6500 \\
16000 \\
24000\end{array}$ & $\begin{array}{l}6.9 \\
6.9 \\
6.7 \\
6.7\end{array}$ & $\begin{array}{l}29.5 \\
30.0 \\
30.0 \\
29.5\end{array}$ & $\begin{array}{c}.53 \\
- \\
-- \\
--\end{array}$ & $\begin{array}{r}5.3 \\
3.4 \\
.0 \\
.5\end{array}$ & $\begin{array}{r}70 \\
46 \\
0 \\
7\end{array}$ \\
\hline $\begin{array}{l}21 \ldots \\
21 \ldots \\
21 \ldots \\
21 \ldots \\
21 \ldots \\
21 \ldots \\
21 \ldots \\
\text { FEB , }\end{array}$ & $\begin{array}{l}1140 \\
1142 \\
1144 \\
1146 \\
1148 \\
1150 \\
1152\end{array}$ & $\begin{array}{l}1.0 \\
5.0 \\
10 \\
15 \\
20 \\
30 \\
44\end{array}$ & $\begin{array}{r}7400 \\
5900 \\
7200 \\
12000 \\
15000 \\
18000 \\
21000\end{array}$ & $\begin{array}{l}6.8 \\
6.9 \\
6.8 \\
6.8 \\
6.9 \\
6.8 \\
6.9\end{array}$ & $\begin{array}{l}32.0 \\
29.0 \\
29.0 \\
29.0 \\
29.0 \\
29.0 \\
29.0\end{array}$ & $\begin{array}{l}.68 \\
-- \\
-- \\
-4 \\
- \\
-- \\
--\end{array}$ & $\begin{array}{l}3.8 \\
3.9 \\
3.2 \\
1.2 \\
1.1 \\
.2 \\
.3\end{array}$ & $\begin{array}{r}53 \\
52 \\
43 \\
16 \\
14 \\
3 \\
4\end{array}$ \\
\hline $\begin{array}{l}07 \ldots \\
07 \ldots \\
07 \ldots \\
07 \ldots\end{array}$ & $\begin{array}{l}1135 \\
1137 \\
1139 \\
1141\end{array}$ & $\begin{array}{l}1.0 \\
10 \\
23 \\
46\end{array}$ & $\begin{array}{l}145 \\
145 \\
200 \\
210\end{array}$ & $\begin{array}{l}6.4 \\
6.4 \\
6.4 \\
6.5\end{array}$ & $\begin{array}{l}7.5 \\
6.5 \\
6.5 \\
6.5\end{array}$ & $\begin{array}{l}.25 \\
-- \\
--\end{array}$ & $\begin{array}{l}11.6 \\
11.9 \\
11.9 \\
11.9\end{array}$ & $\begin{array}{l}97 \\
91 \\
94 \\
97\end{array}$ \\
\hline $\begin{array}{l}\text { MAY } \\
31 \ldots \\
31 \ldots \\
31 \ldots\end{array}$ & $\begin{array}{l}1545 \\
1546 \\
1547\end{array}$ & $\begin{array}{l}1.0 \\
20 \\
42\end{array}$ & $\begin{array}{l}11000 \\
15000 \\
19000\end{array}$ & $\begin{array}{l}6.0 \\
6.7 \\
6.8\end{array}$ & $\begin{array}{l}28.5 \\
28.5 \\
28.5\end{array}$ & $\begin{array}{l}-- \\
--\end{array}$ & $\begin{array}{l}4.3 \\
1.8 \\
1.8\end{array}$ & $\begin{array}{l}58 \\
25 \\
25\end{array}$ \\
\hline
\end{tabular}


Table 1A, -Qual ity of water in the Sabine-Pleches estuary, water years 1977-78--Continued Field Determinations--Cont inued

295842093514900 LINE 214 SITE O2

\begin{tabular}{|c|c|c|c|c|c|c|c|c|}
\hline OATE & TIME & $\begin{array}{l}\text { SAMP- } \\
\text { LING } \\
\text { DEPTH } \\
(F T)\end{array}$ & $\begin{array}{l}\text { SPE- } \\
\text { CIFIC } \\
\text { CON- } \\
\text { DUCT- } \\
\text { ANCE } \\
\text { (MICRQ- } \\
\text { MHOS) }\end{array}$ & $\begin{array}{c}\text { PH } \\
\text { (UNITS) }\end{array}$ & $\begin{array}{l}\text { TEMPER- } \\
\text { ATURE } \\
\text { (DEG C) }\end{array}$ & $\begin{array}{c}\text { TRANS- } \\
\text { PAR- } \\
\text { ENCY } \\
\text { (SECCHI } \\
\text { OISK) } \\
\text { (M). }\end{array}$ & $\begin{array}{c}\text { OXYGEN, } \\
\text { DIS- } \\
\text { SOLVED } \\
\text { (MG } / L)\end{array}$ & $\begin{array}{c}\text { OXYGEN, } \\
\text { DIS- } \\
\text { SOLVED } \\
\text { (PER- } \\
\text { CENT } \\
\text { SATUR- } \\
\text { ATIDS) }\end{array}$ \\
\hline \multicolumn{9}{|c|}{ OCT, 1976} \\
\hline $\begin{array}{l}19 \ldots \\
19 \ldots \\
19 \ldots \\
\text { Nov }\end{array}$ & $\begin{array}{l}1155 \\
1157 \\
1159\end{array}$ & $\begin{array}{l}1.0 \\
20 \\
40\end{array}$ & $\begin{array}{l}19000 \\
31000 \\
34000\end{array}$ & $\begin{array}{l}8.3 \\
8.5 \\
8.4\end{array}$ & $\begin{array}{l}22.5 \\
22.0 \\
22.0\end{array}$ & $\begin{array}{r}.64 \\
-- \\
--\end{array}$ & $\begin{array}{l}6.3 \\
6.3 \\
6.6\end{array}$ & $\begin{array}{l}80 \\
84 \\
88\end{array}$ \\
\hline $\begin{array}{l}16 \ldots \\
16 \ldots \\
16 \ldots \\
0 E C\end{array}$ & $\begin{array}{l}1215 \\
1217 \\
1219\end{array}$ & $\begin{array}{l}1.0 \\
15 \\
35\end{array}$ & $\begin{array}{l}23000 \\
31000 \\
25000\end{array}$ & $\begin{array}{l}7.9 \\
8.0 \\
8.1\end{array}$ & $\begin{array}{l}12.5 \\
12.5 \\
12.0\end{array}$ & $\begin{array}{c}.55 \\
- \\
--\end{array}$ & $\begin{array}{l}7.7 \\
7.6 \\
8.4\end{array}$ & $\begin{array}{l}81 \\
83 \\
88\end{array}$ \\
\hline $\begin{array}{l}15 \ldots \\
15 \ldots \\
15 \ldots \\
15 \ldots \\
15 \ldots \\
15 \ldots \\
\text { FEB }\end{array}$ & $\begin{array}{l}1110 \\
1112 \\
1114 \\
1116 \\
1118 \\
1120 \\
7\end{array}$ & $\begin{array}{l}1.0 \\
10 \\
20 \\
25 \\
30 \\
44\end{array}$ & $\begin{array}{r}7000 \\
10000 \\
20000 \\
21000 \\
23000 \\
30000\end{array}$ & $\begin{array}{l}7.2 \\
7.4 \\
7.8 \\
7.9 \\
8.0 \\
8.1\end{array}$ & $\begin{array}{l}13.0 \\
13.0 \\
13.5 \\
13.5 \\
13.0 \\
12.5\end{array}$ & $\begin{array}{c}.49 \\
-- \\
-- \\
-- \\
-- \\
--\end{array}$ & $\begin{array}{l}8.2 \\
8.2 \\
8.0 \\
8.1 \\
8.4 \\
8.4\end{array}$ & $\begin{array}{l}82 \\
83 \\
85 \\
87 \\
89 \\
91\end{array}$ \\
\hline $01 \ldots$ & 1300 & 1.0 & 13000 & 7.5 & 9.5 & .56 & 9.1 & 86 \\
\hline $0 \mathrm{I}+\ldots$ & 1302 & 10 & 15000 & & 10.0 & -- & 9.0 & 86 \\
\hline $01 \ldots$ & 1304 & 20 & 18000 & 7.3 & 10.0 & $\cdots$ & 8. 9 & 86 \\
\hline $\begin{array}{l}01 \% \cdot \cdots \\
\text { jus }\end{array}$ & \\
\hline $06 \ldots$ & 1440 & 1.0 & 14000 & 7.3 & 31,0 & .50 & 7.9 & 112 \\
\hline $06 \ldots$ & 1442 & 20 & 26000 & 7.6 & 29. & - & 5.3 & $\begin{array}{r}78 \\
.58\end{array}$ \\
\hline $07 \ldots$ & $\begin{array}{l}1444 \\
1000\end{array}$ & $\begin{array}{l}44 \\
1.0\end{array}$ & $\begin{array}{l}36000 \\
14000\end{array}$ & $\begin{array}{l}7.7 \\
7.2\end{array}$ & $\begin{array}{l}29.0 \\
29.0\end{array}$ &.$--\bar{~}$ & $\begin{array}{l}3.8 \\
6.6\end{array}$ & $\begin{array}{l}58 \\
91\end{array}$ \\
\hline $07 \ldots$ & 1002 & 20 & 19000 & 7.2 & 29. & -- & 5.1 & 72 \\
\hline $07 .$. & 1004 & 40 & 26000 & 7.2 & 28.5 & -- & 4.0 & .58 \\
\hline $20 \ldots$ & 1430 & 1.0 & 5900 & -- & 29.5 & .57 & 7.3 & 99 \\
\hline $20 .$. & 1432 & 20 & 7000 & -- & 28. & -- & 7. & 101 \\
\hline $20 .$. & 1434 & 45 & 17000 & $\cdots$ & 29. & 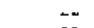 & 7. & 100 \\
\hline 2].. & 0830 & 1.0 & 5000 & -- & 29.0 & .60 & 8.1 & 108 \\
\hline $21 .$. & 0832 & 20 & 5000 & -- & 28 & -- & 5.4 & 71 \\
\hline $\begin{array}{l}21, . \\
A \| G\end{array}$ & \multicolumn{7}{|c|}{ AU, } & 86 \\
\hline 23.. & 1215. & 1.0 & 14000 & 7.1 & 30.0 & .50 & 4.0 & 56 \\
\hline 23.. & $1217^{\circ}$ & 10 & 18000 & 7.1 & 30. & -- & 3.2 & 45 \\
\hline $23 .$. & 1219 & 20 & 22000 & 7.1 & 29. & -- & 2.9 & 41 \\
\hline \\
\hline $21 \ldots$ & 1220 & 1.0 & 15000 & 7.3 & 30.5 & .64 & 4.5 & 63 \\
\hline 21 . & 1222 & 5.0 & 16000 & 7.3 & 30. & -- & 4. & 56 \\
\hline 21. & 1224 & 10 & 19000 & 7.3 & 30 & -- & 3. & 52 \\
\hline 21. & 1226 & 20 & 24000 & 7.5 & 29. & -- & 4. & 58 \\
\hline 21. & 1228 & 30 & 27000 & 7. & 29. & -- & 4. & 58 \\
\hline 21 . & 1230 & 44 & 31000 & 7.7 & & -- & 3. & 55 \\
\hline 21. & 1440 & 1.0 & 14000 & 7.3 & 30. & -- & 4.9 & 69 \\
\hline 21. & 1442 & 10 & 16000 & 7. & 29. & $-m$ & 3. & 55 \\
\hline 21. & 1444 & 20 & 23000 & 7. & 29. & -- & 3. & 55 \\
\hline 21. & 1446 & 44 & 30000 & 7. & 29. & -- & 3.8 & 55 \\
\hline 22. & 1220 & 1.0 & 12000 & & & .70 & 5.1 & 72 \\
\hline 22. & 1221 & 10 & 16000 & 7. & 30. & $=$ & 4.3 & 61 \\
\hline 22. & 122 & 20 & 20000 & 7. & 30 & -- & 4. & 61 \\
\hline $22 \cdots$ & $8^{1223}$ & 44 & 29000 & & & $-\cdots$ & 3.9 & 58 \\
\hline $07 \ldots$ & 1235 & 1.0 & 1800 & 7.0 & 6.5 & .19 & 10.9 & 92 \\
\hline & 1237 & & 3300 & $7 . ?$ & 6. & $=$ & 10 & 92 \\
\hline & 1239 & 41 & 16000 & & & - & 10. & 92 \\
\hline 08. & 1105 & 1.0 & 2100 & 8. & 6. & -- & 11. & 96 \\
\hline 08 . & 1110 & 22 & 17000 & 8. & 6. & -- & 10 & 96 \\
\hline QQBAY & & 44 & 22000 & & & -- & & 108 \\
\hline $31 \ldots$ & 1640 & 1.0 & 17300 & \multicolumn{4}{|c|}{ WAAY } & 83 \\
\hline & I 643 & 23 & 21000 & & & -- & 4.5 & 64 \\
\hline 31. & 1645 & 46 & - & 7.2 & 30.5 & 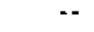 & -- & $=$ \\
\hline \multicolumn{9}{|l|}{ Juy } \\
\hline $01 \ldots$ & 0831 & 20 & 22000 & 7.5 & 29. & - & 4.8 & 68 \\
\hline & 0832 & 40 & 19000 & 7.5 & 29.0 & $\ldots$ & 4.2 & 59 \\
\hline & 1330 & 1.0 & 18000 & 7.4 & 30.5 & $-r$ & 5.6 & 80 \\
\hline & 1333 & 19 & 20000 & 7.3 & 30.0 & -r & 4.8 & 68 \\
\hline & 1335 & & 21000 & 7.4 & 30.0 & - & 4.4 & \\
\hline
\end{tabular}


Table 1A.--Qual ity of water in the Sabine-Neches estuary, water years 1977-78--Continued Field Determinations--Continued

\begin{tabular}{|c|c|c|c|c|c|c|c|c|}
\hline & & 29573 & 36093454600 & DO LINE & 244 SITE & 01 & & \\
\hline DATE & TIME & $\begin{array}{c}\text { SAMP- } \\
\text { LING } \\
\text { DEPTH } \\
\text { (FT) }\end{array}$ & $\begin{array}{l}\text { SPE- } \\
\text { CIFIC. } \\
\text { CON- } \\
\text { DUCT- } \\
\text { AHCE } \\
\text { (HICRO- } \\
\text { MHOS) }\end{array}$ & $\begin{array}{c}\text { PH } \\
\text { (UNITS) }\end{array}$ & $\begin{array}{c}\text { TEMPER- } \\
\text { ATURE } \\
\text { (DEG C) }\end{array}$ & $\begin{array}{l}\text { TRANS- } \\
\text { PAR- } \\
\text { ERCY } \\
\text { (SECCHI } \\
\text { OISK) } \\
\text { (M) }\end{array}$ & $\begin{array}{c}\text { OXYGEN, } \\
\text { OIS- } \\
\text { SOLVED } \\
\text { (MG/L) }\end{array}$ & $\begin{array}{c}\text { OXYGEN, } \\
\text { DIS- } \\
\text { SOLVED } \\
\text { (PER- } \\
\text { CENT } \\
\text { SATUR- } \\
\text { ATION) }\end{array}$ \\
\hline OCT , I & & & & & & & & \\
\hline $\begin{array}{l}18 \ldots \\
18 \ldots \\
\text { nov }\end{array}$ & $\begin{array}{l}1615 \\
1617\end{array}$ & $\begin{array}{l}1.0 \\
5.5\end{array}$ & $\begin{array}{l}15000 \\
19000\end{array}$ & $\begin{array}{l}8.2 \\
7.9\end{array}$ & $\begin{array}{l}19.0 \\
19.0\end{array}$ & $\begin{array}{l}.89 \\
--.\end{array}$ & $\begin{array}{l}6.7 \\
6.2\end{array}$ & $\begin{array}{l}78 \\
74\end{array}$ \\
\hline $\begin{array}{l}16 \ldots \\
16 \ldots \\
D E C\end{array}$ & $\begin{array}{l}1010 \\
1012\end{array}$ & $\begin{array}{l}1.0 \\
6.0\end{array}$ & $\begin{array}{l}19000 \\
19000\end{array}$ & $\begin{array}{l}7.8 \\
7.8\end{array}$ & $\begin{array}{l}8.5 \\
9.0\end{array}$ & $\begin{array}{r}1.46 \\
=-\end{array}$ & $\begin{array}{l}10.1 \\
10.1\end{array}$ & $\begin{array}{l}96 \\
97\end{array}$ \\
\hline $\begin{array}{l}15 \ldots \\
15 \ldots\end{array}$ & $\begin{array}{l}1050 \\
1052\end{array}$ & $\begin{array}{l}1.0 \\
7.0\end{array}$ & $\begin{array}{l}14000 \\
14000\end{array}$ & $\begin{array}{l}7.5 \\
7.5\end{array}$ & $\begin{array}{l}11.0 \\
11.0\end{array}$ & $\begin{array}{l}.99 \\
--\end{array}$ & $\begin{array}{l}9.8 \\
9.8\end{array}$ & $\begin{array}{l}97 \\
97\end{array}$ \\
\hline JUN , 1 & & & & & & & & \\
\hline $\begin{array}{l}07 \ldots \\
07 \ldots \\
21 \ldots \\
21 \ldots\end{array}$ & $\begin{array}{l}1055 \\
1057 \\
0920 \\
0922\end{array}$ & $\begin{array}{l}1.0 \\
6.0 \\
1.0 \\
5.0\end{array}$ & $\begin{array}{r}10000 \\
12000 \\
5800 \\
6000\end{array}$ & $\begin{array}{l}7.7 \\
7.7 \\
7.5 \\
7.5\end{array}$ & $\begin{array}{l}29.0 \\
28.5 \\
28.5 \\
28.5\end{array}$ & $\begin{array}{r}.45 \\
-. \\
.53 \\
--\end{array}$ & $\begin{array}{l}8.4 \\
8.0 \\
6.2 \\
5.8\end{array}$ & $\begin{array}{r}113 \\
108 \\
83 \\
78\end{array}$ \\
\hline $\begin{array}{c}23 \ldots \\
23 \ldots \\
5 E p\end{array}$ & $\begin{array}{l}1345 \\
1347\end{array}$ & $\begin{array}{l}1.0 \\
6.0\end{array}$ & $\begin{array}{l}7600 \\
7700\end{array}$ & $\begin{array}{l}7.8 \\
7.7\end{array}$ & $\begin{array}{l}28.5 \\
28.5\end{array}$ & .56 & $\begin{array}{l}9.1 \\
7.9\end{array}$ & $\begin{array}{l}121 \\
105\end{array}$ \\
\hline $\begin{array}{l}22 \ldots \\
22 \ldots \\
22 \ldots \\
22 \ldots \\
22 \ldots \\
22 \ldots\end{array}$ & $\begin{array}{l}1145 \\
1146 \\
1147 \\
1148 \\
1215 \\
1216\end{array}$ & $\begin{array}{l}1.0 \\
4.0 \\
5.0 \\
6.0 \\
1.0 \\
3.0\end{array}$ & $\begin{array}{r}9700 \\
9800 \\
10000 \\
11000 \\
15000 \\
16000\end{array}$ & $\begin{array}{l}7.9 \\
8.0 \\
7.5 \\
7.3 \\
7.9 \\
7.9\end{array}$ & $\begin{array}{l}29.5 \\
28.0 \\
28.0 \\
28.0 \\
30.5 \\
30.5\end{array}$ & $\begin{array}{c}1.04 \\
-- \\
-- \\
-- \\
.75 \\
-\end{array}$ & $\begin{array}{l}3.5 \\
3.7 \\
2.7 \\
1.7 \\
7.2 \\
7.6\end{array}$ & $\begin{array}{r}47 \\
49 \\
36 \\
23 \\
101 \\
107\end{array}$ \\
\hline JUN , 1 & & & & & & & & \\
\hline $\begin{array}{l}01 \ldots \\
01 \ldots\end{array}$ & $\begin{array}{l}0920 \\
0921\end{array}$ & $\begin{array}{l}1.0 \\
5.0\end{array}$ & $\begin{array}{l}13000 \\
16000\end{array}$ & $\begin{array}{l}7.6 \\
7.4\end{array}$ & $\begin{array}{l}29.5 \\
28.5\end{array}$ & $\begin{array}{l}.85 \\
--\end{array}$ & $\begin{array}{l}9.9 \\
7.4\end{array}$ & $\begin{array}{l}122 \\
103\end{array}$ \\
\hline & & 2957 & 28093464500 & LIHE & 244 SITE & Q2 & & \\
\hline DATE & TIME & $\begin{array}{l}\text { SAMP. } \\
\text { LING } \\
\text { DEPTH } \\
(F T)\end{array}$ & $\begin{array}{l}\text { SPE- } \\
\text { CIFIC } \\
\text { CON- } \\
\text { DUCT- } \\
\text { ANCE } \\
\text { (MICRO- } \\
\text { MHOS) }\end{array}$ & $\begin{array}{c}\mathrm{PH} \\
\text { (UNNITS) }\end{array}$ & $\begin{array}{l}\text { TEMPER- } \\
\text { ATURE } \\
\text { (DEG C) }\end{array}$ & $\begin{array}{c}\text { TRANS- } \\
\text { PAR- } \\
\text { ENCY } \\
\text { (SECCHI } \\
\text { DISK) } \\
(M)\end{array}$ & $\begin{array}{l} \\
\text { OXYGEN, } \\
\text { DIS- } \\
\text { SOLVED } \\
\text { (MG } / L)\end{array}$ & $\begin{array}{c}\text { OXYGEN, } \\
\text { OIS- } \\
\text { SOLYED } \\
\text { (PER- } \\
\text { CENT } \\
\text { SATUR- } \\
\text { ATION) }\end{array}$ \\
\hline oc $\top, 1$ & & & & & & & & \\
\hline $\begin{array}{l}18 \ldots \\
18 \ldots \\
\text { nov }\end{array}$ & $\begin{array}{l}1525 \\
1527\end{array}$ & $\begin{array}{l}1.0 \\
7.5\end{array}$ & $\begin{array}{l}17000 \\
17000\end{array}$ & $\begin{array}{l}8.2 \\
8.2\end{array}$ & $\begin{array}{r}19.5 \\
18.5\end{array}$ & $\begin{array}{r}1.14 \\
--\end{array}$ & $\begin{array}{l}7.7 \\
7.4\end{array}$ & $\begin{array}{l}92 \\
86\end{array}$ \\
\hline $\begin{array}{l}16 \ldots \\
16 \ldots \\
D E C\end{array}$ & $\begin{array}{l}1020 \\
1022\end{array}$ & $\begin{array}{l}1.0 \\
.7 .0\end{array}$ & $\begin{array}{l}22000 \\
22000\end{array}$ & $\begin{array}{l}7.9 \\
7.9\end{array}$ & $\begin{array}{l}9.5 \\
9.0\end{array}$ & $\begin{array}{r}1.84 \\
.-.\end{array}$ & $\begin{array}{r}9.7 \\
10.1\end{array}$ & $\begin{array}{l}96 \\
98\end{array}$ \\
\hline $\begin{array}{l}15 \ldots \\
15 \ldots \\
15 \ldots \\
\text { dISN..1 }\end{array}$ & $\begin{array}{l}1045 \\
1047 \\
1049 \\
7\end{array}$ & $\begin{array}{l}1.0 \\
3.0 \\
7.0\end{array}$ & $\begin{array}{r}9500 \\
9500 \\
13000\end{array}$ & $\begin{array}{l}7.3 \\
7.3 \\
7.1\end{array}$ & $\begin{array}{l}11.0 \\
11.0 \\
11.5\end{array}$ & $\begin{array}{r}.43 \\
-- \\
--\end{array}$ & $\begin{array}{l}9.1 \\
9.1 \\
8.3\end{array}$ & $\begin{array}{l}88 \\
88 \\
82\end{array}$ \\
\hline $\begin{array}{l}07 \ldots \\
07 \ldots \\
21 \ldots \\
21 \ldots \\
\text { AUG }\end{array}$ & $\begin{array}{l}1045 \\
1047 \\
0915 \\
0917\end{array}$ & $\begin{array}{l}1.0 \\
7.0 \\
1.0 \\
6.0\end{array}$ & $\begin{array}{r}13000 \\
14000 \\
8600 \\
8600\end{array}$ & $\begin{array}{l}7.7 \\
7.7 \\
7.5 \\
7.5\end{array}$ & $\begin{array}{l}28.5 \\
28.5 \\
29.0 \\
29.0\end{array}$ & $\begin{array}{r}.70 \\
.- \\
.55 \\
--\end{array}$ & $\begin{array}{l}7.8 \\
7.7 \\
6.1 \\
6.0\end{array}$ & $\begin{array}{r}105 \\
105 \\
83 \\
81\end{array}$ \\
\hline $\begin{array}{l}23 \ldots \\
23 \ldots \\
\text { SEP }\end{array}$ & $\begin{array}{l}1335 \\
1337\end{array}$ & $\begin{array}{l}1.0 \\
6.5\end{array}$ & $\begin{array}{l}7800 \\
9400\end{array}$ & $\begin{array}{l}7.8 \\
7.6\end{array}$ & $\begin{array}{l}28.5 \\
28.5\end{array}$ & $\begin{array}{r}.82 \\
--\end{array}$ & $\begin{array}{l}7.2 \\
6.0\end{array}$ & $\begin{array}{l}96 \\
81\end{array}$ \\
\hline $\begin{array}{l}22 \ldots \\
22 \ldots \\
22 \ldots \\
22 \ldots \\
22 \ldots \\
22 \ldots \\
\text { JUN , } 1\end{array}$ & $\begin{array}{l}1150 \\
1152 \\
1154 \\
1205 \\
1206 \\
1207 \\
8\end{array}$ & $\begin{array}{l}1.0 \\
5.0 \\
7.0 \\
1.0 \\
5.0 \\
7.0\end{array}$ & $\begin{array}{r}9600 \\
9700 \\
11000 \\
9600 \\
9600 \\
14000\end{array}$ & $\begin{array}{l}7.9 \\
7.8 \\
7.4 \\
8.0 \\
7.8 \\
7.1\end{array}$ & $\begin{array}{l}28.0 \\
28.0 \\
28.0 \\
29.5 \\
28.0 \\
29.0\end{array}$ & $\begin{array}{r}1.06 \\
= \\
-- \\
.93 \\
-- \\
-\end{array}$ & $\begin{array}{l}7.3 \\
6.6 \\
4.6 \\
8.2 \\
7.7 \\
3.6\end{array}$ & $\begin{array}{r}97 \\
91 \\
62 \\
111 \\
103 \\
50\end{array}$ \\
\hline $01 \ldots$ & $\begin{array}{l}0910 \\
0911\end{array}$ & $\begin{array}{l}1.0 \\
6.0\end{array}$ & $\begin{array}{l}12000 \\
17000\end{array}$ & $\begin{array}{l}7.5 \\
7.2\end{array}$ & $\begin{array}{l}29.0 \\
29.0\end{array}$ & · $\quad=$ & $\begin{array}{l}8.4 \\
5.6\end{array}$ & $\begin{array}{r}114 \\
78\end{array}$ \\
\hline
\end{tabular}


- Table 1A--QQuality of water in the Sabine-Neches estuary, water years $1977-78--$ Continured Field Determinations--Continued

295720093474500 LINE 244 SITE 03

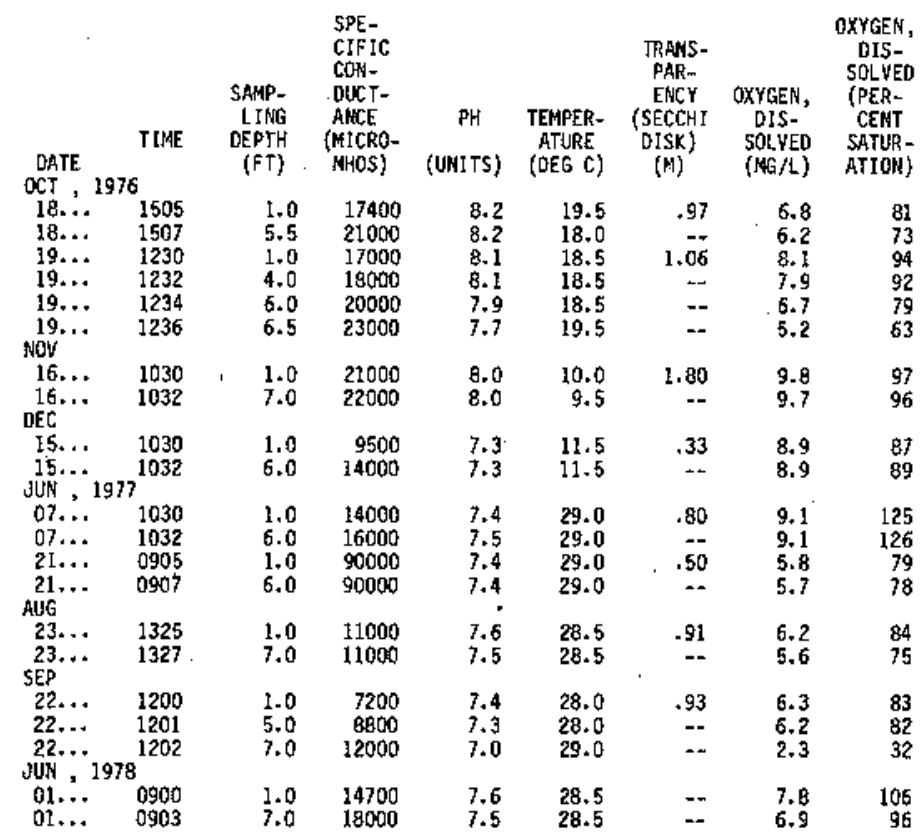

295701093501200 LIRE 244 SITE 04

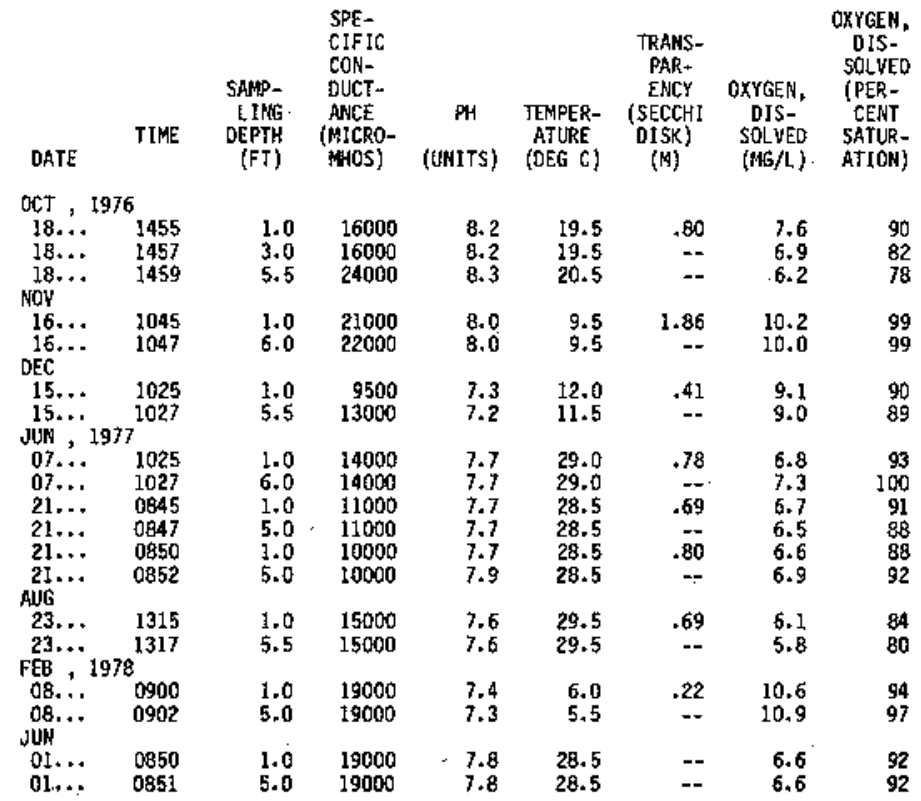


Table 1A,--Quality of water in the Sabine-Heches estuary, water years 1977-78--Continued Field Determinations--Continued

295651093512300 LIHE 244 SITE 05

\begin{tabular}{|c|c|c|c|c|c|c|c|c|}
\hline DATE & TIME & $\begin{array}{c}\text { SAMP- } \\
\text { LJNG } \\
\text { DEPTH } \\
(\mathbf{F r})\end{array}$ & $\begin{array}{l}\text { SPE- } \\
\text { CIFIC } \\
\text { CON- } \\
\text { DUCT- } \\
\text { ANCE } \\
\text { (MICRO- } \\
\text { MFOS) }\end{array}$ & $\begin{array}{c}\text { PH } \\
\text { (UNITS) }\end{array}$ & $\begin{array}{l}\text { TEMPEER- } \\
\text { ATURE } \\
\text { (DEG C) }\end{array}$ & $\begin{array}{l}\text { TRANS- } \\
\text { PAR- } \\
\text { ENCY } \\
\text { (SECCHI } \\
\text { OISK) } \\
\text { (M) }\end{array}$ & $\begin{array}{c}\text { OXYGEHA } \\
\text { DIS- } \\
\text { SOLYED } \\
\text { (MG } / L)\end{array}$ & $\begin{array}{c}\text { OXYGEN, } \\
\text { DIS- } \\
\text { SQLVED } \\
\text { (PER- } \\
\text { CERT } \\
\text { SATUR- } \\
\text { ATIOAE) }\end{array}$ \\
\hline \multicolumn{9}{|c|}{ NOV , 1976} \\
\hline $\begin{array}{l}16 \ldots \\
16 . \cdots \\
\mathrm{DEC}\end{array}$ & $\begin{array}{l}1050 \\
1052\end{array}$ & $\begin{array}{l}1.0 \\
4.0\end{array}$ & $\begin{array}{l}25000 \\
26000\end{array}$ & $\begin{array}{l}8.1 \\
8.1\end{array}$ & $\begin{array}{l}12.0 \\
11.5\end{array}$ & $\begin{array}{r}.79 \\
--\end{array}$ & $\begin{array}{l}8.8 \\
8.6\end{array}$ & $\begin{array}{l}93 \\
91\end{array}$ \\
\hline $\begin{array}{l}15 \ldots \\
15 \ldots \\
\text { JUN }\end{array}$ & $\begin{array}{l}1015 \\
1017 \\
7\end{array}$ & $\begin{array}{l}1.0 \\
4.0\end{array}$ & $\begin{array}{l}8500 \\
8500\end{array}$ & $\begin{array}{l}7.1 \\
7.0\end{array}$ & $\begin{array}{l}12.0 \\
12.0\end{array}$ & $\begin{array}{r}.41 \\
--\end{array}$ & $\begin{array}{l}8.8 \\
9.0\end{array}$ & $\begin{array}{l}87 \\
89\end{array}$ \\
\hline $\begin{array}{l}07 \ldots \\
07 \ldots \\
21 \ldots \\
21 \ldots \\
\text { AUG }\end{array}$ & $\begin{array}{l}1015 \\
1017 \\
0835 \\
0837\end{array}$ & $\begin{array}{l}1.0 \\
3.0 \\
1.0 \\
3.0\end{array}$ & $\begin{array}{r}17000 \\
17000 \\
4900 \\
5000\end{array}$ & $\begin{array}{l}7.6 \\
7.8 \\
7.1 \\
7.1\end{array}$ & $\begin{array}{l}28.5 \\
28.5 \\
28.5 \\
28.5\end{array}$ & $\begin{array}{l}-- \\
-- \\
.52 \\
--\end{array}$ & $\begin{array}{l}7.2 \\
7.4 \\
5.4 \\
5.4\end{array}$ & $\begin{array}{r}95 \\
103 \\
71 \\
77\end{array}$ \\
\hline $\begin{array}{l}23 \ldots \\
23 \ldots \\
\text { JuN }\end{array}$ & $\begin{array}{r}1305 \\
1307 \\
8\end{array}$ & $\begin{array}{l}1.0 \\
4.0\end{array}$ & $\begin{array}{l}16000 \\
18000\end{array}$ & $\begin{array}{l}7.5 \\
7.5\end{array}$ & $\begin{array}{l}30.5 \\
29.0\end{array}$ & $\begin{array}{c}65 \\
-2\end{array}$ & $\begin{array}{l}5.4 \\
4.6\end{array}$ & $\begin{array}{l}73 \\
64\end{array}$ \\
\hline $01 \ldots$ & $\begin{array}{l}0835 \\
0836\end{array}$ & $\begin{array}{l}1.0 \\
4.0\end{array}$ & $\begin{array}{l}20000 \\
20000\end{array}$ & $\begin{array}{l}7.6 \\
7.6\end{array}$ & $\begin{array}{l}29.5 \\
29.0\end{array}$ & -- & $\begin{array}{l}6.1 \\
5.8\end{array}$ & $\begin{array}{l}86 \\
82\end{array}$ \\
\hline
\end{tabular}

295454093460700 LIVE 254 SITE 01

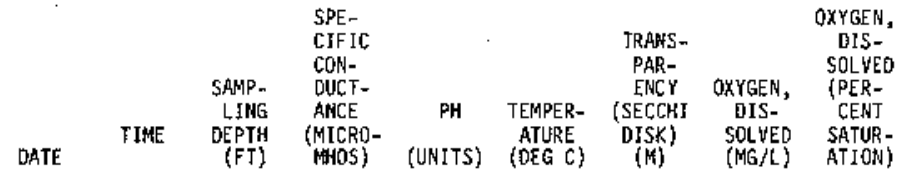

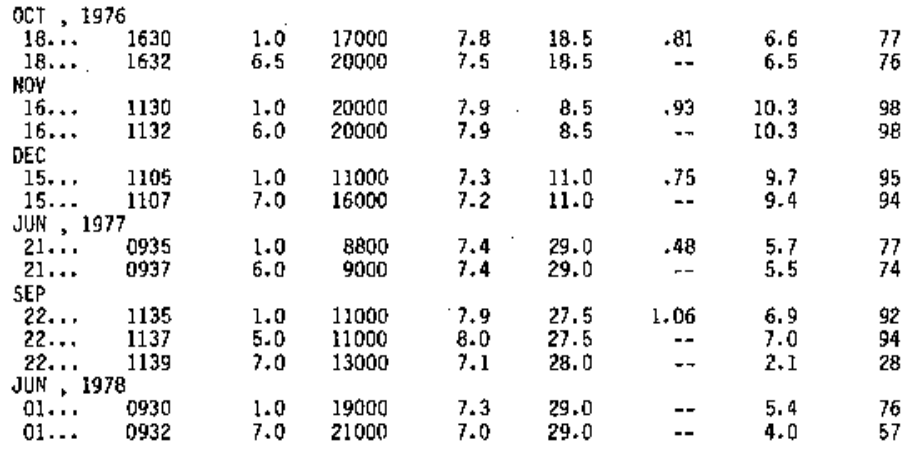

295629093504500 LINE 254 SITE 04

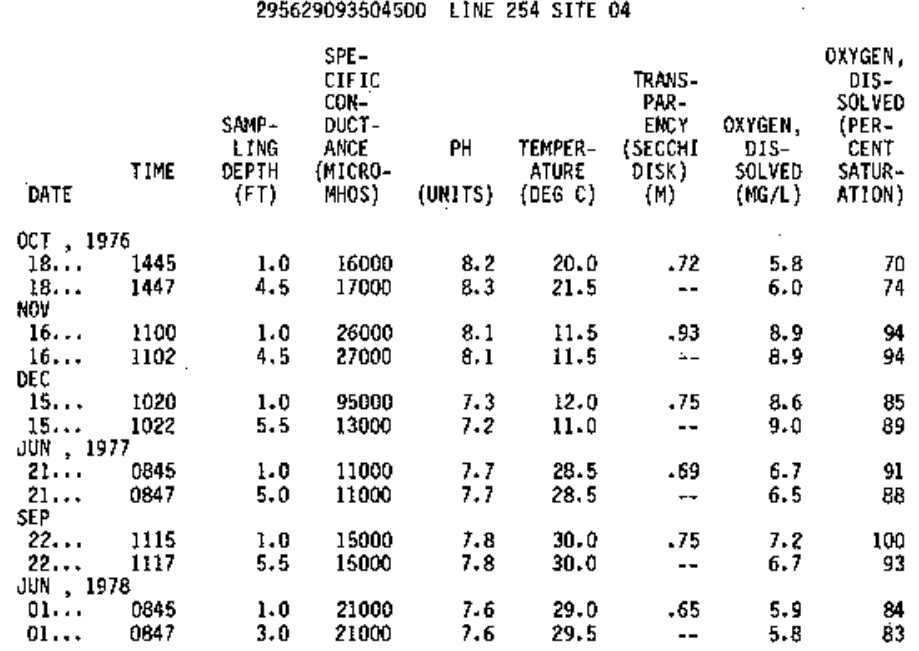


Table 1A.--Quality of water in the Sablne-Heches estuary, water years 1977-78--Continued

Field Determ1nations. - Cont inued

295109093472000 LINE 274 SITE DI

\begin{tabular}{|c|c|c|c|c|c|c|c|c|}
\hline DATE & TIME & $\begin{array}{l}\text { SAAP. } \\
\text { LING } \\
\text { DEPTH } \\
\text { (FT) }\end{array}$ & $\begin{array}{l}\text { SPE. } \\
\text { C1FIC } \\
\text { CON- } \\
\text { DUCT- } \\
\text { AHCE } \\
\text { (PICRO- } \\
\text { MHOS) }\end{array}$ & $\begin{array}{c}\text { PH } \\
\text { (UNITS) }\end{array}$ & $\begin{array}{c}\text { TEMPER- } \\
\text { ATURE } \\
\text { (DEG C) }\end{array}$ & $\begin{array}{l}\text { TRANS- } \\
\text { PAR- } \\
\text { ENCY } \\
\text { (SECCHI } \\
\text { OISK) } \\
(M)\end{array}$ & $\begin{array}{c}\text { OXYGEN, } \\
\text { DIS- } \\
\text { SOLVED } \\
\text { (MG/L) }\end{array}$ & $\begin{array}{c}\text { OXYGEN, } \\
\text { DIS- } \\
\text { SOEVED } \\
\text { (PER- } \\
\text { CENT } \\
\text { SATUR- } \\
\text { ATIOHS) }\end{array}$ \\
\hline $\begin{array}{c}\text { oct , } 1 \\
18 . . . \\
18 . \cdots\end{array}$ & $\begin{array}{l}6 \\
1645 \\
1647\end{array}$ & $\begin{array}{l}1.0 \\
5.0\end{array}$ & $\begin{array}{l}15000 \\
15000\end{array}$ & $\begin{array}{l}7.9 \\
7.7\end{array}$ & $\begin{array}{l}18.5 \\
17.5\end{array}$ & .68 & $\begin{array}{l}6.9 \\
6.7\end{array}$ & $\begin{array}{l}79 \\
76\end{array}$ \\
\hline $\begin{array}{l}16 \ldots \\
16 . . .\end{array}$ & $\begin{array}{l}1220 \\
1222\end{array}$ & $\begin{array}{l}1.0 \\
5.0\end{array}$ & $\begin{array}{l}20000 \\
18000\end{array}$ & $\begin{array}{l}7.9 \\
7.9\end{array}$ & & $\begin{array}{r}.50 \\
--\end{array}$ & $\begin{array}{l}10.4 \\
10.4\end{array}$ & $\begin{array}{l}99 \\
99\end{array}$ \\
\hline $\begin{array}{l}15 \ldots \\
15 \ldots \\
\text { JUN , }\end{array}$ & $\begin{array}{l}1120 \\
7122 \\
7\end{array}$ & $\begin{array}{l}1.0 \\
5.0\end{array}$ & $\begin{array}{l}13000 \\
14000\end{array}$ & $\begin{array}{l}7.5 \\
7.6\end{array}$ & & $\begin{array}{l}.43 \\
--.\end{array}$ & $\begin{array}{r}9.9 \\
10.0\end{array}$ & $\begin{array}{l}97 \\
99\end{array}$ \\
\hline $\begin{array}{l}07 \ldots \\
07 \ldots \\
21 \ldots \\
21 \ldots \\
\text { AUG }\end{array}$ & $\begin{array}{l}1230 \\
1232 \\
0955 \\
0957\end{array}$ & $\begin{array}{l}1.0 \\
6.0 \\
1.0 \\
5.0\end{array}$ & $\begin{array}{r}4000 \\
4000 \\
11000 \\
10000\end{array}$ & $\begin{array}{l}7.3 \\
7.3 \\
7.7 \\
7.6\end{array}$ & $\begin{array}{l}29.5 \\
30.0 \\
29.5 \\
29.0\end{array}$ & $\begin{array}{l}.35 \\
.- \\
.63 \\
--\end{array}$ & $\begin{array}{l}9.4 \\
7.5 \\
6.0 \\
5.7\end{array}$ & $\begin{array}{r}125 \\
109 \\
82 \\
77\end{array}$ \\
\hline $\begin{array}{l}23 . \ldots \\
23 \ldots\end{array}$ & $\begin{array}{l}1415 \\
1417\end{array}$ & $\begin{array}{l}1.0 \\
5.5\end{array}$ & $\begin{array}{l}14000 \\
14000\end{array}$ & $\begin{array}{l}7.6 \\
7.5\end{array}$ & & $\begin{array}{l}.62 \\
--\end{array}$ & $\begin{array}{l}6.4 \\
6.0\end{array}$ & $\begin{array}{l}88 \\
82\end{array}$ \\
\hline $\begin{array}{l}22 \ldots \\
22 \ldots \\
\text { JuN }\end{array}$ & $\begin{array}{l}1030 \\
1032\end{array}$ & $\begin{array}{l}1.0 \\
4.0\end{array}$ & $\begin{array}{l}12000 \\
14000\end{array}$ & $\begin{array}{l}7.2 \\
7.4\end{array}$ & $\begin{array}{l}28.0 \\
28.0\end{array}$ & $\begin{array}{r}.55 \\
--\end{array}$ & $\begin{array}{l}3.9 \\
4.6\end{array}$ & $\begin{array}{l}53 \\
63\end{array}$ \\
\hline $\begin{array}{l}01 \ldots \\
01 \ldots\end{array}$ & $\begin{array}{l}0945 \\
0947\end{array}$ & $\begin{array}{l}1.0 \\
7.0\end{array}$ & $\begin{array}{l}22000 \\
22000\end{array}$ & $\begin{array}{l}7.5 \\
7.4\end{array}$ & $\begin{array}{l}29.0 \\
29.0\end{array}$ & $=$ & $\begin{array}{l}6.4 \\
6.3\end{array}$ & $\begin{array}{l}92 \\
89\end{array}$ \\
\hline
\end{tabular}

295228093492000 LINE 274 SITE 02

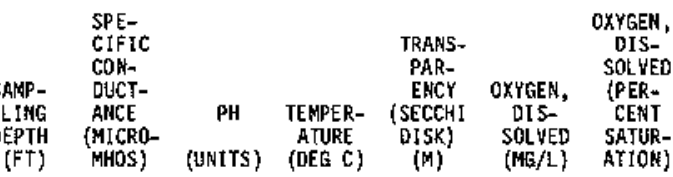

OCT, 1976

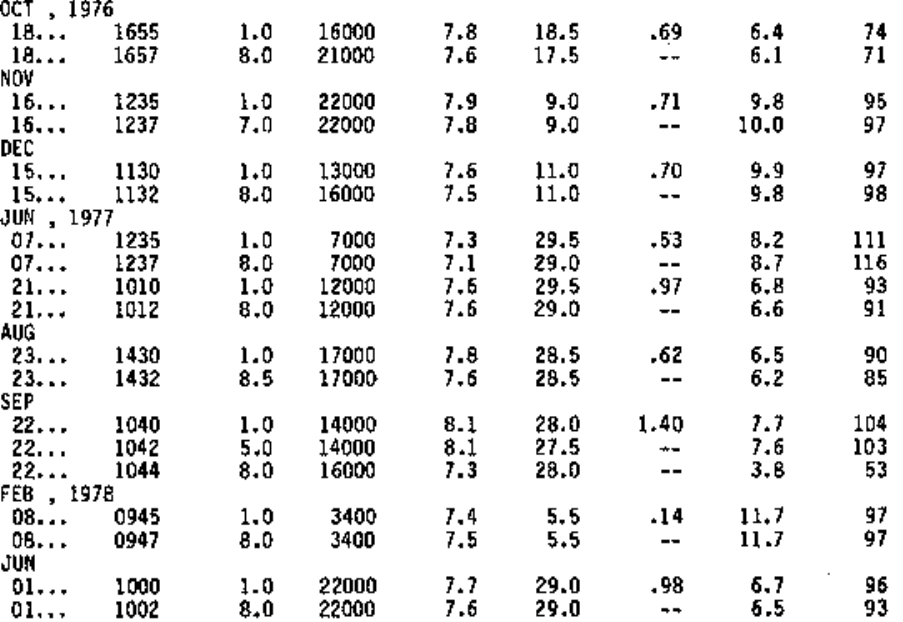


Table IA.-Wualfty of water in the Sabine-fleches estuary, water years 1977-78--Continued Field Determinations--Continued

295329093505500 LISAE 274 SITE 03

\begin{tabular}{|c|c|c|c|c|c|c|c|c|}
\hline DATE & TIME & $\begin{array}{c}\text { SAMP- } \\
\text { LIMG } \\
\text { DEPTH } \\
\text { (FT) }\end{array}$ & $\begin{array}{l}\text { 5PE- } \\
\text { CIFIC } \\
\text { CON- } \\
\text { DUCT- } \\
\text { ANCE } \\
\text { (MICRO- } \\
\text { KHOS) }\end{array}$ & $\begin{array}{c}\text { PH } \\
\text { (UNITS) }\end{array}$ & $\begin{array}{l}\text { TEMPER- } \\
\text { RTURE } \\
\text { (DEG C) }\end{array}$ & $\begin{array}{l}\text { TRAYAS- } \\
\text { PAR- } \\
\text { ENCY } \\
\text { (SECCHI } \\
\text { DISX) } \\
\text { (M) }\end{array}$ & $\begin{array}{l}\text { OXYGEN, } \\
\text { DIS- } \\
\text { SOL VED } \\
\text { (MG } / L)\end{array}$ & $\begin{array}{l}\text { OXYGEN, } \\
\text { DIS- } \\
\text { SOLVEO } \\
\text { (PER- } \\
\text { CENT } \\
\text { SATUR- } \\
\text { ATION) }\end{array}$ \\
\hline \multicolumn{9}{|c|}{ OCT , 1976} \\
\hline $\begin{array}{l}18 \ldots \\
18 \ldots \\
\text { NoW }\end{array}$ & $\begin{array}{l}1705 \\
1707\end{array}$ & $\begin{array}{l}1.0 \\
7.5\end{array}$ & $\begin{array}{l}22000 \\
23000\end{array}$ & $\begin{array}{l}7.9 \\
7.7\end{array}$ & $\begin{array}{l}18.5 \\
18.0\end{array}$ & $\begin{array}{r}.80 \\
--\end{array}$ & $\begin{array}{l}7.9 \\
7.3\end{array}$ & $\begin{array}{l}94 \\
86\end{array}$ \\
\hline $\begin{array}{l}16 \ldots \\
16 \ldots \\
D E C\end{array}$ & $\begin{array}{l}1255 \\
1257\end{array}$ & $\begin{array}{l}1.0 \\
6.0\end{array}$ & $\begin{array}{l}27000 \\
27000\end{array}$ & $\begin{array}{l}8.0 \\
8.0\end{array}$ & $\begin{array}{l}9.5 \\
9.5\end{array}$ & $\begin{array}{r}1.13 \\
-.\end{array}$ & $\begin{array}{l}9.4 \\
9.5\end{array}$ & $\begin{array}{l}95 \\
96\end{array}$ \\
\hline $\begin{array}{l}15 \ldots \\
15 \ldots\end{array}$ & $\begin{array}{l}1140 \\
1142\end{array}$ & $\begin{array}{l}1.0 \\
8.0\end{array}$ & $\begin{array}{l}12000 \\
13000\end{array}$ & $\begin{array}{l}7.5 \\
7.5\end{array}$ & $\begin{array}{l}11.0 \\
11.0\end{array}$ & .65 & $\begin{array}{l}9.9 \\
9.6\end{array}$ & $\begin{array}{l}97 \\
94\end{array}$ \\
\hline \multicolumn{9}{|c|}{ Jun, 1977} \\
\hline $\begin{array}{l}07 \ldots \\
07 \ldots \\
21 \ldots \\
21 \ldots\end{array}$ & $\begin{array}{l}1245 \\
1247 \\
1020 \\
1022\end{array}$ & $\begin{array}{l}1.0 \\
8.0 \\
1.0 \\
8.0\end{array}$ & $\begin{array}{r}9000 \\
9000 \\
13000 \\
13000\end{array}$ & $\begin{array}{l}7.2 \\
7.2 \\
7.6 \\
7.6\end{array}$ & $\begin{array}{l}29.5 \\
29.5 \\
29.0 \\
29.5\end{array}$ & $\begin{array}{r}.55 \\
-34 \\
1.34 \\
-2\end{array}$ & $\begin{array}{l}8.3 \\
7.3 \\
6.4 \\
6.3\end{array}$ & $\begin{array}{r}112 \\
99 \\
88 \\
87\end{array}$ \\
\hline $\mathrm{ANG}$ & & & & & & & & \\
\hline $\begin{array}{l}23 \ldots \\
23 \ldots\end{array}$ & $\begin{array}{l}1440 \\
1442\end{array}$ & $\begin{array}{l}1.0 \\
4.0\end{array}$ & $\begin{array}{l}16000 \\
16000\end{array}$ & $\begin{array}{l}6.0 \\
6.0\end{array}$ & $\begin{array}{r}28.5 \\
28.5\end{array}$ & -97 & $\begin{array}{l}6.9 \\
6.8\end{array}$ & 95 \\
\hline SEP & & & 16000 & & & -+ & & \\
\hline $22 \ldots$ & 1050 & 1.0 & 15000 & 8.1 & 28.8 & 1.22 & 7.6 & 104 \\
\hline $22 \ldots$ & 1054 & 8.0 & $\begin{array}{l}13000 \\
15000\end{array}$ & & $\begin{array}{l}20.0 \\
28.5\end{array}$ & $=$ & 7.4 & $\begin{array}{l}101 \\
100\end{array}$ \\
\hline \multicolumn{9}{|c|}{ JUN , 1978} \\
\hline $\begin{array}{l}01 \ldots \\
01, \ldots\end{array}$ & $\begin{array}{l}1010 \\
1012\end{array}$ & $\begin{array}{l}1.0 \\
6.0\end{array}$ & $\begin{array}{l}22000 \\
22000\end{array}$ & $\begin{array}{l}7.7 \\
7.7\end{array}$ & $\begin{array}{l}28.5 \\
28.5\end{array}$ & $\begin{array}{r}1.27 \\
-.\end{array}$ & $\begin{array}{l}6.8 \\
6.5\end{array}$ & $\begin{array}{l}96 \\
92\end{array}$ \\
\hline
\end{tabular}

295402093514300 . LINE 274 \$ITE 04

\begin{tabular}{|c|c|c|c|c|c|c|c|c|}
\hline DRTE & TIME & $\begin{array}{l}\text { SAMP. } \\
\text { LING } \\
\text { DEPTH } \\
\text { (FT) }\end{array}$ & $\begin{array}{l}\text { SPE- } \\
\text { CIFIC } \\
\text { COH- } \\
\text { DUCT- } \\
\text { APCE } \\
\text { (MICRO- } \\
\text { MHDS) }\end{array}$ & $\begin{array}{c}\text { PH } \\
\text { (UNITS) }\end{array}$ & $\begin{array}{l}\text { TEMPER- } \\
\text { ATURE } \\
\text { (DEG C) }\end{array}$ & $\begin{array}{l}\text { TRANS- } \\
\text { PAR- } \\
\text { ENKY } \\
\text { (SECCHI } \\
\text { DISK) } \\
(M)\end{array}$ & $\begin{array}{c}\text { OXYGEN, } \\
\text { DIS- } \\
\text { SOLYED } \\
\text { (MG } f L)\end{array}$ & $\begin{array}{c}\text { OXYGEN, } \\
\text { DIS- } \\
\text { SOLVED } \\
\text { CPER- } \\
\text { CENT } \\
\text { SATUR- } \\
\text { ATION\} }\end{array}$ \\
\hline \multicolumn{9}{|c|}{ OCT, 1976} \\
\hline $\begin{array}{l}18 . . . \\
18 \ldots \\
\text { NOW }\end{array}$ & $\begin{array}{l}1715 \\
1717\end{array}$ & $\begin{array}{l}1.0 \\
5.5\end{array}$ & $\begin{array}{l}21000 \\
22000\end{array}$ & $\begin{array}{l}7.8 \\
7.6\end{array}$ & $\begin{array}{l}19.0 \\
17.0\end{array}$ & $\begin{array}{r}.79 \\
.\end{array}$ & $\begin{array}{l}7.3 \\
6.5\end{array}$ & $\begin{array}{l}88 \\
76\end{array}$ \\
\hline $\begin{array}{l}16 . . \\
16 . . \\
\text { DEC }\end{array}$ & $\begin{array}{l}1310 \\
1312\end{array}$ & $\begin{array}{l}1.0 \\
5.0\end{array}$ & $\begin{array}{l}23000 \\
22000\end{array}$ & $\begin{array}{l}\text { 8.1 } \\
8.1\end{array}$ & $\begin{array}{l}11.0 \\
11.0\end{array}$ & $\begin{array}{r}1.07 \\
--\end{array}$ & $\begin{array}{l}9.4 \\
9.5\end{array}$ & $\begin{array}{l}96 \\
97\end{array}$ \\
\hline $\begin{array}{l}15 \ldots \\
15 \ldots \\
15 \ldots\end{array}$ & $\begin{array}{l}1145 \\
1147 \\
1149\end{array}$ & $\begin{array}{l}1.0 \\
4.0 \\
6.5\end{array}$ & $\begin{array}{l}12000 \\
12000 \\
12000\end{array}$ & $\begin{array}{l}7.4 \\
7.4 \\
7.3\end{array}$ & $\begin{array}{l}11.0 \\
11.0 \\
11.0\end{array}$ & $\begin{array}{r}.52 \\
\ldots \\
.\end{array}$ & $\begin{array}{l}9 . \\
9 . \\
8 .\end{array}$ & $\begin{array}{l}91 \\
91 \\
82\end{array}$ \\
\hline \multicolumn{9}{|c|}{ JUN, 1977} \\
\hline $\begin{array}{l}07 \ldots \\
07 \ldots \\
21 \ldots \\
21 \ldots\end{array}$ & $\begin{array}{l}1300 \\
1302 \\
1025 \\
1027\end{array}$ & $\begin{array}{l}1.0 \\
7.0 \\
1.0 \\
7.0\end{array}$ & $\begin{array}{r}9000 \\
9000 \\
11000 \\
11000\end{array}$ & $\begin{array}{l}7.1 \\
7.1 \\
7.7 \\
7.7\end{array}$ & $\begin{array}{l}29.5 \\
29.0 \\
29.0 \\
29.0\end{array}$ & $\begin{array}{r}.93 \\
.0 \\
1.00 \\
-.\end{array}$ & $\begin{array}{l}6.6 \\
7.0 \\
6.7 \\
6.6\end{array}$ & $\begin{array}{l}89 \\
95 \\
92 \\
91\end{array}$ \\
\hline \multicolumn{9}{|l|}{ AUG } \\
\hline $\begin{array}{l}23 \ldots \\
23 \ldots \\
\text { SEP }\end{array}$ & $\begin{array}{l}1445 \\
1447\end{array}$ & $\begin{array}{l}1.0 \\
6.5\end{array}$ & $\begin{array}{l}16000 \\
16000\end{array}$ & $\begin{array}{l}5.9 \\
5.8\end{array}$ & $\begin{array}{l}28.5 \\
28.5\end{array}$ & $\begin{array}{r}1.23 \\
-.\end{array}$ & $\begin{array}{l}6.5 \\
6.7\end{array}$ & $\begin{array}{l}91 \\
94\end{array}$ \\
\hline $\begin{array}{l}22 \ldots \\
22 \ldots \\
\text { JUN }\end{array}$ & $\begin{array}{l}1105 \\
1107\end{array}$ & $\begin{array}{l}1.0 \\
6.0\end{array}$ & $\begin{array}{l}10000 \\
10000\end{array}$ & $\begin{array}{l}8.3 \\
8.3\end{array}$ & $\begin{array}{l}29.0 \\
29.0\end{array}$ & $\begin{array}{r}.84 \\
--\end{array}$ & $\begin{array}{l}8.1 \\
7.9\end{array}$ & $\begin{array}{l}109 \\
107\end{array}$ \\
\hline $\begin{array}{l}01 \ldots \\
01 \ldots\end{array}$ & $\begin{array}{l}1018 \\
1020\end{array}$ & $\begin{array}{l}1.0 \\
6.0\end{array}$ & $\begin{array}{l}26600 \\
26600\end{array}$ & $\begin{array}{l}7.7 \\
7.7\end{array}$ & $\begin{array}{l}29.0 \\
29.0\end{array}$ & $\begin{array}{r}1.21 \\
-.-\end{array}$ & $\begin{array}{l}6.4 \\
6.4\end{array}$ & $\begin{array}{l}92 \\
92\end{array}$ \\
\hline
\end{tabular}


294847093525700 LIRE 293 SITE OI

\begin{tabular}{|c|c|c|c|c|c|c|c|c|}
\hline DATE & TIHE & $\begin{array}{l}\text { SAMP. } \\
\text { LING } \\
\text { OEPTH } \\
(\mathrm{FT})\end{array}$ & $\begin{array}{l}\text { SPE- } \\
\text { CIFIC } \\
\text { COHA- } \\
\text { DUCT- } \\
\text { ANCE } \\
\text { (MICRO- } \\
\text { NHIOS) }\end{array}$ & $\begin{array}{c}\mathrm{PH} \\
\text { (UnITS) }\end{array}$ & $\begin{array}{l}\text { TEKPER - } \\
\text { ATURE } \\
\text { (DEG C) }\end{array}$ & $\begin{array}{c}\text { TRAHS- } \\
\text { PAR- } \\
\text { EASCY } \\
\text { (SECCHI } \\
\text { DISK) } \\
\text { (M) }\end{array}$ & $\begin{array}{c}\text { OXYGEN, } \\
\text { DIS- } \\
\text { SOLVED } \\
\text { (MG/L) }\end{array}$ & $\begin{array}{l}\text { OXYGEN, } \\
\text { DIS- } \\
\text { SOLYED } \\
\text { (PER- } \\
\text { CERT } \\
\text { SATUR- } \\
\text { ATION) }\end{array}$ \\
\hline \multicolumn{9}{|c|}{ OC.T, 1976} \\
\hline $\begin{array}{l}18 \ldots \\
18 \ldots \\
\text { NOV }\end{array}$ & $\begin{array}{l}1755 \\
1757\end{array}$ & $\begin{array}{l}1.0 \\
6.0\end{array}$ & $\begin{array}{l}14000 \\
15000\end{array}$ & $\begin{array}{l}7.8 \\
7.6\end{array}$ & $\begin{array}{l}17.0 \\
17.0\end{array}$ & $\begin{array}{r}.52 \\
--\end{array}$ & $\begin{array}{l}7.7 \\
7.7\end{array}$ & $\begin{array}{l}87 \\
87\end{array}$ \\
\hline $\begin{array}{l}16 \ldots \\
16 \ldots \\
\text { DEC }\end{array}$ & $\begin{array}{l}1330 \\
1332\end{array}$ & $\begin{array}{l}1.0 \\
5.0\end{array}$ & $\begin{array}{l}21000 \\
21000\end{array}$ & $\begin{array}{l}7.9 \\
7.9\end{array}$ & $\begin{array}{l}8.5 \\
8.5\end{array}$ & $\begin{array}{r}.84 \\
--\end{array}$ & $\begin{array}{l}10.3 \\
10.3\end{array}$ & $\begin{array}{l}98 \\
98\end{array}$ \\
\hline $\begin{array}{l}15 \ldots \\
15 \ldots \\
\text { JUN . }\end{array}$ & $\begin{array}{l}1225 \\
1227\end{array}$ & $\begin{array}{l}1.0 \\
6.0\end{array}$ & $\begin{array}{l}16000 \\
16000\end{array}$ & $\begin{array}{l}7.6 \\
7.5\end{array}$ & $\begin{array}{l}11.0 \\
11.0\end{array}$ & $\begin{array}{r}.65 \\
-.\end{array}$ & $\begin{array}{l}10.0 \\
10.5\end{array}$ & $\begin{array}{l}100 \\
105\end{array}$ \\
\hline $\begin{array}{l}07 \ldots \\
07 \ldots \\
21 \ldots \\
21 \ldots \\
\text { SEP }\end{array}$ & $\begin{array}{l}1335 \\
1337 \\
1055 \\
1057\end{array}$ & $\begin{array}{l}1.0 \\
6.0 \\
1.0 \\
6.0\end{array}$ & $\begin{array}{r}7000 \\
9000 \\
13000 \\
13000\end{array}$ & $\begin{array}{r}7.5 \\
7.4 \\
-. \\
-.\end{array}$ & $\begin{array}{l}29.5 \\
30.0 \\
28.5 \\
28.5\end{array}$ & $\begin{array}{r}.60 \\
.83 \\
.7\end{array}$ & $\begin{array}{l}8.7 \\
9.4 \\
9.8 \\
9.8\end{array}$ & $\begin{array}{l}117 \\
129 \\
132 \\
132\end{array}$ \\
\hline $\begin{array}{l}22 \ldots . \\
22 \ldots \\
\text { JUN, } 1\end{array}$ & $\begin{array}{l}1010 \\
1012 \\
3\end{array}$ & $\begin{array}{l}1.0 \\
5.5\end{array}$ & $\begin{array}{l}16000 \\
19000\end{array}$ & $\begin{array}{l}7.4 \\
7.5\end{array}$ & $\begin{array}{l}27.5 \\
27.5\end{array}$ & $\begin{array}{r}.80 \\
--\end{array}$ & $\begin{array}{l}5.5 \\
5.6\end{array}$ & $\begin{array}{l}74 \\
78\end{array}$ \\
\hline $\begin{array}{l}01 \ldots \\
01 \ldots\end{array}$ & $\begin{array}{l}1055 \\
1057\end{array}$ & $\begin{array}{l}1.0 \\
6.0\end{array}$ & $\begin{array}{l}21000 \\
21000\end{array}$ & $\begin{array}{l}7.5 \\
7.5\end{array}$ & $\begin{array}{l}28.5 \\
28.5\end{array}$ & -- & $\begin{array}{l}6.5 \\
6.5\end{array}$ & $\begin{array}{l}92 \\
91\end{array}$ \\
\hline
\end{tabular}

294917093534500 LINE 293 SITE 02

\begin{tabular}{|c|c|c|c|c|c|c|c|c|}
\hline DATE & TIME & $\begin{array}{l}\text { SAMP } \\
\text { LING } \\
\text { DEPTH } \\
(F T)\end{array}$ & $\begin{array}{l}\text { SPE- } \\
\text { CIFIC } \\
\text { CON- } \\
\text { DUCT- } \\
\text { ANCE } \\
\text { (MICRO- } \\
\text { MHOS) }\end{array}$ & $\begin{array}{c}P H \\
\text { (UNITS) }\end{array}$ & $\begin{array}{c}\text { TEMPER- } \\
\text { ATURE } \\
\text { (DEG C) }\end{array}$ & $\begin{array}{l}\text { TRANSS- } \\
\text { PAR- } \\
\text { EHCY } \\
\text { (SECCHI } \\
\text { DISK) } \\
\text { (M) }\end{array}$ & $\begin{array}{c}\text { OXYGEN, } \\
\text { OIS- } \\
\text { SOLVED } \\
\text { (MG } / L)\end{array}$ & $\begin{array}{c}\text { OXYGEN, } \\
\text { DIS- } \\
\text { SOEVED } \\
\text { (PER- } \\
\text { CENT } \\
\text { SATUR- } \\
\text { ATION) }\end{array}$ \\
\hline \multicolumn{9}{|c|}{ OCT , 1976} \\
\hline $\begin{array}{l}18 \ldots \\
18 \ldots \\
\text { NOV }\end{array}$ & $\begin{array}{l}1745 \\
1747\end{array}$ & $\begin{array}{l}1.0 \\
5.0\end{array}$ & $\begin{array}{l}17000 \\
17000\end{array}$ & $\begin{array}{l}7.8 \\
7.8\end{array}$ & $\begin{array}{l}18.0 \\
18.0\end{array}$ & $\begin{array}{r}.47 \\
--\end{array}$ & $\begin{array}{l}8.3 \\
8.4\end{array}$ & $\begin{array}{l}97 \\
98\end{array}$ \\
\hline $\begin{array}{l}16 \ldots \\
16 \ldots \\
D E C\end{array}$ & $\begin{array}{l}1340 \\
1342\end{array}$ & $\begin{array}{l}1.0 \\
6.0\end{array}$ & $\begin{array}{l}25000 \\
25000\end{array}$ & $\begin{array}{l}7.9 \\
7.9\end{array}$ & $\begin{array}{l}8.5 \\
9.0\end{array}$ & .48 & $\begin{array}{l}10.1 \\
10.2\end{array}$ & $\begin{array}{l}101 \\
100\end{array}$ \\
\hline $\begin{array}{l}15 \ldots \\
15 \ldots \\
\text { JUM , } 1\end{array}$ & $\begin{array}{l}1215 \\
1217\end{array}$ & $\begin{array}{l}1.0 \\
5.5\end{array}$ & $\begin{array}{l}15000 \\
15000\end{array}$ & $\begin{array}{l}7.5 \\
7.5\end{array}$ & $\begin{array}{l}11.0 \\
11.0\end{array}$ & $\begin{array}{c}.59 \\
--\end{array}$ & $\begin{array}{l}9.8 \\
9.7\end{array}$ & $\begin{array}{l}97 \\
96\end{array}$ \\
\hline $\begin{array}{l}07 \ldots \\
07 \ldots \\
21 \ldots \\
21 \ldots \\
\text { SEP }\end{array}$ & $\begin{array}{l}1325 \\
1327 \\
1045 \\
1047\end{array}$ & $\begin{array}{l}1.0 \\
6.5 \\
1.0 \\
7.0\end{array}$ & $\begin{array}{r}7000 \\
9000 \\
13000 \\
13000\end{array}$ & $\begin{array}{r}7.4 \\
7.2 \\
-. \\
\cdots\end{array}$ & $\begin{array}{l}30.0 \\
29.5 \\
29.0 \\
29.0\end{array}$ & $\begin{array}{l}-- \\
-97 \\
--\end{array}$ & $\begin{array}{l}7.2 \\
8.7 \\
9.4 \\
9.2\end{array}$ & $\begin{array}{r}97 \\
118 \\
129 \\
126\end{array}$ \\
\hline $\begin{array}{c}22 \ldots \\
22 \ldots\end{array}$ & $\begin{array}{l}1005 \\
1006 \\
8\end{array}$ & $\begin{array}{l}1.0 \\
7.0\end{array}$ & $\begin{array}{l}18000 \\
23000\end{array}$ & $\begin{array}{l}7.8 \\
7.6\end{array}$ & $\begin{array}{l}28.5 \\
28.5\end{array}$ & 1.23 & $\begin{array}{l}7.3 \\
5.7\end{array}$ & $\begin{array}{r}101 \\
80\end{array}$ \\
\hline $\begin{array}{l}01, \ldots \\
01, \ldots\end{array}$ & $\begin{array}{l}1047 \\
1049\end{array}$ & $\begin{array}{l}1.0 \\
6.0\end{array}$ & $\begin{array}{l}25000 \\
25000\end{array}$ & $\begin{array}{l}7.7 \\
7.6\end{array}$ & $\begin{array}{l}28.5 \\
28.5\end{array}$ & .80 & $\begin{array}{l}6.5 \\
6.5\end{array}$ & $\begin{array}{l}94 \\
94\end{array}$ \\
\hline
\end{tabular}

294947093543400 LINE 293 SITE 03

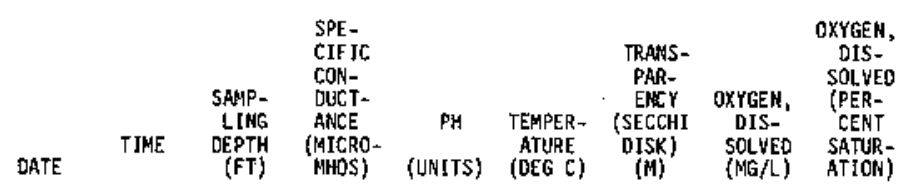

OCT, 1976

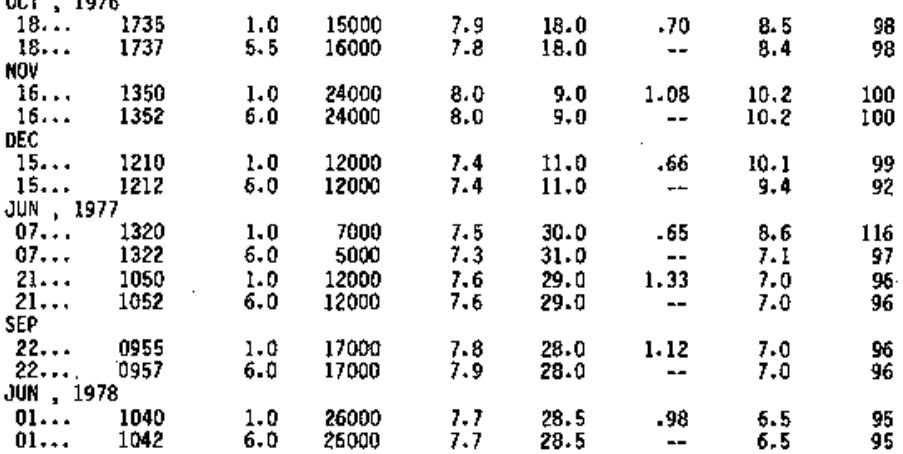


Table 1A.--Qiality of water in the Sabine-Neches estuary, water years 1977-78--Continued Field Determinations--Continued

294735093545900 LIRE 300 SITE O2

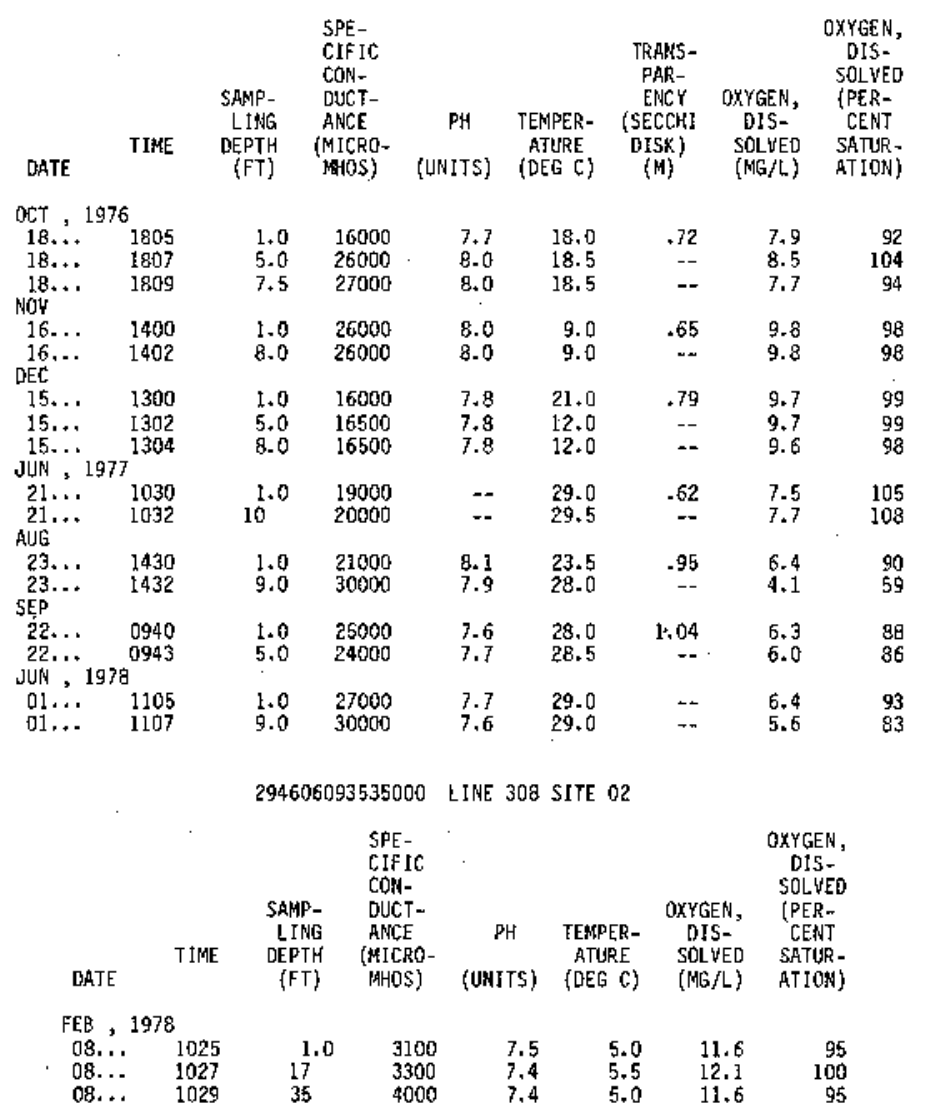


Table 1A.--Qualfty of water in the Sabine-Heches estuary, water years 1977-78--Continued Field Determf nations-uContinued

294945093571700 LINE 339 SITE O2

\begin{tabular}{|c|c|c|c|c|c|c|c|c|}
\hline DATE & TIME & $\begin{array}{l}\text { SALP- } \\
\text { LIHG } \\
\text { DEPTY } \\
\text { (FT) }\end{array}$ & $\begin{array}{l}\text { SPE- } \\
\text { CIFIC } \\
\text { CON- } \\
\text { DUCT- } \\
\text { AKCE } \\
\text { (MICRO- } \\
\text { MHOS) }\end{array}$ & $\begin{array}{c}\text { PH } \\
\text { (UNITS) }\end{array}$ & $\begin{array}{l}\text { TEMPER- } \\
\text { ATURE } \\
\text { (DEG C) }\end{array}$ & $\begin{array}{c}\text { TRANS- } \\
\text { PAR- } \\
\text { ENCY } \\
\text { (SECCHI } \\
\text { OISK) } \\
\text { (N) }\end{array}$ & $\begin{array}{c}\text { OXYGEK, } \\
\text { OIS- } \\
\text { SOLWED } \\
\text { (NG/L) }\end{array}$ & $\begin{array}{c}\text { OXYGEN, } \\
\text { OIS- } \\
\text { SOLYED } \\
\text { (PER- } \\
\text { CEMT } \\
\text { SATLR- } \\
\text { ATION) }\end{array}$ \\
\hline $\begin{array}{l}\text { OCT }, \\
18 . . . \\
18 \ldots \\
18 \ldots \\
18 \ldots \\
\text { NOY... }\end{array}$ & $\begin{array}{l}6 \\
1500 \\
1502 \\
1504 \\
1506\end{array}$ & $\begin{array}{l}1.0 \\
10 \\
20 \\
40\end{array}$ & $\begin{array}{l}25000 \\
31000 \\
37000 \\
37000\end{array}$ & $\begin{array}{l}8.2 \\
8.1 \\
8.1 \\
8.1\end{array}$ & $\begin{array}{l}18.5 \\
18.5 \\
19.5 \\
19.5\end{array}$ & $\begin{array}{r}1.10 \\
-- \\
--\end{array}$ & $\begin{array}{l}8.4 \\
7.6 \\
5.8 \\
6.8\end{array}$ & $\begin{array}{r}102 \\
96 \\
91 \\
91\end{array}$ \\
\hline $\begin{array}{l}16 \ldots \\
16 \ldots \\
16 \ldots \\
16 \ldots \\
D E C\end{array}$ & $\begin{array}{l}1130 \\
1132 \\
1134 \\
1136\end{array}$ & $\begin{array}{l}1.0 \\
10 \\
20 \\
40\end{array}$ & $\begin{array}{l}32000 \\
32500 \\
36000 \\
29000\end{array}$ & $\begin{array}{l}8.0 \\
8.1 \\
8.1 \\
8.1\end{array}$ & $\begin{array}{l}10.0 \\
10.0 \\
11.0 \\
11.5\end{array}$ & $\begin{array}{l}.76 \\
-- \\
-- \\
--\end{array}$ & $\begin{array}{l}8.3 \\
8.9 \\
8.2 \\
8.2\end{array}$ & $\begin{array}{l}86 \\
93 \\
90 \\
87\end{array}$ \\
\hline $\begin{array}{l}15 \ldots \\
15 \ldots \\
15 \ldots \\
15 \ldots \\
15 \ldots \\
15 \ldots \\
\text { FEB. }\end{array}$ & $\begin{array}{l}1145 \\
1147 \\
1149 \\
1151 \\
1153 \\
1155 \\
7\end{array}$ & $\begin{array}{l}1.0 \\
10 \\
15 \\
20 \\
30 \\
43\end{array}$ & $\begin{array}{r}5000 \\
10000 \\
12000 \\
22000 \\
31000 \\
40000\end{array}$ & $\begin{array}{l}7.1 \\
7.4 \\
7.5 \\
8.0 \\
8.2 \\
8.2\end{array}$ & $\begin{array}{l}12.0 \\
11.5 \\
12.0 \\
12.5 \\
13.0 \\
12.5\end{array}$ & $\begin{array}{l}.24 \\
\cdots \\
\cdots \\
\cdots \\
\cdots\end{array}$ & $\begin{array}{l}7.7 \\
7.5 \\
8.0 \\
8.7 \\
8.4 \\
7.9\end{array}$ & $\begin{array}{l}75 \\
74 \\
80 \\
92 \\
93 \\
91\end{array}$ \\
\hline $\begin{array}{l}01 \ldots . . \\
01 \ldots \\
01 \ldots . \\
01 \ldots \\
\text { JiN }\end{array}$ & $\begin{array}{l}1345 \\
1347 \\
1349 \\
1351\end{array}$ & $\begin{array}{l}1.0 \\
20 \\
30 \\
45\end{array}$ & $\begin{array}{l}22000 \\
29000 \\
25000 \\
34000\end{array}$ & $\begin{array}{l}8.0 \\
8.1 \\
8.1 \\
8.1\end{array}$ & $\begin{array}{l}9.0 \\
9.0 \\
9.0 \\
9.0\end{array}$ & $\begin{array}{l}m \\
--\end{array}$ & $\begin{array}{l}9.6 \\
9.2 \\
8.6 \\
8.8\end{array}$ & $\begin{array}{l}93 \\
93 \\
84 \\
91\end{array}$ \\
\hline $\begin{array}{l}07 \ldots . . \\
07 \ldots \\
07 \ldots \\
21 \ldots \\
21 \ldots \\
21 \ldots \\
\text { AUG }\end{array}$ & $\begin{array}{l}1415 \\
1417 \\
1419 \\
0900 \\
0902 \\
0904\end{array}$ & $\begin{array}{l}1.0 \\
20 \\
45 \\
1.0 \\
20 \\
44\end{array}$ & $\begin{array}{r}25000 \\
28000 \\
37000 \\
9000 \\
14000 \\
36000\end{array}$ & $\begin{array}{r}7.1 \\
7.2 \\
7.1 \\
=- \\
=- \\
=-\end{array}$ & $\begin{array}{l}29.5 \\
29.0 \\
28.5 \\
29.0 \\
29.0 \\
28.0\end{array}$ & $\begin{array}{l}-- \\
- \\
-- \\
52 \\
-- \\
--\end{array}$ & $\begin{array}{l}8.5 \\
7.7 \\
5.9 \\
7.8 \\
5.7 \\
7.6\end{array}$ & $\begin{array}{r}124 \\
112 \\
90 \\
105 \\
78 \\
113\end{array}$ \\
\hline $\begin{array}{l}23 . . \\
23 . . \\
23 . \\
23 . . \\
\text { SEP }\end{array}$ & $\begin{array}{l}1300 \\
1302 \\
1304 \\
1306\end{array}$ & $\begin{array}{l}1.0 \\
10 \\
20 \\
39\end{array}$ & $\begin{array}{l}23000 \\
26000 \\
41000 \\
48000\end{array}$ & $\begin{array}{l}7.8 \\
7.8 \\
8.0 \\
8.1\end{array}$ & $\begin{array}{l}29.0 \\
29.0 \\
29.0 \\
29.0\end{array}$ & $\begin{array}{l}.48 \\
-- \\
-- \\
--\end{array}$ & $\begin{array}{l}4.4 \\
3.9 \\
3.7 \\
3.9\end{array}$ & $\begin{array}{l}63 \\
57 \\
57 \\
63\end{array}$ \\
\hline $\begin{array}{l}22 . \\
22 . \\
22 . \\
22 . \\
0 \mathrm{CT} .\end{array}$ & $\begin{array}{l}0811 \\
0812 \\
0814 \\
0816\end{array}$ & $\begin{array}{l}1.0 \\
10 \\
20 \\
44\end{array}$ & $\begin{array}{l}25000 \\
28000 \\
37000 \\
38000\end{array}$ & $\begin{array}{l}7.5 \\
7.6 \\
7.8 \\
7.8\end{array}$ & $\begin{array}{l}28.0 \\
28.0 \\
28.5 \\
28.0\end{array}$ & $\begin{array}{c}.65 \\
-- \\
-=\end{array}$ & $\begin{array}{l}5.5 \\
5.1 \\
5.1 \\
4.9\end{array}$ & $\begin{array}{l}77 \\
74 \\
77 \\
74\end{array}$ \\
\hline $\begin{array}{l}18 . \\
18 . \\
18 . \\
18 . \\
\text { FEB }\end{array}$ & $\begin{array}{l}1500 \\
1502 \\
1504 \\
1506\end{array}$ & $\begin{array}{l}1.0 \\
10 \\
20 \\
40\end{array}$ & $\begin{array}{l}27000 \\
34000 \\
41000 \\
41000\end{array}$ & $\begin{array}{l}8.2 \\
8.1 \\
8.1 \\
8.1\end{array}$ & $\begin{array}{l}18.5 \\
18.5 \\
19.5 \\
19.5\end{array}$ & $\begin{array}{r}1.10 \\
-- \\
=- \\
--\end{array}$ & $\begin{array}{l}8.4 \\
7.6 \\
6.8 \\
6.8\end{array}$ & $\begin{array}{r}102 \\
96 \\
91 \\
91\end{array}$ \\
\hline $\begin{array}{l}08 . \\
08 . \\
08 . \\
\text { JUN }\end{array}$ & $\begin{array}{l}1045 \\
1047 \\
1049\end{array}$ & $\begin{array}{l}1.0 \\
22 \\
44\end{array}$ & $\begin{array}{l}16000 \\
25000 \\
30000\end{array}$ & $\begin{array}{l}8.2 \\
8.2 \\
8.1\end{array}$ & $\begin{array}{l}6.0 \\
5.5 \\
6.0\end{array}$ & $\begin{array}{l}=- \\
-- \\
m\end{array}$ & $\begin{array}{l}11.2 \\
10.7 \\
10.4\end{array}$ & $\begin{array}{l}99 \\
99 \\
97\end{array}$ \\
\hline $\begin{array}{l}01 \ldots \\
01 \ldots \\
01 \ldots\end{array}$ & $\begin{array}{l}1300 \\
1302 \\
1305\end{array}$ & $\begin{array}{l}1.0 \\
22 \\
45\end{array}$ & $\begin{array}{l}27300 \\
28900 \\
28900\end{array}$ & $\begin{array}{l}7.8 \\
7.7 \\
7.7\end{array}$ & $\begin{array}{l}29.5 \\
29.5 \\
29.5\end{array}$ & $\begin{array}{l}-- \\
--\end{array}$ & $\begin{array}{l}6.2 \\
5.5 \\
5.5\end{array}$ & $\begin{array}{l}91 \\
80 \\
80\end{array}$ \\
\hline
\end{tabular}


Table 1A.--Quality of water in the Sabine-Neches estuary, water years 1977-78--Continued Field Determinations-c Continued

294252093512000 LINE 377 SITE 02

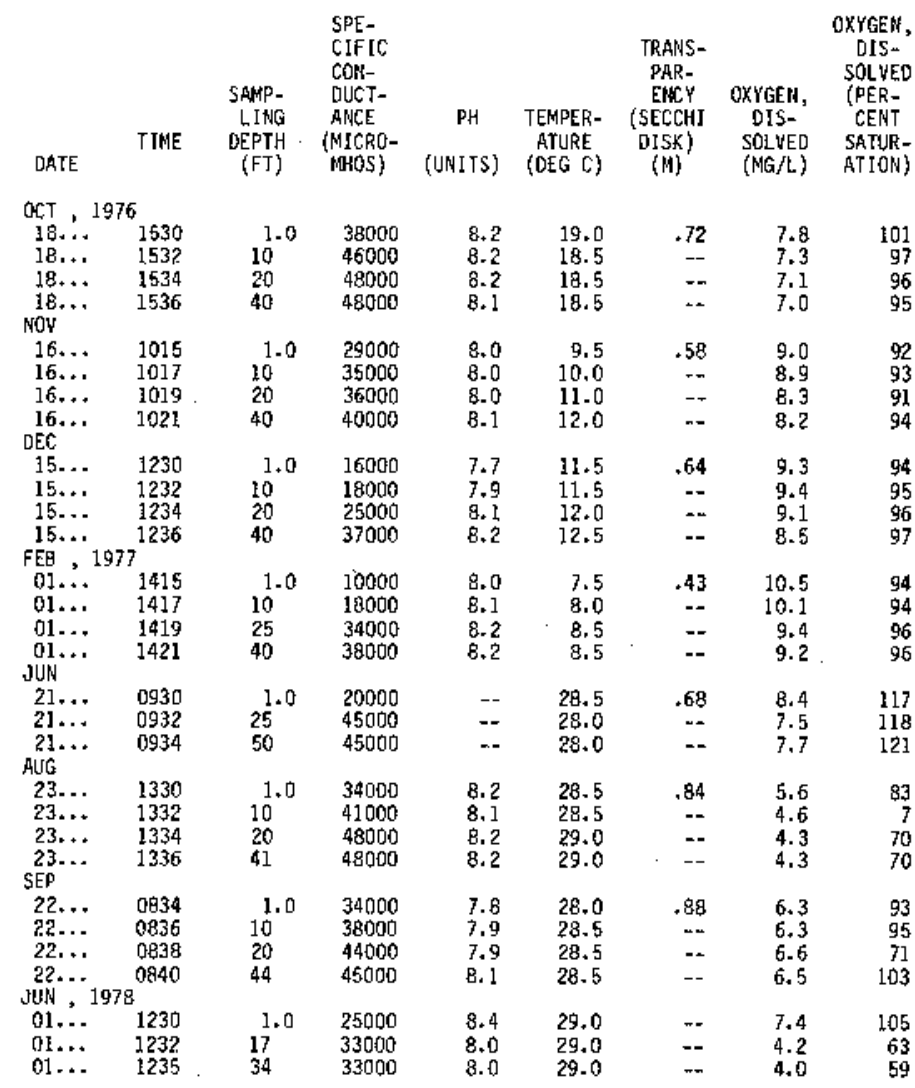

293702093492000 LINE 905 SITE OL

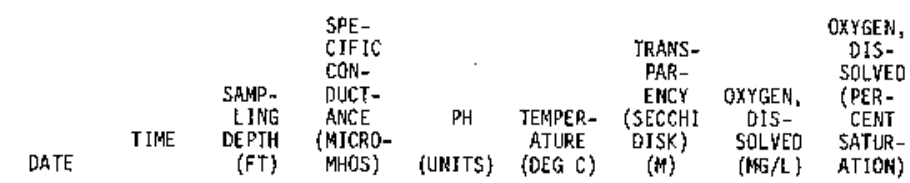

OCT, 1976

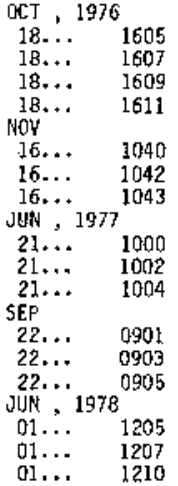

$\begin{array}{cc}1.0 & 4800 \\ 10 & 48000 \\ 20 & 48000 \\ 35 & 48000 \\ 2.0 & 40000 \\ 15 & 45000 \\ 29 & 45000 \\ 1.0 & 4000 \\ 20 & 45000 \\ 42 & 4500 \\ 5.0 & 44000 \\ 20 & 45000 \\ 46 & 45000 \\ 1.0 & 20700 \\ 13 & 22000 \\ 27 & 40000\end{array}$

$\begin{array}{rrrrr}8.3 & 20.0 & 1.25 & 7.0 & 97 \\ 8.3 & 19.5 & -- & 7.1 & 97 \\ 8.2 & 19.5 & -- & 7.0 & 96 \\ 8.2 & 19.5 & -- & 6.8 & 93 \\ 8.1 & 11.5 & 1.80 & 8.2 & 92 \\ 8.1 & 12.5 & -- & 8.0 & 95 \\ 8.1 & 12.5 & -- & 8.2 & 98 \\ -- & 29.0 & .50 & 8.1 & 125 \\ -. & 28.0 & -- & 7.8 & 122 \\ -- & 28.0 & -- & 4.9 & 77 \\ 8.1 & 28.0 & 1.70 & 7.1 & 109 \\ 8.0 & 28.0 & -- & 6.3 & 99 \\ 8.0 & 28.0 & -- & 5.6 & 87 \\ 8.5 & 29.0 & 1.39 & 7.8 & 111 \\ 8.5 & 28.5 & - & 7.3 & 103 \\ 7.8 & 29.0 & -- & 1.5 & 22\end{array}$


Table 13.--Oualịty of water in the Sabine-Neches estuary, water years 1977-78 Nutrient Analyses

( $\mathcal{F} T=$ feet; MG $/ L=$ milligraus per liter $)$

300953093420600 LINC 015 SITE 02

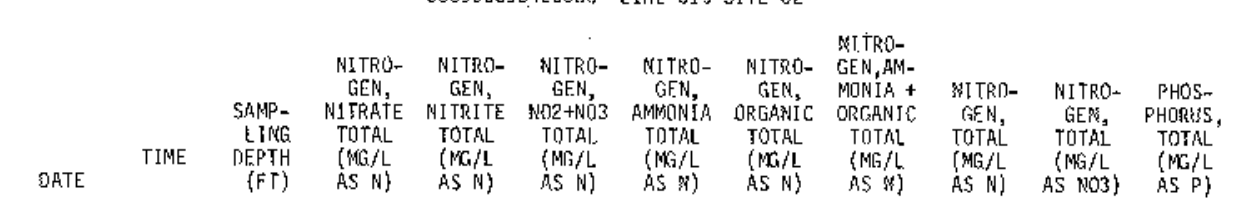

(JCT , 1976

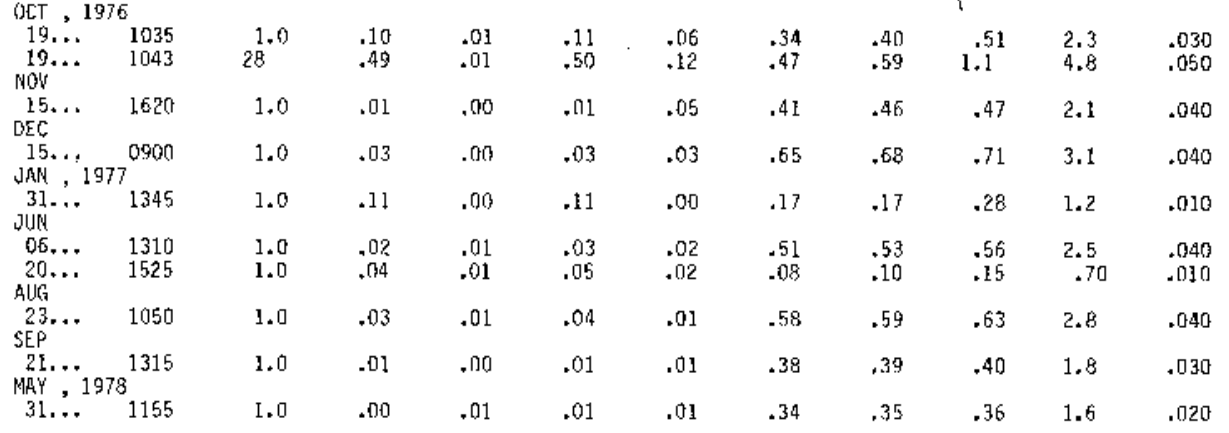

300252093433000 LINE D55 5ITE O2

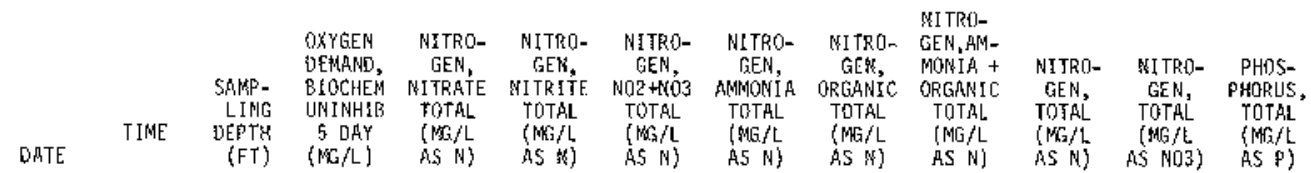

FEB , 1978

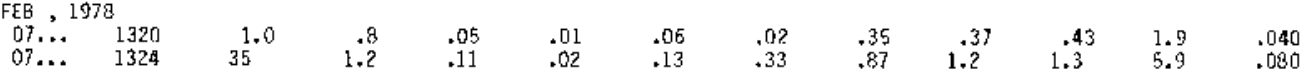

295938093465000 LINE 087 SITE 02

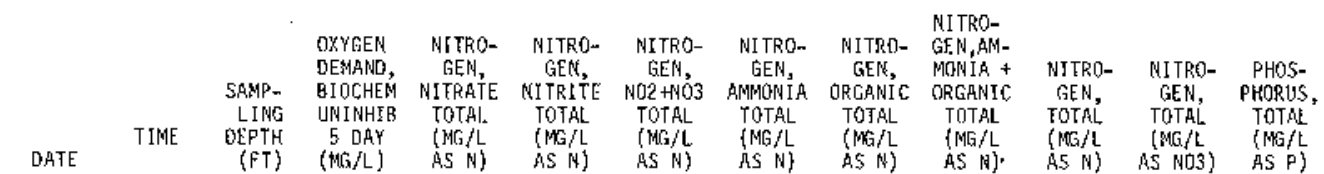

OCT , 1976

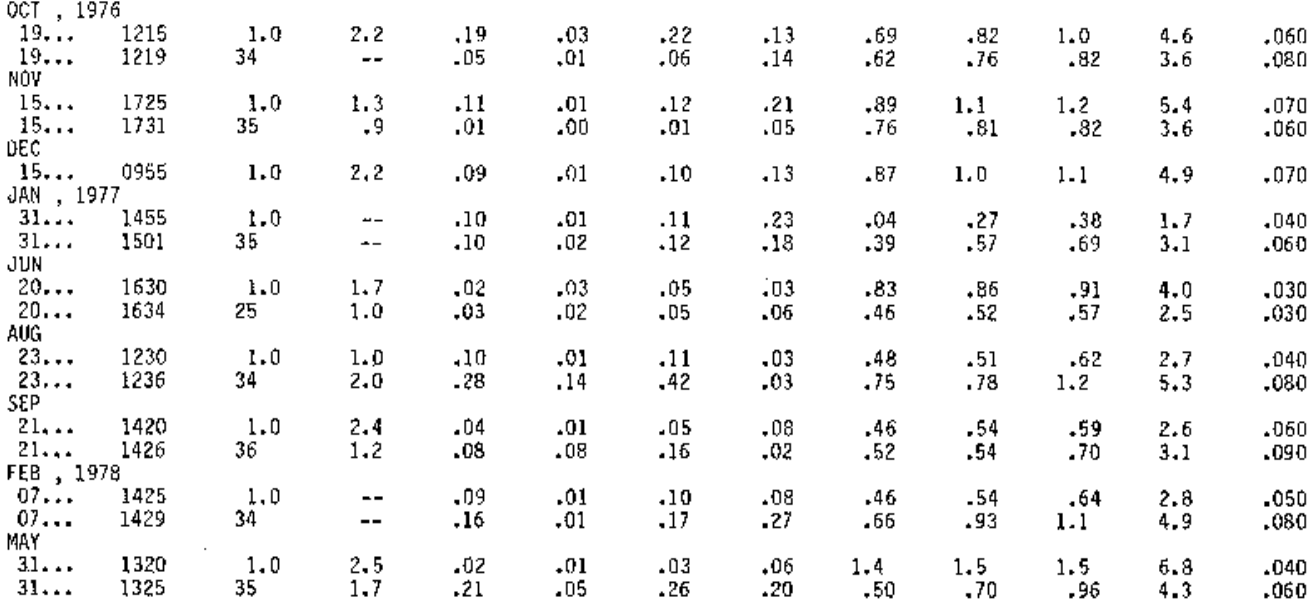


$300922094064900 \quad$ LINE 107 SITE 02

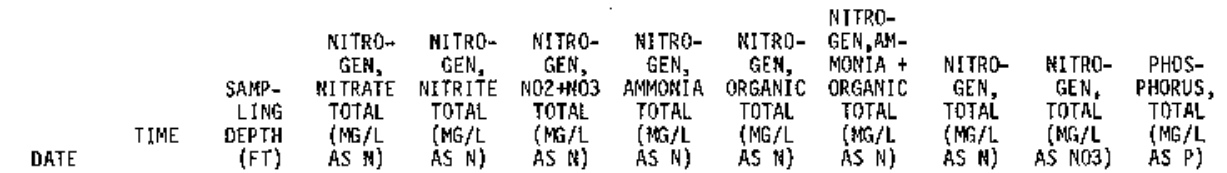

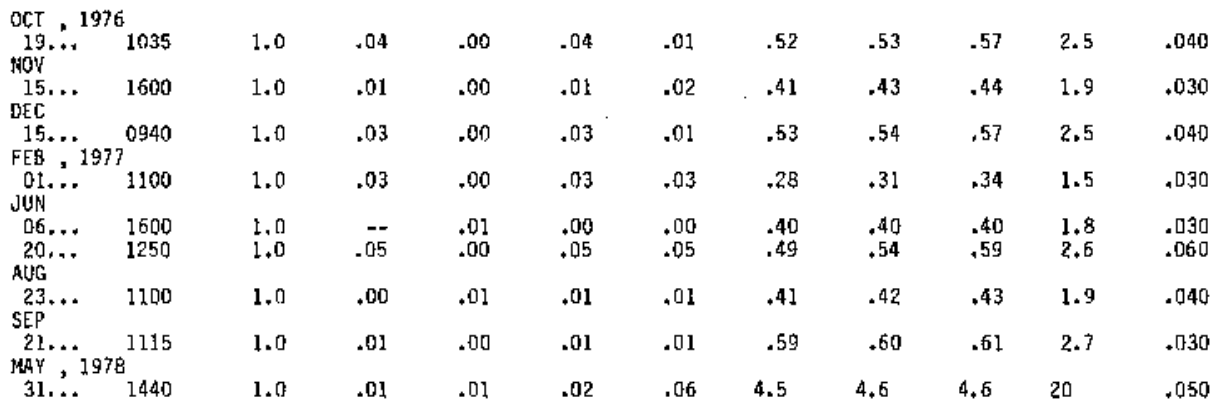

300319094014600 LIME 170 SITE 02

\begin{tabular}{|c|c|c|c|c|c|c|c|c|c|c|c|}
\hline DATE & TIME & $\begin{array}{l}\text { SAMP- } \\
\text { LING } \\
\text { DEPTH } \\
(F T)\end{array}$ & $\begin{array}{l}\text { NITRD- } \\
\text { GEN, } \\
\text { NITRATE } \\
\text { TOTAL } \\
\text { (MG/L } \\
\text { A5 N) }\end{array}$ & $\begin{array}{c}\text { RITRO- } \\
\text { GEN, } \\
\text { NITRIJE } \\
\text { TOTAL } \\
\text { (MG/L } \\
\text { AS N) }\end{array}$ & $\begin{array}{l}\text { NITRQ- } \\
\text { GEN, } \\
\text { NO2 +NO3 } \\
\text { TOTAL } \\
\text { (MG/L } \\
\text { AS N) }\end{array}$ & $\begin{array}{l}\text { NITRO- } \\
\text { GEN, } \\
\text { AMMONIA } \\
\text { TOTAL } \\
\text { (MG/L } \\
\text { AS N) }\end{array}$ & $\begin{array}{l}\text { NITRD- } \\
\text { GER, } \\
\text { ORGANIC } \\
\text { TOTAL } \\
\text { (MG } f L \\
\text { AS N\}) }\end{array}$ & $\begin{array}{l}\text { NITRO- } \\
\text { GEN, } M M- \\
\text { MONIA + } \\
\text { ORGANIL } \\
\text { TOTAL } \\
\text { (MG/L } \\
\text { AS N N) }\end{array}$ & $\begin{array}{l}\text { NITRO- } \\
\text { GEN, } \\
\text { TOTAL } \\
\text { (MG/L } \\
\text { AS RS) }\end{array}$ & $\begin{array}{c}\text { NITRO- } \\
\text { GEN, } \\
\text { TOTAL } \\
\text { (MG/L } \\
\text { AS NOS) }\end{array}$ & $\begin{array}{l}\text { PHDS- } \\
\text { PHORUS, } \\
\text { TOTAL } \\
\text { (NGG/L } \\
\text { AS P) }\end{array}$ \\
\hline
\end{tabular}

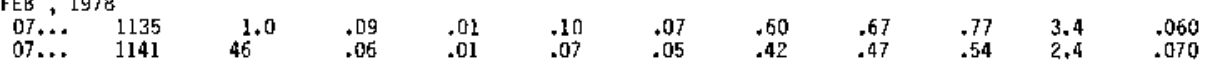

$295842093514900 \quad$ LINE 214 SITE 02

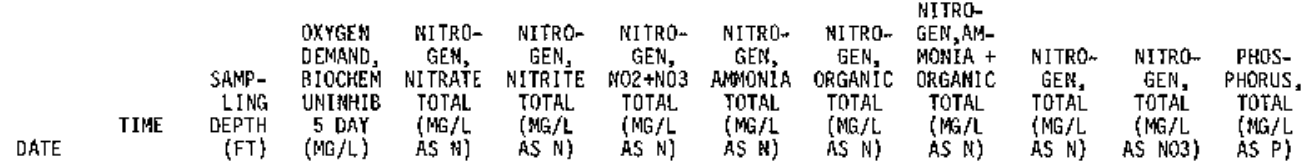

OCT, 1976

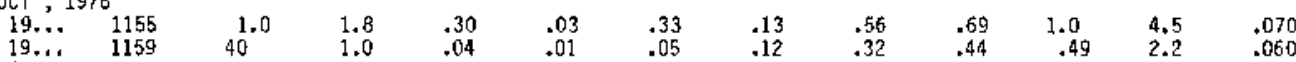

Nov.'1159

16 ... 1215

$16 \ldots \quad 1219$

$15 . \cdots \quad 1110$

$15 \ldots, 112$

01... 1300

$01 . . .1306$

JUN 1440

$06 . . .1444$

$20 . . . \quad 1430$

$\begin{array}{rrr}1.0 & 1.1 & 11 \\ 35 & .9 & .03\end{array}$

$\begin{array}{lll}1.0 & 2.2 & .22 \\ 44 & 1.0 & .10\end{array}$

$\begin{array}{ccc}1.0 & 1.8 & .21 \\ 45 & 1.2 & .00\end{array}$

$\begin{array}{llll}1.0 & 1.8 & .02 & .05\end{array}$

$\begin{array}{ccc}1.0 & 1.8 & .02 \\ 1.0 & 1.8 & .01 \\ & 1.3 & .09\end{array}$

.01
.00
.01
.0
.0
.0
.05
.01
.03

1

.13

.23

.23

1215

45

$1.0 \quad 1.6 \quad .35$

.03

.21

$23 . . .1221$

SEP $21 \ldots \quad 1220$

$21 . \ldots+1230$

$1.0 \quad 1.9 \quad .10$

.04
.18

.07

.07
.04
.10
.18

.00
.09
.16

.39
.38

07... 1235

$1.0 \quad 1.6$

.03
.10

$.13 \quad .06$

$07 \ldots .1239$

31... 1640

$\begin{array}{lll}1.0 & 1.6 & .11\end{array}$

.01

.12

$\begin{array}{lll}1.0 & 1.9 & .46 \\ 46 & 2.2 & .18\end{array}$

.04

$.50 \quad .04$

.56

.72

.80

.03

1.0
.77
1.75

$\begin{array}{cc}1.0 & 1.0 \\ .77 & .8 \\ .75 & .91 \\ 1.2 & 1.4\end{array}$

$\begin{array}{lllll}.82 & .83 & 1.2 & 5.4 & .060\end{array}$

$\begin{array}{lllll}.76 & .82 & .95 & 4.2 & .060 \\ .39 & .44 & .63 & 2.8 & .120\end{array}$

$\begin{array}{lllll}.61 & .74 & .86 & 3.8 & .070 \\ .60 & .80 & .97 & 4.3 & .080\end{array}$

31... 1645

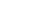

$$
\text { . }
$$

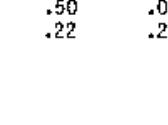

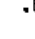$$
\begin{aligned}
& .82 \\
& .86
\end{aligned}
$$ 
Table 1B.--iquality of water in the Sabine-Neches estuary, water years 1977-78--Continued Nutrient Analyses--continued

295728093464500 L.INF. 244 SITE 02

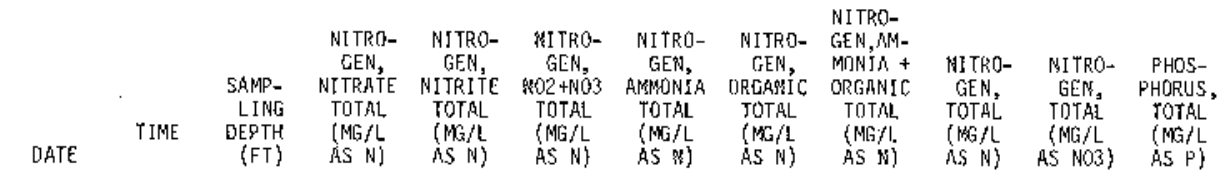

JUN, 1977 O915

.09

.03

295720093474500 LINE. 244 SITE 03

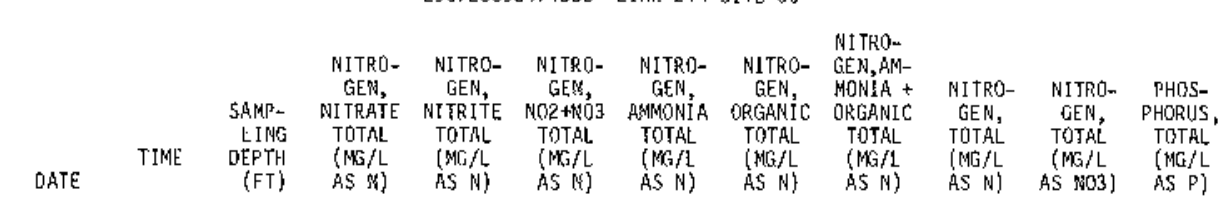
OCT 1976

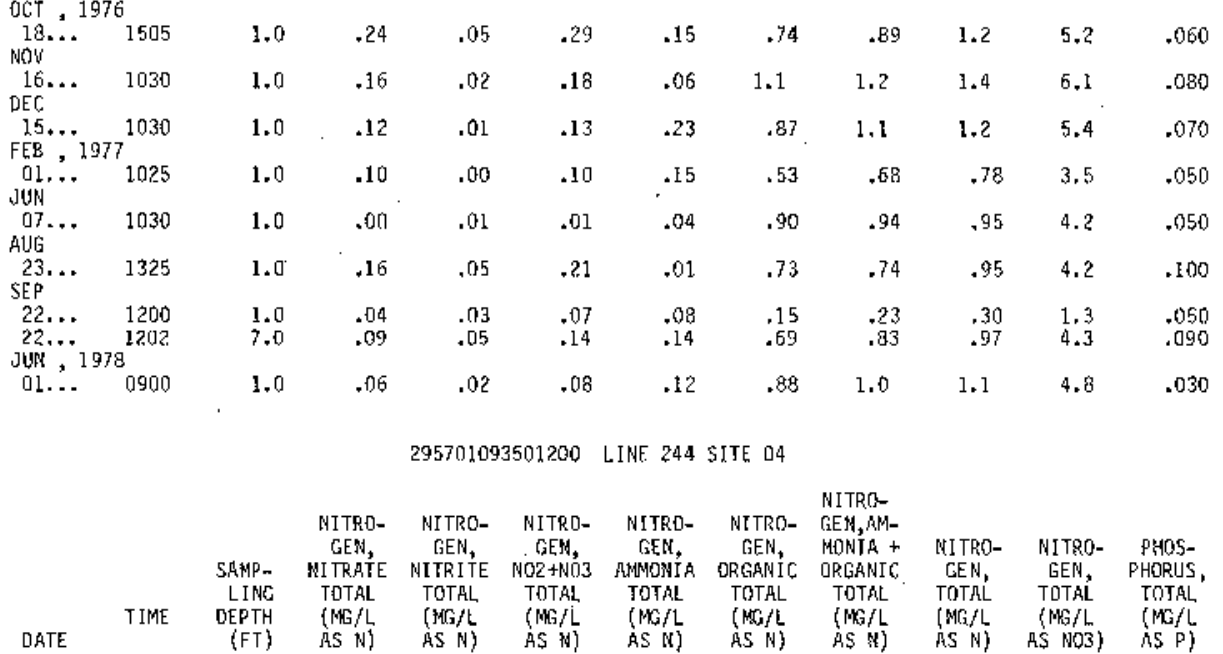
FEB, 1978

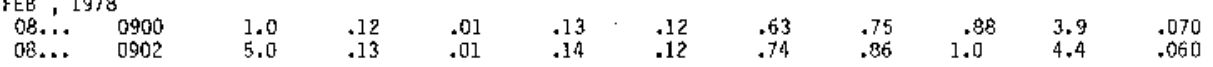
295454093460700 LINE 254 \$ITE DI

\begin{tabular}{|c|c|c|c|c|c|c|c|c|c|c|c|}
\hline & & & & 29545409 & 3460700 & LINE 254 & SITE DI & & & & \\
\hline DATE & TIME & $\begin{array}{l}\text { SAMP- } \\
\text { LING } \\
\text { DEPTH } \\
\text { (FT) }\end{array}$ & $\begin{array}{l}\text { HITRO- } \\
\text { GEN, } \\
\text { NITRATE } \\
\text { TOTAL } \\
\text { (MC } A L \\
\text { AS N) }\end{array}$ & $\begin{array}{c}\text { NITRO- } \\
\text { GEN, } \\
\text { NITRITE } \\
\text { TOTAL } \\
\text { (MG } / L \\
\text { AS N) }\end{array}$ & $\begin{array}{l}\text { RITRO- } \\
\text { GEN, } \\
\text { NO2+NO3 } \\
\text { TOTAL } \\
\text { (MG } / L \\
\text { AS N }\end{array}$ & $\begin{array}{l}\text { NITRO- } \\
\text { GEN, } \\
\text { ANAMONIA } \\
\text { TOTAL } \\
\text { (NAG } / L \\
\text { AS 扵) }\end{array}$ & $\begin{array}{c}\text { NITRO- } \\
\text { GEN, } \\
\text { ORGAHIC } \\
\text { TOTAL } \\
(N A / L \\
\text { AS N })\end{array}$ & $\begin{array}{l}\text { NITRO- } \\
\text { GEN,AMM- } \\
\text { MONIA + } \\
\text { ORGANIC } \\
\text { TOTAL } \\
\text { (MG } / L \\
\text { AS NI) }\end{array}$ & $\begin{array}{l}\text { NLTRO- } \\
\text { GEN, } \\
\text { TOTAL } \\
(N \mathrm{G} / \mathrm{L} \\
\text { AS N })\end{array}$ & $\begin{array}{l}\text { NITRO- } \\
\text { GEN, } \\
\text { TDTAL } \\
\text { (NHG/L } \\
\text { AS ND3) }\end{array}$ & $\begin{array}{l}\text { PHOS- } \\
\text { PHORUS, } \\
\text { TOTAL } \\
\text { (MG } / L \\
\text { AS P) }\end{array}$ \\
\hline OCT, & ${ }_{1630}^{6}$ & 1.0 & .04 & .01 & .05 & .13 & .58 & .71 & .76 & 3.4 & .050 \\
\hline NÔ & & & & & & & & & & & \\
\hline $\begin{array}{l}16 \ldots \\
\mathrm{DEC}\end{array}$ & 1130 & 1.0 & .09 & .00 & .09 & .09 & .88 & .97 & 1.1 & 4.7 & .060 \\
\hline $15 \ldots$ & $7^{1105}$ & 1.0 & .16 & .02 & .18 & .27 & .72 & .99 & 1.2 & 5.2 & .060 \\
\hline נUN & 1110 & 1.0 & .10 & .00 & .10 & .13 & .77 & .90 & 1.0 & 4.4 & ] \\
\hline$\underset{\text { SEP }}{21 . \cdots}$ & 0935 & 1.0 & -- & .01 & .00 & .07 & .61 & .68 & .68 & 3.0 & .030 \\
\hline गUN, & $8^{1135}$ & 1.0 & .00 & .01 & .01 & .05 & .19 & .24 & .25 & 1.1 & .050 \\
\hline $01 \ldots$ & 0930 & 1.0 & .20 & .03 & .23 & .06 & .57 & .63 & .86 & 3.8 & .030 \\
\hline
\end{tabular}


295629093504500 LINE 254 SITE 04

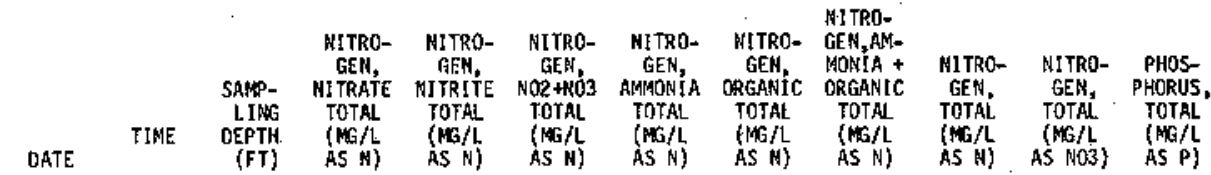

OCT, 1976

\begin{tabular}{|c|c|c|c|c|c|c|c|c|c|c|c|}
\hline $18 \ldots$ & 1445 & 1.0 & .15 & .03 & .18 & .09 & .60 & .69 & .97 & 3.9 & .050 \\
\hline $16 . .$. & 1100 & 1.0 & .12 & .01 & .13 & .19 & .62 & .81 & +94 & 4.2 & .070 \\
\hline fE8... 1977 & $7^{1020}$ & 1.0 & .21 & 01 & .22 &, 34 & .86 & 1.2 & 1.4 & 6.3 & .070 \\
\hline JUN & 1000 & 1.0 & .12 & .00 & .12 & .15 & .45 & .60 & .72 & 3.2 & .040 \\
\hline SEP & 0845 & 1.0 & .00 & .01 & .01 & .03 & .35 & .38 & .39 & 1.7 & .010 \\
\hline JUN, . 1978 & $8^{1115}$ & 1.0 & .05 & .03 & .08 & .05 & .64 & .69 & .77 & 3.4 & .070 \\
\hline
\end{tabular}

295109093472000 LLAE 274 S1TE 01

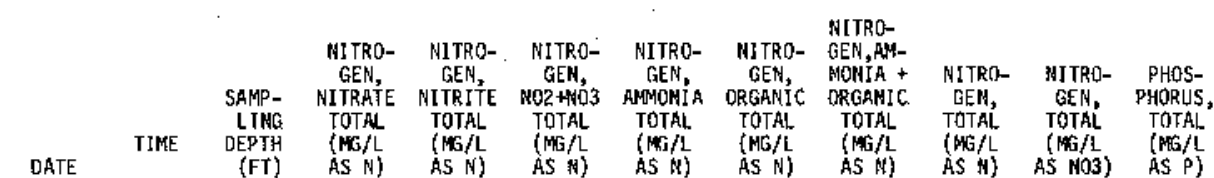

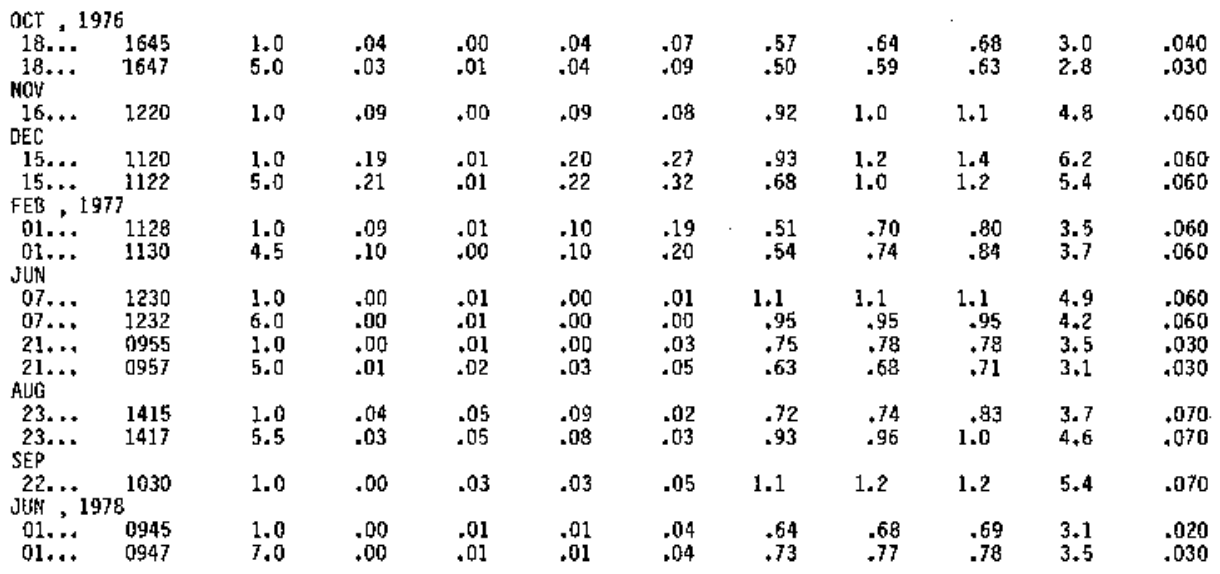

295228093492000 LINE 274 SITE 02

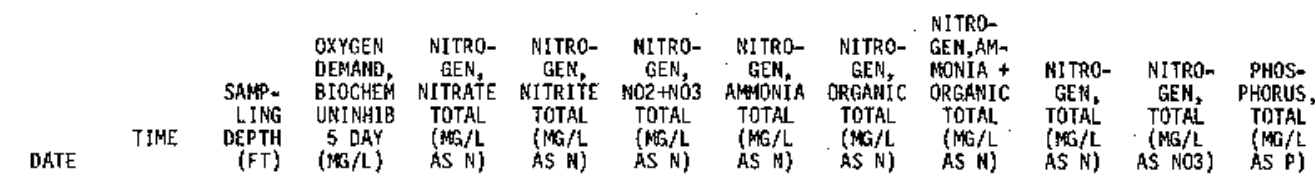

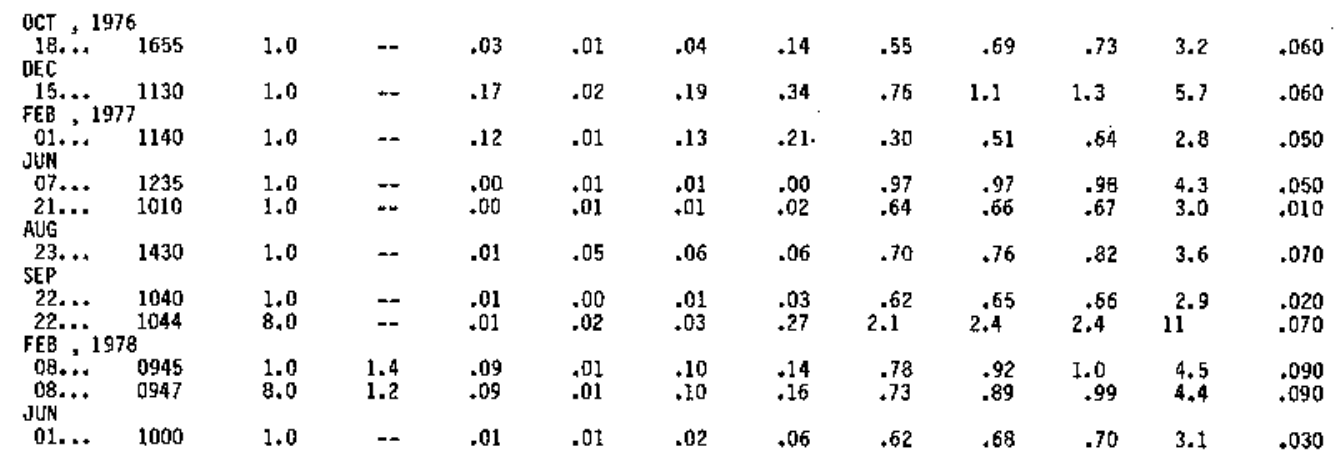


295329093505500 LINE 274 SITE 03

\begin{tabular}{|c|c|c|c|c|c|c|c|c|c|c|c|}
\hline & & & & 29532909 & 3505500 & LINE 274 & SITE 0३ & & & & \\
\hline DATE & TIME & $\begin{array}{l}\text { SAMP - } \\
\text { LING } \\
\text { DEPTH } \\
\text { (FT) }\end{array}$ & $\begin{array}{c}\text { NITRO- } \\
\text { GEN, } \\
\text { NITRATE } \\
\text { TOTALL } \\
\text { (MG/L } \\
\text { AS } M \text { ) }\end{array}$ & $\begin{array}{l}\text { NITRO- } \\
\text { GEN, } \\
\text { NITRITE } \\
\text { TOTAL } \\
\text { (MG } / \text { L } \\
\text { AS N) }\end{array}$ & $\begin{array}{c}\text { NITRO- } \\
\text { GER, } \\
\text { NO2 +NO3 } \\
\text { TDTAL } \\
\text { (MG } / L \\
\text { AS N) }\end{array}$ & $\begin{array}{l}\text { NITRO- } \\
\text { GEN, } \\
\text { AMMONIA } \\
\text { TOTAL } \\
\text { (MG/L } \\
\text { AS N) }\end{array}$ & $\begin{array}{c}\text { NITRO- } \\
\text { GEN, } \\
\text { ORGANIC } \\
\text { JOTAL } \\
\text { (MG } / L \\
\text { AS N) }\end{array}$ & $\begin{array}{l}\text { NITRO- } \\
\text { GEN, AM- } \\
\text { MONIA + } \\
\text { ORGANIC } \\
\text { TOTAL } \\
\text { (MG/L } \\
\text { AS N) }\end{array}$ & $\begin{array}{l}\text { NITRO- } \\
\text { GEN, } \\
\text { TUTAL } \\
\text { (MG/L } \\
\text { AS N) }\end{array}$ & $\begin{array}{l}\text { NITRO- } \\
\text { GEN, } \\
\text { TOTAL } \\
\text { (WGA } A \\
\text { AS NO3) }\end{array}$ & $\begin{array}{c}\text { PHOS- } \\
\text { PHCORUS, } \\
\text { TOTAL } \\
\text { (MG/L } \\
\text { AS P) }\end{array}$ \\
\hline oct, & & & & & & & & & & & \\
\hline & 1705 & 1.0 & .20 & .04 & .24 & .12 & .53 & .65 & .89 & 3.9 & .060 \\
\hline & 1255 & 1.0 & .12 & .01 & .13 & .18 & .82 & 1.0 & 1.1 & 5.0 & .600 \\
\hline $\begin{array}{l}15 \ldots \\
\text { FEB , }\end{array}$ & 1140 & 1.0 & .15 & .01 & .16 & .29 & $.7 \mathrm{I}$ & 1.0 & 1.2 & 5.1 & .060 \\
\hline JUN & $\$ 155$ & 1.0 & .12 & .00 & .12 & .16 & .45 & .61 & .73 & 3.2 & .050 \\
\hline $\begin{array}{l}07 \ldots \\
21 \ldots \\
\text { AUG }\end{array}$ & $\begin{array}{l}1245 \\
1020\end{array}$ & $\begin{array}{l}1.0 \\
1.0\end{array}$ & .00 & .01 & .01 & .02 & $\begin{array}{c}1.5 \\
.75\end{array}$ & $\begin{array}{c}1.5 \\
.80\end{array}$ & $\begin{array}{c}1.5 \\
.81\end{array}$ & $\begin{array}{l}6.7 \\
3.6\end{array}$ & $\begin{array}{r}.050 \\
.010\end{array}$ \\
\hline$\underset{S E P}{23} \ldots$ & 1440 & 1.0 & .00 & .03 & .03 & .00 & .24 & .24 & .27 & 1.2 & .030 \\
\hline 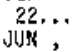 & $8^{1050}$ & 1.0 & .00 & .01 & .01 & .05 & .21 & .26 & .27 & 1.2 & .040 \\
\hline $01 .$. & 1010 & 1.0 & .02 & .01 & .03 & .08 & .63 & .71 & .74 & 3.3 & .030 \\
\hline
\end{tabular}

295402093514300 LINE 274 SITE 04

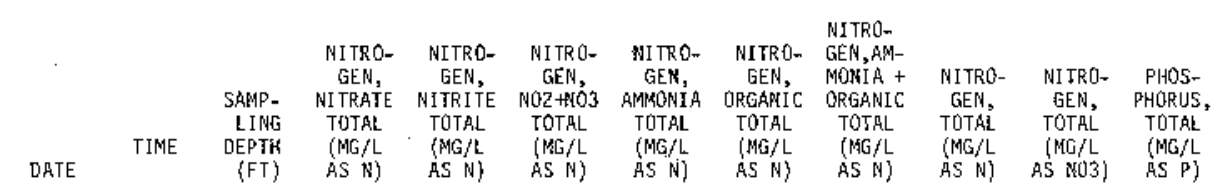

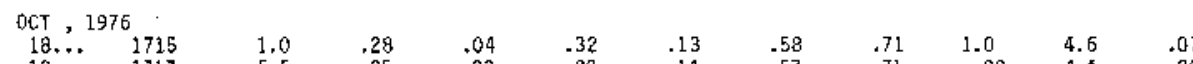

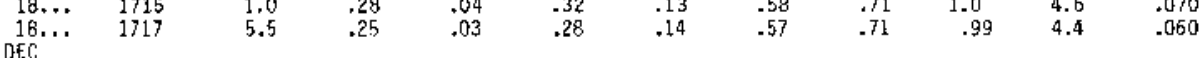

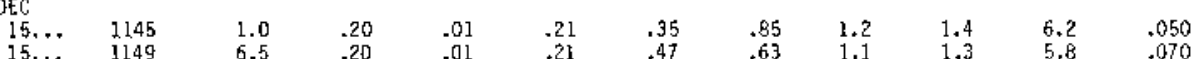

FEB, 1977

01... 1210

1.0

$\begin{array}{llllllll}.01 & .12 & .17 & .13 & .30 & .42 & 1.9 & .040\end{array}$

JUN 1212

$07 \ldots 1300$

$07 . .1302$

$21 \ldots .1025$

$21 \cdots \quad 1027$

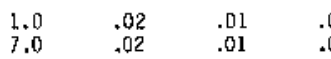

$23 . . . \quad 1445$

SEP

22... 110

$\begin{array}{ll}16 \ldots & 1310 \\ 16 . \ldots & 1312\end{array}$

$16 . \cdots{ }^{1312}$

$01, . .1018$

$\begin{array}{lll}1.0 & .00 & .00 \\ 7.0 & .00 & .00\end{array}$

21

$1.0 \quad-0.0$

.01

$1.0 \quad .01 \quad .00$

$\begin{array}{lllllll}.03 & .04 & .96 & 1.0 & 1.0 & 4.6 & .030\end{array}$

$\begin{array}{lllllll}.03 & .06 & 1.2 & 1.3 & 1.3 & 5.9 & .050 \\ .00 & .02 & .74 & .76 & .76 & 3.4 & .040 \\ .00 & .02 & .83 & .85 & .85 & 3.8 & .040\end{array}$

$01 . .102$

$\begin{array}{lll}1.0 & .11 & .01 \\ 6.0 & .15 & .02\end{array}$

$\begin{array}{lll}1.0 & .00 & .01 \\ 6.0 & .00 & .01\end{array}$

294735093545900 LINE 300 SITE 02

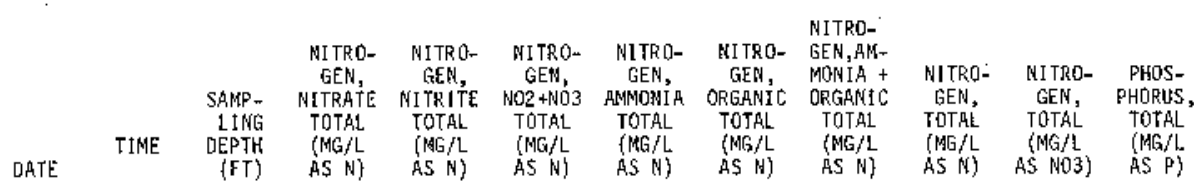

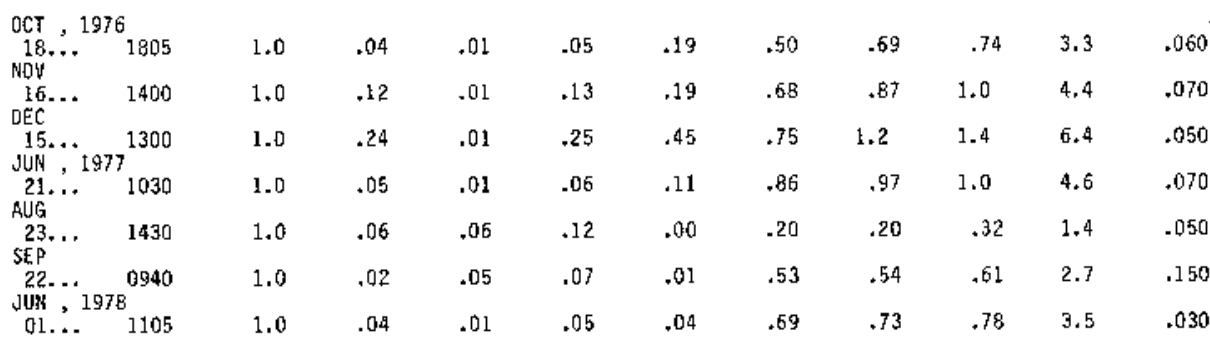


294605093535000 LIHE 308 SITE 02

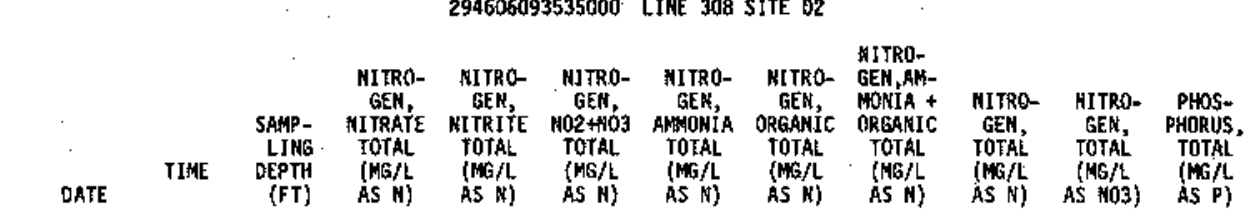

294945093571700 LINE 339 SITE 02

\begin{tabular}{|c|c|c|c|c|c|c|c|c|c|c|c|c|}
\hline ATE & TJME. & $\begin{array}{l}\text { SAMP- } \\
\text { LIMG } \\
\text { DEPTH } \\
\text { (FF) }\end{array}$ & $\begin{array}{l}\text { OXYGEN } \\
\text { DEMAND, } \\
\text { BIOCHEM } \\
\text { UNINHIB } \\
5 \text { OAY } \\
\text { (MG/L) }\end{array}$ & $\begin{array}{l}\text { NITRO- } \\
\text { GEN, } \\
\text { NITRATE } \\
\text { TOTAL } \\
\text { (NG/L } \\
\text { AS N) }\end{array}$ & $\begin{array}{l}\text { HITRO- } \\
\text { GEN, } \\
\text { NITRITE } \\
\text { TOTAL } \\
\text { (MG/L } \\
\text { AS N) }\end{array}$ & $\begin{array}{l}\text { NITRO- } \\
\text { GEN, } \\
\text { NO2+NO3 } \\
\text { TOTAL } \\
\text { (MR/L } \\
\text { AS N) }\end{array}$ & $\begin{array}{l}\text { NITRO- } \\
\text { GEK, } \\
\text { AKWONIA } \\
\text { TOTAL } \\
\text { (MG/L } \\
\text { AS H) - }\end{array}$ & $\begin{array}{c}\text { NITRO- } \\
\text { GEN, } \\
\text { ORGANIC } \\
\text { TOTAL } \\
\text { (MG/L } \\
\text { - AS N) }\end{array}$ & $\begin{array}{l}\text { NITRO- } \\
\text { GEN,AM- } \\
\text { MONIA + } \\
\text { OKGANIC } \\
\text { TOTAL } \\
\text { (NG/L } \\
\text { AS N) }\end{array}$ & $\begin{array}{l}\text { NITRO- } \\
\text { GEN, } \\
\text { TOTAL } \\
\text { (NG/L } \\
\text { AS N) }\end{array}$ & $\begin{array}{l}\text { NITRO- } \\
\text { GEH, } \\
\text { TOTAL } \\
\text { (MGG/L } \\
\text { AS NO3) }\end{array}$ & $\begin{array}{c}\text { PHOS- } \\
\text { PHORUS, } \\
\text { TOTAL } \\
\text { (MG/L } \\
\text { AS P) }\end{array}$ \\
\hline
\end{tabular}

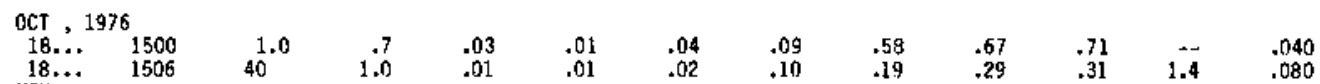

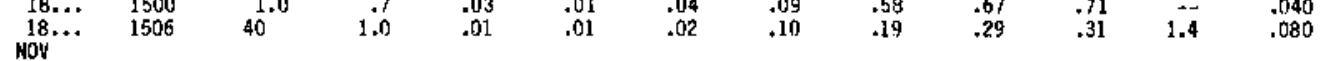

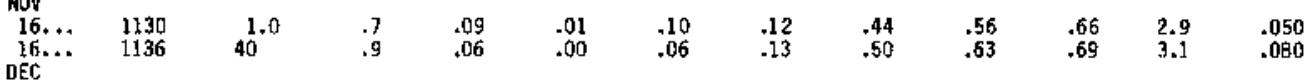

DEC

$15 . . . \quad 1145$

$\begin{array}{llll}1.0 & 1.5 & .09 & .02 \\ 43 & 1.4 & .04 & .01\end{array}$

$1.0 \quad--\quad .12$

01... 1345

JUH

07... 1415

$07 . . . \quad 1419$

$\begin{array}{ll}21 \ldots . . & 0900 \\ 21 \ldots & 0904\end{array}$

AUG 1300

$\begin{array}{ll}23 . \ldots & 1300 \\ 23 . \ldots & 1306\end{array}$

SEP 0811

$\begin{array}{ll}22 \ldots . . & 0811 \\ 22 . . . & 0816\end{array}$

FEB , 1978

OB... 1045

68... 1049

$\begin{array}{ll}01 \ldots . & 1300 \\ 01 \ldots . & 1305\end{array}$

$1.0 \quad 1.4 \quad .0$

$\begin{array}{ccc}1.0 & 1.4 & .03 \\ 45 & 1.0 & .01 \\ 44 & 2.2 & .15 \\ 4.0 & 1.9 & .06\end{array}$

.01

.011

$\begin{array}{llllll}.46 & 1.0 & 1.5 & 1.6 & 7.1 & .160\end{array}$

$\begin{array}{lll}1.0 & 1.0 & .2 \\ 39 & 2.2 & .07\end{array}$

$\begin{array}{llll}1.0 & 1.3 & .06 & .07 \\ 44^{1.3} & 1.3 & .04 & .04\end{array}$

$\begin{array}{llll}1.0 & .7 & .26 & .01 \\ 44 & 1.2 & .26 & .01\end{array}$

.13

$\begin{array}{lllll}.67 & .83 & .88 & 3.9 & .130\end{array}$

$\begin{array}{ccc}1.0 & 1.7 & .13 \\ 45 & 1.4 & .09\end{array}$

$294252093512000 \quad$ LINE 377 SITE 02

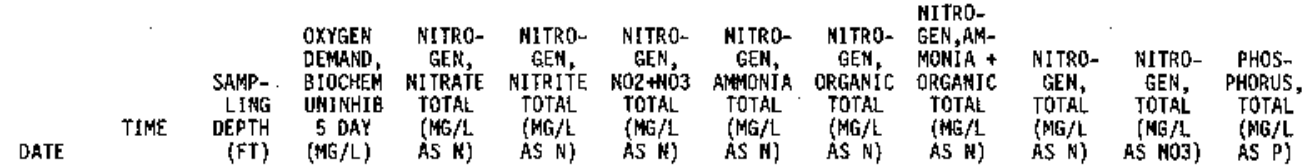

OCT , 1976

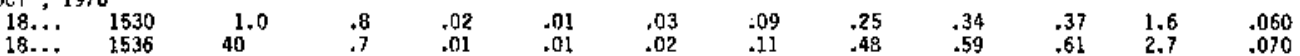

$18 . . \quad 1536$

$16 . .1015$

$\begin{array}{llll}1.0 & -.0 & .11 & .01 \\ 40 & -. & .03 & .01\end{array}$

$16 . . . \quad 102$

DEC $15 . . \quad 1230$

15... 1236

01... 1415

01... 1421

21... 0930

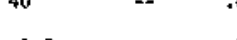

$\begin{array}{lll}1.0 & -- & .18 \\ 40 & -- & .0\end{array}$

$\begin{array}{lll}1.0 & -- & .14\end{array}$

$\begin{array}{ccc}1.0 & \cdots & .08\end{array}$

$\begin{array}{llll}1.0 & -- & .07 & .10 \\ 41 & - & .06 & .08\end{array}$

$\begin{array}{llll}1.00 & \because & .07 & .10 \\ 41 & \because .06 & .08\end{array}$

23... 1330

23... 1336

22... 0834

22. 4.0840

ol... 1230

$\begin{array}{llll}1.0 & -- & .02 & .05 \\ 44 & -- & .01 & .02\end{array}$

$\begin{array}{lll}1.0 & -. & .1 \\ 34 & - & .1\end{array}$

$\begin{array}{lllllll}.03 & .09 & .25 & .34 & .37 & 1.6 & .060 \\ .02 & .11 & .48 & .59 & .61 & 2.7 & .070 \\ .12 & .16 & .60 & .75 & .88 & 3.9 & .060 \\ .04 & .09 & .44 & .53 & .57 & 2.5 & .080 \\ .19 & .38 & .92 & 1.3 & 1.5 & 6.6 & .070 \\ .07 & .15 & .44 & .59 & .66 & 2.9 & .080 \\ .15 & .20 & .31 & .51 & .66 & 2.9 & .060 \\ .02 & .16 & .08 & .24 & .26 & 1.2 & .130 \\ .10 & .11 & .64 & .75 & .85 & 3.8 & .070 \\ .03 & .06 & 1.6 & 1.7 & 1.7 & 7.7 & .300 \\ .17 & .00 & .00 & .00 & .17 & .80 & .060 \\ .14 & .00 & .14 & .14 & .28 & 1.2 & .080 \\ .07 & .05 & .02 & .07 & .14 & .60 & .060 \\ .03 & .07 & .- & .03 & .06 & .30 & .060 \\ .19 & .12 & .30 & .42 & .61 & 2.7 & .020 \\ .12 & .20 & .80 & 1.0 & 1.1 & 5.0 & .040\end{array}$


Table IR.--Qudily of water in the Sabine-Neches estuary, water years 1977-78--Continued Nutrient Analyses--Continued

293702093492000 LINE 905 SITE 01

\begin{tabular}{|c|c|c|c|c|c|c|c|c|c|c|c|}
\hline ATE & TIME. & $\begin{array}{c}\text { SAMP- } \\
\text { LING } \\
\text { DEPTH } \\
\{F T\}\end{array}$ & $\begin{array}{l}\text { NITRO- } \\
\text { CEN, } \\
\text { NITRATE } \\
\text { TOTRL } \\
\text { (MG/L } \\
\text { MS N) }\end{array}$ & $\begin{array}{l}\text { NITRO- } \\
\text { GES, } \\
\text { NITRITE. } \\
\text { TOTAL } \\
\text { (MALL } \\
\text { AS NS) }\end{array}$ & $\begin{array}{c}\text { NITRO- } \\
\text { GLN, } \\
\text { NO2 + NOB } \\
\text { TOTAL } \\
\text { (ML/L } \\
\text { AS N) }\end{array}$ & $\begin{array}{c}\text { NITRO- } \\
\text { GEN, } \\
\text { ANMONIA } \\
\text { TOTAL } \\
(\mathrm{ME} / \mathrm{L} \\
\text { AS } \mathrm{N})\end{array}$ & $\begin{array}{c}\text { NITRO- } \\
\text { CEN, } \\
\text { ORCANIC } \\
\text { TOTAL } \\
\left(K K_{2} / L\right. \\
\text { ASN }\end{array}$ & $\begin{array}{l}\text { NITRO- } \\
\text { GEN,AM- } \\
\text { MONIA + } \\
\text { ORGRNIC } \\
\text { TOTAL. } \\
\text { (MG/L } \\
\text { AS N) }\end{array}$ & $\begin{array}{l}\text { NITRO- } \\
\text { GEN, } \\
\text { TOTAL } \\
\text { (MG/L } \\
\text { AS N) }\end{array}$ & $\begin{array}{c}\text { NITRO- } \\
\text { GLN;: } \\
\text { TDTAL } \\
\text { (NG/L } \\
\text { AS NO3) }\end{array}$ & $\begin{array}{c}\text { PHOS- } \\
\text { PHORUS, } \\
\text { TOTAL } \\
\text { (MG/L } \\
\text { AS P) }\end{array}$ \\
\hline
\end{tabular}

OCT , 1976

\begin{tabular}{|c|c|c|c|c|c|c|c|c|c|c|c|}
\hline OCT, 1 & & & & & & & & & & & \\
\hline $\begin{array}{l}18 \ldots \\
18 \ldots \\
\text { NoN }\end{array}$ & $\begin{array}{l}1605 \\
1611\end{array}$ & $\frac{1.0}{35}$ & $\begin{array}{l}.00 \\
.00\end{array}$ & $\begin{array}{l}.01 \\
.01\end{array}$ & $\begin{array}{l}.01 \\
.01\end{array}$ & $\begin{array}{l}.11 \\
.12\end{array}$ & $\begin{array}{l}.69 \\
.55\end{array}$ & $\begin{array}{l}.80 \\
.67\end{array}$ & .81 & $\begin{array}{l}3.6 \\
3.0\end{array}$ & $\begin{array}{l}.040 \\
.060\end{array}$ \\
\hline $\begin{array}{l}16 \ldots \\
16 \ldots\end{array}$ & $\begin{array}{l}1040 \\
1043\end{array}$ & 29 & $\begin{array}{l}.02 \\
.00\end{array}$ & $\begin{array}{l}.01 \\
.01\end{array}$ & $\begin{array}{l}.03 \\
.01\end{array}$ & $\begin{array}{l}.07 \\
.06\end{array}$ & $\begin{array}{r}.38 \\
.71\end{array}$ & $\begin{array}{l}.45 \\
.77\end{array}$ & .48 & $\begin{array}{l}2.1 \\
3.5\end{array}$ & $\begin{array}{l}.060 \\
.110\end{array}$ \\
\hline JUN , & & & & & & & & & & & \\
\hline $\begin{array}{l}21 \ldots \\
21 . \ldots\end{array}$ & $\begin{array}{l}1000 \\
1004\end{array}$ & $\frac{1.0}{42}$ & .00 & $\begin{array}{l}.01 \\
.03\end{array}$ & $\begin{array}{l}.01 \\
.03\end{array}$ & $\begin{array}{l}.03 \\
.09\end{array}$ & $\begin{array}{r}.32 \\
.82\end{array}$ & .35 & $\begin{array}{l}.36 \\
.94\end{array}$ & $\begin{array}{l}1.6 \\
4.2\end{array}$ & $\begin{array}{r}080 \\
.120\end{array}$ \\
\hline $\begin{array}{r}22 \ldots \\
22 . \ldots\end{array}$ & $\begin{array}{l}0901 \\
0905\end{array}$ & $\begin{array}{l}5.0 \\
46\end{array}$ & .00 & $\begin{array}{l}.01 \\
.01\end{array}$ & $\begin{array}{l}.01 \\
.03\end{array}$ & $\begin{array}{l}.03 \\
.05\end{array}$ & $\begin{array}{l}.02 \\
.43\end{array}$ & $\begin{array}{l}.05 \\
.48\end{array}$ & $\begin{array}{l}.06 \\
.51\end{array}$ & 2.30 & $\begin{array}{l}.040 \\
.090\end{array}$ \\
\hline JUN , 1 & & & & & & & & & & & \\
\hline $\begin{array}{l}01 \ldots . \\
01 . .\end{array}$ & $\begin{array}{l}1205 \\
1210\end{array}$ & $27^{1.0}$ & $\begin{array}{l}.22 \\
.04\end{array}$ & $\begin{array}{l}01 \\
.01\end{array}$ & $\begin{array}{l}.23 \\
.05\end{array}$ & $\begin{array}{l}.12 \\
.35\end{array}$ & $\begin{array}{l}.56 \\
.47\end{array}$ & $\begin{array}{r}.68 \\
.82\end{array}$ & $\begin{array}{l}.91 \\
.87\end{array}$ & $\begin{array}{l}4.0 \\
3.9\end{array}$ & $\begin{array}{l}.040 \\
.040\end{array}$ \\
\hline
\end{tabular}




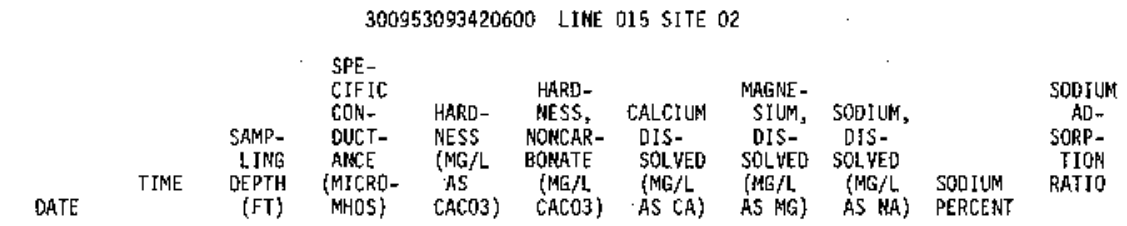

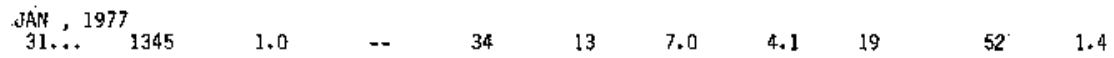

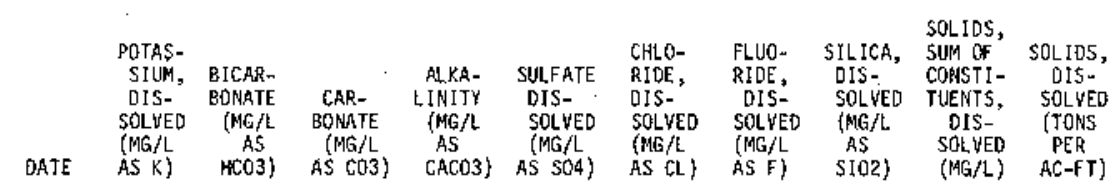

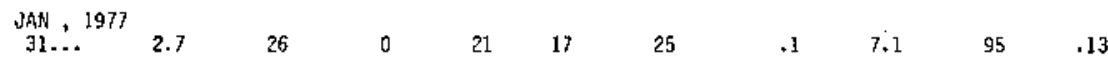
295938093465000 LINE 087 SITE $02^{\circ}$

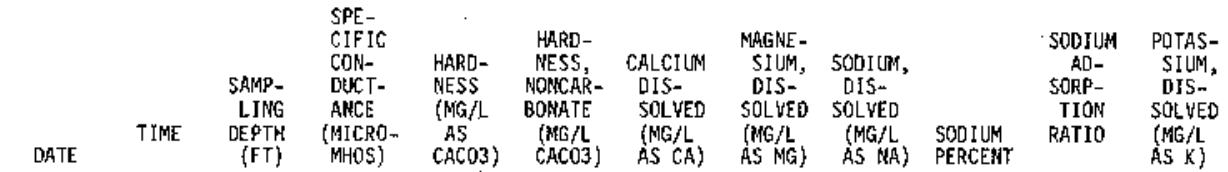

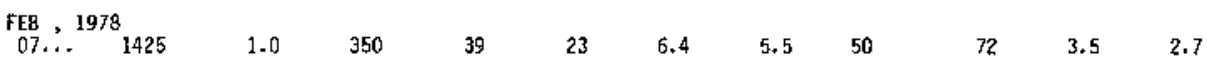

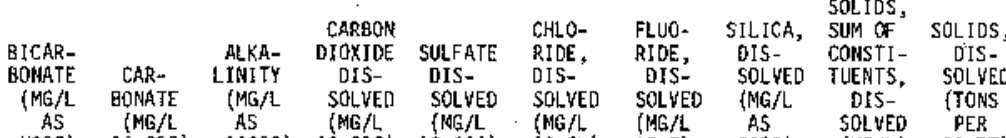

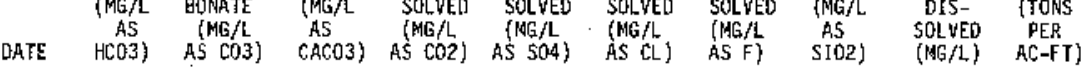

$\begin{array}{lllllllllll}\text { FEB }, 1978 & & & & & & & & & \\ 07 . . & 19 & 0 & 16 & 4.8 & 14 & 89 & .0 & 7.5 & 184 & .25\end{array}$

300319094014600 LINE 170 SITE 02

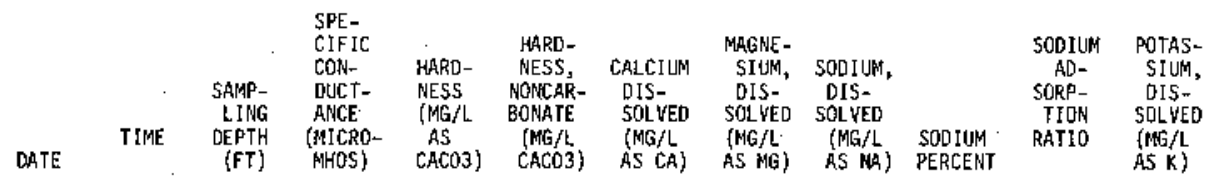

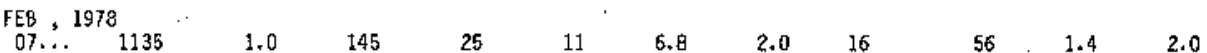

\begin{tabular}{|c|c|c|c|c|c|c|c|c|c|c|}
\hline LTE & $\begin{array}{c}\text { BICAR- } \\
\text { BOMATE } \\
\text { (MG/L } \\
\text { AS } \\
\text { HCOA) } \\
\text { (00440) }\end{array}$ & $\begin{array}{c}\text { CAR- } \\
\text { BOONATE } \\
(M G / L \\
\text { AS CO3) } \\
(00445)\end{array}$ & $\begin{array}{c}\text { ALKA- } \\
\text { LINITY } \\
\text { (MG } \\
\text { AS } \\
\text { CACO3) } \\
(00410)\end{array}$ & $\begin{array}{c}\text { CARBOH } \\
\text { DIOXIDE } \\
\text { OIS } \\
\text { SOLVED } \\
\text { (MG/L } \\
\text { AS CO2) } \\
\text { (OOA05) }\end{array}$ & $\begin{array}{l}\text { SHLFATE } \\
\text { DIS- } \\
\text { SOLVED } \\
\text { (MG/L } \\
\text { AS SO4) } \\
(00945)\end{array}$ & $\begin{array}{l}\text { CHLO- } \\
\text { RIDE, } \\
\text { OIS- } \\
\text { SOLVEO } \\
(N G / L \\
\text { AS CL) } \\
\text { (ODO40) }\end{array}$ & $\begin{array}{l}\text { FLUO- } \\
\text { RIDE, } \\
\text { DIS- } \\
\text { SOLVED } \\
\text { (NG/L } \\
\text { AS F) } \\
(00950)\end{array}$ & $\begin{array}{l}\text { SILICA, } \\
\text { DIS- } \\
\text { SOLVED } \\
\text { (MG/L } \\
\text { AS } \\
\text { SID2) } \\
(00955)\end{array}$ & $\begin{array}{l}\text { SOL IOS, } \\
\text { SUM OF } \\
\text { CONST! - } \\
\text { TUENTS, } \\
\text { DIS- } \\
\text { SOEVED } \\
\text { (MG } / 1) \\
(70301)\end{array}$ & $\begin{array}{c}\text { SOL IDS, } \\
\text { DIS- } \\
\text { SOLVED } \\
\text { (TONS } \\
\text { PER } \\
\text { AC-FT) } \\
(70303)\end{array}$ \\
\hline
\end{tabular}

$\begin{array}{lllllllllll}\text { FEB }, 1978 & 17 & 0 & 14 & 11 & 15 & 28 & .0 & 9.9 & 88 & .12 \\ 07 . . & 17 & 0 & \end{array}$

295728093464500 LINE 244 SITE 02

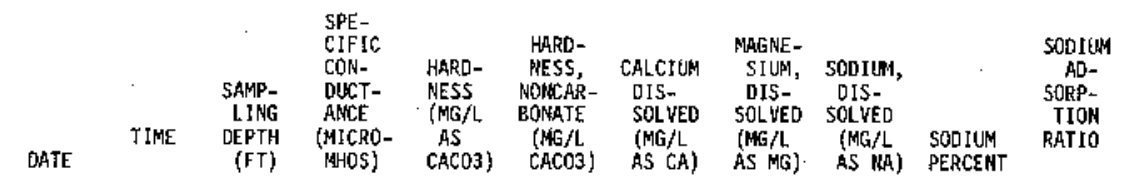

$\begin{array}{llllllllll}\text { JUN } . & 1977 \\ 21 . . & & & & & & & & & \\ 015 & 1.0 & 8600 & 810 & 780 & 61 & 160 & 1400 & 78 & 21\end{array}$ 
295729093464500 LINE 244 SITE 02--Continued

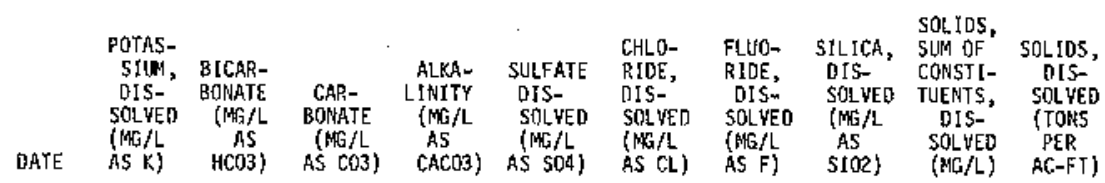

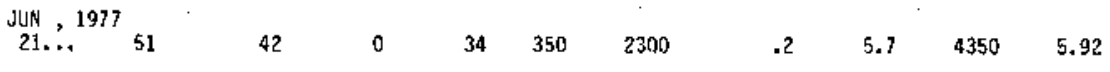

295720093474500 LIRE 244 SITE 03

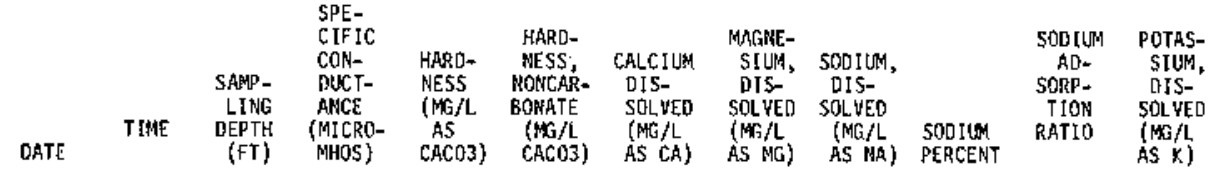

\begin{tabular}{|c|c|c|c|c|c|c|c|c|c|c|c|}
\hline $\begin{array}{l}\text { OCT }, 1976 \\
18 . . .\end{array}$ & 1505 & 1.0 & 17400 & 1700 & 1600 & 110 & 340 & 2800 & 77 & 30 & 110 \\
\hline & 1030 & 1.0 & 21000 & 2800 & 2700 & 200 & 550 & 4600 & 77 & 38 & 180 \\
\hline FEB , 1977 & 1030 & 1.0 & 9500 & 960 & 920 & 70 & 190 & 1800 & 79 & 25 & 64 \\
\hline נด1.'" & 1025 & 1.0 & $=$ & 570 & 540 & 45 & 110 & 1000 & 78 & 18 & 42 \\
\hline AUG & 1030 & 1.0 & 14000 & 1400 & 1400 & 100 & 230 & 2300 & 77 & 27 & 85 \\
\hline 23... & 1325 & 1.0 & 11090 & 1100 & 1000 & 80 & 210 & 2000 & 79 & 27 & 73 \\
\hline $01 . .$. & 0900 & 1.0 & 14700 & 1600 & 1500 & 110 & 310 & 2500 & 76 & 28 & 110 \\
\hline
\end{tabular}

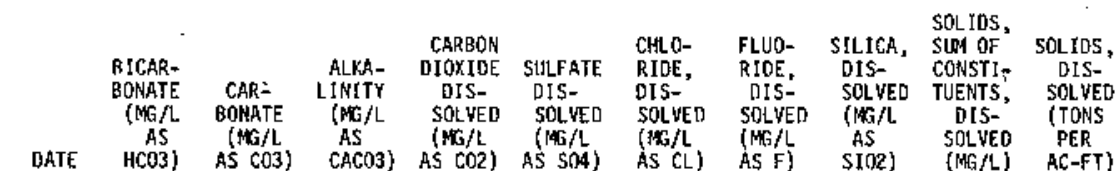

\begin{tabular}{|c|c|c|c|c|c|c|c|c|c|c|}
\hline${ }^{0 C \top}, 1976$ & 70 & 0 & 57 & -- & 690 & 5200 & .6 & 5.5 & 9290 & 12.6 \\
\hline DEC & 92 & 0 & 75 & -- & 1100 & 7900 & .6 & 3.7 & 14600 & 19.9 \\
\hline 15... & 46 & 0 & 38 & -- & 430 & 3000 & .3 & 6.2 & 5580 & 7.59 \\
\hline O1 & 33 & 0 & 27 & $=$ & 240 & 1800 & .2 & 7.1 & 3260 & 4.43 \\
\hline$\underset{\text { AUG }}{07 \ldots}$ & 59 & 0 & 48 & - & 630 & 4300 & .4 & 5.9 & 7730 & 10.5 \\
\hline JUN, 1972 & 56 & 0 & 46 & -- & 480 & 3400 & .3 & 7.1 & 6280 & 8.54 \\
\hline $01 \ldots$ & 68 & 0 & 56 & 2.7 & 680 & 4100 & .5 & 5.6 & 7850 & 10.7 \\
\hline
\end{tabular}

295402093514300 LINE 274 SITE 04

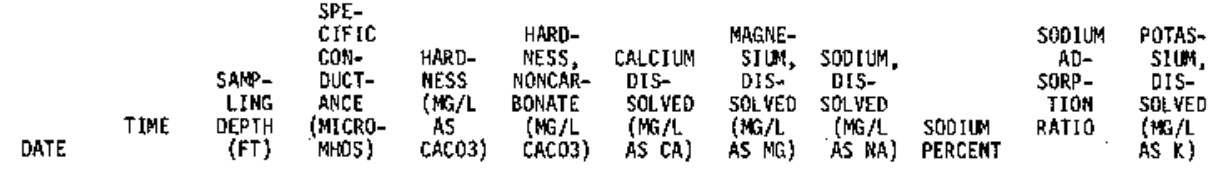

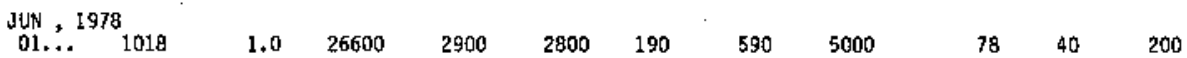

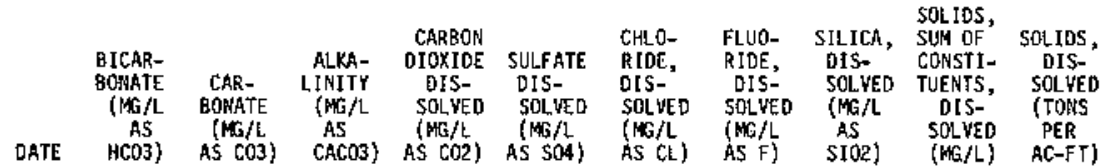

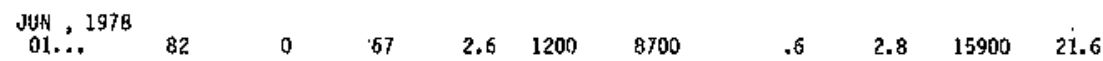


294735093545900 LINE $3 D 0$ SITE 02

\begin{tabular}{|c|c|c|c|c|c|c|c|c|c|c|c|}
\hline DATE & TIME & $\begin{array}{l}\text { SAMP- } \\
\text { LING } \\
\text { DEPTH } \\
(F T)\end{array}$ & $\begin{array}{l}\text { SPE- } \\
\text { CIFIC } \\
\text { CON- } \\
\text { DUCT- } \\
\text { ANCE } \\
\text { (MICRO- } \\
\text { MHOS) }\end{array}$ & $\begin{array}{l}\text { HARD- } \\
\text { NESS } \\
\text { (MG/L } \\
\text { AS } \\
\text { CACO3) }\end{array}$ & $\begin{array}{l}\text { HARQ- } \\
\text { NESS, } \\
\text { NONCAR- } \\
\text { BONATE } \\
\text { (MG } / L \\
\text { CACO3) }\end{array}$ & $\begin{array}{l}\text { CALCIUM } \\
\text { DIS- } \\
\text { SOL VEO } \\
\text { (MG/L } \\
\text { AS CA) }\end{array}$ & $\begin{array}{l}\text { MAGKE- } \\
\text { SIUM, } \\
\text { DIS- } \\
\text { SOLVED } \\
\text { (MG/L } \\
\text { AS MG) }\end{array}$ & $\begin{array}{l}\text { SODIUM, } \\
\text { DIS- } \\
\text { SOLVED } \\
\text { (MG } / L \text {. } \\
\text { AS NA) }\end{array}$ & $\begin{array}{l}\text { SODIUM } \\
\text { PERCERT }\end{array}$ & $\begin{array}{l}\text { SODIUM } \\
\text { AD- } \\
\text { SORP- } \\
\text { TION } \\
\text { RATIO }\end{array}$ & $\begin{array}{l}\text { POTAS- } \\
\text { SIUH, } \\
\text { DIS- } \\
\text { SOLYED } \\
\text { (MG/L } \\
\text { AS K) }\end{array}$ \\
\hline $\begin{array}{l}\text { OCT }, \\
19 . .\end{array}$ & ${ }^{6}{ }_{1805}$ & 1.0 & 16000 & 1600 & 1500 & 110 & 320 & 2600 & 77 & 28 & 100 \\
\hline $\begin{array}{l}16 . . . \\
\text { DEC }\end{array}$ & 1400 & 1.0 & 26000 & 3200 & 3100 & 220 & 650 & 5200 & 76 & 40 & 210 \\
\hline JUN :. & $7^{1300}$ & 1.0 & 16000 & 1700 & 1600 & 110 & 340 & 2900 & 77 & 31 & 130 \\
\hline AUG & 1030 & 1.0 & 19000 & 1800 & 1800 & 130 & 370 & 3300 & 78 & 33 & 110 \\
\hline SEP $23 . \cdots$ & 1430 & 1.0 & 21000 & 2200 & 2100 & 150 & 440 & 3000 & 73 & 28 & 140 \\
\hline $22 \ldots$ & 0940 & 1.0 & $\cdots$ & 2300 & 2300 & 160 & 470 & 3800 & 77 & 34 & 150 \\
\hline
\end{tabular}

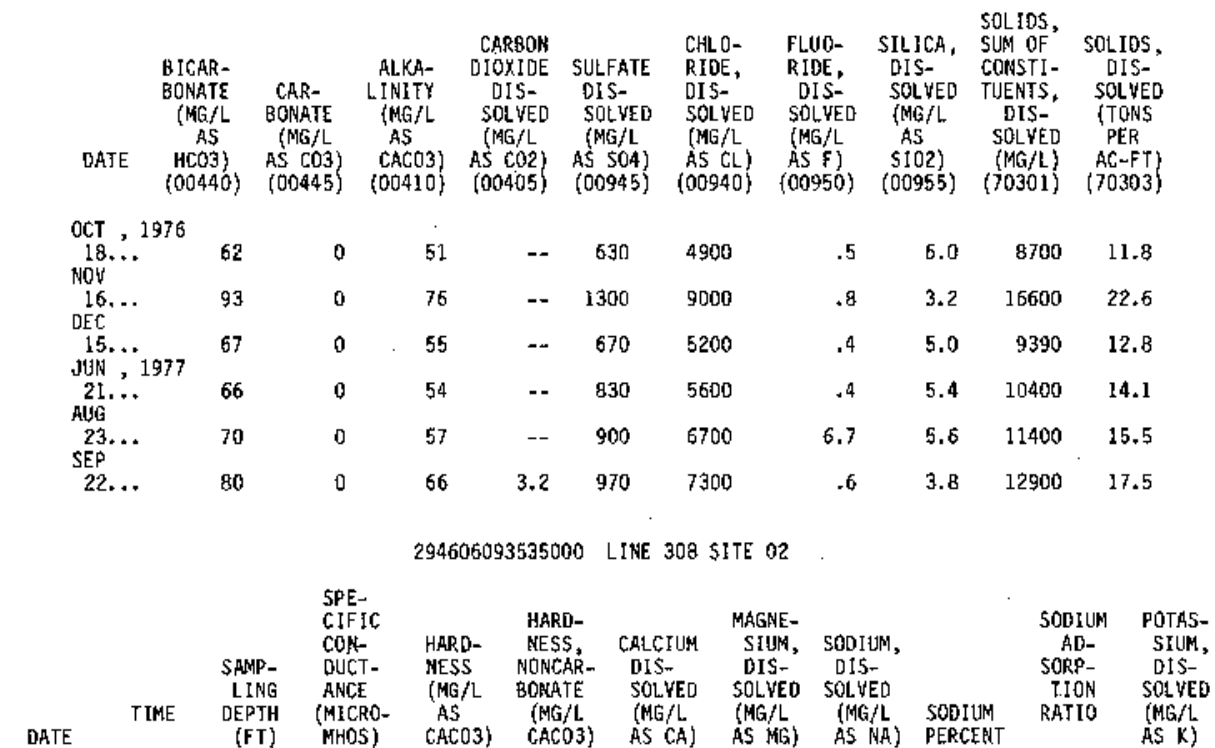

$\begin{array}{ccccccccccc}\text { FEB , } 1978 \\ \text { O8... } 1025 & -- & - & 310 & 290 & 25 & 61 & 510 . & 77 & 13 & 21\end{array}$

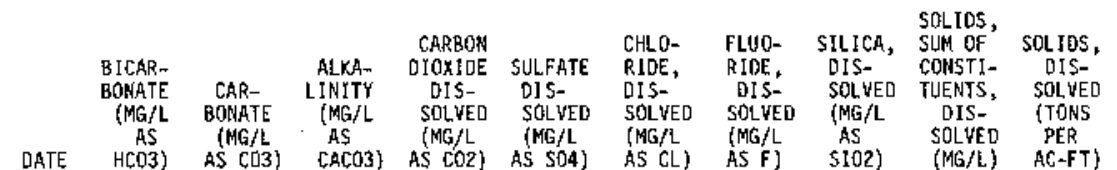

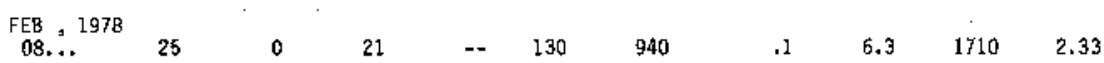

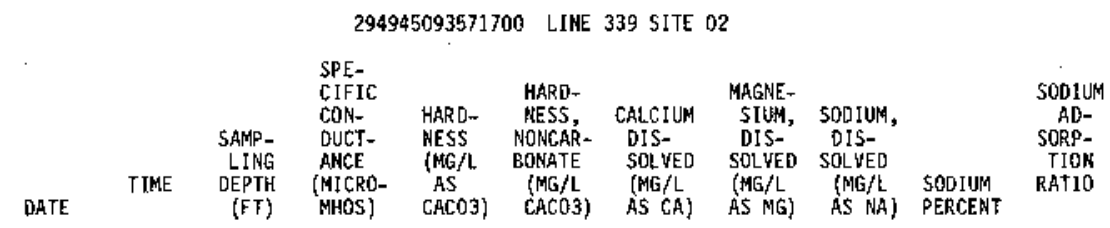

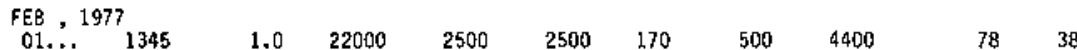


Table 1C.-4Quality of water in the Sabine-Neches estuary, water years 1977-78--Cont inued Chemical Analyses--Continued

294945093571700 LINE 339 SITE O2--Cont inued

\begin{tabular}{|c|c|c|c|c|c|c|c|c|c|c|}
\hline DATE & $\begin{array}{l}\text { POTAS- } \\
\text { SIUM, } \\
\text { DIS- } \\
\text { SOLYED } \\
\text { (MG } / L \\
\text { AS K) } \\
(00935)\end{array}$ & $\begin{array}{c}\text { BICAR- } \\
\text { BONATE } \\
\text { (MG } L L \\
\text { AS } \\
\text { HCO3) } \\
(00440)\end{array}$ & $\begin{array}{c}\text { CAR- } \\
\text { BONATE } \\
(16 G / L \\
\text { AS CO3) } \\
(00445)\end{array}$ & $\begin{array}{c}\text { ALKA- } \\
\text { LINITY } \\
\text { (MGA } \\
\text { A.S } \\
\text { CACO3) } \\
(00410)\end{array}$ & $\begin{array}{l}\text { SHLFATE } \\
\text { DIS- } \\
\text { SOLWED } \\
\text { (NG/L } \\
\text { AS SO4) } \\
\text { (00945) }\end{array}$ & $\begin{array}{l}\text { CHLO- } \\
\text { RIDE, } \\
\text { DIS- } \\
\text { SDLVED } \\
\text { (MG } / L \\
\text { AS CL) } \\
\text { (ODO40) }\end{array}$ & $\begin{array}{c}\text { FLUD- } \\
\text { RIDE, } \\
\text { DIS- } \\
\text { SOLWEO } \\
(\text { SQA L } \\
\text { AS F }) \\
(00950)\end{array}$ & $\begin{array}{l}\text { SILICA, } \\
\text { DIS- } \\
\text { SOLVED } \\
\text { (MLG/L } \\
\text { AS } \\
\text { S102) } \\
\text { (00955) }\end{array}$ & 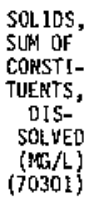 & $\begin{array}{c}\text { SOLIDS, } \\
\text { DIS- } \\
\text { SOLVED } \\
\text { (TOUS } \\
\text { PER } \\
\text { AC-FT) } \\
(70303)\end{array}$ \\
\hline 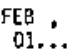 & 180 & 7 & 0 & 6 & 1000 & 7500 & .6 & 4.8 & 13890 & 18.8 \\
\hline
\end{tabular}




\section{Trinity-San Jacinto Estuary}

The Trinity-San Jacinto estuary, which has an area of about 590 square miles $\left(1,530 \mathrm{~km}^{2}\right)$, consists of the tidal parts of the Trinity and San Jacinto Rivers and other tributaries, the Houston Ship Channel, part of the Intracoastal Waterway, Galveston Bay, East Bay, West Bay, and Trinity Bay (Figure 3). Water depth at mean low water is less than 10 feet $(3.0 \mathrm{~m})$ in East Bay, West Bay, and Trinity Bay. Galveston Bay is generally less than 10 feet $(3.0 \mathrm{~m})$ deep except near Bolivar Road where the depth increases to about 40 feet $(12.2 \mathrm{~m})$. The Houston Ship Channel is more than 40 feet $(12.2 \mathrm{~m})$ deep, and the Intracoastal Waterway is about 15 feet deep $(4.6 \mathrm{~m})$.

Water-quality data (Table 2) were collected during October-December 1976; February and April-September 1977; and January, February, and June 1978. Data for the San Jacinto River and for the upper part of the Houston Ship Channel are being collected by other agencies. 



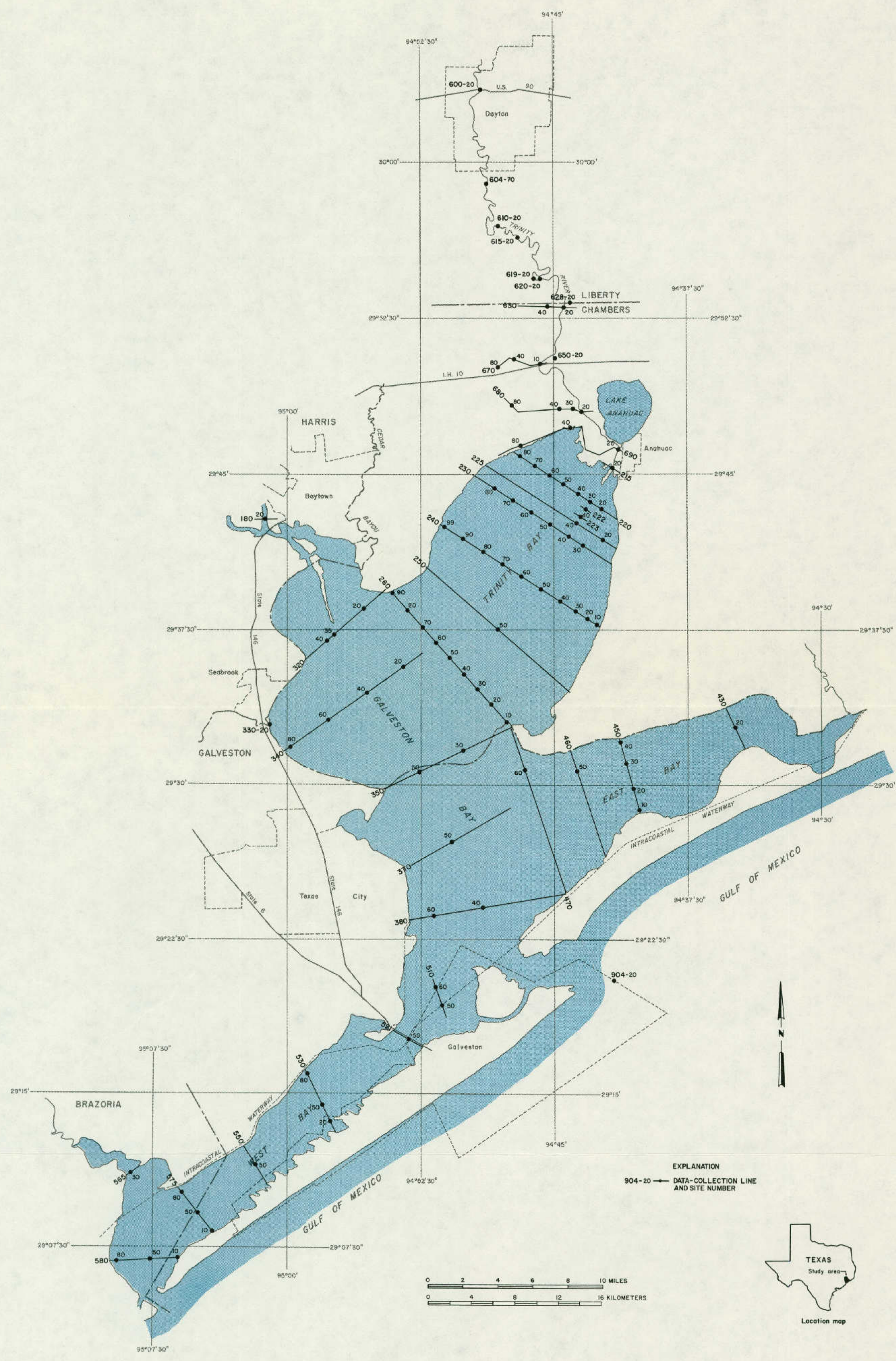

Figure 3

Data-Collection Sites in the Trinity-San Jacinto Estuary 

(FT = feet; MICROMHOS = micromhos per centimeter at $25^{\circ}$ Celsfus; DEG $C=$ degrees Celsius; $M=$ meters: $\mathrm{M}_{\mathrm{a}} / \mathrm{L}=$ milligrants per liter

294255095011300 LINE 180 SITE 20

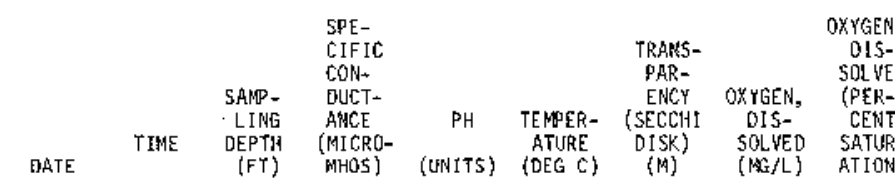

OCT, 1976

$\begin{array}{rr}\text { OCT. } 1976 \\ 21 \ldots & 1315 \\ 21 \ldots & 1317 \\ 21 \ldots & 1319 \\ \text { NOV } & \\ 18 . . & 1240 \\ 18 \ldots & 1242 \\ 18 . . & 1244 \\ 18 . . & 1246 \\ \text { FEB }, 1977 \\ \text { O3... } 1445\end{array}$

1.0 .22000

$$
\text { (OEG C) }
$$$$
\text { (M) (M) }
$$$$
\begin{array}{llllll}
1.0 & 22000 & 8.2 & 18.0 & .56 & - \\
20 & 23000 & 8.2 & 18.0 & -- & --
\end{array}
$$$$
40 \quad 27000 \quad 8.3 \quad 18.0 \quad--\quad 0--
$$$$
\begin{array}{ccccccc}
1.0 & 25000 & -- & 12.5 & .93 & 8.3 & 88 \\
10 & 28000 & -- & 12.5 & -- & 7.6 & 82
\end{array}
$$$$
\begin{array}{lllllll}
10 & 28000 & - & 12.5 & -- & 7.6 & 82 \\
25 & 37000 & -- & 12.0 & -- & 7.8 & 89 \\
42 & 39000 & -- & 12.0 & -- & 7.9 & 89
\end{array}
$$

$03 \ldots .1447$

03... 1449

$03 \ldots 1451$

गU1 $23 . . . \quad 1315$

$23 . . . \quad 1317$

$23 \ldots .1319$

AUG

$\begin{array}{ll}25 \ldots . . & 1230 \\ 25 . \ldots & 1232\end{array}$

$25 . . . \quad 1234$

$25 \ldots .1236$

FEB , 1978

$08 . . . \quad 1150$

$\begin{array}{ll}08 \ldots & 1152 \\ 08 . . . & 1154\end{array}$

JUN

07... 1131

$10.0 \quad 22000$

$\begin{array}{ll}10 & 24000 \\ 20 & 25000 \\ 42 & 30000\end{array}$

$\begin{array}{ll}7.6 & 10.5 \\ 7.6 & 10.5\end{array}$

10.5
10.5

$\begin{array}{ll}7.6 & 10.0 \\ 7.8 & 10.0\end{array}$

$\begin{array}{cc}1.0 & 18000 \\ 10 & 18000 \\ 25 & 18000\end{array}$

8.0
8.0
7.9
8.0

29.5

29.0
29.0
29.5

5

$59 \quad 8.4$

--

8.0
8.0

$--\quad 8.2$

$.38 \quad 5.6 \quad 79$
.59

$\begin{array}{ll}25 & 18000 \\ 45 & 19000\end{array}$

$1.0 \quad 24000$

$\begin{array}{ll}10 & 25000 \\ 20 & 26000\end{array}$

7.630 .5

$7.6 \quad 30.5$

$7.7 \quad 30.0$

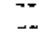

$\begin{array}{ll}- & 4.8 \\ - & 5.0\end{array}$

$.47 \quad 3.7$

$1.0-19100$

$\begin{array}{cc}1.0 & 19100 \\ 20 & 20000 \\ 42 & 23000\end{array}$

7.9
7.9
9.0

30.0

--

1.016500

$\begin{array}{ll}20 & 16500 \\ 45 & 19400\end{array}$

i::

7.5
7.5
7.5

$\begin{array}{ll}-- & 9.6 \\ - & 9.8\end{array}$

55

$07 . . \quad 1136$

294429094421800 LINE 215 SITE 20

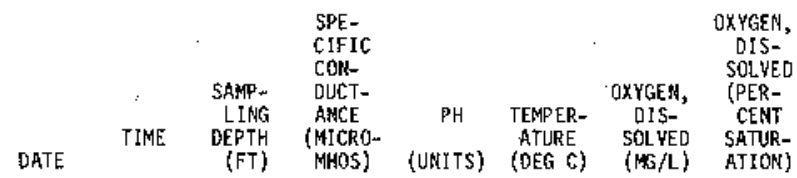

MAY 1977

$10 . . .1410$

140

$$
\begin{array}{llll}
6.0 & 320 & 7.5 & 24.5 \\
12 & 320 & 7.5 & 24.5
\end{array}
$$

$\begin{array}{ll}6.5 & 79 \\ 6.5 & 79\end{array}$

294330094421700 LINE 220 SITE 20

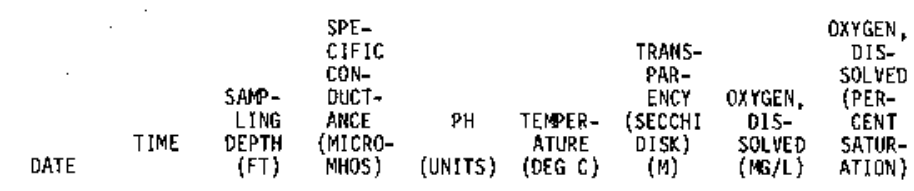

$\begin{array}{cc}\text { DEC , } 1976 \\ 10 . . . & 1120 \\ 10 . \ldots & 1122 \\ 13 . . . & 1320 \\ 13 \ldots . & 1322 \\ 29 . \ldots & 1100 \\ 29 . . & 1102\end{array}$

$\begin{array}{rr}1.0 & 16000 \\ 4.0 & 16000 \\ 1.0 & 10000 \\ 5.0 & 10000 \\ 1.0 & 1300 \\ 2.5 & 1300\end{array}$

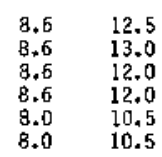

$\begin{array}{rr}.69 & 10.1 \\ -41 & 9.8 \\ .41 & 11.5 \\ .- & 11.4 \\ .19 & 10.2 \\ -- & 10.0\end{array}$

104
102
114
113
95
93 
TabTe 2A.--Quality of water in the Tr1nity-5an Jacinto estuary, water years 1977-78--Continued Field Determinations--Continued

294349094424800 L. INE 220 SITE 30

\begin{tabular}{|c|c|c|c|c|c|c|c|c|}
\hline DATE & T [HEE & $\begin{array}{l}\text { SAFP- } \\
\text { LING } \\
\text { DEPTH } \\
(\mathbf{F T})\end{array}$ & $\begin{array}{l}\text { SPE } \\
\text { CIFIC } \\
\text { COH- } \\
\text { DUCT- } \\
\text { ANCE } \\
\text { (AICRO- } \\
\text { MHOS) }\end{array}$ & $\begin{array}{c}\text { PH } \\
\text { (UNITS) }\end{array}$ & $\begin{array}{l}\text { TEMPER- } \\
\text { ATURE } \\
\text { (OEG C) }\end{array}$ & $\begin{array}{c}\text { TRANS- } \\
\text { PAR- } \\
\text { EACCY } \\
\text { (SECCHI } \\
\text { D!SK) } \\
(M)\end{array}$ & $\begin{array}{c}\text { OXYGEN, } \\
\text { OIS- } \\
\text { SOLYED } \\
\text { (MG/L) }\end{array}$ & $\begin{array}{c}\text { OXYGEN, } \\
\text { OLS- } \\
\text { SOLVED } \\
\text { (PER* } \\
\text { CENT } \\
\text { SATUR* } \\
\text { ATIOKN) }\end{array}$ \\
\hline \multicolumn{9}{|c|}{ OCT , 1976} \\
\hline $\begin{array}{l}21 \ldots . . \\
21 \ldots . . \\
21 \ldots\end{array}$ & $\begin{array}{l}1445 \\
1447 \\
1449\end{array}$ & $\begin{array}{l}1.0 \\
5.0 \\
8.0\end{array}$ & $\begin{array}{r}800 \\
1500 \\
9000\end{array}$ & $\begin{array}{l}8.3 \\
8.3 \\
8.2\end{array}$ & $\begin{array}{l}16.5 \\
16.0 \\
14.5\end{array}$ & $\begin{array}{r}.49 \\
-- \\
.\end{array}$ & $\begin{array}{l}9.4 \\
8.6 \\
7.1\end{array}$ & $\begin{array}{l}99 \\
91 \\
74\end{array}$ \\
\hline $\begin{array}{l}18 \ldots \\
18 \ldots . .\end{array}$ & $\begin{array}{l}1225 \\
1227\end{array}$ & $\begin{array}{l}1.0 \\
8.0\end{array}$ & $\begin{array}{l}27000 \\
42000\end{array}$ & $=$ & $\begin{array}{l}10.5 \\
10.5\end{array}$ & $\begin{array}{r}.34 \\
--\end{array}$ & $\begin{array}{l}10.2 \\
10.0\end{array}$ & $\begin{array}{l}95 \\
94\end{array}$ \\
\hline $\begin{array}{l}10 \ldots \\
10 \ldots \\
13 \ldots \\
13 \ldots \\
28 \ldots \\
28 \ldots \\
29 \ldots \\
29 \ldots \\
\text { vuL... }\end{array}$ & $\begin{array}{l}1330 \\
1332 \\
1420 \\
1422 \\
1205 \\
1207 \\
1230 \\
1232 \\
7\end{array}$ & $\begin{array}{l}1.0 \\
8.0 \\
1.0 \\
8.0 \\
1.0 \\
9.0 \\
1.0 \\
8.0\end{array}$ & $\begin{array}{l}220 \\
220 \\
250 \\
250 \\
330 \\
330 \\
330 \\
330\end{array}$ & $\begin{array}{l}7.7 \\
7.8 \\
8.0 \\
8.0 \\
7.8 \\
7.8 \\
7.0 \\
7.1\end{array}$ & $\begin{array}{l}11.5 \\
11.5 \\
11.5 \\
11.5 \\
11.0 \\
11.0 \\
11.0 \\
11.0\end{array}$ & $\begin{array}{l}-- \\
\overline{.13} \\
.13 \\
\overline{.33} \\
\overline{.-} \\
.25 \\
--\end{array}$ & $\begin{array}{r}9.6 \\
9.7 \\
10.1 \\
10.1 \\
9.4 \\
9.5 \\
9.3 \\
9.6\end{array}$ & $\begin{array}{l}91 \\
92 \\
95 \\
95 \\
88 \\
89 \\
87 \\
90\end{array}$ \\
\hline $\begin{array}{l}06 \ldots \\
06 \ldots \\
06 . \ldots\end{array}$ & $\begin{array}{l}1410 \\
1412 \\
1414\end{array}$ & $\begin{array}{l}1.0 \\
5.0 \\
12\end{array}$ & $\begin{array}{l}1250 \\
2200 \\
2400\end{array}$ & $\begin{array}{l}8.2 \\
8.1 \\
8.1\end{array}$ & $\begin{array}{l}31.0 \\
30.5 \\
30.0\end{array}$ & $\begin{array}{l}.52 \\
- \\
--\end{array}$ & $\begin{array}{l}7.0 \\
7.0 \\
6.2\end{array}$ & $\begin{array}{l}95 \\
95 \\
84\end{array}$ \\
\hline $\begin{array}{l}20 \ldots \\
20 \ldots \\
20 \ldots \\
20 \ldots\end{array}$ & $\begin{array}{l}1200 \\
1202 \\
1204 \\
1206\end{array}$ & $\begin{array}{r}1.0 \\
3.0 \\
5.0 \\
10\end{array}$ & $\begin{array}{l}12000 \\
16000 \\
18000 \\
17000\end{array}$ & $\begin{array}{l}8.3 \\
8.2 \\
8.2 \\
8.2\end{array}$ & $\begin{array}{l}27.5 \\
27.5 \\
27.5 \\
27.5\end{array}$ & $\begin{array}{l}.45 \\
- \\
- \\
-\end{array}$ & $\begin{array}{l}7.1 \\
5.8 \\
4.0 \\
4.0\end{array}$ & $\begin{array}{l}95 \\
78 \\
54 \\
54\end{array}$ \\
\hline
\end{tabular}

294416034433300 LJNE $2205 I T E 40$

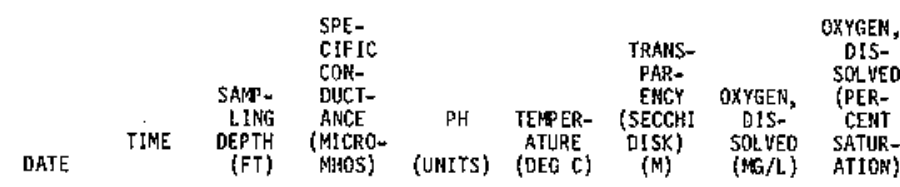

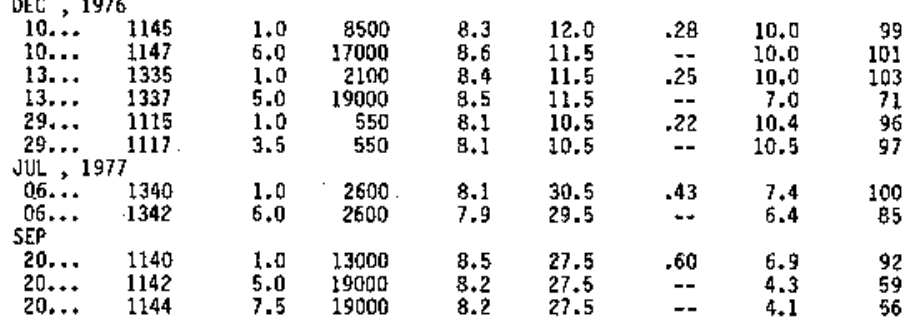

294443094441700 LINE 220 SITE 50

\begin{tabular}{|c|c|c|c|c|c|c|c|c|}
\hline DATE & TIME & $\begin{array}{r}\text { SAMP- } \\
\text { LING } \\
\text { DEPTH } \\
\text { (FT) }\end{array}$ & $\begin{array}{l}\text { SPE- } \\
\text { CIFIC } \\
\text { CON- } \\
\text { DUCT- } \\
\text { ANCE } \\
\text { (MICRO- } \\
\text { MHOS) }\end{array}$ & $\begin{array}{c}\mathrm{PH} \\
\text { (UN!TS) }\end{array}$ & $\begin{array}{l}\text { TEIPER- } \\
\text { ATURE } \\
\text { (DEG C) }\end{array}$ & $\begin{array}{l}\text { TRANS- } \\
\text { PAR- } \\
\text { ENCY } \\
\text { (SECCHI } \\
\text { DISK) } \\
\text { (M) }\end{array}$ & $\begin{array}{c}\text { OXYGEN, } \\
\text { DIS- } \\
\text { SOLLED } \\
\text { (YG/L) }\end{array}$ & $\begin{array}{l}\text { OXYGEN, } \\
\text { OIS- } \\
\text { SOLYED } \\
\text { (PER- } \\
\text { CENT } \\
\text { SATUR- } \\
\text { ATION) }\end{array}$ \\
\hline $\begin{array}{l}\text { OCT, } \\
21 . \cdots \\
21 . \cdots \\
\text { HON }\end{array}$ & $\begin{array}{l}6 \\
1535 \\
1537\end{array}$ & $\begin{array}{l}1.0 \\
6.0\end{array}$ & $\begin{array}{l}10000 \\
17000\end{array}$ & $\begin{array}{l}8.6 \\
8.4\end{array}$ & $\begin{array}{l}15.5 \\
15.0\end{array}$ & .37 & $\begin{array}{r}11.0 \\
8.7\end{array}$ & $\begin{array}{r}117 \\
95\end{array}$ \\
\hline $\begin{array}{l}18 . . \\
18 . .\end{array}$ & $\begin{array}{l}1210 \\
1212\end{array}$ & $\begin{array}{l}1.0 \\
6.0\end{array}$ & $\begin{array}{l}17000 \\
21000\end{array}$ & -- & $\begin{array}{l}10.0 \\
10.0\end{array}$ & $\begin{array}{r}1.16 \\
-2\end{array}$ & $\begin{array}{r}11.0 \\
9.8\end{array}$ & $\begin{array}{r}107 \\
97\end{array}$ \\
\hline
\end{tabular}




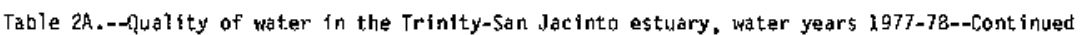
Field Determinations--Cont inued

294513094450300 LIIHE 220 \$ITE 60

\begin{tabular}{|c|c|c|c|c|c|c|c|c|}
\hline ATE & TIME & $\begin{array}{c}\text { SAPP- } \\
\text { LING } \\
\text { DEPTH } \\
\text { (FT) }\end{array}$ & $\begin{array}{l}\text { SPE- } \\
\text { CIFIC } \\
\text { CON- } \\
\text { DUICT- } \\
\text { ANCE } \\
\text { (MICRO- } \\
\text { MHOS) }\end{array}$ & $\begin{array}{c}\mathrm{PH} \\
\text { (UNITS) }\end{array}$ & $\begin{array}{l}\text { TEMPER- } \\
\text { ATURE } \\
\text { (DEG C) }\end{array}$ & $\begin{array}{l}\text { TRANS- } \\
\text { PAR- } \\
\text { ENCY } \\
\text { (SECCHI } \\
\text { DISK) } \\
\text { (M) }\end{array}$ & $\begin{array}{c}\text { OXYGEN, } \\
\text { OIS- } \\
\text { SOLVE[] } \\
\text { (MG/L) }\end{array}$ & $\begin{array}{c}\text { OXYGEN, } \\
\text { DIS- } \\
\text { SOLVED } \\
\text { (PER- } \\
\text { CENT } \\
\text { SATUR- } \\
\text { ATION) }\end{array}$ \\
\hline
\end{tabular}

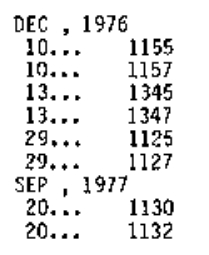

$\begin{array}{rrrrrrr}1.0 & 11000 & 8.3 & 12.0 & .56 & 10.1 & 101 \\ 6.0 & 14000 & 8.4 & 12.0 & -- & 9.8 & 99 \\ 1.0 & 8000 & 8.4 & 12.5 & .30 & 10.8 & 108 \\ 6.0 & 10000 & 8.4 & 12.0 & -- & 10.0 & 99 \\ 1.0 & 720 & 8.2 & 10.5 & .23 & 10.4 & 96 \\ 4.0 & 930 & 8.2 & 10.5 & -2 & 10.0 & 93 \\ & & & & & & \\ 1.0 & 15000 & 8.5 & 27.5 & .40 & 6.5 & 87 \\ 5.5 & 17090 & 8.2 & 27.5 & \ldots & 4.1 & 55\end{array}$

294541094454900 LINE 220 SITE 70

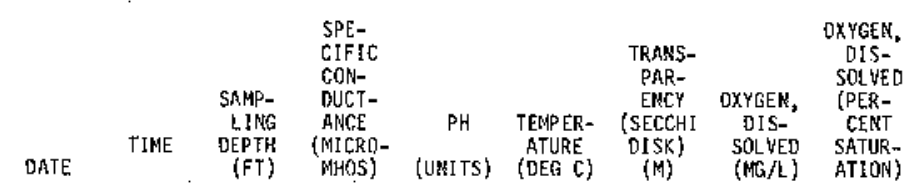

\begin{tabular}{rrrrrrrrr}
\multicolumn{2}{c}{ OCT, 1976} & & & & & & & \\
$21 \ldots$ & 1545 & 1.0 & 15000 & 8.6 & 16.0 & .52 & 11.8 & 130 \\
$21 \ldots$ & 1547 & 6.0 & 16000 & 8.4 & 15.0 & -- & 9.4 & 102 \\
NOW & &. & & & & & & \\
$18 \ldots$ & 1200 & 1.0 & 18900 &.- & 10.0 & .94 & 10.8 & 105 \\
$18 \ldots$ & 1202 & 5.0 & 20000 & -- & 10.0 &.- & 9.5 & 94
\end{tabular}

294611094163800 LINE 220 SITE 80

\begin{tabular}{|c|c|c|c|c|c|c|c|c|}
\hline DATE & T [ME & $\begin{array}{l}\text { SAIP- } \\
\text { LING } \\
\text { DEPTH } \\
\text { (FT) }\end{array}$ & $\begin{array}{l}\text { SPE- } \\
\text { CIFIC } \\
\text { CON- } \\
\text { DUCT- } \\
\text { ANCE } \\
\text { (MICRO- } \\
\text { MHOS) }\end{array}$ & $\begin{array}{c}\mathrm{PH} \\
\text { (UhITS) }\end{array}$ & $\begin{array}{l}\text { TEMPER- } \\
\text { ATURE } \\
\text { (DEG C) }\end{array}$ & $\begin{array}{l}\text { TRANS- } \\
\text { PAR- } \\
\text { ENCY } \\
\text { (SECCHI } \\
\text { ElSK) } \\
\text { (M) }\end{array}$ & $\begin{array}{c}\text { OXYGEN, } \\
\text { DIS } \\
\text { SOLVED } \\
\text { (MG } / L)\end{array}$ & $\begin{array}{c}\text { OXYGEN } \\
\text { DIS- } \\
\text { SOLYE } \\
\text { (PER- } \\
\text { CENT } \\
\text { SATUR } \\
\text { ATION }\end{array}$ \\
\hline
\end{tabular}

$\begin{array}{rr}1.0 & 9000 \\ 5.0 & 10000 \\ 1.0 & 9000 \\ 3.0 & 10000 \\ 2.0 & 600\end{array}$

$1.0 \quad 1500$ $\begin{array}{ll}8.4 & 1 \\ 8.4 & 1 \\ 8.4 & 1 \\ 8.5 & 1 \\ 8.3 & 1\end{array}$

$8.5 \quad 31.5$

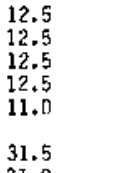

2.5
2.5
2.5
2.5
1.0
1.5
1.0

294322094430700 LINE 222 SITE 40

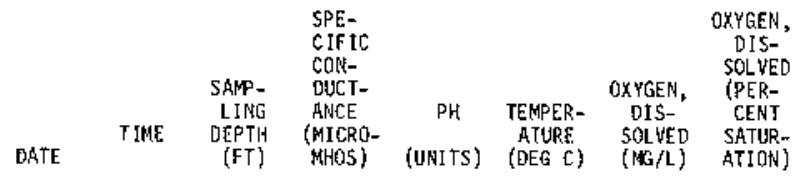

$$
\text { DEC , } 1976
$$

DEC, 1976

$10 \ldots \quad 1400$

$$
\begin{array}{rrrrrr}
1.0 & 3000 & 7.8 & 11.5 & 9.5 & 91 \\
4.0 & 4500 & 8.0 & 12.0 & 9.8 & 95 \\
6.0 & 12000 & 8.4 & 12.0 & 9.8 & 98
\end{array}
$$

\begin{tabular}{|c|c|c|c|c|c|c|c|c|}
\hline QATE & TIME & $\begin{array}{l}\text { SAMP } \\
\text { L1WG } \\
\text { DEPTH } \\
\text { (FT) }\end{array}$ & $\begin{array}{l}\text { SPE- } \\
\text { CIFIC } \\
\text { CON- } \\
\text { DUCT- } \\
\text { ANCE } \\
\text { (MICRO- } \\
\text { MHOS) }\end{array}$ & $\begin{array}{c}\mathrm{PH} \\
\text { (UNITS) }\end{array}$ & $\begin{array}{c}\text { TEMPER- } \\
\text { ATURE } \\
\text { (OEG C) }\end{array}$ & $\begin{array}{l}\text { TRANS- } \\
\text { PAR- } \\
\text { ENCY } \\
\text { (SECCHI } \\
\text { DISK) } \\
\text { (MI) }\end{array}$ & $\begin{array}{c}\text { OXYGEN, } \\
\text { DIS- } \\
\text { SDE VED } \\
\{\text { MO } / \mathrm{L}\}\end{array}$ & $\begin{array}{l}\text { OXYGEN, } \\
\text { DIS- } \\
\text { SOLVED } \\
\text { (PER- } \\
\text { CENT } \\
\text { SATUR- } \\
\text { ATION) }\end{array}$ \\
\hline & & & & & & & & \\
\hline 10. & $\begin{array}{l}1350 \\
1352\end{array}$ & $\begin{array}{l}1.0 \\
8.0\end{array}$ & $\begin{array}{l}15000 \\
16000\end{array}$ & $\begin{array}{l}8.5 \\
8.4\end{array}$ & $\begin{array}{l}13.0 \\
11.5\end{array}$ &. & $\begin{array}{r}10.6 \\
9.2\end{array}$ & $\begin{array}{r}109 \\
93\end{array}$ \\
\hline
\end{tabular}

294256094432600 LINE 223 SITE 40 
Table 2A,--Quality of water in the Trinity-San Jacinto estuary, water years $1977-78--C o n t$ inued Ffeld Determinations=-Cont 1 nued

29414909442240D LINE 225 SITE 20

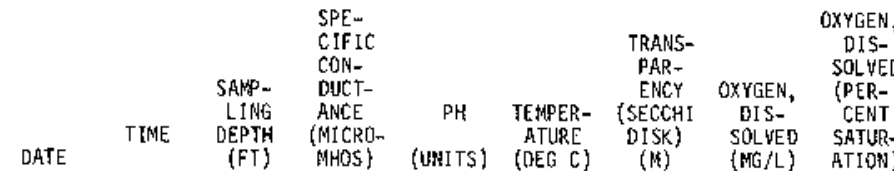
DEC, 1976

$\begin{array}{lllllllll}10 . . . & 1315 & 1.0 & 19000 & 8.6 & 12.0 & .41 & 10.2 & 105 \\ 10 . . & 13.7 & 6.5 & 19000 & 8.6 & 12.0 & -. & 10.2 & 105\end{array}$

294232094434400 LINE 225 SITE 40

\begin{tabular}{|c|c|c|c|c|c|c|c|c|}
\hline DATE & TIME & $\begin{array}{r}\text { SAMP- } \\
\text { LING } \\
\text { DEPTH } \\
\text { (FT) }\end{array}$ & $\begin{array}{l}\text { SPE- } \\
\text { CIFIC } \\
\text { CON- } \\
\text { DUCT- } \\
\text { ANCE } \\
\text { (MICRO- } \\
\text { MHOS) }\end{array}$ & $\begin{array}{c}\mathrm{PH} \\
\text { (UNITS) }\end{array}$ & $\begin{array}{c}\text { TEMPER- } \\
\text { ATURE } \\
\text { (OEG C) }\end{array}$ & $\begin{array}{l}\text { TRANS- } \\
\text { PAR- } \\
\text { ENCY } \\
\text { (SECCHI } \\
\text { DISK) } \\
\text { (M) }\end{array}$ & $\begin{array}{c}\text { OXYGER, } \\
\text { OIS- } \\
\text { SOLVED } \\
\text { (MG/L) }\end{array}$ & $\begin{array}{l}\text { OXYGEN, } \\
\text { OIS- } \\
\text { SDLYED } \\
\text { (PER- } \\
\text { CENT } \\
\text { SATUR- } \\
\text { ATION) }\end{array}$ \\
\hline
\end{tabular}
DEC , 1976

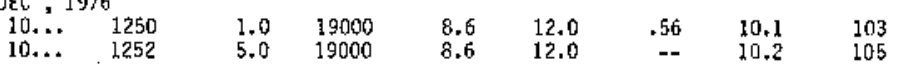

294122094424400 LINE 230 SITE 20

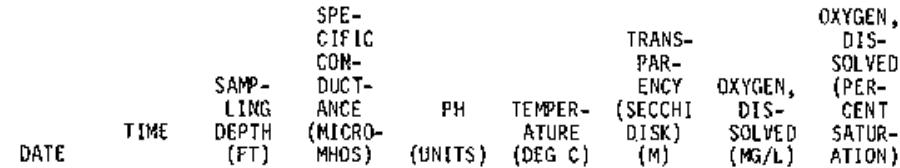

DEC , 1976

DEC, 1976

$13 . . .1300$

$13 \ldots .1304$

$29 . . . \quad 1215$

29... 1217

APR , 1977

26... 1345

$26 \ldots \quad 1347$

$26 \ldots 1349$

MAY

$04 . . .1315$

$04 . . . \quad 1317$

JUN 1300

$16 \ldots .1302$

JUL

$06 . . .1105$

06... 1107

06.

$\begin{array}{lllllllll}20 \ldots . . & 1040 & 1.0 & 21000 & 8.2 & 27.5 & .58 & 6.4 & 89 \\ 20 . . & 1042 & 7.0 & 21000 & 8.2 & 27.0 & -- & 5.5 & 75\end{array}$

$\begin{array}{rr}1.0 & 8000 \\ 5.0 & 9000 \\ 7.0 & 21000 \\ 1.0 & 650 \\ 5.5 & 2200 \\ 1.0 & 390\end{array}$

1.0390

$\begin{array}{ll}3.5 & 420 \\ 7.0 & 450\end{array}$

M)

294143094432200 LINE 230 SITE 30

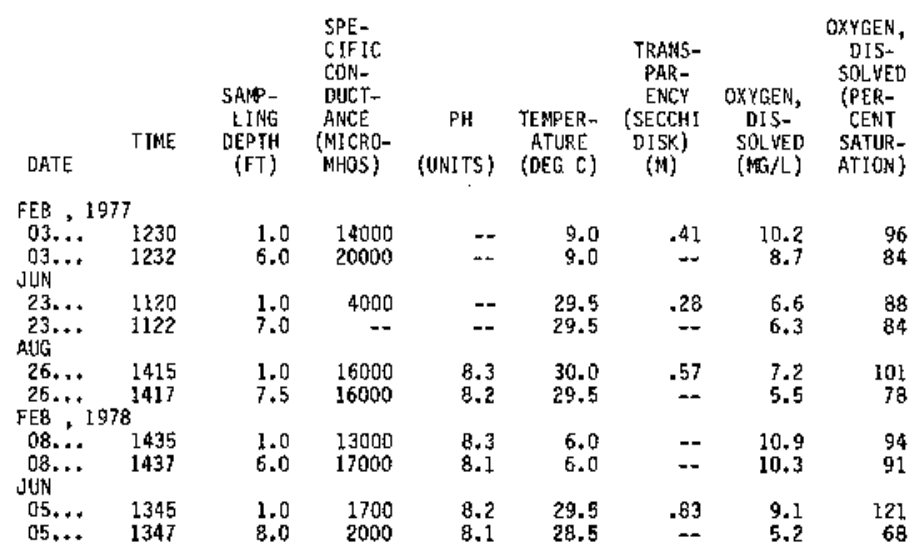


Table 2A.--Quallty of water in the Trinity-San Jacinto estuary, water years 1977-70--Continued Field Determinations--Continused

294205094440400 LINE 230 SITE 40

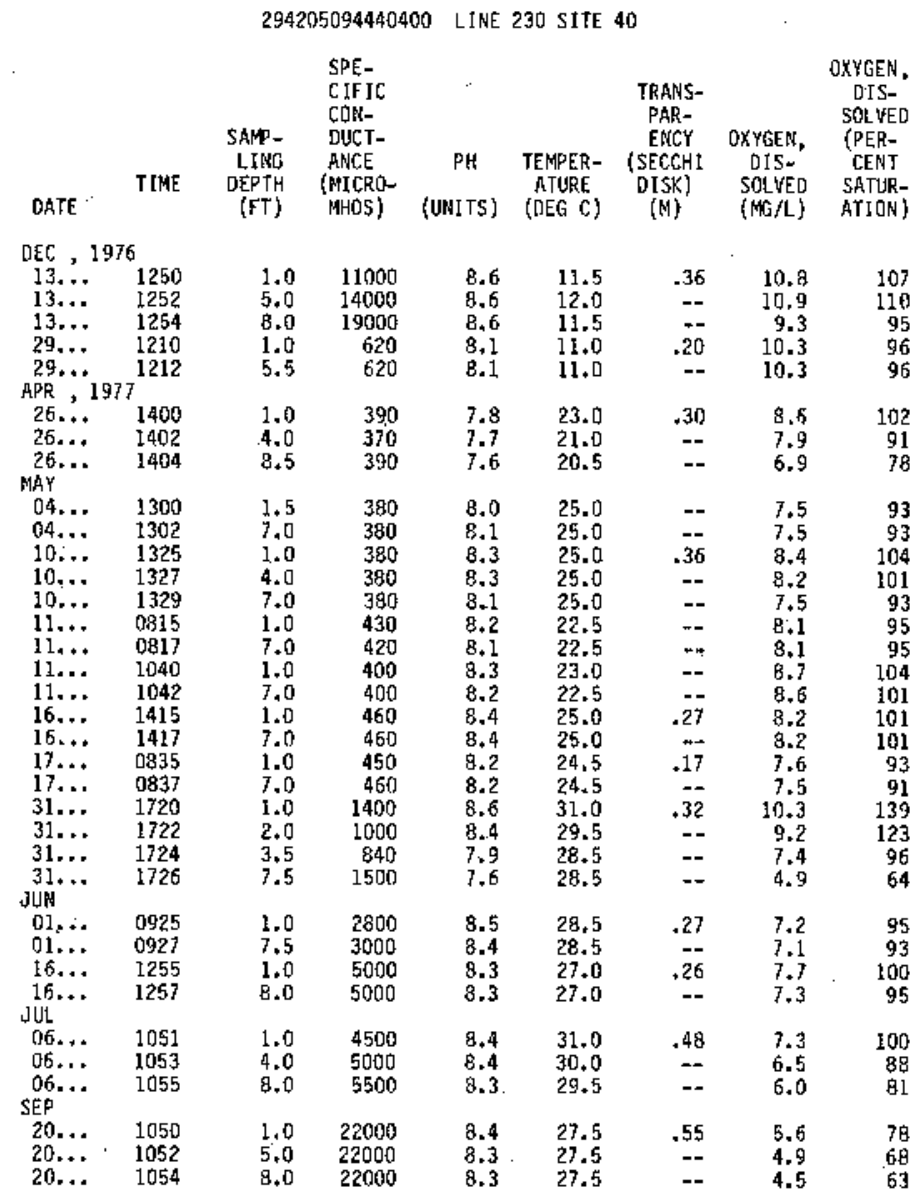

294239094450700 LJWE 230 SITE 50

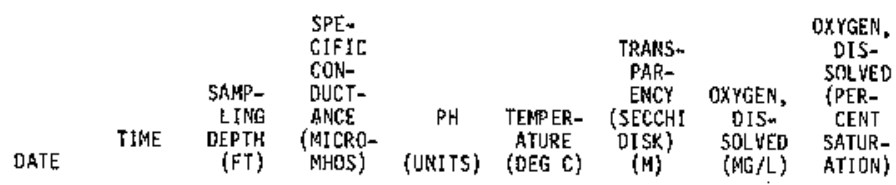

\begin{tabular}{|c|c|c|c|c|c|c|c|c|}
\hline \multicolumn{9}{|c|}{ FEB , 1977} \\
\hline $03 \ldots$ & 1245 & 1.0 & 13000 & $m$ & 9.0 & .46 & 10.0 & 93 \\
\hline$\underset{\text { JUN }}{03 . . .}$ & 1247 & 6.0 & 20000 & $\omega$ & 9.0 & -- & 9.0 & 86 \\
\hline $\begin{array}{l}23, \ldots \\
23, \ldots\end{array}$ & $\begin{array}{l}1130 \\
1132\end{array}$ & $\begin{array}{l}1.0 \\
9.0\end{array}$ & $\begin{array}{l}4500 \\
4500\end{array}$ & $=$ & $\begin{array}{l}29.0 \\
29.5\end{array}$ & $\begin{array}{r}.23 \\
--\end{array}$ & $\begin{array}{l}7.2 \\
6.5\end{array}$ & \\
\hline AUGG & & & & & & & & \\
\hline $\begin{array}{l}26 \ldots \\
26 \ldots \\
\text { FEB... }\end{array}$ & $\begin{array}{l}1400 \\
1402 \\
8\end{array}$ & $\begin{array}{l}1.0 \\
8.0\end{array}$ & $\begin{array}{l}14000 \\
14000\end{array}$ & $\begin{array}{l}8.2 \\
8.2\end{array}$ & $\begin{array}{l}30.0 \\
29.5\end{array}$ & .39 & $\begin{array}{l}7.3 \\
6.7\end{array}$ & \\
\hline $08, \ldots$ & $\begin{array}{l}1420 \\
1422\end{array}$ & $\begin{array}{l}1.0 \\
6.0\end{array}$ & $\begin{array}{l}12000 \\
17000\end{array}$ & $\begin{array}{l}8.5 \\
8.2\end{array}$ & $\begin{array}{l}6.5 \\
7.0\end{array}$ & $\begin{array}{l}-- \\
--\end{array}$ & $\begin{array}{l}10.9 \\
10.4\end{array}$ & \\
\hline $05 \ldots$ & 1334 & 1.0 & 1700 & 8.1 & 28.0 & .86 & 85 & \\
\hline $05 \ldots$ & 1336 & 9.0 & 1900 & 7.8 & 28.5 & $=$ & 4.7 & \\
\hline
\end{tabular}


Table 2A.--Quatity of water in the Trinity-San Jacinto estuary, water years 1977-78--Continued

Field Determinations--Cont inued

294313094460800 LINE 230 SITE 60

\begin{tabular}{|c|c|c|c|c|c|c|c|c|}
\hline DATE & TIME & $\begin{array}{c}\text { SAME- } \\
\text { LING } \\
\text { DEPTH } \\
\text { (FT) }\end{array}$ & $\begin{array}{l}\text { SPE- } \\
\text { CIFIC } \\
\text { CON- } \\
\text { DUCT- } \\
\text { ANCE } \\
\text { (MICRO- } \\
\text { MHOS) }\end{array}$ & $\begin{array}{c}\text { PH } \\
\text { (UNITS) }\end{array}$ & $\begin{array}{l}\text { TEMPER- } \\
\text { ATURE } \\
\text { (DEG C) }\end{array}$ & $\begin{array}{c}\text { TRANS- } \\
\text { PAR- } \\
\text { ENCY } \\
\text { (SECCHI } \\
\text { DISK) } \\
\text { (M). }\end{array}$ & $\begin{array}{c}\text { OXYGEN, } \\
\text { DIS- } \\
\text { SOLYED } \\
\text { (MG } / L \text { ) }\end{array}$ & $\begin{array}{c}\text { OXYGER, } \\
\text { OIS- } \\
\text { SDLYED } \\
\text { SPER- } \\
\text { CENT } \\
\text { SATUR- } \\
\text { ATION) }\end{array}$ \\
\hline
\end{tabular}

BEC, 1976

10.21230

$10, \ldots 1232$

$13 . . . \quad 1242$

$29 . . .1200$

$29 . . .1202$

26... 1410

$26 . . . \quad 1412$

$26 . . . \quad 1414$

MAY 1330

$04 \ldots 1330$

$04 . . .1332$

$10 \ldots 1345$

$17 \ldots .0845$

$17 . . . \quad 0847$

JUNN * D.

$\begin{array}{ll}16 . . . & 1230 \\ 16 . . . & 1232\end{array}$

JUL 1120

$06 . . . \quad 1120$

$06 \ldots . \quad 1122$

SEP

20... 1100

20... 1102

$20 . . .1104$

1.020000

$\begin{array}{ll}7.5 & 20000 \\ 1.0 & 15000\end{array}$

$7.0 \quad 16000$

$1.0 \quad 1300$

$\begin{array}{ll}8.6 & 12.0 \\ 8.6 & 12.0 \\ 8.6 & 12.0 \\ 8.6 & 11.5 \\ 8.3 & 11.0\end{array}$

$\begin{array}{lll}.51 & 10.7 \quad 110\end{array}$

$1.0 \quad 340$

$8.3 \quad 11.0$

$\begin{array}{lll}.73 & 10.6 & 109 \\ .43 & 11.1 & 112\end{array}$

$4.0 \quad 340$

$\begin{array}{lllll}\text { B.0 } & 23.5 & .27 & 9.8 & 118\end{array}$

$8.0 \quad 360$

7

$\begin{array}{ll}1.5 & 350 \\ 8.0 & 350\end{array}$

$1.0 \quad 340$

$\begin{array}{rr}8.0 & 350 \\ 1.0 & 2400\end{array}$

$9.0 \quad 2500$

$1.0 \quad 5200$

$8.0 \quad 5500$

$\begin{array}{ll}1.0 & 6000 \\ 5.0 & 6700 \\ 8.0 & 7500\end{array}$

7.6

21.

$\begin{array}{rrrrr}8.0 & 25.0 & -5 & 7.5 & 94 \\ 8.2 & 25.5 & .29 & 8.2 & 102\end{array}$

$8.1 \quad 25.0 \quad-7 \quad 7.5 \quad 93$

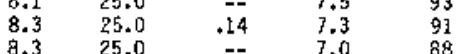

$8.3 \quad 27.0 \quad-2 \quad 7.3 \quad 95$

$8.3 \quad 27.0 \quad--\quad 7.1 \quad 92$

$\begin{array}{llllll}6700 & 8.4 & 30.5 & .61 & 7.1 & 99 \\ 7500 & 8.3 & 30.5 & \ldots & 6.2 & 85 \\ & & & & 5.8 & 79\end{array}$

$\begin{array}{ll}1.0 & 20000 \\ 3.0 & 20000\end{array}$

$5.0 \quad 21000$

$\begin{array}{lllll}8.3 & 27.5 & .48 & 6.0 & 82 \\ 8.4 & 27.5 & -. & 5.4 & 74\end{array}$

$20, \ldots \quad 1106$

294346094470800 . LINE 230 SITE 70.

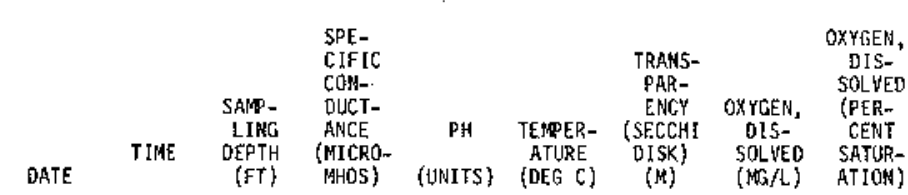

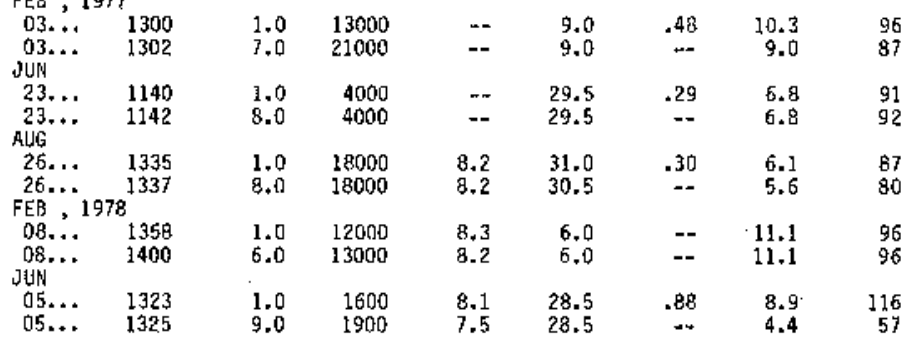


Table $2 A,-$-quality of water in the Trinfty-San Jacinto estuary, water years 1977-78--Continued Field Determinations--Cont inuẹd

294420094480900 LIRE 230 SITE 80

\begin{tabular}{|c|c|c|c|c|c|c|c|c|}
\hline DATE & TIME & $\begin{array}{l}\text { SAMP. } \\
\text { LING } \\
\text { DEPTH } \\
\text { (FT) }\end{array}$ & $\begin{array}{l}\text { SPE- } \\
\text { CIEIC } \\
\text { CON- } \\
\text { DUCT- } \\
\text { ANCE } \\
\text { (MICRO- } \\
\text { MHOS) }\end{array}$ & $\begin{array}{c}\text { PH } \\
\text { (UNITS) }\end{array}$ & $\begin{array}{l}\text { TEIPER } \\
\text { ATURE } \\
\text { (OEG C) }\end{array}$ & $\begin{array}{c}\text { TRARS- } \\
\text { PAR- } \\
\text { ENCY } \\
\text { (SECECHI } \\
\text { DISK) } \\
\text { (M) }\end{array}$ & $\begin{array}{c}\text { OXYGEA, } \\
\text { DJS- } \\
\text { SOLVED } \\
\text { (ME } / L \text { ) }\end{array}$ & $\begin{array}{l}\text { OXYGEN, } \\
\text { DIS- } \\
\text { SOLYED } \\
\text { (PER- } \\
\text { CENT } \\
\text { SATUR- } \\
\text { ATION) }\end{array}$ \\
\hline $\begin{array}{l}\text { DEC , } \\
10 . . . \\
10 \ldots \\
13 \ldots \\
13 . . . \\
29 . . \\
\text { APR , }\end{array}$ & $\begin{array}{l}6 \\
1215 \\
1217 \\
1230 \\
1232 \\
1142 \\
7\end{array}$ & $\begin{array}{l}1.0 \\
6.0 \\
1.0 \\
5.5 \\
4.0\end{array}$ & $\begin{array}{r}4500 \\
12000 \\
12000 \\
15000 \\
8100\end{array}$ & $\begin{array}{l}8.4 \\
8.3 \\
8.6 \\
8.6 \\
8.0\end{array}$ & $\begin{array}{l}12.5 \\
12.5 \\
12.0 \\
12.0 \\
13.5\end{array}$ & $\begin{array}{l}- \\
- \\
.66 \\
-- \\
--\end{array}$ & $\begin{array}{r}10.0 \\
9.4 \\
11.1 \\
11.1 \\
9.9\end{array}$ & $\begin{array}{r}90 \\
95 \\
111 \\
112 \\
102\end{array}$ \\
\hline $\begin{array}{l}26 . \ldots \\
26 \ldots \\
26 \ldots \\
26 \ldots \\
\text { MAY }\end{array}$ & $\begin{array}{l}1430 \\
1432 \\
1434 \\
1436\end{array}$ & $\begin{array}{l}1.0 \\
4.5 \\
3.0 \\
6.0\end{array}$ & $\begin{array}{r}360 \\
450 \\
370 \\
6800\end{array}$ & $\begin{array}{l}7.8 \\
7.5 \\
7.5 \\
7.8\end{array}$ & $\begin{array}{l}24.0 \\
23.0 \\
23.0 \\
23.0\end{array}$ & $\begin{array}{l}= \\
\cdots \\
--\end{array}$ & $\begin{array}{l}8.7 \\
6.9 \\
6.7 \\
7.1\end{array}$ & $\begin{array}{r}106 \\
82 \\
\quad 80 \\
87\end{array}$ \\
\hline $\begin{array}{l}04 \ldots \\
04 \ldots \\
\text { JUA }\end{array}$ & $\begin{array}{l}1350 \\
1352\end{array}$ & $\begin{array}{l}1.5 \\
7.0\end{array}$ & $\begin{array}{l}400 \\
400\end{array}$ & $\begin{array}{l}7.5 \\
7.5\end{array}$ & $\begin{array}{l}25.0 \\
25.0\end{array}$ & $=$ & $\begin{array}{l}7.1 \\
7.2\end{array}$ & $\begin{array}{l}88 \\
89\end{array}$ \\
\hline $\begin{array}{l}16 . . . \\
16 . . . \\
\text { Jut }\end{array}$ & $\begin{array}{l}1210 \\
1212\end{array}$ & $\begin{array}{l}1.0 \\
6.0\end{array}$ & $\begin{array}{l}7000 \\
8000\end{array}$ & $\begin{array}{l}8.6 \\
8.5\end{array}$ & $\begin{array}{l}27.5 \\
27.0\end{array}$ & $\begin{array}{r}.37 \\
--\end{array}$ & $\begin{array}{l}8.8 \\
8.3\end{array}$ & $\begin{array}{l}114 \\
109\end{array}$ \\
\hline $\begin{array}{l}06 . \ldots \\
06 . \ldots \\
06 . \ldots \\
\text { SEP }\end{array}$ & $\begin{array}{l}1140 \\
1142 \\
1144\end{array}$ & $\begin{array}{l}1.0 \\
4.0 \\
5.0\end{array}$ & $\begin{array}{r}3500 \\
3800 \\
10000\end{array}$ & $\begin{array}{l}8.5 \\
8.5 \\
8.3\end{array}$ & $\begin{array}{l}32.5 \\
32.5 \\
32.5\end{array}$ & $\begin{array}{l}.44 \\
-- \\
--\end{array}$ & $\begin{array}{l}7.5 \\
7.5 \\
6.4\end{array}$ & $\begin{array}{r}104 \\
104 \\
92\end{array}$ \\
\hline $\begin{array}{l}20 \ldots \\
20 \ldots \\
20 \ldots\end{array}$ & $\begin{array}{l}1120 \\
1122 \\
1124\end{array}$ & $\begin{array}{l}1.0 \\
5.0 \\
7.5\end{array}$ & $\begin{array}{l}21000 \\
23000 \\
23000\end{array}$ & $\begin{array}{l}8.4 \\
8.3 \\
8.3\end{array}$ & $\begin{array}{l}28.0 \\
27.5 \\
27.5\end{array}$ & $\begin{array}{r}.53 \\
\cdots \\
--\end{array}$ & $\begin{array}{l}7.0 \\
5.3 \\
5.4\end{array}$ & $\begin{array}{l}99 \\
73 \\
74\end{array}$ \\
\hline
\end{tabular}

293746094433300 LINE 240 SITE 20

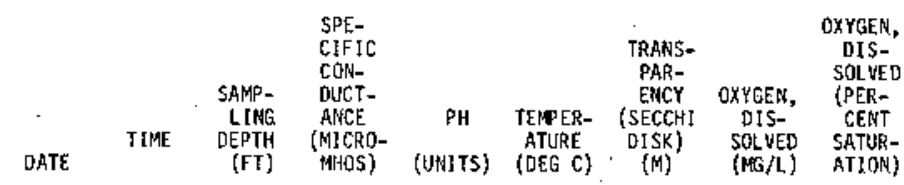

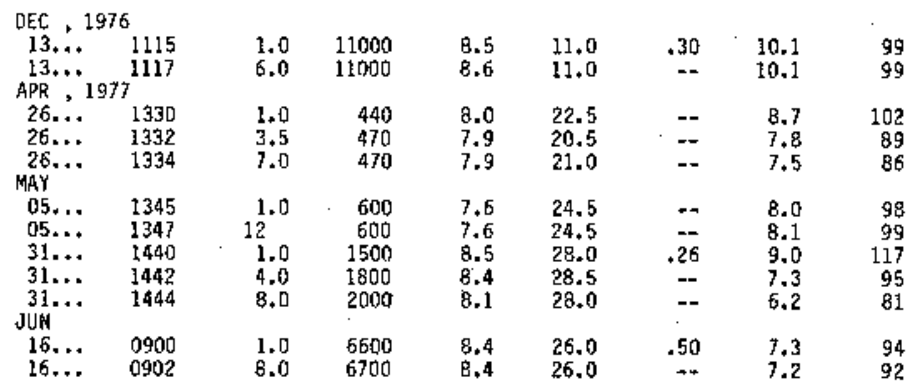

293910094441100 LINE 240 SITE 30

\begin{tabular}{|c|c|c|c|c|c|c|c|c|}
\hline DATE & TIME & $\begin{array}{l}\text { 5ANP- } \\
\text { LING } \\
\text { DEPTH } \\
\text { (FT) }\end{array}$ & $\begin{array}{l}\text { SPE- } \\
\text { CIFIC } \\
\text { CON- } \\
\text { OUCT- } \\
\text { ANCE } \\
\text { (MICRO- } \\
\text { MHOS) }\end{array}$ & $\begin{array}{c}\text { PH } \\
\text { (UNITS) }\end{array}$ & $\begin{array}{l}\text { TEFPER- } \\
\text { ATURE } \\
\text { (DEG C) }\end{array}$ & $\begin{array}{c}\text { TRANS- } \\
\text { PAR- } \\
\text { ENCY } \\
\text { (SECCHI } \\
\text { DISK) } \\
(M)\end{array}$ & $\begin{array}{c}\text { OXYGEN, } \\
\text { D1S- } \\
\text { SOLWEO } \\
\text { (MGS } / L)\end{array}$ & $\begin{array}{c}\text { OXYGEK, } \\
\text { DIS- } \\
\text { SOLVED } \\
\text { (PER- } \\
\text { CENT } \\
\text { SATUR- } \\
\text { ATIOK) }\end{array}$ \\
\hline CT, 1 & & & & & & & & \\
\hline $\begin{array}{l}21 \ldots \\
21 \ldots \\
21 \ldots \\
\text { NOW }\end{array}$ & $\begin{array}{l}1425 \\
1427 \\
1429\end{array}$ & $\begin{array}{l}1.0 \\
5.0 \\
7.0\end{array}$ & $\begin{array}{l}14000 \\
19000 \\
25000\end{array}$ & $\begin{array}{l}8.5 \\
8.4 \\
8.3\end{array}$ & $\begin{array}{l}15.5 \\
15.5 \\
15.5\end{array}$ & $\begin{array}{r}.46 \\
-- \\
--\end{array}$ & $\begin{array}{l}9.3 \\
8.4 \\
7.4\end{array}$ & $\begin{array}{r}101 \\
93 \\
84\end{array}$ \\
\hline $\begin{array}{l}18 . \cdots \\
18 . . . \\
\text { FEB } 1\end{array}$ & $\begin{array}{l}1115 \\
1117 \\
7\end{array}$ & $\begin{array}{l}1.0 \\
9.0\end{array}$ & $\begin{array}{l}17000 \\
25000\end{array}$ & $=$ & $\begin{array}{r}9.5 \\
10.0\end{array}$ & $\begin{array}{r}1.10 \\
-=\end{array}$ & $\begin{array}{r}10.9 \\
9.6\end{array}$ & $\begin{array}{r}105 \\
97\end{array}$ \\
\hline $\begin{array}{l}03 . . . \\
03 . . \\
\text { JuA }\end{array}$ & $\begin{array}{l}1430 \\
1432\end{array}$ & $\begin{array}{l}1.0 \\
8.0\end{array}$ & $\begin{array}{l}18000 \\
21000\end{array}$ & -- & $\begin{array}{l}9.0 \\
9.0\end{array}$ & .41 & $\begin{array}{l}9.9 \\
8.7\end{array}$ & $\begin{array}{l}94 \\
84\end{array}$ \\
\hline $\begin{array}{l}23 \ldots . . \\
23 \ldots \\
\text { AUG }\end{array}$ & $\begin{array}{l}1300 \\
1302\end{array}$ & $\begin{array}{l}1.0 \\
9.0\end{array}$ & $\begin{array}{l}6000 \\
6000\end{array}$ & $\begin{array}{l}-= \\
m=\end{array}$ & $\begin{array}{l}29.0 \\
29.0\end{array}$ & $\begin{array}{r}.40 \\
--\end{array}$ & $\begin{array}{l}7.5 \\
7.1\end{array}$ & $\begin{array}{r}100 \\
95\end{array}$ \\
\hline $\begin{array}{c}26 \ldots . . \\
26 \ldots \\
\text { FEB } .1\end{array}$ & $\begin{array}{l}1240 \\
1242 \\
8\end{array}$ & $\begin{array}{l}1.0 \\
9.5\end{array}$ & $\begin{array}{l}21000 \\
24000\end{array}$ & $\begin{array}{l}8.4 \\
8.3\end{array}$ & $\begin{array}{l}30.0 \\
29.5\end{array}$ & $\begin{array}{r}.66 \\
--\end{array}$ & $\begin{array}{l}7.0 \\
5.8\end{array}$ & $\begin{array}{r}101 \\
82\end{array}$ \\
\hline $\begin{array}{l}08 . \ldots \\
08 . . . \\
\text { JUN }\end{array}$ & $\begin{array}{l}1445 \\
1447\end{array}$ & $\begin{array}{l}1.0 \\
6.0\end{array}$ & $\begin{array}{l}19000 \\
19000\end{array}$ & $\begin{array}{l}8.2 \\
8.2\end{array}$ & $\begin{array}{l}6.0 \\
6.0\end{array}$ & $\begin{array}{l}m \\
=-\end{array}$ & $\begin{array}{l}10.7 \\
10.6\end{array}$ & $\begin{array}{l}96 \\
94\end{array}$ \\
\hline $\begin{array}{l}05 \ldots \\
05 \ldots\end{array}$ & $\begin{array}{l}1243 \\
1245\end{array}$ & $10^{1.0}$ & $\begin{array}{l}1800 \\
2300\end{array}$ & $\begin{array}{l}8.2 \\
8.0\end{array}$ & $\begin{array}{l}28.5 \\
28.0\end{array}$ & : & $\begin{array}{l}8.8 \\
5.6\end{array}$ & $\begin{array}{r}114 \\
73\end{array}$ \\
\hline
\end{tabular}


293850094451500 LINE 240 SITE 40

\begin{tabular}{|c|c|c|c|c|c|c|c|c|}
\hline DATE & TIME & $\begin{array}{l}\text { SAMP- } \\
\text { LIKG } \\
\text { DEPTH } \\
\text { (FT) }\end{array}$ & $\begin{array}{l}\text { SPE- } \\
\text { CIFIC } \\
\text { CON- } \\
\text { DUCT- } \\
\text { ANCE } \\
\text { (MICRO- } \\
\text { MHOS) }\end{array}$ & $\begin{array}{c}\text { PH } \\
\text { (UNITS) }\end{array}$ & $\begin{array}{l}\text { TEMPER- } \\
\text { ATURE } \\
\text { (DEG C) }\end{array}$ & $\begin{array}{l}\text { TRANS- } \\
\text { PAR- } \\
\text { ENCY } \\
\text { (SECCHI } \\
\text { DISK) } \\
\text { (M) }\end{array}$ & $\begin{array}{c}\text { OXYGEN, } \\
\text { DIS= } \\
\text { SOLVED } \\
\text { (MG/L) }\end{array}$ & $\begin{array}{l}\text { OXYGEN, } \\
\text { DIS- } \\
\text { SOLWED } \\
\text { (PER- } \\
\text { CENT } \\
\text { SATUR- } \\
\text { ATIDN) }\end{array}$ \\
\hline $\begin{array}{l}\text { DEC, } \\
13 . . \\
13, \ldots \\
\text { APR, }\end{array}$ & $\begin{array}{l}6 \\
1130 \\
7^{1132}\end{array}$ & $\begin{array}{l}1.0 \\
8.5\end{array}$ & $\begin{array}{l}15000 \\
15000\end{array}$ & $\begin{array}{l}8.6 \\
8.6\end{array}$ & $\begin{array}{l}11.5 \\
11.0\end{array}$ & .46 & $\begin{array}{l}10.2 \\
10.1\end{array}$ & $\begin{array}{l}102 \\
100\end{array}$ \\
\hline $\begin{array}{l}26 \ldots \\
26, \ldots \\
26 \ldots \\
26 \ldots \\
\text { JuN }\end{array}$ & $\begin{array}{l}1315 \\
1317 \\
1319 \\
1321\end{array}$ & $\begin{array}{l}1.0 \\
2.5 \\
4.0 \\
8.5\end{array}$ & $\begin{array}{l}420 \\
500 \\
520 \\
530\end{array}$ & $\begin{array}{l}8.1 \\
7.9 \\
7.9 \\
7.8\end{array}$ & $\begin{array}{l}23.5 \\
21.0 \\
20.5 \\
21.0\end{array}$ & $\begin{array}{l}.15 \\
-- \\
= \\
--\end{array}$ & $\begin{array}{l}9.1 \\
7.8 \\
7.5 \\
7.3\end{array}$ & $\begin{array}{r}110 \\
900 \\
85 \\
84\end{array}$ \\
\hline $\begin{array}{l}16 \ldots \\
16 \ldots \\
\text { JuL }\end{array}$ & $\begin{array}{l}0905 \\
090 \%\end{array}$ & $10^{1.0}$ & $\begin{array}{l}8700 \\
8700\end{array}$ & $\begin{array}{l}8.5 \\
8.5\end{array}$ & $\begin{array}{l}26.0 \\
26.0\end{array}$ & $\begin{array}{r}.39 \\
--\end{array}$ & $\begin{array}{l}7.3 \\
7.3\end{array}$ & $\begin{array}{l}94 \\
94\end{array}$ \\
\hline $\begin{array}{l}06 . . \\
06 . . \\
06 . .\end{array}$ & $\begin{array}{l}1305 \\
1307 \\
1309\end{array}$ & $\begin{array}{l}1.0 \\
5.0 \\
9.0\end{array}$ & $\begin{array}{r}5400 \\
10000 \\
10000\end{array}$ & $\begin{array}{l}8.6 \\
8.5 \\
8.5\end{array}$ & $\begin{array}{l}31.5 \\
31.0 \\
30.5\end{array}$ & $\begin{array}{l}.60 \\
-- \\
--\end{array}$ & $\begin{array}{l}7.6 \\
7.2 \\
6.4\end{array}$ & $\begin{array}{r}106 \\
100 \\
89\end{array}$ \\
\hline
\end{tabular}

293927094461100 LINE 2410 SITE 50

\begin{tabular}{|c|c|c|c|c|c|c|c|c|}
\hline CARTE & TIME & $\begin{array}{l}\text { SAMP. } \\
\text { LING } \\
\text { DEPTH } \\
\text { (FT) }\end{array}$ & $\begin{array}{l}\text { SPE- } \\
\text { CIFIC } \\
\text { CON- } \\
\text { DUCT- } \\
\text { ANCE } \\
\text { (MICRO- } \\
\text { MHOS) }\end{array}$ & $\begin{array}{c}\mathrm{PH} \\
\text { (ON1TS\}) }\end{array}$ & $\begin{array}{l}\text { TEMPER- } \\
\text { ATURE } \\
\text { (DEG C) }\end{array}$ & $\begin{array}{c}\text { TRANS- } \\
\text { PAR- } \\
\text { ENCY } \\
\text { (SECCHI } \\
\text { DISK) } \\
\text { (MI) }\end{array}$ & $\begin{array}{c}\text { OXYGEN, } \\
\text { OIS- } \\
\text { SOLWED } \\
\text { (MG/L) }\end{array}$ & $\begin{array}{l}\text { OXYGEN, } \\
\text { DIS- } \\
\text { SOLVED } \\
\text { (PER- } \\
\text { CENT } \\
\text { SATUR- } \\
\text { ATION- }\end{array}$ \\
\hline
\end{tabular}

OCT , 1976

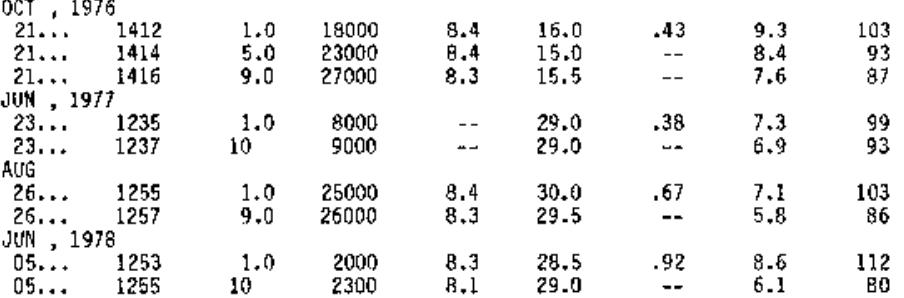

294004094470900 LINE 240 SITE 60

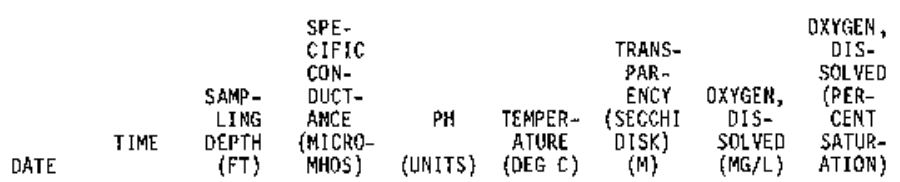

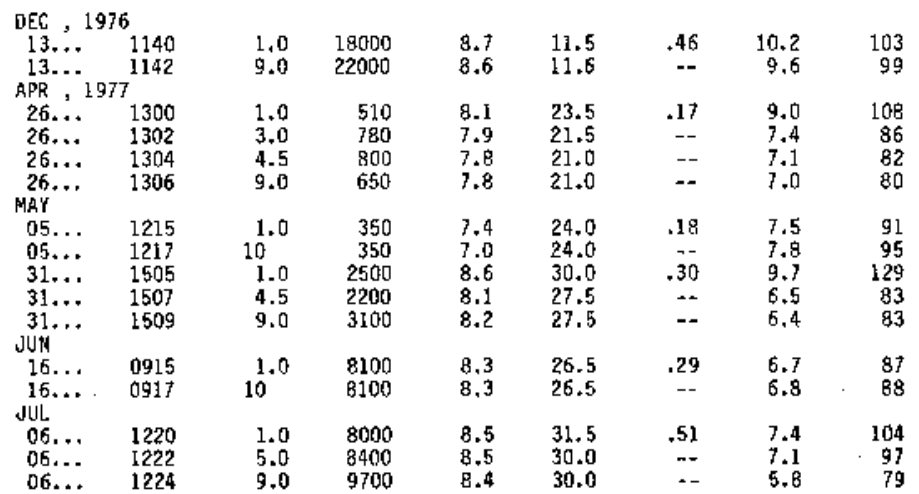


Table 2A.- Quality of water in the Trinity-San Jacinto estuary, water years 1977-76--Cont inued Field Detenuf nations--Cont inued

294042094480700 LIME 240 \$ITE 70

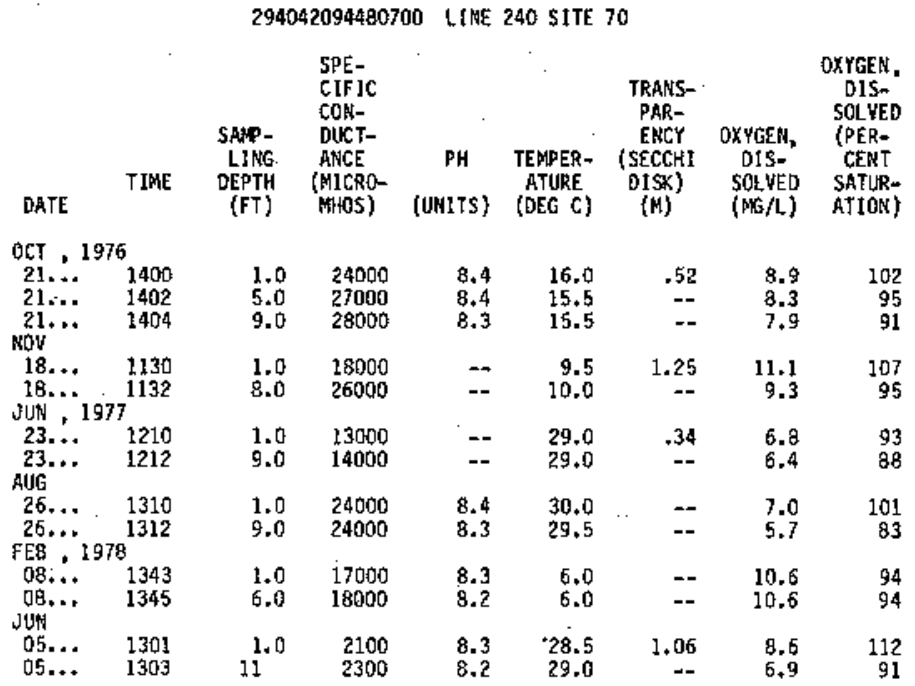

294120094490600 LINE 240 SITE 90

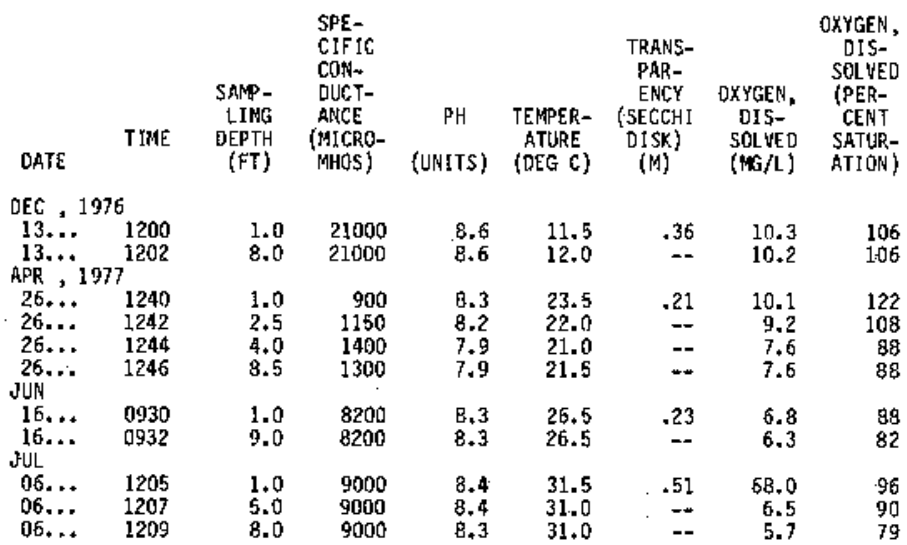

294158094500500 LINE 240 SIJE 90

\begin{tabular}{|c|c|c|c|c|c|c|c|c|}
\hline DATE & TIME & $\begin{array}{l}\text { SAMP- } \\
\text { LING } \\
\text { DEPTH } \\
\text { (FT) }\end{array}$ & $\begin{array}{l}\text { SPE- } \\
\text { CIFIC } \\
\text { COR- } \\
\text { DUCT- } \\
\text { ARCE } \\
\text { (MICRO- } \\
\text { MHOS) }\end{array}$ & $\begin{array}{c}P H \\
\text { (UNIIS) }\end{array}$ & $\begin{array}{c}\text { TEMPER- } \\
\text { ATURE } \\
\text { (DEG C) }\end{array}$ & $\begin{array}{l}\text { JRANS- } \\
\text { PAR- } \\
\text { ENCY } \\
\text { (SECCHI } \\
\text { DISK) } \\
\text { (M) }\end{array}$ & $\begin{array}{c}\text { OXYGEN, } \\
\text { DIS- } \\
\text { SOLVED } \\
\text { (MGLL) }\end{array}$ & $\begin{array}{c}\text { OXYGEN, } \\
\text { OIS- } \\
\text { SOLVED } \\
\text { (PER- } \\
\text { CENT } \\
\text { SATUR- } \\
\text { ATION) }\end{array}$ \\
\hline \multicolumn{9}{|c|}{ OCT, 1976} \\
\hline $\begin{array}{l}21 \ldots \\
21 \ldots\end{array}$ & $\begin{array}{l}1350 \\
1352\end{array}$ & $\begin{array}{l}1.0 \\
3.0\end{array}$ & $\begin{array}{l}22000 \\
25000\end{array}$ & $\begin{array}{l}8.4 \\
8.4\end{array}$ & $\begin{array}{l}16.0 \\
16.0\end{array}$ & .55 & $\begin{array}{l}9.1 \\
8.3\end{array}$ & $\begin{array}{r}103 \\
95\end{array}$ \\
\hline $\begin{array}{l}18 \ldots \\
18 \ldots \\
\text { FEB. }\end{array}$ & $\begin{array}{l}1140 \\
1142 \\
7\end{array}$ & $\begin{array}{l}1.0 \\
8.0\end{array}$ & $\begin{array}{l}20000 \\
27000\end{array}$ & -- & $\begin{array}{r}9.5 \\
10.0\end{array}$ & $\begin{array}{r}1.28 \\
--\end{array}$ & $\begin{array}{r}11.0 \\
9.4\end{array}$ & $\begin{array}{r}107 \\
96\end{array}$ \\
\hline $\begin{array}{c}03 . \cdots \\
03 \ldots \\
\text { JUN }\end{array}$ & $\begin{array}{l}1315 \\
1317\end{array}$ & $\begin{array}{l}1.0 \\
6.0\end{array}$ & $\begin{array}{l}10000 \\
12000\end{array}$ & -- & $\begin{array}{c}9.0 \\
--\end{array}$ & $\begin{array}{r}.43 \\
--\end{array}$ & $\begin{array}{r}10.7 \\
9.7\end{array}$ & $\begin{array}{l}99 \\
91\end{array}$ \\
\hline $\begin{array}{l}23 . \ldots \\
23 . \cdots \\
\text { AUG }\end{array}$ & $\begin{array}{l}1200 \\
1202\end{array}$ & $\begin{array}{l}1.0 \\
8.0\end{array}$ & $\begin{array}{l}13000 \\
13000\end{array}$ & $=$ & $\begin{array}{l}29.0 \\
29.0\end{array}$ & .20 & $\begin{array}{l}6.5 \\
6.5\end{array}$ & $\begin{array}{l}89 \\
89\end{array}$ \\
\hline $\begin{array}{l}26 . . \\
26 . \ldots \\
F E B, \cdots\end{array}$ & $\begin{array}{l}1320 \\
1322 \\
8\end{array}$ & $\begin{array}{l}1.0 \\
8.0\end{array}$ & $\begin{array}{l}16000 \\
16000\end{array}$ & $\begin{array}{l}8.4 \\
8.4\end{array}$ & $\begin{array}{r}30.0 \\
.30 .0\end{array}$ & $\begin{array}{l}.50 \\
-.\end{array}$ & $\begin{array}{l}7.2 \\
6.9\end{array}$ & $\begin{array}{r}101 \\
97\end{array}$ \\
\hline $\begin{array}{l}08 . . . \\
08 . . . \\
\text { JuM }\end{array}$ & $\begin{array}{l}1340 \\
1342\end{array}$ & $\begin{array}{l}1.0 \\
6.0\end{array}$ & $\begin{array}{l}160000 \\
16000\end{array}$ & $\begin{array}{l}8.3 \\
8.3\end{array}$ & $\begin{array}{l}7.0 \\
7.0\end{array}$ & $m$ & $\begin{array}{l}10.6 \\
10.4\end{array}$ & $\begin{array}{l}97 \\
95\end{array}$ \\
\hline $\begin{array}{l}05 . \\
05 . .\end{array}$ & $\begin{array}{l}1314 \\
1316\end{array}$ & $\begin{array}{l}1.0 \\
9.0\end{array}$ & $\begin{array}{l}1900 \\
2000\end{array}$ & $\begin{array}{l}8.2 \\
8.1\end{array}$ & $\begin{array}{l}28.5 \\
29.0\end{array}$ & .90 & $\begin{array}{l}8.4 \\
6.7\end{array}$ & $\begin{array}{r}109 \\
88\end{array}$ \\
\hline
\end{tabular}


Table 2h.--Quality of water in the Trinity-5an Jacinto estuary, water years 1977-78--Continued Field Determinations--Continuęd

294236094510400 LINE. 240 SITE 99

\begin{tabular}{|c|c|c|c|c|c|c|c|c|}
\hline DATE & TIME & $\begin{array}{c}\text { SANP- } \\
\text { LING } \\
\text { DEPTH } \\
(F T)\end{array}$ & $\begin{array}{l}\text { SPE- } \\
\text { CIFIC } \\
\text { CONA- } \\
\text { DUCC- } \\
\text { ANCE } \\
\text { (MICRO- } \\
\text { MHOS) }\end{array}$ & $\begin{array}{c}\text { PH } \\
\text { (UNITS) }\end{array}$ & $\begin{array}{l}\text { TEMPER- } \\
\text { ATURE } \\
\text { (DEG C) }\end{array}$ & $\begin{array}{c}\text { TRANS- } \\
\text { PAR- } \\
\text { ENCY } \\
\text { (SECCKI } \\
\text { OISK) } \\
\text { (M) }\end{array}$ & $\begin{array}{c}\text { OXYGEN, } \\
\text { DIS- } \\
\text { SOLVEO } \\
\text { (MG/L) }\end{array}$ & $\begin{array}{l}\text { OXYGEN, } \\
\text { DIS- } \\
\text { SOLVED } \\
\text { (PER- } \\
\text { CERT } \\
\text { SATUR- } \\
\text { RTION) }\end{array}$ \\
\hline \multicolumn{9}{|c|}{$D E C, 1976$} \\
\hline $\begin{array}{l}13 \ldots \\
13 \ldots \\
\text { APR }, 1\end{array}$ & $\begin{array}{l}1215 \\
7^{1217}\end{array}$ & 6.0 & $\begin{array}{l}16000 \\
16000\end{array}$ & $\begin{array}{l}8.6 \\
8.6\end{array}$ & $\begin{array}{l}17.0 \\
12.0\end{array}$ & .56 & $\begin{array}{l}10.6 \\
10.5\end{array}$ & $\begin{array}{r}108 \\
101\end{array}$ \\
\hline $\begin{array}{c}26 \ldots \\
26, \ldots \\
\text { MAY }\end{array}$ & $\begin{array}{l}1225 \\
1227\end{array}$ & $\begin{array}{l}1.0 \\
5.0\end{array}$ & $\begin{array}{l}1800 \\
3000\end{array}$ & $\begin{array}{l}8.1 \\
8.1\end{array}$ & $\begin{array}{l}21.5 \\
21.5\end{array}$ & $\begin{array}{r}.27 \\
--\end{array}$ & $\begin{array}{l}9.7 \\
8.7\end{array}$ & $\begin{array}{l}113 \\
102\end{array}$ \\
\hline $\begin{array}{l}05 \ldots \\
05 \ldots \\
31 \ldots \\
31 \ldots \\
\text { JuN }\end{array}$ & $\begin{array}{l}1145 \\
1147 \\
1525 \\
1527\end{array}$ & $\begin{array}{l}1.0 \\
6.0 \\
1.0 \\
5.0\end{array}$ & $\begin{array}{l}1200 \\
1200 \\
5500 \\
5500\end{array}$ & $\begin{array}{l}7.8 \\
7.9 \\
8.5 \\
8.4\end{array}$ & $\begin{array}{l}24.0 \\
24.0 \\
29.5 \\
29.5\end{array}$ & $\begin{array}{l}-. \\
. \overline{-} \\
.40 \\
--\end{array}$ & $\begin{array}{l}7.4 \\
7.8 \\
8.3 \\
7.9\end{array}$ & $\begin{array}{r}90 \\
93 \\
112 \\
107\end{array}$ \\
\hline $\begin{array}{l}16 \ldots \\
16 \ldots\end{array}$ & $\begin{array}{l}0945 \\
0947\end{array}$ & $\begin{array}{l}1.0 \\
6.0\end{array}$ & $\begin{array}{l}13000 \\
13000\end{array}$ & $\begin{array}{l}8.4 \\
8.2\end{array}$ & $\begin{array}{l}26.5 \\
2.6 .5\end{array}$ & .24 & $\begin{array}{l}7.0 \\
6.5\end{array}$ & $\begin{array}{l}92 \\
86\end{array}$ \\
\hline
\end{tabular}

$293754094480600 \quad$ LINE 250 SITE 50

\begin{tabular}{|c|c|c|c|c|c|c|c|c|}
\hline DATE & TIME. & $\begin{array}{r}\text { SAMP- } \\
\text { LING } \\
\text { OEPTH } \\
(F T)\end{array}$ & $\begin{array}{l}\text { SPE- } \\
\text { CIFIC } \\
\text { CON- } \\
\text { COUCT- } \\
\text { ANCE } \\
\text { (MICRO- } \\
\text { MH(IS) }\end{array}$ & $\begin{array}{c}\text { PH } \\
\text { (UNITS) }\end{array}$ & $\begin{array}{c}\text { TEMPER- } \\
\text { ATGRE } \\
\text { (DEG C) }\end{array}$ & $\begin{array}{l}\text { TRANS- } \\
\text { PAR- } \\
\text { ENCY } \\
\text { \{SECCHI } \\
\text { [SISK) } \\
\text { (M) }\end{array}$ & $\begin{array}{c}\text { OXYGLN, } \\
\text { OIS- } \\
\text { SOLVED } \\
\{M G / L\}\end{array}$ & $\begin{array}{c}\text { OXYGEN, } \\
\text { DIS- } \\
\text { SOLVED } \\
\text { (PER- } \\
\text { CENT } \\
\text { SATUR- } \\
\text { ATION }\end{array}$ \\
\hline
\end{tabular}

JUإ, , 1977

$06 . .1225$

$\begin{array}{ll}06 \ldots . . & 1227 \\ 06 \ldots & 1229\end{array}$

$\begin{array}{rrrrrrr}1.0 & 13000 & 8.6 & 31.5 & .76 & 7.8 & 109 \\ 5.0 & 13000 & 8.4 & 30.5 & -- & 6.2 & 87 \\ 10 & 13000 & 8.4 & 30.0 & -m & 5.8 & 80\end{array}$

293348094482800 LIME 260 SITE, 20

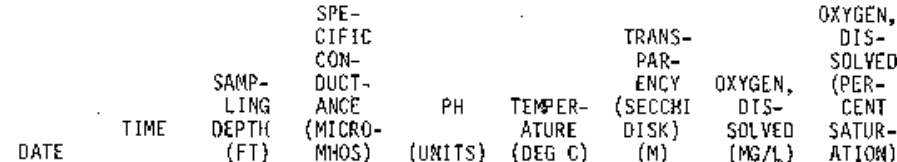

OCT, 1976

$\begin{array}{rr}21 \ldots & 1255 \\ 21 \ldots & 1257\end{array}$

1.022000

$18 \ldots 1030$

$\begin{array}{ll}1.0 & 22000 \\ 6.0 & 22000\end{array}$

$\begin{array}{lll}8.3 & 15.0 \\ 8.3 & 15.0\end{array}$

1.016000

6.019000

$18 \ldots 1032$

$13 . \ldots 1030$

$13, \cdots, 1032$

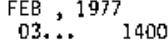

03... 1402

APR

2E. .. 105

$26 \ldots .1057$

$26 \ldots \quad 1059$

$26 \ldots \quad 1101$

MAY

$05 . . . \quad 1020$

$05 \ldots .102 ?$

11... 0910

$11 \ldots .0912$

$17 . . . \quad 1030$

$17 \ldots .1032$

$19 . . .0930$

$19 . . . \quad 0932$

31... 1610

$31 \cdots \quad 1612$

JuN

$\begin{array}{ll}16 \ldots . . & 1115 \\ 16 . . & 1117\end{array}$

16... 1117

$26 \ldots 1220$

$26 \cdots{ }_{1978}^{1222}$

EB.. 19781510

$08 . . . \quad 1512$

गN

$07 . . \quad 0947$

1.0 19000

$6.0 \quad 19000$

$\begin{array}{ll}1.0 & 17000 \\ 4.0 & 19000\end{array}$

1.04600

$\begin{array}{ll}1.0 & 4000 \\ 2.5 & 5200 \\ 4.0 & 5600\end{array}$

$\begin{array}{lll}- & 9.5 \\ - & 9.5\end{array}$

$8.6 \quad 11.5-0250.4$

8.06200

$1.5 \quad 2800$

$\begin{array}{rr}7.0 & 2800 \\ 1.0 & 720\end{array}$

$\begin{array}{rr}7.0 & 740 \\ 1.0 & 7800\end{array}$

$7.0 \quad 8800$

$1.0 \quad 4700$

8.07600

$\begin{array}{ll}1.0 & 4200 \\ 3.5 & 4400\end{array}$

$7.0 \quad 4800$

$1.0 \quad 8000$

8.08000

1.029000

8.029000

$\begin{array}{ll}1.0 & 18000 \\ 7.0 & 19000\end{array}$

$1.0 \quad 27000$

$6.0 \quad 32000$

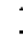

$--$

$\begin{array}{lllll}8.1 & 21.0 & .17 & 8.3 & 97\end{array}$

$\begin{array}{lllll}8.1 & 21.0 & -- & 7.3 & 86 \\ 8.0 & 21.5 & -- & 6.9 & 82\end{array}$

$\begin{array}{lllll}7.8 & 23.5 & - & 7.4 & 90\end{array}$

$\begin{array}{lllll}7.8 & 23.5 & -- & 7.9 & 96 \\ 8.3 & 23.5 & -- & 7.5 & 90\end{array}$

$\begin{array}{rrrrr}8.4 & 23.5 & - & 7.5 & 90 \\ 8.4 & 24.5 & .18 & 7.9 & 100\end{array}$

$8.3 \quad 24.5 \quad-5 \quad 7.5$

8.325 .0

$8.2 \quad 25.0 \quad \ldots$

$\begin{array}{llll}8.5 & 30.0 & .34 & 6.3 \\ 8.2 & 28.5 & -. & 7.5\end{array}$

$\begin{array}{llll}8.2 & 28.5 & -- & 7.5 \\ 8.0 & 28.0 & -- & 6.0\end{array}$

$\begin{array}{llll}8.5 & 26.5 & .25 & 7.1\end{array}$

8.426 .5

8.530 .007 .075

$\begin{array}{lllll}8.5 & 30.0 & .45 & 7.2 & 108 \\ 8.5 & 29.5 & -- & 6.8 & 100\end{array}$

$\begin{array}{lllll}8.2 & 7.0 & -- & 10.6 & 97 \\ 8.2 & 7.0 & -- & 10.5 & 97\end{array}$

$\begin{array}{lllll}8.0 & 27.0 & .21 & 6.7 & 94 \\ 8.0 & 27.0 & -. & 6.5 & 94\end{array}$ 
293519094500800 LINE 260 SITE 40

\begin{tabular}{|c|c|c|c|c|c|c|c|c|}
\hline DATE & TIME & $\begin{array}{c}\text { SAPP- } \\
\text { LING } \\
\text { DEPTH } \\
\text { (FT) }\end{array}$ & $\begin{array}{l}\text { SPE- } \\
\text { CIFIC } \\
\text { CON- } \\
\text { DULCT- } \\
\text { ANCE } \\
\text { (MICRO- } \\
\text { MHDS) }\end{array}$ & $\begin{array}{c}\mathrm{PH} \\
\text { (UNITS) }\end{array}$ & $\begin{array}{l}\text { TEMPER- } \\
\text { ATURE } \\
\text { (DEG C) }\end{array}$ & $\begin{array}{l}\text { JRANS- } \\
\text { PAR- } \\
\text { ENCY } \\
\text { (SECCCHI } \\
\text { DISK) } \\
\text { (M) }\end{array}$ & $\begin{array}{c}\text { OXYGEN, } \\
\text { DJS- } \\
\text { SOLWED } \\
\text { (MG } / L)\end{array}$ & $\begin{array}{l}\text { OXYGEN, } \\
\text { OIS- } \\
\text { SOLYED } \\
\text { (PER- } \\
\text { CENT } \\
\text { SATUR- } \\
\text { ATION) }\end{array}$ \\
\hline \multicolumn{9}{|c|}{ CT, 1976} \\
\hline $\begin{array}{l}21 \ldots \\
21 \ldots\end{array}$ & $\begin{array}{l}1305 \\
1307\end{array}$ & $\begin{array}{l}1.0 \\
7.0\end{array}$ & $\begin{array}{l}19000 \\
23000\end{array}$ & $\begin{array}{l}8.4 \\
8.3\end{array}$ & $\begin{array}{l}15.5 \\
15.5\end{array}$ & $\begin{array}{r}.52 \\
-.\end{array}$ & $\begin{array}{l}8.2 \\
7.5\end{array}$ & $\begin{array}{l}91 \\
88\end{array}$ \\
\hline $\begin{array}{l}18 \ldots \\
18 . . . \\
D F C\end{array}$ & $\begin{array}{l}1045 \\
1047\end{array}$ & $\begin{array}{l}1.0 \\
7.0\end{array}$ & $\begin{array}{l}23000 \\
24000\end{array}$ & $m$ & $\begin{array}{l}9.5 \\
9.5\end{array}$ & $\begin{array}{r}1.49 \\
-\end{array}$ & $\begin{array}{l}10.6 \\
10.5\end{array}$ & $\begin{array}{l}105 \\
105\end{array}$ \\
\hline $\begin{array}{l}13 . . . \\
13 \ldots \\
\text { APR , } 1\end{array}$ & $\begin{array}{l}0950 \\
0952 \\
7\end{array}$ & $\begin{array}{l}1.0 \\
9.0\end{array}$ & $\begin{array}{l}22000 \\
22000\end{array}$ & $\begin{array}{l}8.6 \\
8.5\end{array}$ & $\begin{array}{l}11.5 \\
11.5\end{array}$ & $\begin{array}{l}.30 \\
--\end{array}$ & $\begin{array}{l}9.8 \\
9.8\end{array}$ & $\begin{array}{l}101 \\
101\end{array}$ \\
\hline $\begin{array}{l}26 \ldots \\
26 \ldots \\
26 \ldots \\
26 . \ldots \\
\text { MAY }\end{array}$ & $\begin{array}{l}1110 \\
1112 \\
1114 \\
1116\end{array}$ & $\begin{array}{l}1.0 \\
2.5 \\
4.0 \\
8.0\end{array}$ & $\begin{array}{l}2000 \\
3700 \\
5100 \\
5400\end{array}$ & $\begin{array}{l}8.2 \\
8.2 \\
8.3 \\
8.3\end{array}$ & $\begin{array}{l}21.5 \\
21.5 \\
21.0 \\
21.0\end{array}$ & $\begin{array}{l}.18 \\
=- \\
=-\end{array}$ & $\begin{array}{l}8.9 \\
8.4 \\
8.0 \\
8.0\end{array}$ & $\begin{array}{r}103 \\
99 \\
94 \\
94\end{array}$ \\
\hline $\begin{array}{l}05 \ldots \\
05 \ldots \\
11 \ldots \\
11 \ldots \\
19 \ldots \\
19 \ldots \\
31 \ldots \\
31 \ldots \\
\text { JUN }\end{array}$ & $\begin{array}{l}1030 \\
1032 \\
0920 \\
0922 \\
0945 \\
0947 \\
1620 \\
1622\end{array}$ & $\begin{array}{r}1.5 \\
8.0 \\
1.0 \\
8.5 \\
1.0 \\
10 \\
1.0 \\
9.5\end{array}$ & $\begin{array}{l}1300 \\
1200 \\
1100 \\
1100 \\
1500 \\
1400 \\
5700 \\
7200\end{array}$ & $\begin{array}{l}7.8 \\
7.7 \\
8.4 \\
8.4 \\
8.4 \\
8.3 \\
8.5 \\
7.9\end{array}$ & $\begin{array}{l}24.0 \\
24.0 \\
24.0 \\
24.0 \\
24.5 \\
25.0 \\
30.0 \\
27.5\end{array}$ & $\begin{array}{r}.10 \\
.11 \\
. \overline{.11} \\
.- \\
.34 \\
.-\end{array}$ & $\begin{array}{l}7.5 \\
7.9 \\
7.5 \\
7.6 \\
7.3 \\
7.2 \\
9.4 \\
5.9\end{array}$ & $\begin{array}{r}91 \\
96 \\
91 \\
93 \\
90 \\
89 \\
127 \\
77\end{array}$ \\
\hline $\begin{array}{l}16 . . \\
16 \ldots \\
\text { AUG }\end{array}$ & $\begin{array}{l}1100 \\
1102\end{array}$ & $\begin{array}{l}1.0 \\
9.5\end{array}$ & $\begin{array}{l}13000 \\
13000\end{array}$ & $\begin{array}{l}8.3 \\
8.4\end{array}$ & $\begin{array}{l}26.5 \\
26.5\end{array}$ & .35 & $\begin{array}{l}6.8 \\
6.8\end{array}$ & $\begin{array}{l}90 \\
90\end{array}$ \\
\hline $\begin{array}{l}26 \ldots \\
26 \ldots .\end{array}$ & $\begin{array}{l}1205 \\
8^{1207}\end{array}$ & $\begin{array}{l}1.0 \\
9.0\end{array}$ & $\begin{array}{l}28000 \\
28000\end{array}$ & $\begin{array}{l}8.4 \\
8.4\end{array}$ & $\begin{array}{l}29.5 \\
29.5\end{array}$ & $\begin{array}{r}45 \\
--\end{array}$ & $\begin{array}{l}7.3 \\
6.9\end{array}$ & $\begin{array}{l}108 \\
101\end{array}$ \\
\hline $\begin{array}{l}07 \ldots \\
07 \ldots\end{array}$ & $\begin{array}{l}1003 \\
1005\end{array}$ & $\begin{array}{l}1.0 \\
7.0\end{array}$ & $\begin{array}{l}26000 \\
26000\end{array}$ & $\begin{array}{l}8.0 \\
8.1\end{array}$ & $\begin{array}{l}27.0 \\
27.0\end{array}$ & .20 & $\begin{array}{l}6.6 \\
6.5\end{array}$ & $\begin{array}{l}92 \\
91\end{array}$ \\
\hline
\end{tabular}

293650094514800 LINE 260 SITE 60

\begin{tabular}{|c|c|c|c|c|c|c|c|c|}
\hline DATE & TIME & $\begin{array}{l}\text { SANP- } \\
\text { LING } \\
\text { DEPTH } \\
\text { (FT) }\end{array}$ & $\begin{array}{l}\text { SPE- } \\
\text { CIFIC } \\
\text { CDN- } \\
\text { DUCT- } \\
\text { ANCE } \\
\text { (MICRO- } \\
\text { MHOS) }\end{array}$ & $\begin{array}{c}\mathrm{PH} \\
\text { (UNITS) }\end{array}$ & $\begin{array}{l}\text { TEMPER- } \\
\text { ATURE } \\
\text { (DEG C) }\end{array}$ & $\begin{array}{l}\text { TRARS- } \\
\text { PAR- } \\
\text { ENCY } \\
\text { (SECCHI } \\
\text { DISK) } \\
(M)\end{array}$ & $\begin{array}{c}\text { OXYGEN, } \\
\text { OIS* } \\
\text { SOLYEO } \\
\text { (MG } / L)\end{array}$ & $\begin{array}{l}\text { OXYGEN, } \\
\text { DIS- } \\
\text { SOLVED } \\
\text { (PER- } \\
\text { CENT } \\
\text { SATUR- } \\
\text { ATIOK) }\end{array}$ \\
\hline \multicolumn{9}{|c|}{ OCT, 1976} \\
\hline $\begin{array}{l}21 \ldots \\
21 \ldots \\
21 \ldots \\
\text { Now }\end{array}$ & $\begin{array}{l}1315 \\
1317 \\
1319\end{array}$ & $\begin{array}{l}1.0 \\
5.0 \\
9.0\end{array}$ & $\begin{array}{l}25000 \\
25000 \\
29000\end{array}$ & $\begin{array}{l}8.3 \\
8.3 \\
8.2\end{array}$ & $\begin{array}{l}16.0 \\
15.5 \\
16.0\end{array}$ & $\begin{array}{l}.58 \\
-- \\
--\end{array}$ & $\begin{array}{l}8.2 \\
8.0 \\
7.3\end{array}$ & $\begin{array}{l}94 \\
91 \\
86\end{array}$ \\
\hline $\begin{array}{l}18 . . . \\
18 \ldots \\
\text { oEc }\end{array}$ & $\begin{array}{l}1110 \\
1112\end{array}$ & $\begin{array}{l}1.0 \\
9.0\end{array}$ & $\begin{array}{l}24000 \\
29000\end{array}$ & -- & $\begin{array}{l}10.0 \\
10.5\end{array}$ & 1.28 & $\begin{array}{r}10.5 \\
9.5\end{array}$ & $\begin{array}{r}106 \\
99\end{array}$ \\
\hline $\begin{array}{l}13 \ldots \\
13 E B\end{array}$ & $\begin{array}{r}0930 \\
0932 \\
7\end{array}$ & $\begin{array}{l}3.0 \\
9.0\end{array}$ & $\begin{array}{l}21400 \\
22000\end{array}$ & $\begin{array}{l}8.3 \\
7.2\end{array}$ & $\begin{array}{l}11.5 \\
11.5\end{array}$ & .46 & $\begin{array}{l}9.4 \\
9.5\end{array}$ & $\begin{array}{l}97 \\
98\end{array}$ \\
\hline $\begin{array}{l}03 . \cdots \\
03 . \cdots \\
\text { APR }\end{array}$ & $\begin{array}{l}1345 \\
1347\end{array}$ & $\begin{array}{l}1.0 \\
7.0\end{array}$ & $\begin{array}{l}18000 \\
22000\end{array}$ & $=$ & $\begin{array}{l}9.0 \\
9.0\end{array}$ & .48 & $\begin{array}{r}10.0 \\
8.9\end{array}$ & $\begin{array}{l}95 \\
86\end{array}$ \\
\hline $\begin{array}{l}26 \ldots \\
26 \ldots \\
26 \ldots \\
26 \ldots \\
\text { MAY }\end{array}$ & $\begin{array}{l}1120 \\
1122 \\
1124 \\
1126\end{array}$ & $\begin{array}{l}1.0 \\
3.0 \\
4.5 \\
9.0\end{array}$ & $\begin{array}{l}6550 \\
6300 \\
6600 \\
6600\end{array}$ & $\begin{array}{l}8.7 \\
8.6 \\
8.5 \\
8.5\end{array}$ & $\begin{array}{l}21.5 \\
21.5 \\
21.0 \\
21.0\end{array}$ & $\begin{array}{l}.34 \\
- \\
- \\
4 .\end{array}$ & $\begin{array}{r}11.1 \\
10.5 \\
8.8 \\
8.5\end{array}$ & $\begin{array}{l}133 \\
126 \\
103 \\
100\end{array}$ \\
\hline $\begin{array}{l}05 \ldots \\
05 \ldots \\
11 \ldots \\
11 \ldots \\
17 \ldots \\
17 \ldots \\
19 \ldots \\
19 \ldots \\
31 \ldots \\
31 \ldots \\
31 \ldots \\
\text { JuN }\end{array}$ & $\begin{array}{l}1045 \\
1047 \\
0940 \\
0942 \\
1045 \\
1047 \\
0955 \\
0957 \\
1635 \\
1637 \\
1639\end{array}$ & $\begin{array}{c}1.5 \\
9.0 \\
1.0 \\
10 \\
1.0 \\
11 \\
1.0 \\
10 \\
1.0 \\
5.0 \\
10\end{array}$ & $\begin{array}{r}1200 \\
1000 \\
5300 \\
5500 \\
1470 \\
1200 \\
3200 \\
3200 \\
6600 \\
6700 \\
9500\end{array}$ & $\begin{array}{l}7.9 \\
7.9 \\
8.4 \\
8.4 \\
8.5 \\
8.6 \\
8.2 \\
8.2 \\
8.5 \\
8.2 \\
8.0\end{array}$ & $\begin{array}{l}24.0 \\
24.0 \\
24.5 \\
24.5 \\
24.5 \\
24.5 \\
24.5 \\
24.5 \\
30.0 \\
27.5 \\
27.5\end{array}$ & $\begin{array}{l}-- \\
.- \\
.15 \\
.09 \\
.09 \\
.12 \\
. \overline{-} \\
.4 \\
--\end{array}$ & $\begin{array}{l}7.8 \\
7.6 \\
7.4 \\
7.9 \\
7.6 \\
7.5 \\
7.0 \\
6.9 \\
9.1 \\
7.0 \\
5.6\end{array}$ & $\begin{array}{r}95 \\
93 \\
92 \\
99 \\
94 \\
93 \\
86 \\
85 \\
123 \\
92 \\
75\end{array}$ \\
\hline $\begin{array}{l}16 . \ldots \\
16 . . . \\
\text { AUG }\end{array}$ & $\begin{array}{l}1020 \\
1022\end{array}$ & $10^{1.0}$ & $\begin{array}{l}17900 \\
18000\end{array}$ & $\begin{array}{l}8.5 \\
8.4\end{array}$ & $\begin{array}{l}26.5 \\
26.5\end{array}$ & .28 & $\begin{array}{l}6.7 \\
6.6\end{array}$ & $\begin{array}{l}90 \\
87\end{array}$ \\
\hline $\begin{array}{l}26 . \ldots \\
26 . \ldots \\
F E B\end{array}$ & $\begin{array}{l}1140 \\
1142 \\
8\end{array}$ & $\begin{array}{l}1.0 \\
9.5\end{array}$ & $\begin{array}{l}21000 \\
21000\end{array}$ & $\begin{array}{l}8.3 \\
8.3\end{array}$ & $\begin{array}{l}29.5 \\
29.0\end{array}$ & $\begin{array}{r}.55 \\
--\end{array}$ & $\begin{array}{l}6.2 \\
5.6\end{array}$ & $\begin{array}{l}88 \\
80\end{array}$ \\
\hline $\begin{array}{l}08 . . . \\
08 . \cdots \\
\text { JUN }\end{array}$ & $\begin{array}{l}1310 \\
1312\end{array}$ & $\begin{array}{l}1.0 \\
9.0\end{array}$ & $\begin{array}{l}24000 \\
23000\end{array}$ & $\begin{array}{l}8.1 \\
8.1\end{array}$ & $\begin{array}{l}6.5 \\
6.0\end{array}$ & $=$ & $\begin{array}{l}10.3 \\
10.5\end{array}$ & $\begin{array}{l}96 \\
94\end{array}$ \\
\hline $\begin{array}{l}07 . .4 \\
07 \ldots\end{array}$ & $\begin{array}{l}1015 \\
1017\end{array}$ & $\begin{array}{l}1.0 \\
9.0\end{array}$ & $\begin{array}{l}27400 \\
27200\end{array}$ & $\begin{array}{l}8.1 \\
8.1\end{array}$ & $\begin{array}{l}27.0 \\
27.0\end{array}$ & 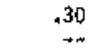 & $\begin{array}{l}6.7 \\
5.5\end{array}$ & $\begin{array}{l}94 \\
91\end{array}$ \\
\hline
\end{tabular}


Table 2A..+Quality of water in the Trintty-5an Jacinto estuary, water years 1977-78--Continued

Fie1d Determinations--Continued

293821094532900 LINE 260 SITE 80

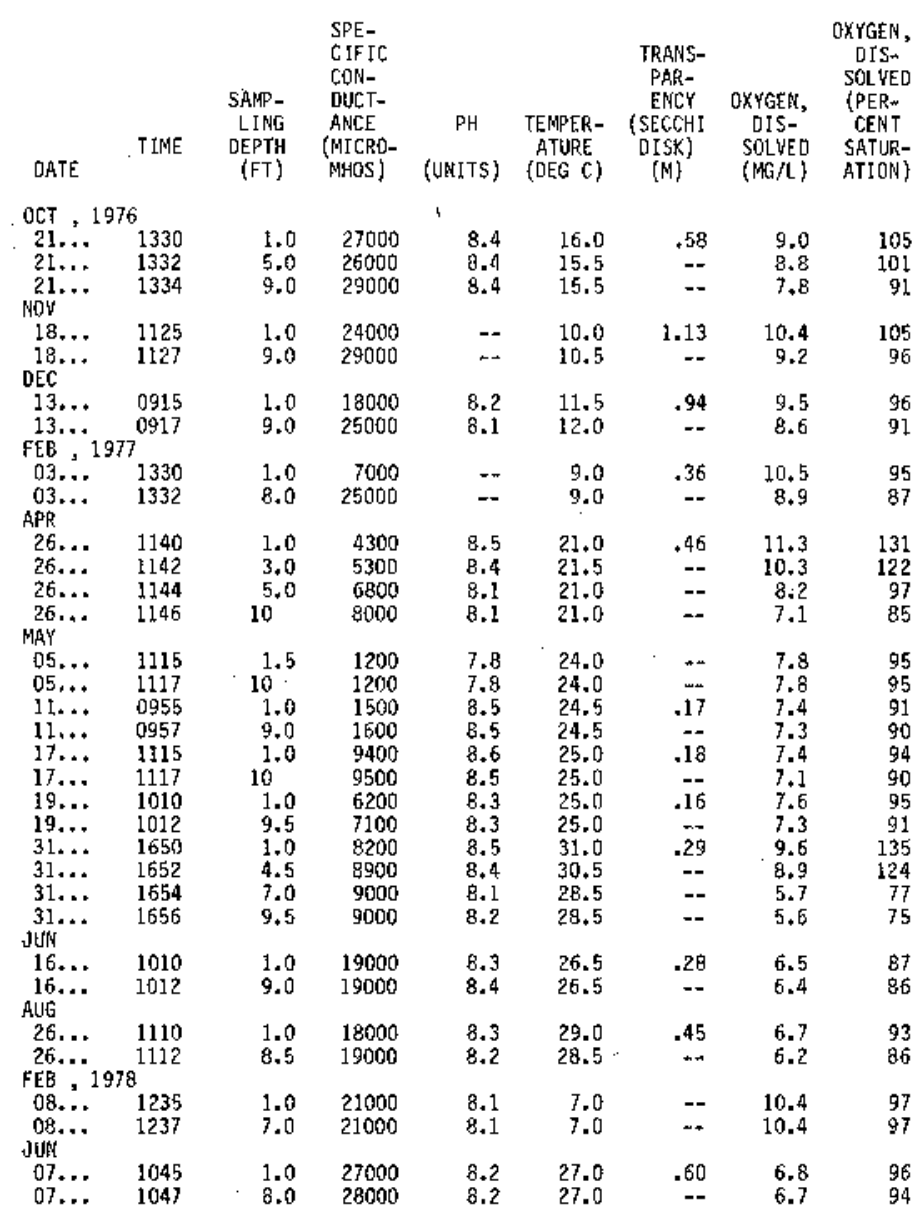

$293829094560900 \quad$ LINE $320 \cdot$ SITE 20

\begin{tabular}{|c|c|c|c|c|c|c|c|c|}
\hline DATE & TIME & $\begin{array}{c}\text { SAPAP- } \\
\text { EING } \\
\text { DEPTH } \\
\text { (FT) }\end{array}$ & $\begin{array}{l}\text { SPE- } \\
\text { CIFIC } \\
\text { CON- } \\
\text { OUCT- } \\
\text { ANCE } \\
\text { (MICRO- } \\
\text { MHOS) }\end{array}$ & $\begin{array}{c}\mathrm{PH} \\
\text { (UผITS) }\end{array}$ & $\begin{array}{l}\text { TEMPER- } \\
\text { ATURE } \\
\text { (DEG C) }\end{array}$ & $\begin{array}{l}\text { TRANS- } \\
\text { PAR- } \\
\text { ENCY } \\
\text { (SECCHI } \\
\text { DISK) } \\
\text { (M) }\end{array}$ & $\begin{array}{c}\text { OXYGER, } \\
\text { DIS- } \\
\text { SOLVED } \\
\text { (MG } / L)\end{array}$ & $\begin{array}{c}\text { OXYGEN, } \\
\text { DIS- } \\
\text { SOLUED } \\
\text { (PER- } \\
\text { CENT } \\
\text { SATUR- } \\
\text { ATION) }\end{array}$ \\
\hline \multicolumn{9}{|c|}{ ROV , 1976} \\
\hline $\begin{array}{l}18 \ldots \\
18 . . \\
\text { FEB }\end{array}$ & $\begin{array}{l}1245 \\
1247 \\
7\end{array}$ & $\begin{array}{l}1.0 \\
6.0\end{array}$ & $\begin{array}{l}27000 \\
28000\end{array}$ & $=$ & $\begin{array}{l}11.5 \\
12.0\end{array}$ & $\begin{array}{r}.75 \\
-+\end{array}$ & $\begin{array}{l}8.9 \\
2.7\end{array}$ & $\begin{array}{l}91 \\
93\end{array}$ \\
\hline $\begin{array}{l}03 . . \\
\text { RU... } \\
\text { RUG }\end{array}$ & $\begin{array}{l}1355 \\
1357\end{array}$ & $\begin{array}{l}1.0 \\
8.0\end{array}$ & $\begin{array}{l}20000 \\
21000\end{array}$ & $\begin{array}{l}8.7 \\
8.7\end{array}$ & $\begin{array}{l}9.0 \\
9.0\end{array}$ & $\begin{array}{r}.38 \\
--\end{array}$ & $\begin{array}{l}12+0 \\
12.3\end{array}$ & $\begin{array}{l}115 \\
110\end{array}$ \\
\hline $\begin{array}{l}26 \ldots \\
26 \ldots \\
\text { FEB } .\end{array}$ & $\begin{array}{l}1120 \\
1122 \\
B\end{array}$ & $\begin{array}{l}1.0 \\
8.5\end{array}$ & $\begin{array}{l}19000 \\
19000\end{array}$ & $\begin{array}{l}8.3 \\
8.3\end{array}$ & $\begin{array}{l}29.5 \\
29.5\end{array}$ & $\begin{array}{r}.51 \\
--\end{array}$ & $\begin{array}{l}6.4 \\
6.1\end{array}$ & $\begin{array}{l}91 \\
86\end{array}$ \\
\hline $\begin{array}{l}08 . . . \\
\text { JUN... }\end{array}$ & $\begin{array}{l}1225 \\
1227\end{array}$ & $\begin{array}{l}1.0 \\
6.0\end{array}$ & $\begin{array}{l}22000 \\
22000\end{array}$ & $\begin{array}{l}8.2 \\
8.1\end{array}$ & $\begin{array}{l}7.0 \\
7.5\end{array}$ & $=$ & $\begin{array}{l}10.2 \\
10.1\end{array}$ & $\begin{array}{l}94 \\
96\end{array}$ \\
\hline $\begin{array}{l}07 \ldots \\
07 \ldots\end{array}$ & $\begin{array}{l}1058 \\
1100\end{array}$ & $\begin{array}{l}1.0 \\
6.0\end{array}$ & $\begin{array}{l}26000 \\
27000\end{array}$ & $\begin{array}{l}8.1 \\
8.1\end{array}$ & $\begin{array}{l}27.0 \\
27.0\end{array}$ & $\begin{array}{l}.50 \\
=-\end{array}$ & $\begin{array}{l}6.6 \\
6.5\end{array}$ & $\begin{array}{l}92 \\
91\end{array}$ \\
\hline
\end{tabular}

293722094573200 LINE 320 SITE 35

\begin{tabular}{|c|c|c|c|c|c|c|c|c|}
\hline DATE & TIME & $\begin{array}{c}\text { SALP- } \\
\text { EING } \\
\text { DEPTH } \\
\text { (FT) }\end{array}$ & $\begin{array}{l}\text { SPE- } \\
\text { CIFIC } \\
\text { CON- } \\
\text { DULT- } \\
\text { ANCE } \\
\text { (MICRO- } \\
\text { MHOS) }\end{array}$ & $\begin{array}{c}\text { PH } \\
\text { (UNITS) }\end{array}$ & $\begin{array}{c}\text { TEMPER- } \\
\text { ATURE } \\
\text { (DEG C) }\end{array}$ & $\begin{array}{c}\text { TRANS- } \\
\text { PAR- } \\
\text { ENCY } \\
\text { (SECCHI } \\
\text { D1SX) } \\
\text { (M) }\end{array}$ & $\begin{array}{c}\text { OXYGEN, } \\
\text { DIS- } \\
\text { SOLWED } \\
\text { (MG/L) }\end{array}$ & $\begin{array}{l}\text { OXYGEN, } \\
\text { DIS- } \\
\text { SOLVED } \\
\text { (PER- } \\
\text { CENT } \\
\text { SATUR- } \\
\text { ATION) }\end{array}$ \\
\hline $\begin{array}{r}\text { JUN }, \\
23 \ldots \\
23 \ldots \\
23 \ldots \\
23 \ldots\end{array}$ & $\begin{array}{l}7 \\
1245 \\
1247 \\
1249 \\
1251\end{array}$ & $\begin{array}{l}1.0 \\
10 \\
20 \\
34\end{array}$ & $\begin{array}{l}19000 \\
20000 \\
23000 \\
23000\end{array}$ & $\begin{array}{l}8.0 \\
8.0 \\
7.9 \\
7.9\end{array}$ & $\begin{array}{l}29.0 \\
29.0 \\
29.0 \\
29.5\end{array}$ & $\begin{array}{c}.40 \\
-- \\
--\end{array}$ & $\begin{array}{l}5.8 \\
5.4 \\
5.0 \\
4.4\end{array}$ & $\begin{array}{l}82 \\
76 \\
71 \\
63\end{array}$ \\
\hline
\end{tabular}


Table 2A.--Quality of water in the Trinity-San Jacinto estuary, water years 1977-78--Continued

Field Deterninations--Continued

293707094574800 LINE 320 SITE 40

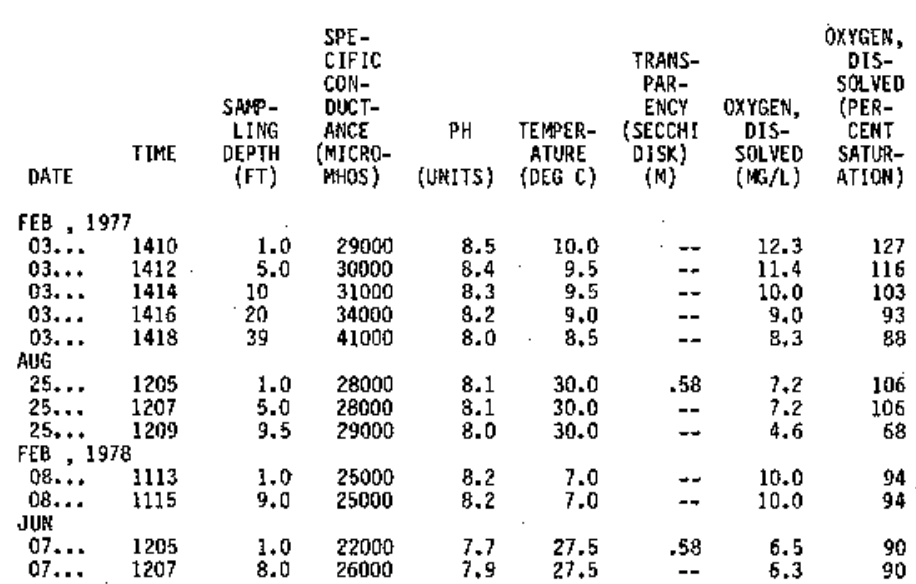

293253095010400 LINE 330 SITE 20

\begin{tabular}{|c|c|c|c|c|c|c|c|c|}
\hline DATE & TIME & $\begin{array}{l}\text { SAlP- } \\
\text { LiNG } \\
\text { OEPTH } \\
\text { (FT) }\end{array}$ & $\begin{array}{l}\text { SPE- } \\
\text { CIF IC } \\
\text { CDN- } \\
\text { DUCT- } \\
\text { ANCE } \\
\text { (MICRO- } \\
\text { MHOS) }\end{array}$ & $\begin{array}{c}\text { PH } \\
\text { (UNITS) }\end{array}$ & $\begin{array}{c}\text { TEMPER- } \\
\text { ATURE } \\
\text { (DEG C) }\end{array}$ & $\begin{array}{c}\text { TRANS- } \\
\text { PAR- } \\
\text { ENCY } \\
\text { (SECCHI } \\
\text { DISK) } \\
\text { (M) }\end{array}$ & $\begin{array}{c}\text { OXYGEN, } \\
\text { DIS- } \\
\text { SOLVED } \\
\text { (NG } / L)\end{array}$ & $\begin{array}{l}\text { OXYGEN, } \\
\text { DIS- } \\
\text { SOLVED } \\
\text { (PER- } \\
\text { CENT } \\
\text { SATUR- } \\
\text { ATION) }\end{array}$ \\
\hline OCT, & & & & & & & & \\
\hline $\begin{array}{l}21 \ldots \\
21 \ldots \ldots \\
21 \ldots \\
\text { MOH }\end{array}$ & $\begin{array}{l}1220 \\
1222 \\
1224\end{array}$ & $\begin{array}{r}2.0 \\
5.0 \\
17\end{array}$ & $\begin{array}{l}24000 \\
24000 \\
24000\end{array}$ & $\begin{array}{l}6.7 \\
6.7 \\
7.5\end{array}$ & $\begin{array}{l}17.0 \\
17.0 \\
17.0\end{array}$ & $\begin{array}{r}.63 \\
-2 \\
-\end{array}$ & $\begin{array}{l}-- \\
-- \\
--\end{array}$ & $\begin{array}{l}-- \\
--\end{array}$ \\
\hline $\begin{array}{l}18 . . . \\
18, \ldots \\
18 \ldots \\
\text { FEB , }\end{array}$ & $\begin{array}{l}1335 \\
1337 \\
1339 \\
7\end{array}$ & $\begin{array}{l}1.0 \\
8.0 \\
17\end{array}$ & $\begin{array}{l}22000 \\
24000 \\
26000\end{array}$ & $\begin{array}{l}-- \\
--\end{array}$ & $\begin{array}{l}12.0 \\
11.5 \\
11.0\end{array}$ & $\begin{array}{l}.68 \\
\cdots \\
-2\end{array}$ & $\begin{array}{r}10.6 \\
9.9 \\
9.2\end{array}$ & $\begin{array}{l}110 \\
103 \\
102\end{array}$ \\
\hline $\begin{array}{l}03 . . . \\
03 . . . \\
03 \ldots \\
\text { JUN }\end{array}$ & $\begin{array}{l}1305 \\
1307 \\
1309\end{array}$ & $\begin{array}{c}1.0 \\
5.0 \\
15\end{array}$ & $\begin{array}{l}19000 \\
20000 \\
20000\end{array}$ & $\begin{array}{l}8.6 \\
8.6 \\
8.6\end{array}$ & $\begin{array}{l}10.5 \\
10.5 \\
10.5\end{array}$ & $\begin{array}{l}.40 \\
-- \\
--\end{array}$ & $\begin{array}{l}10.6 \\
10.6 \\
10.6\end{array}$ & $\begin{array}{l}106 \\
106 \\
106\end{array}$ \\
\hline $\begin{array}{l}23 \ldots \\
23 \ldots \\
23 \ldots \\
\text { AUG }\end{array}$ & $\begin{array}{l}1155 \\
1157 \\
1159\end{array}$ & $\begin{array}{l}1.0 \\
10 \\
20\end{array}$ & $\begin{array}{l}22000 \\
23000 \\
23000\end{array}$ & $\begin{array}{l}8.2 \\
8.3 \\
8.3\end{array}$ & $\begin{array}{l}29.0 \\
29.0 \\
30.0\end{array}$ & .42 & $\begin{array}{l}6.3 \\
6.4 \\
6.3\end{array}$ & $\begin{array}{l}89 \\
91 \\
91\end{array}$ \\
\hline $\begin{array}{r}25 \ldots \\
25 \ldots \\
25 \ldots \\
\text { FEB } .\end{array}$ & $\begin{array}{l}1.125 \\
1127 \\
1129 \\
8\end{array}$ & $\begin{array}{l}1.0 \\
10 \\
19\end{array}$ & $\begin{array}{l}280 \text { 여 } \\
28000 \\
28000\end{array}$ & $\begin{array}{l}8.2 \\
8.2 \\
8.2\end{array}$ & $\begin{array}{l}30.0 \\
29.5 \\
29.5\end{array}$ & $\begin{array}{l}.33 \\
-- \\
--\end{array}$ & $\begin{array}{l}6.7 \\
5.2 \\
4.7\end{array}$ & $\begin{array}{l}99 \\
76 \\
68\end{array}$ \\
\hline $\begin{array}{l}08 . . \\
08 . .\end{array}$ & $\begin{array}{l}0945 \\
0947\end{array}$ & $18^{1.0}$ & $\begin{array}{l}18000 \\
20000\end{array}$ & $\begin{array}{l}8.4 \\
8.3\end{array}$ & $\begin{array}{l}7.0 \\
6.5\end{array}$ & $=-$ & $\begin{array}{r}9.9 \\
10.0\end{array}$ & $\begin{array}{l}90 \\
91\end{array}$ \\
\hline $\begin{array}{l}07 \ldots \\
07 \ldots \\
07 \ldots\end{array}$ & $\begin{array}{l}1305 \\
1307 \\
1309\end{array}$ & $\begin{array}{l}1.0 \\
10 \\
23\end{array}$. & $\begin{array}{l}26000 \\
26000 \\
27000\end{array}$ & $\begin{array}{l}8.2 \\
8.2 \\
8.3\end{array}$ & $\begin{array}{l}27.5 \\
27.5 \\
27.5\end{array}$ & $\begin{array}{l}.35 \\
--\end{array}$ & $\begin{array}{l}6.3 \\
6.4 \\
6.3\end{array}$ & $\begin{array}{l}90 \\
91 \\
90\end{array}$ \\
\hline
\end{tabular}

293549094535000 LINE 340 SITE 20

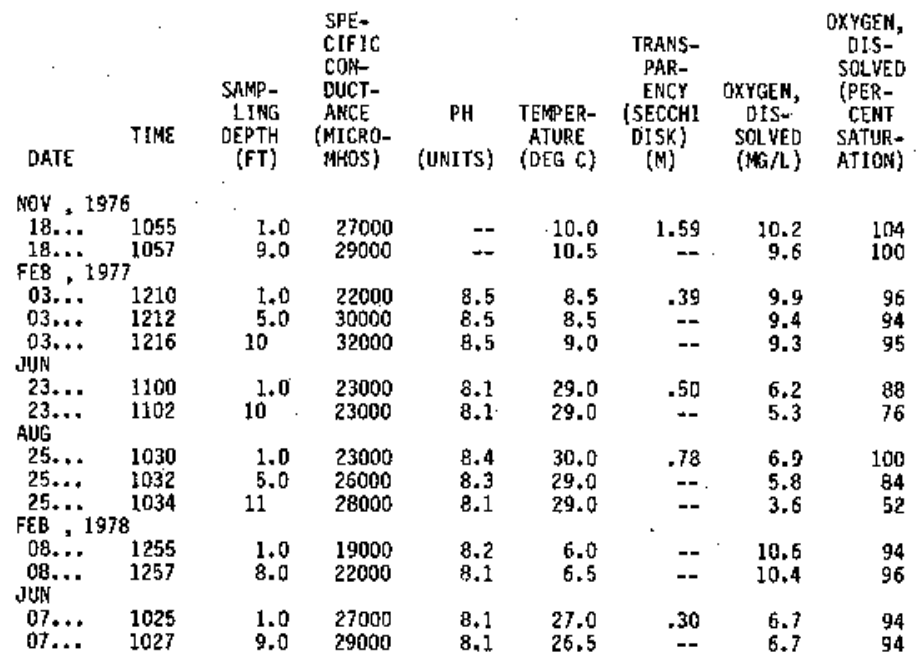


Table 2A.-nquality of water in the TrinitywSan Jacinto estuary, water years 1977-73-nContinued Field Determinations--Contrinued

293428094553800 LINE 340 SITE 40

\begin{tabular}{|c|c|c|c|c|c|c|c|c|}
\hline DATE & TIME & $\begin{array}{l}\text { SAMP- } \\
\text { LING } \\
\text { DEPTH } \\
\text { (FT) }\end{array}$ & $\begin{array}{l}\text { SPE- } \\
\text { CIFIC } \\
\text { CON- } \\
\text { DUCT- } \\
\text { ANCE } \\
\text { (MICRO- } \\
\text { MHOS) }\end{array}$ & $\begin{array}{c}\text { PH } \\
\text { \{UNITS\} }\end{array}$ & $\begin{array}{l}\text { TEMPER- } \\
\text { ATURE } \\
\text { (DEG C) }\end{array}$ & $\begin{array}{l}\text { TRANS- } \\
\text { PAR- } \\
\text { ENCY } \\
\text { (SECCHI } \\
\text { DISK) } \\
\text { (M) }\end{array}$ & $\begin{array}{c}\text { OXYGEN, } \\
\text { BIS- } \\
\text { SOL } \\
\text { (MG } / L)\end{array}$ & $\begin{array}{c}\text { OXYGEN, } \\
\text { OIS- } \\
\text { SOLVED } \\
\text { (PER- } \\
\text { CERT } \\
\text { SRTUR- } \\
\text { ATION) }\end{array}$ \\
\hline \multicolumn{9}{|c|}{ OCT, 1976} \\
\hline $21 \ldots$ & 1155 & 2.0 & $=-$ & 8.4 & 17.0 & .56 & -- & -- \\
\hline $\begin{array}{l}21 \ldots \\
21 . .\end{array}$ & $\begin{array}{l}1157 \\
1159\end{array}$ & $\begin{array}{l}\text { I0 } \\
20\end{array}$ & $\begin{array}{l}29000 \\
30000\end{array}$ & $\begin{array}{l}8.4 \\
8.3\end{array}$ & $\begin{array}{l}17.0 \\
17.0\end{array}$ & $=$ & $=$ & $=$ \\
\hline $21 \ldots$ & 1201 & 38 & 35000 & 8.4 & 17.5 & - & $\because$ & 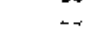 \\
\hline $18 \ldots$ & 1035 & 1.0 & 28000 & -- & 10.3 & 1.14 & 9.9 & 102 \\
\hline $18 \ldots$ & 1037 & 20 & 37000 & -- & 11.0 & $\sim$ & 8.6 & 95 \\
\hline FEB & $\gamma^{1039}$ & 44 & 44000 & $\infty$ & 11.5 & $m$ & 8.2 & 95 \\
\hline $03 .$. & 1145 & 1.0 & 28000 & 8.9 & 9.0 & $=\pi$ & 11.7 & 117 \\
\hline $03 . .$. & 1147 & 5.0 & 30000 & a.7 & 9.0 & -- & 10,4 & 105 \\
\hline 03. & 1149 & 10 & 32000 & 8.7 & 9.0 & -- & 10.1 & 103 \\
\hline 03. & 1151 & 20 & 35000 & 8.5 & 8.5 & -- & 9.2 & 94 \\
\hline 03. & 1153 & 40 & 39000 & 8.3 & 8.5 & -- & 8.8 & 92 \\
\hline \multicolumn{9}{|l|}{ JUN } \\
\hline 23. & 1045 & 1.0 & 22100 & 8.1 & 29.0 & .40 & 5.4 & 78 \\
\hline 23. & 1047 & 10 & 23000 & 8.1 & 29. & - & 5. & 75 \\
\hline 23. & 1049 & 20 & 24000 & 8.0 & 29. & r & 5.2 & 74 \\
\hline $23 .$. & $105 !^{\prime}$ & 33 & 26000 & 8.0 & 29.0 & $m$ & 4.4 & 63 \\
\hline \multicolumn{9}{|l|}{ AUG } \\
\hline $25 \ldots$ & 1010 & 1.0 & 25000 & 8.2 & 29.0 & - & 5.7 & 82 \\
\hline $25 \ldots$ & 1014 & 20 & 38000 & 8.2 & 29. & - & $\begin{array}{l}4.9 \\
5.0\end{array}$ & $\begin{array}{l}74 \\
78\end{array}$ \\
\hline & 1016 & 45 & 42000 & 8.1 & 29.5 & - & 3.8 & 61 \\
\hline \multicolumn{9}{|c|}{ FEB, 1978} \\
\hline $08 \ldots$ & 1045 & 1.0 & 22000 & 8,3 & 6.5 & -- & 10.4 & 96 \\
\hline 08. & 1047 & 20 & 28000 & 8.3 & 6. & -- & 10.2 & 94 \\
\hline $08 .$. & 1049 & 48 & 38000 & 8.5 & 6.0 & - & 9.4 & 93 \\
\hline \multicolumn{9}{|l|}{ JUN } \\
\hline $07 \ldots$ & 1225 & 1.0 & 30100 & 8,1 & 27.5 & .50 & 6.6 & 95 \\
\hline & 1227 & 20 & 33000 & 8.1 & 28.0 & -- & 6.4 & 94 \\
\hline & 1229 & 45 & 30800 & B.I & 28.0 & -- & 6.3 & 92 \\
\hline
\end{tabular}

293301094573200 LINE 340 SIYE 60

\begin{tabular}{|c|c|c|c|c|c|c|c|c|}
\hline DATE & TIME & $\begin{array}{l}\text { SAMP- } \\
\text { LING } \\
\text { DEPTH } \\
\text { (FT) }\end{array}$ & $\begin{array}{l}\text { SPE- } \\
\text { CIFIC } \\
\text { CON- } \\
\text { DUCT- } \\
\text { ANCE } \\
\text { (MICRO- } \\
\text { MHOS) }\end{array}$ & $\begin{array}{c}\text { PH } \\
\text { (UHITS) }\end{array}$ & $\begin{array}{c}\text { TEMPER- } \\
\text { ATLRE } \\
\text { (DEG C) }\end{array}$ & $\begin{array}{c}\text { TRANS- } \\
\text { PAR- } \\
\text { ENCY } \\
\text { (SECCHI } \\
\text { DISK) } \\
\text { (M) }\end{array}$ & $\begin{array}{c}\text { OXYGEN, } \\
\text { DIS- } \\
\text { SOLVED } \\
\text { (MG/L) }\end{array}$ & $\begin{array}{c}\text { OXYGEN, } \\
\text { OIS- } \\
\text { SOLYED } \\
\text { (PER- } \\
\text { CENT } \\
\text { SATUR- } \\
\text { ATION) }\end{array}$ \\
\hline \multicolumn{9}{|c|}{ NOH , 1976} \\
\hline $\begin{array}{l}18 \ldots \\
18 \ldots \\
\text { FEB },\end{array}$ & $\begin{array}{l}1005 \\
7^{1007}\end{array}$ & $\begin{array}{l}1.0 \\
9.0\end{array}$ & $\begin{array}{l}28000 \\
29000\end{array}$ & -- & $\begin{array}{l}10.5 \\
10.5\end{array}$ & $\begin{array}{r}1.12 \\
--\end{array}$ & $\begin{array}{l}10.1 \\
10.0\end{array}$ & $\begin{array}{l}104 \\
104\end{array}$ \\
\hline $\begin{array}{l}03 . \cdots \\
03 . \cdots \\
03 . \cdots \\
\text { JuN }\end{array}$ & $\begin{array}{l}1230 \\
1232 \\
1234\end{array}$ & $\begin{array}{l}1.0 \\
5.0 \\
10\end{array}$ & $\begin{array}{l}28000 \\
28000 \\
29000\end{array}$ & $\begin{array}{l}8.7 \\
8.7 \\
8.6\end{array}$ & $\begin{array}{l}9.0 \\
9.0 \\
9.0\end{array}$ & $\begin{array}{l}.48 \\
-- \\
--\end{array}$ & $\begin{array}{l}10.7 \\
10.3 \\
10.1\end{array}$ & $\begin{array}{l}107 \\
103 \\
102\end{array}$ \\
\hline $\begin{array}{l}23 \ldots \\
23 \ldots \\
\text { AUG }\end{array}$ & $\begin{array}{l}1120 \\
1122\end{array}$ & 11.0 & $\begin{array}{l}23000 \\
23000\end{array}$ & $\begin{array}{l}8.1 \\
8.1\end{array}$ & $\begin{array}{l}28.5 \\
29.0\end{array}$ & $\begin{array}{r}.47 \\
--\end{array}$ & $\begin{array}{l}6.2 \\
6.0\end{array}$ & $\begin{array}{l}87 \\
86\end{array}$ \\
\hline $\begin{array}{l}25 \ldots \\
25 \ldots \\
25 \ldots \\
25 \ldots \\
\text { FEB }\end{array}$ & $\begin{array}{l}1100 \\
1102 \\
1104 \\
1106\end{array}$ & $\begin{array}{r}1.0 \\
3.0 \\
5.0 \\
11\end{array}$ & $\begin{array}{l}29000 \\
29000 \\
29000 \\
29000\end{array}$ & $\begin{array}{l}8.3 \\
8.4 \\
8.3 \\
8.2\end{array}$ & $\begin{array}{l}30.0 \\
30.0 \\
29.5 \\
29.0\end{array}$ & $\begin{array}{l}.50 \\
-. \\
--\end{array}$ & $\begin{array}{l}8.3 \\
7.9 \\
5.9 \\
4.8\end{array}$ & $\begin{array}{r}124 \\
118 \\
87 \\
71\end{array}$ \\
\hline $\begin{array}{l}08 . . . \\
\text { OB... } \\
\text { JUN }\end{array}$ & $\begin{array}{l}1025 \\
1027\end{array}$ & 1.0 & $\begin{array}{l}25000 \\
27000\end{array}$ & $\begin{array}{l}8.4 \\
8.3\end{array}$ & $\begin{array}{l}6.0 \\
5.5\end{array}$ & $=$ & $\begin{array}{l}10.3 \\
10.2\end{array}$ & $\begin{array}{l}94 \\
93\end{array}$ \\
\hline $\begin{array}{l}07 \ldots \\
07 \ldots\end{array}$ & $\begin{array}{l}1243 \\
1245\end{array}$ & $10^{1.0}$ & $\begin{array}{l}27000 \\
28000\end{array}$ & $\begin{array}{l}8.1 \\
8.1\end{array}$ & $\begin{array}{l}27.5 \\
27.0\end{array}$ & .45 & $\begin{array}{l}6.7 \\
6.6\end{array}$ & $\begin{array}{l}96 \\
92\end{array}$ \\
\hline
\end{tabular}


Table 2A.--Quality of water in the Trinity-San Jacinto estuary, water years 1977-78--Continued Field Determinations--Cont inued

293133094592900 LINE 340 SITE BO

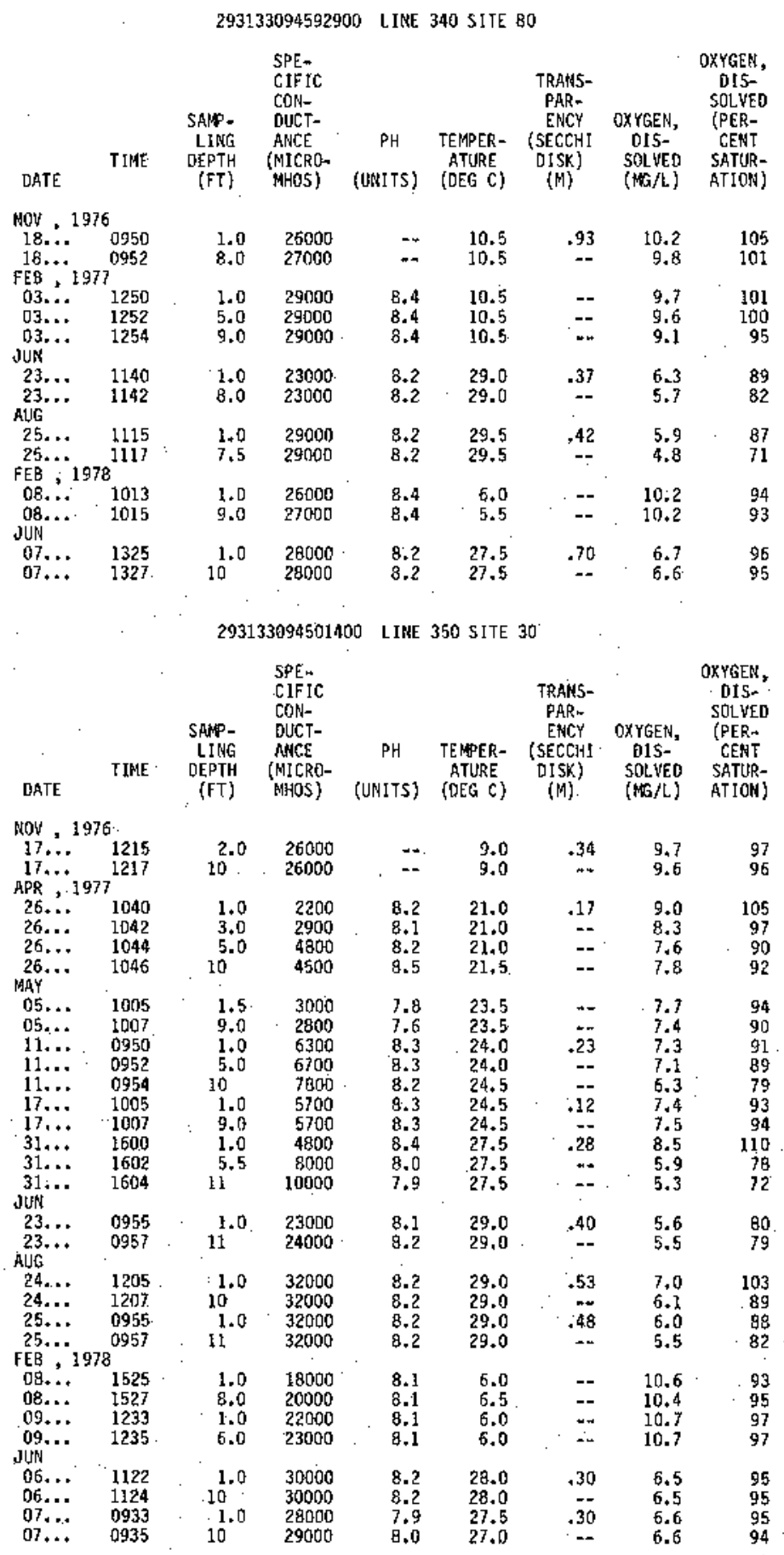


Table 2A.--Quality of water in the Trinity-San Jacinto estuary, water years 1977-78--Continued Field Determi nations--Continued

$293030094523500 \quad$ LINE 350 SITE 50

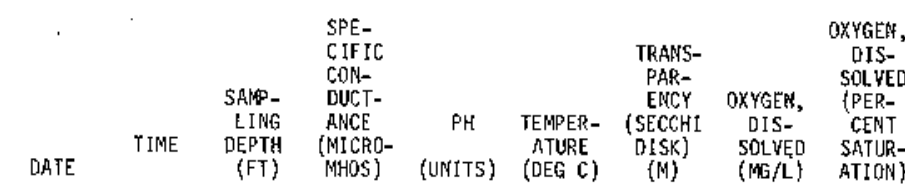

OCT , 1976

$21 . .21125$

21
21 $\quad 1127$

$2.0 \quad 27000$

$\begin{array}{lllll}8.3 & 16.7 & .63 & -- & --\end{array}$

$21 \ldots \quad 1131$

$\begin{array}{ll}10 & 28000 \\ 20 & 31000\end{array}$

$\begin{array}{lllll}8.3 & 17.0 & -- & -- & - \\ 8.3 & 17.0 & - & -- & - \\ 8.3 & 17.5 & - & -- & \end{array}$

$17 . . . \quad 1225$

$17 . \cdots \quad 1227$

$\begin{array}{ll}17 \ldots & 1229 \\ 17 \ldots & 1231\end{array}$

Q3... 1100

03... 1100

$03 . . . \quad 1104$

$\begin{array}{ll}03 . . . & 1106 \\ 03 . . & 1108\end{array}$

JUN

23... 1015

23... 1017

23... 1019

23... 1021

AtlG ... 0935

$25 \ldots .0937$

25... 0939

25... 1978

$09 . . . \quad 1300$

$09 . . . \quad 1302$

JUN... 1304

06... 1140

$06 \ldots .114$

$46 \quad 36000$

- 10.0

$\begin{array}{ll}2.0 & 32000 \\ 10 & 32000 \\ 20 . & 36000\end{array}$

$\begin{array}{lllll}-2 & 10.0 & 1.16 & 9.9 & 103\end{array}$

$40 \quad 40000$

-- 11.0

$\begin{array}{rrr}16 & 9.9 & 103 \\ - & 9.9 & 103\end{array}$

1.030000

$\begin{array}{cc}1.0 & 30000 \\ 10 & 31000 \\ 20 & 32000 \\ 45 & 45000\end{array}$

$\begin{array}{lllll}8.5 & 8.5 & .76 & 9.0 & 90 \\ 8.5 & 8.5 & -7 & 8.8 & 89\end{array}$

$20 \quad 34000$

$8.5 \quad 8.5$

$\begin{array}{ll}8.4 & 8.5 \\ 8.2 & 8.5\end{array}$

--

$1.0 \quad 28000$

$\begin{array}{ll}10 & 28000 \\ 25 & 34000\end{array}$

8.229 .0

8.20

$\begin{array}{ll}8.2 & 29.0 \\ 8.1 & 29.0\end{array}$

53

$53 \quad 5.9 \quad 86$

$\begin{array}{ll}25 & 34000 \\ 40 & 39000\end{array}$

$1.0 \quad 34000$

$10 \quad 37000$

$37 \quad 44000$

8.329 .5

$8.2 \quad 29.5 \quad .46$

$\begin{array}{ll}8.2 & 30.0 \\ 8.2 & 30.0\end{array}$

46

$6.5 \quad 99$

1.025000

$\begin{array}{ll}1.0 & 25000 \\ 40 & 27000\end{array}$

$\begin{array}{lllll}8.1 & 6.5 & - & 10.8 & 100 \\ 8.1 & 6.5 & -- & 10.7 & 100\end{array}$

35000

8.2 7

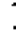

$\begin{array}{cc}2.0 & 33000 \\ 20 & 34000\end{array}$

$\begin{array}{ll}8.2 & 28.5 \\ 8.2 & 28.5\end{array}$

$\begin{array}{lll}.40 & 6.4 & 96 \\ -- & 6.5 & 97 \\ -- & 6.5 & 96\end{array}$

292653094503200 LINE 370 SITE 50

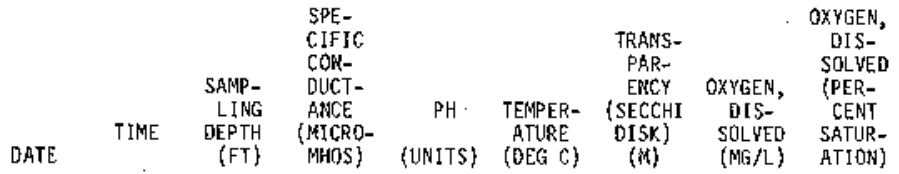

OCT, 1976

21... 1100

$\begin{array}{ll}21 \ldots . . & 1100 \\ 21 \ldots & 1102 \\ 21 \ldots & 1104\end{array}$ NOV

$17 \ldots \quad 1250$

$17 \ldots \quad 1252$

$17 \ldots . .1254$

FEB . 1977

03... 1035

$03 \ldots . \quad 1037$

$03 . . . \quad 1039$

$03 . . .1043$

JUN

23... 0935

23... 0937

$23 \ldots .0939$

$23 \ldots$ 094

RLJG 0920

$25 \ldots+\quad 0920$

$25 \ldots .0922$

$25 \ldots \quad 0926$

FEB , 1978

$09 . .11320$

$09 . . . \quad 1322$

juN

$06 . . . \quad 1100$

$2.0 \quad 31000$

$\begin{array}{ll}20 & 36000 \\ 42 & 36000\end{array}$

.

(MG $/$ )

IDN)

$\begin{array}{ll}2.0 & 32000 \\ 10 & 32000 \\ 20 & 34000\end{array}$

$\begin{array}{lllll}8.4 & 18.5 & -55 & = & = \\ 8.5 & 18.5 & -- & = & =\end{array}$

$\begin{array}{lllll}-- & 10.0 & - & 9.8 & 102\end{array}$

$40 \quad 40000$

$\begin{array}{ll}1.0 & 20000 \\ 5.0 & 30000\end{array}$

$\begin{array}{ll}1.0 & 30000 \\ 10 & 34000\end{array}$

$\begin{array}{ll}10 & 34000 \\ 20 & 36000 \\ 45 & 45000\end{array}$

$=11.0$

$\begin{array}{lllll}0.6 & 9.0 & .46 & 9.8 & 94\end{array}$

1.029000

$\begin{array}{ll}10 & 31000 \\ 25 & 39000\end{array}$

$42 \quad 42000$

1.037000

$\begin{array}{ll}10 & 46000 \\ 20 & 50000\end{array}$

4151000

$\begin{array}{lllll}8.4 & 8.5 & .46 & 9.8 & 94 \\ 8.3 & 8.5 & -- & 9.0 & 90 \\ 8.3 & 8.5 & -- & 8.8 & 89 \\ 8.2 & 8.5 & - & 8.6 & 8.5\end{array}$

$06 . . .1104$

$\begin{array}{cc}1.0 & 27000 \\ 20 & 32000 \\ 43 & 35000\end{array}$

$43 \quad 35000$

$2.0 \quad 32000$

$\begin{array}{lllll}8.2 & 29.0 & .57 & 5.8 & 86\end{array}$

$\begin{array}{lllll}8.2 & 29.0 & - & 5.5 & 90 \\ 8.1 & 29.0 & -- & 4.6 & 71 \\ 8.1 & 29.0 & -- & 4.6 & 71\end{array}$

$\begin{array}{lllll}8.3 & 29.5 & .59 & 5.6 & 86\end{array}$

$\begin{array}{lllll}8.2 & 29.5 & -- & 5.1 & 82 \\ 8.1 & 30.0 & -- & 5.2 & 87 \\ 8.1 & 29.5 & -- & 5.4 & 89\end{array}$

$\begin{array}{lllll}8.2 & 6.0 & - & 10.9 & 101 \\ 8.2 & 6.5 & - & 10.5 & 101\end{array}$

$\begin{array}{lllll}8.2 & 6.5 & 4- & 10.5 & 101 \\ 8.2 & 6.5 & \ldots & 10.4 & 101\end{array}$

$\begin{array}{lllll}8.4 & 28.5 & .40 & 6.5 & 96 \\ 8.3 & 28.5 & -- & 6.5 & 97\end{array}$ 
Table 2A.--Quality of water in the Trinity-San Jacinto estuary, water years 1977-78--Continued Field Determinations--Continued

292427094463700 LINE 380 SITE 20

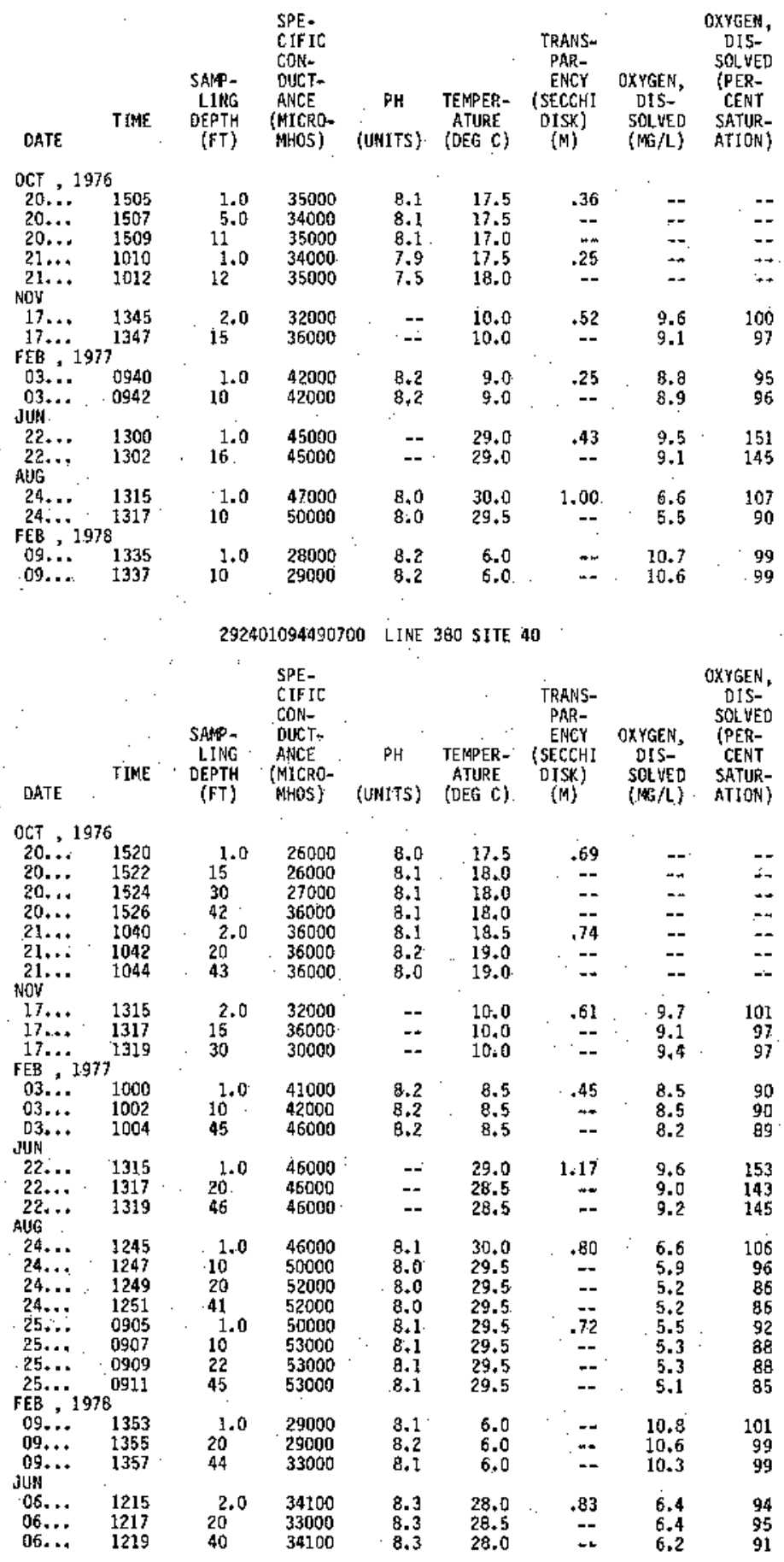


Table 2A.--Quality of water in the Ir1nity-5an Jacinto estuary, water years 1977-78--Cont inued Field Deterninations--Continued

\section{LIKE 380 SITE 60}

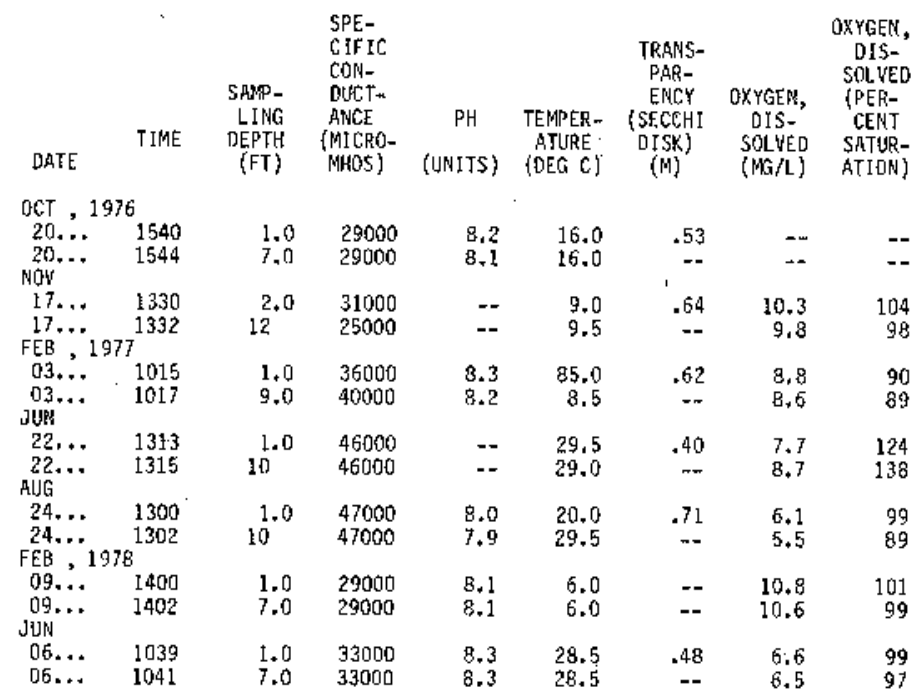

$293243094345200 \quad$ LINE 430 SITE 20

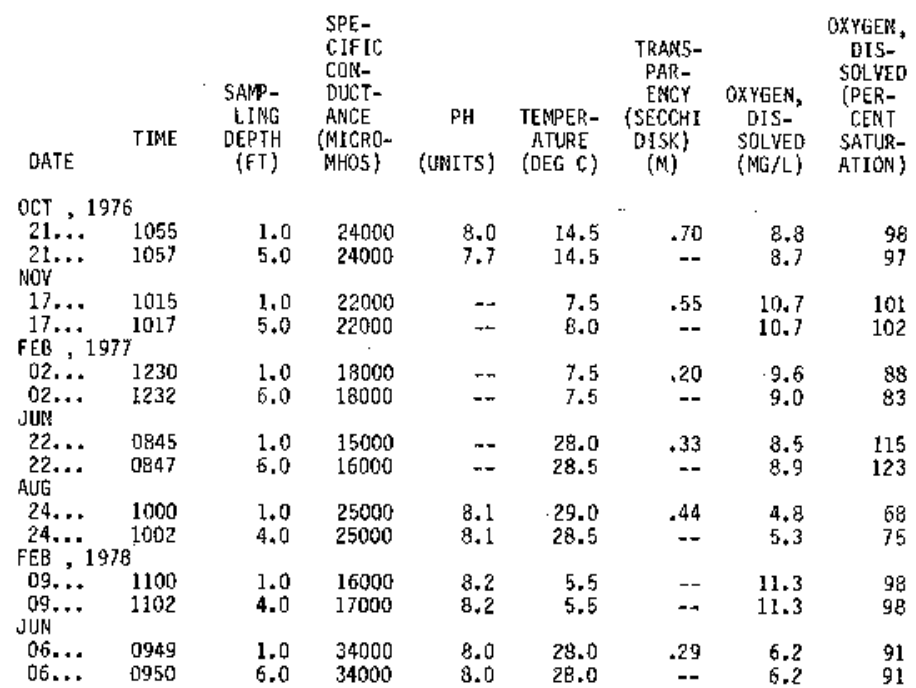

292841094402900 LIAHE 450 SITE 10

\begin{tabular}{|c|c|c|c|c|c|c|c|c|}
\hline DATE & TIME & $\begin{array}{l}\text { SANP- } \\
\text { LING } \\
\text { DEPTH } \\
\text { (FT) }\end{array}$ & $\begin{array}{l}\text { SPE- } \\
\text { CIFIC } \\
\text { CON- } \\
\text { DUCT- } \\
\text { ANCE } \\
\text { (MICRO- } \\
\text { MHOS) }\end{array}$ & $\begin{array}{c}\text { PH } \\
\text { (UNITS) }\end{array}$ & $\begin{array}{l}\text { TEIPER- } \\
\text { ATURE } \\
\text { (DEG C) }\end{array}$ & $\begin{array}{c}\text { TRANS- } \\
\text { PAR- } \\
\text { ENCY } \\
\text { (SECCHI } \\
\text { DISK) } \\
(M)\end{array}$ & $\begin{array}{c}\text { OXYGEN, } \\
\text { DIS- } \\
\text { SOL VED } \\
\text { (HW } / L)\end{array}$ & $\begin{array}{c}\text { OXYGEN, } \\
\text { OIS- } \\
\text { SQLVED } \\
\text { (PER- } \\
\text { CENT } \\
\text { SATUR } \\
\text { ATION) }\end{array}$ \\
\hline \multicolumn{9}{|c|}{ ОСТ , 1976} \\
\hline $\begin{array}{l}21 \ldots \\
21 \ldots \\
\text { NOW }\end{array}$ & $\begin{array}{l}1125 \\
1127\end{array}$ & $\begin{array}{l}1.0 \\
3.0\end{array}$ & $\begin{array}{l}24000 \\
24000\end{array}$ & $\begin{array}{l}8.3 \\
8.3\end{array}$ & $\begin{array}{l}14.5 \\
14.5\end{array}$ & .37 & $\begin{array}{l}8.6 \\
8.5\end{array}$ & $\begin{array}{l}96 \\
94\end{array}$ \\
\hline FEB & $7^{1105}$ & 3.0 & 25000 & -- & 8.0 & .52 & 10.8 & 104 \\
\hline $\begin{array}{l}\text { 02... } \\
\text { d2... } \\
\text { Juश }\end{array}$ & $\begin{array}{l}1300 \\
1302\end{array}$ & $\begin{array}{l}1.0 \\
5.0\end{array}$ & $\begin{array}{l}21000 \\
21000\end{array}$ & 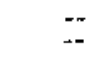 & $\begin{array}{l}7.5 \\
8.0\end{array}$ &. & $\begin{array}{l}9.7 \\
9.7\end{array}$ & $\begin{array}{l}92 \\
92\end{array}$ \\
\hline $\begin{array}{l}22 \ldots \\
22 \ldots \\
\text { QUJG }\end{array}$ & $\begin{array}{l}0915 \\
0917\end{array}$ & $\begin{array}{l}1.0 \\
5.0\end{array}$ & $\begin{array}{l}20000 \\
20000\end{array}$ & -- & $\begin{array}{l}28.5 \\
28.5\end{array}$ & $\begin{array}{l}.50 \\
-.\end{array}$ & $\begin{array}{l}11.7 \\
11.7\end{array}$ & $\begin{array}{l}162 \\
162\end{array}$ \\
\hline $\begin{array}{l}24 \ldots \\
24 \ldots \\
F E B .\end{array}$ & $9^{1115}$ & $\begin{array}{l}1.0 \\
5.0\end{array}$ & $\begin{array}{l}25000 \\
25000\end{array}$ & $\begin{array}{l}8.2 \\
8.2\end{array}$ & $\begin{array}{l}28.5 \\
28.5\end{array}$ & $\begin{array}{r}.57 \\
-.\end{array}$ & $\begin{array}{l}6.3 \\
5.6\end{array}$ & $\begin{array}{l}90 \\
81\end{array}$ \\
\hline $\begin{array}{l}09 . . . \\
09 . . . \\
\text { JUW }\end{array}$ & $\begin{array}{l}1128 \\
1130\end{array}$ & $\begin{array}{l}1.0 \\
3.0\end{array}$ & $\begin{array}{l}18000 \\
18000\end{array}$ & $\begin{array}{l}8.3 \\
8.2\end{array}$ & $\begin{array}{l}6.0 \\
6.0\end{array}$ & $=$ & $\begin{array}{l}11.4 \\
11.2\end{array}$ & $\begin{array}{r}101 \\
99\end{array}$ \\
\hline $06, \ldots$ & $\begin{array}{l}0900 \\
0902\end{array}$ & $\begin{array}{l}1.0 \\
4.0\end{array}$ & $\begin{array}{l}33000 \\
330000\end{array}$ & $\begin{array}{l}8.0 \\
8.0\end{array}$ & $\begin{array}{l}27.5 \\
27.5\end{array}$ & $\begin{array}{r}.33 \\
--\end{array}$ & $\begin{array}{l}6.2 \\
6.1\end{array}$ & $\begin{array}{l}91 \\
90\end{array}$ \\
\hline
\end{tabular}


Table 2A.--Quality of water in the Trinity-San Jacinto estuary, water years $1977+78-$-Continued Field Deterninations--Cont inued

292950094404100 LINE 450 SITE 20

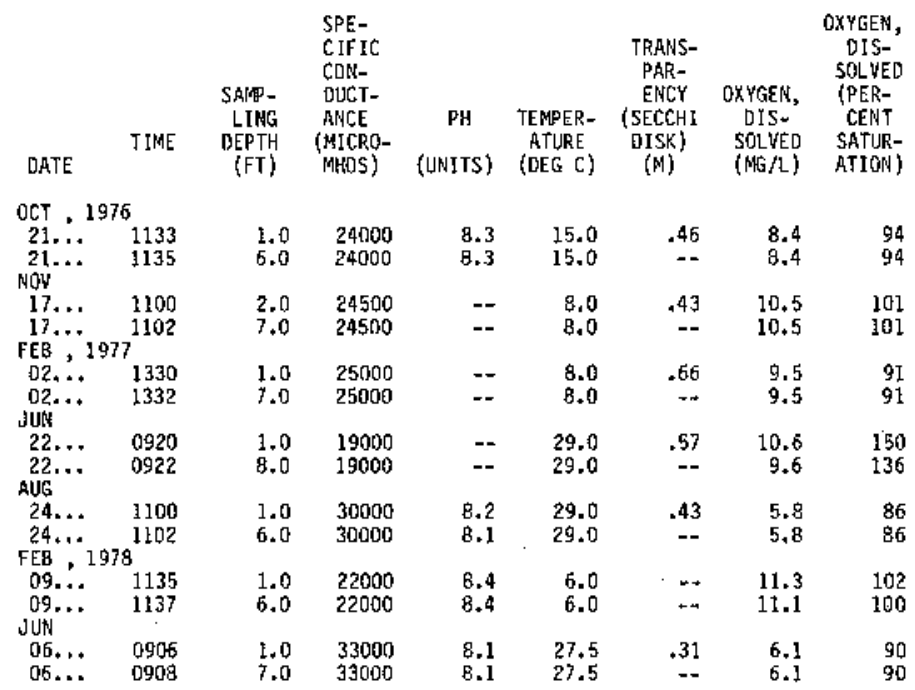

293058094405400 LINE 450 SITE 30

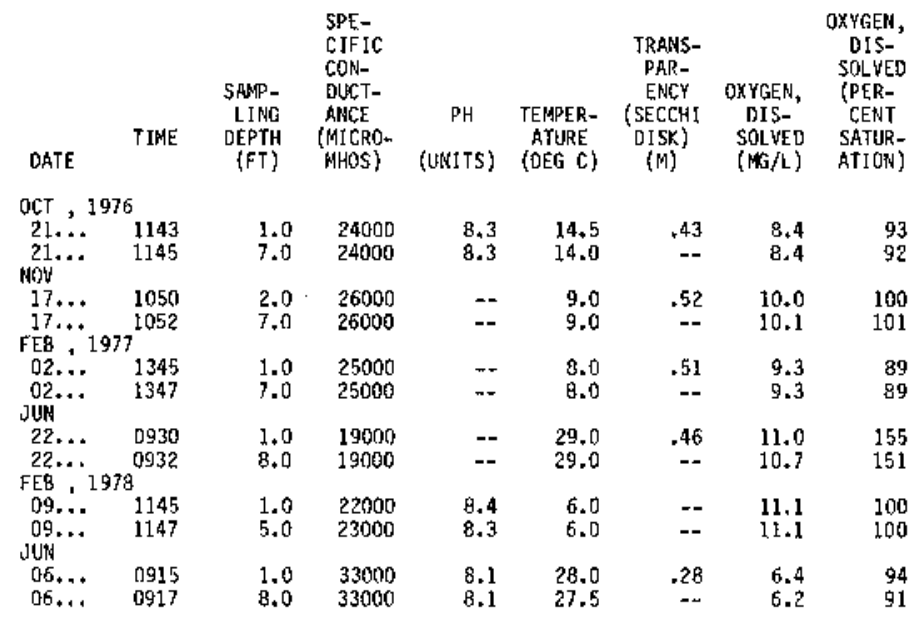

293206094410600 LINE 450 SITE 40

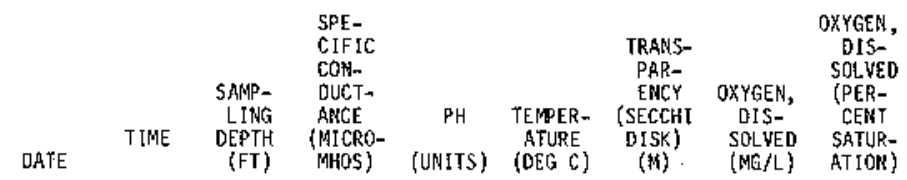

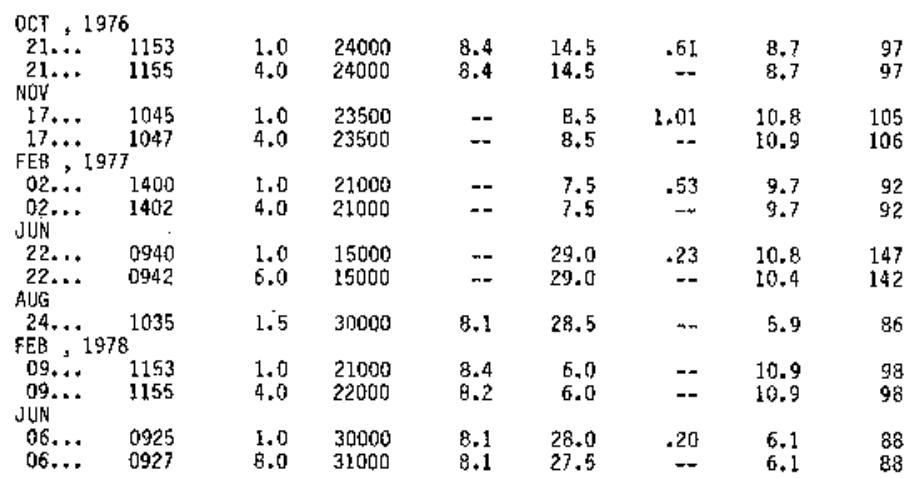


Table 2A.--Q̨uality of water in the Trinfty-San Jacinto estuary, water years 1977-78--Continued

Field Determinations--Continued

292720094451300 LINE 470 SITE 30

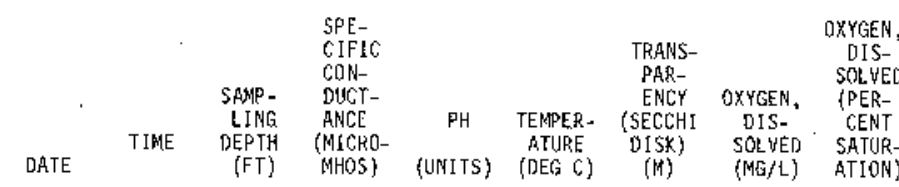

OCT , 1976

\begin{tabular}{|c|c|c|c|c|c|c|c|c|}
\hline $\begin{array}{l}21 . \\
21 . \\
\text { nov }\end{array}$ & $\begin{array}{l}1220 \\
1222\end{array}$ & $\begin{array}{l}1.0 \\
6.0\end{array}$ & $\begin{array}{l}24000 \\
2.4000\end{array}$ & $\begin{array}{l}8.3 \\
8.3\end{array}$ & $\begin{array}{l}15.0 \\
15.0\end{array}$ & .40 & $\begin{array}{l}8.1 \\
7.8\end{array}$ & $\begin{array}{l}91 \\
88\end{array}$ \\
\hline 17. & 1130 & 2.0 & 27000 & -- & 9.0 & .46 & 10.0 & 100 \\
\hline $17 \ldots$ & 1132 & 7.0 & 27000 & -- & 9.0 & - & 9.9 & 99 \\
\hline \multicolumn{9}{|c|}{ FEB, 1977} \\
\hline 02. & 1430 & 1.0 & 32000 & -- & B.D & .51 & 9.2 & 92 \\
\hline $\begin{array}{c}\text { D2... } \\
\text { JUN }\end{array}$ & \multicolumn{7}{|c|}{ JUN } & 92 \\
\hline $22 .$. & 1150 & 1.0 & 19000 & -- & 29.5 & .54 & 10.7 & 151 \\
\hline $22 .$. & 1152 & 6.0 & 19000 & -- & 29.0 & -- & 10.5 & 114 \\
\hline \multicolumn{9}{|l|}{ AUIG } \\
\hline $\begin{array}{l}24 . . \\
24, .\end{array}$ & 1130 & 1.0 & 34000 & 8.2 & 29.0 & .53 & 6.2 & 92 \\
\hline \multicolumn{9}{|l|}{ JUN } \\
\hline 06. & 0818 & 1.0 & 33000 & 8.2 & 28.0 & .27 & 6.7 & 99 \\
\hline 06. & 0820 & 8.0 & 33000 & 8.1 & 27.5 & - & 6.6 & 97 \\
\hline
\end{tabular}

293029094462800 LINE 470 SITE 60

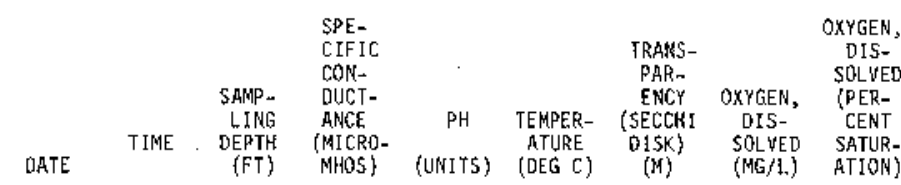

OCT , 1976

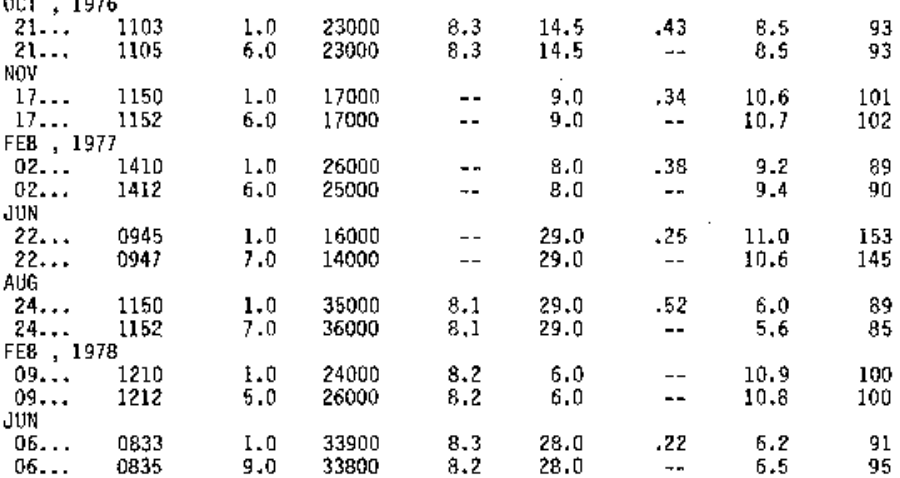

$291926094511000 \quad$ LIRE 510 SITE 50

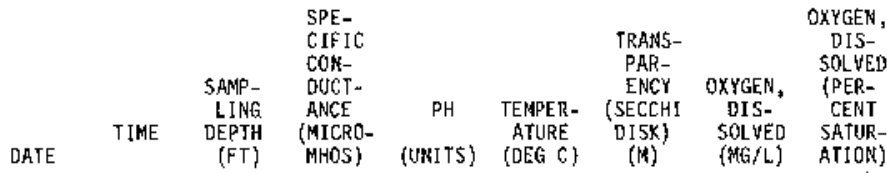
FEB , 1977

$\begin{array}{lllllllll}02 \ldots & 0915 & 1.0 & 38000 & 8.3 & 7.5 & .99 & 9.2 & 94 \\ 02 \ldots & 0917 & 7.0 & 40000 & 8.3 & 7.5 & -- & 9.2 & 94\end{array}$

292013094513400 LINE 510 SITE 60

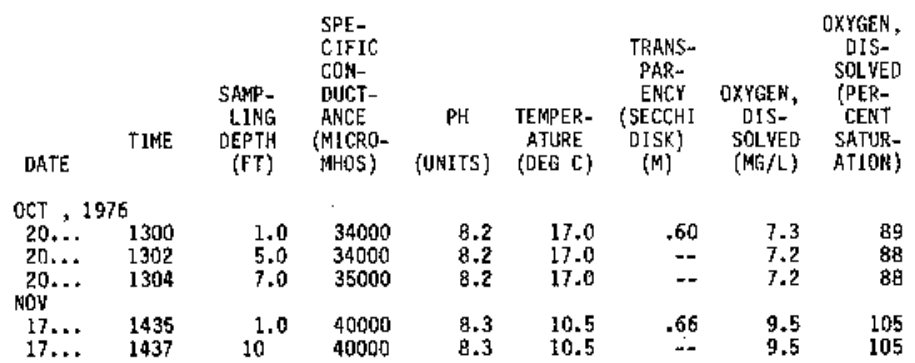


291744094531200 LINE 521 SITE 50

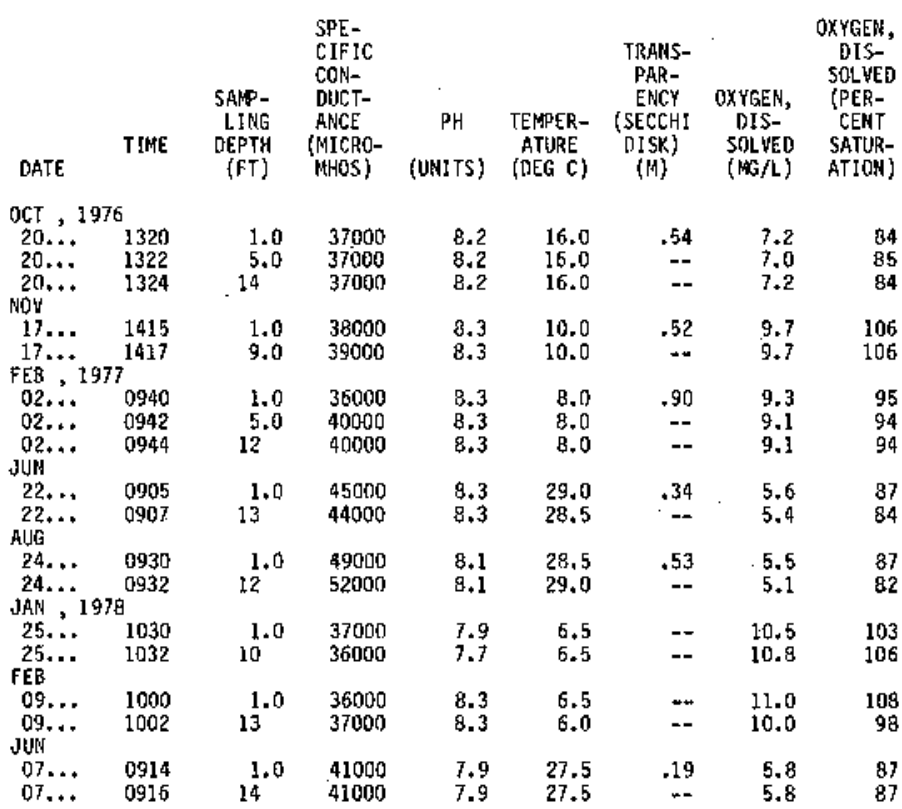

291339094573400 LINE 530 SITE 20

\begin{tabular}{|c|c|c|c|c|c|c|c|c|}
\hline DATE & TIME & $\begin{array}{l}\text { SANP- } \\
\text { LING } \\
\text { DEPTH } \\
\text { (FT) }\end{array}$ & $\begin{array}{l}\text { SPE- } \\
\text { CIFIC } \\
\text { CON- } \\
\text { DUCT- } \\
\text { ANCE } \\
\text { (MICRO- } \\
\text { MAHOS) }\end{array}$ & $\begin{array}{c}\text { PH } \\
\text { (UNITS) }\end{array}$ & $\begin{array}{c}\text { TEMPER- } \\
\text { ATURE } \\
\text { (DEG C) }\end{array}$ & $\begin{array}{l}\text { TRANS- } \\
\text { PAR - } \\
\text { ENCY } \\
\text { (SECCH1 } \\
\text { DISK) } \\
(M)\end{array}$ & $\begin{array}{c}\text { OXYGEN, } \\
\text { DIS- } \\
\text { SOLYED } \\
\text { (MG } / L \text { ) }\end{array}$ & $\begin{array}{c}\text { QXYGEN, } \\
\text { DIS- } \\
\text { SOLVED } \\
\text { (PER- } \\
\text { CENT } \\
\text { SATUR- } \\
\text { ATION) }\end{array}$ \\
\hline \multicolumn{9}{|c|}{ ОСТ, 1976} \\
\hline $\begin{array}{l}20 . \ldots \\
20 \ldots \\
\text { NOY }\end{array}$ & $\begin{array}{l}1350 \\
1352\end{array}$ & $\begin{array}{l}1.0 \\
2.5\end{array}$ & $\begin{array}{l}38000 \\
38000\end{array}$ & $\begin{array}{l}\text { 8.3. } \\
8.3\end{array}$ & $\begin{array}{l}16.0 \\
16.0\end{array}$ & $\begin{array}{l}.64 \\
--\end{array}$ & $\begin{array}{l}8.2 \\
8.1\end{array}$ & $\begin{array}{l}101 \\
100\end{array}$ \\
\hline FEB & $7^{1045}$ & 2.0 & 36000 & 8.3 & 9.0 & 2.00 & 10.3 & 107 \\
\hline $\begin{array}{c}02 . \cdots \\
\text { AUG }\end{array}$ & 1035 & 2.0 & 36000 & 8.1 & 8.0 & .76 & 9.6 & 98 \\
\hline $\begin{array}{l}24 . . \\
24 \ldots \\
\text { FEB }\end{array}$ & $\begin{array}{l}1030 \\
1032 \\
8\end{array}$ & $\begin{array}{l}1.0 \\
4.0\end{array}$ & $\begin{array}{l}52000 \\
52000\end{array}$ & $\begin{array}{l}8.2 \\
8.2\end{array}$ & $\begin{array}{r}29.5 \\
29.5\end{array}$ & .47 & $\begin{array}{l}5.6 \\
5.5\end{array}$ & $\begin{array}{l}92 \\
31\end{array}$ \\
\hline $\begin{array}{l}09 . . . \\
\text { JUN }\end{array}$ & $\begin{array}{l}1050 \\
1052\end{array}$ & $\begin{array}{l}1.0 \\
4.0\end{array}$ & $\begin{array}{l}37000 \\
37000\end{array}$ & $\begin{array}{l}8.4 \\
8.4\end{array}$ & $\begin{array}{l}6.5 \\
6.0\end{array}$ & $\begin{array}{r}.75 \\
-.\end{array}$ & $\begin{array}{l}10.8 \\
10.0\end{array}$ & $\begin{array}{r}106 \\
98\end{array}$ \\
\hline $\begin{array}{l}07 \ldots \\
07 \ldots\end{array}$ & $\begin{array}{l}0959 \\
1000\end{array}$ & $\begin{array}{l}1.0 \\
4.0\end{array}$ & $\begin{array}{l}42000 \\
42000\end{array}$ & $\begin{array}{l}7.9 \\
7.9\end{array}$ & $\begin{array}{l}27.0 \\
27.0\end{array}$ & .21 & $\begin{array}{l}5.8 \\
5.8\end{array}$ & $\begin{array}{l}87 \\
87\end{array}$ \\
\hline
\end{tabular}

291428094575900 LIME 530 SITE 50

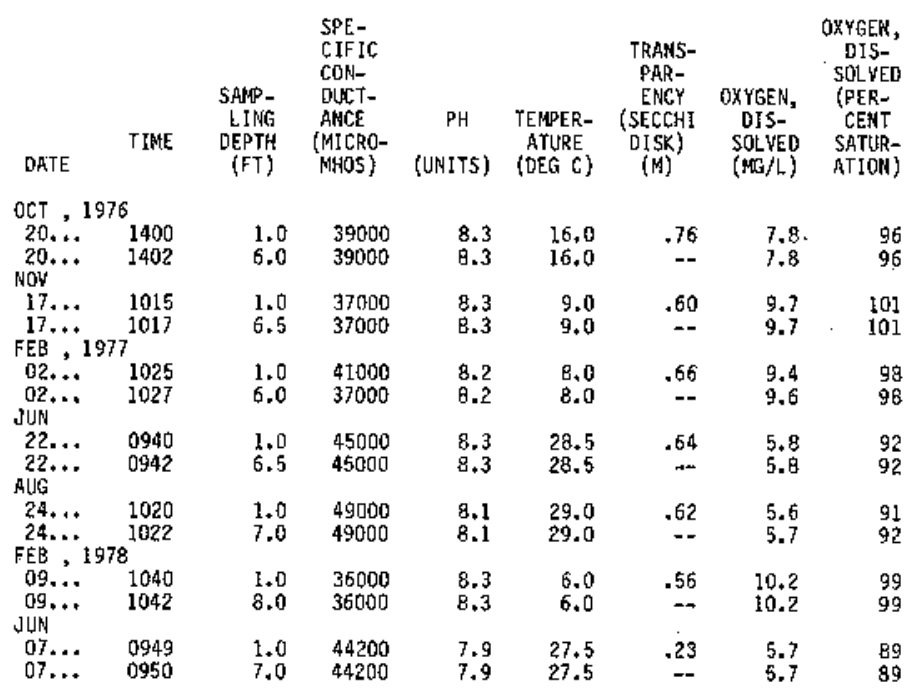


Table 2A.--Quality of water in the Trinity-San Jacinto estiary, water years 1977-78--Continued field Determinations--Continuces

291601094585200 LINL 530 SITE 80

\begin{tabular}{|c|c|c|c|c|c|c|c|c|}
\hline OATF, & TIME & $\begin{array}{l}\text { SAMP. } \\
\text { LING } \\
\text { DEPTH } \\
\text { (FT) }\end{array}$ & $\begin{array}{l}\text { SPE- } \\
\text { CIFIC } \\
\text { CON- } \\
\text { DUCT- } \\
\text { ANCE } \\
\text { (MICRO- } \\
\text { MHOS) }\end{array}$ & $\begin{array}{c}\text { PH } \\
\text { [UNITS) }\end{array}$ & $\begin{array}{l}\text { TEWOER- } \\
\text { ATURE } \\
\text { (OEG C) }\end{array}$ & $\begin{array}{l}\text { TKANS- } \\
\text { PAR- } \\
\text { ENCY } \\
\text { (SECCHI } \\
\text { DISK) } \\
\text { (M) }\end{array}$ & $\begin{array}{c}\text { OXYGEN, } \\
\text { DI S- } \\
\text { SOLVED } \\
\text { (MG/L) }\end{array}$ & $\begin{array}{c}\text { OXYGES } \\
\text { DIS- } \\
\text { SOLVE } \\
\text { LPER- } \\
\text { CERN } \\
\text { SATUP } \\
\text { RTIOS }\end{array}$ \\
\hline
\end{tabular}

UCT , 1976

$\begin{array}{ll}20 . \cdots & 141.5 \\ 20 . . & 1417\end{array}$

$\begin{array}{rrrrrrr}1.0 & 39000 & 8.4 & 16.0 & .72 & 8.1 & 100 \\ 4.0 & 39000 & 8.4 & 16.0 & -- & 8.4 & 104 \\ 1.0 & 36000 & 8.3 & 8.5 & 1.42 & 9.8 & 101 \\ 5.0 & 36000 & 8.3 & 8.5 & -- & 10.6 & 110 \\ 1.0 & 40000 & 8.2 & 7.5 & .44 & 9.5 & 97 \\ 4.5 & 40000 & 8.2 & 8.0 & -- & 9.4 & 97 \\ 1.0 & 39000 & 8.3 & 28.5 & .24 & 6.0 & 91 \\ 4.5 & 40600 & 8.3 & 28.5 & -- & 6.0 & 91 \\ 1.0 & 47000 & 8.1 & 29.0 & .35 & 5.9 & 93 \\ 3.5 & 47000 & 8.1 & 28.5 & -. & 5.9 & 94 \\ 1.0 & 35000 & 8.4 & 6.0 & .66 & 10.7 & 103 \\ 5.5 & 35000 & 8.4 & 5.5 & -- & 10.3 & 97 \\ 1.0 & 35000 & 8.0 & 27.5 & .40 & 5.8 & 87 \\ 4.0 & 41000 & 8.0 & 27.5 & -- & 5.6 & 87\end{array}$

291127095015500 LINE: 550 SITE 50

$17 \ldots 1000$

$17 . . .1002$

FEB , 1977

02... 1012

JUN

$\begin{array}{ll}22 \ldots . & 0925 \\ 22 . . & 0927\end{array}$

AGS

24... 1010

24,.. 1012

FE8 , 1978

09... 1030

Jus

$07 \ldots \quad 0940$

$07 \ldots .0942$

\begin{tabular}{|c|c|c|c|c|c|c|c|c|}
\hline DATE & TIME & $\begin{array}{c}\text { SAMP- } \\
\text { LING } \\
\text { DEPTH } \\
(F T)\end{array}$ & $\begin{array}{l}\text { SPE- } \\
\text { CIF IC } \\
\text { CON- } \\
\text { DUCT- } \\
\text { ANCE } \\
\text { (MICRO- } \\
\text { MHOS) }\end{array}$ & $\begin{array}{c}\mathrm{PH} \\
\text { (GNITS) }\end{array}$ & $\begin{array}{l}\text { TEMPER- } \\
\text { ATURE } \\
\text { (DEG C) }\end{array}$ & $\begin{array}{l}\text { TRANS- } \\
\text { PAR- } \\
\text { ENCY } \\
\text { SSECCHI } \\
\text { OISK) } \\
\text { (M) }\end{array}$ & $\begin{array}{c}\text { OXYUEN, } \\
\text { DIS- } \\
\text { SOLLYED } \\
\text { \{MG } / L \text { \} }\end{array}$ & $\begin{array}{l}\text { OXYGEN, } \\
\text { OIS- } \\
\text { SOLVEU } \\
\text { (PER- } \\
\text { CENT } \\
\text { SATUR- } \\
\text { ATLON) }\end{array}$ \\
\hline ICT, 1 & & - & & & & & & \\
\hline $\begin{array}{l}20 \ldots \\
20 \%\end{array}$ & $\begin{array}{l}1450 \\
1452\end{array}$ & $\begin{array}{l}1.0 \\
6.0\end{array}$ & $\begin{array}{l}39000 \\
39000\end{array}$ & $\begin{array}{l}8.4 \\
8.4\end{array}$ & $\begin{array}{l}16.5 \\
16.5\end{array}$ & $\begin{array}{l}-61 \\
--\end{array}$ & $\begin{array}{l}7.7 \\
7.7\end{array}$ & $\begin{array}{l}96 \\
96\end{array}$ \\
\hline $\begin{array}{l}17 \\
17 \\
F E B\end{array}$ & $\begin{array}{l}1115 \\
1117 \\
77\end{array}$ & $\begin{array}{l}1.0 \\
6.5\end{array}$ & $\begin{array}{l}36000 \\
36000\end{array}$ & $\begin{array}{l}8.3 \\
8.3\end{array}$ & $\begin{array}{l}8.5 \\
8.5\end{array}$ & .55 & $\begin{array}{l}10.0 \\
10.1\end{array}$ & $\begin{array}{l}103 \\
104\end{array}$ \\
\hline $\begin{array}{l}02 \ldots . \\
02 \ldots \\
\text { JUN }\end{array}$ & $\begin{array}{l}1100 \\
1102\end{array}$ & $\begin{array}{l}1.0 \\
6.0\end{array}$ & $\begin{array}{l}40000 \\
39000\end{array}$ & $\begin{array}{l}8.3 \\
8.2\end{array}$ & $\begin{array}{l}8.0 \\
8.0\end{array}$ & $\begin{array}{r}.62 \\
--\end{array}$ & $\begin{array}{l}9.5 \\
9.5\end{array}$ & $\begin{array}{l}98 \\
98\end{array}$ \\
\hline $\begin{array}{l}22 \ldots \\
22 \ldots \\
\text { RUG }\end{array}$ & $\begin{array}{l}1010 \\
1012\end{array}$ & $\begin{array}{l}1.0 \\
6.0\end{array}$ & $\begin{array}{l}45000 \\
45000\end{array}$ & $\begin{array}{l}8.4 \\
8.4\end{array}$ & $\begin{array}{l}28.5 \\
28.5\end{array}$ & .40 & $\begin{array}{l}5.4 \\
5.4\end{array}$ & $\begin{array}{l}36 \\
90\end{array}$ \\
\hline $\begin{array}{l}24 \ldots \\
24 \ldots \\
F E B, 1\end{array}$ & $\begin{array}{l}1055 \\
1057 \\
78\end{array}$ & $\begin{array}{l}\mathrm{I} .0 \\
6.0\end{array}$ & $\begin{array}{l}48000 \\
52000\end{array}$ & $\begin{array}{l}8.2 \\
8.1\end{array}$ & $\begin{array}{l}29.5 \\
29.0\end{array}$ & .61 & $\begin{array}{l}6.0 \\
5.4\end{array}$ & $\begin{array}{l}97 \\
87\end{array}$ \\
\hline $\begin{array}{l}09 . . . \\
\text { JUN }\end{array}$ & $\begin{array}{l}1110 \\
1112\end{array}$ & $\begin{array}{l}\text { I. } 0 \\
5.0\end{array}$ & $\begin{array}{l}34000 \\
34000\end{array}$ & $\begin{array}{l}8.3 \\
8.3\end{array}$ & $\begin{array}{l}6.0 \\
6.0\end{array}$ & $\begin{array}{l}51 \\
--\end{array}$ & $\begin{array}{l}10.5 \\
10.3\end{array}$ & $\begin{array}{r}101 \\
99\end{array}$ \\
\hline $\begin{array}{l}07 \ldots \\
07 \ldots\end{array}$ & $\begin{array}{l}1022 \\
1024\end{array}$ & $\begin{array}{l}1.0 \\
7.0\end{array}$ & $\begin{array}{l}37000 \\
39000\end{array}$ & $\begin{array}{l}8.1 \\
8.1\end{array}$ & $\begin{array}{l}27.5 \\
27.5\end{array}$ & .38 & $\begin{array}{l}6.0 \\
5.8\end{array}$ & $\begin{array}{l}90 \\
86\end{array}$ \\
\hline
\end{tabular}


Table 2A.- quality of water in the Trinity-San Jacinto estuary, water years 1977-78--Continued F1eld Deteminations--Cont inued

291106095084200 LINE 565 SITE 30

\begin{tabular}{|c|c|c|c|c|c|c|c|c|}
\hline DATE & TIME & $\begin{array}{l}\text { SAMP- } \\
\text { LING } \\
\text { DEPTH } \\
\text { (FT.) }\end{array}$ & $\begin{array}{l}\text { SPE- } \\
\text { CIF IC } \\
\text { CON- } \\
\text { DUCT- } \\
\text { ARCE } \\
\text { (MICRO- } \\
\text { MHOS) }\end{array}$ & $\begin{array}{c}\text { PH } \\
\text { (UNITS) }\end{array}$ & $\begin{array}{l}\text { TENPER- } \\
\text { ATURE } \\
\text { (DEG C) }\end{array}$ & $\begin{array}{c}\text { TRANS- } \\
\text { PAR- } \\
\text { ENCY } \\
\text { (SECCH] } \\
\text { DISK) } \\
\text { (M) }\end{array}$ & $\begin{array}{c}\text { OXYGEN, } \\
\text { DIS- } \\
\text { SOLVED } \\
\text { (MG/L) }\end{array}$ & $\begin{array}{c}\text { OXYGEK, } \\
\text { DIS- } \\
\text { SOLVED } \\
\text { (PER- } \\
\text { CENT } \\
\text { SATUR- } \\
\text { ATJON) }\end{array}$ \\
\hline $\begin{array}{l}\text { OCT }, 1 \\
20 \ldots \ldots \\
20 \ldots \ldots \\
20 \ldots \\
20 \ldots\end{array}$ & $\begin{array}{l}6 \\
1545 \\
1547 \\
1549 \\
1551\end{array}$ & $\begin{array}{r}1.0 \\
5.0 \\
8.0 \\
10\end{array}$ & $\begin{array}{l}360000 \\
37000 \\
37000 \\
38000\end{array}$ & $\begin{array}{l}8.2 \\
8.2 \\
8.2 \\
8.2\end{array}$ & $\begin{array}{l}15.5 \\
15.5 \\
15.5 \\
15.5\end{array}$ & $\begin{array}{l}58 \\
-- \\
--\end{array}$ & $\begin{array}{l}8.3 \\
8.1 \\
7.4 \\
6.8\end{array}$ & $\begin{array}{r}100 \\
98 \\
89 \\
70\end{array}$ \\
\hline $\begin{array}{l}\text { NoV } \\
17 \ldots \\
17 \ldots \\
\text { FEB... }\end{array}$ & $7^{1310}$ & $10^{1.0}$ & & & $\begin{array}{l}9.5 \\
9.0\end{array}$ & $\begin{array}{r}.52 \\
--\end{array}$ & $\begin{array}{l}10.2 \\
10.1\end{array}$ & $\begin{array}{l}106 \\
104\end{array}$ \\
\hline $\begin{array}{l}02 \ldots \\
02 . . . \\
02 . . \\
\text { JuN }\end{array}$ & $\begin{array}{l}1305 \\
1307 \\
1309\end{array}$ & $\begin{array}{l}1.0 \\
5.0 \\
11\end{array}$ & $\begin{array}{l}30000 \\
33000 \\
26000\end{array}$ & $\begin{array}{l}8.4 \\
8.3 \\
8.3\end{array}$ & $\begin{array}{l}9.0 \\
9.0 \\
8.5\end{array}$ & $\begin{array}{l}.52 \\
-- \\
--\end{array}$ & $\begin{array}{r}9.9 \\
9.7 \\
10.7\end{array}$ & $\begin{array}{l}100 \\
100 \\
105\end{array}$ \\
\hline $\begin{array}{l}22 \ldots \\
22 \ldots \\
22 \ldots \\
\text { AUG }\end{array}$ & $\begin{array}{l}1140 \\
1142 \\
1144\end{array}$ & $\begin{array}{l}1.0 \\
5.0 \\
10\end{array}$ & $\begin{array}{l}20000 \\
20000 \\
40000\end{array}$ & $\begin{array}{l}8.6 \\
8.6 \\
8.4\end{array}$ & $\begin{array}{r}29.0 \\
2.9 \\
29.0\end{array}$ & $\begin{array}{l}.30 \\
\cdots\end{array}$ & $\begin{array}{l}6.4 \\
6.4 \\
4.5\end{array}$ & $\begin{array}{l}91 \\
91 \\
70\end{array}$ \\
\hline $\begin{array}{l}24 \ldots \\
24 \ldots \\
24, \ldots \\
24 \ldots \\
\text { FEB , }\end{array}$ & $\begin{array}{l}1125 \\
1127 \\
1245 \\
1247 \\
8\end{array}$ & $\begin{array}{r}1.0 \\
7.0 \\
1.0 \\
11\end{array}$ & $\begin{array}{l}54000 \\
53000 \\
49000 \\
49000\end{array}$ & $\begin{array}{l}8.2 \\
8.2 \\
8.1 \\
8.0\end{array}$ & $\begin{array}{l}29.5 \\
29.5 \\
29.0 \\
29.0\end{array}$ & $\begin{array}{l}.45 \\
.- \\
.48 \\
--\end{array}$ & $\begin{array}{l}5.7 \\
5.6 \\
4.6 \\
4.7\end{array}$ & $\begin{array}{r}95 \\
93 \\
7 \\
76\end{array}$ \\
\hline $\begin{array}{l}09 . . \\
09 . .\end{array}$ & $\begin{array}{l}1300 \\
1302\end{array}$ & $\begin{array}{l}1.0 \\
7.0\end{array}$ & $\begin{array}{l}20000 \\
35000\end{array}$ & $\begin{array}{l}B .6 \\
8.6\end{array}$ & $\begin{array}{l}7.0 \\
6.5\end{array}$ & .56 & $\begin{array}{l}10.9 \\
10.0\end{array}$ & $\begin{array}{r}100 \\
97\end{array}$ \\
\hline $\begin{array}{l}07 \ldots \\
07 \ldots\end{array}$ & $\begin{array}{l}1208 \\
1210\end{array}$ & $12^{1.0}$ & $\begin{array}{l}15000 \\
21000\end{array}$ & $\begin{array}{l}8.0 \\
8.0\end{array}$ & $\begin{array}{l}26.5 \\
26.5\end{array}$ & .20 & $\begin{array}{l}6.4 \\
5.6\end{array}$ & $\begin{array}{l}85 \\
80\end{array}$ \\
\hline
\end{tabular}

290823095040200 LINE 575 SITE 10

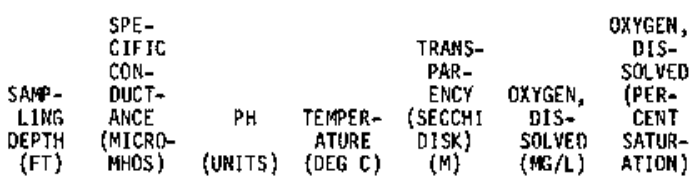

OCT , 1976

\begin{tabular}{|c|c|c|c|c|c|c|c|c|}
\hline \multicolumn{9}{|c|}{ OCT , 1976} \\
\hline $\begin{array}{l}20, \cdots \\
20, \ldots \\
\text { NOV }\end{array}$ & $\begin{array}{l}1510 \\
1512\end{array}$ & $\begin{array}{l}1.0 \\
7.0\end{array}$ & $\begin{array}{l}41000 \\
41000\end{array}$ & $\begin{array}{l}8.3 \\
8.3\end{array}$ & $\begin{array}{l}16.0 \\
16.5\end{array}$ & $\begin{array}{r}.77 \\
--\end{array}$ & $\begin{array}{l}7.7 \\
7.7\end{array}$ & $\begin{array}{l}96 \\
96\end{array}$ \\
\hline $\begin{array}{l}17 \ldots \\
17 \ldots \ldots \\
\text { FEB }, 1977\end{array}$ & $7^{1140}$ & $\begin{array}{l}1.0 \\
8.0\end{array}$ & $\begin{array}{l}37000 \\
37000\end{array}$ & $\begin{array}{l}8.2 \\
8.2\end{array}$ & $\begin{array}{l}8.5 \\
8.5\end{array}$ & $\begin{array}{r}.80 \\
.\end{array}$ & $\begin{array}{l}10.1 \\
10.1\end{array}$ & $\begin{array}{r}104 \\
95\end{array}$ \\
\hline $\begin{array}{l}02 \ldots \\
02 \ldots \\
\text { JUA }\end{array}$ & $\begin{array}{l}1125 \\
1127\end{array}$ & $\begin{array}{l}1.0 \\
7.0\end{array}$ & $\begin{array}{l}32000 \\
41000\end{array}$ & $\begin{array}{l}8.2 \\
8.1\end{array}$ & $\begin{array}{l}8.5 \\
9.0\end{array}$ & $\begin{array}{r}81 \\
--\end{array}$ & $\begin{array}{r}10.0 \\
8.4\end{array}$ & $\begin{array}{r}101 \\
90\end{array}$ \\
\hline $\begin{array}{l}28 \ldots \\
28 . . . \\
\text { AUG }\end{array}$ & $\begin{array}{l}1030 \\
1032\end{array}$ & $\begin{array}{l}1.0 \\
8.0\end{array}$ & $\begin{array}{l}48000 \\
48000\end{array}$ & $\begin{array}{l}8.4 \\
8.4\end{array}$ & $\begin{array}{l}28.5 \\
28.5\end{array}$ & $\begin{array}{r}.47 \\
--\end{array}$ & $\begin{array}{l}5.3 \\
5.2\end{array}$ & $\begin{array}{l}84 \\
83\end{array}$ \\
\hline $\begin{array}{l}24 \ldots \\
24 \cdots \\
F E B, 1978\end{array}$ & $8^{1115}$ & $\begin{array}{l}1.0 \\
8.0\end{array}$ & $\begin{array}{l}51000 \\
51000\end{array}$ & $\begin{array}{l}8.2 \\
8.1\end{array}$ & $\begin{array}{l}29.5 \\
29.5\end{array}$ & $\begin{array}{r}.53 \\
--\end{array}$ & $\begin{array}{l}5.7 \\
5.5\end{array}$ & $\begin{array}{l}93 \\
91\end{array}$ \\
\hline $\begin{array}{l}09 . \cdots \\
09 \ldots \\
\text { dUN }\end{array}$ & $\begin{array}{l}1128 \\
1130\end{array}$ & $\begin{array}{l}1.0 \\
9.0\end{array}$ & $\begin{array}{l}36000 \\
37000\end{array}$ & $\begin{array}{l}8.6 \\
8.5\end{array}$ & $\begin{array}{l}6.5 \\
6.0\end{array}$ & $\begin{array}{l}.60 \\
--\end{array}$ & $\begin{array}{l}10.3 \\
10.2\end{array}$ & $\begin{array}{r}101 \\
99\end{array}$ \\
\hline $\begin{array}{l}07 \ldots \\
07 \ldots\end{array}$ & $\begin{array}{l}1039 \\
1041\end{array}$ & $\begin{array}{l}1.0 \\
8.0\end{array}$ & $\begin{array}{l}35000 \\
35000\end{array}$ & $\begin{array}{l}8.2 \\
8.2\end{array}$ & $\begin{array}{l}27.0 \\
27.0\end{array}$ & $\begin{array}{r}.54 \\
--\end{array}$ & $\begin{array}{l}6.2 \\
6.0\end{array}$ & $\begin{array}{l}90 \\
87\end{array}$ \\
\hline
\end{tabular}


Table 2.A.--Quality of water in the Trinity-San Jacinto estuary, water yoars 1977-78--Continued Field Deterilinations - Cont inued

290924095045100 LINE: 575 SITE 50

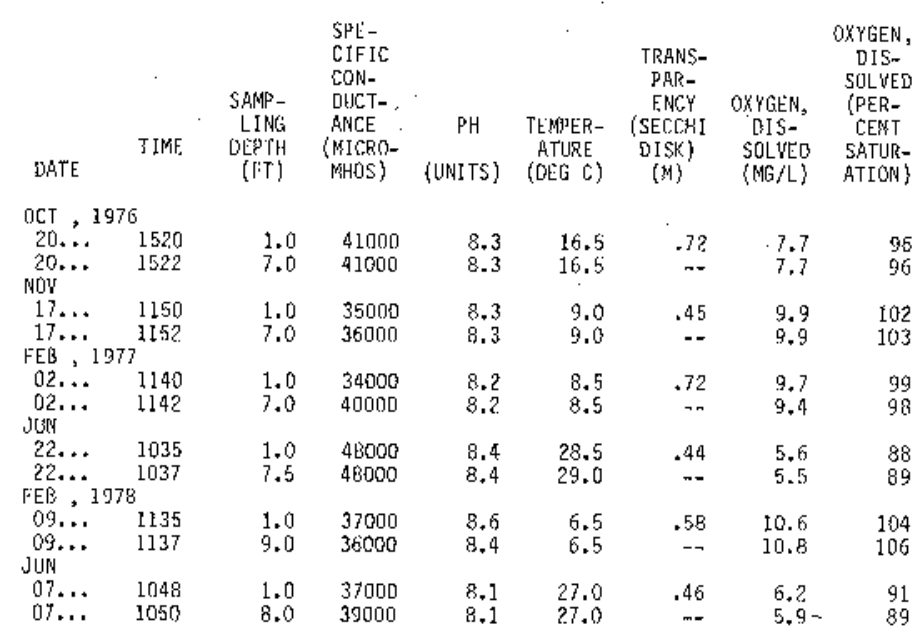

291023095053900 LINE 57551 TTE 80

\begin{tabular}{|c|c|c|c|c|c|c|c|c|}
\hline DATE & TIME & $\begin{array}{c}\text { SAMP- } \\
\text { EING } \\
\text { D):PT1 } \\
\text { (FT) }\end{array}$ & $\begin{array}{l}\text { SPE- } \\
\text { CIF IC } \\
\text { CON- } \\
\text { DUCT- } \\
\text { ANCE } \\
\text { (MICRO- } \\
\text { MHOS) }\end{array}$ & $\begin{array}{c}\mathrm{PH} \\
\text { (URITS) }\end{array}$ & $\begin{array}{l}\text { TE'MPER- } \\
\text { ATURT. } \\
\text { (DEG E) }\end{array}$ & $\begin{array}{c}\text { TRANS- } \\
\text { PMR- } \\
\text { ENCY } \\
\text { \{SECCHI } \\
\text { OISKS) } \\
\text { (M) }\end{array}$ & $\begin{array}{c}\text { OXYGEN, } \\
\text { DIS- } \\
\text { SOLVED } \\
\text { (MG/L) }\end{array}$ & $\begin{array}{l}\text { OXYGLN, } \\
\text { CIS- } \\
\text { SOLYED } \\
\text { (PER- } \\
\text { CENY } \\
\text { SATUR- } \\
\text { ATION) }\end{array}$ \\
\hline
\end{tabular}

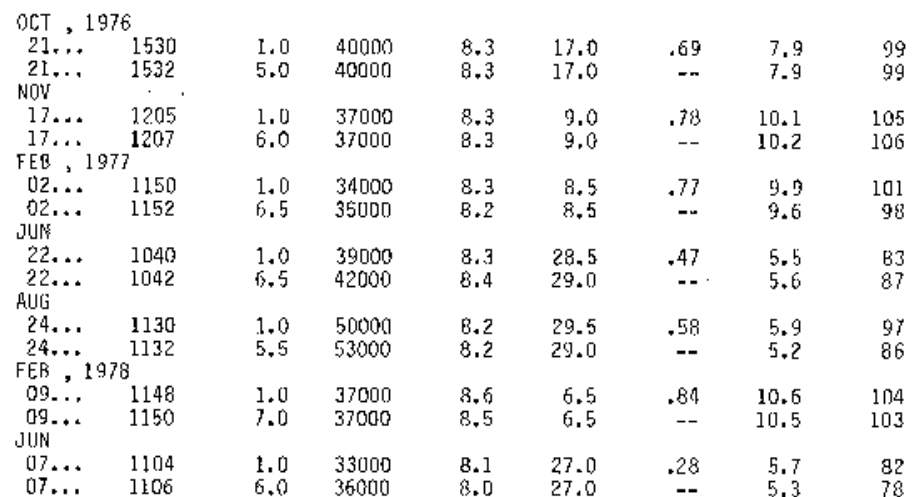

290659095063500 LINE 580 SITE IO

\begin{tabular}{|c|c|c|c|c|c|c|c|c|}
\hline DATE & TIME & $\begin{array}{l}\text { SAMP- } \\
\text { IING } \\
\text { DFPTH } \\
\text { (FT) }\end{array}$ & $\begin{array}{l}\text { SPE- } \\
\text { CIFIC } \\
\text { CON- } \\
\text { DUCT- } \\
\text { ANCE } \\
\text { (MICRO- } \\
\text { MHOS) }\end{array}$ & $\begin{array}{c}\text { PH } \\
\text { (UNITS) }\end{array}$ & $\begin{array}{c}\text { TEMPER- } \\
\text { ATURE } \\
\text { (DEG C) }\end{array}$ & $\begin{array}{l}\text { TRANSS- } \\
\text { PAR- } \\
\text { ENCY } \\
\text { (SFCCHS } \\
\text { DISK) } \\
\text { (N) }\end{array}$ & $\begin{array}{l}\text { OXYGLN, } \\
\text { DIS- } \\
\text { SOLVEL } \\
\{M G / L\}\end{array}$ & $\begin{array}{c}\text { OXYGEN, } \\
\text { OIS- } \\
\text { SOLVED } \\
\text { (PER- } \\
\text { CENT } \\
\text { SATUR- } \\
\text { ATION) }\end{array}$ \\
\hline \multicolumn{9}{|c|}{ OCT, 1976} \\
\hline $\begin{array}{r}20 \ldots \\
20 \ldots\end{array}$ & $\begin{array}{l}1640 \\
1642\end{array}$ & $\begin{array}{l}1.0 \\
5.0\end{array}$ & $\begin{array}{l}46000 \\
46000\end{array}$ & $\begin{array}{l}8.3 \\
8.3\end{array}$ & $\begin{array}{l}17.0 \\
17.0\end{array}$ & $\begin{array}{r}1.06 \\
--\end{array}$ & $\begin{array}{r}8.0 \\
8.0\end{array}$ & $\begin{array}{l}104 \\
104\end{array}$ \\
\hline $\begin{array}{l}17 \ldots \\
17 \ldots\end{array}$ & $\begin{array}{l}1225 \\
1227\end{array}$ & $\begin{array}{l}1.0 \\
4.0\end{array}$ & $\begin{array}{l}36000 \\
37000\end{array}$ & $\begin{array}{l}8.2 \\
8.2\end{array}$ & $\begin{array}{l}8.5 \\
8.5\end{array}$ & $\begin{array}{r}.70 \\
--\end{array}$ & $\begin{array}{l}10 . ? \\
10.3\end{array}$ & $\begin{array}{l}105 \\
106\end{array}$ \\
\hline \multicolumn{9}{|c|}{ FEB, 1977} \\
\hline $\begin{array}{l}02 \ldots \\
02 \ldots \\
\text { dUN }\end{array}$ & $\begin{array}{l}1205 \\
1207\end{array}$ & $\begin{array}{l}1.0 \\
5.5\end{array}$ & $\begin{array}{l}36000 \\
41000\end{array}$ & $\begin{array}{l}8.3 \\
8.3\end{array}$ & $\begin{array}{l}8.5 \\
8.5\end{array}$ & $\begin{array}{l}.54 \\
--\end{array}$ & $\begin{array}{r}10.0 \\
9.6\end{array}$ & $\begin{array}{l}103 \\
102\end{array}$ \\
\hline $\begin{array}{l}22 \ldots \\
22, \ldots \\
\text { RUIG }\end{array}$ & $\begin{array}{l}1100 \\
1102\end{array}$ & $\begin{array}{l}1.0 \\
4.0\end{array}$ & $\begin{array}{l}49000 \\
49000\end{array}$ & $\begin{array}{l}8.3 \\
8.3\end{array}$ & $\begin{array}{l}28.0 \\
28.0\end{array}$ & $\begin{array}{r}.40 \\
--\end{array}$ & $\begin{array}{l}6.2 \\
6.3\end{array}$ & $\begin{array}{r}99 \\
100\end{array}$ \\
\hline $\begin{array}{r}24 \ldots \\
24 \ldots \\
\text { FEB },\end{array}$ & $\begin{array}{l}1150 \\
1152 \\
8\end{array}$ & $\begin{array}{l}1.0 \\
2.5\end{array}$ & $\begin{array}{l}54000 \\
54000\end{array}$ & $\begin{array}{l}8.1 \\
8.1\end{array}$ & $\begin{array}{l}30.0 \\
30.0\end{array}$ & $\begin{array}{r}.37 \\
--\end{array}$ & $\begin{array}{l}6.0 \\
5.9\end{array}$ & $\begin{array}{r}100 \\
99\end{array}$ \\
\hline $\begin{array}{l}09 . . \\
09 . \\
\text { JUN }\end{array}$ & $\begin{array}{l}1204 \\
1206\end{array}$ & $\begin{array}{l}1.0 \\
4.0\end{array}$ & $\begin{array}{l}36000 \\
37000\end{array}$ & $\begin{array}{l}8.6 \\
8.6\end{array}$ & $\begin{array}{l}6.5 \\
6.5\end{array}$ & $\begin{array}{r}.65 \\
--\end{array}$ & $\begin{array}{l}10.6 \\
10.8\end{array}$ & $\begin{array}{l}104 \\
106\end{array}$ \\
\hline $\begin{array}{l}07 \ldots \\
07 \ldots\end{array}$ & $\begin{array}{l}1129 \\
1130\end{array}$ & $\begin{array}{l}1.0 \\
3.0\end{array}$ & $\begin{array}{l}34000 \\
34000\end{array}$ & $\begin{array}{l}8.3 \\
8.3\end{array}$ & $\begin{array}{l}27.0 \\
26.5\end{array}$ & .25 & $\begin{array}{l}6.5 \\
6.6\end{array}$ & $\begin{array}{l}94 \\
95\end{array}$ \\
\hline
\end{tabular}


Table 2A.--Quality of water fn the Trinity-San Jacinto estuary, water years. 1977-78--Cont linued Field Determinations--Cont inted

$2906 \$ 4095075100$ LIHE 580 SITE 50

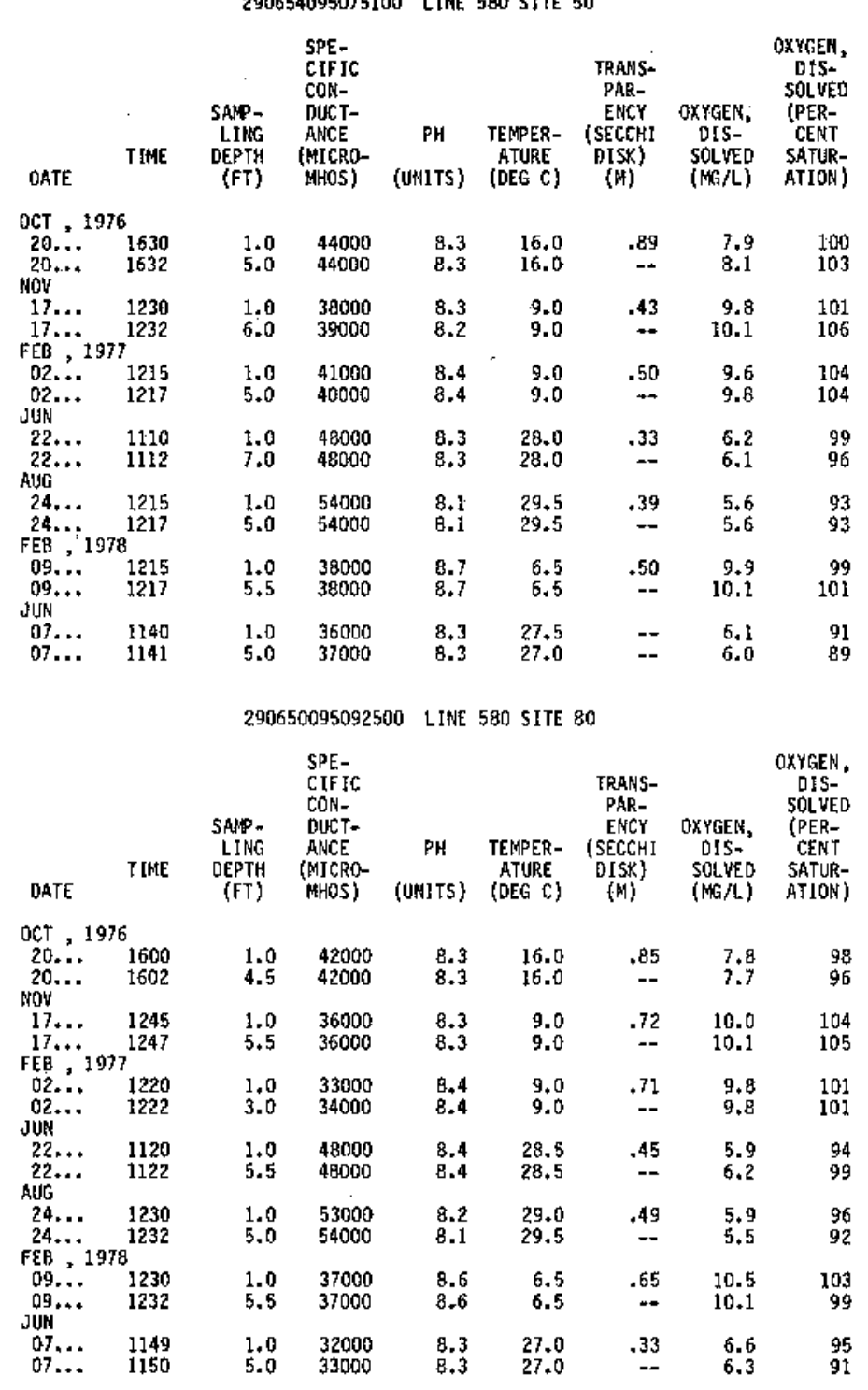


Table 2A.--Quality of water in the Trinity-San jaclnto estiary, water years 197/-78-mContinued Field Determinations-Continued

300328094490500 LINE 600 SITE 20

\begin{tabular}{|c|c|c|c|c|c|c|c|c|}
\hline DATE & TIME & $\begin{array}{c}\text { SAMP- } \\
\text { LING } \\
\text { DEPTH } \\
\text { (FT) }\end{array}$ & $\begin{array}{l}\text { SPE- } \\
\text { CIFIC } \\
\text { CON- } \\
\text { DUCT- } \\
\text { ANCE } \\
\text { (MICRD- } \\
\text { MHOS) }\end{array}$ & $\begin{array}{c}\mathrm{PH} \\
\text { \{UNITS }\end{array}$ & $\begin{array}{l}\text { TEMPER- } \\
\text { ATURE } \\
\text { (DEG C) }\end{array}$ & $\begin{array}{c}\text { TRANS- } \\
\text { PAR- } \\
\text { ENCY } \\
\text { (SECCHI } \\
\text { DISK) } \\
\text { (M) }\end{array}$ & $\begin{array}{c}\text { OXYGEN, } \\
\text { OIS- } \\
\text { SOL YED } \\
\text { (MG/L) }\end{array}$ & $\begin{array}{l}\text { OXYGEN, } \\
\text { OIS- } \\
\text { SOLVED } \\
\text { (PER- } \\
\text { CENT } \\
\text { SATUR- } \\
\text { ATION) }\end{array}$ \\
\hline \multicolumn{9}{|c|}{ Hov , 1976} \\
\hline $30 .$. & 1030 & 1.0 & 320 & 8.3 & 7.5 & - & 11.6 & 100 \\
\hline $30 \ldots$ & 1032 & 8.0 & 320 & 8.3 & 7.5 & -- & 11.6 & 100 \\
\hline 30. . & 1300 & 1.0 & 305 & 8.4 & 8.5 & -- & $11: 6$ & 103 \\
\hline $30 \ldots$ & 1302 & 20 & 300 & 8.5 & 8.5 & -- & 11.6 & 103 \\
\hline $30 \ldots$ & 1600 & 1.0 & 310 & 8.6 & 9.0 & $-\infty$ & 11.6 & 104 \\
\hline $30 .$. & 1602 & 20 & 305 & 8.8 & 9.0 & $=$ & 11.8 & 105 \\
\hline $30 .$. & 1900 & 1.0 & 320 & 8.3 & 9.0 & $\cdots$ & 11.6 & 104 \\
\hline $30 \ldots$ & 1902 & 20 & 320 & 8.4 & 9.0 & -- & 12.0 & 107 \\
\hline $30 .$. & 2200 & 1.0 & 320 & 8.3 & 9.0 & $=-$ & 11.6 & 104 \\
\hline $30 \ldots$ & 2202 & 20 & 320 & 8.3 & 9.0 & 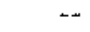 & 11.6 & 104 \\
\hline \multicolumn{9}{|l|}{$\mathrm{DEC}$} \\
\hline $01 \ldots$ & 0100 & 1.0 & 320 & 8.2 & 9.0 & $\cdots$ & 11.4 & 102 \\
\hline $01 \ldots$ & 0102 & 20 & 320 & 8.2 & 9.0 & -- & 11.6 & 104 \\
\hline $01 .$. & 0400 & 1.0 & 370 & 8.1 & 8,0 & - & 11.4 & 99 \\
\hline $01 \ldots$ & 0402 & 20 & 320 & 8.1 & 8.0 & $\ldots$ & 11.4 & 99 \\
\hline $01 .$. & 0700 & 1.0 & 320 & 8.1 & 8.0 & -- & 11.4 & 99 \\
\hline $01 .$. & 0702 & 20 & 320 & 8.1 & 8.0 & -- & 11.4 & 99 \\
\hline $01 .$, & 1000 & 1.0 & 320 & 8.2 & 8.0 & $m$ & 11.4 & 99 \\
\hline $01 .$. & 1002 & 20 & 320 & 8.2 & 8.0 & -- & 11.6 & 101 \\
\hline $08,$. & 1000 & 1.0 & 180 & 7.6 & 11.0 & .10 & 8.9 & 83 \\
\hline \multicolumn{8}{|c|}{ MAY , 1977} & 83 \\
\hline JUL & 1540 & 1.0 & 339 & -- & 25.5 & -- & 8.1 & 101 \\
\hline $20 \ldots$ & 0100 & 1.0 & 380 & 8.2 & 30.5 & -- & 7.5 & 100 \\
\hline 20. & 0102 & 9.0 & 380 & 8.2 & 30.5 & -- & 7.4 & 99 \\
\hline $20 .$. & 0104 & 18 & 375 & 8.2 & 30.5 & $-\infty$ & 7.4 & 99 \\
\hline $20 \ldots$ & 1000 & 1.0 & 360 & 8.2 & 31.0 & -- & 6.5 & $8 B$ \\
\hline $20 .$. & 1002 & 9.0 & 360 & 8.1 & 30.5 & -- & 6.4 & 85 \\
\hline $20 .$. & 1004 & 18 & 365 & 8.1 & 3ก. .5 & $\ldots$ & 6.2 & 83 \\
\hline 20. & 1300 & 1.0 & 360 & 8.1 & 30.0 &.- & 6.9 & 92 \\
\hline $2 \pi .$. & 1302 & 9.0 & 360 & 8,1 & 30.0 & מـ & 6.8 & 91 \\
\hline 20. & 1304 & 18 & 370 & 8.1 & 30.0 & -- & 6.9 & 92 \\
\hline $20 .$. & 1600 & 1.0 & 365 & 8.2 & 30.0 & 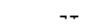 & 7.6 & 101 \\
\hline $20 .$. & 1602 & 9.0 & 365 & 8.2 & 30.0 & -- & 7.6 & 101 \\
\hline $20 .$. & 1604 & 18 & 365 & 8.2 & 30.0 & -- & 7.7 & 103 \\
\hline 20. & 1900 & 1.0 & 360 & 8.3 & 30.5 & -- & 8.2 & 109 \\
\hline 20. & 1902 & 9,0 & 360 & 8.3 & 30.5 & -. & 8.1 & 108 \\
\hline $20 .$. & 1904 & 18 & 360 & 8.3 & 30.5 & -- & 8.2 & 109 \\
\hline 20. & 2200 & 1.0 & 365 & 8.3 & 31.0 & $m$ & 8.6 & 116 \\
\hline 20. & 2202 & 9.0 & 370 & 8.3 & 30.5 & -- & 8.6 & 115 \\
\hline $20 .$. & 2204 & 18 & 370 & 8.3 & 30.5 & -- & 8.9 & 119 \\
\hline 21. & 0400 & 1.0 & 385 & $8 . ?$ & 30.0 & - & 7.2 & 96 \\
\hline 21. & 0402 & 9.0 & 380 & 8.2 & 30.0 & -- & 7.2 & 96 \\
\hline 21. & 0404 & 18 & 385 & 8.2 & 30.0 & 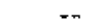 & 7.2 & 96 \\
\hline $21 .$. & 0700 & 1.0 & 370 & 9.2 & 30.0 & -- & 7.0 & 93 \\
\hline 21. & 0702 & 9.0 & 370 & 8.2 & 30. & -- & 7.0 & 93 \\
\hline 21. & 0704 & 18 & 390 & 8.2 & 30.0 & - & 7.0 & 93 \\
\hline 21. & 1000 & 1,0 & 375 & 8.2 & 29.0 & -- & 7.2 & 95 \\
\hline $21 \ldots$ & 1002 & 9.0 & 375 & 8.1 & 29.0 & -- & 7.2 & 95 \\
\hline 21, & 1004 & 18 & 375 & 8.1 & 29.0 & $=$ & 7.3 & 96 \\
\hline \multicolumn{9}{|l|}{ AUIG } \\
\hline $10 \ldots$ & 1000 & I.D & 380 & 8.4 & 31.0 & -- & 6.6 & 89 \\
\hline & 1002 & 8.5 & 380 & 8.3 & 31.0 & -- & 6.6 & 89 \\
\hline 10. & 1004 & 17 & 380 & 8.3 & 31.0 & -- & 6.7 & 91 \\
\hline 10. & 1300 & 1.0 & 390 & 8.5 & 31.0 & . & 7.4 & 100 \\
\hline 10. & 1302 & 8.5 & 38 & 8. & 31.0 & -- & 7. & 100 \\
\hline $10 .$. & 1304 & 17 & 380 & 8.5 & 31.0 & $=$ & 7.4 & 100 \\
\hline & 1600 & 1.0 & 380 & 8.6 & 31.5 & $m$ & 7.9 & 107 \\
\hline 10. & 1602 & 8.5 & 380 & 8.7 & 31.5 & -- & 7.9 & 107 \\
\hline 10. & 1604 & 17 & 380 & 8.7 & 31.5 & -- & 7.9 & 107 \\
\hline & 1900 & 1.0 & 370 & 8. & 32. & -- & 8.3 & 114 \\
\hline $10 .$. & 1902 & 8.5 & 37 & 8.7 & 32.0 & -- & 8.3 & 114 \\
\hline & 1904 & 17 & 370 & 8.7 & 32.0 & -- & $\mathrm{P}, 4$ & 115 \\
\hline $10 .$. & 2200 & 1.0 & 380 & 8.7 & 32.0 & -- & 7.8 & 107 \\
\hline 10. & 2202 & 8.5 & 380 & 8.7 & 32.0 & $\ldots$ & 7.8 & 107 \\
\hline & 2204 & 17 & 38 & 8.7 & 32.0 & -- & 7.8 & 107 \\
\hline 11. & 0100 & 1.0 & 380 & 8.6 & 31.5 & -- & 7.2 & 97 \\
\hline & 0102 & 8.5 & 380 & 8.6 & 31.5 & -- & 7.2 & 97 \\
\hline & 0104 & 17 & 380 & 8.6 & 31.5 & -- & 7.3 & 99 \\
\hline & 0.400 & 1.0 & 380 & 8.3 & 31.5 & $m$ & 6.4 & 86 \\
\hline & 0402 & 8.5 & 380 & 8.3 & 31.5 & -- & 6.4 & 86 \\
\hline & 0404 & 17 & 380 & 8.3 & 31.5 & -1 & 6.4 & 86 \\
\hline & 0700 & 1.0 & 380 & 8.3 & 31.0 & -- & 6.1 & 82 \\
\hline & 0702 & 8.5 & 390 & 8.3 & 31.0 & $\cdots$ & 6.2 & 84 \\
\hline & 0704 & 17 & 380 & 8.3 & 31.0 & -- & 6.3 & 85 \\
\hline & 1000 & 1.0 & 38 & 8.3 & 30.5 & $\because$ & 5.2 & 83 \\
\hline & 1002 & 8.5 & 380 & 8.3 & 30.5 & -- & 6.3 & 84 \\
\hline & $1004^{\prime}$ & 17 & 380 & 8.4 & 30.5 & $m$ & 6.2 & 83 \\
\hline
\end{tabular}


Table 2A.-0quality of water in the Trinity-San Jacinto estuary, water years 1977-78--Continued Field Deterninat fans--Cont inued

295855094485200 LINE 6 OOA SITE 70

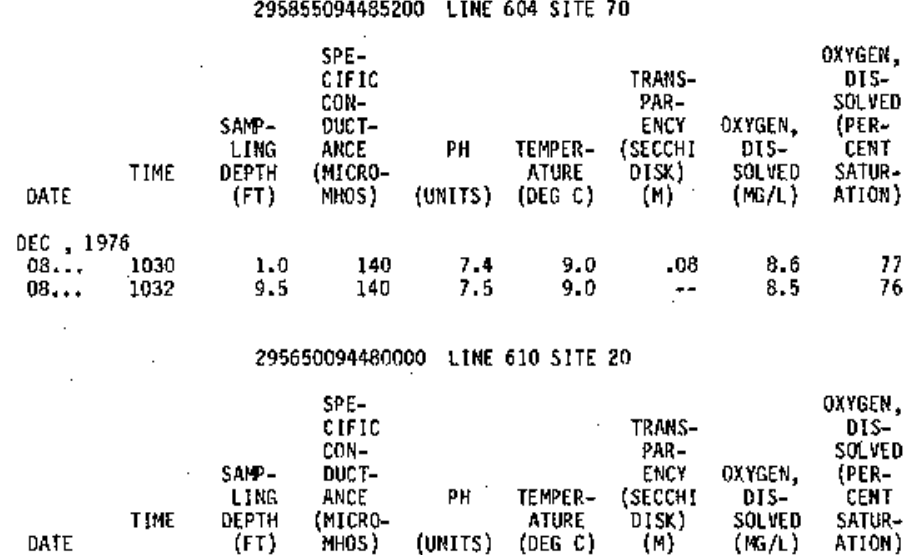

DEC , 1976

$08 . . . \quad 1115$

$\begin{array}{lllllll}1.0 & 120 & 7.5 & 11.0 & .10 & 8.8 & 82 \\ 10 & 180 & 7.5 & 11.0 & -- & 8.7 & 81\end{array}$

295616094465600 LINE 615 SITE $20^{\circ}$

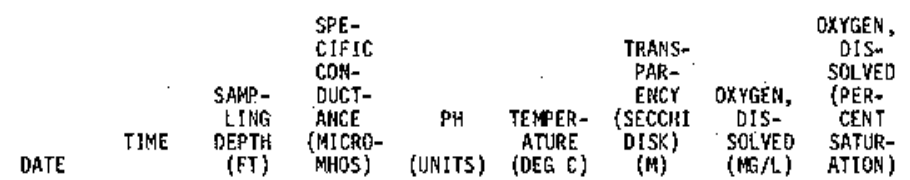

DEC, 1976

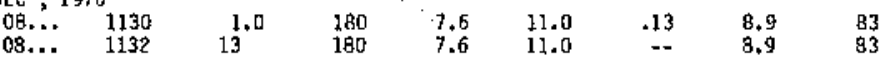


Table 2A.--QQuality of water in the Trinity-San Jacinto estuary, water years 1977-39--Continued

Field Deterusinations-Cont inved

295420094460600 LIVHE 619 SITE 20

\begin{tabular}{|c|c|c|c|c|c|c|c|c|}
\hline DATE & TIME & $\begin{array}{c}\text { SAMP- } \\
\text { LING } \\
\text { DEPTH } \\
(F T)\end{array}$ & $\begin{array}{l}\text { SPE- } \\
\text { CIFIC } \\
\text { CDN- } \\
\text { DUCT- } \\
\text { ANCE } \\
\text { (MICRO- } \\
\text { MHOS) }\end{array}$ & $\begin{array}{c}\mathrm{PH} \\
\text { (UNITS) }\end{array}$ & $\begin{array}{l}\text { TEMPER- } \\
\text { ATURE } \\
\text { (DEG C) }\end{array}$ & $\begin{array}{l}\text { TRAAS- } \\
\text { PAR- } \\
\text { ENCY } \\
\text { (SECCHI } \\
\text { DISK) } \\
\text { (PI) }\end{array}$ & $\begin{array}{c}\text { OXYGES, } \\
\text { DIS- } \\
\text { SOLVED } \\
\text { (MG/L) }\end{array}$ & $\begin{array}{l}\text { OXYGEN, } \\
\text { OIS- } \\
\text { SOLYED } \\
\text { (PER- } \\
\text { CENT } \\
\text { SATUR- } \\
\text { ATION) }\end{array}$ \\
\hline \multicolumn{9}{|c|}{ NDV , 1976} \\
\hline $30 .$. & 1100 & 1.0 & 240 & $\cdots$ & 7.0 & .52 & 10.6 & 90 \\
\hline $30 \ldots$ & 1102 & 3.2 & 280 & -+ & 7.0 & $=$ & 10.8 & 92 \\
\hline $30 .$. & 1104 & 6.5 & 280 & $\omega$ & 6.5 & -- & 10.7 & 90 \\
\hline $30 \ldots$ & 1400 & 1.0 & 190 & - & 8.5 & .48 & 10.5 & 93 \\
\hline $30 \ldots$ & 1402 & 3.5 & 220 & -- & 8,5 & -- & 10.5 & 93 \\
\hline $30 \ldots$ & 1404 & 7.0 & 230 & -- & 8.5 & 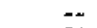 & 10.3 & 91 \\
\hline $30 \ldots$ & 1700 & 1.0 & 220 & - & B. 5 & .51 & 10.5 & 93 \\
\hline $30 \ldots$ & 1702 & 3.5 & 240 & -- & 8.5 & $\sim$ & 10.4 & 92 \\
\hline $30 \ldots$ & 1704 & 7.0 & 200 & -- & 8.5 & $-n$ & 10.4 & 92 \\
\hline $30 \ldots$ & 2000 & 1.0 & 300 & -- & 7.0 & -- & 11.0 & 93 \\
\hline $30 . . .4$ & 2002 & 3.5 & 300 & -- & 6.5 & $=$ & 11.0 & 92 \\
\hline 30. & 2004 & 7.0 & 200 & -- & 6.0 & -- & 11.1 & 92 \\
\hline $30 \ldots$ & 2300 & 1.0 & 290 & -- & 7.0 & $=-$ & 11.0 & 93 \\
\hline 30. & 2302 & 3.5 & 290 & -- & 7.0 & -- & 10.9 & 92 \\
\hline & 92 \\
\hline $01 \ldots$ & 0200 & 1.0 & 300 & n- & 6.5 & -- & 11.1 & 93 \\
\hline $01 \ldots$ & 0202 & 3.5 & 290 & 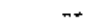 & 6.5 & -- & 11.1 & 93 \\
\hline $01 \ldots$ & 0204 & 7.0 & 290 & $+\infty$ & 6.0 & -- & 11.0 & 91 \\
\hline $01 \ldots$ & 0500 & 1.0 & 300 & $\cdots$ & 7.0 & $\therefore$ & 11.1 & 34 \\
\hline $01 .$. & 0502 & 3.5 & 280 & -- & $6.5^{\circ}$ & -- & 11.2 & 94 \\
\hline $01 .$. & 0504 & 7.0 & 280 & $n$ & 6.0 & $=$ & 11.2 & 93 \\
\hline $01 .$. & 0800 & 1.0 & 280 & -- & 7.0 & $.4 B$ & 11.0 & 93 \\
\hline $01 \ldots$ & 0802 & 3.5 & 300 & -- & 7.0 & -- & 11.0 & 93 \\
\hline $01 \ldots$ & 0804 & 7.0 & 300 & - & 7.0 & $=-$ & 11.0 & 93 \\
\hline $01 \ldots$ & 1100 & 1.0 & 290 & $m$ & 7.5 & .53 & 11.2 & 97 \\
\hline $01 \ldots$ & 1102 & 3.5 & 290 & $-\cdots$ & 7.5 & -- & 11.1 & 96 \\
\hline 아.... & 1104 & 7.0 & 280 & $\because$ & 7.5 & $=$ & 11.1 & 96 \\
\hline $02 \ldots$ & 1100 & 1.0 & 310 & 7.6 & 8.5 & .49 & -- & - \\
\hline $02, \ldots$ & 1102 & 3.7 & 310 & 7.5 & 8.5 & $-n$ & -- & -- \\
\hline $02 \ldots$ & 1104 & 7.5 & 310 & 7.4 & 8.5 & $z$ & $-\infty$ & $=$ \\
\hline $02 \ldots$ & 1400 & 1.0 & 320 & 7.6 & 9.0 & .51 & -- & $=$ \\
\hline $02 \ldots$ & 1402 & 3.7 & 320 & 7.6 & 8.5 & -- & $\sim$ & $=$ \\
\hline $02 \ldots$ & 1404 & 7.5 & 320 & 7.6 & 8.5 & $\overline{44}$ & $=$ & $\cdots$ \\
\hline $\begin{array}{l}02 . \\
02 .\end{array}$ & $\begin{array}{l}1700 \\
1702\end{array}$ & $\begin{array}{l}1.0 \\
3.7\end{array}$ & $\begin{array}{l}315 \\
320\end{array}$ & $\begin{array}{l}7.9 \\
7.9\end{array}$ & $\begin{array}{l}9.0 \\
9.0\end{array}$ & $\begin{array}{r}.44 \\
-\end{array}$ & $=$ & $\begin{array}{l}-- \\
--\end{array}$ \\
\hline 02. & 1704 & 7.5 & 320 & 7.9 & 9.0 & $=$ & $=$ & $=$ \\
\hline $02+$. & 2000 & 1.0 & 320 & 7.9 & 8.5 & $+\infty$ & -- & $=$ \\
\hline $02 \ldots$ & 2002 & 3.7 & 320 & 7.9 & B. 5 & -- & 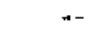 & -- \\
\hline $02 .$. & 2004 & 7.5 & 320 & 7.9 & 8.5 & $=$ & -- & $\rightarrow$ \\
\hline $02 \ldots$ & 2300 & 1.0 & 310 & 7.9 & 8.5 & -- & -- & 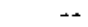 \\
\hline 02. & 2302 & 3.7 & 310 & 8.0 & 8.5 & -- & -- & - \\
\hline 02. & 2304 & 7.5 & 310 & 7.9 & 8.5 & -- & -- & - \\
\hline $03 \ldots$ & 0200 & 1.0 & 300 & 7.9 & 85.0 & $=$ & -- & - \\
\hline 03. & 0202 & 3.7 & 310 & B. 0 & 8.5 & $\ldots$ & $-\cdots$ & - \\
\hline $03 . .$. & 0204 & 7.5 & 310 & 8.0 & 8.0 & -- & $\cdots$ & \\
\hline $03 .$. & 0500 & 1.0 & 300 & 7.9 & 8.5 & -- & -- & 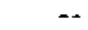 \\
\hline $03 .$. & 0502 & 3.7 & 300 & 7.9 & 8.5 & -- & -- & - \\
\hline $03 .$. & 0504 & 7.5 & 300 & 7.9 & 8.5 & $\rightarrow$ & -- & - \\
\hline 03. & 0800 & 1.0 & 300 & 7.9 & B. 5 & .49 & + & - \\
\hline $03 .$. & 0,002 & 3.7 & 300 & 7.9 & 8.5 & -- & $+\infty$ & -- \\
\hline \multicolumn{9}{|c|}{ JUL , 1977} \\
\hline $20 .$. & 1020 & 2.5 & 350 & 7.7 & 30.5 & .57 & 6,5 & 87 \\
\hline $20+\ldots$ & 1300 & 3.5 & 360 & 7.7 & 30. & .54 & 6.3 & 84 \\
\hline 20. & 1600 & 3.5 & 355 & 7.7 & 30. & .57 & 6.6 & 88 \\
\hline 20. & 1900 & 3.5 & 355 & 7.8 & 30. & .50 & 7.0 & 93 \\
\hline & 2200 & 1.0 & 355 & 7.7 & 30. & -- & 6.5 & 87 \\
\hline $20 .$. & 2202 & 6.0 & 355 & 7.7 & 30. & -- & 6.5 & 87 \\
\hline 20. & 2204 & 3.5 & 35 & 7.7 & 30. & -- & 6.5 & 87 \\
\hline 21. & 0100 & 3.5 & 35 & 7.7 & 30. & -- & 6.3 & 84 \\
\hline & 0400 & 3.5 & 35 & 7.6 & 29. & $\therefore$ & 6.0 & 79 \\
\hline 21. & 0700 & 3.5 & 36 & 7.6 & 29. & $m$ & 6.0 & 79 \\
\hline 21. & 1000 & 3.5 & 36 & & 30.0 & - & 6.0 & 80 \\
\hline \multicolumn{9}{|l|}{$A \cup G$} \\
\hline $10 .$. & 1000 & 1.0 & 410 & 7.6 & 30.5 & -- & 5.3 & 71 \\
\hline 10. & 1002 & 6.0 & 410 & 7.6 & 30. & -- & 5.4 & 72 \\
\hline & 1300 & 1.0 & 400 & 7.8 & 31 . & -- & 6.6 & 89 \\
\hline 10. & 1302 & 6.0 & 400 & 7.8 & 31. & $=$ & 6.5 & 88 \\
\hline 10. & 1600 & 1.0 & 400 & 7.9 & 32. & $n$ & 7.2 & 99 \\
\hline 10. & 1602 & 6.0 & 400 & 7,7 & 31. & -- & 6.3 & 85 \\
\hline & 1900 & 1.0 & 400 & 7.9 & 31. & -- & 6.4 & 86 \\
\hline & 1902 & 6.0 & 400 & 7.8 & 31. & -- & 6.2 & 84 \\
\hline & 2200 & 1.0 & 410 & 7.7 & 30. & -- & 5.7 & 76 \\
\hline 10.. & 2202 & 6.0 & 410 & 7.7 & 30. & -- & 5.8 & 77 \\
\hline & 0100 & 1.0 & 410 & 7.7 & 30. & -- & 5.5 & 73 \\
\hline & 0102 & 6.0 & 410 & 7.7 & 30. & 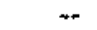 & 5.8 & 77 \\
\hline 11. & 0400 & 1.0 & 410 & 7.7 & 30.5 & $n-$ & 5.4 & 72 \\
\hline & 0402 & 6.0 & 410 & 7.6 & 30. & -- & 5.1 & 68 \\
\hline & 0700 & 1.0 & 410 & 7.7 & 30.5 & -- & 5.2 & 69 \\
\hline & 0702 & 6.0 & 410 & 7.7 & 30. & -- & 5.2 & 69 \\
\hline & 1000 & 1. & 41 & 7.7 & 30. & -- & 5.2 & 69 \\
\hline & 1002 & 6.0 & 410 & & 30.5 & - & 5.3 & 71 \\
\hline
\end{tabular}


Table 2A,--0uality of water in the Trinity-San Jacinto estuary, water years 1977-78--Continued Field Determinations--Continued

295424094454500 LINE 620 SITE 20

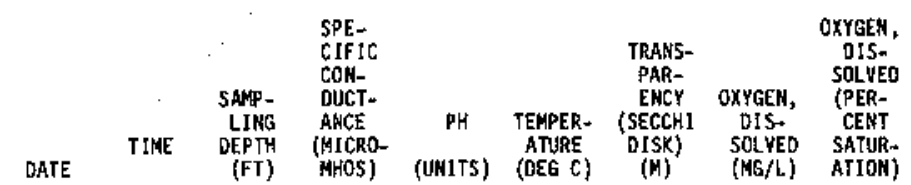

$$
\begin{aligned}
& \text { DEC . } 1976 \\
& \begin{array}{cc}
\text { 08C... } & 1200 \\
08 . . & 1202 \\
\text { JUL . } 1977 & \\
06 . . . & 1610 \\
06 . . . & 1612 \\
06 . . . & 1614 \\
06 . . . & 1616
\end{array}
\end{aligned}
$$

$\begin{array}{llllllr}1.0 & 180 & 7.7 & 11.0 & .13 & 8.9 & 83 \\ 10 & 180 & 7.7 & 11.0 & -- & 8.9 & 83 \\ 1.0 & 410 & 8.2 & 32.5 & .84 & 8.0 & 110 \\ 5.0 & 410 & 7.9 & 31.5 & -- & 6.3 & 85 \\ 10 & 410 & 7.8 & 31.0 & -- & 5.6 & 76 \\ 14 & 410 & 7.8 & 31.5 & -- & 5.3 & 72\end{array}$

295316094440100 LIHE 628 SITE 20

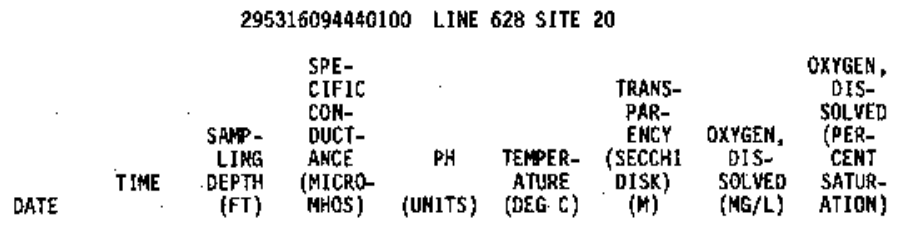

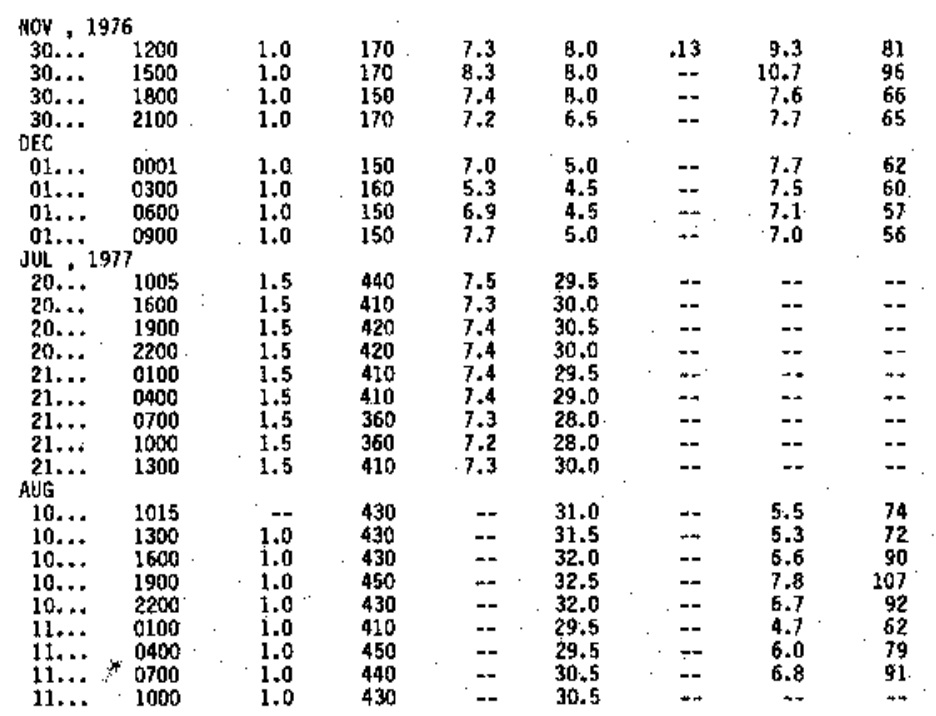

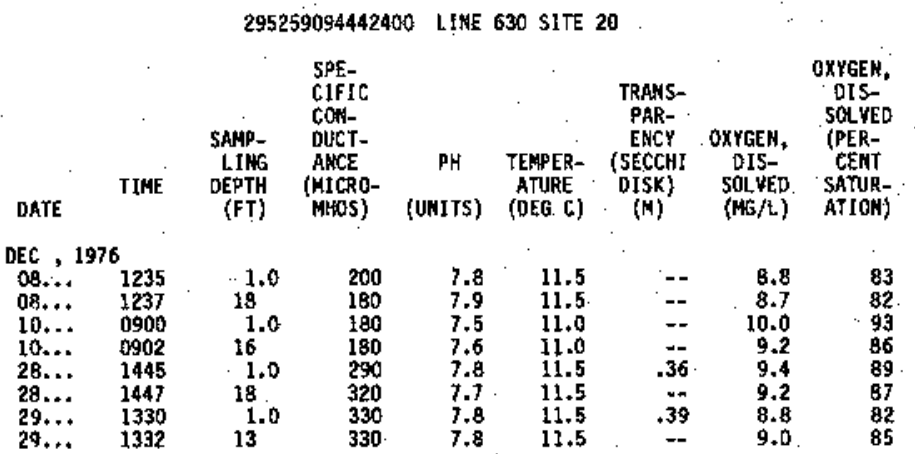


Table $2 A .--Q u a l i t y$ of water in the Trinity-San Jacinto estuary, water years 1977-79--Continued

Field Deterninat ions--.Continued

295310094453200 LIHE 630 SITE 40

\begin{tabular}{|c|c|c|c|c|c|c|c|c|}
\hline DATE & TIME & $\begin{array}{l}\text { SAMP- } \\
\text { LING } \\
\text { DEPTH } \\
\text { (FT) }\end{array}$ & $\begin{array}{l}\text { SPE- } \\
\text { CIFIC } \\
\text { CON- } \\
\text { DUCT- } \\
\text { ANCE } \\
\text { (MICRO- } \\
\text { MHOS) }\end{array}$ & $\begin{array}{c}\text { PH } \\
\text { (UNITS) }\end{array}$ & $\begin{array}{c}\text { TEMPER- } \\
\text { ATURE } \\
\text { (DEG C) }\end{array}$ & $\begin{array}{l}\text { TRAHS- } \\
\text { PAR- } \\
\text { EACY } \\
\text { (SECCHI } \\
\text { DISK) } \\
\text { (M) }\end{array}$ & $\begin{array}{c}\text { OXYGEN, } \\
\text { DIS- } \\
\text { SOLVED } \\
\text { (MG/L) }\end{array}$ & $\begin{array}{l}\text { OXYGER, } \\
\text { DIS- } \\
\text { SOLYED } \\
\text { (PER- } \\
\text { CENT } \\
\text { SATUR- } \\
\text { ATION) }\end{array}$ \\
\hline $\begin{array}{l}\text { OEC , } \\
0 \mathrm{~B} . \ldots \\
08 \ldots \\
10 \ldots \\
10 \ldots \\
28 \ldots \\
28 \ldots \\
\text { SEP }\end{array}$ & $\begin{array}{l}6 \\
1415 \\
1417 \\
0950 \\
0952 \\
1345 \\
1347 \\
7^{134}\end{array}$ & $\begin{array}{l}1.0 \\
5.0 \\
1.0 \\
7.0 \\
1.0 \\
3.0\end{array}$ & $\begin{array}{l}400 \\
400 \\
200 \\
200 \\
330 \\
330\end{array}$ & $\begin{array}{l}7.2 \\
7.2 \\
7.5 \\
7.5 \\
7.8 \\
7.8\end{array}$ & $\begin{array}{r}10.0 \\
8.5 \\
11.5 \\
11.0 \\
11.5 \\
11.5\end{array}$ & $\begin{array}{r}.25 \\
.18 \\
.2 \\
.25 \\
.5\end{array}$ & $\begin{array}{l}8.3 \\
8.0 \\
9.3 \\
9.4 \\
9.0 \\
9.0\end{array}$ & $\begin{array}{l}76 \\
71 \\
88 \\
88 \\
85 \\
85\end{array}$ \\
\hline $20 \ldots$ & $\begin{array}{l}1420 \\
1422\end{array}$ & $\begin{array}{l}1.0 \\
3.5\end{array}$ & $\begin{array}{l}380 \\
460\end{array}$ & $\begin{array}{l}7.7 \\
7.6\end{array}$ & $\begin{array}{l}29.5 \\
28.5\end{array}$ & $\begin{array}{r}.34 \\
--\end{array}$ & $\begin{array}{l}5.5 \\
5.4\end{array}$ & $\begin{array}{l}72 \\
70\end{array}$ \\
\hline \multicolumn{9}{|c|}{295034094450700} \\
\hline DATE & TIME & $\begin{array}{l}\text { SAMP- } \\
\text { LING } \\
\text { DEPTH } \\
\text { (FT) }\end{array}$ & $\begin{array}{l}\text { SPE- } \\
\text { CIFIC } \\
\text { CON- } \\
\text { OUCT- } \\
\text { ANCE } \\
\text { (MICRO- } \\
\text { MHOS) }\end{array}$ & $\begin{array}{c}\text { PH } \\
\text { (UNITS) }\end{array}$ & $\begin{array}{c}\text { TEPPER- } \\
\text { ATURE } \\
\text { (DEG C) }\end{array}$ & $\begin{array}{l}\text { TRANS- } \\
\text { PAR- } \\
\text { ENCY } \\
\text { (SECCHI } \\
\text { OISK) } \\
\text { (M) }\end{array}$ & $\begin{array}{c}\text { OXYGEN, } \\
\text { DIS- } \\
\text { SOLWED } \\
\text { (MG } / L)\end{array}$ & $\begin{array}{c}\text { OXYGEN, } \\
\text { DIS- } \\
\text { 5OLWED } \\
\text { (PER- } \\
\text { CENT } \\
\text { SATUR+ } \\
\text { ATION) }\end{array}$ \\
\hline
\end{tabular}

\begin{tabular}{|c|c|c|c|c|c|c|c|c|}
\hline \multicolumn{9}{|c|}{ DEC, 1976} \\
\hline D8.. & 1300 & 1.0 & 180 & 7.8 & 11.5 & .13 & 8.6 & \\
\hline $08 \ldots$ & 1302 & 6.0 & 180 & 7.8 & 11.5 & $=$ & 8.6 & 81 \\
\hline $10 .$. & 0915 & 1.0 & 220 & 7.6 & 11.0 & .13 & 10.0 & 93 \\
\hline $10 .$. & 0917 & 6.0 & 220 & 7.6 & 11.0 & - & 10.1 & 94 \\
\hline $28,$. & 1510 & 1.0 & 370 & 7.5 & 12.5 & .29 & 8.0 & 78 \\
\hline 28. & 1512 & 6.0 & 370 & 1,5 & 12.5 & $=$ & 8.1 & \\
\hline 29. & 1315 & 1.0 & 400 & 7.5 & 11.5 & .25 & 7.7 & 3 \\
\hline 29. & 1317 & $5.0^{\circ}$ & 400 & 7.5 & & - & 8.0 & 5 \\
\hline
\end{tabular}

295015094454800 LINE 670 SITE 10

\begin{tabular}{|c|c|c|c|c|c|c|c|c|}
\hline DATE & TIME & $\begin{array}{c}\text { SAMP- } \\
\text { LING } \\
\text { DEPTH } \\
(\mathbf{F T}) \\
(00003)\end{array}$ & $\begin{array}{l}\text { SPE- } \\
\text { CIFIC } \\
\text { CON- } \\
\text { DUCT- } \\
\text { ANCE } \\
\text { (MICRO- } \\
\text { (MHOS) } \\
\text { (OOOOS) }\end{array}$ & $\begin{array}{c}\text { PH } \\
\left(\begin{array}{c}\text { UNITS } \\
00400\end{array}\right)\end{array}$ & $\begin{array}{l}\text { TEMPER- } \\
\text { ATURE } \\
\text { (DEG C) } \\
\text { (DOOIO) }\end{array}$ & $\begin{array}{c}\text { TRANS- } \\
\text { PAR- } \\
\text { ENCY } \\
\text { (SECCHI } \\
\text { DISK) } \\
(M) \\
(00078)\end{array}$ & $\begin{array}{c}\text { OXYGEN, } \\
\text { DIS- } \\
\text { SOLYED } \\
\text { (MG } / L) \\
\text { (00300) }\end{array}$ & $\begin{array}{c}\text { OXYGEH, } \\
\text { DIS- } \\
\text { SOLVED } \\
\text { (PER- } \\
\text { CENT } \\
\text { SATUR- } \\
\text { ATION) } \\
\text { (00301) }\end{array}$ \\
\hline
\end{tabular}

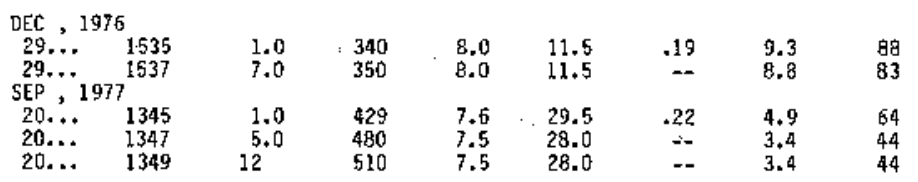


Table 2A.--Quality of water in the Trinity-San Jacinto estuary, water years 1977-78--Continued Field Deterninations--Cont inued

295034094471200 LINE 670 S1TE 40

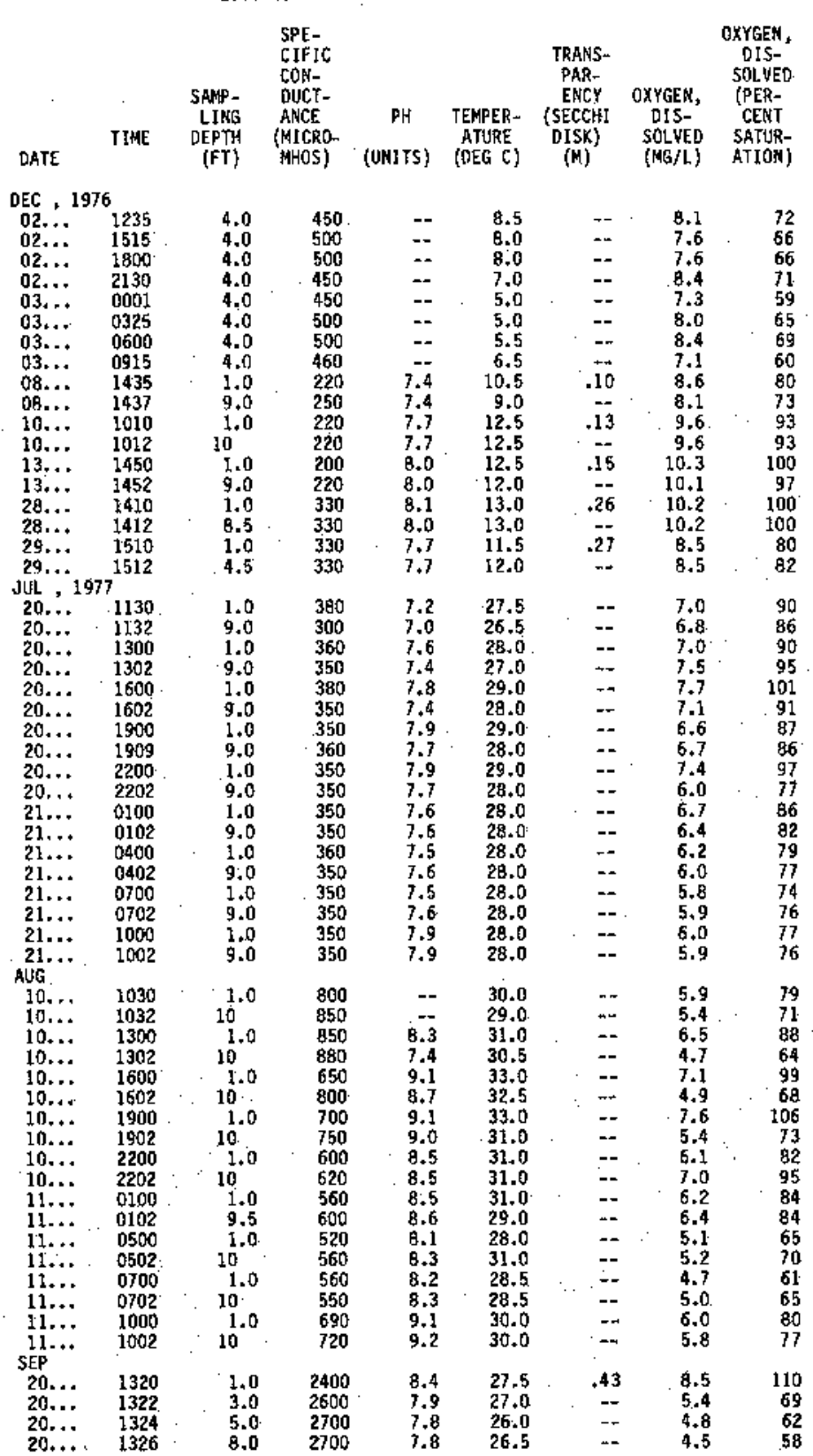


Table 2A.--Quality of water in the Trinity-San Jacinto estuary, water years 1977-78--Continued Field Determiniations-Continued

295003094480700 LIANE 670 SITE 80

\begin{tabular}{|c|c|c|c|c|c|c|c|c|}
\hline DATE & TIME & $\begin{array}{l}\text { SAMP. } \\
\text { LING } \\
\text { DEPFH } \\
\text { (FT) }\end{array}$ & $\begin{array}{l}\text { SPE- } \\
\text { CIFIC } \\
\text { CON- } \\
\text { DUCT- } \\
\text { ANCE } \\
\text { (MICRO- } \\
\text { MHOS) }\end{array}$ & $\begin{array}{c}\text { PH } \\
\text { (UNITS) }\end{array}$ & $\begin{array}{l}\text { TEMPER- } \\
\text { ATURE } \\
\text { (DEG C) }\end{array}$ & $\begin{array}{l}\text { TRAKS- } \\
\text { PAR- } \\
\text { ERCY } \\
\text { (SECCHI } \\
\text { DISK) } \\
\text { (M) }\end{array}$ & $\begin{array}{c}\text { OXYGEN, } \\
\text { DIS- } \\
\text { SOLYED } \\
\text { (MG/L) }\end{array}$ & $\begin{array}{l}\text { OXYGER, } \\
\text { DIS- } \\
\text { SOLYEO } \\
\text { (PER- } \\
\text { CENT } \\
\text { SATUR- } \\
\text { ATIOH) }\end{array}$ \\
\hline
\end{tabular}

DEC, 1976

$02 . \cdots \quad 1200$

$\begin{array}{ll}3.0 & 280 \\ 3.0 & 300 \\ 3.0 & 320 \\ 3.0 & 320 \\ 3.0 & 340 \\ 3.0 & 290 \\ 3.0 & 260 \\ 1.0 & 150 \\ 4.0 & 150 \\ 1.0 & 190 \\ 6.0 & 170 \\ 1.0 & 140 \\ 5.0 & 130 \\ 1.0 & 300 \\ 4.0 & 320 \\ 1.0 & 330 \\ 4.0 & 330\end{array}$

$\begin{array}{lllll}-- & 9.0 & -. & 9.2 & 73 \\ -- & 9.0 & -- & 7.3 & 65 \\ -- & 8.0 & -- & 7.1 & 62 \\ -- & 8.0 & -- & 7.2 & 63 \\ -- & 6.0 & -- & 6.9 & 57 \\ -- & 5.0 & - & 6.9 & 56 . \\ -- & 6.0 & -- & 6.4 & 53 \\ 7.0 & 12.5 & -- & 7.9 & 77 \\ 7.0 & 12.0 & -. & 7.9 & 76 \\ 7.5 & 12.0 & .15 & 9.4 & 90 \\ 7.5 & 12.0 & -. & 9.4 & 90 \\ 7.5 & 13.0 & .13 & 8.8 & 86 \\ 7.5 & 13.0 & -- & 8.8 & 86 \\ 7.8 & 13.0 & .33 & 9.1 & 99 \\ 7.8 & 12.5 & -- & 8.9 & 86 \\ 7.7 & 12.5 & .21 & 6.4 & 82 \\ 7.7 & 12.5 & -- & 8.3 & 81\end{array}$

$29 . . .1440$

juL , 1977

20... 1100

20... 1102

20... 1300

$20 \ldots \quad 1302$

$20 . . . \quad 1402$

$20 . . . \quad 1500$

20... $\quad 1502$

$20 . . .1600$

$20 \ldots 1602$

$20 \ldots .1702$

$20 \ldots 1900$

$20 \ldots \quad 1902$

$20 . . . \quad 2000$

$20 \ldots 2002$

$20 . . .2100$

$20 \ldots \quad 2102$

$20 . . . \quad 2200$

$20 . . . \quad 2300$

$20 . . .2302$

21... 0002

21... 0102

$21 \ldots .0102$

$21 \ldots .0200$

21... 0300

$21 . . .0302$

21... 0400

21... 0402

$21 . . .0502$

$21 . . .0600$

$21 . . \quad 0602$

$21 \ldots \quad 0700$

$21 \ldots 0702$

$21 \ldots 000800$

21... 0900

21... 0902

21... 1002

AUG

$10 . . .0700$

$10 . . .0702$

$10 \ldots \quad 1035$

$10 . . .1037$

$10 . . . \quad 1302$

$10 . . . \quad 1302$

$10 . . .1602$

$10 \ldots .1900$

$10 \ldots . \quad 1902$

$10 . . .2200$

11... 0100

11... 0102

$11 \ldots 00400$

11... $\quad 1000$

11... 1002

1.050

550
550

$\begin{array}{ll}5.5 & 550 \\ 1.0 & 425 \\ 5.5 & 460\end{array}$

$\begin{array}{ll}8.1 & 28.0 \\ 7.9 & 27\end{array}$

28.0
27.0

$\begin{array}{ll}B .1 & 27.0 \\ \text { B.1 } & 25.5\end{array}$

$\begin{array}{ll}\text { B.1 } & 25.5 \\ \text { B.1 } & 27.0\end{array}$

B.1 26.0

$8.0 \quad 27.5$

$7.8 \quad 28.0$

$\begin{array}{ll}8.1 & 28.0 \\ 7.8 & 28.0\end{array}$

8.128 .0

8.028 .0

8.128 .0

$\begin{array}{ll}8.1 & 28.5 \\ 7.9 & 28.0\end{array}$

$7.9 \quad 28.0$

$7.8 \quad 28.0$

7.928 .0

$7.9 \quad 28.0$

$7.8 \quad 28.0$

$7.7 \quad 27.0$

1.927.

$\begin{array}{ll}7.9 & 27.0 \\ 8.1 & 27.5\end{array}$

$8.0 \quad 27.0$

$\begin{array}{ll}8.2 & 27.5 \\ 8.2 & 27.0\end{array}$

8.027 .0

$8.0 \quad 27.0$

$7.7 \quad 27.0$

$7.6 \quad 27.0$

$7.7 \quad 26.0$

$7.6 \quad 25.0$

$7.6 \quad 25.5$

$7.6 \quad 27.0$

$7.5 \quad 27.0$

$\begin{array}{ll}7.5 & 27.0 \\ 7.7 & 27.5\end{array}$

$\begin{array}{ll}20 \ldots . . & 1310 \\ 20 . . & 1312\end{array}$

$\begin{array}{llllr}7.6 & 27.5 & -- & 4.2 & 54 \\ 7.6 & 27.0 & -- & 4.1 & 53 \\ 8.0 & 30.5 & -- & 6.1 & 82 \\ 7.9 & 30.0 & -- & 5.8 & 77 \\ 8.3 & 31.5 & -- & 6.4 & 88 \\ 8.2 & 31.0 & -- & 5.3 & 72 \\ 8.5 & 33.0 & -- & 8.2 & 114 \\ 8.2 & 31.5 & -- & 6.2 & 85 \\ 8.4 & 32.0 & -- & 7.4 & 101 \\ 8.2 & 32.0 & -- & 6.9 & 95 \\ 8.0 & 31.0 & - & 6.6 & 89 \\ 8.1 & 30.5 & - & 6.7 & 91 \\ 7.8 & 29.0 & -- & 5.1 & 67 \\ 7.8 & 29.0 & -- & 5.1 & 67 \\ 7.5 & 28.0 & -- & 3.9 & 50 \\ 7.6 & 28.0 & -- & 3.7 & 47 \\ 8.0 & 28.0 & -- & 5.3 & 69 \\ 8.0 & 28.0 & -- & 5.7 & 74 \\ 8.1 & 28.0 & -- & 7.7 & 100 \\ 7.7 & 26.0 & -- & 4.7 & 59\end{array}$


294759094432700 LINE 680 SITE 20

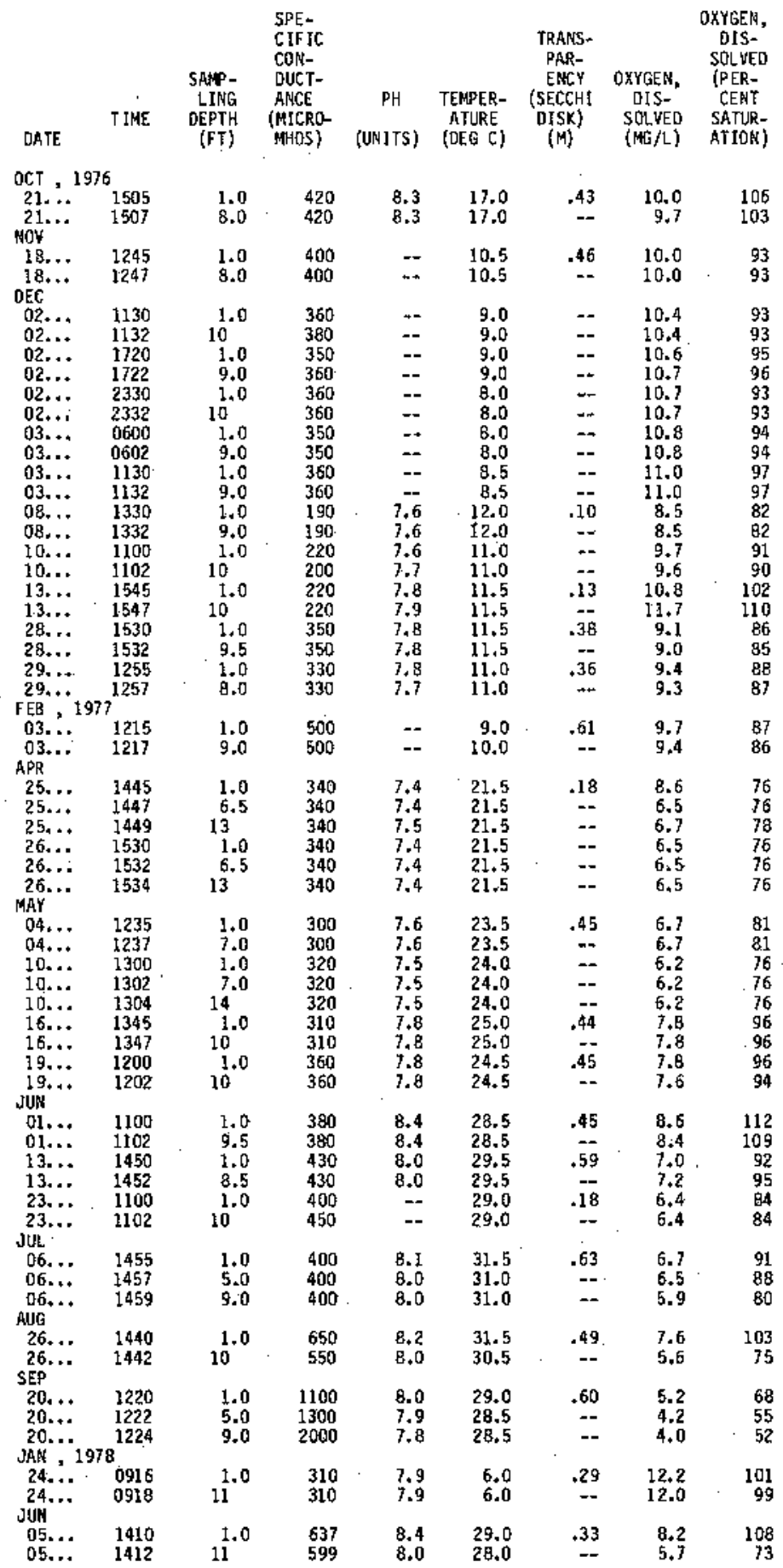


Table 2A.--Qual tty of water in the Trinity-San Jacinto estwary, water years 1977-78-Continued Field Deteminations--Continued

294809094434600 LINE 680 SITE 30

\begin{tabular}{|c|c|c|c|c|c|c|}
\hline DATE & TIME & $\begin{array}{c}\text { SAMP - } \\
\text { LIRG } \\
\text { DEPTH } \\
\text { (FT) }\end{array}$ & $\begin{array}{l}\text { \$PE- } \\
\text { CIFIC } \\
\text { CON- } \\
\text { DUCT- } \\
\text { ANACE } \\
\text { \{MICRO- } \\
\text { JHHOS) }\end{array}$ & $\begin{array}{l}\text { TEMPER- } \\
\text { ATURE } \\
\text { (DEG C) }\end{array}$ & $\begin{array}{c}\text { OXYGEN, } \\
\text { DIS- } \\
\text { SOLVED } \\
\text { (IHAL) }\end{array}$ & $\begin{array}{l}\text { OXYGEN, } \\
\text { DIS- } \\
\text { SOLYED } \\
\text { (PER- } \\
\text { CENT } \\
\text { SATUR- } \\
\text { ATIDN) }\end{array}$ \\
\hline \multicolumn{7}{|c|}{ DEC , 1976} \\
\hline $\begin{array}{l}02 \ldots . \\
02 \ldots \\
02 \ldots \\
02 \ldots \\
02 \ldots \\
02 \ldots \\
03 \ldots \\
03 \ldots \\
03 \ldots \\
03 \ldots\end{array}$ & $\begin{array}{l}1100 \\
1102 \\
1700 \\
1702 \\
2300 \\
2302 \\
0515 \\
0517 \\
1100 \\
1102\end{array}$ & $\begin{array}{c}1.0 \\
11 \\
1.0 \\
11 \\
1.0 \\
10 \\
1.0 \\
9.0 \\
1.0 \\
9.0\end{array}$ & $\begin{array}{l}350 \\
350 \\
360 \\
360 \\
360 \\
360 \\
350 \\
350 \\
360 \\
360\end{array}$ & $\begin{array}{l}9.0 \\
9.0 \\
9.0 \\
9.0 \\
8.0 \\
8.0 \\
8.0 \\
0.0 \\
8.5 \\
8.5\end{array}$ & $\begin{array}{l}10.4 \\
10.2 \\
10.7 \\
10.8 \\
10.7 \\
10.7 \\
10.7 \\
10.7 \\
10.9 \\
11.0\end{array}$ & $\begin{array}{l}93 \\
91 \\
96 \\
96 \\
93 \\
93 \\
93 \\
93 \\
96 \\
97\end{array}$ \\
\hline
\end{tabular}

294815094444200 LINE 680 SITE 40

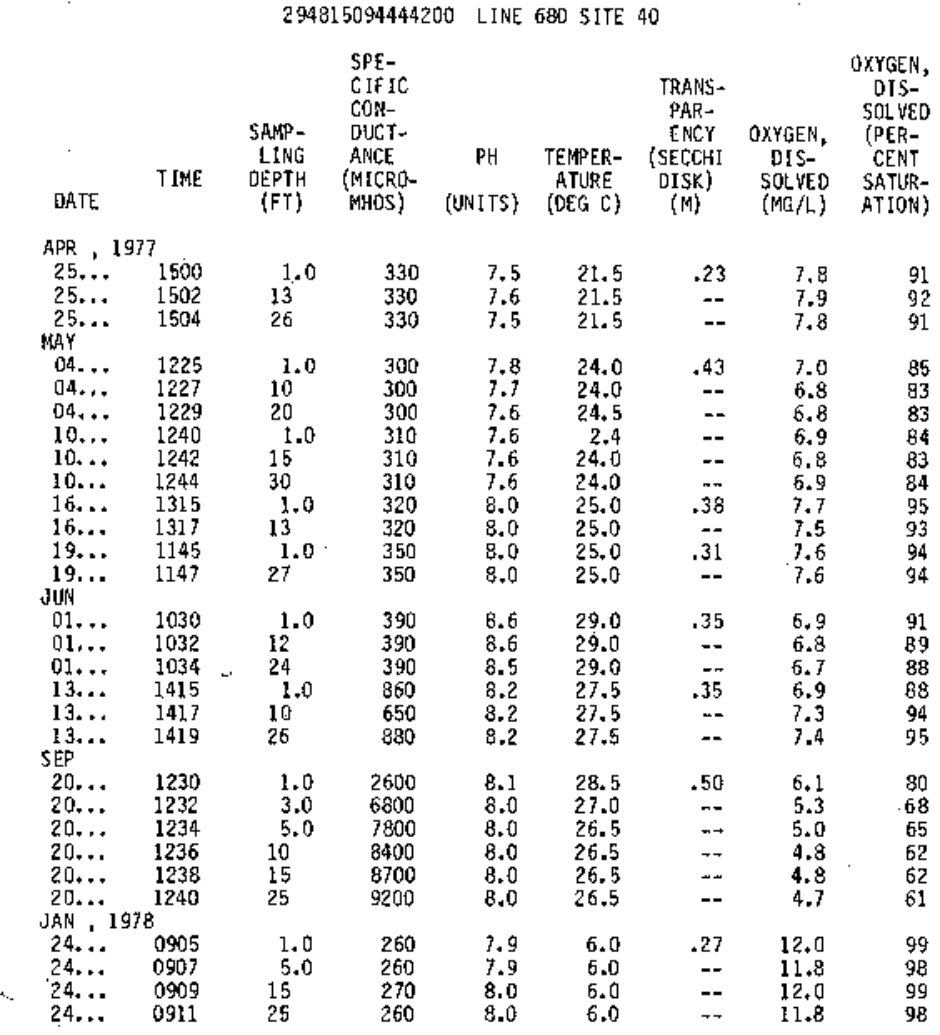

294813094471300 LINE 680 SITE, 80
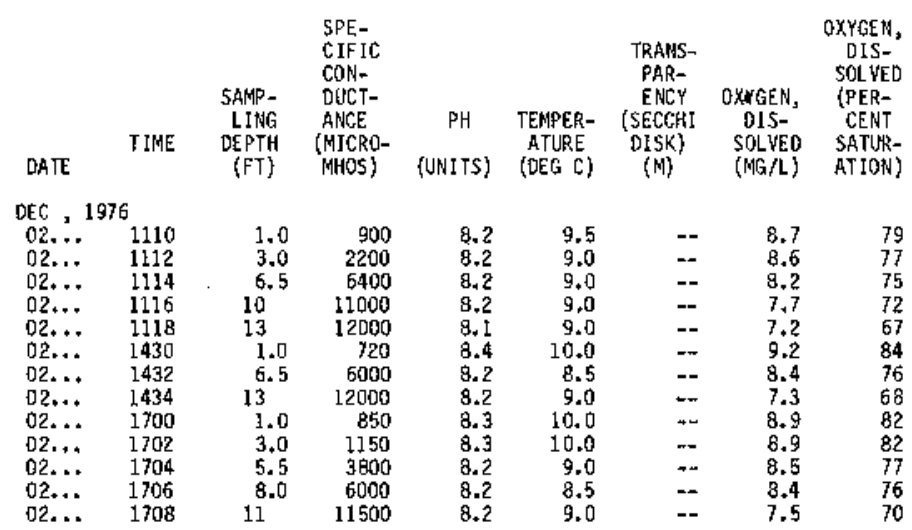

$\begin{array}{lrlll}8.2 & 9.5 & -- & 8.7 & 79 \\ 8.2 & 9.0 & -- & 8.6 & 77 \\ 8.2 & 9.0 & -- & 8.2 & 75 \\ 8.2 & 9.0 & -- & 7.7 & 72 \\ 8.1 & 9.0 & -- & 7.2 & 67 \\ 8.4 & 10.0 & -- & 9.2 & 84 \\ 8.2 & 8.5 & -- & 8.4 & 76 \\ 8.2 & 9.0 & - & 7.3 & 68 \\ 8.3 & 10.0 & - & 8.9 & 82 \\ 8.3 & 10.0 & -- & 8.9 & 82 \\ 8.2 & 9.0 & -- & 8.5 & 77 \\ 8.2 & 8.5 & -- & 3.4 & 76 \\ 8.2 & 9.0 & -- & 7.5 & 70\end{array}$


Table 2A.--Quality of water in the Trinity-San Jacinto estuary, water years 1977-78--Continued Field Deteminations-rContinued

294813094471300 LIME 680 S1TE 80 -Continued

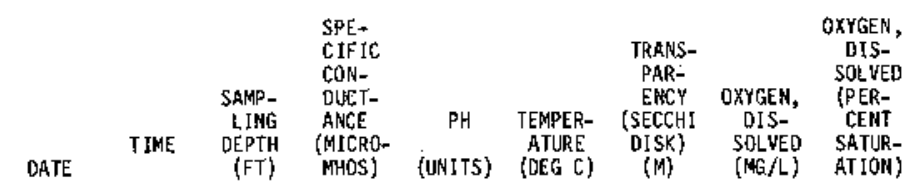

\begin{tabular}{|c|c|c|c|c|c|c|c|c|}
\hline \multicolumn{9}{|c|}{ DEC, $1976--$ Continued } \\
\hline $02 .$. & 2000 & 1.0 & 1100 & 8.2 & 10.0 & $m$ & 8.8 & 81 \\
\hline $02 \ldots$ & 2002 & 5.5 & 2900 & 8.2 & 9.0 & -- & 8.8 & 79 \\
\hline $02 \ldots$ & 2004 & 11. & 12000 & 8.0 & 8.5 & -- & 6.9 & 63 \\
\hline $02 \ldots$ & 2300 & 1.0 & 800 & 8.0 & 9.5 & -- & 8.4 & 76 \\
\hline $02 x+$ & 2302 & 5.5 & 1300 & 8.0 & 9.0 & -- & B. 2 & 74 \\
\hline $02 \ldots$ & 2304 & 11 & 12000 & 8.2 & 8.5 & -- & 7.2 & 6.6 \\
\hline $03 .$. & 0220 & 1.0 & 540 & 8.1 & 9.0 & -- & 8.9 & 79 \\
\hline $03 . .$. & 0222 & 5.0 & 2600 & 8.1 & 9.0 & $=$ & 6.7 & 78 \\
\hline $03 \ldots$ & 0224 & 11 & 11500 & 8.0 & 8.5 & $m$ & 7.0 & 64 \\
\hline $03 . .$. & 0500 & 1.0 & 720 & B.1 & 9.0 & - & 9.2 & 82 \\
\hline $03 . .$. & 0502 & 5.5 & 2600 & 8.1 & 9.5 & -- & 8.9 & 81 \\
\hline $03 \ldots$ & 0504 & 11 & $11500^{\circ}$ & 8.0 & 8.2 & -- & 7.3 & 66 \\
\hline $03 \ldots$ & $O B O Q$ & 1.0 & 900 & 8.0 & 9.0 & -- & 9.1 & 82 \\
\hline $03 \ldots$ & $\begin{array}{l}0802 \\
0804\end{array}$ & $\begin{array}{l}5.5 \\
8.0\end{array}$ & $\begin{array}{l}2900 \\
4300\end{array}$ & $\begin{array}{l}8.0 \\
8.0\end{array}$ & $\begin{array}{l}9.0 \\
9.0\end{array}$ & $=$ & 9.1 & 82 \\
\hline 0 & 0806 & $11^{0.10}$ & 11500 & 8.0 & 8.5 & - & $\begin{array}{l}9.3 \\
7.2\end{array}$ & $\begin{array}{l}84 \\
66\end{array}$ \\
\hline $03 .$. & 1000 & 1.0 & 650 & 7.9 & 9.0 & 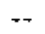 & 8.2 & 73 \\
\hline $03 . .$. & 1002 & 5.5 & 3000 & 8.1 & 9.5 & - & 8.7 & 80 \\
\hline $03 . .$. & 1004 & 11 & 11000 & 8.0 & 9.0 & -- & 7.0 & 65 \\
\hline पB... & 1515 & 1.0 & 320 & 7.9 & 11,5 & .15 & 10.7 & 101 \\
\hline $08 . .$. & 1517 & 11 & 400 & 7.5 & 11.5 & -- & 9.7 & 92 \\
\hline $10 \ldots$ & 1030 & 1.0 & 200 & 7.7 & 12.0 & $=$ & 9.9 & 95 \\
\hline $10 \ldots$ & 1032 & 12 & 200 & 7.7 & 11.5 & 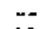 & 9.8 & 92 \\
\hline $13 .$. & 1515 & 1.0 & 140 & 7.5 & 13.0 & 13 & 9.9 & 97 \\
\hline $13 . \ldots$ & 1517 & 11 & 140 & 7.5 & 12.5 & מ & 9.9 & 96 \\
\hline $28 . \ldots$ & 1300 & 1.0 & 330 & 8.0 & 12.5 & .33 & 10.2 & 99 \\
\hline $28 .$. & 1302 & 11 & 330 & 8.0 & 12.5 & -- & 10.0 & 97 \\
\hline $29 .$. & 1550 & 1.0 & 360 & 8.1 & 12.5 & .19 & 9.2 & 89 \\
\hline $29 \ldots$ & 1552 & 10 & 360 & B.1 & 12.5 & $=$ & 9.0 & 87 \\
\hline \multicolumn{9}{|c|}{ APR , 1977} \\
\hline $25 .$. & 1520 & 1.0 & 330 & 7.5 & 21.5 & .24 & 7.6 & 88 \\
\hline $25 . \ldots$ & 1522 & 8.0 & 330 & 7.5 & 21.5 & -- & 7,6 & 88 \\
\hline $25 \ldots$ & 1524 & 16 & 330 & 7.5 & 22.0 & -- & 7.6 & 89 \\
\hline \multicolumn{9}{|l|}{ MAY } \\
\hline $04 \ldots$ & 1210 & 1.0 & 300 & 7.5 & 25.0 & .34 & 5.4 & 67 \\
\hline $04 \ldots$ & 1212 & 7.0 & 300 & 7.6 & 24.5 & $-*$ & 5.6 & 68 \\
\hline $04 \ldots$ & 1214 & 15 & 300 & 7.5 & 25.0 & -. & 5.4 & 67 \\
\hline $10 \ldots$ & 1230 & 1.0 & 280 & 7.4 & 24.0 & $=$ & 5.1 & 62 \\
\hline $10 \ldots$ & 1232 & 7.0 & 280 & 7.3 & 24.0 & -- & 5.0 & 61 \\
\hline $10 . \ldots$ & 1234 & 13 & 280 & 7.3 & 24.0 & - & 5.0 & 61 \\
\hline $16 .$. & 1300 & 1.0 & 310 & 8.2 & 25.5 & .33 & 7.7 & 96 \\
\hline $16 \ldots$ & 1302 & 13 & 310 & 8.0 & 25.5 & $m$ & 7.1 & 89 \\
\hline $19 .$. & 1130 & 1.0 & 360 & 8.1 & 25.5 & .24 & 7.1 & 89 \\
\hline $19 . .$. & 1132 & 15 & 370 & 8.1 & 25.0 & -- & 6.8 & 84 \\
\hline JUK & & & & & & & & \\
\hline $01, \ldots$ & 1000 & 1.0 & 390 & 8.3 & 28.5 & .30 & 5.9 & 76 \\
\hline $01 \ldots$ & .1002 & 13 & 390 & 8.3 & 28.0 & $=-$ & 6.0 & 77 \\
\hline $13 \ldots$ & 1200 & 1.0 & 1400 & 8.1 & 28.0 & .28 & 6.5 & 84 \\
\hline $13 \ldots$ & 1202 & 13 & 3200 & 7.9 & 28.0 & mer & 5.7 & 74 \\
\hline $\begin{array}{l}\text { JUL } \\
06 . . .\end{array}$ & & & & 8.6 & 32.5 & & 7.9 & \\
\hline $\begin{array}{l}06 . . . \\
06 . .\end{array}$ & $\begin{array}{l}1515 \\
1517\end{array}$ & 3.0 & 670 & 8.5 & 32.0 & $\because 4$ & 7.5 & 103 \\
\hline $06 .$. & 1519 & 5.0 & 1500 & 7.8 & 30.5 & -- & 3.9 & 53 \\
\hline $06 \ldots$ & 1521 & 11 & 2200 & 7.6 & 30.0 & -- & 2.5 & 33 \\
\hline $20 \ldots$ & 1015 & 1.0 & 850 & 8.4 & 29.0 & -- & 5.3 & 70 \\
\hline $20 .$. & 1017 & 11 & 1000 & 8.4 & 29.0 & $m$ & 4.8 & 63 \\
\hline $20 . \ldots$ & 1315 & 1.0 & 700 & 8.4 & 28.5 & $m$ & 6.2 & 81 \\
\hline $20 \ldots$ & 1317 & 12 & 700 & 8.4 & 28.5 & $\mu$ & 6.1 & 79 \\
\hline $20 \ldots$ & 1615 & 1.0 & 700 & 8.3 & 29.0 & -- & 7.0 & 92 \\
\hline $20 .$. & 1617 & 11 & 700 & 8.2 & 28.5 & -- & 6.5 & 84 \\
\hline $20+\ldots$ & 1915 & 1.0 & 900 & 8.3 & 28.5 & -- & 6.5 & 84 \\
\hline $20 \ldots$ & 1917 & 11 & 1700 & 8.2 & 28.5 & $-r$ & 5.2 & 68 \\
\hline $20 \ldots$ & 2205 & 1.0 & 1300 & 8.3 & 28.0 & $\rightarrow$ & 6.0 & 79 \\
\hline $20 . .$. & 2207 & 11 & 1300 & 8.3 & 28.5 & - & 5.7 & 74 \\
\hline & 0115 & 1.0 & 2200 & 8.5 & 28.0 & -- & 5.7 & 86 \\
\hline $21 . .$. & 0117 & 11 & 2200 & 8.5 & 28.0 & -- & 6.7 & 86 \\
\hline & 0415 & 1.0 & 2000 & 8.4 & 28.0 & -- & 6.0 & 77 \\
\hline 21. & 0417 & 11 & 2200 & 8.4 & 28.0 & $\ldots$ & 6.1 & 77 \\
\hline 21. & 0715 & 1.0 & 1700 & 8.3 & 27.0 & $\cdots$ & 9.6 & 71 \\
\hline $21 \ldots$ & 0717 & 11 & 1800 & 8.3 & 27.0 & -- & 5.5 & 70 \\
\hline $21 . \ldots$ & 1000 & 1.0 & 1300 & 8.2 & 27.0 & -- & 5,9 & 75 \\
\hline & 1002 & 12 & 2200 & 8.2 & 28.0 & -- & 4.5 & 58. \\
\hline AUG & & & & & & & & \\
\hline $10 .$. & 1000 & 1,0 & 3900 & 8.3 & 31.0 & +- & 4.6 & 63 \\
\hline $10 \ldots$ & 1002 & 6.0 & 5300 & 8.3 & 31.0 & -- & 3.9 & 53 \\
\hline $10 .$. & 1004 & 13 & 6000 & 7.7 & 30.0 & -- & 4.0 & 54 \\
\hline $10 \ldots$ & 1320 & 1.0 & 3800 & 8.5 & 31.5 & -- & 5.1 & 70 \\
\hline 10. & 1322 & 7.0 & 5800 & 8.2 & 30.5 & -- & 3.1 & 42 \\
\hline $10 .$. & 1324 & 14 & 6000 & 7.9 & 30.0 & -- & 2.5 & 34 \\
\hline $10 . \ldots$ & 1600 & 1.0 & 3500 & 8.6 & 33.5 & -- & 6.7 & 94 \\
\hline 10. & 1602 & 6.0 & 5700 & 8.1 & 31.0 & -- & 4.5 & 62 \\
\hline 10. & 1604 & 13 & 6000 & 8.1 & 31.0 & -- & 6.1 & 84 \\
\hline 10. & 1900 & 1.0 & 4400 & 8.5 & 32.0 & -- & 5.4 & 75 \\
\hline & 1902 & 7.0 & 5500 & 8.1 & 31.0 & -- & 3.7 & 51 \\
\hline & 1904 & 15 & 6000 & 8.1 & 30.5 & - & 4.2 & 58 \\
\hline
\end{tabular}


Table 2h--Quality of water in the Trinity-San Jacinto estuary, water years 1977-78--Continued Field Determinations=-Cont inued

\begin{tabular}{|c|c|c|c|c|c|c|c|c|}
\hline DATE & TIME & $\begin{array}{c}\text { SAMP - } \\
\text { LING } \\
\text { DEPTH } \\
\text { (FT) }\end{array}$ & $\begin{array}{l}\text { SPE- } \\
\text { CIFIC } \\
\text { CON- } \\
\text { DULT- } \\
\text { ANCE- } \\
\text { (MICRO- } \\
\text { MHOS) }\end{array}$ & $\begin{array}{c}\text { PH } \\
\text { (UNITS) }\end{array}$ & $\begin{array}{l}\text { TEMPER- } \\
\text { MTURE } \\
\text { (DEG C) }\end{array}$ & $\begin{array}{l}\text { TRARAS- } \\
\text { PAR- } \\
\text { EKCY } \\
\text { (SECCHI } \\
\text { DISK) } \\
\text { ( } 1 \text { (A) }\end{array}$ & $\begin{array}{c}\text { OXYGEN, } \\
\text { DIS- } \\
\text { SOLVED } \\
\text { \{KG } / L\}\end{array}$ & $\begin{array}{l}\text { OXYGEN, } \\
\text { DIS- } \\
\text { SOLVED } \\
\text { (PER- } \\
\text { CENT } \\
\text { SATUR- } \\
\text { ATION) }\end{array}$ \\
\hline \multicolumn{9}{|c|}{ AUG , 1977-Continued } \\
\hline $10,$. & 2200 & 1,0 & 4500 & 8.4 & 30.5 & $=-$ & 4.5 & 61 \\
\hline $10 \ldots$ & 2202 & 7.0 & 5400 & 8.3 & 30. & $-r$ & 3.4 & 47 \\
\hline $10 . .$. & 2204 & 14 & 6100 & 8.0 & 29. & - & 2.6 & 35 \\
\hline $11 \ldots$ & 0100 & 1.0 & 4000 & 8.4 & 30. & -- & 4.2 & 56 \\
\hline $11 \ldots$ & 0102 & 7.0 & 5500 & 8,2 & 30. & $\cdots$ & & 39 \\
\hline 11. & 0104 & 14 & 6000 & ?. & 29 & 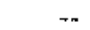 & 2. & 30 \\
\hline 11. . & 0400 & 1.0 & 4600 & 8.3 & 29. & $m$ & 4.0 & 53 \\
\hline 11 . & $040 ?$ & 7.0 & 5500 & 8.2 & 30 & $\sim$ & & 35 \\
\hline 11.. & 0404 & 14 & 6000 & 7.9 & 29 & -- & 1. & 20 \\
\hline $11 .$, & 0700 & 1.0 & 3800 & 8.2 & 29 & -- & 3.9 & 51 \\
\hline $11 .$. & 0702 & 7.0 & 55 & 7. & 29. & -- & & 30 \\
\hline $11 \ldots$ & 0704 & 14 & 6000 & 7.8 & 29. & $-\infty$ & & 28 \\
\hline $11 \ldots$ & 1090 & 1.0 & 3800 & 8.2 & & - & & \\
\hline . & 1002 & 7.0 & 5400 & 8.1 & & -- & 2 & 34 \\
\hline $11 \ldots$ & 1004 & 15 & 6000 & 7.8 & 29.5 & - & 2.3 & 31 \\
\hline \multicolumn{9}{|c|}{5300} \\
\hline & 1252 & 3.0 & 7300 & 7.8 & & $\begin{array}{r}.48 \\
-7\end{array}$ & 4.3 & $\begin{array}{l}94 \\
56\end{array}$ \\
\hline 2 & 1254 & 5.0 & 8200 & 7. & & - & 4.0 & 53 \\
\hline $20 . .$. & 1256 & 11 & 9800 & 7.8 & 27.0 & $m$ & & 45 \\
\hline
\end{tabular}

294605094412400 LINE 690 SITE 20

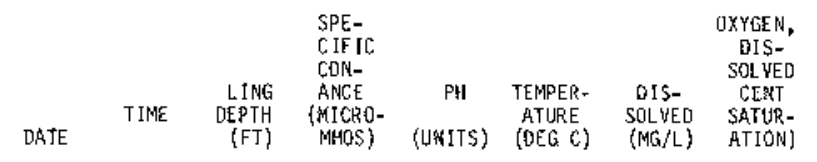

JUN, 1977

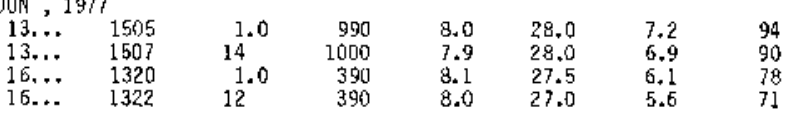

294712094440200 LINE 690 SITE 40

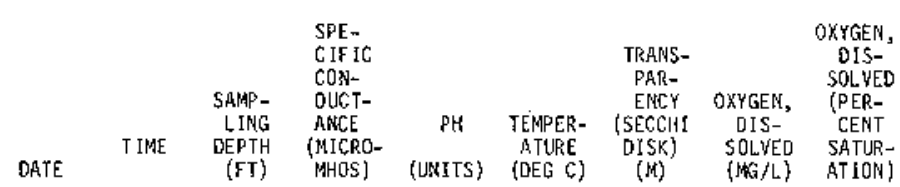

DEC , 1976

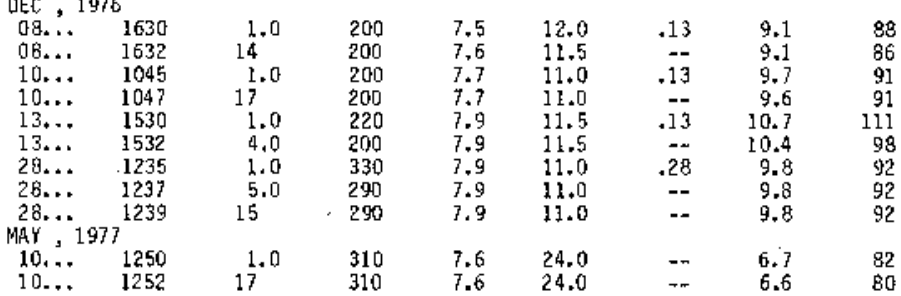

294629094465400 LINE 690 SITE 80

\begin{tabular}{|c|c|c|c|c|c|c|c|}
\hline DATE & T IME & $\begin{array}{l}\text { SAMP - } \\
\text { LING } \\
\text { DEPTH } \\
\text { (FT) }\end{array}$ & $\begin{array}{l}\text { SPE- } \\
\text { CIFYC } \\
\text { CON- } \\
\text { DUCT- } \\
\text { ANCE } \\
\text { (MICRO- } \\
\text { MHOS) }\end{array}$ & $\begin{array}{c}\mathrm{PH} \\
\text { (UN1TS) }\end{array}$ & $\begin{array}{l}\text { TEMPER- } \\
\text { ATURE } \\
\text { (DEG C) }\end{array}$ & $\begin{array}{c}\text { OXYGEN, } \\
\text { OIS- } \\
\text { SOLWED } \\
\text { (MG/L) }\end{array}$ & $\begin{array}{c}\text { OXYGE } \\
\text { OIS- } \\
\text { SOLYED } \\
\text { (PER- } \\
\text { CENT } \\
\text { SATUR- } \\
\text { ATION) }\end{array}$ \\
\hline
\end{tabular}

DEC , 1976

$\begin{array}{llllllll}08 . . . & 1545 & 1.0 & 400 & 7.7 & 10.0 & 10.2 & 94 \\ 08 . . . & 1547 & 7.0 & 400 & 7.7 & 10.5 & 10.2 & 94\end{array}$ 
Table 2A,--Qual ity of water in the Trinity-SaA Jacinto est uary, water years 1971-79--Continued Field Deteminations--Cantinued

291929094393300 LINE 904 SITE 20

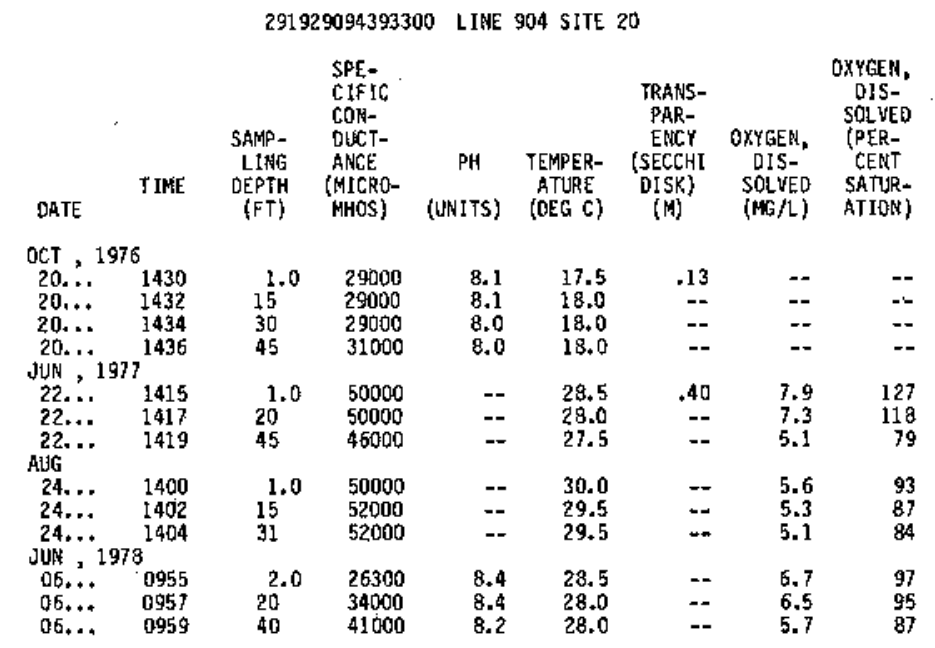


(FT = feet; $M G / L=$ milligrams per 11 ter $)$

294255095011300 LINE I8O SITE 20

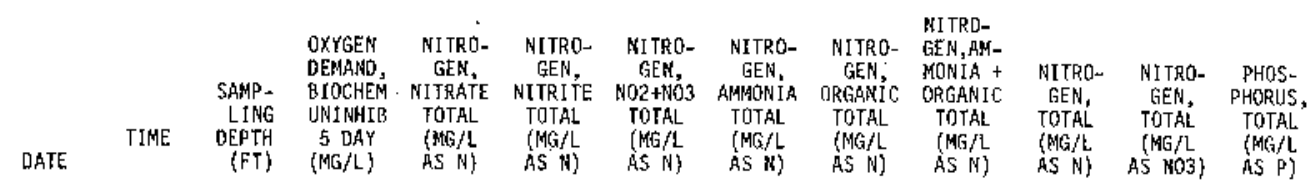

OCT , 1976

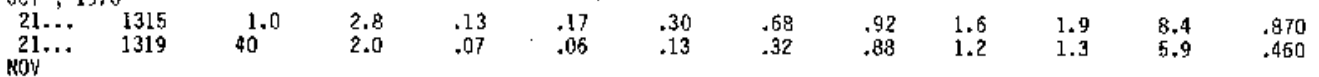

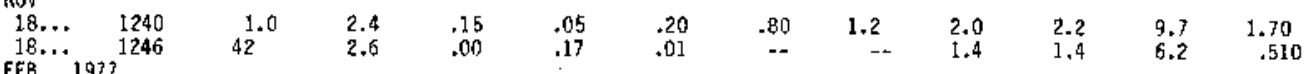

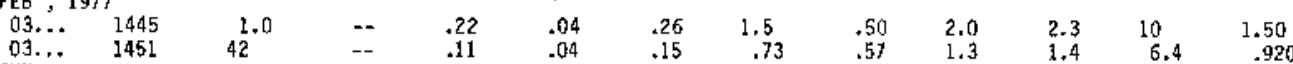

$23, \ldots \quad 1315$

$23 \ldots .1321$

25... 1230

$25 \ldots . .1230$

FEB , $1979^{1236}$

$\begin{array}{ll}0 . . . & 1150 \\ 09 . . & 1154\end{array}$

JUN

$\begin{array}{ccc}1.0 & 2.0 \\ 45 & 1.9 & .09\end{array}$

$.09 \quad .15$

$\begin{array}{ll}1.0 & 2.8 \\ 41 & 2.0\end{array}$

$.22 \quad .98$

$\begin{array}{ll}.25 & .38 \\ .23 & .38\end{array}$

$\begin{array}{lll}. ? & .45 \\ .46 & .22\end{array}$

$\begin{array}{lll}1.0 & 2.0 & .32 \\ 42 & 1.6 & .26\end{array}$

$\begin{array}{ll}.32 & .06 \\ .26 & .06\end{array}$

$\begin{array}{cc}.38 & 1.1 \\ .32 & .77\end{array}$

$07 \ldots . \quad 1136$

$\begin{array}{ll}1.0 & 7 \\ 45 & 7\end{array}$

.11
$.20 \quad 1.0$

$\begin{array}{lll}1.0 & 1.6 & 1.4 \\ 1.2 & 1.1 & 1.2\end{array}$

294330094421700 LINE 220 SITE 20

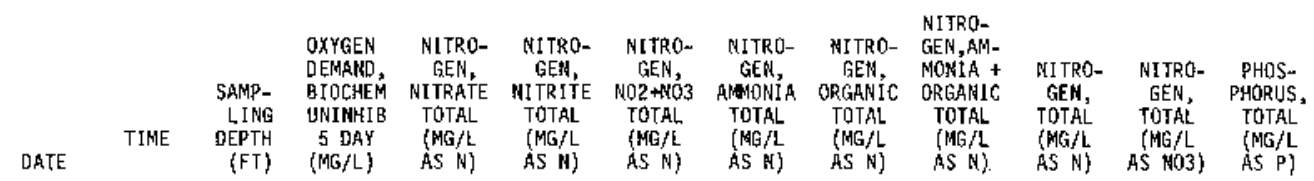

DEC , 1976

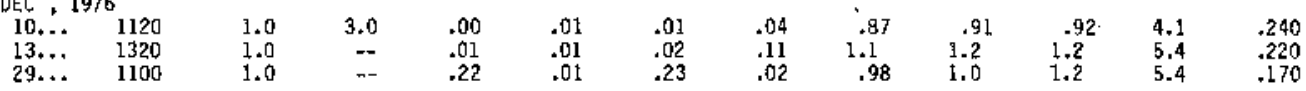

294349094424800 LINE 220 SITE 30

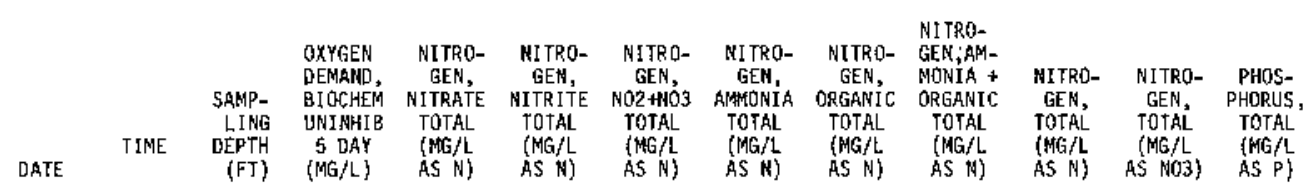

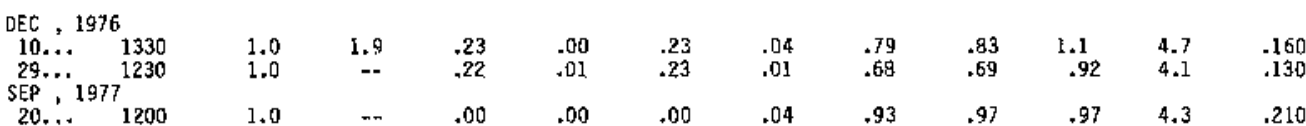

294416094433300 LINE 220 SITE 40

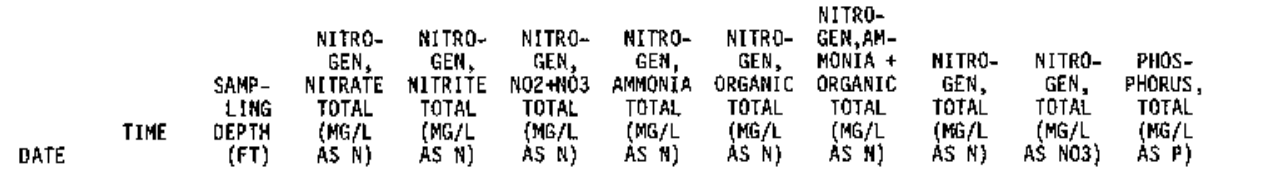

OEC, 1976

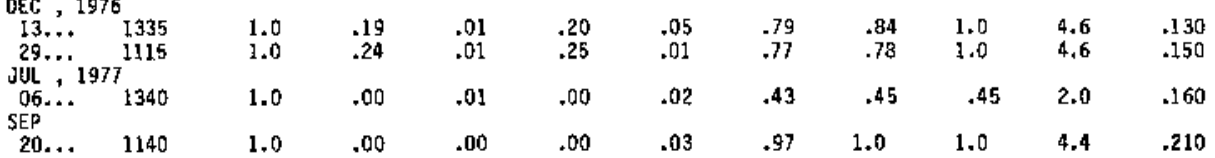


294443094441700 L1NE 220 S1TE 50

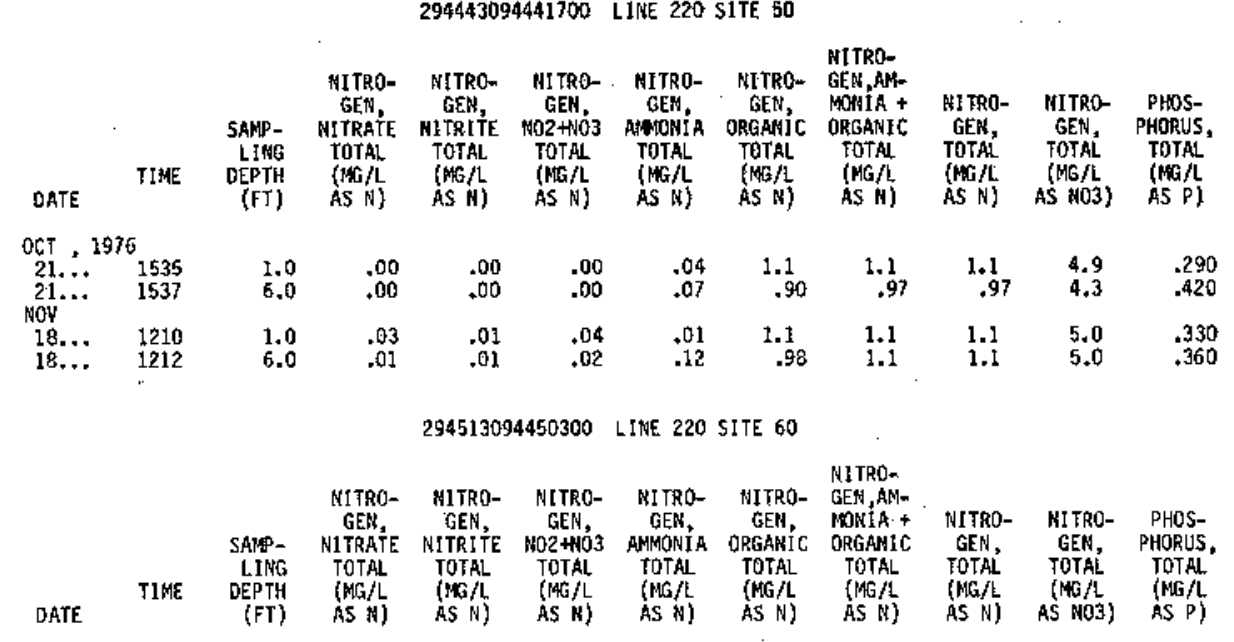

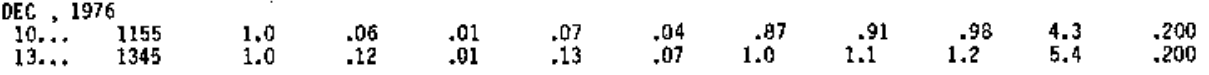

294322094430700 LINE 222 SITE 40

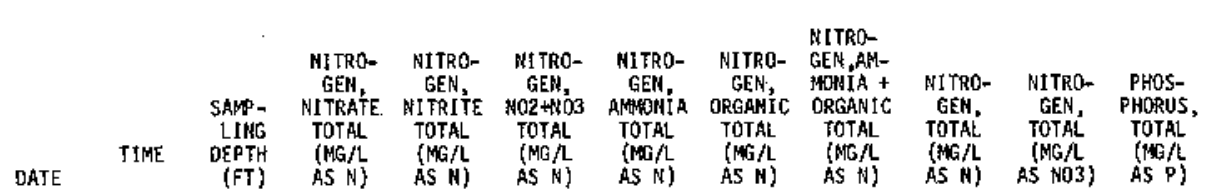

DEC , 1976

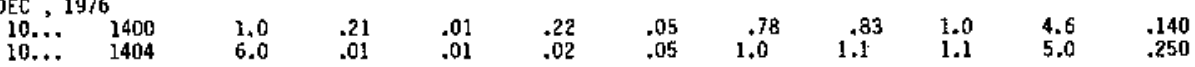

294149094422400 LINE 225 SITE 20

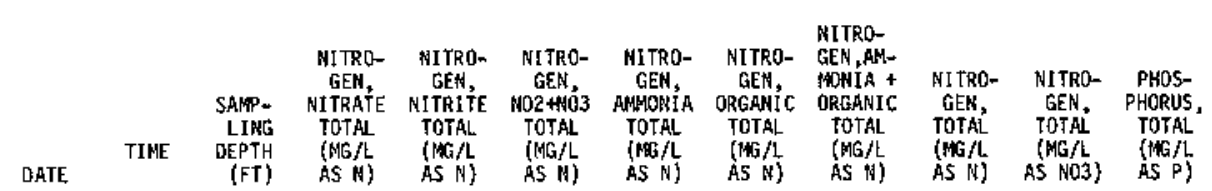

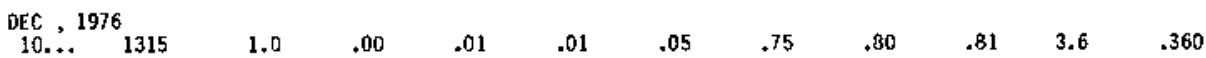

294232094434400 LINE 225 SITE 40

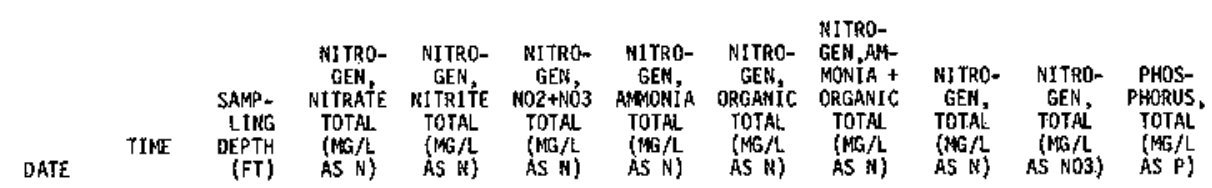

$\begin{array}{lllllllllllll}\text { OEC }, & 1976 \\ 10 .,{ }^{1050} & 1.0 & .00 & .01 & .01 & .02 & .87 & .89 & .90 & 4.0 & .330\end{array}$

294122094424400 LINE 230 SITE 20

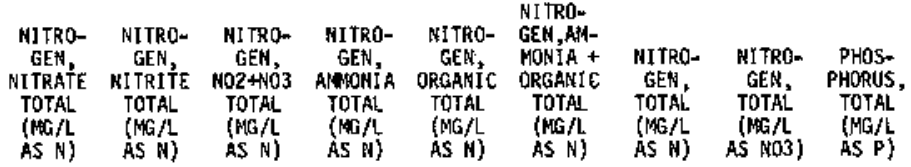

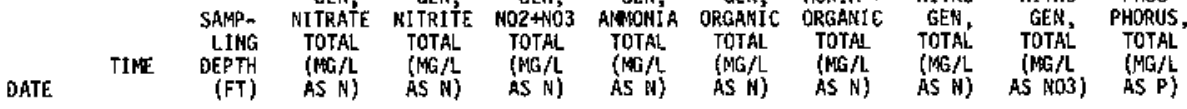

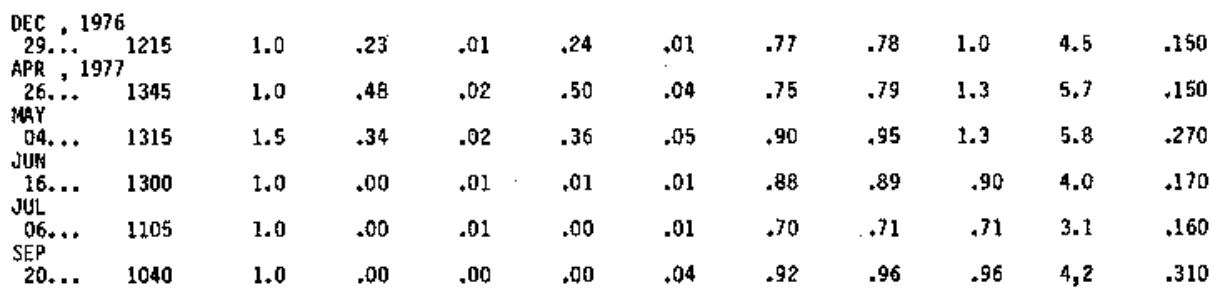


294239094450700 LINE 230 SITË 50

\begin{tabular}{|c|c|c|c|c|c|c|c|c|c|c|c|}
\hline DATE & TIME & $\begin{array}{c}\text { SAMP- } \\
\text { LING } \\
\text { DEPTH } \\
\text { (FT) }\end{array}$ & $\begin{array}{l}\text { "ITRO- } \\
\text { GEN, } \\
\text { NITRATE } \\
\text { TOTAL } \\
\text { (MA } / L \\
\text { RS N) }\end{array}$ & $\begin{array}{l}\text { NITRO- } \\
\text { GEN, } \\
\text { NIYRITE } \\
\text { TDTAL } \\
\text { (MG/L } \\
\text { AS N }\end{array}$ & $\begin{array}{l}\text { NITRO- } \\
\text { GEN, } \\
\text { NO2+NO3 } \\
\text { TOTAL } \\
(M G / 4 \\
\text { ASN) }\end{array}$ & $\begin{array}{l}\text { NITRO- } \\
\text { GEN, } \\
\text { AMMONIA } \\
\text { TOTAL } \\
\text { (MKA } / \text { L } \\
\text { AS N) }\end{array}$ & $\begin{array}{l}\text { NITRO- } \\
\text { GEN, } \\
\text { ORGANIC } \\
\text { TOTAL } \\
\text { (MG/L } \\
\text { AS N) }\end{array}$ & $\begin{array}{l}\text { NITRO- } \\
\text { GEN,AM- } \\
\text { MONIA + } \\
\text { ORGAAIC } \\
\text { TOTAL } \\
\text { (NG/L } \\
\text { AS N }\end{array}$ & $\begin{array}{l}\text { NITRO- } \\
\text { GEN, } \\
\text { TUTAL } \\
\text { (MG/L } \\
\text { AS N) }\end{array}$ & $\begin{array}{c}\text { NITRRO- } \\
\text { GEN, } \\
\text { TOTAL } \\
\text { (MG/L } \\
\text { AS N03) }\end{array}$ & $\begin{array}{c}\text { PHOS- } \\
\text { PHORIUS, } \\
\text { TOTAL } \\
\text { (MG/L } \\
\text { AS P) }\end{array}$ \\
\hline \multicolumn{12}{|c|}{ FEB , 1977} \\
\hline $\begin{array}{l}03, \ldots \\
03 \ldots \\
\text { JUN }\end{array}$ & $\begin{array}{l}1245 \\
1247\end{array}$ & $\begin{array}{l}1.0 \\
5.0\end{array}$ & .01 & $\begin{array}{l}.00 \\
.00\end{array}$ & .01 & $\begin{array}{l}.10 \\
.08\end{array}$ & $\begin{array}{l}.73 \\
.36\end{array}$ & $\begin{array}{l}.83 \\
.44\end{array}$ & $\begin{array}{l}.84 \\
.45\end{array}$ & $\begin{array}{l}3.7 \\
2.0\end{array}$ & $\begin{array}{l}.510 \\
.460\end{array}$ \\
\hline $\begin{array}{l}23 . \cdots \\
23 . \ldots \\
\text { AUG }\end{array}$ & $\begin{array}{l}1130 \\
1132\end{array}$ & $\begin{array}{l}1.0 \\
9.0\end{array}$ & .00 & .02 & .01 & $\begin{array}{l}.04 \\
.08\end{array}$ & $\begin{array}{l}.69 \\
.85\end{array}$ & $\begin{array}{l}.73 \\
.93\end{array}$ & $\begin{array}{r}.74 \\
.94\end{array}$ & $\begin{array}{l}3.3 \\
4.2\end{array}$ & $\begin{array}{l}.190 \\
.230\end{array}$ \\
\hline $\begin{array}{r}26 \ldots \\
26 \ldots \\
\text { FEB }\end{array}$ & $\begin{array}{l}1400 \\
1402 \\
8\end{array}$ & $\begin{array}{l}1.0 \\
8.0\end{array}$ & .00 & $\begin{array}{l}.01 \\
.01\end{array}$ & .01 & .04 & $\begin{array}{l}.61 \\
.78\end{array}$ & $\begin{array}{l}.65 \\
.82\end{array}$ & $\begin{array}{l}.66 \\
.83\end{array}$ & $\begin{array}{l}2.9 \\
3.7\end{array}$ & $\begin{array}{r}.310 \\
.320\end{array}$ \\
\hline $\begin{array}{l}08 . . . \\
\text { 08... } \\
\text { JUN }\end{array}$ & $\begin{array}{l}1420 \\
1422\end{array}$ & $\begin{array}{l}1.0 \\
6.0\end{array}$ & $\begin{array}{l}.05 \\
.27\end{array}$ & $\begin{array}{r}.04 \\
.06\end{array}$ & $\begin{array}{l}.09 \\
.33\end{array}$ & $\begin{array}{r}.23 \\
.23\end{array}$ & $\begin{array}{l}.72 \\
.71\end{array}$ & $\begin{array}{r}.95 \\
.94\end{array}$ & $\begin{array}{l}1.0 \\
1.3\end{array}$ & $\begin{array}{l}4.6 \\
5.6\end{array}$ & $\begin{array}{r}.210 \\
.300\end{array}$ \\
\hline $\begin{array}{l}05 . . \\
05, \ldots\end{array}$ & $\begin{array}{l}1334 \\
1336\end{array}$ & $\begin{array}{l}1.0 \\
9.0\end{array}$ & $\begin{array}{l}.00 \\
.00\end{array}$ & .01 & .01 & $\begin{array}{l}.01 \\
.05\end{array}$ & $\begin{array}{l}.53 \\
.61\end{array}$ & $\begin{array}{l}.54 \\
.66\end{array}$ & $\begin{array}{l}.55 \\
.67\end{array}$ & $\begin{array}{l}2.4 \\
3.0\end{array}$ & $\begin{array}{r}.120 \\
.190\end{array}$ \\
\hline
\end{tabular}

294313094460800 LINE 230 SITE 60

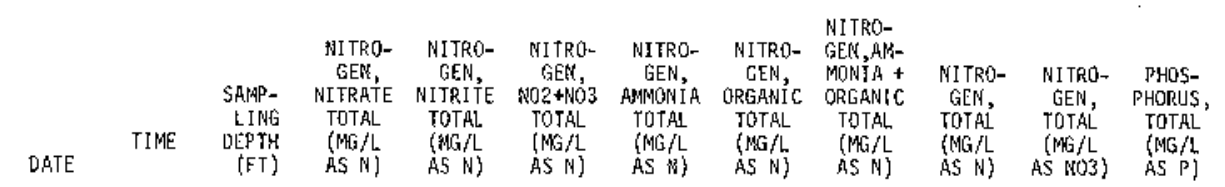

DEC , 1976

$10 . \therefore 1230$

$10 . . . \quad 1232$

29... 1200

29... 1202

26... 1410

$26 . . .1414$

1.0

$\begin{array}{llllllllll}1.0 & .00 & .00 & .00 & .04 & .70 & .74 & .74 & 3.3 & .350 \\ 7.5 & .00 & .01 & .01 & .03 & .97 & 1.0 & 1.0 & 4.5 & .360 \\ 1.0 & .22 & .01 & .23 & .01 & .87 & .88 & 1.1 & 4.9 & .190 \\ 6.0 & .21 & .01 & .22 & .01 & 1.2 & 1.2 & 1.4 & 6.3 & .250\end{array}$

MAY 1414

$1.0-45$

$.45 \quad .01$

04... $\quad 1330$

04... 133?

$10 \ldots \quad 1345$

$\begin{array}{ll}10 \ldots . & 1347 \\ 17 \ldots & 0845\end{array}$

17... 0847

JUN $16, \ldots, 1230$

16...

06... 1120

06... 1124

20... 1100

8,0

$.01 \quad .46$

1.5
8.0

.39
.42

$\begin{array}{ll}39 & .02 \\ 42 & .00\end{array}$

$1.0 \quad .36$

8.0

1.0
9.0

.04
.06

.02
.00
.00

.00

$\begin{array}{llccccc}.46 & .02 & .71 & .73 & 1.2 & 5.3 & .130 \\ .47 & .07 & 1.1 & 1.2 & 1.7 & 7.4 & .170\end{array}$

$1.0 \quad .02$

.06

$\begin{array}{ll}.41 & .04 \\ .42 & .04 \\ .36 & .04 \\ .34 & .04 \\ .04 & .0 \\ .06 & .12\end{array}$

1.0 $\quad-$

.01

.03

.04
.04
.04
.04
.06
.12
.05

.78
.25
.29
.57
1.5
1.3

$\begin{array}{llll}.82 & 1.2 & 5.4 \quad .120\end{array}$

$-\overline{0} \quad .01$

$.01 \quad .02$

$.00: .01$

294420094480900 LINE 230 SITE 80

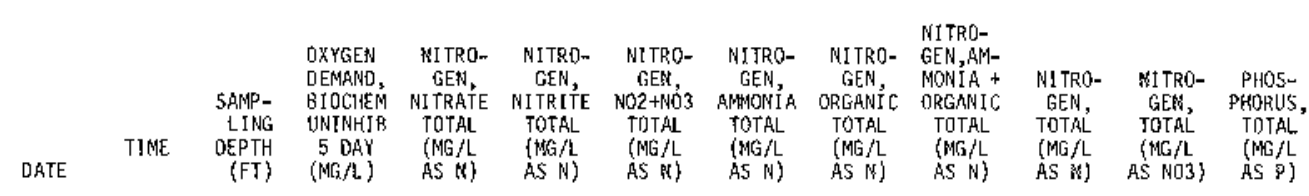

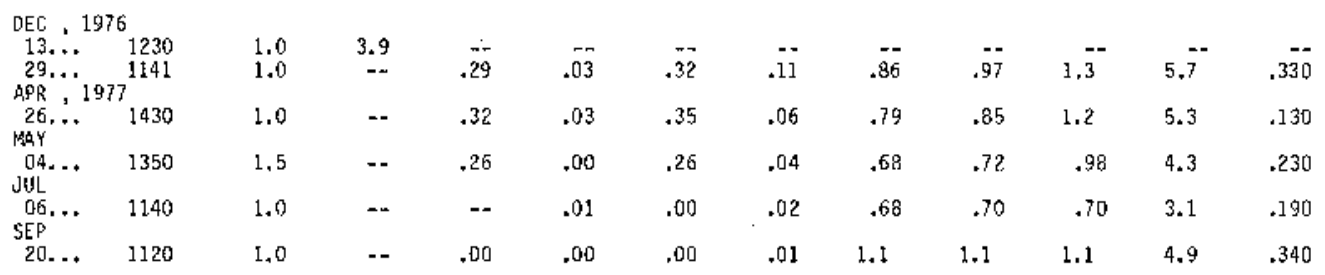


$293810094441100 \quad$ L, 1HE 240 SITE 30

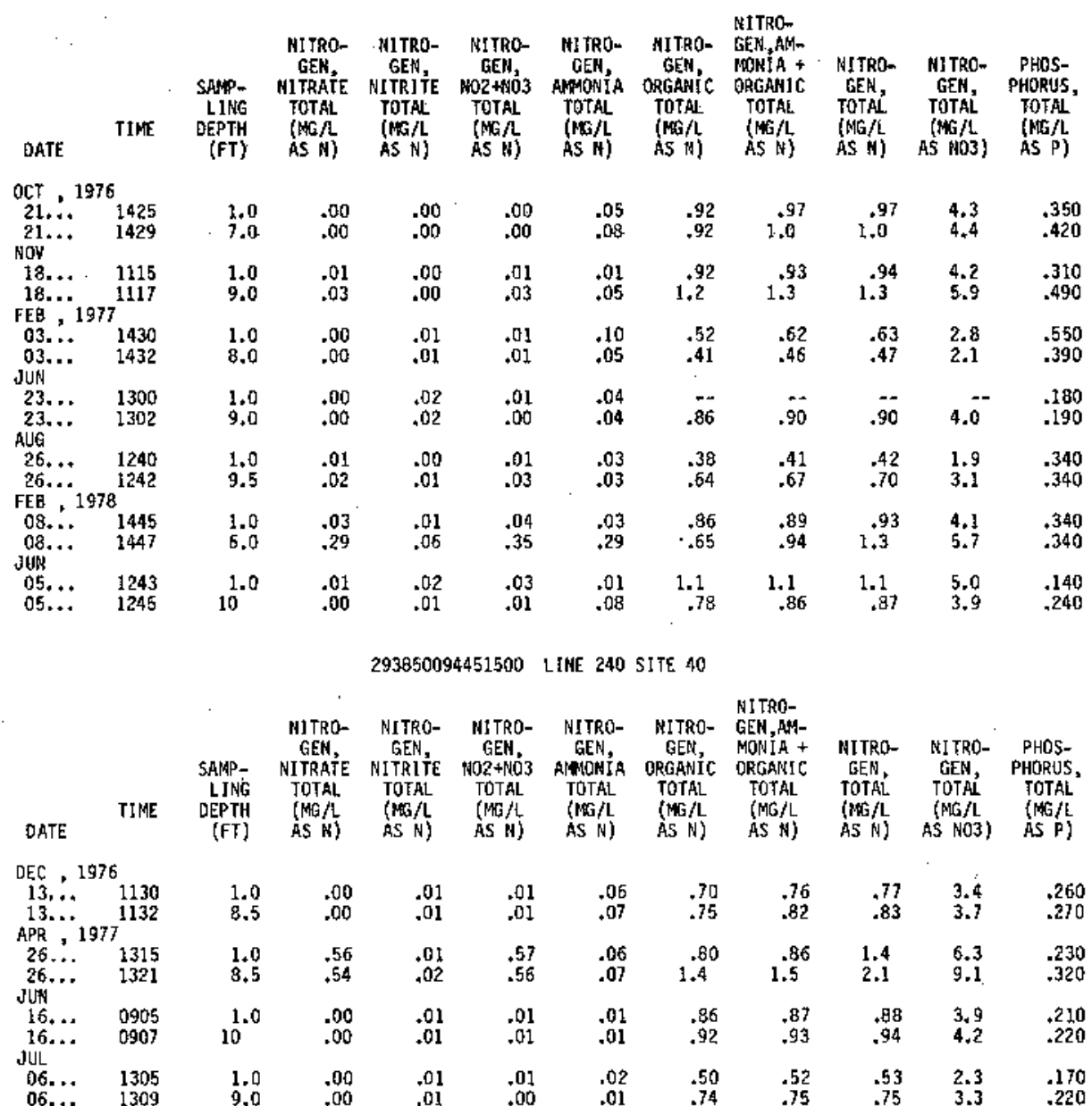

294004094470900 LINE 240 SITE 60

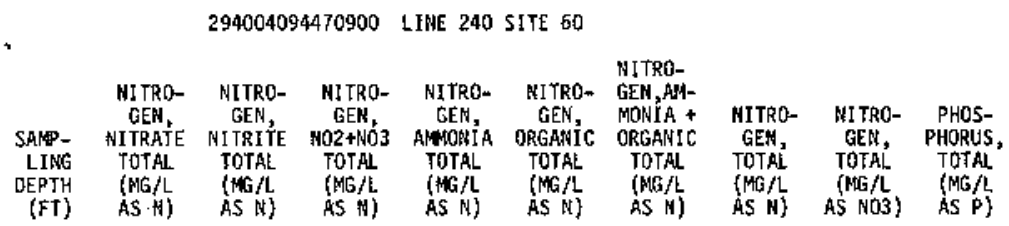

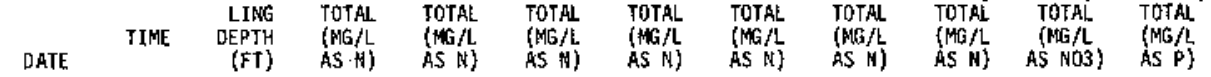

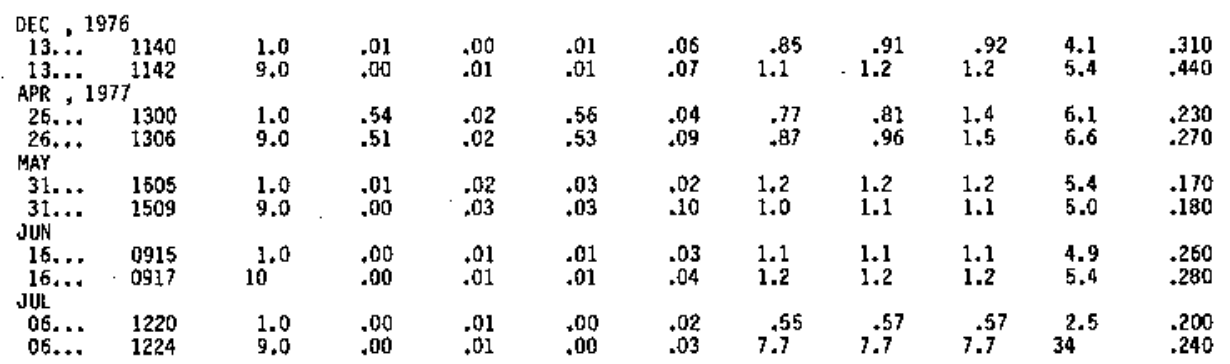


Table 2b:-Quality of water in the Trigity-San dacinto esturary, water year 1977-78--Continued Rutrient Analyses--Continued

294120094490600 LINE 240 SITE 80

\begin{tabular}{|c|c|c|c|c|c|c|c|c|c|c|c|}
\hline DATE & TIME & $\begin{array}{l}\text { SAMP- } \\
\text { LI\$SG } \\
\text { DEPTH } \\
\text { (FT) }\end{array}$ & $\begin{array}{l}\text { NITRQ- } \\
\text { GEN, } \\
\text { NITRATE } \\
\text { TOTAL } \\
(M G / L \\
\text { AS N })\end{array}$ & $\begin{array}{l}\text { NITRO- } \\
\text { GEN, } \\
\text { NITRITE } \\
\text { TDTAL } \\
\text { (MG/L } \\
\text { ASN) }\end{array}$ & $\begin{array}{l}\text { NITRD- } \\
\text { GEN, } \\
\text { NO2 HNO33 } \\
\text { TOTAL } \\
\text { (AC } / L \\
\text { AS N N) }\end{array}$ & 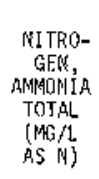 & $\begin{array}{c}\text { NITRO- } \\
\text { GEN, } \\
\text { ORGANIC } \\
\text { TDTAL } \\
\text { (MGIL } \\
\text { AS } M \text { S) }\end{array}$ & $\begin{array}{l}\text { EITRO- } \\
\text { GEN, AM- } \\
\text { MONIA + } \\
\text { ORAIANIC } \\
\text { TOTAL } \\
\{\text { MCL/L } \\
\text { AS N })\end{array}$ & $\begin{array}{l}\text { NITRO- } \\
\text { GEN, } \\
\text { TDTAL } \\
\text { (MG./L } \\
\text { AS NI) }\end{array}$ & $\begin{array}{l}\text { NITRO- } \\
\text { CEN, } \\
\text { TOTAL } \\
\text { \{NG/L } \\
\text { A5 NO3) }\end{array}$ & $\begin{array}{l}\text { PHOS- } \\
\text { PHORUS, } \\
\text { TDTAL } \\
\text { (MG/L } \\
\text { AS P) }\end{array}$ \\
\hline \multicolumn{12}{|c|}{ OEC, 1976} \\
\hline $\begin{array}{r}13 . \ldots \\
13 \ldots \\
\text { APR }\end{array}$ & $7^{1200}$ & $\begin{array}{l}1.0 \\
8.0\end{array}$ & $\begin{array}{l}.01 \\
.00\end{array}$ & $\begin{array}{l}00 \\
.01\end{array}$ & $\begin{array}{l}01 \\
.01\end{array}$ & $\begin{array}{l}.08 \\
.07\end{array}$ & $\begin{array}{l}1.1 \\
.93\end{array}$ & $\begin{array}{l}1.2 \\
1.0\end{array}$ & $\begin{array}{l}1.2 \\
1.0\end{array}$ & $\begin{array}{l}5.4 \\
4.5\end{array}$ & $\begin{array}{r}.440 \\
.470\end{array}$ \\
\hline $\begin{array}{c}26 \ldots \\
26 \ldots \\
\text { JUN }\end{array}$ & $\begin{array}{l}1240 \\
1246\end{array}$ & $\begin{array}{l}1.0 \\
8.5\end{array}$ & $\begin{array}{l}.56 \\
.49\end{array}$ & $\begin{array}{l}.02 \\
.02\end{array}$ & $\begin{array}{l}.58 \\
.51\end{array}$ & $\begin{array}{l}.07 \\
.13\end{array}$ & $\begin{array}{r}.93 \\
.83\end{array}$ & $\begin{array}{l}1.0 \\
.96\end{array}$ & $\begin{array}{l}1.6 \\
1.5\end{array}$ & $\begin{array}{l}7.0 \\
6.5\end{array}$ & $\begin{array}{l}.200 \\
.230\end{array}$ \\
\hline $\begin{array}{l}16, \ldots \\
16, \ldots \\
\text { JusL }\end{array}$ & $\begin{array}{l}0930 \\
0932\end{array}$ & $\begin{array}{l}1.0 \\
9.0\end{array}$ & $\begin{array}{l}.01 \\
.00\end{array}$ & .01 & $\begin{array}{l}02 \\
.02 \\
.01\end{array}$ & $\begin{array}{l}.05 \\
.05\end{array}$ & 1.93 & 1.98 & $\begin{array}{l}1.0 \\
1.5\end{array}$ & $\begin{array}{l}4.4 \\
6.7\end{array}$ & $\begin{array}{l}.280 \\
.320\end{array}$ \\
\hline $\begin{array}{l}06 . . \\
06 . .\end{array}$ & $\begin{array}{l}1205 \\
1209\end{array}$ & $\begin{array}{l}1.0 \\
8.0\end{array}$ & $\begin{array}{l}00 \\
.00\end{array}$ & $\begin{array}{l}.01 \\
.01\end{array}$ & $\begin{array}{l}0100 \\
.01\end{array}$ & .01 & $\begin{array}{l}.99 \\
.84\end{array}$ & $\begin{array}{c}1.0 \\
.87\end{array}$ & $\begin{array}{c}1.0 \\
.88\end{array}$ & $\begin{array}{l}4.4 \\
3.9\end{array}$ & $\begin{array}{l}.220 \\
.250\end{array}$ \\
\hline
\end{tabular}

29A15B094500500 LINE 240 SITE 90

\begin{tabular}{|c|c|c|c|c|c|c|c|c|c|c|c|}
\hline & & & & 29415809 & 4500500 & LINE 240 & SITE 90 & & & & \\
\hline DATE & TIME & $\begin{array}{l}\text { SAMP- } \\
\text { LING } \\
\text { DEPTH } \\
(F T)\end{array}$ & $\begin{array}{l}\text { NITRO- } \\
\text { GEN, } \\
\text { MITRAJE } \\
\text { TOTAL } \\
\text { (MG/L } \\
\text { AS N) }\end{array}$ & $\begin{array}{l}\text { NI JRO- } \\
\text { GEN, } \\
\text { NIJRITE } \\
\text { TOTAL } \\
\text { (MG } / L \\
\text { AS N) }\end{array}$ & $\begin{array}{c}\text { NITRO- } \\
\text { GEN, } \\
\text { NO2+NO3 } \\
\text { TOTAL } \\
\text { (MG } / L \\
\text { AS N) }\end{array}$ & $\begin{array}{l}\text { NITRO- } \\
\text { GEN, } \\
\text { AMMONIA } \\
\text { TOTAL } \\
\text { (MG } / L \\
\text { AS N) }\end{array}$ & $\begin{array}{l}\text { NITRO- } \\
\text { GEN, } \\
\text { ORGANIC, } \\
\text { TOTAL } \\
\text { (MG/L } \\
\text { AS N) }\end{array}$ & $\begin{array}{l}\text { NITRO- } \\
\text { GEN,AM- } \\
\text { MONIA + } \\
\text { ORGANIC } \\
\text { TOTA!. } \\
\text { (MG L } \\
\text { AS NS) }\end{array}$ & $\begin{array}{l}\text { NITKO- } \\
\text { GEN, } \\
\text { TOTAL } \\
(N G / L \\
\text { AS N })\end{array}$ & $\begin{array}{l}\text { NITRO- } \\
\text { GEN, } \\
\text { TOTAL } \\
\text { (MG } / L \\
\text { AS NO3) }\end{array}$ & $\begin{array}{c}\text { PHOS- } \\
\text { PHORUS, } \\
\text { TUTAL } \\
\{M G / L \\
\text { AS P })\end{array}$ \\
\hline NDY , & & & & & & & & & & & \\
\hline $\begin{array}{l}18 \ldots \\
18 . . \\
\text { FE, }\end{array}$ & $\begin{array}{r}1140 \\
1142 \\
7\end{array}$ & $\begin{array}{l}1.0 \\
8.0\end{array}$ & $\begin{array}{l}.00 \\
.08\end{array}$ & $\begin{array}{l}.00 \\
.02\end{array}$ & $\begin{array}{l}.00 \\
.10\end{array}$ & $\begin{array}{l}.02 \\
.11\end{array}$ & $\begin{array}{l}.95 \\
.82\end{array}$ & $\begin{array}{r}.97 \\
.93\end{array}$ & $\begin{array}{l}.97 \\
1.0\end{array}$ & $\begin{array}{l}4.3 \\
4.6\end{array}$ & $\begin{array}{l}.360 \\
.410\end{array}$ \\
\hline $\begin{array}{l}03 . \cdots \\
03 . . . \\
\text { JWN }\end{array}$ & $\begin{array}{l}1315 \\
1317\end{array}$ & $\begin{array}{l}1.0 \\
6.0\end{array}$ & $\begin{array}{l}.00 \\
.00\end{array}$ & .00 & $\begin{array}{l}.00 \\
.01\end{array}$ & $\begin{array}{l}.09 \\
.07\end{array}$ & $\begin{array}{c}1.0 \\
.12\end{array}$ & $\begin{array}{c}1.1 \\
.19\end{array}$ & $\begin{array}{l}1.1 \\
.20\end{array}$ & $\begin{array}{l}4.9 \\
.90\end{array}$ & $\begin{array}{r}.380 \\
.380\end{array}$ \\
\hline $\begin{array}{l}23 . \ldots \\
23 \ldots \\
\text { AUG }\end{array}$ & $\begin{array}{l}1200 \\
1202\end{array}$ & $\begin{array}{l}1,0 \\
8.0\end{array}$ & $\begin{array}{l}00 \\
.00 \\
\end{array}$ & $\begin{array}{l}.02 \\
.02\end{array}$ & .00 & $\begin{array}{l}.05 \\
.05\end{array}$ & $\begin{array}{l}.95 \\
.66\end{array}$ & $\begin{array}{l}1.0 \\
.71\end{array}$ & $\begin{array}{l}1.0 \\
.72\end{array}$ & $\begin{array}{l}4.4 \\
3.2\end{array}$ & $\begin{array}{l}.240 \\
.270\end{array}$ \\
\hline $\begin{array}{l}26 \ldots \\
26 \ldots \\
\text { FEB }\end{array}$ & $\begin{array}{l}1320 \\
8^{1322}\end{array}$ & $\begin{array}{l}1.0 \\
8.0\end{array}$ & $\begin{array}{l}.00 \\
.00\end{array}$ & .01 & $\begin{array}{l}01 \\
.01\end{array}$ & $\begin{array}{l}.03 \\
.04\end{array}$ & $\begin{array}{l}.79 \\
.58\end{array}$ & $\begin{array}{l}.82 \\
.62\end{array}$ & $\begin{array}{l}.83 \\
.63\end{array}$ & $\begin{array}{l}3.7 \\
2.8\end{array}$ & $\begin{array}{l}.360 \\
.360\end{array}$ \\
\hline $\begin{array}{l}08 . . \\
08 \ldots \\
\text { duN }\end{array}$ & $\begin{array}{l}1340 \\
1342\end{array}$ & $\begin{array}{l}1.0 \\
6.0\end{array}$ & $\begin{array}{l}.17 \\
.17\end{array}$ & $\begin{array}{l}.04 \\
.04\end{array}$ & $\begin{array}{l}.21 \\
.21\end{array}$ & $\begin{array}{l}.23 \\
.23\end{array}$ & $\begin{array}{l}.70 \\
.65\end{array}$ & $\begin{array}{l}.93 \\
.88\end{array}$ & $\begin{array}{l}1.1 \\
1.1\end{array}$ & $\begin{array}{l}5.0 \\
4.8\end{array}$ & $\begin{array}{l}.270 \\
.270\end{array}$ \\
\hline $\begin{array}{l}05 . . \\
05 \ldots\end{array}$ & $\begin{array}{l}1314 \\
1316\end{array}$ & $\begin{array}{l}1.0 \\
9.0\end{array}$ & סט. & .01 & $\begin{array}{l}01 \\
.01\end{array}$ & .01 & $\begin{array}{r}.61 \\
.97\end{array}$ & $\begin{array}{l}.62 \\
1.0\end{array}$ & 1.03 & $\begin{array}{l}2.8 \\
4.5\end{array}$ & $\begin{array}{l}.170 \\
.220\end{array}$ \\
\hline
\end{tabular}

293348094482800 LINE 260 SITE 20

\begin{tabular}{|c|c|c|c|c|c|c|c|c|c|}
\hline $\begin{array}{l}\text { SARPP- } \\
\text { LING } \\
\text { DEPTH } \\
\text { (FT) }\end{array}$ & $\begin{array}{l}\text { MITRO- } \\
\text { GER, } \\
\text { NITRATE } \\
\text { TOTAL } \\
\text { (MG/L } \\
\text { AS N) }\end{array}$ & $\begin{array}{l}\text { NITRQ- } \\
\text { GEN, } \\
\text { MITRITE } \\
\text { TOTAL } \\
\text { (MGA } \\
\text { AS N) }\end{array}$ & $\begin{array}{l}\text { NITRO- } \\
\text { GEN, } \\
\text { NO2+ND3 } \\
\text { TOTAL } \\
\text { (MG/L } \\
\text { AS N) }\end{array}$ & $\begin{array}{l}\text { NITRO- } \\
\text { GEN, } \\
\text { AMMONÍ } \\
\text { TOTAL } \\
\text { (MG/L } \\
\text { AS N) }\end{array}$ & $\begin{array}{l}\text { NITRO- } \\
\text { GEN, } \\
\text { ORGANIC } \\
\text { TOTAI } \\
\text { (MG/L } \\
\text { AS } \nVdash \text { ) }\end{array}$ & $\begin{array}{l}\text { NI TRO- } \\
\text { GEN, AM- } \\
\text { MONIA + } \\
\text { ORGANIC } \\
\text { JOTAL } \\
(M G / 2 \\
\text { A5N) }\end{array}$ & $\begin{array}{l}\text { NITRO- } \\
\text { GEN, } \\
\text { TOTAL } \\
\text { (MG } / L \\
\text { AS N) }\end{array}$ & $\begin{array}{c}\text { NI TRO- } \\
\text { GENA, } \\
\text { TOTAL } \\
\text { \{MG/L } \\
\text { AS NO3\} }\end{array}$ & $\begin{array}{l}\text { PHOS- } \\
\text { PHORUS, } \\
\text { TOTAL } \\
\text { (MG /L } \\
\text { AS } P \text { ) }\end{array}$ \\
\hline
\end{tabular}

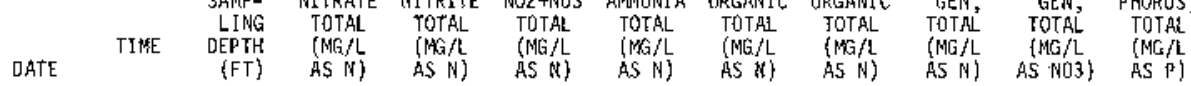

OCT , 1976

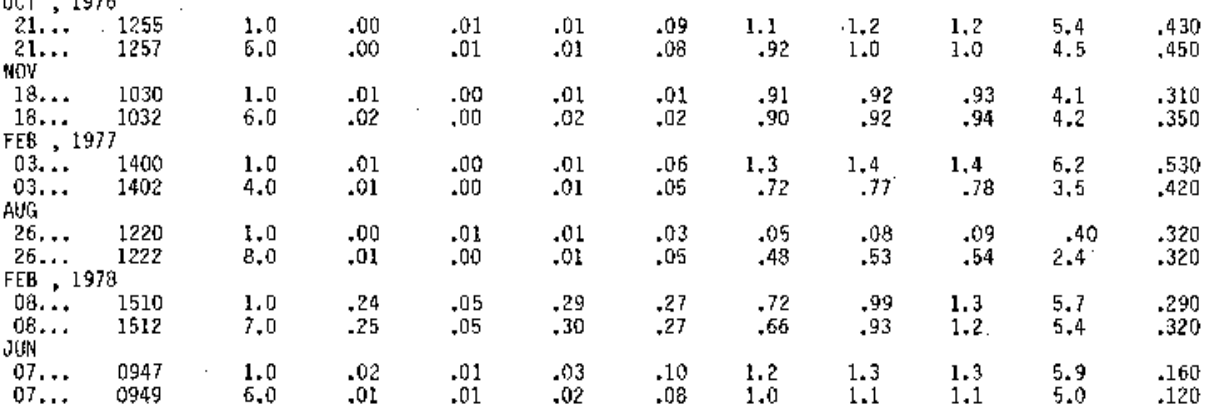


293519094500800 LINE 260 SITE 40

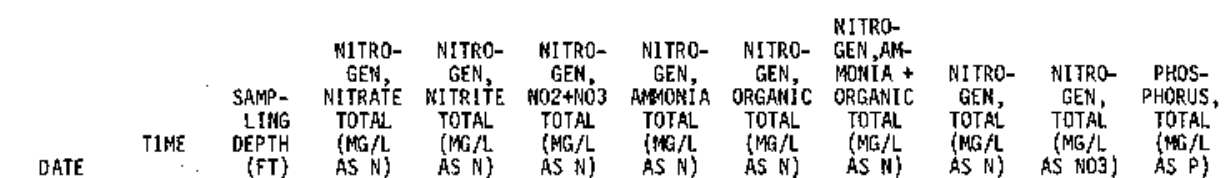

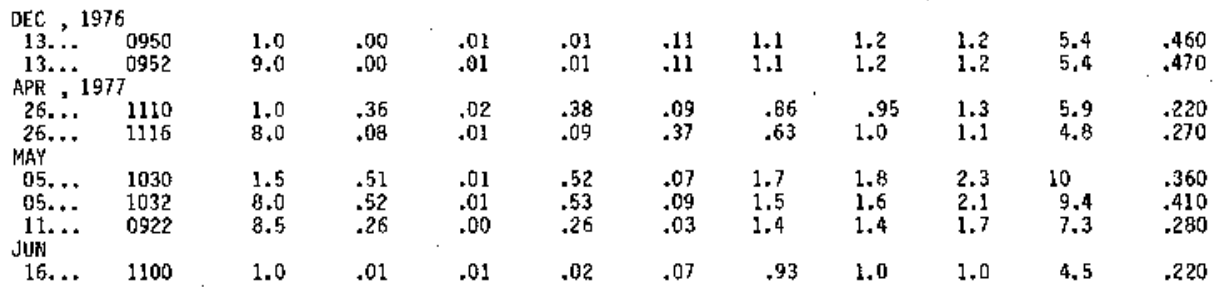

293650094514800 LIPAE 260 SITE 60

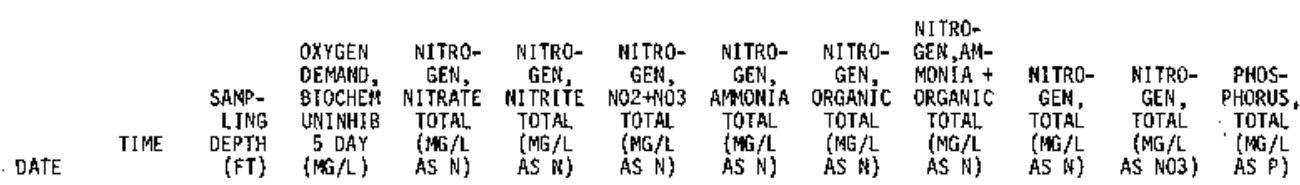

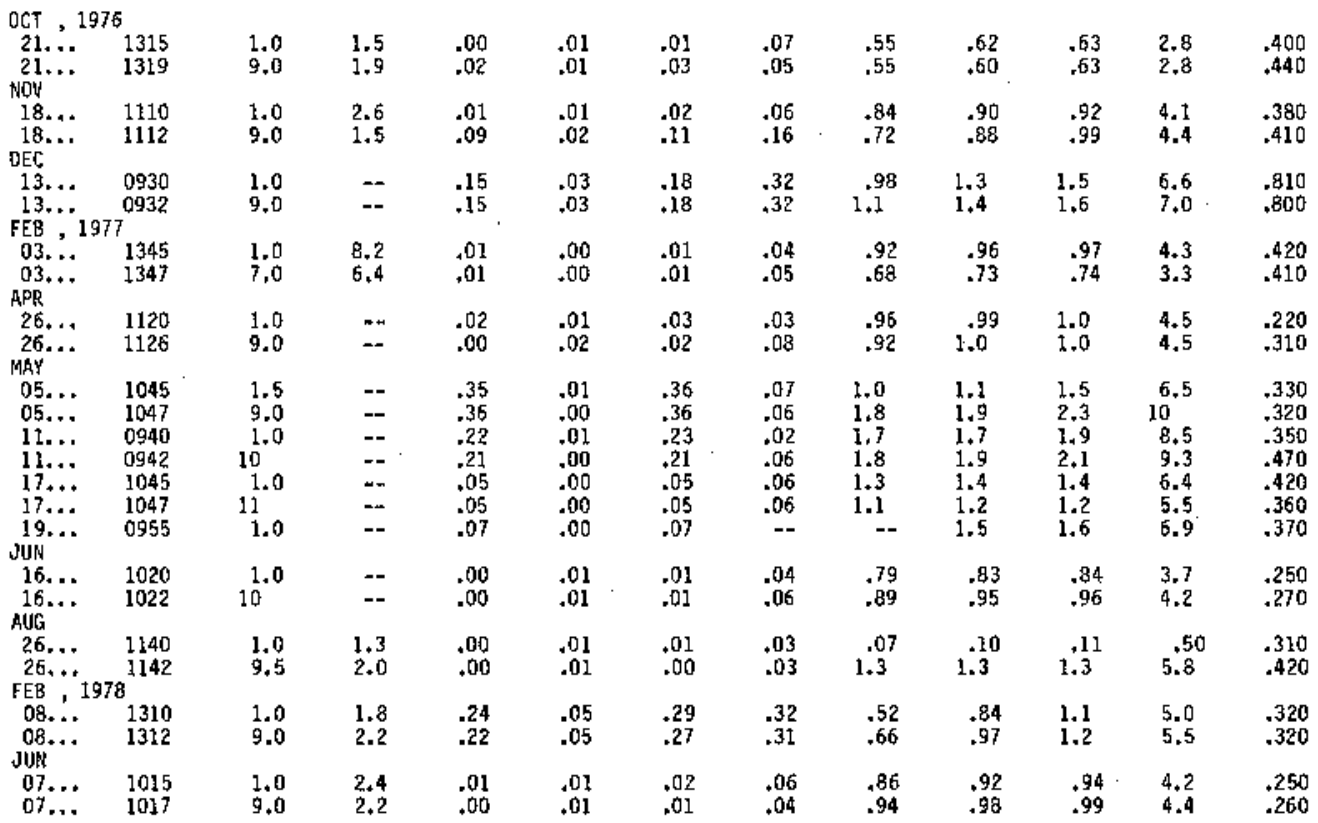


293821094532900 LINE 260 SITE 80

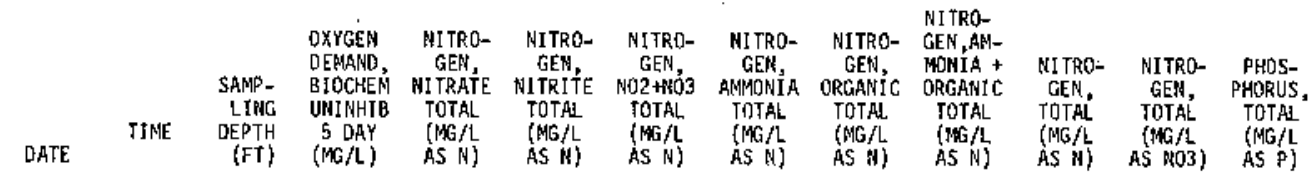

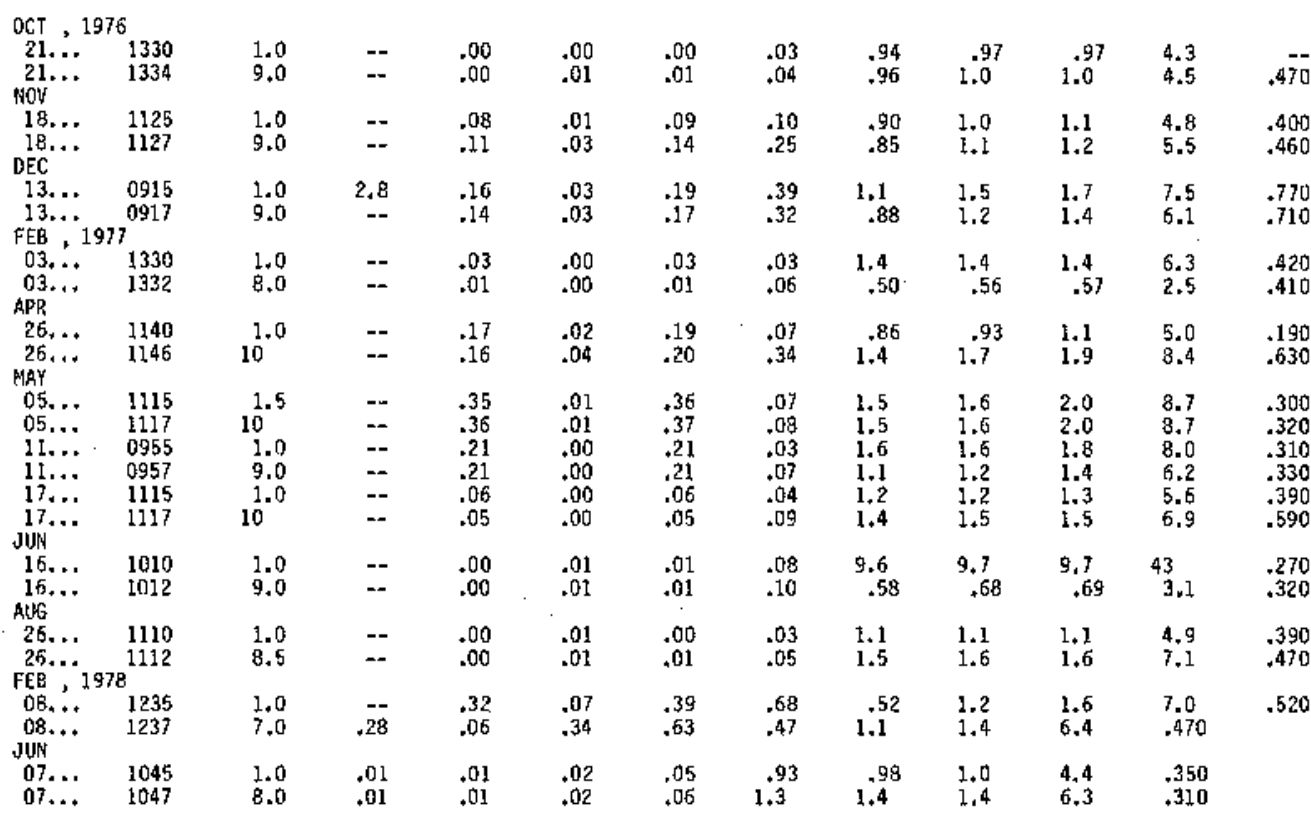

293253095010400 LINE 330 SITE 20

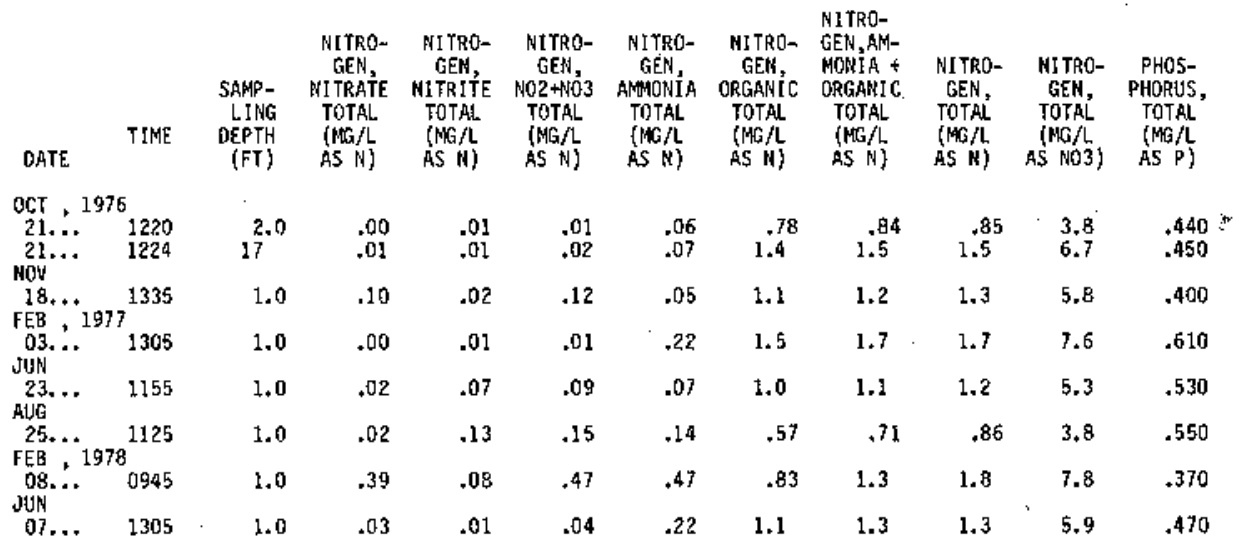




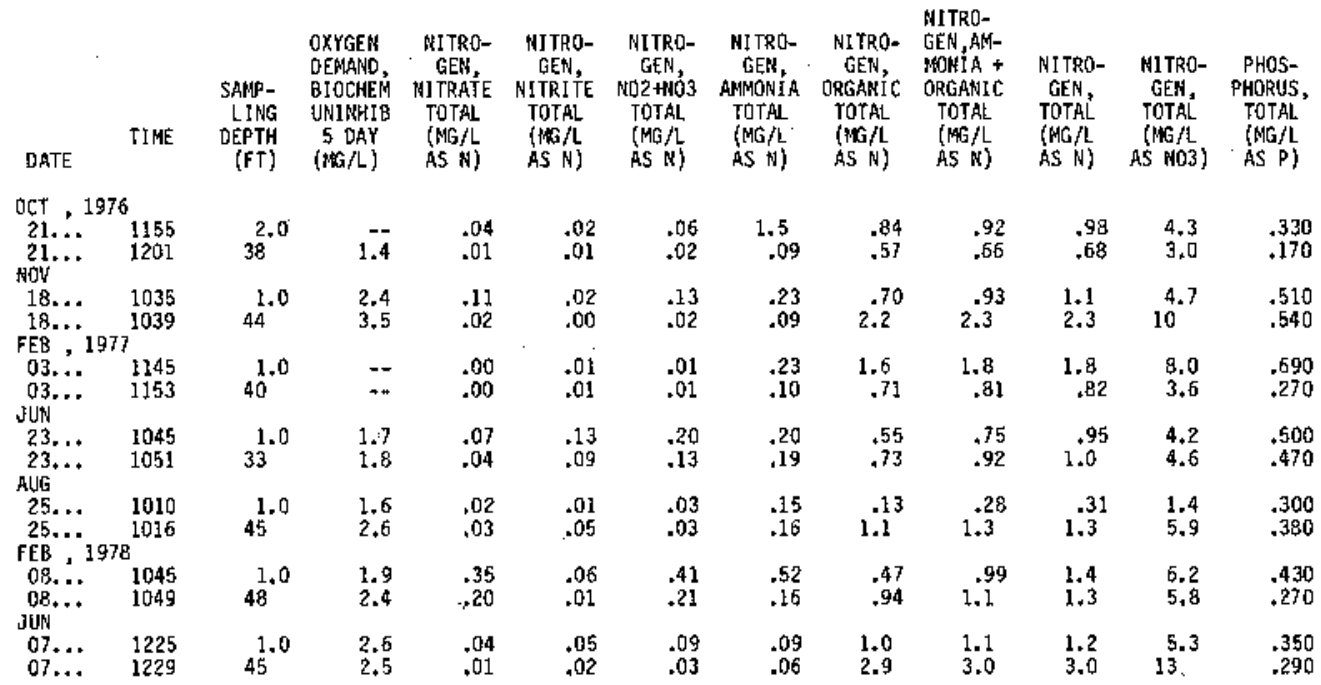

293133094501400 LINE 350 SITE 30

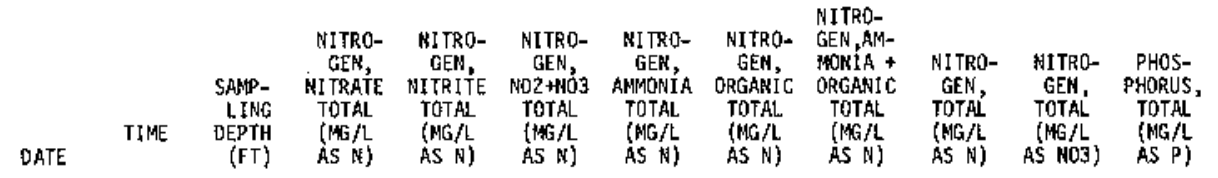

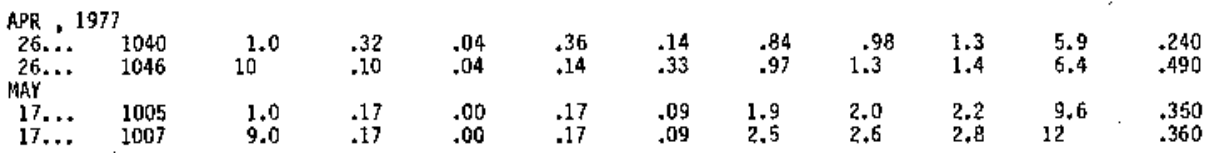

293030094523500 LINE 350 SITE 50

\begin{tabular}{|c|c|c|c|c|c|c|c|c|c|c|c|}
\hline DATE & TIME & $\begin{array}{l}\text { SAPP- } \\
\text { LING } \\
\text { DEPTH } \\
\text { (FT) }\end{array}$ & $\begin{array}{l}\text { NITRO- } \\
\text { GEN, } \\
\text { NITRATE } \\
\text { TOTAL } \\
\text { (MG/L } \\
\text { AS N) }\end{array}$ & $\begin{array}{l}\text { NITRQm } \\
\text { GE } \\
\text { NITRIYE } \\
\text { TOTAL } \\
\text { (MG } / \\
\text { AS N) }\end{array}$ & $\begin{array}{c}\text { NITRO- } \\
\text { GEN, } \\
\text { MO2+NO3 } \\
\text { TOTAL } \\
\text { (MG/L } \\
\text { AS N N) }\end{array}$ & $\begin{array}{l}\text { MITRO- } \\
\text { GEN, } \\
\text { ANWONIA } \\
\text { TOTAL } \\
\text { (NWH } / L \\
\text { AS N }\end{array}$ & $\begin{array}{l}\text { NITRO- } \\
\text { GEN, } \\
\text { ORGANIC } \\
\text { TOTAL } \\
\text { (NG/L } \\
\text { AS N) }\end{array}$ & $\begin{array}{l}\text { NITRO- } \\
\text { GEN,AM- } \\
\text { MOMIA + } \\
\text { ORGANIC } \\
\text { TQTAL. } \\
\text { (MG } / L \\
\text { AS N) }\end{array}$ & $\begin{array}{l}\text { BITRO- } \\
\text { GEN, } \\
\text { TOTAL } \\
\text { (MS } / L \\
\text { AS N) }\end{array}$ & $\begin{array}{c}\text { NITRO- } \\
\text { GEN, } \\
\text { TDTAL } \\
\text { (MG/L } \\
\text { AS NOS) }\end{array}$ & $\begin{array}{c}\text { PHOS- } \\
\text { PHORUS, } \\
\text { TOTAL } \\
\text { (NG } / L \\
\text { AS P) }\end{array}$ \\
\hline \multicolumn{12}{|c|}{ OCT, 1976} \\
\hline $\begin{array}{l}21 \ldots \\
21 \ldots \\
\text { NON }\end{array}$ & $\begin{array}{l}1125 \\
1131\end{array}$ & $4_{46}^{2.0}$ & .00 & .01 & $\begin{array}{l}.01 \\
.01\end{array}$ & $\begin{array}{l}.08 \\
.11\end{array}$ & $\begin{array}{l}.40 \\
.87\end{array}$ & $\begin{array}{l}.48 \\
.98\end{array}$ & $\begin{array}{r}.49 \\
.99\end{array}$ & $\begin{array}{l}2.2 \\
4.4\end{array}$ & $\begin{array}{r}290 \\
.190\end{array}$ \\
\hline $\begin{array}{l}17 . \ldots \\
17 . \ldots\end{array}$ & $\begin{array}{l}1225 \\
1231 \\
7\end{array}$ & $40^{2.0}$ & $\begin{array}{l}.00 \\
.01\end{array}$ & .01 & $\begin{array}{l}01 \\
.01\end{array}$ & .04 & $\begin{array}{r}.75 \\
.26\end{array}$ & $\begin{array}{r}.79 \\
.33\end{array}$ & $\begin{array}{r}.80 \\
.34 \\
.34\end{array}$ & $\begin{array}{l}3.5 \\
1.5\end{array}$ & $\begin{array}{r}.260 \\
.130\end{array}$ \\
\hline $\begin{array}{r}03 . \ldots \\
03 . \ldots \\
\text { JuN }\end{array}$ & $\begin{array}{l}1100 \\
1108\end{array}$ & $4^{1.0}$ & $\begin{array}{l}.00 \\
.00\end{array}$ & .01 & $\begin{array}{l}01 \\
.01\end{array}$ & $\begin{array}{l}.05 \\
.15\end{array}$ & $\begin{array}{l}.48 \\
.80\end{array}$ & $\begin{array}{l}.53 \\
.95\end{array}$ & $\begin{array}{r}+54 \\
.96\end{array}$ & $\begin{array}{l}2.4 \\
4.2\end{array}$ & $\begin{array}{r}.290 \\
.340\end{array}$ \\
\hline $\begin{array}{r}23 \ldots \\
23+\ldots \\
A E G G\end{array}$ & $\begin{array}{l}1015 \\
1021\end{array}$ & $40^{1.0}$ & .00 & $\begin{array}{l}.04 \\
.03\end{array}$ & $\begin{array}{l}.03 \\
.01\end{array}$ & .04 & $\begin{array}{r}.57 \\
+54\end{array}$ & .61 & $\begin{array}{l}.64 \\
.66\end{array}$ & $\begin{array}{l}2.8 \\
2.9\end{array}$ & $\begin{array}{l}.350 \\
.210\end{array}$ \\
\hline $\begin{array}{r}25 \ldots \\
25 \ldots \\
\text { FEB. }\end{array}$ & $\begin{array}{l}0935 \\
0941 \\
8\end{array}$ & 37 & .01 & .00 & .01 & $\begin{array}{l}.05 \\
.05\end{array}$ & $\begin{array}{r}.03 \\
--\end{array}$ & $\begin{array}{l}.08 \\
.04\end{array}$ & $\begin{array}{l}.09 \\
.05\end{array}$ & $\begin{array}{l}.40 \\
.20\end{array}$ & $\begin{array}{l}.320 \\
.210\end{array}$ \\
\hline $\begin{array}{l}09 . \ldots \\
09 . \ldots \\
\text { Juk }\end{array}$ & $\begin{array}{l}1300 \\
1304\end{array}$ & ${ }_{40}^{1.0}$ & $\begin{array}{l}.23 \\
.19\end{array}$ & $\begin{array}{l}.05 \\
.01\end{array}$ & $\begin{array}{r}.28 \\
.20\end{array}$ & .29 & 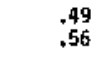 & $\begin{array}{r}.78 \\
.69\end{array}$ & 1.18 & $\begin{array}{l}4.7 \\
3.9\end{array}$ & $\begin{array}{l}.270 \\
.140\end{array}$ \\
\hline $\begin{array}{l}06 . . . \\
06, \ldots\end{array}$ & $\begin{array}{l}1140 \\
1144\end{array}$ & $42^{2.0}$ & .00 & $\begin{array}{l}.01 \\
.01\end{array}$ & $\begin{array}{l}.01 \\
.01\end{array}$ & $\begin{array}{l}03 \\
.01\end{array}$ & $\stackrel{.58}{1.1}$ & 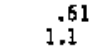 & $\begin{array}{l}.62 \\
1.1\end{array}$ & $\begin{array}{l}2.7 \\
4.9\end{array}$ & $\begin{array}{r}.120 \\
.130\end{array}$ \\
\hline
\end{tabular}


$292401094490 \% 00$ LIKE 380 S1TE 40

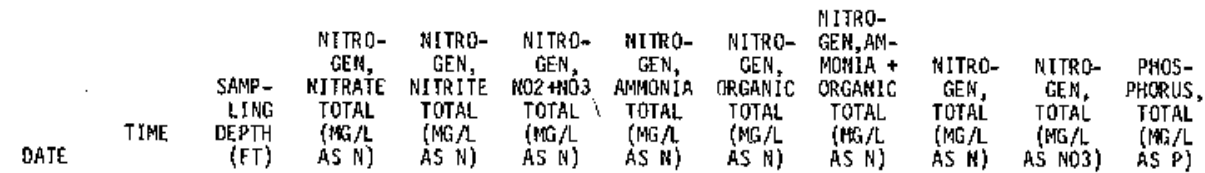

OCT . 1976

$20 . \therefore \quad 1520$

$20 \ldots . .1526$

$21 \ldots . \quad 1044$

NOY

$\begin{array}{ccc}1.0 & .00 & .0 \\ 42 & .00 & .0 \\ 43 & .00 & .0\end{array}$

$\begin{array}{ll}.01 & .01 \\ .01 & .01 \\ .01 & .01 \\ .01 & .01\end{array}$

$\begin{array}{ll}.05 & .3 \\ .06 & .7 \\ .09 & .3 \\ .08 & .30\end{array}$

$\begin{array}{ll}.30 & .35 \\ .72 & .78 \\ .34 & .43 \\ .30 & .38\end{array}$

$\begin{array}{llll}.35 & .36 & 1.6 & .130 \\ .78 & .79 & 3.5 & .180\end{array}$

$17 . . . \quad 1315$

$\begin{array}{llll}2.0 & .01 & .00 & .01\end{array}$

$\begin{array}{llllll}.04 & .60 & .64 & .65 & 2.9 & .230 \\ .05 & .57 & .62 & .63 & 2.9 & .150\end{array}$

GEB 1977

$03 . .21000$

JUN

$22 \ldots . .1319$

AUA $24 . \cdots \quad 1245$

24... 1245

FEB , 1978

$30 \quad .01 \quad .00$

$.01 \quad .05$

$\begin{array}{lll}1.0 & .00 & .01 \\ 45 & .01 & .01\end{array}$

.01

.09

$\begin{array}{lll}1.0 & -0 & .03 \\ 46 & .00 & .03\end{array}$

.01

$\begin{array}{lll}1.0 & .00 & .01 \\ 41 & .02 & .01\end{array}$

.01

Jun 2353

$\begin{array}{lll}1.0 \quad .07 & .03\end{array}$

$.10 \quad .13$

$12 \quad .11$

06... 1215

$\begin{array}{lll}2.0 & .10 & .01 \\ 40^{2} & .10 & .01\end{array}$

.11

$.08 \quad .12$

293243094345200 LINE 430 SITE 20

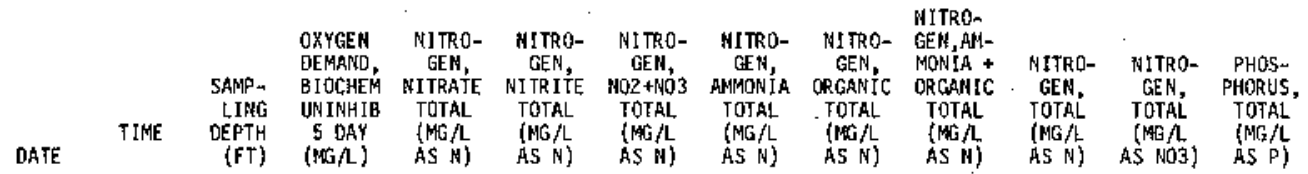

OCT , 1976

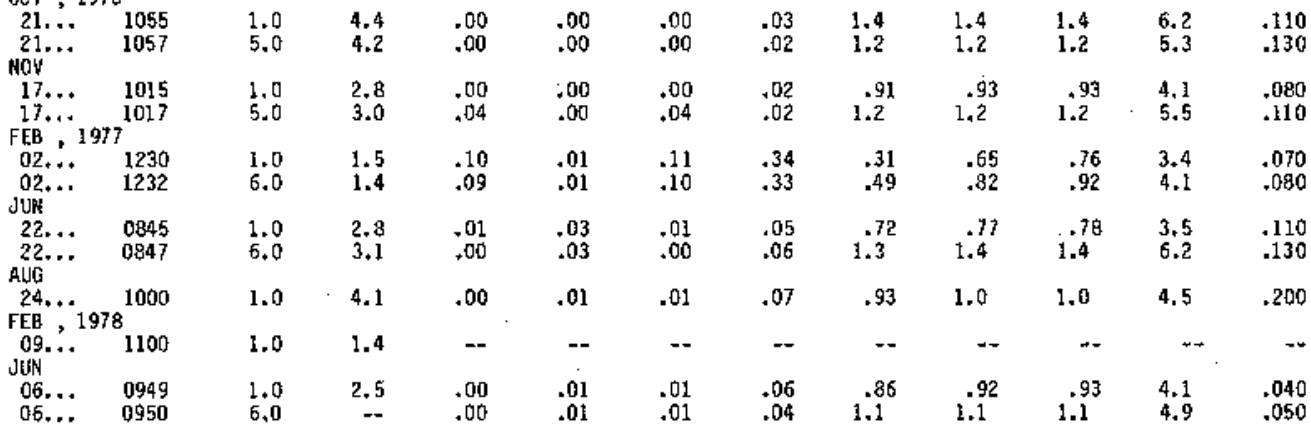

292720094451300 LINE 470 SITE 30

\begin{tabular}{|c|c|c|c|c|c|c|c|c|c|c|c|}
\hline DATE & IIME & $\begin{array}{l}\text { SANP- } \\
\text { LING } \\
\text { DEPTH } \\
\text { (FT) }\end{array}$ & $\begin{array}{l}\text { NITRO- } \\
\text { QENN, } \\
\text { M!TRATE } \\
\text { TOTAL } \\
\text { (MG/L } \\
\text { AS N) }\end{array}$ & $\begin{array}{c}\text { NITRO- } \\
\text { GEN, } \\
\text { NI TR ITE } \\
\text { TOTAL } \\
\text { (TAO /L } \\
\text { AS N) }\end{array}$ & $\begin{array}{l}\text { HITRO- } \\
\text { GEN, } \\
\text { NO2 +NO3 } \\
\text { TOTAL } \\
\text { (MGAL } \\
\text { AS N) }\end{array}$ & $\begin{array}{l}\text { MITRO- } \\
\text { GEK, } \\
\text { AMONONIA } \\
\text { TOTAL } \\
\text { (MG/L } \\
\text { AS N) }\end{array}$ & $\begin{array}{l}\text { NITRQ- } \\
\text { GEN, } \\
\text { ORGANIC } \\
\text { TOTAL } \\
\text { (MGG A } \\
\text { AS : }\end{array}$ & $\begin{array}{l}\text { MITRO- } \\
\text { GEN,AN- } \\
\text { PONIA 4 } \\
\text { DRGANIC } \\
\text { TOTAL } \\
\text { (MG } / L \\
\text { AS N) }\end{array}$ & $\begin{array}{l}\text { NITRO- } \\
\text { CEN, } \\
\text { TOTAL } \\
\text { (MG } / \mathrm{L} \\
\text { AS N) }\end{array}$ & $\begin{array}{l}\text { NITRO- } \\
\text { GEN, } \\
\text { TOTAL } \\
\text { (MGA } \\
\text { AS NO3) }\end{array}$ & $\begin{array}{l}\text { PHOS- } \\
\text { PHORUS, } \\
\text { TOTAL } \\
(\$ 4 G / L \\
\text { AS P) }\end{array}$ \\
\hline
\end{tabular}

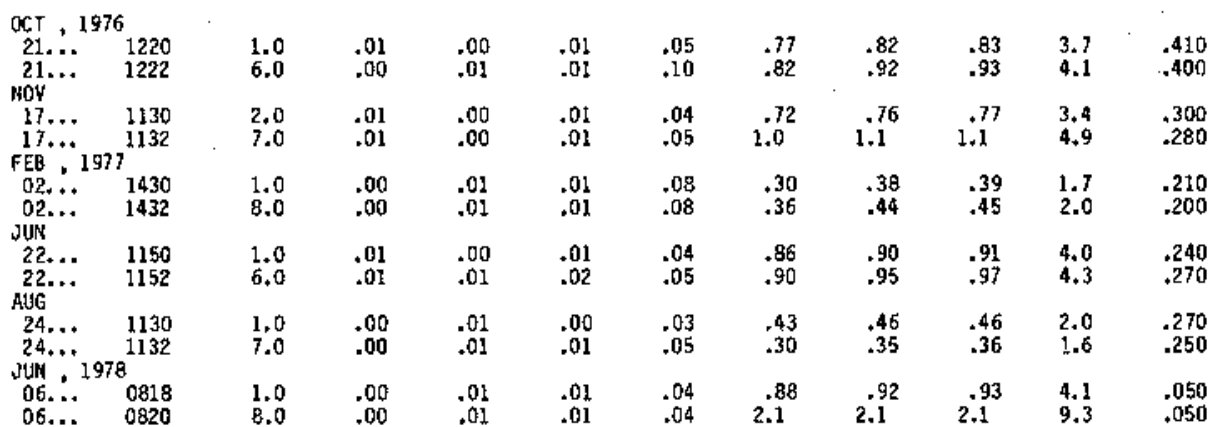


293029094462800 LINE 470 SITE 60

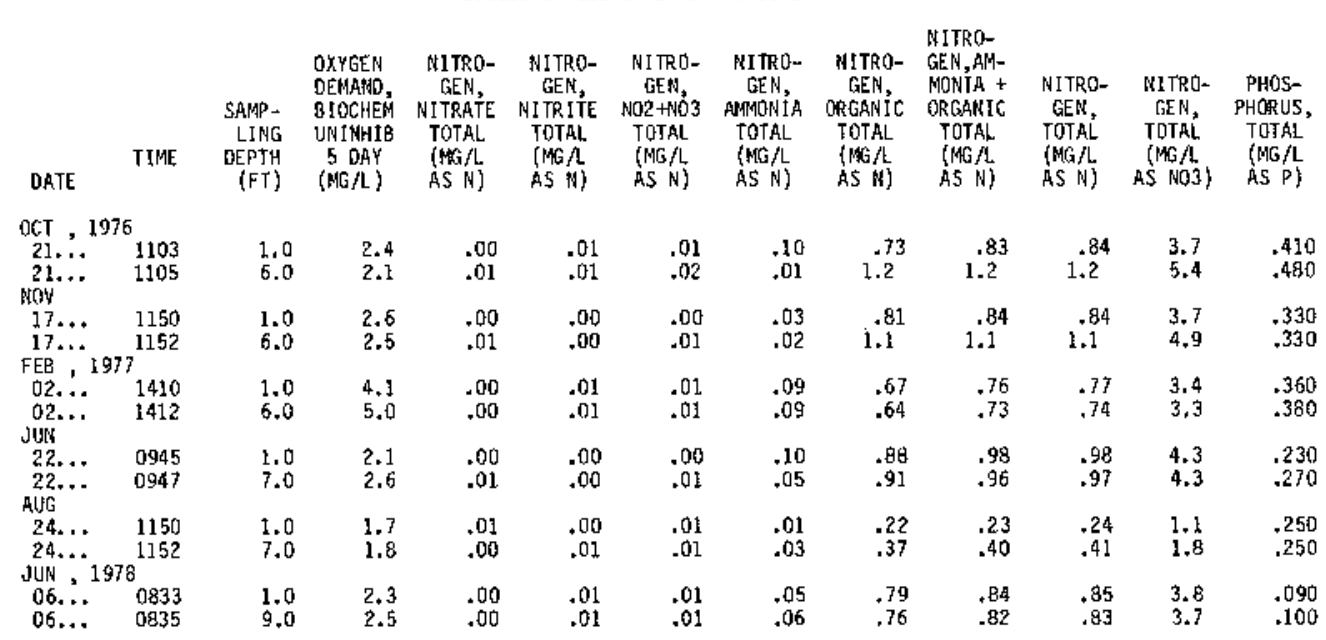

291744094531200 LINE 521 SITE 50

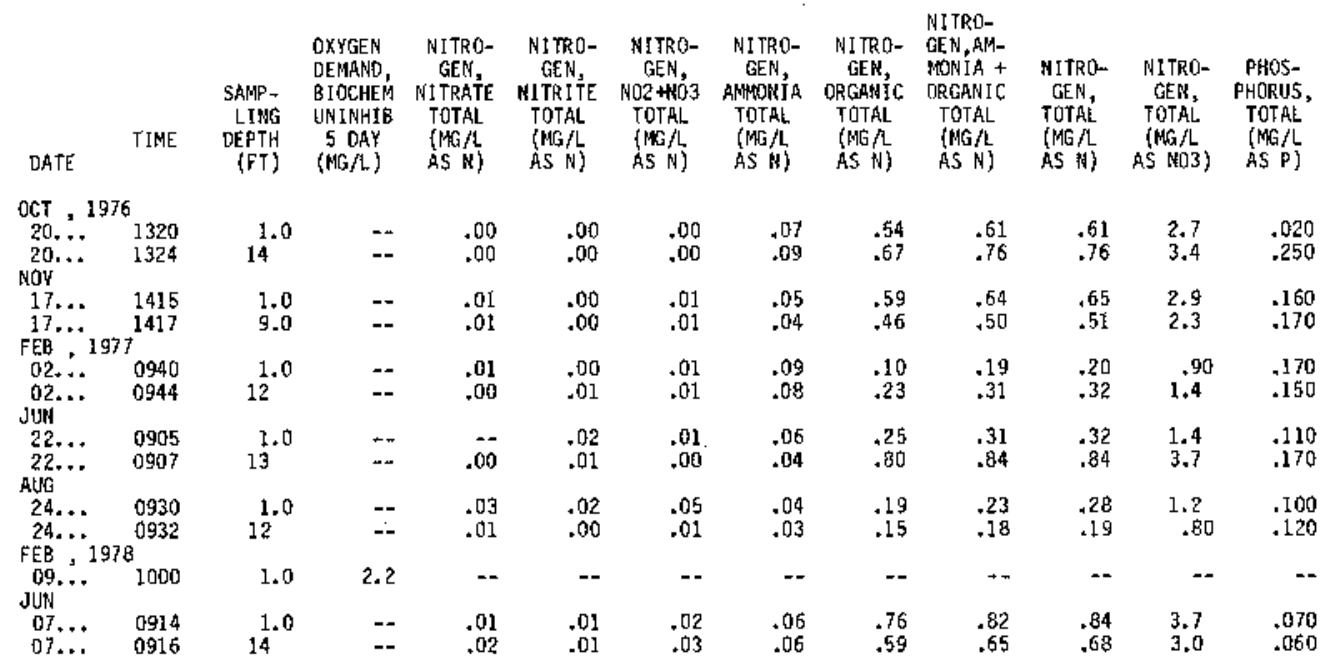

291428094575900 LINE 530 SITE 50

\begin{tabular}{|c|c|c|c|c|c|c|c|c|c|c|c|}
\hline DATE & TIME & $\begin{array}{r}\text { SAMP- } \\
\text { LING } \\
\text { DEPTH } \\
\text { (FT) }\end{array}$ & $\begin{array}{c}\text { NITRO- } \\
\text { GEM, } \\
\text { NITRATE } \\
\text { TOTAL } \\
\text { (MG } / L \\
\text { AS N) }\end{array}$ & $\begin{array}{c}\text { NITRO- } \\
\text { GEN, } \\
\text { NITRITE } \\
\text { TOTAL } \\
\text { (MUA } / L \\
\text { AS N) }\end{array}$ & $\begin{array}{l}\text { NITRO- } \\
\text { GEN, } \\
\text { MO2+NO3 } \\
\text { TOTAL } \\
\text { (MG } / \\
\text { AS N) }\end{array}$ & $\begin{array}{l}\text { NIIRO- } \\
\text { GEN, } \\
\text { AMMONIA } \\
\text { TOTAL } \\
\text { (NGLL } \\
\text { AS N) }\end{array}$ & $\begin{array}{l}\text { MITRQ- } \\
\text { GEN, } \\
\text { ORGANIC } \\
\text { TOTAL } \\
\text { (NAL } \\
\text { AS N) }\end{array}$ & $\begin{array}{l}\text { NITRO- } \\
\text { GEN, AM- } \\
\text { MONIA + } \\
\text { ORGAMIC } \\
\text { TOTAL } \\
\text { (MG/L } \\
\text { AS RS) }\end{array}$ & $\begin{array}{l}\text { NITRO- } \\
\text { GEN, } \\
\text { TOTAL } \\
\text { (MG/L } \\
\text { AS N) }\end{array}$ & $\begin{array}{l}\text { RITRO- } \\
\text { GEN, } \\
\text { TDTAL } \\
\text { (ME } / L \\
\text { AS } N 03)\end{array}$ & $\begin{array}{l}\text { PHOS- } \\
\text { PHORUS, } \\
\text { TOTAL } \\
\text { (MG/L } \\
\text { AS P) }\end{array}$ \\
\hline \multicolumn{12}{|c|}{ OCT, 1976} \\
\hline $\begin{array}{l}20 . \ldots \\
20 . \ldots \\
\text { NOW }\end{array}$ & $\begin{array}{l}1400 \\
1402\end{array}$ & $\begin{array}{l}1.0 \\
6.0\end{array}$ & $\begin{array}{l}.00 \\
.00\end{array}$ & $\begin{array}{l}.00 \\
.00\end{array}$ & $\begin{array}{l}.00 \\
.00\end{array}$ & $\begin{array}{l}.06 \\
.07\end{array}$ & $\begin{array}{l}.47 \\
.63\end{array}$ & $\begin{array}{r}.53 \\
.70\end{array}$ & $\begin{array}{r}.53 \\
.70\end{array}$ & $\begin{array}{l}2.3 \\
3.1\end{array}$ & $\begin{array}{l}.160 \\
.150\end{array}$ \\
\hline $\begin{array}{l}17 \ldots \\
17 \ldots\end{array}$ & $\begin{array}{l}1015 \\
1017 \\
7\end{array}$ & $\begin{array}{l}1.0 \\
6.5\end{array}$ & .01 & .00 & $\begin{array}{l}.01 \\
.01\end{array}$ & $\begin{array}{l}.04 \\
.04\end{array}$ & $\begin{array}{r}.51 \\
.50\end{array}$ & $\begin{array}{l}.55 \\
.54\end{array}$ & $\begin{array}{l}.56 \\
.55\end{array}$ & $\begin{array}{l}2.5 \\
2.4\end{array}$ & $\begin{array}{l}.180 \\
.180\end{array}$ \\
\hline $\begin{array}{c}02, \cdots \\
02 \ldots \\
\text { Juk }\end{array}$ & $\begin{array}{l}1025 \\
1027\end{array}$ & $\begin{array}{l}1.0 \\
6.0\end{array}$ & $\begin{array}{l}.01 \\
.00\end{array}$ & $\begin{array}{l}.00 \\
.01\end{array}$ & $\begin{array}{l}.01 \\
.01\end{array}$ & $\begin{array}{r}.07 \\
.07\end{array}$ & $\begin{array}{l}.06 \\
.02\end{array}$ & $\begin{array}{l}13 \\
.09\end{array}$ & $\begin{array}{r}.14 \\
.10\end{array}$ & $\begin{array}{l}.60 \\
.40\end{array}$ & $\begin{array}{l}.130 \\
.130\end{array}$ \\
\hline $\begin{array}{l}22 \ldots \\
22 \ldots \\
\text { AUG }\end{array}$ & $\begin{array}{l}0940 \\
0942\end{array}$ & $\begin{array}{l}1.0 \\
6.5\end{array}$ & .00 & .01 & .00 & $\begin{array}{l}.04 \\
.04\end{array}$ & .45 & $\begin{array}{l}.49 \\
.35\end{array}$ & $\begin{array}{r}.49 \\
.35\end{array}$ & $\begin{array}{l}2.2 \\
1.5\end{array}$ & $\begin{array}{l}.110 \\
.110\end{array}$ \\
\hline $\begin{array}{r}24 \ldots \\
24 \ldots\end{array}$ & $\begin{array}{l}1020 \\
1022\end{array}$ & $\begin{array}{l}1.0 \\
7.0\end{array}$ & $\begin{array}{l}.00 \\
.00\end{array}$ & $\begin{array}{l}.01 \\
.01\end{array}$ & $\begin{array}{l}.00 \\
.00\end{array}$ & $\begin{array}{l}.00 \\
.05\end{array}$ & $\begin{array}{r}.14 \\
.30\end{array}$ & .14 & $\begin{array}{r}.14 \\
.35\end{array}$ & .60 & $\begin{array}{l}.080 \\
.130\end{array}$ \\
\hline $\begin{array}{r}07 . . \\
07 \ldots\end{array}$ & $\begin{array}{l}0949 \\
0950\end{array}$ & $\begin{array}{l}1.0 \\
7.0\end{array}$ & $\begin{array}{l}.01 \\
.02\end{array}$ & $\begin{array}{l}.01 \\
.01\end{array}$ & $\begin{array}{l}.02 \\
.03\end{array}$ & $\begin{array}{l}.08 \\
.06\end{array}$ & $\begin{array}{l}.67 \\
.50\end{array}$ & $\begin{array}{l}.75 \\
.56\end{array}$ & .77 & $\begin{array}{l}3.4 \\
2.6\end{array}$ & $\begin{array}{l}.060 \\
.050\end{array}$ \\
\hline
\end{tabular}


Table 2B.--Quality of water in the Trinity-San Jacinto estuary, water year 1977-78--Continued

Nutrient Analyses--Continued

291127095015500 LINE 550 SITE 50

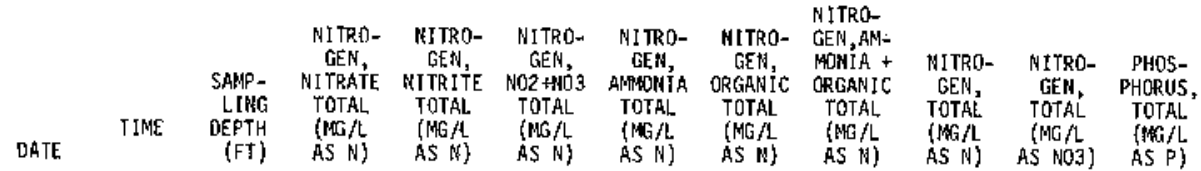

OCT , 1976

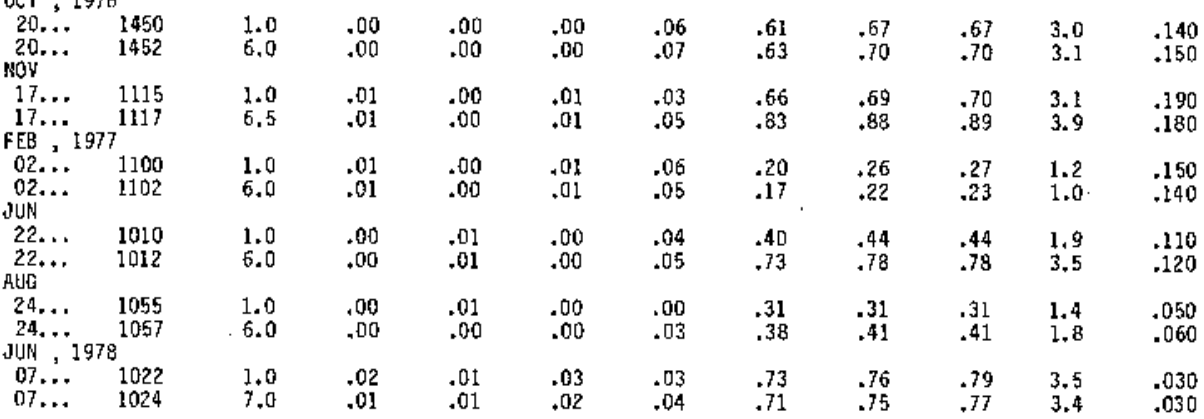

291106095084200 LINE 565 SITE 30

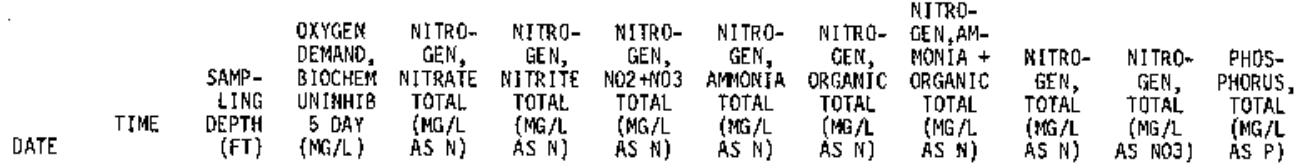

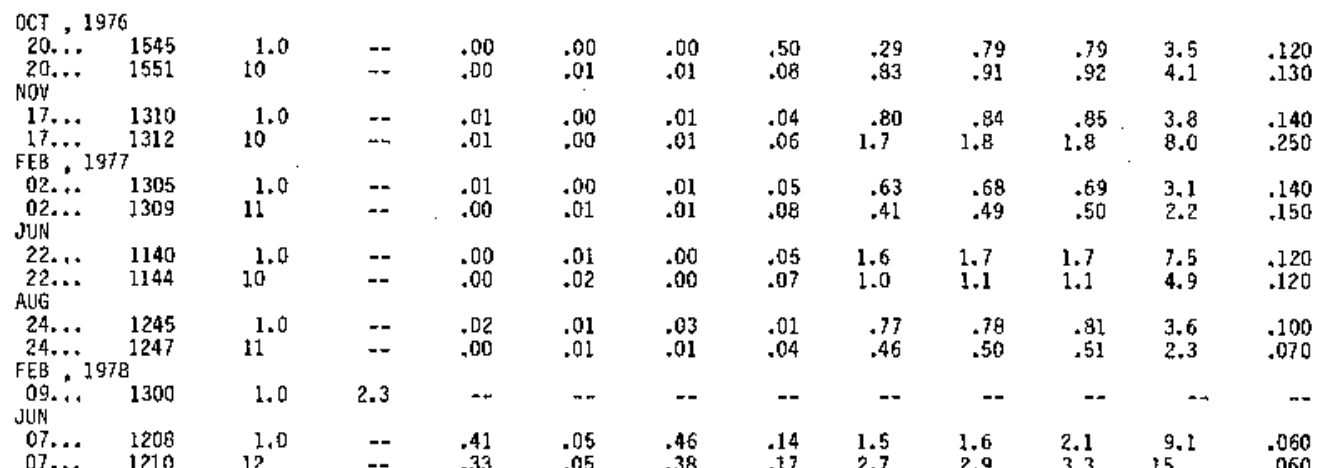

290654095075100 LIME 580 SITE 50

\begin{tabular}{|c|c|c|c|c|c|c|c|c|c|c|c|}
\hline DATE & TIME & $\begin{array}{r}\text { SAMP- } \\
\text { LING } \\
\text { DEPTH } \\
\text { (FT) }\end{array}$ & $\begin{array}{l}\text { NAITRO- } \\
\text { GEN, } \\
\text { NITRATE } \\
\text { TOTAL } \\
\text { (MG } \\
\text { AS N) }\end{array}$ & $\begin{array}{l}\text { NITRO- } \\
\text { GEN, } \\
\text { NITRIIE } \\
\text { TOTAL } \\
\text { (IWG } / L \\
\text { AS N) }\end{array}$ & $\begin{array}{l}\text { NITRO- } \\
\text { GEN, } \\
\text { NO2 +NDB3 } \\
\text { TOTAL } \\
\text { (MG } A \\
\text { AS MI) }\end{array}$ & $\begin{array}{l}\text { NITRQ- } \\
\text { GEN, } \\
\text { ATNONIA } \\
\text { TOTAL } \\
\text { (NAG } \\
\text { ASN) }\end{array}$ & $\begin{array}{l}\text { NITRO- } \\
\text { GEN, } \\
\text { ORGANIC } \\
\text { TOTAL } \\
\text { (NGG } / \text { L } \\
\text { AS N) }\end{array}$ & $\begin{array}{l}\text { NITRO- } \\
\text { GEN, AM- } \\
\text { MONIA + } \\
\text { ORGANIC } \\
\text { TOTAL } \\
\text { (MG/L } \\
\text { AS N) }\end{array}$ & $\begin{array}{l}\text { NJTRD- } \\
\text { GEN, } \\
\text { TOTAL } \\
\text { (MG } / L \\
\text { AS N }\end{array}$ & $\begin{array}{c}\text { NITRO- } \\
\text { GEN, } \\
\text { TOTAL } \\
\text { (MG } / \text { A } \\
\text { AS \$03) }\end{array}$ & $\begin{array}{c}\text { PHOS- } \\
\text { PHORUS, } \\
\text { TOTAL } \\
\text { (MG/L } \\
\text { AS P) }\end{array}$ \\
\hline OCT . 1976 & & & & & & & & & & & \\
\hline $\begin{array}{l}20 . . . \\
20 . .\end{array}$ & $\begin{array}{l}1630 \\
1632\end{array}$ & $\begin{array}{l}1.0 \\
5.0\end{array}$ & $\begin{array}{l}.00 \\
.00\end{array}$ & .01 & $\begin{array}{l}.01 \\
.01\end{array}$ & $\begin{array}{r}.08 \\
.08\end{array}$ & $\begin{array}{l}.51 \\
.44\end{array}$ & $\begin{array}{l}.59 \\
.52\end{array}$ & .60 & $\begin{array}{l}2.7 \\
2.3\end{array}$ & $\begin{array}{l}.100 \\
.110\end{array}$ \\
\hline $\begin{array}{l}17 \ldots \\
17 \ldots \\
F E B\end{array}$ & $\begin{array}{l}1230 \\
1232 \\
7\end{array}$ & $\begin{array}{l}1.0 \\
6.0\end{array}$ & .01 & $\begin{array}{l}.00 \\
.00\end{array}$ & .01 & $\begin{array}{r}.05 \\
.03\end{array}$ & $\begin{array}{l}.71 \\
.53\end{array}$ & $\begin{array}{l}.76 \\
.56\end{array}$ & .77 & $\begin{array}{l}3.4 \\
2.5\end{array}$ & $\begin{array}{l}.160 \\
.160\end{array}$ \\
\hline $\begin{array}{l}02 \ldots \\
02 \ldots \\
\text { JuN }\end{array}$ & $\begin{array}{l}1215 \\
1217\end{array}$ & $\begin{array}{l}1.0 \\
5.0\end{array}$ & .00 & .01 & .01 & .08 & $\begin{array}{l}.08 \\
.52\end{array}$ & $\begin{array}{l}.16 \\
.59\end{array}$ & .17 & 2.80 & $\begin{array}{l}.150 \\
.170\end{array}$ \\
\hline $\begin{array}{l}22 . . . \\
22 . .\end{array}$ & $\begin{array}{l}1110 \\
1112\end{array}$ & $\begin{array}{l}1.0 \\
7.0\end{array}$ & $\begin{array}{l}.00 \\
.01\end{array}$ & $\begin{array}{l}.02 \\
.02\end{array}$ & $\begin{array}{r}.02 \\
.03\end{array}$ & .05 & $\begin{array}{l}.54 \\
.49\end{array}$ & $\begin{array}{l}.59 \\
.56\end{array}$ & $\begin{array}{r}.61 \\
.59\end{array}$ & $\begin{array}{l}2.7 \\
2.6\end{array}$ & $\begin{array}{l}.090 \\
.110\end{array}$ \\
\hline $\begin{array}{l}24 \ldots \\
24 \ldots . .\end{array}$ & $\begin{array}{l}1215 \\
1217\end{array}$ & $\begin{array}{l}1.0 \\
5.0\end{array}$ & .01 & $\begin{array}{l}02 \\
.01\end{array}$ & $\begin{array}{l}.03 \\
.03\end{array}$ & $\begin{array}{l}.00 \\
.00\end{array}$ & $\begin{array}{r}.00 \\
.04\end{array}$ & .00 & .03 & $\begin{array}{r}.10 \\
.30\end{array}$ & $\begin{array}{l}.030 \\
.040\end{array}$ \\
\hline $\begin{array}{l}07 \ldots \\
07 \ldots\end{array}$ & $\begin{array}{l}1140 \\
1141\end{array}$ & $\begin{array}{l}1.0 \\
5.0\end{array}$ & $\begin{array}{l}.05 \\
.05\end{array}$ & $\begin{array}{l}.00 \\
.01\end{array}$ & .05 & .04 & 2.1 & 2.1 & $\begin{array}{l}2.2 \\
.64\end{array}$ & $\begin{array}{l}9.5 \\
2.8\end{array}$ & $\begin{array}{l}.010 \\
.010\end{array}$ \\
\hline
\end{tabular}




\begin{tabular}{|c|c|c|c|c|c|c|c|c|c|c|c|c|}
\hline DATE & TIME & $\begin{array}{l}\text { SAMP } \\
\text { LING } \\
\text { DEPTH } \\
\text { (FT) }\end{array}$ & $\begin{array}{l}\text { OXYGEN } \\
\text { DEMAND, } \\
\text { BIOCHEM } \\
\text { UHINHIS } \\
5 \text { OAY } \\
\text { (NAL } / L)\end{array}$ & $\begin{array}{l}\text { NITRO- } \\
\text { GER, } \\
\text { NI TRATE } \\
\text { TOTAL } \\
\text { (MGA } \\
\text { AS N) }\end{array}$ & $\begin{array}{l}\text { NITRO- } \\
\text { GEN, } \\
\text { MITRITE } \\
\text { TOTAL } \\
\text { (MG } / L \\
\text { AS N }\end{array}$ & $\begin{array}{l}\text { NITRO- } \\
\text { GEN, } \\
\text { NO2 +NO3 } \\
\text { TOTAL } \\
\text { (NGG } / 2 \\
\text { AS N) }\end{array}$ & $\begin{array}{l}\text { NITRO- } \\
\text { GEN, } \\
\text { AMNONIA } \\
\text { TOTAL } \\
\text { (MG/L } \\
\text { AS N) }\end{array}$ & $\begin{array}{l}\text { NITRO- } \\
\text { CEN, } \\
\text { ORGANIC } \\
\text { TOTAL } \\
\text { (MGA } \\
\text { AS N) }\end{array}$ & $\begin{array}{l}\text { NITRO- } \\
\text { GER, AM- } \\
\text { MONIA + } \\
\text { ORGANIC } \\
\text { TOTRL } \\
\text { (MG } A \\
\text { AS N) }\end{array}$ & $\begin{array}{l}\text { NITRO- } \\
\text { GEN, } \\
\text { TOTAL } \\
\text { (NG/L } \\
\text { RS RI) }\end{array}$ & $\begin{array}{l}\text { MITRO- } \\
\text { GEN, } \\
\text { TOJAL } \\
\text { (MG } A \text { L } \\
\text { AS NO3) }\end{array}$ & $\begin{array}{l}\text { PHOS- } \\
\text { PHORUS, } \\
\text { TOTAL } \\
\text { (HWG } / L \\
\text { AS P) }\end{array}$ \\
\hline $\begin{array}{l}\text { OEC , } \\
\text { DB... } \\
\text { APR. }\end{array}$ & $7^{6} 1000$ & 1.0 & 2.0 & .23 & .00 & .23 & .06 & 1.1 & 1.2 & 1.4 & 6.3 & .26 \\
\hline $\begin{array}{l}26 . \cdots \\
M A Y\end{array}$ & 0900 & - & - & .55 & .01 & .56 & .08 & .65 & .74 & 1.3 & 5.8 & .10 \\
\hline $\begin{array}{l}04 \ldots \\
10 . . \\
16 . . \\
19 . .\end{array}$ & $\begin{array}{l}1600 \\
1540 \\
1615 \\
1345\end{array}$ & $\begin{array}{r}-- \\
1.0 \\
--\end{array}$ & $\begin{array}{r}2.5 \\
=- \\
=-\end{array}$ & $\begin{array}{l}.64 \\
.54 \\
.61 \\
.71\end{array}$ & $\begin{array}{l}.00 \\
.00 \\
.00 \\
.00\end{array}$ & $\begin{array}{l}.64 \\
.54 \\
.61 \\
.71\end{array}$ & $\begin{array}{l}.01 \\
.04 \\
.04 \\
.02\end{array}$ & $\begin{array}{l}.21 \\
.52 \\
.65 \\
.31\end{array}$ & $\begin{array}{l}.22 \\
.56 \\
.59 \\
.33\end{array}$ & $\begin{array}{l}.86 \\
1.1 \\
1.3 \\
1.0\end{array}$ & $\begin{array}{l}3.8 \\
4.9 \\
5.8 \\
4.6\end{array}$ & $\begin{array}{l}.180 \\
.210 \\
.180 \\
.170\end{array}$ \\
\hline $\begin{array}{l}\text { JUN } \\
01 \ldots \\
16 . .\end{array}$ & $\begin{array}{l}0745 \\
1500\end{array}$ & -- & $=$ & .00 & .01 & $\begin{array}{l}.01 \\
.08\end{array}$ & $\begin{array}{l}.02 \\
.02\end{array}$ & $\begin{array}{l}.63 \\
.76\end{array}$ & $\begin{array}{l}.65 \\
.80\end{array}$ & $\begin{array}{l}.66 \\
.88\end{array}$ & $\begin{array}{l}2.9 \\
3.9\end{array}$ & .1 \\
\hline
\end{tabular}

295855094485200 LINE 604 SITE 70

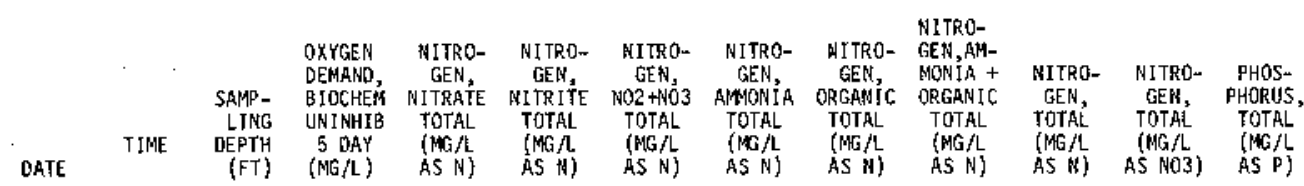

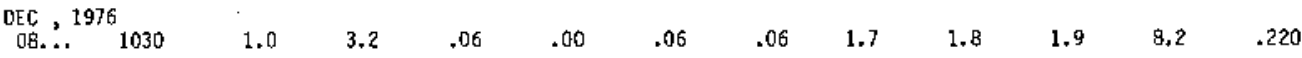

295424094454500 LINE 620 SITE 20

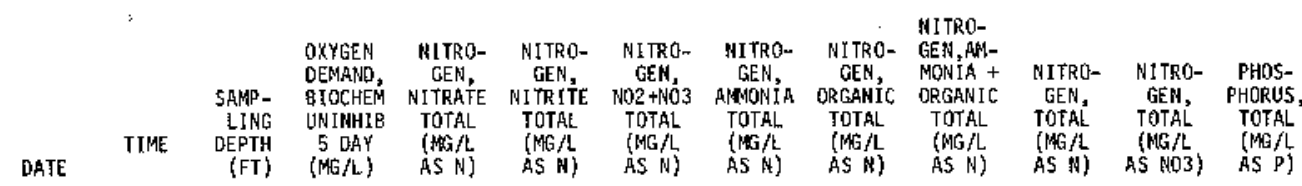

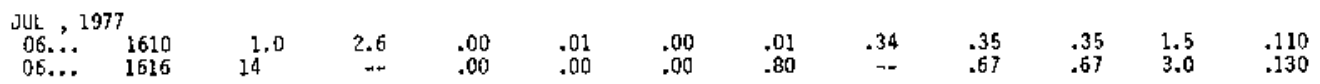

295259094442400 LINE 630 5ITE 20

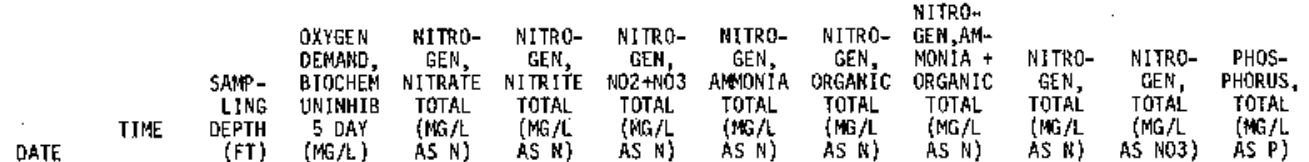

DEC , 1976

Q8... 1235

$08 \ldots .1237$

$10 . . \quad 0900$

$28 . . . \quad 1330$

$\begin{array}{rrr}1.0 & 2.0 & .16 \\ 18 & -16 & .16 \\ 1.0 & -. & .22 \\ 1.0 & 1.6 & .33 \\ 1.0 & -- & .32\end{array}$

$\begin{array}{ll}.01 & .17 \\ .01 & .17 \\ .01 & .23 \\ .01 & .34 \\ .01 & .33\end{array}$

$\begin{array}{ll}.17 & .02 \\ .17 & .03 \\ .23 & .05 \\ .34 & .02 \\ .33 & .01\end{array}$

$\begin{array}{lll}1.1 & 1.1 & 1.3 \\ 1.2 & 1.2 & 1.4 \\ .69 & .74 & .97 \\ .61 & .63 & .97\end{array}$

$\begin{array}{ll}5.6 & .190 \\ 6.1 & .220 \\ 4.3 & .160 \\ 4.3 & .150 \\ 4.2 & .140\end{array}$

295310094453200 LINE 630 SITE 4D

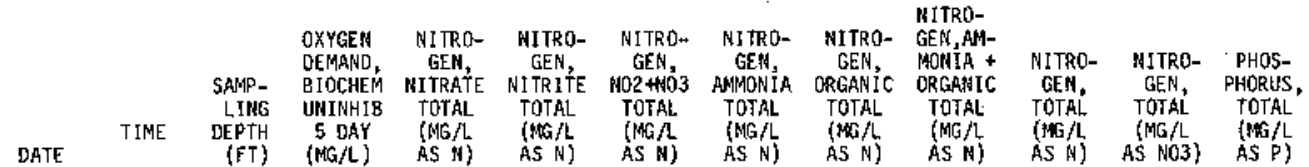

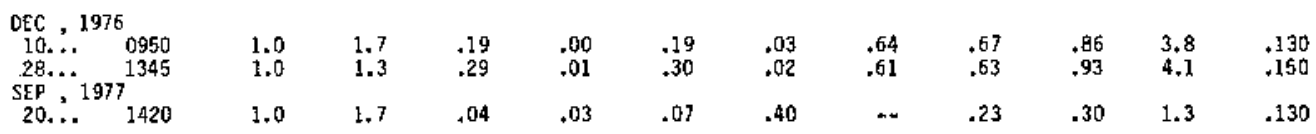


295034094450700 LIIAE 650 SITE 20

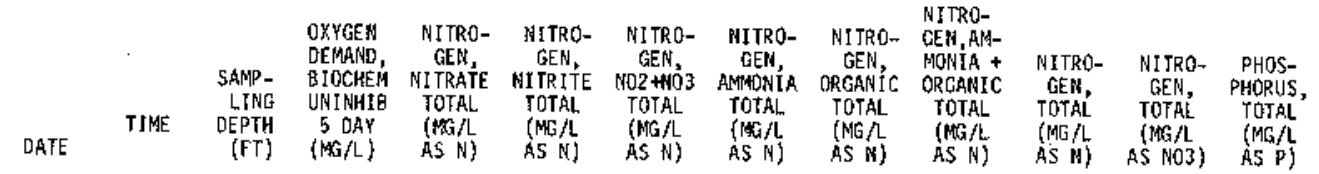

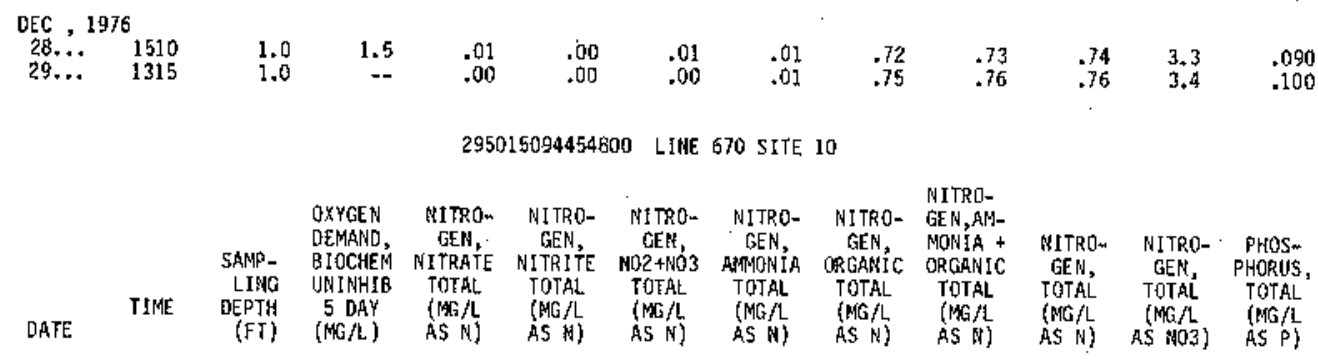

DEC , 1976

$08 .+1976$

$08 . . . \quad 1437$

$10 . .11010$

$13 \ldots .1450$

29... 1510

$$
\begin{array}{rrrr}
1.0 & - & .05 & .00 \\
9.0 & - & .05 & .01 \\
1.0 & - & .10 & .01 \\
1.0 & -- & .15 & .01 \\
1.0 & 1.9 & .15 & .01 \\
1.0 & -- & .24 & .01
\end{array}
$$

$\begin{array}{ll}.05 & .02 \\ .06 & .03 \\ .11 & .05 \\ .16 & .04 \\ .16 & .00 \\ .25 & .01\end{array}$

$\begin{array}{cccccc}.02 & 1.2 & 1.2 & 1.2 & 5.5 & .130 \\ .03 & 1.4 & 1.4 & 1.5 & 6.5 & .130 \\ .05 & .79 & .84 & .95 & 4.2 & .120 \\ .04 & .72 & .76 & .92 & 4.1 & .130 \\ .00 & .72 & .72 & .88 & 3.9 & .130 \\ .01 & .74 & .75 & 1.0 & 4.4 & .130 \\ .03 & 1.5 & 1.5 & 1.5 & 6.7 & .170\end{array}$

$20 . . .1320$

1.0

.00

295003094480700 LINE 670 SITE 80

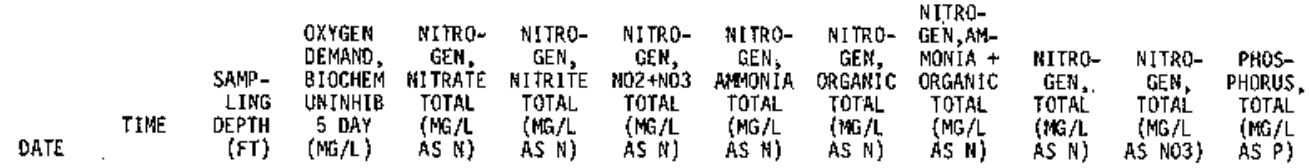

DEC, 1976

DEC. 1976

$10 \ldots 1020$

$13 . . . \quad 1500$

28... 1315

$29 \ldots 1440$

SEP , I977

$\begin{array}{llll}1.0 & -- & .03 & .01 \\ 1.0 & -- & .17 & .01 \\ 1.0 & -- & .07 & .01 \\ 1.0 & 1.9 & .14 & .01 \\ 1.0 & -. & .15 & .01\end{array}$

1.0 $\begin{array}{lr}-+ & .15 \\ -- & .00\end{array}$ $\begin{array}{ll}.01 & .99 \\ .06 & .- \\ .02 & .99 \\ .01 & .66 \\ .03 & .72\end{array}$

1.5
1.0
.03
.92
.67
.75

1.0
.21
1.0
.82
.91

$\begin{array}{ll}4.6 & .120 \\ .90 & .120 \\ 4.4 & .140 \\ 3.6 & .120 \\ 4.0 & .130\end{array}$

1.51.

$6.6 \quad .190$ 
294759094432700 LINE 680 SITE 20

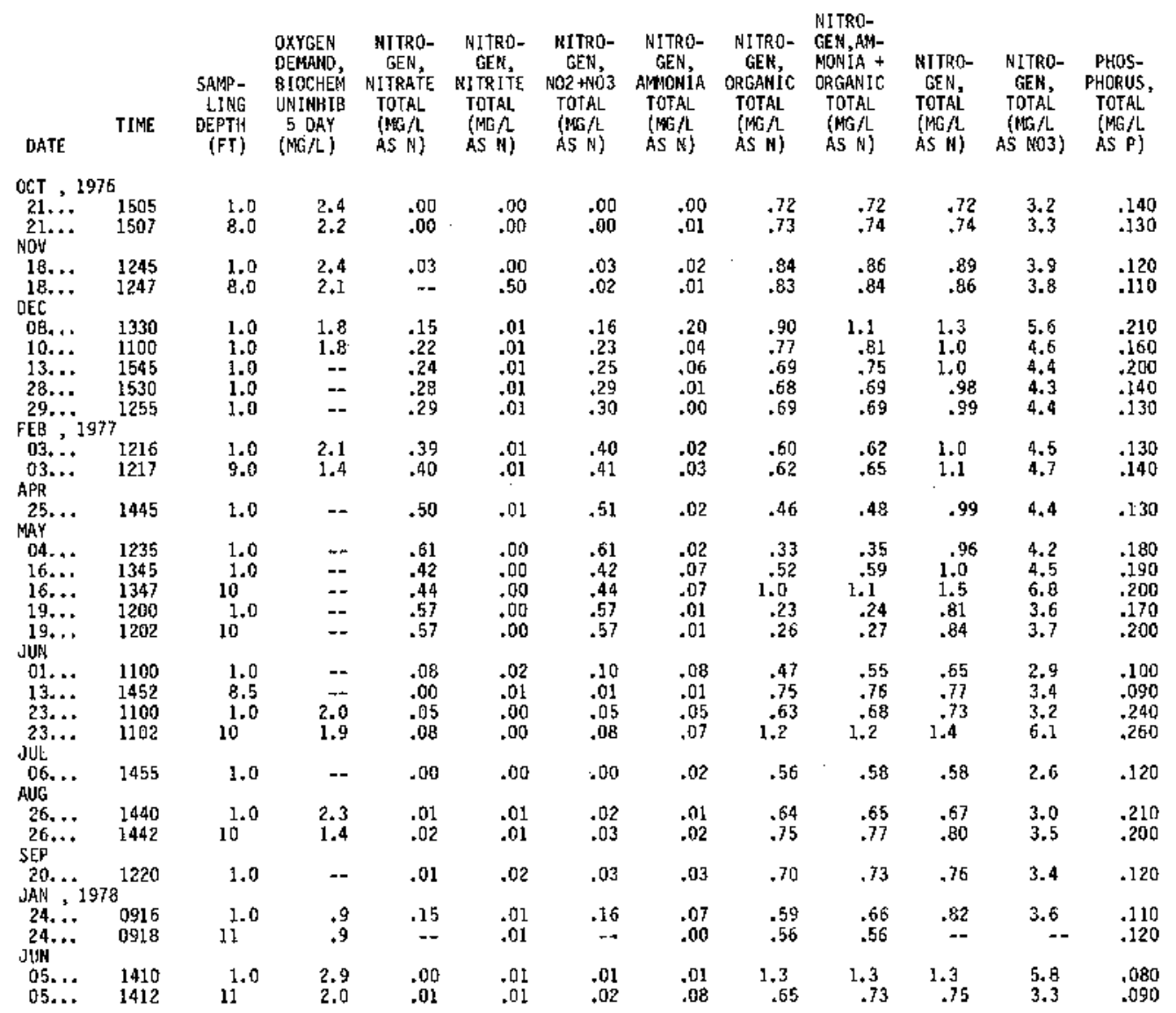

294813094471300 LINE 680 SITE 80

\begin{tabular}{|c|c|c|c|c|c|c|c|c|c|c|c|}
\hline DATE & TIME & $\begin{array}{r}\text { SAMP- } \\
\text { LING } \\
\text { DEPTH } \\
(F T)\end{array}$ & $\begin{array}{l}\text { HJTRO- } \\
\text { GEN, } \\
\text { MITRATE } \\
\text { TOTAL } \\
\text { (MG /L } \\
\text { AS N) }\end{array}$ & $\begin{array}{l}\text { IIITRO- } \\
\text { GEN, } \\
\text { NITRI IE } \\
\text { FOTAL } \\
\text { (HN } / L \\
\text { AS N) }\end{array}$ & $\begin{array}{l}\text { NITRO- } \\
\text { GEN, } \\
\text { MO2+HOB } \\
\text { TOTAL } \\
\text { (MG/L } \\
\text { AS N) }\end{array}$ & $\begin{array}{l}\text { RITRO- } \\
\text { GEN, } \\
\text { AISONIA } \\
\text { TOTAL } \\
\text { (NGA } \\
\text { AS N) }\end{array}$ & $\begin{array}{c}\text { NITRO } \\
\text { GEN, } \\
\text { ORGARIC } \\
\text { TOTAL } \\
\text { (MGA } \\
\text { AS N }\end{array}$ & $\begin{array}{l}\text { NITRO- } \\
\text { GEN,AN- } \\
\text { MONIA + } \\
\text { ORGANIC } \\
\text { TOTAL } \\
(M G G / L \\
\text { AS N) }\end{array}$ & $\begin{array}{l}\text { NITRO- } \\
\text { GENA, } \\
\text { TOTAL } \\
\text { (NG/L } \\
\text { AS NS) }\end{array}$ & $\begin{array}{c}\text { NITRO- } \\
\text { GEN, } \\
\text { TOTAL } \\
\text { (MGL } \\
\text { AS ND3) }\end{array}$ & $\begin{array}{l}\text { PHOS- } \\
\text { PHORUS, } \\
\text { TOTAL } \\
(M G A \\
\text { AS P) }\end{array}$ \\
\hline 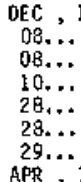 & $\begin{array}{l}6 \\
1515 \\
1517 \\
1030 \\
1300 \\
1302 \\
1550\end{array}$ & $\begin{array}{c}1.0 \\
11 \\
1.0 \\
1.0 \\
11 \\
1.0\end{array}$ & $\begin{array}{l}.06 \\
.00 \\
.22 \\
.14 \\
.14 \\
.12\end{array}$ & $\begin{array}{l}.01 \\
.01 \\
.01 \\
.01 \\
.01 \\
.01\end{array}$ & $\begin{array}{l}.07 \\
.01 \\
.23 \\
.15 \\
.15 \\
.13\end{array}$ & $\begin{array}{l}.01 \\
.01 \\
.06 \\
.00 \\
.01 \\
.01\end{array}$ & $\begin{array}{r}.91 \\
1.1 \\
.58 \\
.69 \\
.69 \\
.76\end{array}$ & $\begin{array}{r}.92 \\
1.1 \\
.64 \\
.69 \\
.70 \\
.77\end{array}$ & $\begin{array}{l}.99 \\
1.1 \\
.87 \\
.84 \\
.85 \\
.90\end{array}$ & $\begin{array}{l}4.4 \\
4.9 \\
3.9 \\
3.7 \\
3.8 \\
4.0\end{array}$ & $\begin{array}{l}.130 \\
.120 \\
.120 \\
.110 \\
.110 \\
.130\end{array}$ \\
\hline $\begin{array}{l}25 . \cdots \\
25 \ldots\end{array}$ & $\begin{array}{l}1520 \\
1524\end{array}$ & 16 & $\begin{array}{r}.50 \\
.51\end{array}$ & .02 & $\begin{array}{l}.52 \\
.53\end{array}$ & $\begin{array}{r}.06 \\
.07\end{array}$ & $\begin{array}{l}.51 \\
.79\end{array}$ & $\begin{array}{l}.57 \\
.86\end{array}$ & $\begin{array}{l}1.1 \\
1.4\end{array}$ & $\begin{array}{l}4.8 \\
6.2\end{array}$ & $\begin{array}{l}.160 \\
.200\end{array}$ \\
\hline $\begin{array}{l}13 . \ldots \\
13 \ldots \\
\text { JUL }\end{array}$ & $\begin{array}{l}1200 \\
1202\end{array}$ & $13^{1.0}$ & $\begin{array}{l}.00 \\
.00\end{array}$ & .01 & .01 & 101 & $\begin{array}{l}1.4 \\
1.5\end{array}$ & $\begin{array}{l}1.4 \\
1.5\end{array}$ & $\begin{array}{l}1.4 \\
1.5\end{array}$ & $\begin{array}{l}6.2 \\
6.7\end{array}$ & $\begin{array}{l}.180 \\
.240\end{array}$ \\
\hline $\begin{array}{l}06, \ldots \\
06 . . . \\
\text { SEP }\end{array}$ & $\begin{array}{l}1515 \\
1521\end{array}$ & $11^{1.0}$ & $\begin{array}{l}.89 \\
.00\end{array}$ & $\begin{array}{r}.01 \\
.01\end{array}$ & $\begin{array}{l}.90 \\
.01\end{array}$ & $\begin{array}{l}.07 \\
.32\end{array}$ & 1.76 & $\frac{.83}{1.4}$ & $\begin{array}{l}1.7 \\
1.4\end{array}$ & $\begin{array}{l}7.7 \\
5.2\end{array}$ & $\begin{array}{r}.190 \\
.220\end{array}$ \\
\hline $\begin{array}{l}20 \ldots \\
20 \ldots\end{array}$ & $\begin{array}{l}1250 \\
1256\end{array}$ & $11^{1.0}$ & $\begin{array}{l}.01 \\
.00\end{array}$ & .00 & $\begin{array}{l}.01 \\
.00\end{array}$ & .01 & $\begin{array}{l}1.3 \\
1.1\end{array}$ & $\begin{array}{l}1.3 \\
1.1\end{array}$ & $\begin{array}{l}1.3 \\
1.1\end{array}$ & $\begin{array}{l}5.8 \\
4.9\end{array}$ & $\begin{array}{l}.150 \\
.220\end{array}$ \\
\hline
\end{tabular}


Table 2B.--Quality of water in the Trinity-San Jacinto estuary, water year 1977+78--Continued

Nutrient Aualyses--Cont inued

294712094440200 LINE 690 SITE 40

\begin{tabular}{|c|c|c|c|c|c|c|c|c|c|c|c|c|}
\hline DATE & TIME & $\begin{array}{l}\text { SAMP- } \\
\text { LING } \\
\text { DEPTH } \\
(F T)\end{array}$ & $\begin{array}{l}\text { OXYGEN } \\
\text { OEMAND, } \\
\text { BIOCHEM } \\
\text { UNINHIB } \\
5 \text { DAY } \\
(M G / L)\end{array}$ & $\begin{array}{l}\text { NITRO- } \\
\text { CENA, } \\
\text { NITRATE } \\
\text { TDTAL } \\
\text { (MG } / \\
\text { AS N) }\end{array}$ & $\begin{array}{l}\text { MITRO- } \\
\text { GEN, } \\
\text { NITRITE } \\
\text { TOTAL } \\
\text { (MG } / L \\
\text { AS H) }\end{array}$ & $\begin{array}{l}\text { NITRO- } \\
\text { GEN, } \\
\text { MO2 +NO3 } \\
\text { TOTAL } \\
\text { (MO } / L \\
\text { AS N) }\end{array}$ & $\begin{array}{l}\text { MITRO- } \\
\text { GEN, } \\
\text { AMMONIA } \\
\text { TOTAL } \\
\text { (MG } A L \\
\text { AS } N \text { ) }\end{array}$ & $\begin{array}{l}\text { NITRO- } \\
\text { GEH, } \\
\text { ORGANIC } \\
\text { TOTAL } \\
\text { (MG } / L \\
\text { AS N) }\end{array}$ & $\begin{array}{l}\text { NITRO- } \\
\text { GEN, AM- } \\
\text { MONIA + } \\
\text { ORGANIC } \\
\text { TOTAL } \\
\text { (MG } \\
\text { AS NS) }\end{array}$ & $\begin{array}{l}\text { NITRO- } \\
\text { GEN, } \\
\text { TOTAL } \\
\text { (MGG } / L \\
\text { AS N N) }\end{array}$ & $\begin{array}{l}\text { NITRO- } \\
\text { GEN, } \\
\text { TOTAL } \\
\text { (NAS/L } \\
\text { AS NOS })\end{array}$ & $\begin{array}{l}\text { PHOS- } \\
\text { PHORUS, } \\
\text { TOTAL } \\
\text { (MG/L } \\
\text { AS P) }\end{array}$ \\
\hline
\end{tabular}

$\mathrm{DEC}, 1976$

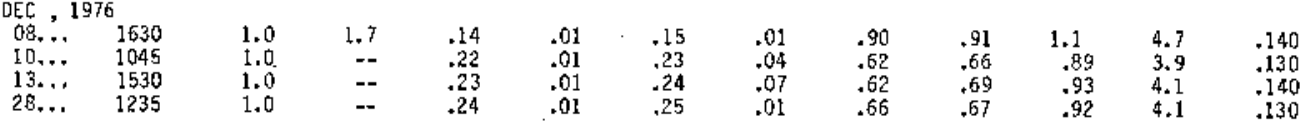

294629094465400 LINE $69051 T E 80$

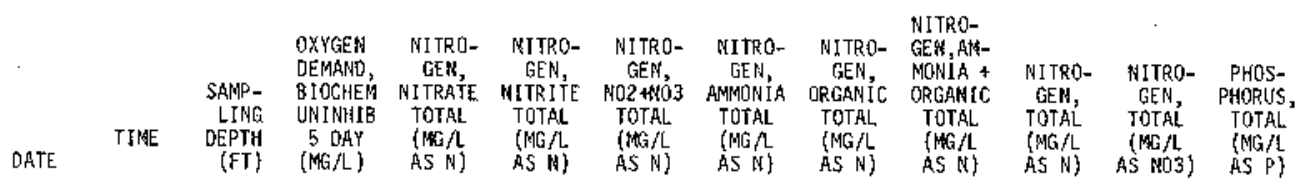

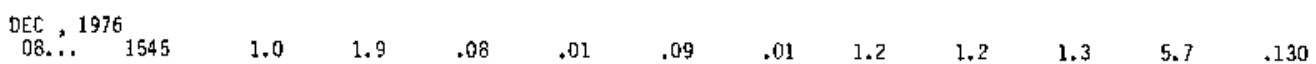

291929094393300 LINE 904 SITE 20

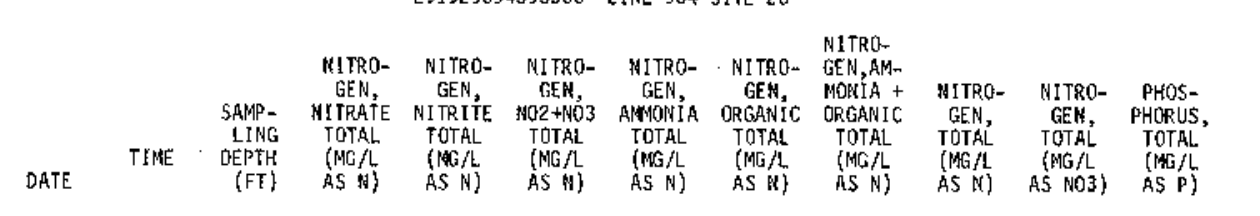

OCT , 1976

\begin{tabular}{|c|c|c|c|c|c|c|c|c|c|c|c|}
\hline $20 . \cdots$ & $\begin{array}{r}1430 \\
1436\end{array}$ & $\frac{1.0}{45^{2}}$ & .00 & $\begin{array}{l}01 \\
.01 \\
.01\end{array}$ & $\begin{array}{l}.01 \\
.02\end{array}$ & .11 & $\begin{array}{r}.31 \\
.81\end{array}$ & $\begin{array}{l}.42 \\
.94\end{array}$ & $\begin{array}{l}.43 \\
.96\end{array}$ & $\begin{array}{l}1.9 \\
4.2\end{array}$ & $\begin{array}{l}.070 \\
.170\end{array}$ \\
\hline JUN , 197 & & & & & & & & & & & \\
\hline $\begin{array}{l}22 \ldots \\
22 \ldots \\
\text { AUG }\end{array}$ & $\begin{array}{l}1415 \\
1419\end{array}$ & $\begin{array}{l}1.0 \\
45\end{array}$ & $\begin{array}{l}.00 \\
.03\end{array}$ & .01 & .01 & .04 & $\begin{array}{l}.26 \\
.59\end{array}$ & .30 &. .31 & $\begin{array}{l}1.4 \\
3.1\end{array}$ & $\begin{array}{l}.070 \\
.070\end{array}$ \\
\hline $\begin{array}{l}24 \ldots \\
24 \ldots \\
\text { JUN, }, 197\end{array}$ & $\begin{array}{l}1400 \\
8^{1404}\end{array}$ & 31.0 & .02 & $\begin{array}{l}.02 \\
.03\end{array}$ & $\begin{array}{l}.04 \\
.04\end{array}$ & .00 & $\begin{array}{l}.00 \\
.25\end{array}$ & $\begin{array}{l}.00 \\
.27\end{array}$ & $\begin{array}{l}.04 \\
.31\end{array}$ & .20 & $\begin{array}{l}.020 \\
.040\end{array}$ \\
\hline $\begin{array}{l}06 . . \\
06 . .\end{array}$ & $\begin{array}{l}0955 \\
0959\end{array}$ & $\begin{array}{l}2.0 \\
40^{-0}\end{array}$ & .09 & $\begin{array}{l}.01 \\
.01\end{array}$ & $\begin{array}{l}.10 \\
.04\end{array}$ & $\begin{array}{l}.12 \\
.16\end{array}$ & $\begin{array}{l}1.5 \\
.70\end{array}$ & $\begin{array}{l}1.6 \\
.86\end{array}$ & $\begin{array}{l}1.7 \\
.90\end{array}$ & $\begin{array}{l}7.5 \\
4.0\end{array}$ & $\begin{array}{l}.050 \\
.020\end{array}$ \\
\hline
\end{tabular}


Table 2C.--Quality of water in the Trinity-San Jacinto estuary, water years 1977-78 Chemical Analyses

(FT = feet; MICROMtHOS = micromhos per centimeter at $25^{\circ}$ Celsius; MG $/ \mathrm{L}=$ milligrams per 1 fter; AC-FT = acre-feet)

294255095011300 LINE 180 SITE 20

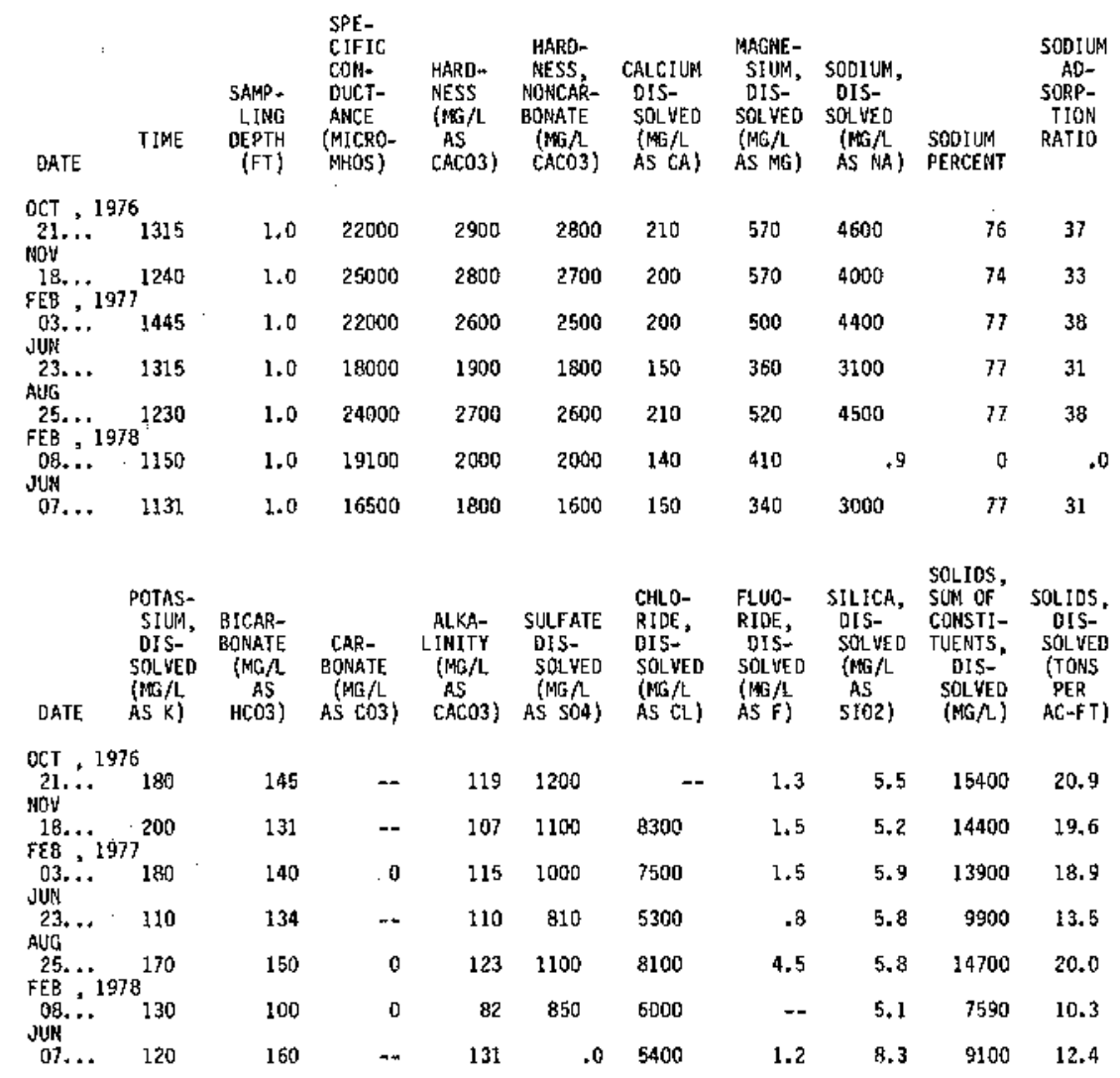

294416094433300 LINE 220 SITE 40

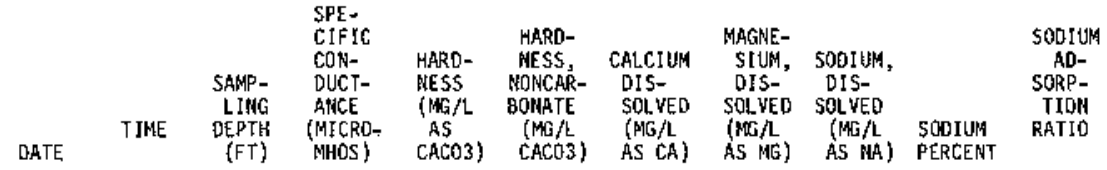

DEC, 1976

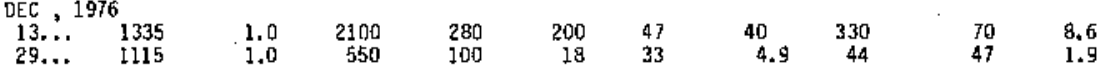

\begin{tabular}{|c|c|c|c|c|c|c|c|c|c|c|}
\hline DATE & $\begin{array}{l}\text { POTAS- } \\
\text { SIUM, } \\
\text { DIS- } \\
\text { SOLYED } \\
\text { (MG /L } \\
\text { AS X) }\end{array}$ & $\begin{array}{l}\text { BICAR- } \\
\text { BONRTE } \\
\text { (MG } / L \\
\text { AS } \\
\text { HCO3) }\end{array}$ & $\begin{array}{l}\text { CAR- } \\
\text { BONATE } \\
\text { (MG } / L \\
\text { AS CO3) }\end{array}$ & $\begin{array}{l}\text { ALKA- } \\
\text { LIAITY } \\
\text { (MGA } A \\
\text { AS } \\
\text { CACO3) }\end{array}$ & $\begin{array}{l}\text { SULFATE } \\
\text { OIS- } \\
\text { SOLVED } \\
\text { (MG/L } \\
\text { AS 5O4) }\end{array}$ & $\begin{array}{l}\text { CHEO- } \\
\text { RIOE, } \\
\text { DIS- } \\
\text { SOLVED } \\
\text { (MG } / L \\
\text { AS CL) }\end{array}$ & $\begin{array}{l}\text { FLUO- } \\
\text { RIDE, } \\
\text { OIS- } \\
\text { SOLVED } \\
\text { (UTS } / L \\
\text { AS F) }\end{array}$ & $\begin{array}{l}\text { SILICA, } \\
\text { D1S- } \\
\text { SOL WED } \\
\text { (MG } / L \\
\text { AS } \\
\text { SIO2) }\end{array}$ & $\begin{array}{l}\text { SOLIDS, } \\
\text { SUA OF } \\
\text { CONSTI- } \\
\text { TUENTS, } \\
\text { DIS- } \\
\text { SOLVED } \\
\text { (MG/L) }\end{array}$ & $\begin{array}{c}\text { SOLIDS, } \\
\text { DIS- } \\
\text { SOLVEO } \\
\text { (TOUS } \\
\text { PER } \\
\text { AC-FT) }\end{array}$ \\
\hline
\end{tabular}

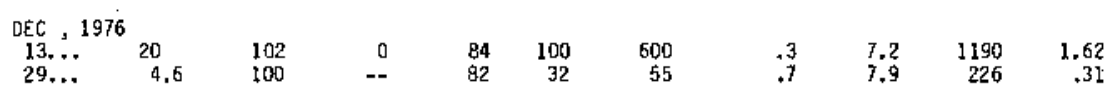

294322094430700 L.INE 222 SITE 40

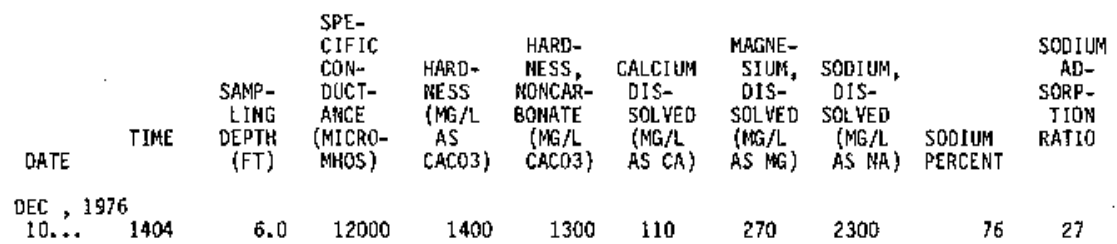


Table 2C. -Quality of water in the Trinity-San Jacinto estuary, water years 1977-78--Continued Chemical Andlyses--Continued

294322094430700 LINE 222 SITE 40--Continued

\begin{tabular}{|c|c|c|c|c|c|c|c|c|}
\hline $\begin{array}{l}\text { POTAS- } \\
\text { SIUM, } \\
\text { OIS- } \\
\text { SOLVED } \\
\text { (MG } / L \\
\text { AS K) }\end{array}$ & $\begin{array}{c}\text { BICAR- } \\
\text { BONATE } \\
\text { (MG/L } \\
\text { AS } \\
\text { HCO })\end{array}$ & $\begin{array}{l}\text { ALKA- } \\
\text { LINITY } \\
\text { (MGAL } \\
\text { AS. } \\
\text { CACO3) }\end{array}$ & $\begin{array}{l}\text { SULFATE } \\
\text { OIS- } \\
\text { SOL VED } \\
\text { (WA /L } \\
\text { AS SO4) }\end{array}$ & $\begin{array}{l}\text { CHLD- } \\
\text { RIDE, } \\
\text { DIS- } \\
\text { SOLVED } \\
\text { (MG } / L \\
\text { AS CL) }\end{array}$ & $\begin{array}{l}\text { FLUO- } \\
\text { RIDE, } \\
\text { OIS- } \\
\text { SOLUED } \\
\text { (WTA } / \\
\text { AS F) }\end{array}$ & $\begin{array}{l}\text { SILICA, } \\
\text { OIS- } \\
\text { SOL WED } \\
\text { (MG } / \text { } \\
\text { AS } \\
\text { SIOL) }\end{array}$ & $\begin{array}{l}\text { SOLIDS, } \\
\text { SUM OF } \\
\text { CORSTI- } \\
\text { TUENTS, } \\
\text { DIS- } \\
\text { SOL VED } \\
\text { (MG } / \text { ) }\end{array}$ & $\begin{array}{c}\text { SOLIDS, } \\
\text { DI5- } \\
\text { SOLVEO } \\
\text { \{TONS } \\
\text { PER } \\
\text { AC-FT) }\end{array}$ \\
\hline
\end{tabular}

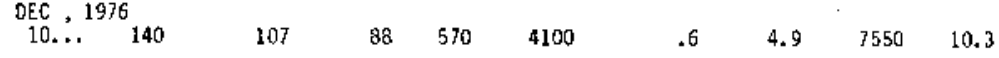

294313094460800 LINE 230 SITE 60

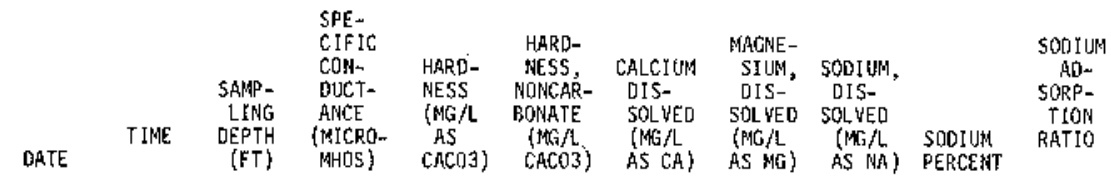

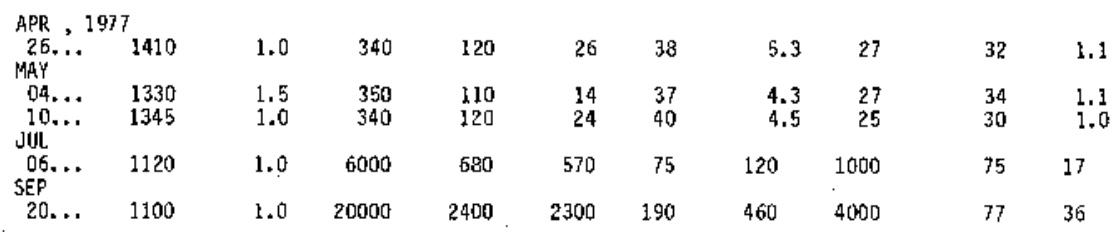

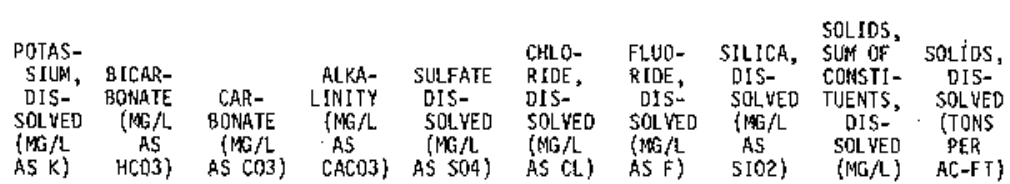

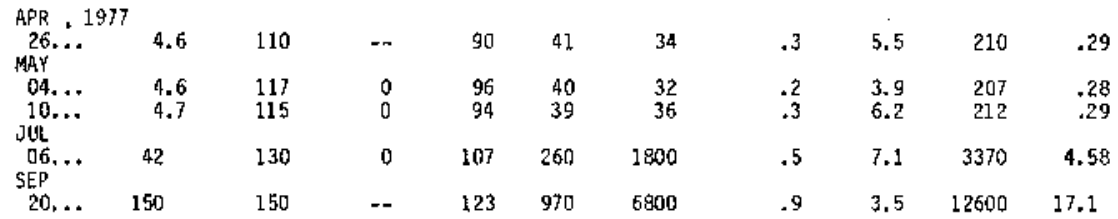

293650094514800 LIRE 260 SITE 60

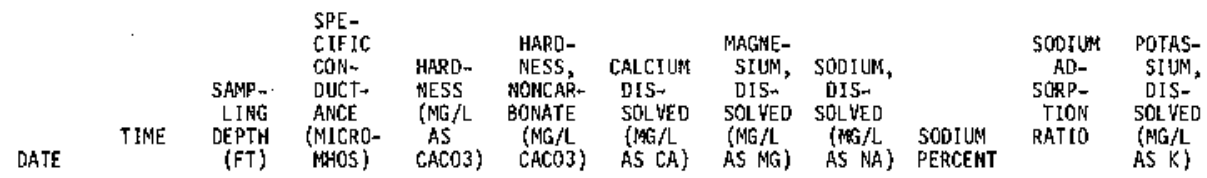

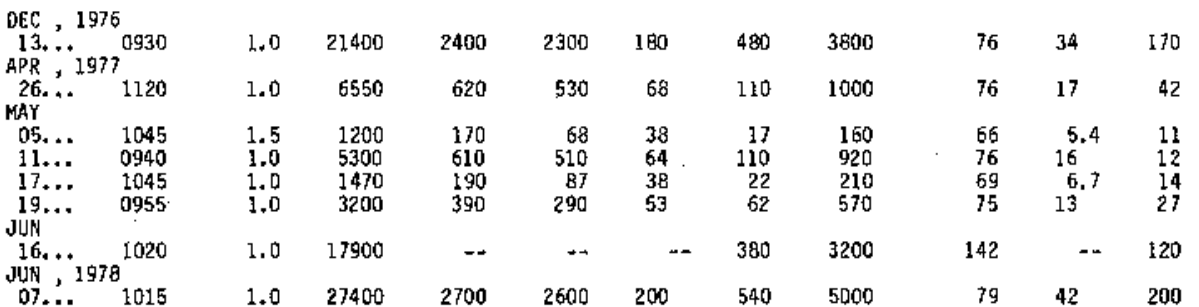


293650094514800 LINE 260 SITE 60--Continued

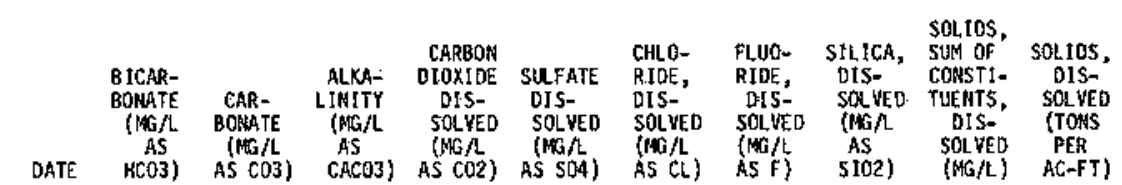

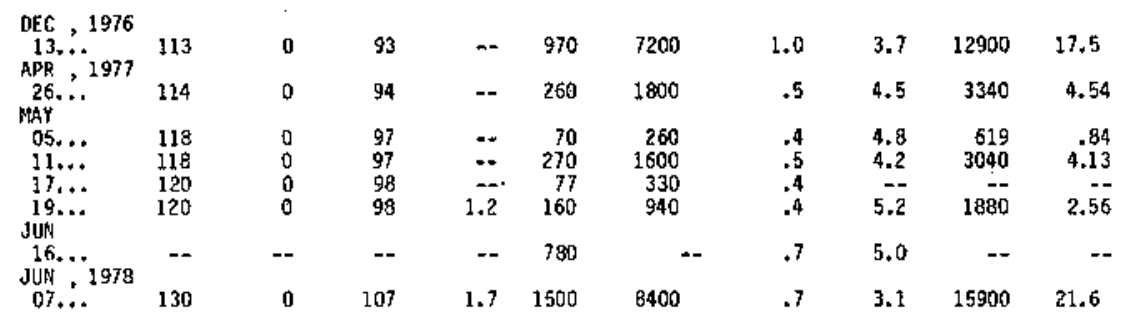

293428094553800 L.INE 340 SITE 40

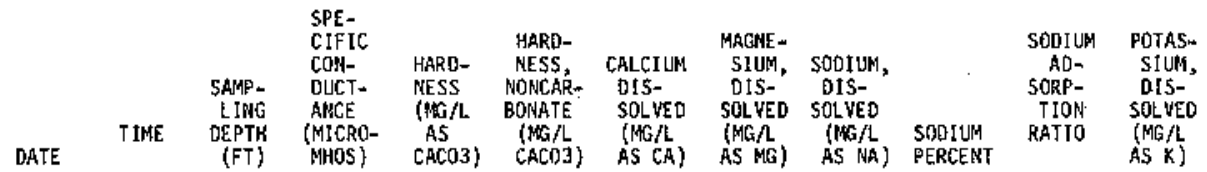

\begin{tabular}{|c|c|c|c|c|c|c|c|c|c|c|c|}
\hline $\begin{array}{l}\text { OCT }, 197 \\
21, \ldots\end{array}$ & 6 & 2. 0 & -- & 3900 & 3800 & 270 & 780 & 4400 & 70 & 31 & 230 \\
\hline L8. $\cdots$ & $7^{1035}$ & 1.0 & 28000 & 3300 & 3200 & 200 & 680 & 5400 & 77 & $41^{\circ}$ & 220 \\
\hline 23... & 1045 & 1.0 & 22100 & 2300 & 2200 & 180 & 450 & 4100 & 78 & 37 & 150 \\
\hline FEB $\cdots$ & $8^{1010}$ & 1.0 & 25000 & 2700 & 2600 & 200 & 540 & 4400 & 76 & 37 & 200 \\
\hline Ju: & 1045 & 1.0 & 22000 & 2500 & 2400 & 170 & 510 & 4700 & 77 & 36 & 170 \\
\hline 07 & 1225 & 1.0 & 30100 & 3500 & 3300 & 230 & 700 & 5800 & 77 & 43 & 230 \\
\hline
\end{tabular}

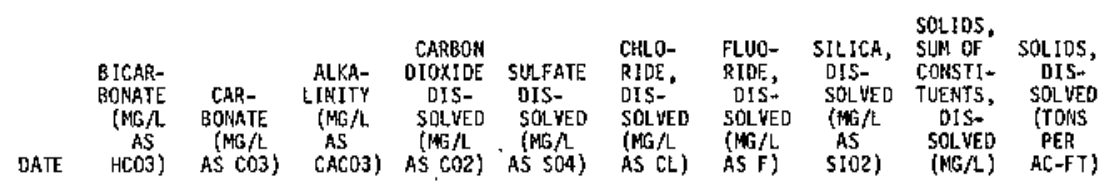

\begin{tabular}{|c|c|c|c|c|c|c|c|c|c|c|}
\hline$\underset{21 . ;}{\text { OCT }} 1976$ & 142 & 0 & 116 & - & 1200 & 11000 & 1.1 & 2.8 & 18000 & 24.5 \\
\hline 18... & 150 & 0 & 123 & -- & 1300 & 9700 & 1.1 & 3.5 & 17600 & 23.9 \\
\hline AUG & 139 & 0 & 114 & $=$ & 1000 & 7000 & .8 & 4.7 & 13000 & 17.7 \\
\hline FEB 25,1979 & 150 & 0 & 123 & $\cdots$ & 1200 & 2400 & .7 & 5.4 & 15000 & 20.4 \\
\hline JUN & 120 & 0 & 98 & $=$ & 1000 & 7800 & .7 & 3.9 & 13800 & 18.8 \\
\hline $07 \ldots$ & 140 & 0 & 115 & 1.8 & 980 & 11000 & .9 & 2.1 & 19000 & 25.8 \\
\hline
\end{tabular}

293133094501400 LINE 350 SITE 30

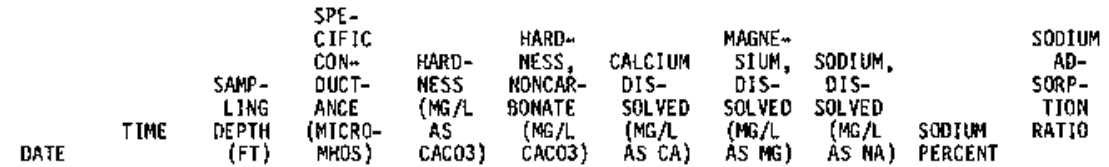

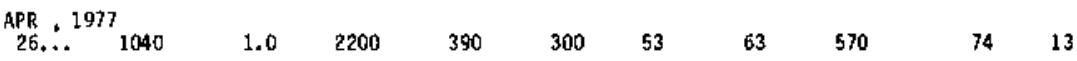

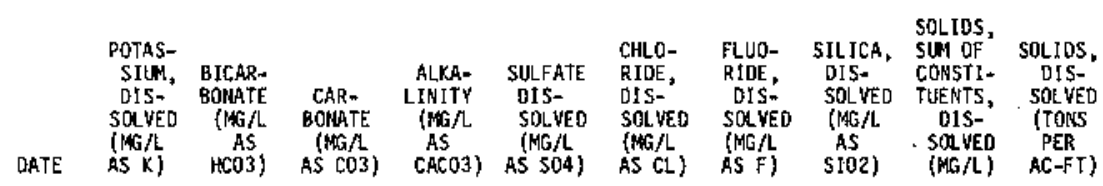

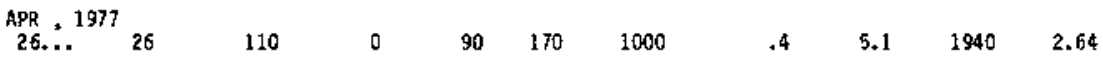


293243094345200 LINE 430 SITE 20

\begin{tabular}{|c|c|c|c|c|c|c|c|c|c|c|c|}
\hline DATE & TIME & $\begin{array}{l}\text { SAMP- } \\
\text { LING } \\
\text { DFPTH } \\
(F T)\end{array}$ & $\begin{array}{l}\text { SPE- } \\
\text { CIFIC } \\
\text { CON- } \\
\text { OUCT- } \\
\text { ANCE } \\
\text { (MICRO- } \\
\text { (AHOS) }\end{array}$ & $\begin{array}{l}\text { MARD - } \\
\text { MESS } \\
\text { (MA } / L \\
\text { AS } \\
\text { CACO3) }\end{array}$ & $\begin{array}{l}\text { HARD- } \\
\text { NESS, } \\
\text { NONCAR- } \\
\text { BONATE } \\
\text { (WHAL } \\
\text { CACO3) }\end{array}$ & $\begin{array}{l}\text { CALCIUM } \\
\text { DIS- } \\
\text { SOLVED } \\
\text { (MG/L } \\
\text { AS CA) }\end{array}$ & $\begin{array}{l}\text { MAGNE- } \\
\text { SIUM, } \\
\text { DIS- } \\
\text { SOLVED } \\
\text { (MG/L } \\
\text { AS MG) }\end{array}$ & $\begin{array}{l}\text { SODIUNA, } \\
\text { DIS- } \\
\text { SOLWED } \\
\text { (MG/L } \\
\text { AS NA) }\end{array}$ & $\begin{array}{l}\text { SOOIUM } \\
\text { PERCENT }\end{array}$ & $\begin{array}{l}\text { SODIOHA } \\
\text { ADD- } \\
\text { SORP- } \\
\text { TION } \\
\text { RATIO }\end{array}$ & $\begin{array}{l}\text { POTAS- } \\
\text { SIUM, } \\
\text { DIS- } \\
\text { SOL UED } \\
\text { (MEG } / L \\
\text { AS K) }\end{array}$ \\
\hline
\end{tabular}

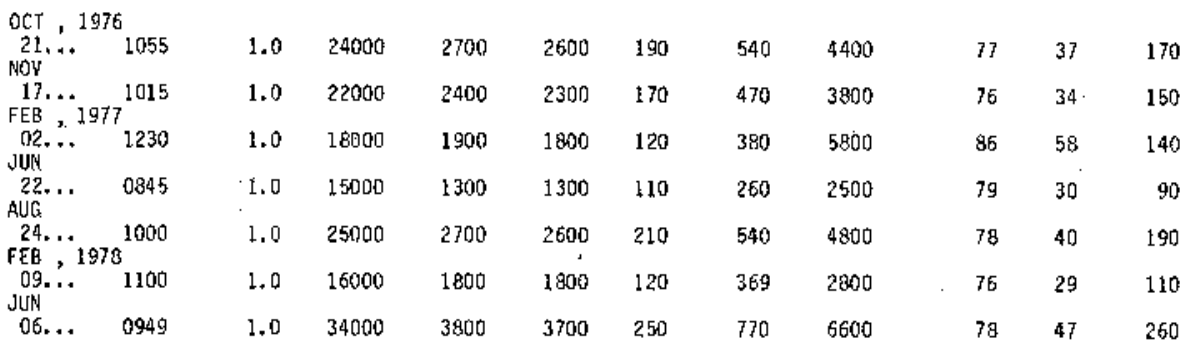

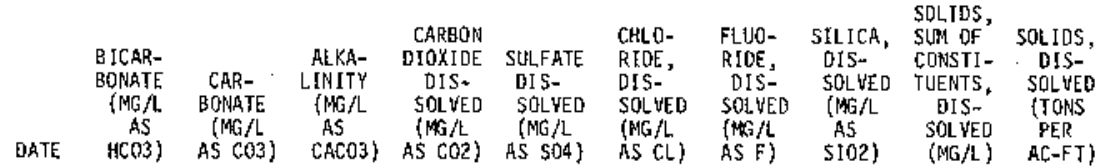

\begin{tabular}{|c|c|c|c|c|c|c|c|c|c|c|}
\hline $\begin{array}{l}\text { OCT }, 1976 \\
21 . . . \\
\text { KOW }\end{array}$ & 112 & 0 & 92 & -- & 1100 & 8100 & .7 & $\rightarrow$ & - &.- \\
\hline $17 \ldots$ & 90 & 0 & 74 & - & 930 & 6900 & .6 & 3.9 & 12500 & 17.0 \\
\hline dUN & 76 & 0 & 62 & -- & 800 & $\therefore-$ & .5 & -- & -- &.- \\
\hline AUG & 88 & 0 & 72 & -- & 610 & 4100 & .5 & $4-$ & -- & - \\
\hline $\begin{array}{l}24 \ldots \\
F E B, 1978\end{array}$ & 130 & 0 & 107 & - & 1100 & 8300 & .6 & 5.3 & 15200 & 20.7 \\
\hline JuN & 78 & 0 & 64 & - & 720 & 5300 & .4 & 2.7 & 9460 & 12.9 . \\
\hline $06 .$. & 120 & 0 & 98 & 1.9 & 1600 & 12000 & .7 & 3.9 & 21500 & 29.2 \\
\hline
\end{tabular}

293029094462800 LINE 470 SITE 60

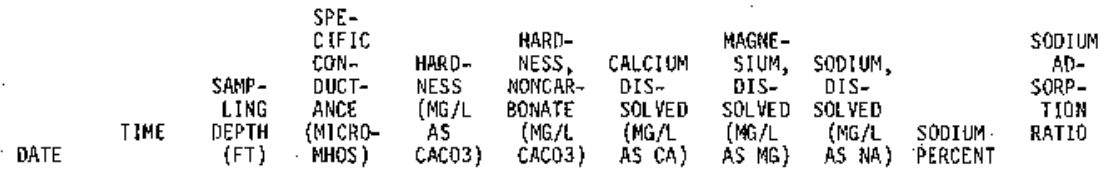

JUN , 1977

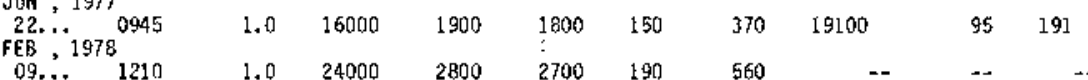

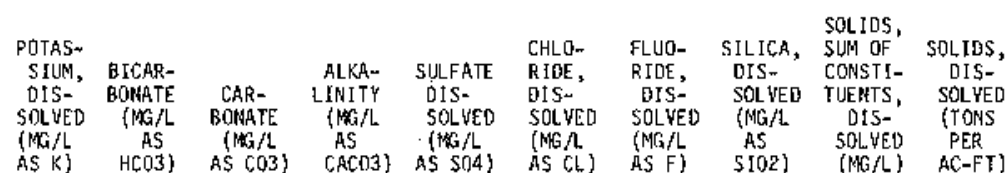

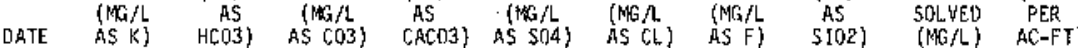

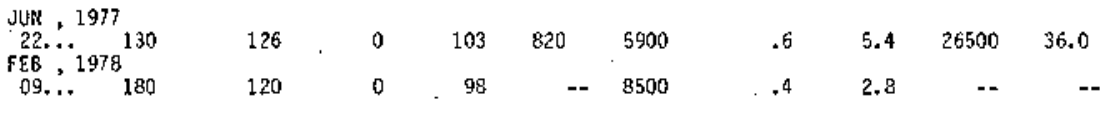
291744094531200 LINE 52151 TE 50

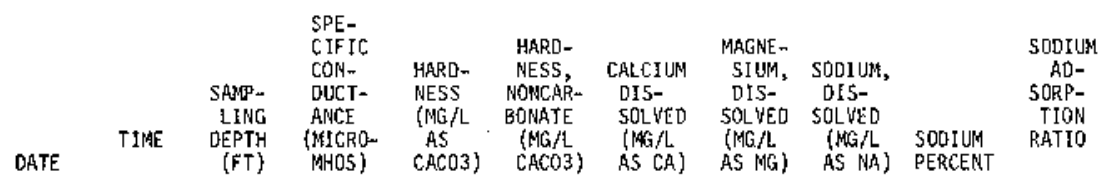

FEB , 1978.

$\begin{array}{llllllll}1.0 & 36000 & 4100 \cdot 4000: & 260 & 840 & 6700 & 78 & 45\end{array}$ 
291744094531200 LIHE 521 \$1TE 50--Continued

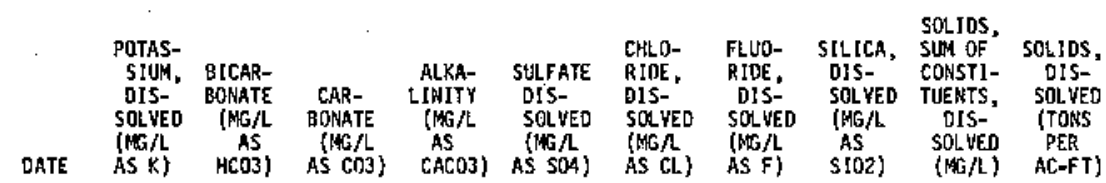

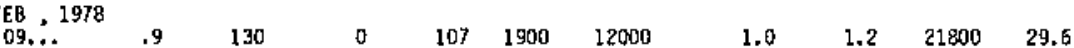

291428094575900 LINE 530 SITE 50

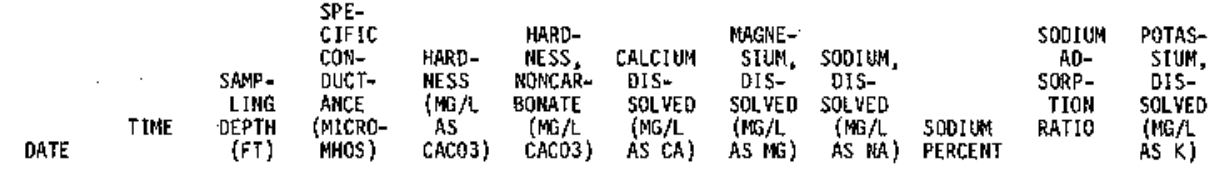

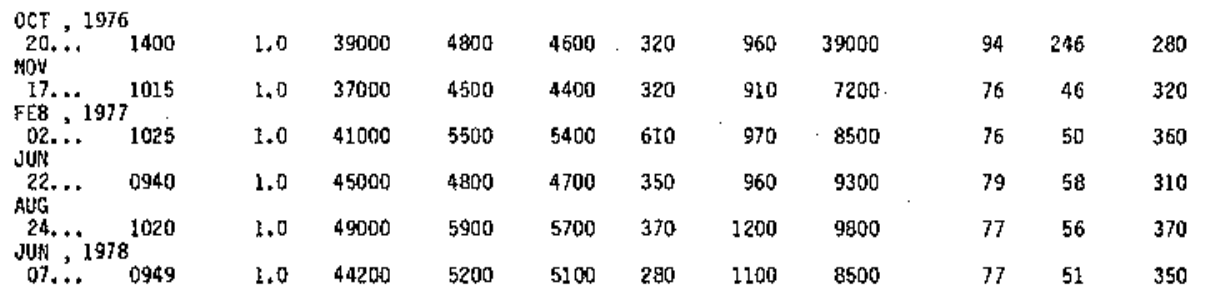

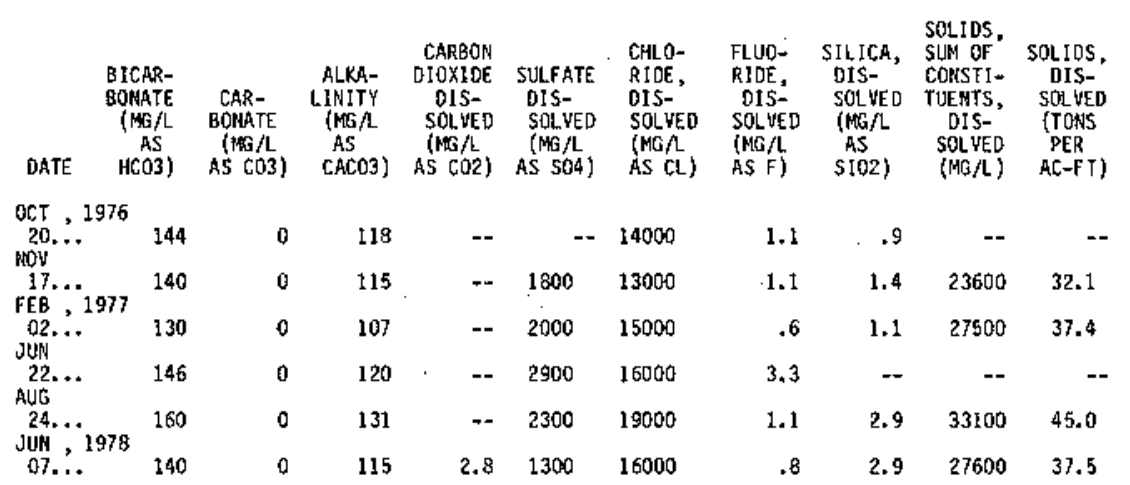

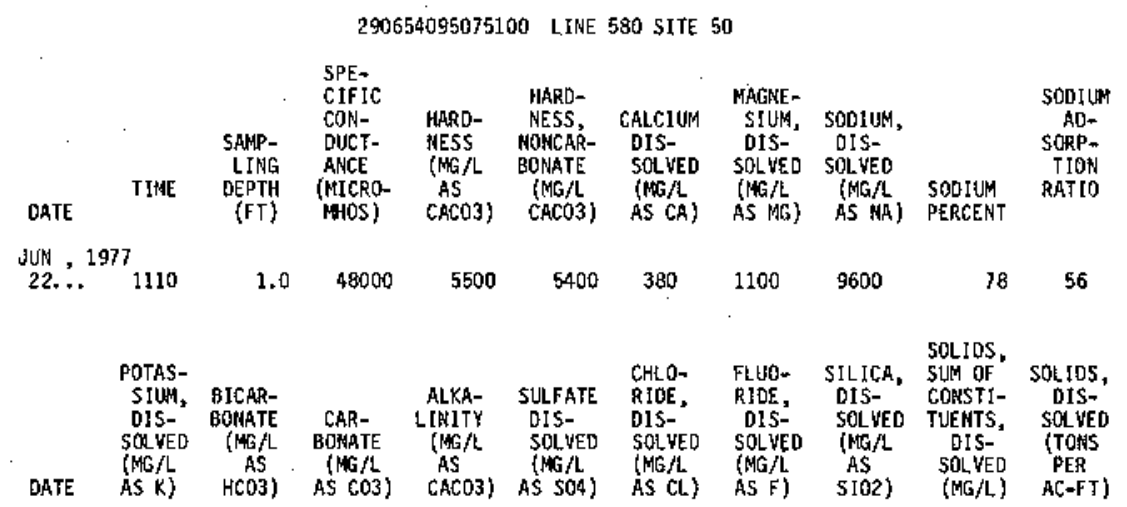


TabTe 2C,--Quality of water in the Trinity-San Jacinto estuary, water years 1977-78--Cont inued Chemical Analyses--Contínued.

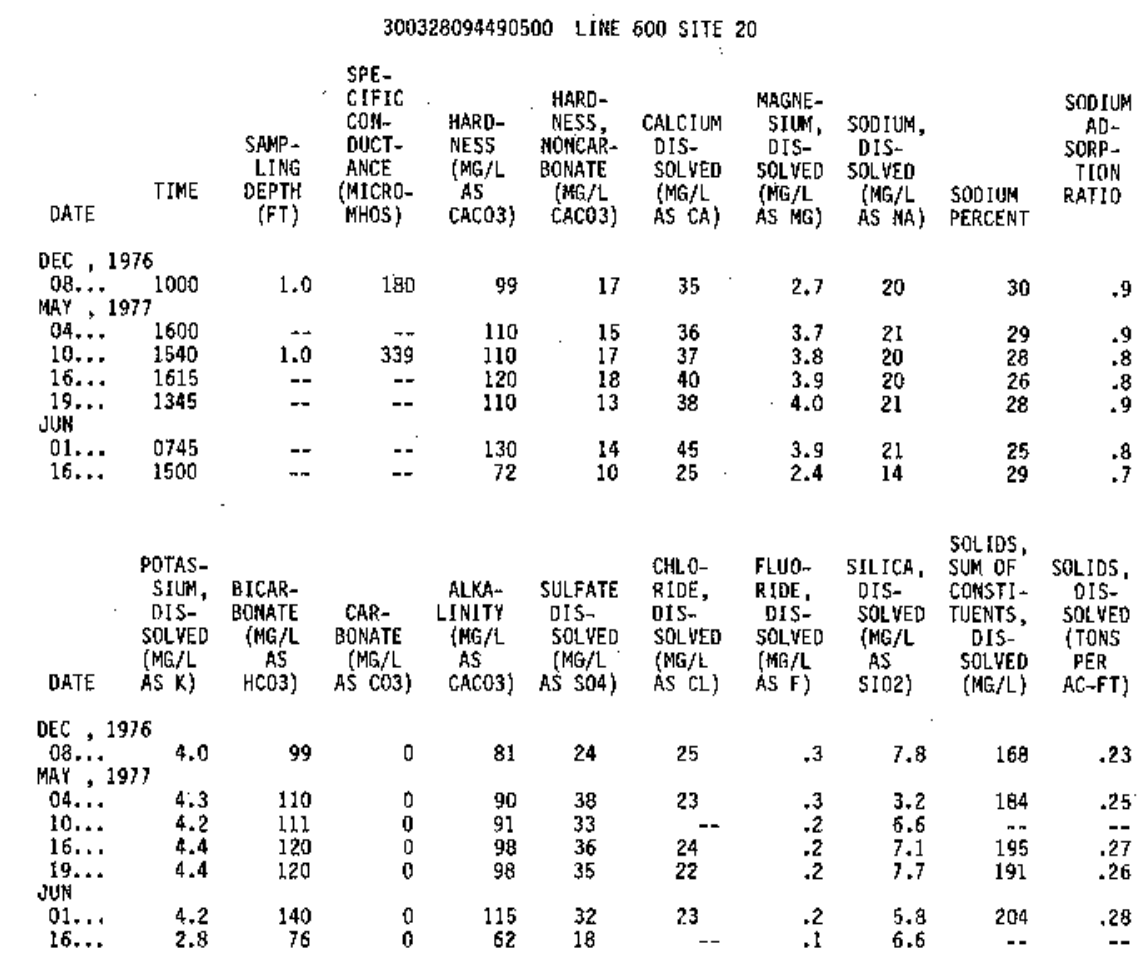

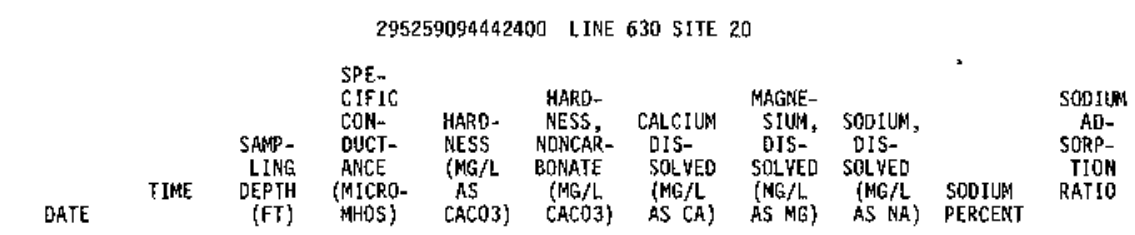

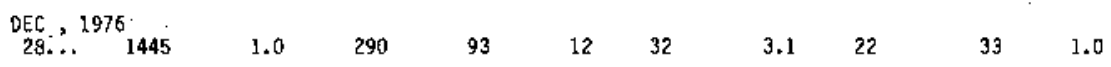

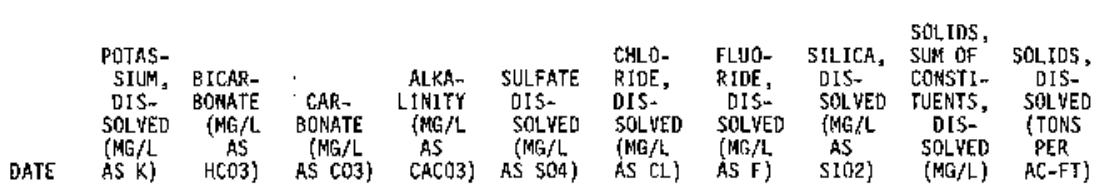

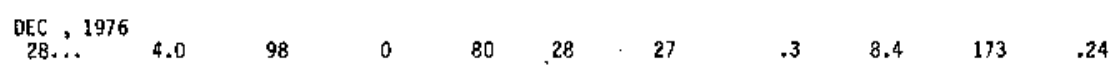

295034094471200 LINE 670 SITE 40

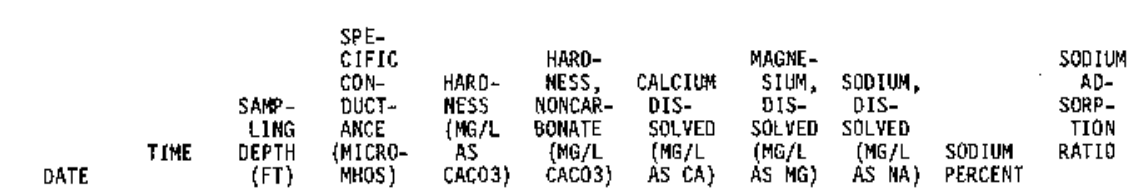

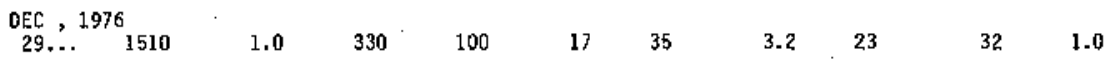

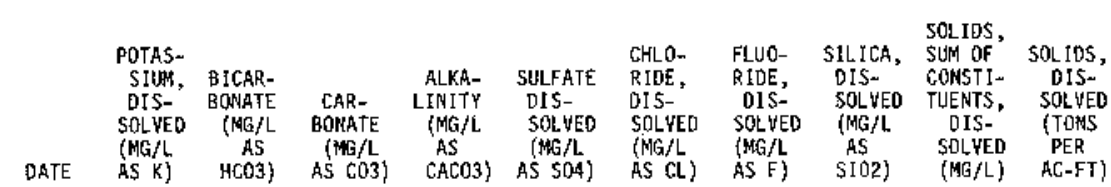

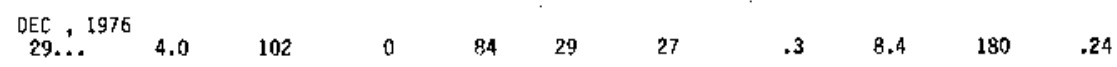


294759094432700 LINE 680 SITE 20

$$
\begin{aligned}
& \text { SPE- } \\
& \text { CIFIC HARD- }
\end{aligned}
$$

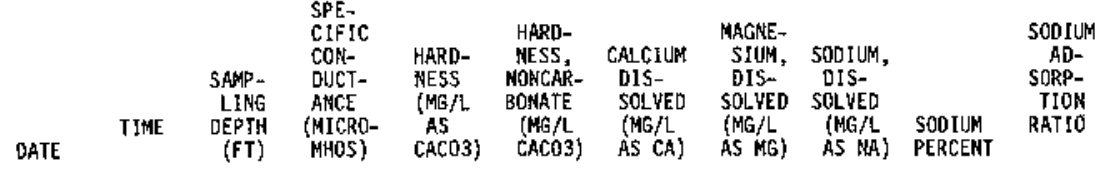

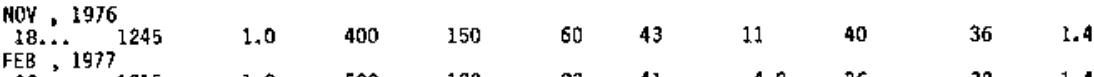

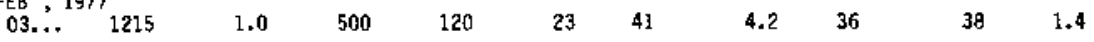

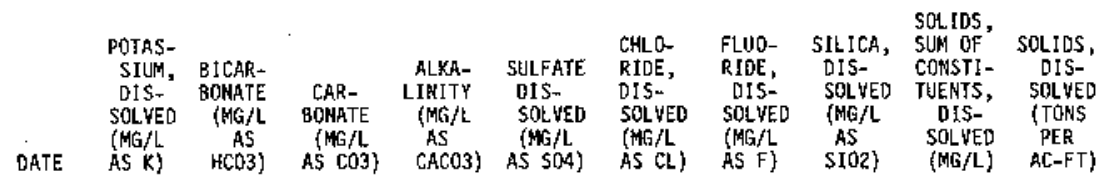

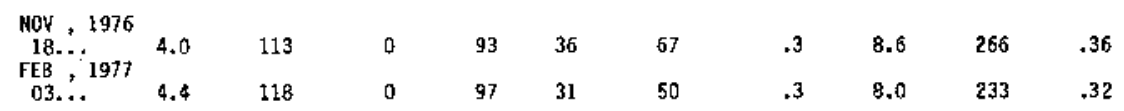

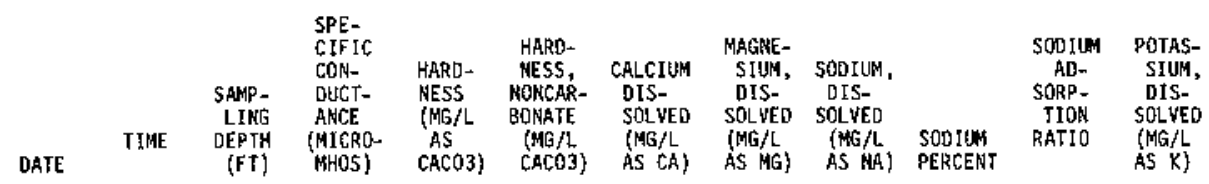

\begin{tabular}{|c|c|c|c|c|c|c|c|c|c|c|c|}
\hline \multicolumn{12}{|c|}{ MAY, 1977} \\
\hline JUN & 1200 & 1.0 & 360 & 110 & 12 & 38 & 3.8 & 24 & 31 & 1.0 & 4.4 \\
\hline $\begin{array}{r}01 \ldots \\
23 \ldots \\
\text { AUG }\end{array}$ & $\begin{array}{l}1100 \\
1100\end{array}$ & $\begin{array}{l}1.0 \\
1.0\end{array}$ & $\begin{array}{l}380 \\
400\end{array}$ & $\begin{array}{l}130 \\
120\end{array}$ & $\begin{array}{l}20 \\
16\end{array}$ & $\begin{array}{l}44 \\
41\end{array}$ & $\begin{array}{l}4.1 \\
4.0\end{array}$ & $\begin{array}{l}31 \\
23\end{array}$ & $\begin{array}{l}34 \\
29\end{array}$ & $\begin{array}{r}1.2 \\
.9\end{array}$ & $\begin{array}{l}4.3 \\
4.1\end{array}$ \\
\hline JAK, & $78^{1440}$ & 1.0 & 650 & 150 & 22 & 49 & 5.6 & 54 & 44 & 1.9 & 4. 7 \\
\hline JUN & 0916 & 1.0 & 310 & 110 & 99 & 3) & 3.9 & 27 & 34 & 1.1 & 4.0 \\
\hline $05 \ldots$ & 1410 & 1.0 & 637 & 140 & 32 & 43 & 1.5 & 67 & 50 & 2.5 & 5.9 \\
\hline
\end{tabular}
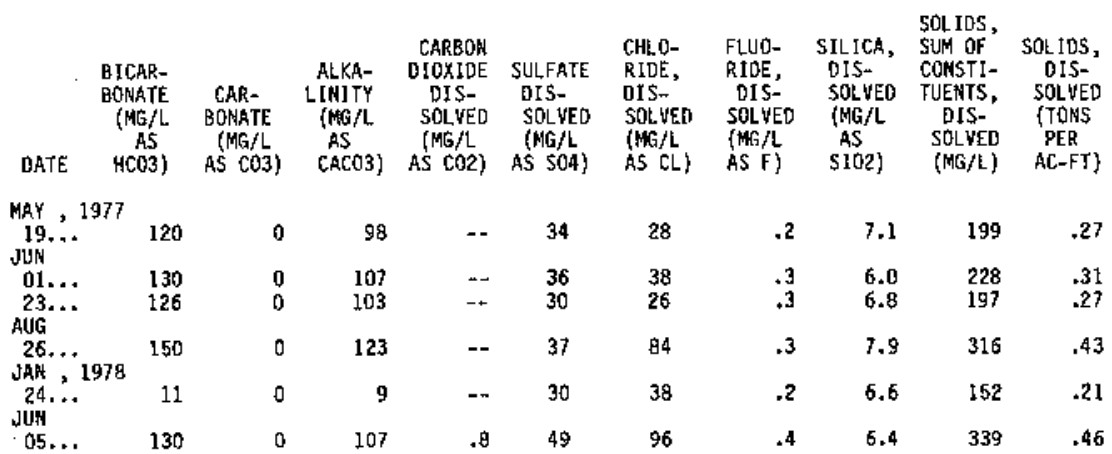

294712094440200 LINE 690 SITE 40

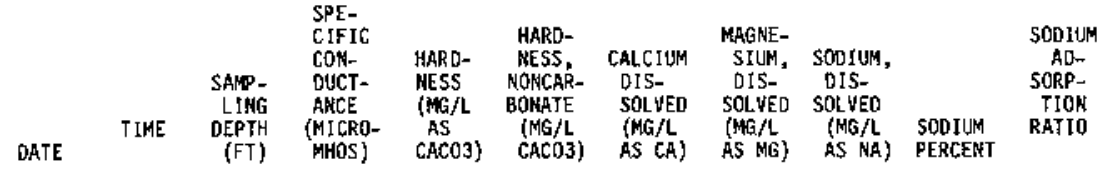

$\begin{array}{ccccccccccc}\text { OEC . } & 1976 & & & & & & & \\ 10 . . . & 1045 & 1.0 & 200 & 91 & 20 & 31 & 3.4 & 28 & 39 & 1.3 \\ 28 \ldots & 1235 & 1.0 & 330 & 97 & 16 & 33 & 3.5 & 28 & 37 & 1.2\end{array}$

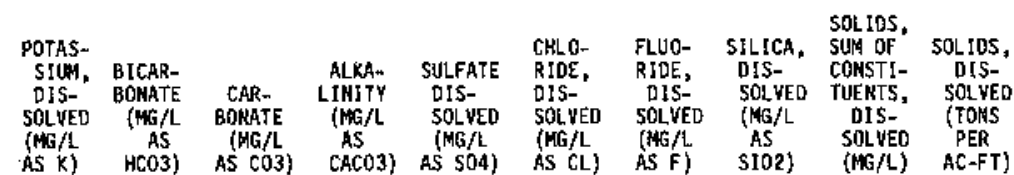

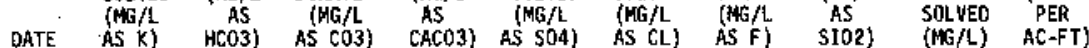

$\begin{array}{ccccccccccc}\text { OEC . } 1976 & & & & & & & & & & \\ 10 . \ldots & 3.9 & 87 & 0 & 71 & 24 & 35 & .2 & 7.8 & 176 & .24 \\ 28 . . . & 4.0 & 99 & 0 & 81 & 33 & 35 & .3 & 8.1 & 194 & .26\end{array}$ 


\section{Brazos Estuary}

The Brazos estuary, which has an area of about 3 square miles $\left(8 \mathrm{~km}^{2}\right)$ consists of the tidal parts of the Brazos River and parts of the Intracoastal Waterway (Figure 4). Although Freeport Harbor is not directly connected with the estuary, wastes from industrial operations around the harbor are discharged into the estuary. Water depth at mean low water is about 10 feet $(3.0 \mathrm{~m})$ in the river and about 15 feet $(4.6 \mathrm{~m})$ in the Intracoastal Waterway.

Water-quality data (Table 3) were collected during February and June 1977 and February and May 1978. 


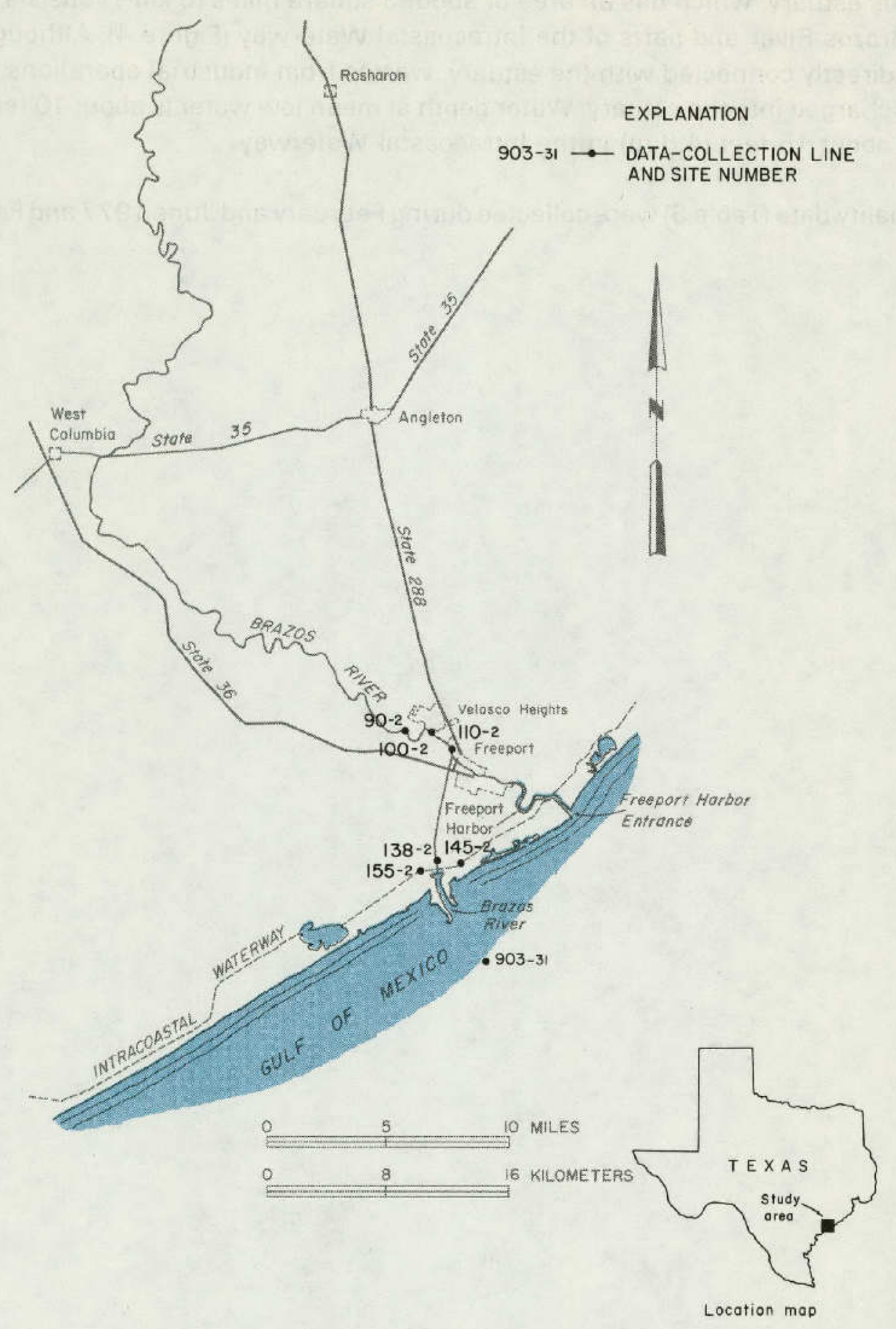

Figure 4

Data-Collection Sites in the Brazos Estuary 
$\left(F T=\right.$ feet; MICROMHOS = micromhos per centimeter at $25^{\circ}$ Celsius; OEG $C=$ degrees Celsius; $M=$ meters; ia $/ \mathrm{L}=$ milligrams per liter

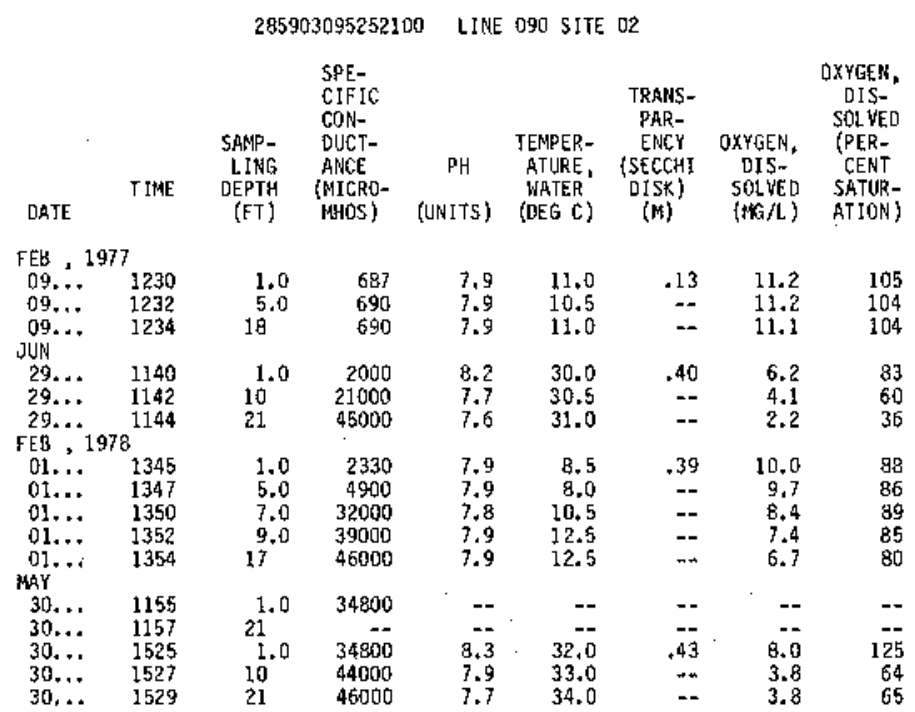

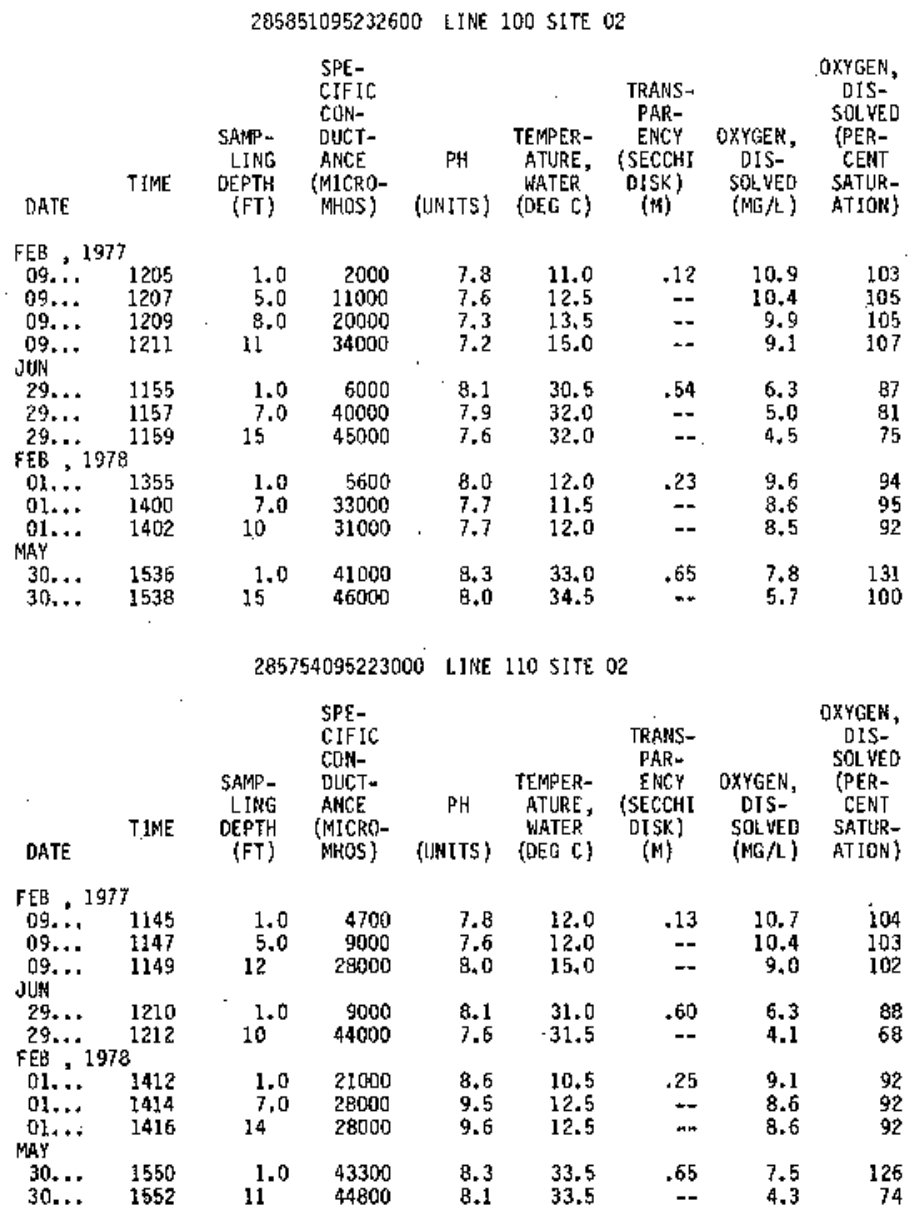


Table 3A,--Qualfty of water in the Brazos estuary, water year 1977-7B--Continued Field Deteminations-mCont inued

285430095231100 LINE 138 SITE 02

\begin{tabular}{|c|c|c|c|c|c|c|c|c|}
\hline DATE & T IME. & $\begin{array}{l}\text { SAYPS- } \\
\text { LIMG } \\
\text { DEPTH } \\
\text { (FT) }\end{array}$ & $\begin{array}{l}\text { SPE- } \\
\text { CIFIC } \\
\text { CON- } \\
\text { OUCT- } \\
\text { ANCE } \\
\text { (MICRO. } \\
\text { PHOS) }\end{array}$ & $\begin{array}{c}\text { PH } \\
\text { (UNITS) }\end{array}$ & $\begin{array}{l}\text { TEMPER- } \\
\text { ATURE, } \\
\text { WATER } \\
\text { (OEG C) }\end{array}$ & $\begin{array}{l}\text { TRAMS- } \\
\text { PAR- } \\
\text { ENCY } \\
\text { (SECCHI } \\
\text { D]SK) } \\
\text { (M) }\end{array}$ & $\begin{array}{c}\text { OXYGEN, } \\
\text { DIS- } \\
\text { SOLVEO } \\
\text { (NG } / L \text { ) }\end{array}$ & $\begin{array}{c}\text { OXYGEN, } \\
\text { DIS- } \\
\text { SOLVEO } \\
\text { (PER- } \\
\text { CENT } \\
\text { SATUR- } \\
\text { AT ION) }\end{array}$ \\
\hline \multicolumn{9}{|c|}{ FEB , 1977} \\
\hline $\begin{array}{l}09 . . . \\
09 . . \\
09 . . \\
09 . . \\
\text { JUN }\end{array}$ & $\begin{array}{l}1045 \\
1047 \\
1049 \\
1051\end{array}$ & $\begin{array}{l}1.0 \\
5.0 \\
10 \\
20\end{array}$ & $\begin{array}{r}8000 \\
9000 \\
12000 \\
18000\end{array}$ & $\begin{array}{l}6.9 \\
6.7 \\
7.5 \\
7.9\end{array}$ & $\begin{array}{l}12.0 \\
12.5 \\
13.0 \\
13.5\end{array}$ & $\begin{array}{r}21 \\
-- \\
--\end{array}$ & $\begin{array}{l}11.1 \\
10.9 \\
10.6 \\
10.1\end{array}$ & $\begin{array}{l}111 \\
109 \\
108 \\
106\end{array}$ \\
\hline $\begin{array}{r}29 . \ldots \\
29 . \ldots \\
29 \ldots \\
F E 8, \ldots\end{array}$ & $\begin{array}{l}1225 \\
1227 \\
1229 \\
8\end{array}$ & $\begin{array}{l}1.0 \\
10^{21}\end{array}$ & $\begin{array}{l}15000 \\
40000 \\
50000\end{array}$ & $\begin{array}{l}8.0 \\
7.8 \\
7.9\end{array}$ & $\begin{array}{l}30.5 \\
31.0 \\
30.0\end{array}$ & $\begin{array}{l}.78 \\
-- \\
--\end{array}$ & $\begin{array}{l}6.4 \\
4.2 \\
4.2\end{array}$ & $\begin{array}{l}91 \\
66 \\
69\end{array}$ \\
\hline $\begin{array}{l}01 \ldots \\
01 \ldots . . \\
01 \ldots\end{array}$ & $\begin{array}{l}1420 \\
1422 \\
1424\end{array}$ & $\begin{array}{l}1.0 \\
9.0 \\
17\end{array}$ & $\begin{array}{l}19000 \\
26000 \\
26000\end{array}$ & $\begin{array}{l}8.6 \\
8.1 \\
8.1\end{array}$ & $\begin{array}{l}10.5 \\
10.0 \\
10.0\end{array}$ & $\begin{array}{l}.51 \\
-- \\
--\end{array}$ & $\begin{array}{l}8.8 \\
8.8 \\
9.1\end{array}$ & $\begin{array}{l}88 \\
90 \\
93\end{array}$ \\
\hline $\begin{array}{l}30 \ldots \\
30 \ldots \\
30 . .\end{array}$ & $\begin{array}{l}1605 \\
1607 \\
1609\end{array}$ & $\begin{array}{l}1.0 \\
10 \\
20\end{array}$ & $\begin{array}{l}44000 \\
44000 \\
44000\end{array}$ & $\begin{array}{l}8.1 \\
8.0 \\
8.0\end{array}$ & $\begin{array}{l}34.0 \\
34.0 \\
33.5\end{array}$ & $\begin{array}{l}.77 \\
-. \\
-\end{array}$ & $\begin{array}{l}5.3 \\
5.2 \\
4.1\end{array}$ & $\begin{array}{l}90 \\
87 \\
69\end{array}$ \\
\hline
\end{tabular}

\section{LIPE 145 SITE 02}

\begin{tabular}{|c|c|c|c|c|c|c|c|c|}
\hline DATE & TIME & $\begin{array}{l}\text { SAMP- } \\
\text { LING } \\
\text { DEPTH } \\
(F T)\end{array}$ & $\begin{array}{l}\text { SPE- } \\
\text { CIFIC } \\
\text { COR- } \\
\text { DUCT- } \\
\text { ANCE } \\
\text { (MICRO- } \\
\text { MHOS) }\end{array}$ & $\begin{array}{c}\mathrm{PH} \\
\text { (UNITS) }\end{array}$ & $\begin{array}{l}\text { TEPPERR- } \\
\text { ATURE. } \\
\text { WATER } \\
\text { (DEG C) }\end{array}$ & $\begin{array}{l}\text { TRANS- } \\
\text { PAR- } \\
\text { ENCY } \\
\text { (SECCHI } \\
\text { DISK) } \\
\text { (M) }\end{array}$ & $\begin{array}{c}\text { OXYGEN, } \\
\text { OIS- } \\
\text { SOLVED } \\
\text { (ME } / L \text { ) }\end{array}$ & $\begin{array}{c}\text { OXYGEH, } \\
\text { DIS- } \\
\text { SOLVED } \\
\text { (PER- } \\
\text { CENT } \\
\text { SATUR- } \\
\text { ATION) }\end{array}$ \\
\hline FEB , 1 & & & & & & & & \\
\hline $\begin{array}{c}09 . . . \\
09 . \ldots \\
09 . . . \\
\text { JUN }\end{array}$ & $\begin{array}{l}1015 \\
1017 \\
1019\end{array}$ & $\begin{array}{l}1.0 \\
5.0 \\
14\end{array}$ & $\begin{array}{l}14000 \\
14000 \\
21000\end{array}$ & $\begin{array}{l}8.1 \\
7.9 \\
7.8\end{array}$ & $\begin{array}{l}12.0 \\
12.0 \\
11.0\end{array}$ & $\begin{array}{l}.18 \\
-- \\
--\end{array}$ & $\begin{array}{r}10.7 \\
10.2 \\
9.3\end{array}$ & $\begin{array}{r}108 \\
103 \\
95\end{array}$ \\
\hline $\begin{array}{l}29 . \ldots \\
29 . \ldots \\
\text { FEB }, 1\end{array}$ & $\begin{array}{l}1300 \\
1302\end{array}$ & $11^{1.0}$ & $\begin{array}{l}32000 \\
50000\end{array}$ & $\begin{array}{l}7.9 \\
7.8\end{array}$ & & $\begin{array}{r}.47 \\
--\end{array}$ & & $\begin{array}{l}23 \\
67\end{array}$ \\
\hline $\begin{array}{l}01 \ldots . . \\
01 . . . \\
01 \ldots\end{array}$ & $\begin{array}{l}1445 \\
1447 \\
1449\end{array}$ & $\begin{array}{l}1.0 \\
8.0 \\
16\end{array}$ & $\begin{array}{l}26000 \\
25000 \\
27000\end{array}$ & $\begin{array}{l}8.1 \\
8.2 \\
8.1\end{array}$ & $\begin{array}{l}10.0 \\
10.5 \\
10.0\end{array}$ & 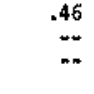 & $\begin{array}{l}8.9 \\
8.7 \\
8.7\end{array}$ & $\begin{array}{l}91 \\
89 \\
99\end{array}$ \\
\hline $\begin{array}{l}30 \ldots . . \\
30 \ldots\end{array}$ & $\begin{array}{l}1627 \\
1629\end{array}$ & $15^{1.0}$ & $\begin{array}{l}41000 \\
42000\end{array}$ & $\begin{array}{l}8.0 \\
7.9\end{array}$ & $\begin{array}{l}33.5 \\
32.0\end{array}$ & $\begin{array}{r}.62 \\
--\end{array}$ & $\begin{array}{l}5.8 \\
5.3\end{array}$ & $\begin{array}{l}97 \\
86\end{array}$ \\
\hline
\end{tabular}

285355095235300 LIAE 155 SITE 02

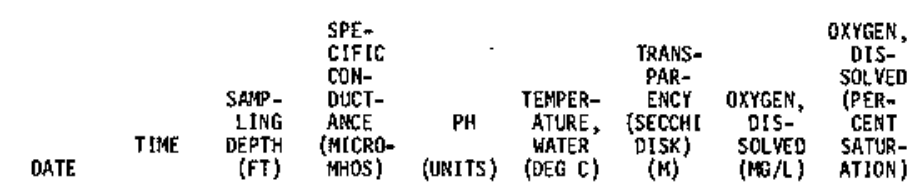

\begin{tabular}{|c|c|c|c|c|c|c|c|c|}
\hline \multicolumn{9}{|c|}{ FEB , 1977} \\
\hline $\begin{array}{l}09 . . \\
09 . \ldots \\
09 . .\end{array}$ & $\begin{array}{l}1030 \\
1032 \\
1034\end{array}$ & $\begin{array}{r}1.0 \\
5.0 \\
14^{-0}\end{array}$ & $\begin{array}{l}15000 \\
20000 \\
22000\end{array}$ & $\begin{array}{l}7.7 \\
8.0 \\
7.7\end{array}$ & $\begin{array}{l}13.0 \\
11.5 \\
11.5\end{array}$ & $\begin{array}{l}.38 \\
-- \\
--\end{array}$ & $\begin{array}{l}10.7 \\
10.4 \\
10.4\end{array}$ & $\begin{array}{l}111 \\
107 \\
108\end{array}$ \\
\hline JUß & & & & & & & & \\
\hline $\begin{array}{r}29 . \ldots \\
29 \ldots \\
\text { FEB... }\end{array}$ & $\begin{array}{l}1240 \\
1242 \\
8\end{array}$ & $10^{1.0}$ & $\begin{array}{l}32000 \\
40000\end{array}$ & $\begin{array}{l}7.9 \\
8.1\end{array}$ & $\begin{array}{l}30.5 \\
30.0\end{array}$ & .55 & $\begin{array}{l}5.5 \\
5.2\end{array}$ & $\begin{array}{l}83 \\
81\end{array}$ \\
\hline $\begin{array}{c}01 \ldots \\
01 . . .\end{array}$ & $\begin{array}{l}1435 \\
1437\end{array}$ & 12.0 & $\begin{array}{l}38000 \\
39000\end{array}$ & $\begin{array}{l}8.2 \\
8.2\end{array}$ & $\begin{array}{l}10.5 \\
10.0\end{array}$ & .40 & $\begin{array}{l}8.4 \\
8.2\end{array}$ & $\begin{array}{l}92 \\
89\end{array}$ \\
\hline $\begin{array}{l}30 . . \\
30 . . \\
30 . .\end{array}$ & $\begin{array}{l}1619 \\
1621 \\
1623\end{array}$ & $\begin{array}{l}1.0 \\
8.0 \\
18\end{array}$ & $\begin{array}{l}42000 \\
42000 \\
43000\end{array}$ & $\begin{array}{l}8.2 \\
8.1 \\
8.2\end{array}$ & $\begin{array}{l}34.0 \\
32.5 \\
31.5\end{array}$ & $\begin{array}{r}.37 \\
-- \\
-\end{array}$ & $\begin{array}{l}7.5 \\
6.1 \\
4.8\end{array}$ & $\begin{array}{r}127 \\
100 \\
76\end{array}$ \\
\hline
\end{tabular}

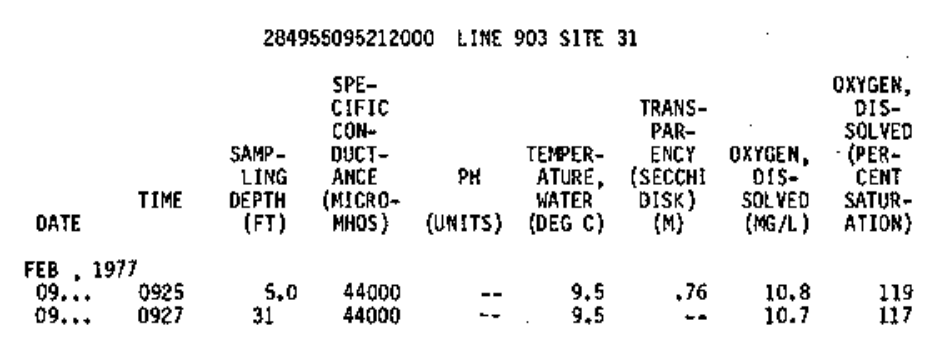


$(F T=$ feet; MG/L $=$ ril11\{grams per liter $)$

285903095252100 LINE 090 SITE 02

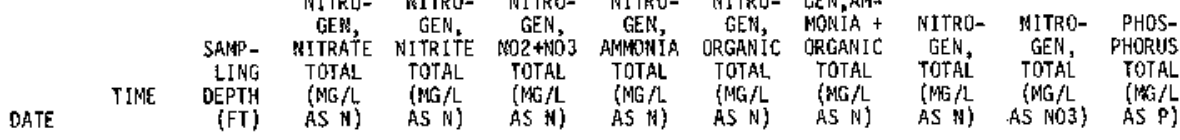

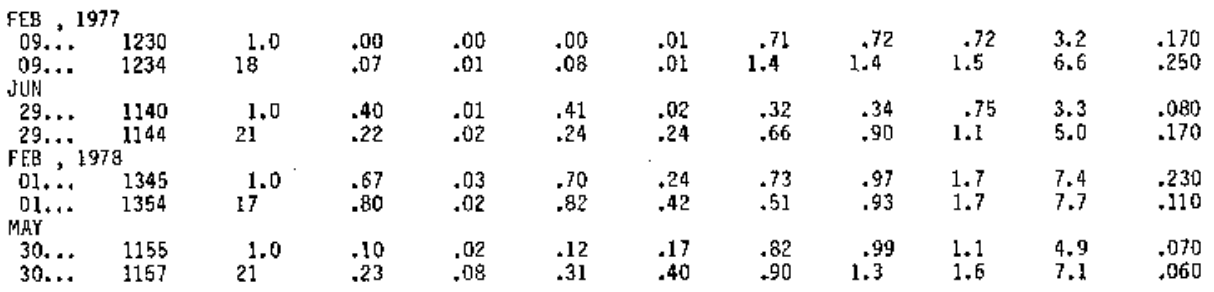

285754095223000 LINE 110 SITE 02

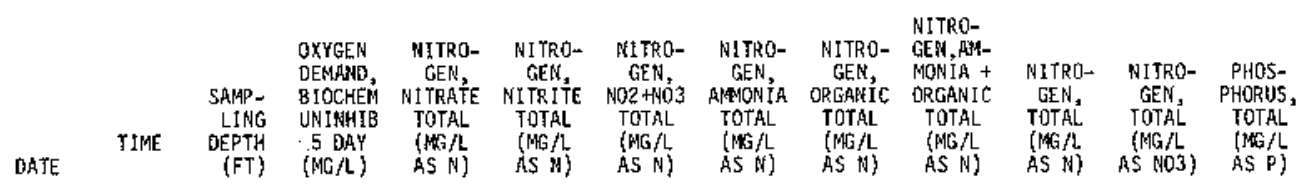

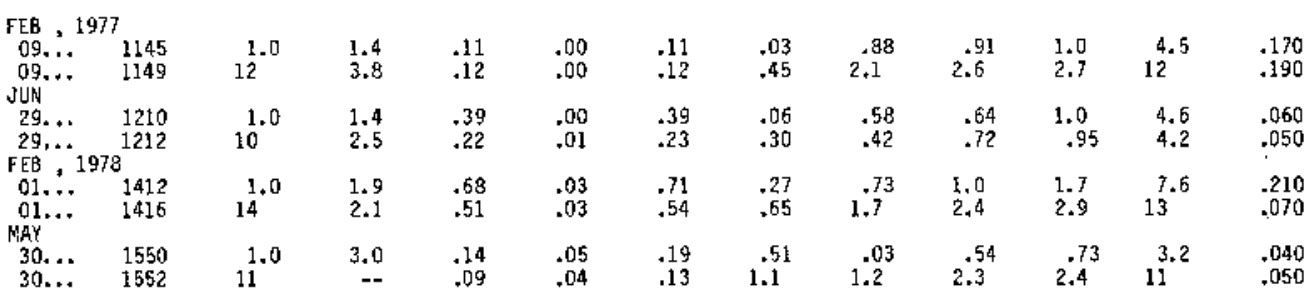

284955095212000 LIME 903 SITE 31

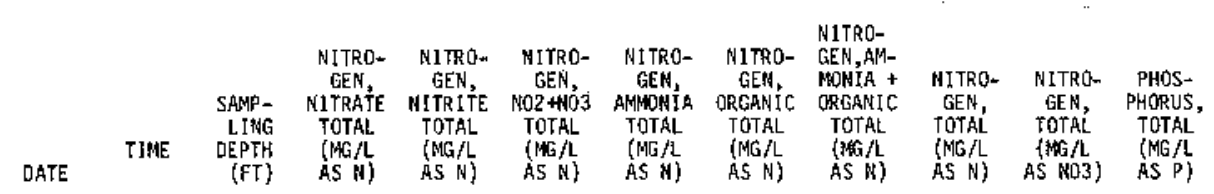

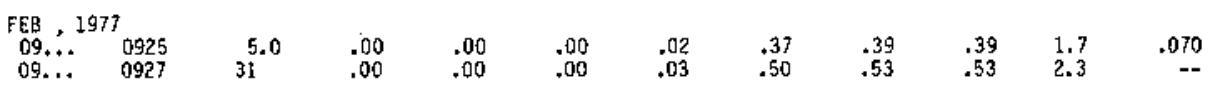


(FT = feet; MICROMHOS = micromhos per centimeter at $25^{\circ}$ Celsius; MG $/ L$ m milligrans per liter; AC-FT - acre-feet)

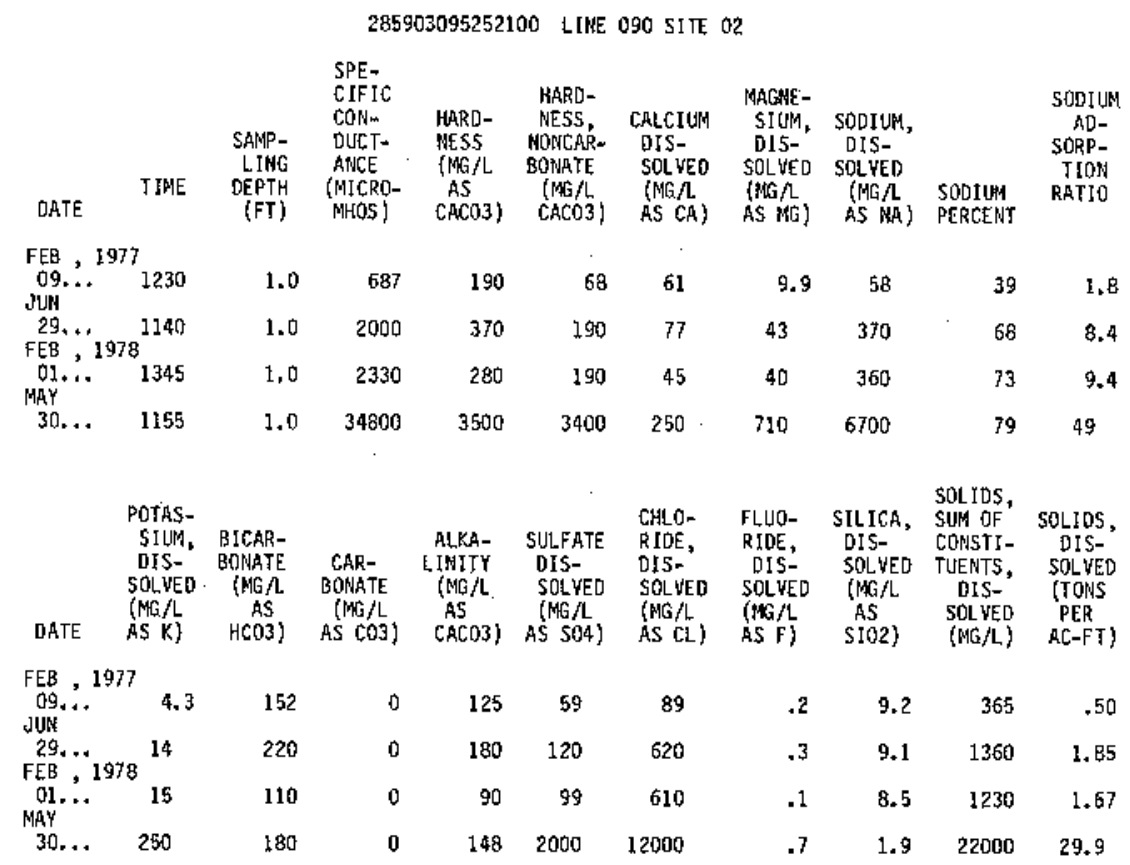




\section{East Matagorda Estuary}

The East Matagorda estuary, which has an area of about 56 square miles $\left(145 \mathrm{~km}^{2}\right)$, consists of East Matagorda Bay, part of the Intracoastal Waterway, the tidal reaches of Caney Creek and Live Oak Bayou, and the tidal part of small tributaries (Figure 5). The maximum water depth at mean low water is 5 feet $(1.5 \mathrm{~m})$ in East Matagorda Bay and about 15 feet $(4.6 \mathrm{~m})$ in the Intracoastal Waterway.

Water-quality data (Table 4) were collected during February and April-June 1977 and February and May 1978.

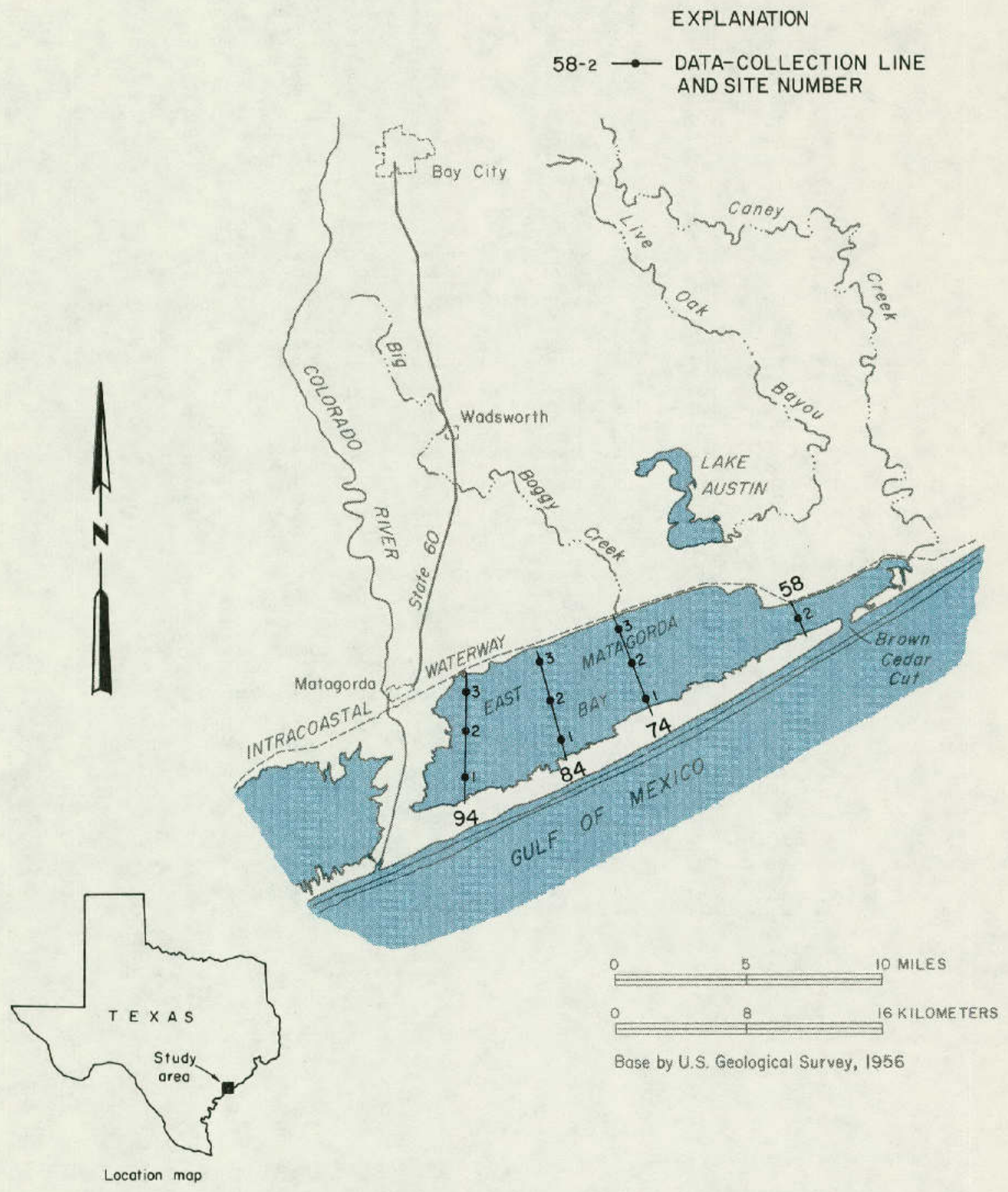

Figure 5.-Data-Collection Sites in the East Matagorda Estuary 
Table 4A.--Quality of water in the East Matagorda estuary, water years 1977-78 Field Deteminations

$\left(F T=\right.$ feet; MICROKHOS $=$ micromhos per centimeter at $25^{\circ}$ Celsius; DEG $C=$ degrees Celsius; $M=$ meters;

$\mathrm{MG} / \mathrm{L}=$ milligrans per 1 iter)

284349095433000 LINE 058 SITE 02

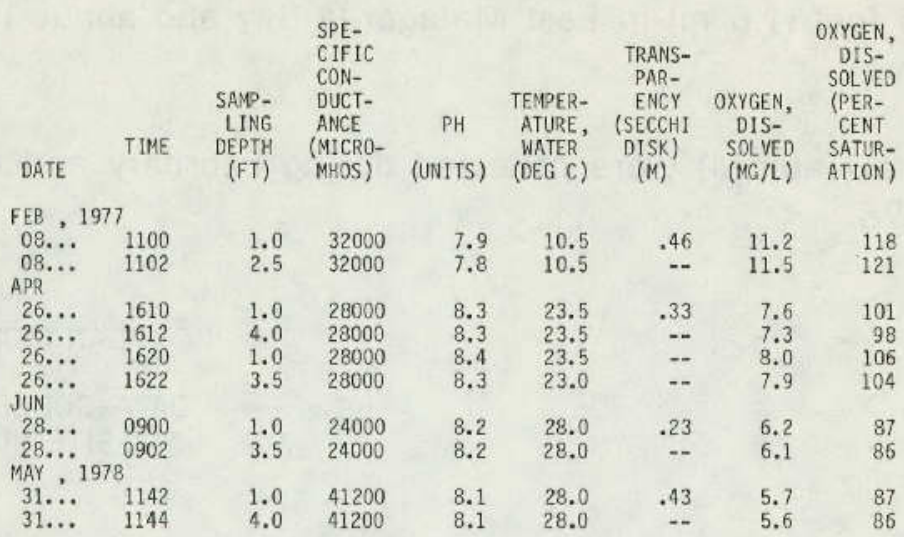

284123095490200 LINE 074 SITE 01

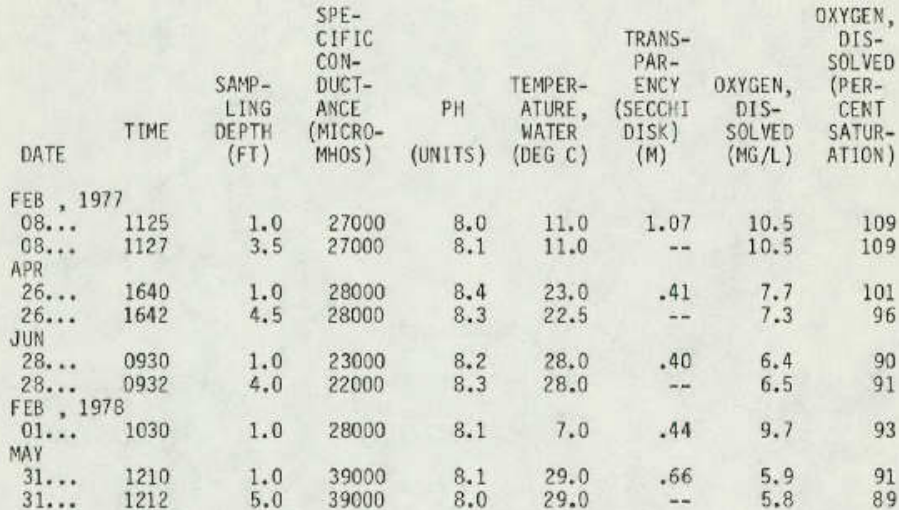

284219095492900 LINE 074 SITE 02

\begin{tabular}{|c|c|c|c|c|c|c|c|c|}
\hline DATE & TIME & $\begin{array}{l}\text { SAMP- } \\
\text { LING } \\
\text { DEPTH } \\
\text { (FT) }\end{array}$ & $\begin{array}{l}\text { SPE- } \\
\text { CIFIC } \\
\text { CON- } \\
\text { OUCT- } \\
\text { ANCE } \\
\text { (MICRO- } \\
\text { MHOS) }\end{array}$ & $\begin{array}{c}\text { PH } \\
\text { (UNITS) }\end{array}$ & $\begin{array}{l}\text { TEMPER- } \\
\text { ATURE, } \\
\text { WATER } \\
\text { (DEG C) }\end{array}$ & $\begin{array}{l}\text { TRANS- } \\
\text { PAR- } \\
\text { ENCY } \\
\text { (SECCHI } \\
\text { DISK) } \\
\text { (M) }\end{array}$ & $\begin{array}{c}\text { OXYGEN, } \\
\text { DIS- } \\
\text { SOLVED } \\
(M G / L)\end{array}$ & $\begin{array}{l}\text { OXYGEN, } \\
\text { OIS- } \\
\text { SOLVED } \\
\text { (PER- } \\
\text { CENT } \\
\text { SATUR- } \\
\text { ATION) }\end{array}$ \\
\hline EB , & 1977 & & & & & & & \\
\hline $\begin{array}{l}08 \ldots \\
08 \ldots\end{array}$ & $\begin{array}{l}1135 \\
\cdot \quad 1137\end{array}$ & $\begin{array}{l}1.0 \\
4.5\end{array}$ & $\begin{array}{l}26000 \\
26000\end{array}$ & $\begin{array}{l}8.0 \\
8.0\end{array}$ & & .87 & $\begin{array}{l}10.6 \\
10.6\end{array}$ & $\begin{array}{l}110 \\
110\end{array}$ \\
\hline $\begin{array}{l}26 \ldots \\
26 \ldots \\
\text { JUN }\end{array}$ & $\begin{array}{l}1635 \\
1637\end{array}$ & $\begin{array}{l}1.0 \\
5.0\end{array}$ & $\begin{array}{l}28000 \\
28000\end{array}$ & $\begin{array}{l}8.4 \\
8.4\end{array}$ & $\begin{array}{l}23.0 \\
22.0\end{array}$ & .46 & $\begin{array}{l}7.7 \\
7.1\end{array}$ & $\begin{array}{r}101 \\
92\end{array}$ \\
\hline $\begin{array}{r}28 \ldots \\
28, \ldots \\
F E B\end{array}$ & $\begin{array}{r}0940 \\
-\quad{ }^{0942}\end{array}$ & $\begin{array}{l}1.0 \\
4.0\end{array}$ & $\begin{array}{l}26000 \\
26000\end{array}$ & $\begin{array}{l}8.2 \\
8.2\end{array}$ & & .25 & $\begin{array}{l}6.1 \\
6.2\end{array}$ & \\
\hline MAY & - $\quad 1045$ & 1.0 & 30000 & 8.2 & 6.5 & .39 & 9.5 & 90 \\
\hline $\begin{array}{l}31 \ldots \\
31 \ldots\end{array}$ & $\begin{array}{l}1218 \\
1220\end{array}$ & $\begin{array}{l}1.0 \\
5.0\end{array}$ & $\begin{array}{l}40000 \\
40000\end{array}$ & $\begin{array}{l}8.1 \\
8.1\end{array}$ & $\begin{array}{r}29.5 \\
29.0\end{array}$ & .67 & $\begin{array}{l}6.2 \\
6.1\end{array}$ & \\
\hline
\end{tabular}


Table 4h.--Quality of water in the East Matagorda estuary, water years 1977,78--itontinued Field Deterninations--Continued

294331095500300 LINE 074 SITE 03

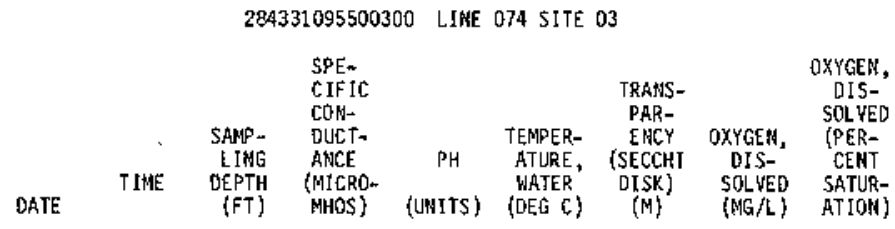

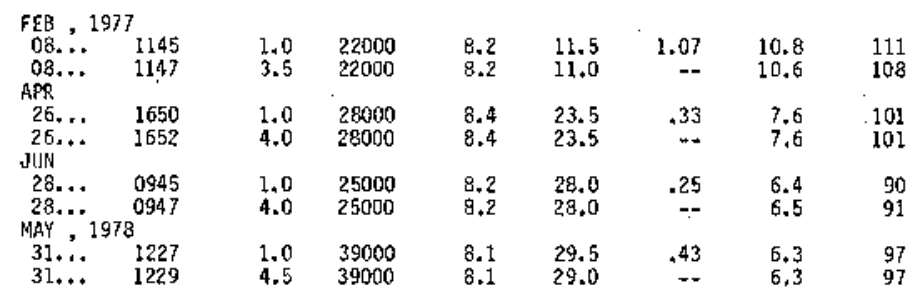

283945095515700 LINE 094 SITE 01

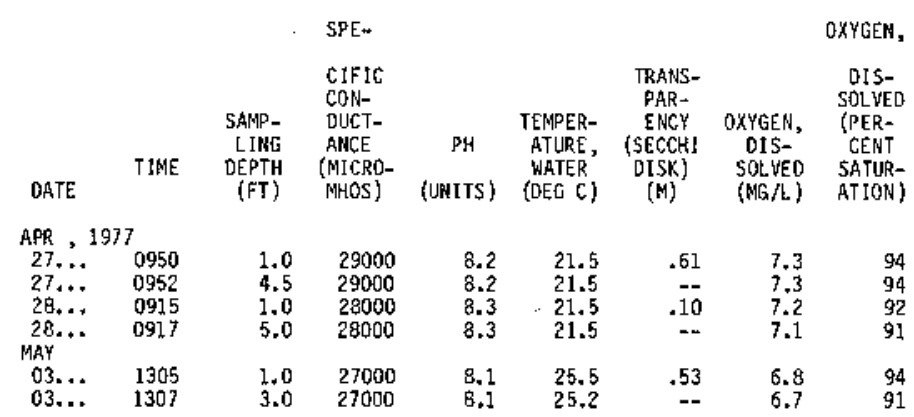

284058095522600 LINE 084 SITE 02

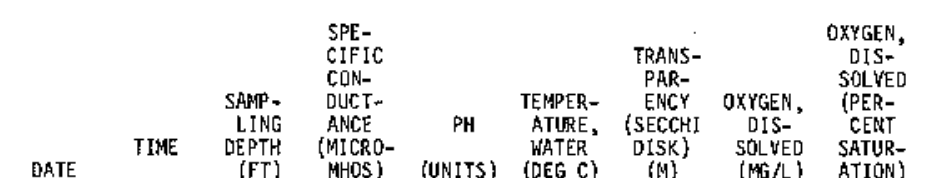

FEB , 1977

$\begin{array}{ccccccccc}\text { FEB }, 1977 & & & & & & \\ 08 . . . & 1245 & 1.0 & 21000 & 8.1 & 11.5 & .79 & 11.0 & 113 \\ 08 \ldots & 1247 & 2.5 & 21000 & 8.1 & 11.5 & -. & 11.0 & 113 \\ \text { APR } & & & & & & & & \\ 27 . . . & 1000 & 1.0 & 28000 & 8.3 & 22.0 & .30 & 7.4 & 96 \\ 27 \ldots & 1002 & 4.0 & 28000 & 8.3 & 22.0 & -. & 7.4 & 96 \\ 28 \ldots . & 0905 & 1.0 & 28000 & 8.3 & 21.5 & .10 & 7.1 & 91 \\ 28 \ldots & 0907 & 4.0 & 28000 & 8.3 & 21.5 & -. & 7.1 & 91\end{array}$

284208095525600 LINE 084 SITE 03

\begin{tabular}{|c|c|c|c|c|c|c|c|c|}
\hline DATE & TIME & $\begin{array}{r}\text { SAMP- } \\
\text { LIMG } \\
\text { DEPTH } \\
\text { (FT) }\end{array}$ & $\begin{array}{l}\text { SPE- } \\
\text { CIFIC } \\
\text { CON- } \\
\text { DUCT- } \\
\text { AHCE } \\
\text { (MICRD- } \\
\text { MHOS) }\end{array}$ & $\begin{array}{c}P H \\
\text { (UNITS) }\end{array}$ & $\begin{array}{l}\text { TEMPER- } \\
\text { ATURE, } \\
\text { WATER } \\
\text { (DES C) }\end{array}$ & $\begin{array}{l}\text { TRANS- } \\
\text { PAR- } \\
\text { ENCY } \\
\text { (SECCHI } \\
\text { OISX) } \\
\text { (M) }\end{array}$ & $\begin{array}{c}\text { OXYGEN, } \\
\text { DIS* } \\
\text { SOL VED } \\
\text { (MG/L) }\end{array}$ & $\begin{array}{c}\text { OXYGEN, } \\
\text { OIS- } \\
\text { SOLVED } \\
\text { (PER- } \\
\text { CENT } \\
\text { SATUR- } \\
\text { ATION) }\end{array}$ \\
\hline PR , 1977 & & & & & & & & \\
\hline $\begin{array}{l}27 \ldots \\
27 \ldots \\
28 \ldots \\
28 \ldots \\
M A Y\end{array}$ & $\begin{array}{l}1010 \\
1012 \\
0900 \\
0902\end{array}$ & $\begin{array}{l}1.0 \\
4.0 \\
1.0 \\
4.0\end{array}$ & $\begin{array}{l}22000 \\
28000 \\
19000 \\
19000\end{array}$ & $\begin{array}{l}8.4 \\
8.3 \\
8.4 \\
8.3\end{array}$ & $\begin{array}{l}22.0 \\
21.5 \\
21.5 \\
21.5\end{array}$ & $\begin{array}{r}.38 \\
. \overline{10} \\
.-\end{array}$ & $\begin{array}{l}7.8 \\
7.0 \\
7.1 \\
7.1\end{array}$ & $\begin{array}{l}99 \\
90 \\
89 \\
89\end{array}$ \\
\hline $\begin{array}{l}03 \ldots \\
03 . \cdots \\
J 1 N\end{array}$ & $\begin{array}{l}1250 \\
1252\end{array}$ & $\begin{array}{l}1.0 \\
3.0\end{array}$ & $\begin{array}{l}13000 \\
15000\end{array}$ & $\begin{array}{l}8.2 \\
8.1\end{array}$ & $\begin{array}{l}26.0 \\
24.7\end{array}$ & $\begin{array}{l}.46 \\
--\end{array}$ & $\begin{array}{l}7.6 \\
6.6\end{array}$ & $\begin{array}{l}99 \\
84\end{array}$ \\
\hline $\begin{array}{l}28 \ldots \\
28 \ldots \\
\text { FEB...1978 }\end{array}$ & $\begin{array}{l}1030 \\
1032 \\
8\end{array}$ & $\begin{array}{l}1.0 \\
3.5\end{array}$ & $\begin{array}{l}15000 \\
21000\end{array}$ & $\begin{array}{l}8.1 \\
8.1\end{array}$ & $\begin{array}{l}29.0 \\
28.0\end{array}$ & .32 & $\begin{array}{l}6.2 \\
5.8\end{array}$ & $\begin{array}{l}86 \\
82\end{array}$ \\
\hline $01 \ldots$ & 1055 & 1.0 & 31000 & 8.2 & 6.0 & .52 & 9.3 & 89 \\
\hline $\begin{array}{l}31 \ldots . . \\
31 . \ldots\end{array}$ & $\begin{array}{l}1241 \\
1243\end{array}$ & $\begin{array}{l}1.0 \\
4.0\end{array}$ & $\begin{array}{l}36300 \\
36300\end{array}$ & $\begin{array}{l}8.1 \\
8.1\end{array}$ & $\begin{array}{l}29.5 \\
29.0\end{array}$ & $\begin{array}{r}.37 \\
--\end{array}$ & $\begin{array}{l}6.2 \\
6.4\end{array}$ & $\begin{array}{l}95 \\
97\end{array}$ \\
\hline
\end{tabular}


Tabte 4A.--Quality of water in the East Matagorda estuary, water years 1977-78--Cont inued field 0eterminations--Continued

283852095545100 . LINE 094 SITE ON

\begin{tabular}{|c|c|c|c|c|c|c|c|c|}
\hline DATE & TIME & $\begin{array}{c}\text { SAMP- } \\
\text { LING } \\
\text { DEPTH } \\
\text { (FT) }\end{array}$ & $\begin{array}{l}\text { SPE- } \\
\text { CIFIC } \\
\text { CON- } \\
\text { DUCT- } \\
\text { ANCE } \\
\text { (MICRO- } \\
\text { MHOS) }\end{array}$ & $\begin{array}{c}\text { PH } \\
\text { (UNITS) }\end{array}$ & $\begin{array}{c}\text { TEMPER- } \\
\text { ATURE, } \\
\text { WATER } \\
\text { (DEG C) }\end{array}$ & $\begin{array}{l}\text { TRANS- } \\
\text { PAR- } \\
\text { ENCY } \\
\text { (SECCHI } \\
\text { OISK) } \\
\text { (M) }\end{array}$ & $\begin{array}{c}\text { OXYGEN, } \\
\text { DIS- } \\
\text { SOL VED } \\
\text { (MG/L) }\end{array}$ & $\begin{array}{c}\text { OXYGEN, } \\
\text { OIS- } \\
\text { SOLWED } \\
\text { (PER- } \\
\text { CENT } \\
\text { SATUR- } \\
\text { ATION) }\end{array}$ \\
\hline \multicolumn{9}{|c|}{ FEB , 1977} \\
\hline $\begin{array}{l}08 . . . \\
08 . \ldots \\
\text { APR }\end{array}$ & $\begin{array}{l}1225 \\
1227\end{array}$ & $\begin{array}{l}1.0 \\
4.5\end{array}$ & $\begin{array}{l}22000 \\
22000\end{array}$ & $\begin{array}{l}7.9 \\
7.9\end{array}$ & $\begin{array}{l}11.5 \\
11.5\end{array}$ & .32 & $\begin{array}{l}10,3 \\
10.4\end{array}$ & $\begin{array}{l}107 \\
108\end{array}$ \\
\hline $\begin{array}{c}26 . \ldots \\
26 \ldots \\
26 \ldots \\
26 \ldots \\
27 \ldots \\
27 \ldots \\
27 \ldots \\
27 \ldots \\
28 \ldots \\
28 . \ldots \\
\text { MAY }\end{array}$ & $\begin{array}{l}1720 \\
1722 \\
1724 \\
1726 \\
0930 \\
0932 \\
1700 \\
1702 \\
0935 \\
0937\end{array}$ & $\begin{array}{l}1.0 \\
3.0 \\
4.0 \\
4.5 \\
1.0 \\
4.5 \\
1.0 \\
5.0 \\
1.0 \\
4.0\end{array}$ & $\begin{array}{l}13000 \\
13000 \\
14000 \\
19100 \\
14000 \\
20000 \\
16000 \\
16000 \\
24000 \\
24000\end{array}$ & $\begin{array}{l}8.5 \\
8.5 \\
8.5 \\
8.1 \\
8.3 \\
8.3 \\
8.3 \\
8.3 \\
8.3 \\
8.3\end{array}$ & $\begin{array}{l}23.3 \\
23.5 \\
23.5 \\
23.0 \\
21.5 \\
22.0 \\
24.0 \\
24.0 \\
21.0 \\
21.0\end{array}$ & $\begin{array}{l}.66 \\
-- \\
-- \\
.56 \\
.66 \\
.30 \\
.71 \\
.7\end{array}$ & $\begin{array}{r}9.9 \\
10.1 \\
9.8 \\
5.0 \\
8.4 \\
7.5 \\
8.8 \\
8.4 \\
6.9 \\
6.8\end{array}$ & $\begin{array}{r}124 \\
127 \\
124 \\
64 \\
102 \\
95 \\
112 \\
107 \\
87 \\
86\end{array}$ \\
\hline $\begin{array}{l}03 . . \\
03 \ldots \\
11 \ldots \\
11 \ldots \\
\text { Juk }\end{array}$ & $\begin{array}{l}1320 \\
1322 \\
1320 \\
1322\end{array}$ & $\begin{array}{l}1.0 \\
4.0 \\
1.0 \\
4.0\end{array}$ & $\begin{array}{l}24000 \\
26000 \\
19000 \\
19000\end{array}$ & $\begin{array}{l}8.1 \\
8.0 \\
8.1 \\
8.1\end{array}$ & $\begin{array}{l}25.0 \\
24.0 \\
24.0 \\
24.0\end{array}$ & $\begin{array}{r}.66 \\
. .4 \\
.13 \\
-.\end{array}$ & $\begin{array}{l}6.8 \\
6.2 \\
6.9 \\
6.8\end{array}$ & $\begin{array}{l}91 \\
84 \\
90 \\
88\end{array}$ \\
\hline $\begin{array}{l}28, \ldots \\
28 . \ldots \\
\text { FEB. }\end{array}$ & $\begin{array}{l}1000 \\
1002 \\
8\end{array}$ & $\begin{array}{l}1.0 \\
4.0\end{array}$ & $\begin{array}{l}24000 \\
24000\end{array}$ & $\begin{array}{l}8.2 \\
8.2\end{array}$ & $\begin{array}{l}28.0 \\
28.5\end{array}$ & .38 & $\begin{array}{l}6.4 \\
6.2\end{array}$ & $\begin{array}{l}90 \\
88\end{array}$ \\
\hline MAY.". & 1025 & 1.0 & 30000 & 8.1 & 7.5 & +48 & 9.7 & 95 \\
\hline $\begin{array}{l}31 \ldots \\
31 \ldots\end{array}$ & $\begin{array}{l}1313 \\
1315\end{array}$ & $\begin{array}{l}1.0 \\
4.5\end{array}$ & $\begin{array}{l}32000 \\
32000\end{array}$ & $\begin{array}{l}8.0 \\
8.0\end{array}$ & $\begin{array}{l}29.5 \\
29.5\end{array}$ & $\begin{array}{r}.65 \\
--\end{array}$ & $\begin{array}{l}6.7 \\
6.7\end{array}$ & $\begin{array}{l}100 \\
100\end{array}$ \\
\hline
\end{tabular}

294000095545700 LIAE 094 SITE 02

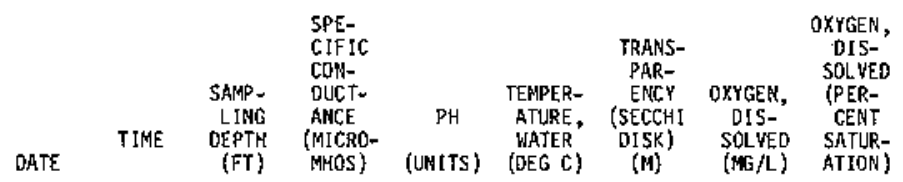

FEB , 1977

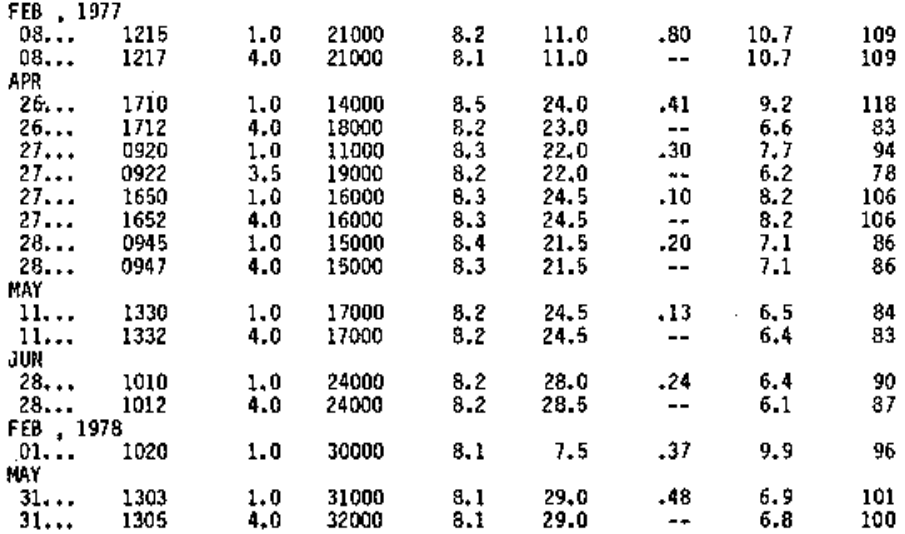


Table 4A. - Ruality of water in the East Matagorda estuary, water years 1977-78--Continued

Field Determinations--Continued

284115095550400 LINE 094 SITE 03

\begin{tabular}{|c|c|c|c|c|c|c|c|c|}
\hline DATE & TIME. & $\begin{array}{l}\text { SAMP- } \\
\text { LING } \\
\text { DEPTH } \\
\text { (FT) }\end{array}$ & $\begin{array}{l}\text { SPE- } \\
\text { CIFIC } \\
\text { CON- } \\
\text { DUCT- } \\
\text { ANCE } \\
\text { (MICRO- } \\
\text { MHOS) }\end{array}$ & $\begin{array}{c}\mathrm{PH} \\
\text { (UNITS) }\end{array}$ & $\begin{array}{l}\text { TEMAPER- } \\
\text { ATURE, } \\
\text { WATER } \\
\text { (DEG C) }\end{array}$ & $\begin{array}{l}\text { TRANS- } \\
\text { PAR- } \\
\text { ENCY } \\
\text { (SECCH! } \\
\text { DISK) } \\
\text { (A) }\end{array}$ & $\begin{array}{c}\text { OXYGEN, } \\
\text { OIS } \\
\text { SOLYEO } \\
\text { (MG } / L)\end{array}$ & $\begin{array}{c}\text { OXYGEN, } \\
\text { DIS- } \\
\text { SOLVED } \\
\text { (PER- } \\
\text { CENT } \\
\text { SATUR- } \\
\text { ATION) }\end{array}$ \\
\hline \multicolumn{9}{|c|}{ FEB , 1977} \\
\hline $\begin{array}{l}08 . . . \\
\text { OP... } \\
\text { APR }\end{array}$ & $\begin{array}{l}1205 \\
1207\end{array}$ & $\begin{array}{l}1.0 \\
3.0\end{array}$ & $\begin{array}{l}21000 \\
21000\end{array}$ & $\begin{array}{l}7.6 \\
7.6\end{array}$ & $\begin{array}{l}11.0 \\
11.0\end{array}$ & .91 & $\begin{array}{l}11.2 \\
11.0\end{array}$ & $\begin{array}{l}114 \\
112\end{array}$ \\
\hline $\begin{array}{l}26 \ldots \\
26 \ldots \\
27 \ldots \\
27 \ldots \\
27 \ldots \\
27 \ldots \\
28 \ldots \\
28 \ldots \\
\text { MAY }\end{array}$ & $\begin{array}{l}1740 \\
1742 \\
0910 \\
0912 \\
1635 \\
1637 \\
0950 \\
0952\end{array}$ & $\begin{array}{l}1.0 \\
3.0 \\
1.0 \\
3.0 \\
1.0 \\
3.0 \\
1.0 \\
3.0\end{array}$ & $\begin{array}{l}18000 \\
22000 \\
13000 \\
14000 \\
13000 \\
13000 \\
14000 \\
14000\end{array}$ & $\begin{array}{l}8.5 \\
8.4 \\
8.4 \\
8.3 \\
8.4 \\
8.3 \\
8.4 \\
8.4\end{array}$ & $\begin{array}{l}24.0 \\
24.5 \\
22.0 \\
22.0 \\
25.0 \\
24.5 \\
21.5 \\
21.5\end{array}$ & $\begin{array}{r}.61 \\
.- \\
.46 \\
.10 \\
.20 \\
.20 \\
-.\end{array}$ & $\begin{array}{l}9.7 \\
6.6 \\
8.2 \\
7.7 \\
9.0 \\
9.2 \\
7.4 \\
7.4\end{array}$ & $\begin{array}{r}124 \\
115 \\
100 \\
95 \\
115 \\
116 \\
91 \\
91\end{array}$ \\
\hline $\begin{array}{l}03 \ldots \\
03 \ldots \\
11 \ldots \\
11 \ldots \\
11 \ldots\end{array}$ & $\begin{array}{l}1335 \\
1337 \\
1340 \\
1342 \\
1344\end{array}$ & $\begin{array}{l}1.0 \\
3.0 \\
1.0 \\
3.0 \\
6.0\end{array}$ & $\begin{array}{l}19000 \\
19000 \\
23000 \\
23000 \\
25000\end{array}$ & $\begin{array}{l}8.1 \\
8.1 \\
8.1 \\
8.0 \\
7.9\end{array}$ & $\begin{array}{l}26.0 \\
26.0 \\
24.5 \\
24.5 \\
24.5\end{array}$ & $\begin{array}{l}.46 \\
.36 \\
.36 \\
--\end{array}$ & $\begin{array}{l}7.2 \\
7.3 \\
7.0 \\
6.6 \\
5.2\end{array}$ & $\begin{array}{l}96 \\
98 \\
94 \\
88 \\
70\end{array}$ \\
\hline $\begin{array}{l}\text { JUN } \\
28, \ldots \\
28, \ldots\end{array}$ & $\begin{array}{r}1020 \\
1022\end{array}$ & $\begin{array}{l}1.0 \\
3.5\end{array}$ & $\begin{array}{l}24000 \\
24000\end{array}$ & $\begin{array}{l}8.2 \\
8.2\end{array}$ & $\begin{array}{l}28.0 \\
28.0\end{array}$ & .38 & $\begin{array}{l}6.6 \\
6.5\end{array}$ & $\begin{array}{l}92 \\
91\end{array}$ \\
\hline $\begin{array}{c}\text { FEB }, \\
\text { Ol. } .\end{array}$ & & & & & & & & \\
\hline$\underset{\text { MAY }}{01 . . .}$ & 1000 & 1.0 & 30000 & 8.1 & 7.5 & .40 & 10.1 & 98 \\
\hline $\begin{array}{l}31 \ldots \\
31 \ldots\end{array}$ & $\begin{array}{l}1254 \\
1256\end{array}$ & $\begin{array}{l}1.0 \\
3.0\end{array}$ & $\begin{array}{l}35000 \\
35000\end{array}$ & $\begin{array}{l}8.1 \\
8.1\end{array}$ & $\begin{array}{l}29.5 \\
29.5\end{array}$ & $\begin{array}{r}.47 \\
.7\end{array}$ & $\begin{array}{l}6.6 \\
6.5\end{array}$ & $\begin{array}{r}100 \\
99\end{array}$ \\
\hline
\end{tabular}


Table 48,--Quality of water in the East Matagorda estuary, water years 1977-78 Nutrient Analyses

(FT = feet; : $G / L=$ milligrans per 1 iter)

284349095433000 LINE 058 SITE D2

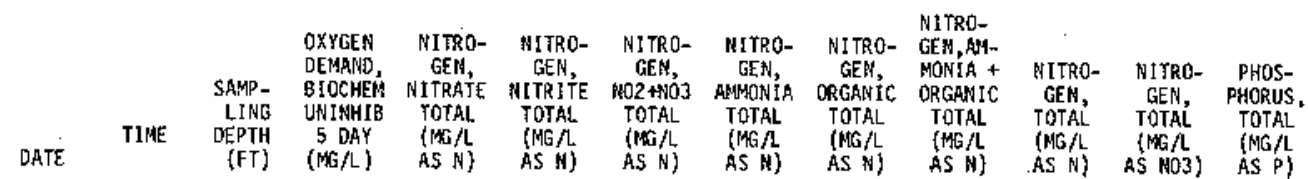

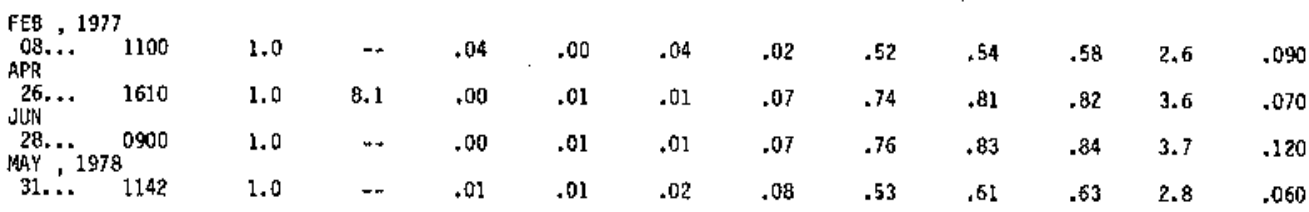

284123095490200 LINE 074 SITE 01

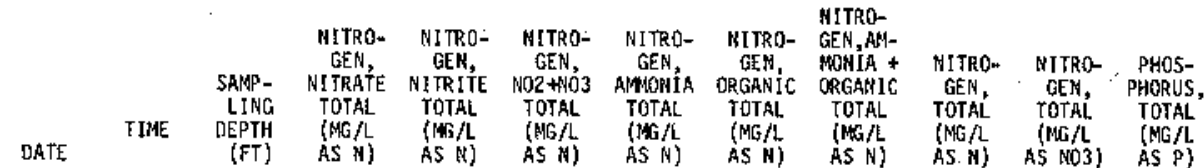

\begin{tabular}{|c|c|c|c|c|c|c|c|c|c|c|c|}
\hline $\begin{array}{l}\text { FEB }, \\
\text { OB. } \\
\text { APR }\end{array}$ & $7_{1125}$ & 1.0 & .00 & .00 & .00 & .03 & .42 & .45 & .45 & 2.0 & .060 \\
\hline $\begin{array}{c}26 . \cdots \\
\text { JUN }\end{array}$ & 1640 & 1.0 & .01 & .01 & .02 & .05 & .43 & .48 & .50 & 2.2 & .060 \\
\hline $\begin{array}{l}28 . \cdots \\
\text { FEB , }\end{array}$ & $7^{0930}$ & 1.0 & .00 & .01 & .00 & .04 & .58 & .62 & .62 & 2.7 & .090 \\
\hline $\begin{array}{c}01 \\
\text { MAY }\end{array}$ & 1030 & 1.0 & .01 & .03 & .04 & .69 & -- & .65 & .69 & 3.1 & .110 \\
\hline $31 . \ldots$ & 1210 & 1.0 & .00 & .01 & .01 & .01 & .54 & .55 & .56 & 2.5 & .040 \\
\hline
\end{tabular}

284331095500300 LIRE 074 SITE 03

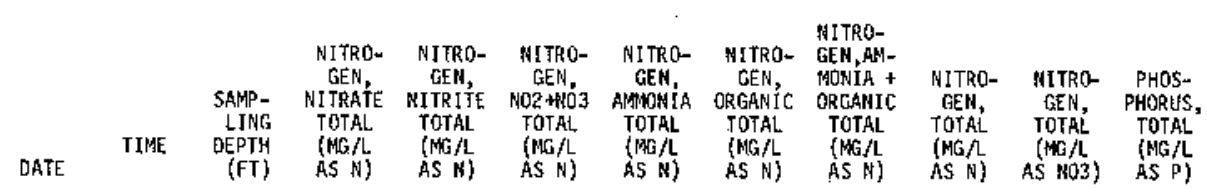

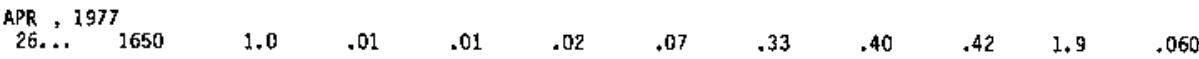

293945095515700 LINE 084 SITE O1

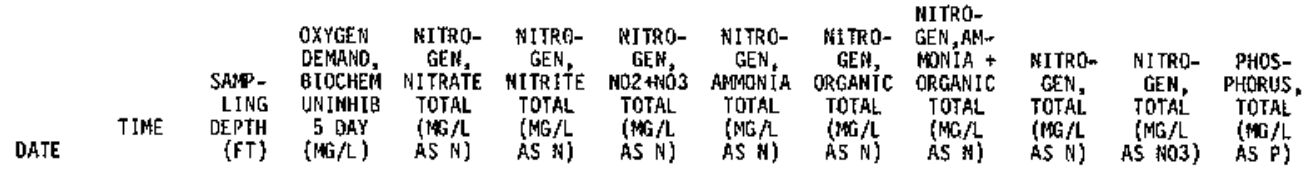

$\begin{array}{lllllllllllll}\text { MAY. } & 1977 \\ 03 . . . & 1305 & 1.0 & 1.3 & .00 & .01 & .01 & .05 & .30 & .35 & .36 & 1.6 & .050\end{array}$

294058095522600 L.JNE 084 SITE 02

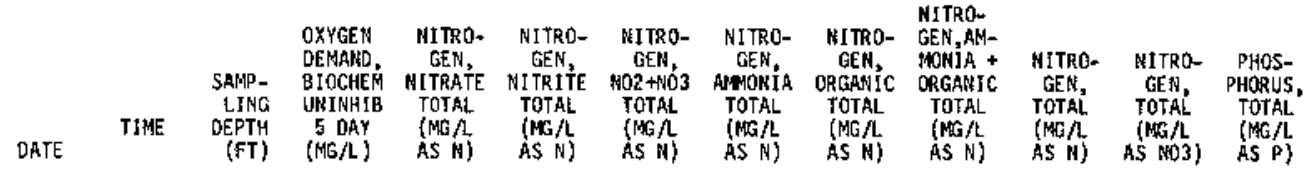

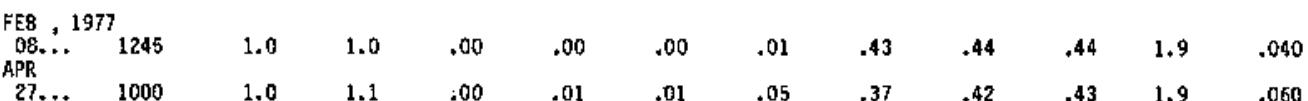




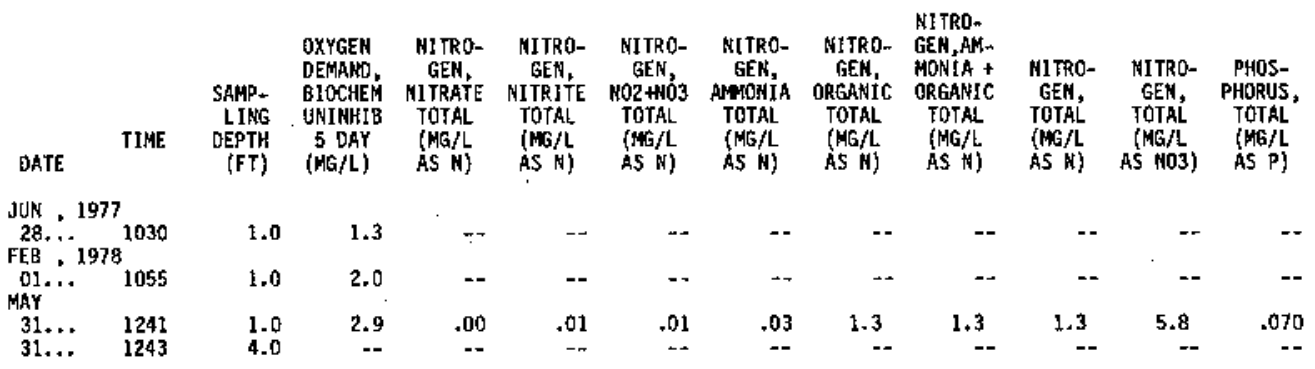

283852095545100 LINE 094 SIFE OI

\begin{tabular}{|c|c|c|c|c|c|c|c|c|c|c|c|c|}
\hline DATE & TIME & $\begin{array}{r}\text { SAMP- } \\
\text { LIMG } \\
\text { DEPTH } \\
\text { (FT) }\end{array}$ & $\begin{array}{c}\text { OXYGEN } \\
\text { DEMAKD, } \\
\text { BIOCHEM } \\
\text { UNIHHIB } \\
5 \text { OAY } \\
\text { (NMG } / L)\end{array}$ & $\begin{array}{l}\text { NITRO- } \\
\text { GEN, } \\
\text { NITRATE } \\
\text { TOTAL } \\
\text { (MG/L } \\
\text { AS N) }\end{array}$ & $\begin{array}{l}\text { NITRQ- } \\
\text { GEN, } \\
\text { NITR1TE } \\
\text { TOTAL } \\
\text { (NG/L } \\
\text { AS M) }\end{array}$ & $\begin{array}{c}\text { MITRO- } \\
\text { GER, } \\
\text { N02+HO3 } \\
\text { TOTAL } \\
\text { (MG/L } \\
\text { AS N) }\end{array}$ & $\begin{array}{l}\text { HITRO- } \\
\text { GEN, } \\
\text { AMMHONIA } \\
\text { TOTAL } \\
\text { (MG/L } \\
\text { AS N) }\end{array}$ & $\begin{array}{l}\text { NLTRO- } \\
\text { GEN, } \\
\text { ORGANLC } \\
\text { TOTAL } \\
\text { (MG/L } \\
\text { AS M) }\end{array}$ & $\begin{array}{l}\text { HITRO- } \\
\text { GEN,AM- } \\
\text { MONAA + } \\
\text { ORGANIC } \\
\text { TOTAL } \\
\text { (MGG/L } \\
\text { AS N) }\end{array}$ & $\begin{array}{l}\text { NITRO- } \\
\text { GEN, } \\
\text { TQTAL } \\
(M G / L \\
\text { AS N } N)\end{array}$ & $\begin{array}{l}\text { NJTRO- } \\
\text { GEN, } \\
\text { IOTAL } \\
\text { (NGG/L } \\
\text { AS NOS })\end{array}$ & $\begin{array}{c}\text { PHOS- } \\
\text { PHORUS, } \\
\text { TOTAL } \\
\text { (MG/L } \\
\text { AS P) }\end{array}$ \\
\hline $\begin{array}{l}\text { APR } . \\
26 \ldots \\
26 \ldots \\
27 \ldots \\
27 \ldots \\
\text { MAY }\end{array}$ & $\begin{array}{l}7 \\
1720 \\
1726 \\
0930 \\
0932\end{array}$ & $\begin{array}{l}1.0 \\
4.5 \\
1.0 \\
4.5\end{array}$ & $\begin{array}{l}2.5 \\
1.3 \\
1.0 \\
1.1\end{array}$ & $\begin{array}{l}.07 \\
.12 \\
.09 \\
.00\end{array}$ & $\begin{array}{l}.03 \\
.01 \\
.01 \\
.01\end{array}$ & $\begin{array}{l}.10 \\
.13 \\
.10 \\
.01\end{array}$ & $\begin{array}{l}.05 \\
.10 \\
.01 \\
.05\end{array}$ & $\begin{array}{l}.58 \\
.46 \\
.58 \\
.60\end{array}$ & $\begin{array}{l}.63 \\
.56 \\
.59 \\
.65\end{array}$ & $\begin{array}{l}.73 \\
.69 \\
.69 \\
.66\end{array}$ & $\begin{array}{l}3.2 \\
3.1 \\
3.1 \\
2.9\end{array}$ & $\begin{array}{l}.070 \\
.090 \\
.030 \\
.050\end{array}$ \\
\hline $\begin{array}{l}03 \ldots \\
03 \ldots \\
11 \ldots \\
11 \ldots\end{array}$ & $\begin{array}{l}1320 \\
1322 \\
1320 \\
1322\end{array}$ & $\begin{array}{l}1.0 \\
4.0 \\
1.0 \\
4.0\end{array}$ & $\begin{array}{l}.8 \\
-2 \\
--\end{array}$ & $\begin{array}{l}.01 \\
.00 \\
.04 \\
.04\end{array}$ & $\begin{array}{l}.01 \\
.01 \\
.00 \\
.00\end{array}$ & $\begin{array}{l}.02 \\
.01 \\
.04 \\
.04\end{array}$ & $\begin{array}{l}.00 \\
.01 \\
.01 \\
.02\end{array}$ & $\begin{array}{c}.38 \\
.39 \\
1.6 \\
.69\end{array}$ & $\begin{array}{r}.38 \\
.40 \\
1.6 \\
.71\end{array}$ & $\begin{array}{r}.40 \\
.41 \\
1.6 \\
.75\end{array}$ & $\begin{array}{l}1.8 \\
1.8 \\
7.3 \\
3.3\end{array}$ & $\begin{array}{l}.050 \\
.070 \\
.110 \\
.120\end{array}$ \\
\hline
\end{tabular}

284000095545700 LIAE 094 SITE 02

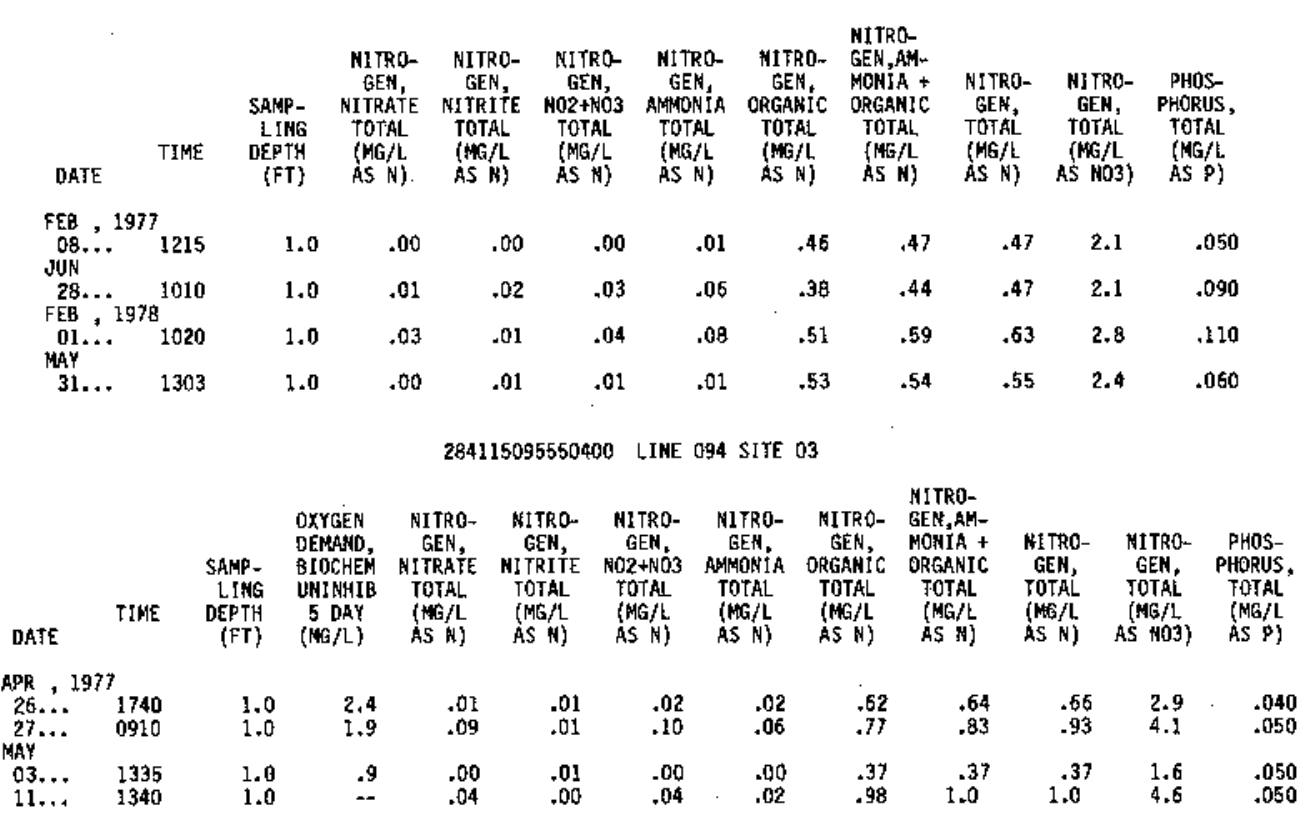


(FT = feet; MICROAtHOS a micromhos per centílieter at $25^{\circ}$ Celsius; MC $/ L=$ milligraris per liter; AC-FT = acre- feet )

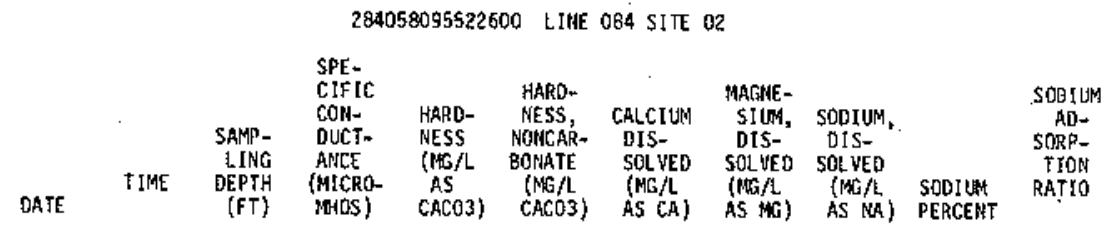

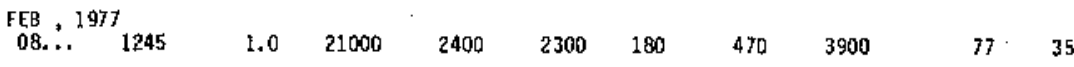

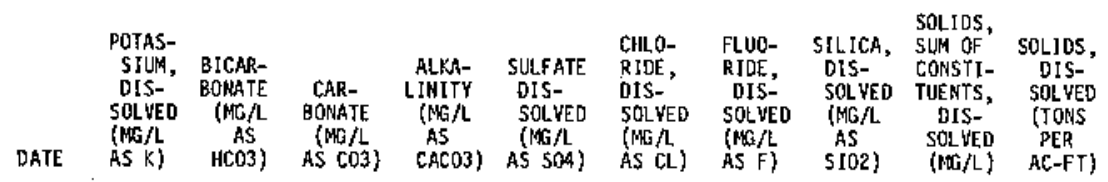

$\begin{array}{lllllllllll}\text { FEB } & \times 1977 \\ 08 & 170 & 136 & 0 & 112 & 990 & 7100 & .4 & 2.6 & 12900 & 17.5\end{array}$

284208095525600 LINE 084 SITE 03

\begin{tabular}{|c|c|c|c|c|c|c|c|c|c|c|c|}
\hline DATE & TIME & $\begin{array}{l}\text { SAYP- } \\
\text { LING } \\
\text { DEPTH } \\
\text { (FT) }\end{array}$ & $\begin{array}{l}\text { SPE- } \\
\text { CIFIC } \\
\text { CON- } \\
\text { DUCT- } \\
\text { ANCE } \\
\text { (MICRO- } \\
\text { MHOS) }\end{array}$ & $\begin{array}{l}\text { HARO- } \\
\text { HESS } \\
\text { (MG/A } \\
\text { AS } \\
\text { CACO3) }\end{array}$ & $\begin{array}{l}\text { HARD- } \\
\text { NESS, } \\
\text { NONCAR- } \\
\text { BONATE } \\
\text { (MG/L } \\
\text { CACO3) }\end{array}$ & $\begin{array}{l}\text { CALCIUIA } \\
\text { DFS- } \\
\text { SOL } Y E D \\
\text { (MG/L } \\
\text { AS CA) }\end{array}$ & $\begin{array}{l}\text { MAGNE- } \\
\text { SIUM, } \\
\text { DIS- } \\
\text { SOL.VED } \\
\text { (MG/L } \\
\text { AS MG) }\end{array}$ & $\begin{array}{l}\text { SODIUM, } \\
\text { DIS- } \\
\text { SOLVED } \\
\text { (AG/L } \\
\text { AS NA) }\end{array}$ & $\begin{array}{l}\text { SQDIUM } \\
\text { PERCEUT }\end{array}$ & $\begin{array}{l}\text { SODIUA } \\
\text { AD- } \\
\text { SORP- } \\
\text { TION } \\
\text { RATIO }\end{array}$ & $\begin{array}{l}\text { POTAS- } \\
\text { SIUM, } \\
\text { DIS- } \\
\text { SOLVED } \\
\text { (MG/L } \\
\text { AS K) }\end{array}$ \\
\hline $\begin{array}{l}\text { JEN , } \\
28 . \cdots \\
\text { FEB },\end{array}$ & ${ }_{8}^{7} 1030$ & 1.0 & 15000 & 1500 & 1300 & 130 & -- & 2700 & $=$ & -- & \\
\hline MAY & 1055 & 1.0 & 31000 & 3600 & 3500 & 220 & 730 & 5700 & .76 & 42 & 220 \\
\hline $31 . \ldots$ & 1241 & 1.0 & 36300 & 4500 & 4400 & 270 & 930 & 7300 & 76 & 47 & 300 \\
\hline
\end{tabular}

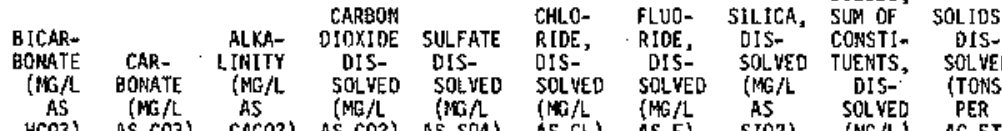

OATE HCO3) AS CO3) CACO3) ASCO2) AS SO4) AS CL) ASF) SIO2) (MG/L) AC-FT)

\begin{tabular}{|c|c|c|c|c|c|c|c|c|c|c|}
\hline $\begin{array}{l}\text { JUN , } 1977 \\
28 .: 7 \\
F E B, 1978\end{array}$ & 190 & 0 & 156 & -- & 520 & 4700 & .4 & 8.2 & 8640 & 11.8 \\
\hline MAY... & 160 & 0 & 131 & $=$ & 1500 & 10000 & .8 & 3,8 & 18500 & 25.2 \\
\hline $31 \ldots$ & 170 & 0 & 139 & 2.2 & 1700 & 13000 & .7 & 4.4 & 23600 & 32.1 \\
\hline
\end{tabular}

283852095545100 LINE 094 SITE 0I

\begin{tabular}{|c|c|c|c|c|c|c|c|c|c|c|}
\hline & TIME & $\begin{array}{l}\text { SARP- } \\
\text { LING } \\
\text { DEPTH }\end{array}$ & $\begin{array}{l}\text { SPE- } \\
\text { CIFIC } \\
\text { CON- } \\
\text { DUCT- } \\
\text { ANCE } \\
\text { (MICRO- }\end{array}$ & $\begin{array}{l}\text { HARQ- } \\
\text { MESS } \\
\text { (MG } / L \\
\text { AS }\end{array}$ & $\begin{array}{l}\text { HARD- } \\
\text { NESS, } \\
\text { NONCAR- } \\
\text { BONATE } \\
\text { (MC/L }\end{array}$ & $\begin{array}{l}\text { CALCIUM } \\
\text { DIS- } \\
\text { SOLVED } \\
\text { (MG/L }\end{array}$ & $\begin{array}{l}\text { MAGRE- } \\
\text { SIUA, } \\
\text { DIS- } \\
\text { SOL VED } \\
\left(M x_{1} /\right. \\
\text { AS }\end{array}$ & $\begin{array}{l}\text { SODIUM, } \\
\text { DIS- } \\
\text { SOLVED } \\
\text { (IAG/L }\end{array}$ & SODIN & $\begin{array}{l}\text { SODIUH } \\
\text { AD- } \\
\text { SORP- } \\
\text { JION } \\
\text { RATIO }\end{array}$ \\
\hline & & & & & & & & & PERCENT & \\
\hline
\end{tabular}

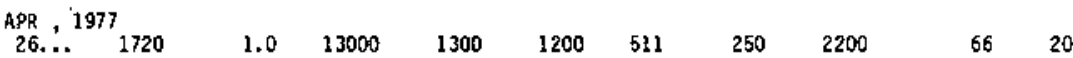

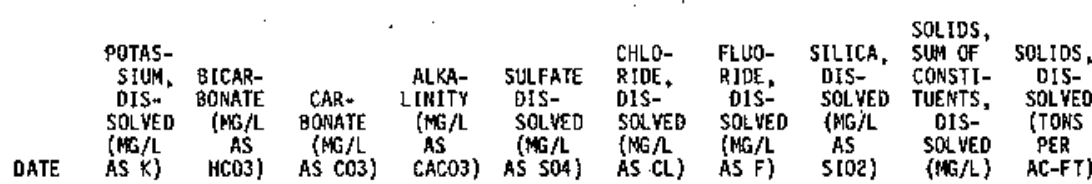

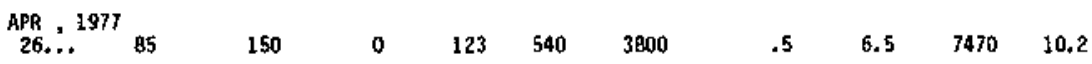




\section{Colorado Estuary}

The Colorado estuary, which has an area of about 2 square miles $\left(5 \mathrm{~km}^{2}\right)$, consists of the tidal part of the Colorado River and part of the Intracoastal Waterway (Figure 6). The minimum depth at mean low water is about 6 feet $(1.8 \mathrm{~m})$ in the river channel and about 15 feet $(4.6 \mathrm{~m})$ in the Intracoastal Waterway.

Water-quality data (Table 5) were collected during February and April-July 1977 and February and May 1978. 


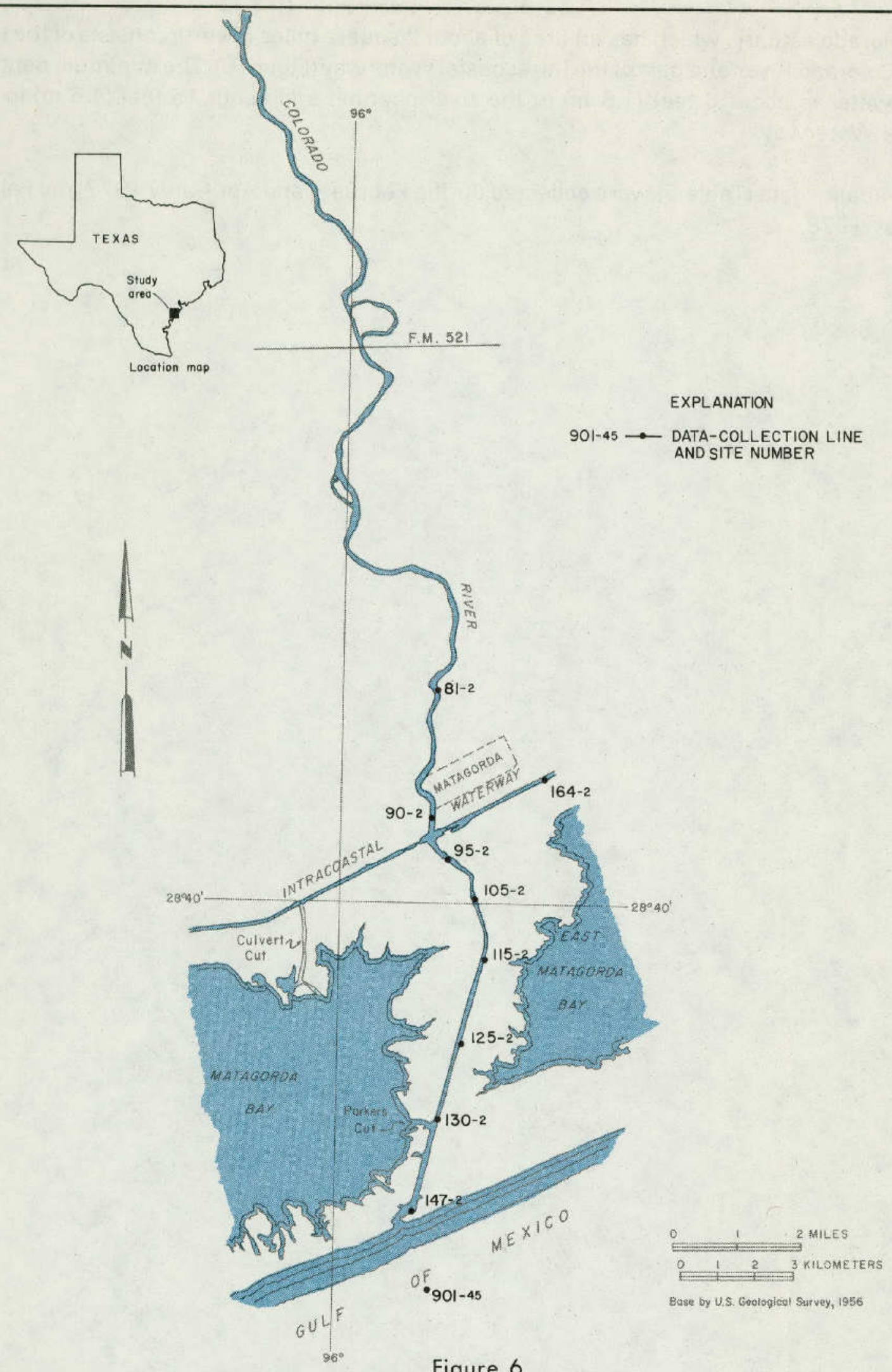

Figure 6

Data-Collection Sites in the Colorado Estuary 
(FT = feet; MICROMHOS a micromhos per cent ineter at $25^{\circ}$ Celstus; DEG C - degrees Celsíus; $M$ - meters; in $/ L$ - milligrans per liter)

284255095583500 LIME 081 SITE 02

\begin{tabular}{|c|c|c|c|c|c|c|c|c|}
\hline DATE & I IME & $\begin{array}{l}\text { SAMP- } \\
\text { LIHG } \\
\text { OEPTH } \\
\text { (FT) }\end{array}$ & $\begin{array}{l}\text { SPE- } \\
\text { C1FIC } \\
\text { CON- } \\
\text { DUCT- } \\
\text { ANCE } \\
\text { (MICRO- } \\
\text { NHOS) }\end{array}$ & $\begin{array}{c}\text { PH } \\
\text { (UNITS) }\end{array}$ & $\begin{array}{l}\text { TEMPER- } \\
\text { ATURE, } \\
\text { WATER } \\
\text { (DEG C) }\end{array}$ & $\begin{array}{l}\text { TRANS- } \\
\text { PAR- } \\
\text { ENCY } \\
\text { (SECCH' } \\
\text { DISK') } \\
\text { (M) }\end{array}$ & $\begin{array}{c}\text { OXYGEK, } \\
\text { OIS- } \\
\text { SOLWED } \\
\text { (MG/L) }\end{array}$ & $\begin{array}{c}\text { OXYGEN, } \\
\text { DIS- } \\
\text { SOLVED } \\
\text { (PERn } \\
\text { CENT } \\
\text { SATUR- } \\
\text { AIION) }\end{array}$ \\
\hline
\end{tabular}

FEB , 1977

07... 1330

07... 1332

$\begin{array}{ll}07 \ldots & 1334 \\ 07 \ldots & 1338\end{array}$

MAY

11... 1420

i1... 1422

$\begin{array}{ll}11 \ldots . . & 1424 \\ 11 \ldots . & 1426\end{array}$

$\begin{array}{ll}11 \ldots . & 1426 \\ 11 . . & 1428\end{array}$

JUN

28... 1115

$28 . . . \quad 1117$

$28 . . \quad 1119$

$28 . . . \quad 1121$

$28, \ldots \quad 1125$

28... 1978

FEB , 19780920

02... 0920

MAY ... 0922

$\begin{array}{ll}31 . \ldots & 1.452 \\ 31 . . . & 1454\end{array}$

31.... 1456

$\begin{array}{ll}31 . . . & 1458 \\ 31 . . & 1500\end{array}$

$\begin{array}{cc}1.0 & 600 \\ 10 & 600 \\ 20 & 600 \\ 45 & 600 \\ 1.0 & 650 \\ 5.0 & 650 \\ 10 & 650 \\ 20 & 650 \\ 29 & 650\end{array}$

$\begin{array}{lllll}7.6 & 11.5 & .13 & 9.9 & 93 \\ 7.6 & 12.0 & -- & 9.9 & 95 \\ 7.5 & 11.5 & \ldots & 9.9 & 93 \\ 7.8 & 11.5 & -. & 9.8 & 92\end{array}$

$\begin{array}{ll}1.0 & 1000 \\ 5.0 & 1100 \\ 7.0 & 16000 \\ 10 & 38000 \\ 20 & 48000\end{array}$

20
40

84.5

$\begin{array}{llll}8.3 & 24.5 & .30 & 7.7\end{array}$

$\begin{array}{llll}24.5 & - & 7.7 & 94\end{array}$

$\begin{array}{lllll}8.3 & 24.5 & -- & 7.7 & 94 \\ 8.4 & 24.5 & -- & 7.7 & 94\end{array}$

$\begin{array}{lllll}8.4 & 24.5 & -- & 7.7 & 94 \\ 8.4 & 24.5 & -- & 7.6 & 93\end{array}$

$\begin{array}{ll}1.0 & 14000 \\ 21 & 17000\end{array}$

1.013300

$\begin{array}{rr}5.0 & 24000 \\ 10 & 32000 \\ 20 & 37000 \\ 41 & 37000\end{array}$

$8.4 \quad 29.0 \quad .46$

$8.2 \quad 29.0$

$7.6 \quad 29.0$

$7.7 \quad 29.0$

$46 \quad 6.9$

$\begin{array}{ll}-- & 6.4 \\ - & 4.5\end{array}$

$\begin{array}{rr}4.0 & 62 \\ -\quad 2.9 & 47\end{array}$

$\begin{array}{lllll}8.0 & 8.5 & .98 & 8.5 & 79 \\ 8.0 & 8.5 & -- & 8.2 & 78\end{array}$

284107095583400 LINE OSO SITE 02

\begin{tabular}{|c|c|c|c|c|c|c|c|c|}
\hline DATE & FIME & $\begin{array}{r}\text { SAMP- } \\
\text { LING } \\
\text { DEPTH } \\
\text { (FT) }\end{array}$ & $\begin{array}{l}\text { SPE- } \\
\text { CIFIC } \\
\text { CON- } \\
\text { DUCT- } \\
\text { ANCE } \\
\text { (MICRO- } \\
\text { MHOS) }\end{array}$ & $\begin{array}{c}\text { PH } \\
\text { (UNITS) }\end{array}$ & $\begin{array}{l}\text { TEMPER } \\
\text { ATLRE, } \\
\text { WATER } \\
\text { (DEG C) }\end{array}$ & $\begin{array}{l}\text { TRANS- } \\
\text { PAR- } \\
\text { ENCY } \\
\text { (SECCHI } \\
\text { OISK) } \\
\text { (M) }\end{array}$ & $\begin{array}{c}\text { OXYGEN, } \\
\text { DIS* } \\
\text { SOLVEO } \\
\text { (NG/L.) }\end{array}$ & $\begin{array}{l}\text { OXYGEN, } \\
\text { DIS- } \\
\text { SOLVED } \\
\text { (PER- } \\
\text { CENT } \\
\text { SATUR- } \\
\text { ATION) }\end{array}$ \\
\hline \multicolumn{9}{|c|}{ WhY , 1977} \\
\hline $25 . \cdots$ & 1200 & -1.0 & 500 & 8.1 & 25.0 & -- & 8.0 & 99 \\
\hline $25 \ldots$ & 1202 & 10 & 2700 & 8.2 & 25.0 & $=$ & 7.8 & 98 \\
\hline 25. & 1204 & 15 & 12000 & 8.1 & 25. & $\cdots$ & 7.1 & 91 \\
\hline 25. & 1206 & 20 & 20000 & 8.1 & 26. & - & 8.9 & 94 \\
\hline 25. & 1500 & 1.0 & 550 & 8.2 & 25 & .26 & 8.0 & 100 \\
\hline $25 .$. & 1502 & 8.0 & 800 & 8.2 & 25. & $=$ & 8.0 & 100 \\
\hline 25. & 1504 & 15 & 7500 & 8. & 25. & 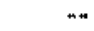 & 7.5 & 95 \\
\hline & 1506 & 20 & 10000 & 8. & 26. & $=$ & 7 & 99 \\
\hline 25. & 1800 & 1.0 & 500 & 8. & 25. & -- & 8.0 & 100 \\
\hline 25. & 1802 & 10 & 550 & B. & 25 & -- & 8. & 100 \\
\hline 25. & 1804 & 15 & 4500 & 8.2 & 26. & 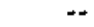 & 7.7 & $9 ?$ \\
\hline 25. & 1806 & 20 & 13000 & 8.1 & 26. & +- & 7.5 & 99 \\
\hline & 2100 & 1.0 & 500 & 8.3 & 25. & -- & 8. & 100 \\
\hline 25. & 2102 & 10 & 760 & 8.2 & 25.5 & $=$ & 7.8 & $\begin{array}{l}98 \\
07\end{array}$ \\
\hline $\begin{array}{l}25 . \\
25 .\end{array}$ & $\begin{array}{l}2104 \\
2106\end{array}$ & $\begin{array}{l}15 \\
20\end{array}$ & $\begin{array}{r}7000 \\
18000\end{array}$ & $\begin{array}{l}8.1 \\
8.1\end{array}$ & $\begin{array}{l}25 . \\
26 .\end{array}$ & $=$ & $\begin{array}{l}7.7 \\
7.2\end{array}$ & $\begin{array}{l}97 \\
96\end{array}$ \\
\hline & 0001 & 1.0 & 510 & 8.1 & 25 . & - & 8.0 & 100 \\
\hline 26. & 0002 & 10 & 600 & 8. & 25 & $=$ & 8.1 & 101 \\
\hline 26. & 0004 & 15 & 7100 & 8.1 & 25.5 & - & 7.7 & 97 \\
\hline $\begin{array}{l}26 . \\
26 .\end{array}$ & 0006 & $\begin{array}{l}20 \\
1.0\end{array}$ & $\begin{array}{r}18000^{\circ} \\
500^{\circ}\end{array}$ & $\begin{array}{l}8 . \\
8 .\end{array}$ & $\begin{array}{l}25 \\
25\end{array}$ & $\cdots$ & $\begin{array}{l}7.1 \\
8.1\end{array}$ & $\begin{array}{r}94 \\
101\end{array}$ \\
\hline & 0302 & 10 & 550 & 8. & 25 & - & 8.0 & 100 \\
\hline 26. & 0304 & 15 & 9000 & 8. & & -- & 7. & 96 \\
\hline 25. & 0306 & 20 & 18000 & 8.1 & 25. & $=$ & 7,0 & 92 \\
\hline $\begin{array}{l}26 . \\
26 .\end{array}$ & $\begin{array}{l}0600 \\
0602\end{array}$ & 1.0 & $\begin{array}{l}510 \\
550\end{array}$ & $\begin{array}{l}8.1 \\
8.1\end{array}$ & $\begin{array}{l}25 . \\
25 .\end{array}$ & $=$ & $\begin{array}{l}8.1 \\
8.0\end{array}$ & $\begin{array}{l}100 \\
100\end{array}$ \\
\hline & 0604 & 15 & 8000 & 8.1 & 25 & - & 7.6 & 98 \\
\hline & 0606 & 20 & 18000 & 8. & 25 & -- & 7. & 95 \\
\hline 26. & 0900 & 1.0 & 520 & & & -- & 8 & 102 \\
\hline & 09 & 10 & 1800 & 8. & 25 & -- & g. & 101 \\
\hline & & 15 & 10000 & 8. & & מ & & 99 \\
\hline 26. & 090 & 19 & 19000 & 8. & 26. & سـ & 7 & 100 \\
\hline & 1200 & 1.0 & 590 & 8.2 & 25 & $m$ & 8. & 102 \\
\hline & 1202 & 10 & 1100 & 8. & & -- & & 101 \\
\hline & 120 & 15 & 760 & 8. & & -- & & 99 \\
\hline & 1206 & & 15000 & & 26. & & & 96 \\
\hline \multicolumn{9}{|l|}{ JuL } \\
\hline 27. & 1600 & 1.0 & 4100 & 8.2 & 31.5 & $=m$ & 7. & 104 \\
\hline & 160 & 9.5 & & 8. & & -- & 4. & 73 \\
\hline & & 19 & 45000 & 8.0 & 30.0 & -- & & 87 \\
\hline
\end{tabular}




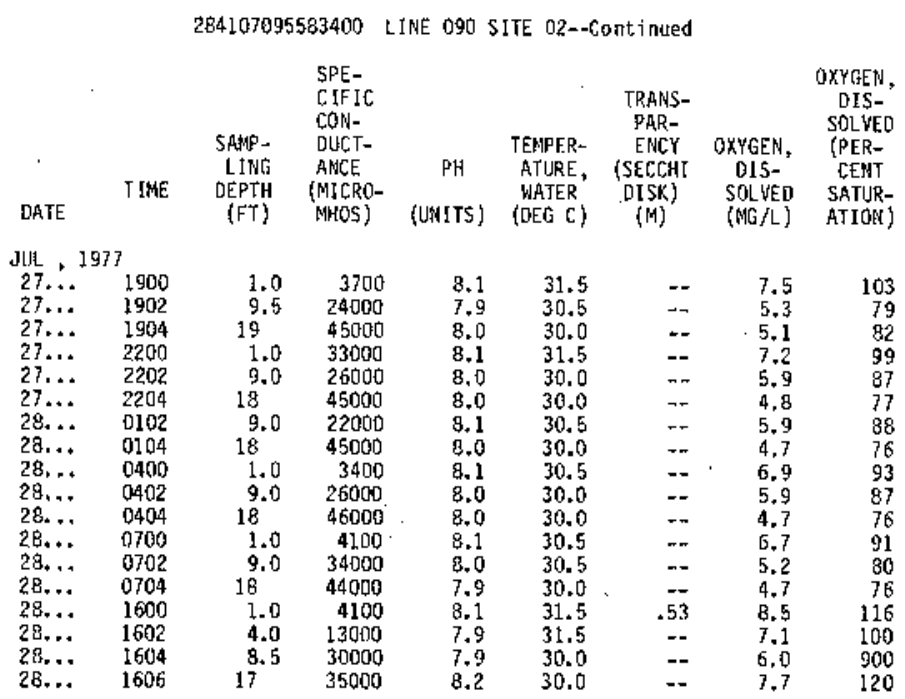

284038095582100 LINE 095 SITE 02

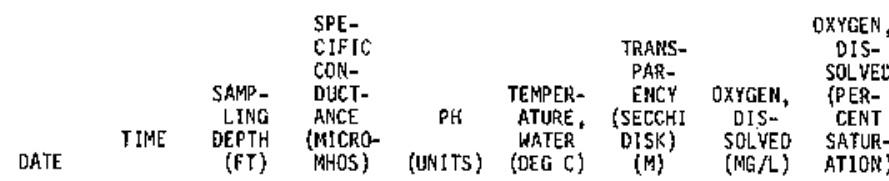

FEB, 1977

\begin{tabular}{|c|c|c|c|c|c|c|c|c|}
\hline $\begin{array}{l}\text { } \\
07 \ldots \\
07 \ldots \\
07 \ldots \\
07 \ldots\end{array}$ & $\begin{array}{l}1255 \\
1257 \\
1259 \\
1301\end{array}$ & $\begin{array}{l}1.0 \\
5.0 \\
10 \\
15\end{array}$ & $\begin{array}{r}2000 \\
2000 \\
4000 \\
28000\end{array}$ & $\begin{array}{l}8.0 \\
7.7 \\
7.7 \\
8.0\end{array}$ & $\begin{array}{l}12.0 \\
11.5 \\
12.0 \\
12.0\end{array}$ & $\begin{array}{l}.13 \\
-. \\
- \\
--\end{array}$ & $\begin{array}{l}9.9 \\
9.8 \\
9.8 \\
8.9\end{array}$ & $\begin{array}{l}95 \\
93 \\
95 \\
94\end{array}$ \\
\hline JUN & & & & & & & & \\
\hline $\begin{array}{l}28 . \ldots \\
28 . \ldots \\
28 . \ldots \\
F E B, 1978\end{array}$ & $\begin{array}{l}1210 \\
1212 \\
1214\end{array}$ & $\begin{array}{l}1.0 \\
6.0 \\
13\end{array}$ & $\begin{array}{r}6000 \\
16000 \\
48000\end{array}$ & $\begin{array}{l}8.2 \\
8.1 \\
8.0\end{array}$ & $\begin{array}{l}30.0 \\
29.0 \\
29.0\end{array}$ & $\begin{array}{c}.60 \\
\ldots+ \\
\cdots\end{array}$ & $\begin{array}{l}7.4 \\
6.8 \\
5.3\end{array}$ & $\begin{array}{r}100 \\
95 \\
86\end{array}$ \\
\hline $\begin{array}{l}02 \ldots \\
02 \ldots\end{array}$ & $\begin{array}{l}1140 \\
1142\end{array}$ & $15^{1.0}$ & $\begin{array}{l}41000 \\
41000\end{array}$ & $\begin{array}{l}7.9 \\
8.0\end{array}$ & $\begin{array}{l}6.5 \\
6.0\end{array}$ & $\begin{array}{l}.60 \\
--\end{array}$ & $\begin{array}{l}9.0 \\
8.9\end{array}$ & $\begin{array}{l}90 \\
89\end{array}$ \\
\hline $\begin{array}{l}31 \ldots \\
31 \ldots \\
31 \ldots\end{array}$ & $\begin{array}{l}1711 \\
1713 \\
1715\end{array}$ & $\begin{array}{l}1.0 \\
7.0\end{array}$ & $\begin{array}{l}18000 \\
29000 \\
41000\end{array}$ & $\begin{array}{l}8.3 \\
8.0 \\
7.9\end{array}$ & $\begin{array}{l}30.5 \\
29.0 \\
29.0\end{array}$ & $\begin{array}{r}.67 \\
-- \\
--\end{array}$ & $\begin{array}{l}7.7 \\
5.1 \\
3.8\end{array}$ & $\begin{array}{r}109 \\
75 \\
58\end{array}$ \\
\hline
\end{tabular}

284005095575400 LINE 105 SITE 02

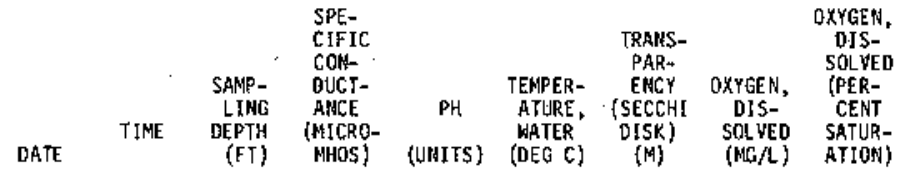

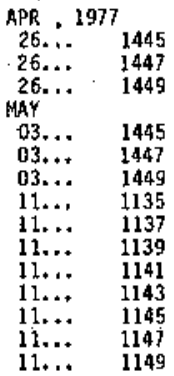

$\begin{array}{cr}1.0 & 550 \\ 5.0 & 550 \\ 10 & 550 \\ 1.0 & 700 \\ 8.0 & 800 \\ 15 & 900 \\ 1.0 & 1000 \\ 5.0 & 1100 \\ 7.0 & 1300 \\ 10 & 7000 \\ 11 & 34000 \\ 12 & 38000 \\ 15 & 41000 \\ 18 & 41000\end{array}$

.20

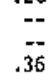

.36

--

--

--

$\begin{array}{ll}7.4 & 89 \\ 7.5 & 90 \\ 7.6 & 93 \\ 7.8 & 95 \\ 7.7 & 94 \\ 7.7 & 94 \\ 7.5 & 93 \\ 6.3 & 88 \\ 6.1 & 88 \\ 6.1 & 88 \\ 6.1 & 88\end{array}$



Table 5A:--Quality of water in the Colorado estuary, water years 1977-78--Continued
Field Determinations-Cont inued

283916095574500 LINE 1.15 SITE OZ

\begin{tabular}{|c|c|c|c|c|c|c|c|c|}
\hline DATE & TINE & $\begin{array}{l}\text { SAMP. } \\
\text { EING } \\
\text { DEPTHA } \\
\text { (FT) }\end{array}$ & $\begin{array}{l}\text { SPE- } \\
\text { CIFIC } \\
\text { CON- } \\
\text { OUCT- } \\
\text { ANCE } \\
\text { (MICRO- } \\
\text { MHOS) }\end{array}$ & $\begin{array}{c}\text { PH } \\
\text { (UNITS) }\end{array}$ & $\begin{array}{l}\text { TEAPER- } \\
\text { ATURE, } \\
\text { WATER } \\
\text { (DEG C) }\end{array}$ & $\begin{array}{l}\text { TRANS- } \\
\text { PAR- } \\
\text { ENCY } \\
\text { (SECCHI } \\
\text { DISK) } \\
\text { (N) }\end{array}$ & $\begin{array}{c}\text { OXYGEN, } \\
\text { DIS- } \\
\text { SOLWED } \\
\text { (WEA } A)\end{array}$ & $\begin{array}{c}\text { OXYGEN, } \\
\text { DIS- } \\
\text { SOLVEO } \\
\text { (PER- } \\
\text { CENT } \\
\text { SATUR- } \\
\text { ATIOS) }\end{array}$ \\
\hline \multicolumn{9}{|c|}{ FEB, 1977} \\
\hline $\begin{array}{l}07 \ldots \\
07 \ldots \\
07 \ldots \\
07 \ldots \\
\text { JUN }\end{array}$ & $\begin{array}{l}1240 \\
1242 \\
1244 \\
1246\end{array}$ & $\begin{array}{l}1.0 \\
5.0 \\
10 \\
17\end{array}$ & $\begin{array}{r}1000 \\
2000 \\
24000 \\
45000\end{array}$ & $\begin{array}{l}7.7 \\
7.5 \\
7.7 \\
7.6\end{array}$ & $\begin{array}{l}12.0 \\
11.5 \\
12.0 \\
12.0\end{array}$ & $\begin{array}{c}.12 \\
-- \\
--\end{array}$ & $\begin{array}{r}10.0 \\
9.8 \\
9.0 \\
8.2\end{array}$ & $\begin{array}{l}96 \\
93 \\
94 \\
96\end{array}$ \\
\hline $\begin{array}{l}28 \ldots \\
28 \ldots \\
28 \ldots \\
\text { FEB... }\end{array}$ & $\begin{array}{l}1220 \\
1222 \\
1224 \\
\end{array}$ & $\begin{array}{l}1.0 \\
10 \\
19\end{array}$ & $\begin{array}{l}28000 \\
48000 \\
48000\end{array}$ & $\begin{array}{r}8.1 \\
8.0 \\
8.0\end{array}$ & $\begin{array}{l}29.0 \\
28.5 \\
29.0\end{array}$ & $\begin{array}{l}.71 \\
-.- \\
--\end{array}$ & $\begin{array}{l}7.0 \\
5.6 \\
5.3\end{array}$ & $\begin{array}{r}101 \\
88 \\
86\end{array}$ \\
\hline $\begin{array}{l}02 \ldots . . \\
02 \ldots \\
\text { MRY }\end{array}$ & $\begin{array}{l}1147 \\
1149\end{array}$ & 15 & $\begin{array}{l}23000 \\
37000\end{array}$ & $\begin{array}{l}8.0 \\
7.9\end{array}$ & $\begin{array}{l}6.5 \\
6.5\end{array}$ & $\begin{array}{r}.79 \\
\ldots\end{array}$ & $\begin{array}{r}10.1 \\
3.1\end{array}$ & $\begin{array}{l}92 \\
89\end{array}$ \\
\hline $\begin{array}{l}31 \ldots \\
31 \ldots \\
31 \ldots \\
31 \ldots\end{array}$ & $\begin{array}{l}1723 \\
1725 \\
1727 \\
1729\end{array}$ & $\begin{array}{l}1.0 \\
5.0 \\
10 \\
19\end{array}$ & $\begin{array}{l}25000 \\
25000 \\
41000 \\
41000\end{array}$ & $\begin{array}{l}8.2 \\
8.0 \\
7.9 \\
7.8\end{array}$ & $\begin{array}{l}30.5 \\
30.0 \\
29.0 \\
28.5\end{array}$ & $\begin{array}{l}.71 \\
-- \\
-- \\
--\end{array}$ & $\begin{array}{l}7.8 \\
6.2 \\
4.4 \\
3.2\end{array}$ & $\begin{array}{r}115 \\
89 \\
68 \\
49\end{array}$ \\
\hline
\end{tabular}

283807095589700 LINE 125 SITE 02

MAY , 1977

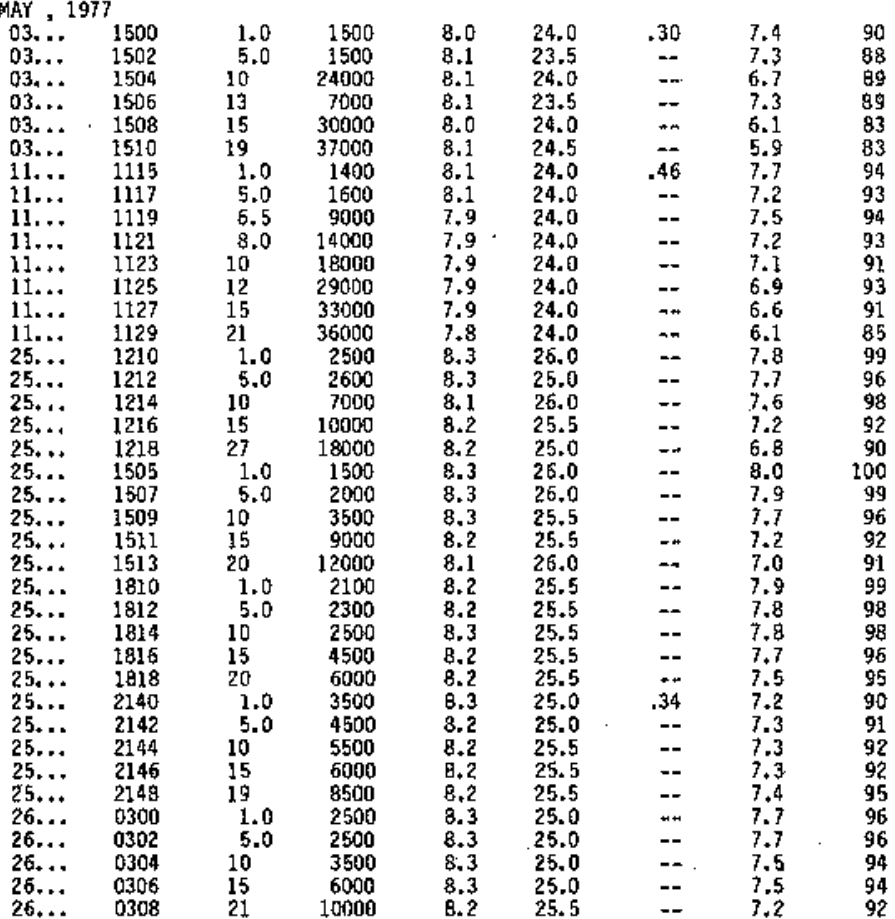


Table 5A. m Q Field Determinations--Cont inated

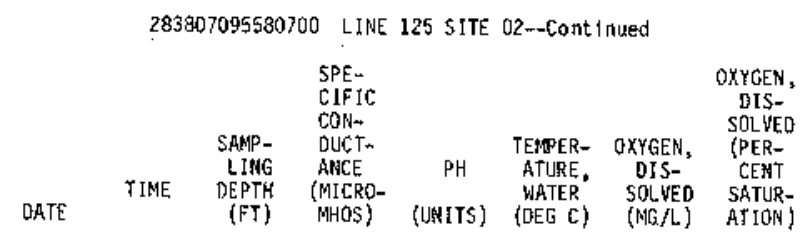

MAY 1977

MAY, 19770435

$26 . . .0437$

$26 . . .0439$

$26 \ldots \quad 0441$

$26 . .0443$

$26 . \ldots .0617$

$26 . . \quad 0619$

$26 . . . \quad 0621$

$26 \ldots \quad 0623$

$26 . . .0900$

$26 . . .0902$

$\begin{array}{ll}26 . \ldots & 0904 \\ 26 . \ldots & 0906\end{array}$

26... 0908

$25 . . . \quad 0910$

$26 . \cdots 1220$

$26 . . .1224$

$26 . \cdots 1226$

$26 . . .1228$

$26 \ldots 1230$

ग)

27... 1600

$27 \ldots \quad 1602$

$27 . \cdots \quad 1604$

27... 1902

$27 . . . \quad 1904$

$27 . \cdots \quad 2200$

$27+\cdots+2202$

$\begin{array}{ll}27 . . . & 2204 \\ 28 . . . & 0100\end{array}$

28... 0102

$28 . . . \quad 0104$

28... 0400

28... 0402

28... 0404

28... 0700

28... 0702

$28 . .0704$

$28 . .1000$

28... 1002

28... 1300

28... 1302

$28 . . .1304$

$28 . . .1600$

$\begin{array}{ll}28 . . . & 1602 \\ 28, . . & 1604\end{array}$

$\begin{array}{ll}1.0 & 3500 \\ 5.0 & 3500 \\ 10 & 6000 \\ 15 & 8900 \\ 24 & 8500 \\ 1.0 & 2500 \\ 5.0 & 2500 \\ 10 & 3000 \\ 15 & 5000\end{array}$

20

$1.0 \quad 2500$

5.03000

15

20

$25 \quad 7500$

2.0 7500

1.0000

15

$20 \quad 9000$

9000
9000

8.325 .0

$7.7-96$

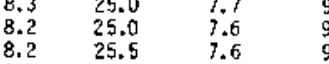

$8.2 \quad 25.3 \quad 6.6$

$8.2 \quad 25.1$

8.325 .0

8.325.

$8.2 \quad 25.0$

$8.2 \quad 25.0$

$\begin{array}{ll}8.2 & 25.0 \\ 8.3 & 25.0\end{array}$

3.325 .0

$8.2 \quad 25.0$

$8.2 \quad 25.0$

8.326 .0

$8.3 \quad 25.5$

$8.2 \quad 25.0$

$\begin{array}{ll}8.2 & 25.5 \\ 8.2 & 25.5\end{array}$

$1.0 \quad 16000$

$\begin{array}{cc}8.0 & 46000 \\ 15 & 50000 \\ 1.0 & 17000\end{array}$

$\begin{array}{cc}1.0 & 17000 \\ 1.0 & 20000 \\ 14 & 44000\end{array}$

$1.0 \quad 19000$

$7.0 \quad 26500$

15

$\begin{array}{rr}1.0 & 22000 \\ 7.5 & 27000\end{array}$

$15 \quad 29000$

$1.0 \quad 25000$

$17.5 \quad 51000$

$\begin{array}{cc}1.0 & 28000 \\ 12 & 50000\end{array}$

$24 \quad 50000$

$1.0 \quad 42000$

12

24
$1.0 \quad 30000$

$10 \quad 48000$

$20 \quad 50000$

1.018000

$\begin{array}{rl}8.5 & 41000 \\ 17 & 50000\end{array}$

$\begin{array}{llll}8.2 & 32.0 & 8.0 & 119 \\ 8.2 & 30.5 & 6.3 & 103\end{array}$

8.130 .5

8.0 31.5

$8.0 \quad 30.5$

$8.0 \quad 31.0$

$8.0 \quad 31.0$

8. 0 30.5

$\begin{array}{ll}8.1 & 31.0 \\ 8.5 & 32.0\end{array}$

$8.0 \quad 32.0$

8.131 .0

$8.1 \quad 30.0$

8.1 30.0

8.130 .0

8. $\quad 29.5$

B.1 29.5

$8.0 \quad 29.5$

$\begin{array}{ll}8.0 & 29.5 \\ 8.0 & 30.5\end{array}$

$\begin{array}{ll}8.0 & 30.5 \\ 8.0 & 29.5\end{array}$

$8.0 \quad 29.5$

8.2 32.0

$\begin{array}{ll}8.2 & 30.0 \\ 8.1 & 29.5\end{array}$

6.2
7.6

7.6
7.6

7.5

7.4
7.7

7.6
7.5

7.3

7.3

8.0

7.4

7.2

283706095582500 LINE 130 SITE O2

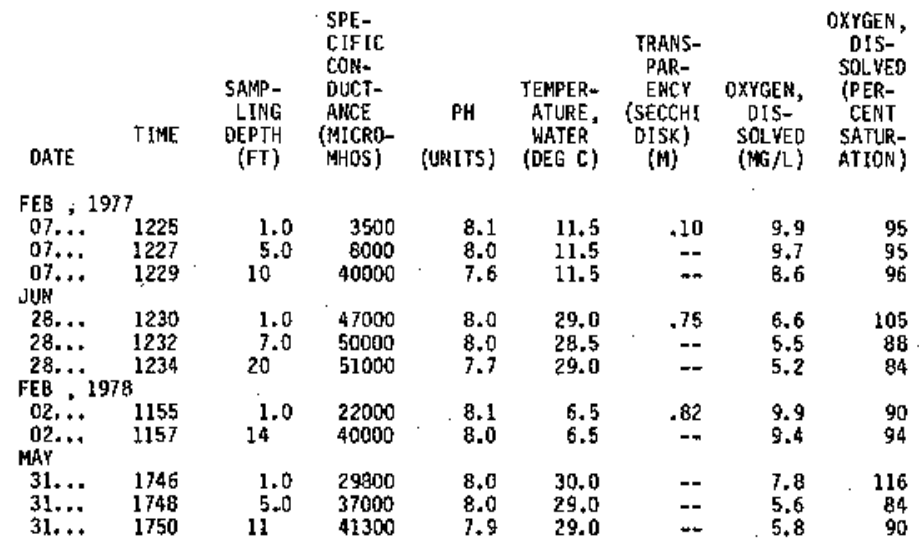


Table 5h.--Quality of water in the colorado estuary, water years 1977-79--Continued Field Determinations--Continued

293555095584400 LINE 147 SITE 02

\begin{tabular}{|c|c|c|c|c|c|c|c|c|}
\hline DATE & TIME & $\begin{array}{r}\text { SANP - } \\
\text { LING } \\
\text { DEPTH } \\
\text { (FT) }\end{array}$ & $\begin{array}{l}\text { SPE- } \\
\text { C1FIC } \\
\text { CON- } \\
\text { OUCT- } \\
\text { ANCE } \\
\text { (MICRO- } \\
\text { MOSS) }\end{array}$ & $\begin{array}{c}\mathrm{PH} \\
\text { (UNITS) }\end{array}$ & $\begin{array}{l}\text { TEMPER- } \\
\text { ATURE, } \\
\text { WATER } \\
\text { (DEG C) }\end{array}$ & $\begin{array}{c}\text { TRANS- } \\
\text { PAR- } \\
\text { ENCY } \\
\text { (SECCHI } \\
\text { DISK) } \\
\text { (M) }\end{array}$ & $\begin{array}{l}\text { OXYGEN, } \\
\text { OIS- } \\
\text { SOLVED } \\
\text { (MG } / L)\end{array}$ & $\begin{array}{l}\text { OXYGEN, } \\
\text { DIS- } \\
\text { SOLVED } \\
\text { CPER- } \\
\text { CENT } \\
\text { SATUR- } \\
\text { ATION }\end{array}$ \\
\hline
\end{tabular}

FEB , 1977

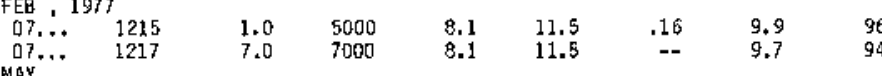

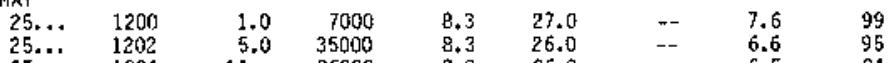

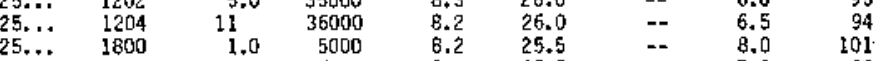

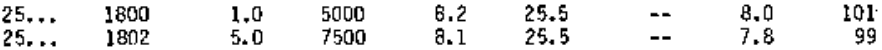

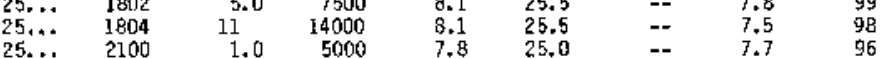

$\begin{array}{lllllllll}25 \ldots & 2100 & 1.0 & 5000 & 7.8 & 25.0 & -- & 7.7 & 96 \\ 25 \ldots & 2102 & 11 & 5500 & 7.8 & 25.0 & -- & 7.6 & 95 \\ 26 . \ldots & 0001 & 1.0 & 5500 & 8.2 & 25.0 & -. & 7.4 & 92\end{array}$

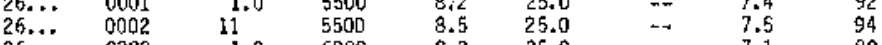

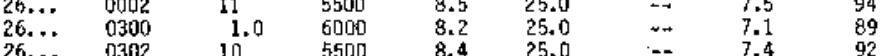

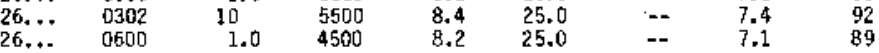

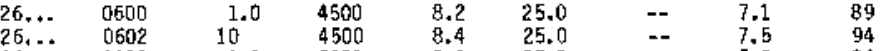

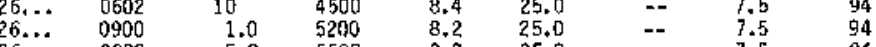

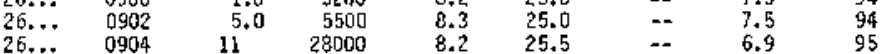

$26, \ldots 1200$

26... 1500

$26 . . .1502$

26... 1503

J... 1625

$27, \ldots \quad 1627$

$27 . \ldots \quad 1629$

27... 2225

$27 \ldots \quad 2227$

$28 . . \quad 0115$

$28 . . \quad 0117$

$28, \ldots \quad 0119$

28... 0400

28... 0402

28... 0406

$28, \cdots \quad 0700$

$28 . . . \quad 0704$

$28 \ldots-072$

$28 . . . \quad 0722$

$28 . . . \quad 0724$

28... 1000

$28 . . . \quad 100$

28... 1300

$28 . . .1302$

$28 . . .1304$

$28 \ldots \quad 1615$

$28, \ldots .1619$

$$
11
$$

$\begin{array}{rr}11.0 & 5300 \\ 11 & 34000\end{array}$

$1.0 \quad 6000$

$11^{5.0} \quad 35000$

8.3 25.5

$+7.6$

94

$\begin{array}{cc}1.0 & 27000 \\ 5.0 & 27000 \\ 10 & 48000 \\ 1.0 & 21000 \\ 5.0 & 30000\end{array}$

9.540000

$1.0 \quad 55000$

5.055000

$9.0 \quad 55000$

$\begin{array}{rr}5.0 & 55000 \\ 10 & 55000\end{array}$

$\begin{array}{lr}10 & 55000 \\ 1.0 & 4100\end{array}$

$\begin{array}{cc}9.0 & 34000 \\ 18 & 44000 \\ 1.0 & 55000\end{array}$

$1.0 \quad 55000$

10

55000
55000

$5.0 \quad 55000$

10.55000

1.0
5.0
10

$10 \quad 55000$

$\begin{array}{ll}1.0 & 35000 \\ 5.0 & 47000\end{array}$

$\begin{array}{lllll}8.3 & 31.0 & -- & 7.8 & 116 \\ 8.2 & 31.0 & -- & 7.3 & 109\end{array}$

$\begin{array}{lllll}8.2 & 31.0 & -- & 7.3 & 109 \\ 8.3 & 30.5 & -- & 6.2 & 101\end{array}$

$7.7 \quad 30.5$

30,5

30.5

30.0

$7.7 \quad 30.0$

$\begin{array}{ll}7.7 & 39.5\end{array}$

$7.7 \quad 29.5$

$\begin{array}{ll}7.7 & 29.0 \\ 8.1 & 30.5 \\ 8.0 & 30.5\end{array}$

$\begin{array}{ll}8.0 & 30.5 \\ 7.9 & 30.0\end{array}$

$\begin{array}{ll}7.9 & 30.0 \\ 7.7 & 29.0\end{array}$

$7.7 \quad 29.0$

7.5
7.6

$7.6 \quad 29.0$

$7.9 \quad 30.5$

$7.9 \quad 30.0$

$\begin{array}{ll}7.9 & 30.0 \\ 8.2 & 31.5 \\ 8.2 & 31.0\end{array}$

-- 5.1

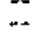

-
--

$--$

$-$

in 5

$=-\quad 6.2$

$\begin{array}{ll}-- & 5.7 \\ -- & 4.7\end{array}$

-4.7
$-\quad 46$

$\begin{array}{rrr}- & 4.8 & 79 \\ - & 5.1 & 84\end{array}$

$\begin{array}{rrr}- & 5.1 & 84 \\ - & 5.2 & 85 \\ - & 4.9\end{array}$

$\begin{array}{lll}- & 4.9 & 80 \\ - & 5.1 & 83\end{array}$

\begin{tabular}{rrr}
- & 5.1 & 83 \\
.54 & 5.0 & 82 \\
\hline & 5.3 & 89
\end{tabular}

$\begin{array}{rrr}.54 & 5.3 & 89 \\ - & 5.1 & 87 \\ - & 5.9 & 87\end{array}$

$-\quad 4.9$

$10 \quad 52000$

$\begin{array}{ll}8.1 & 30.5\end{array}$

284149095563300 LINE 164 SITE 02

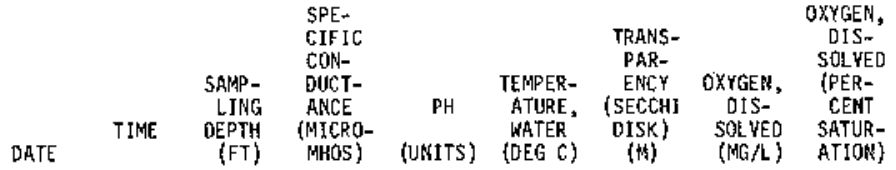

FEB , 1977

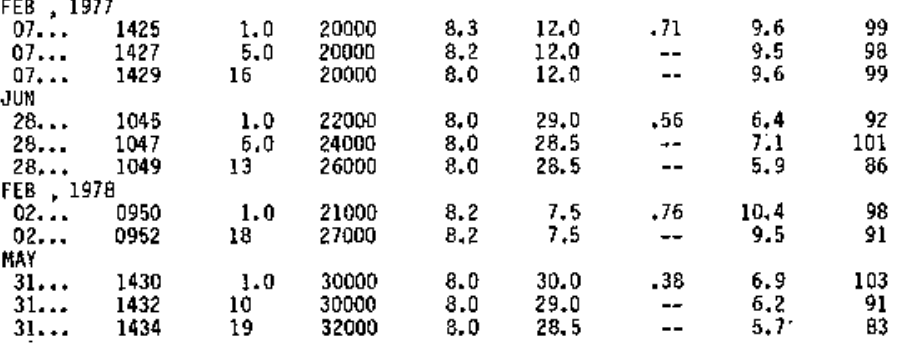


Table 5A.--Quality of water in the Colorado estuary, water years 1977-78--Continued Field Deteminat fons--Continued

283454095583400 LIKE 901 SITE 45

\begin{tabular}{|c|c|c|c|c|}
\hline $\begin{array}{l}\text { SPE- } \\
\text { CIFIC } \\
\text { CON- } \\
\text { DUCT- } \\
\text { ARCE } \\
\text { (MICRO- } \\
\text { (MHOS) }\end{array}$ & $\begin{array}{c}\text { PH } \\
\text { (UNA1TS) }\end{array}$ & $\begin{array}{l}\text { TEMPER- } \\
\text { ATURE, } \\
\text { WATER } \\
\text { (DEG C) }\end{array}$ & $\begin{array}{c}\text { OXYGEN, } \\
\text { DIS- } \\
\text { SOLVED } \\
\text { (MG } / L)\end{array}$ & $\begin{array}{c}\text { DXYGEN, } \\
\text { DIS- } \\
\text { SOLVED } \\
\text { (PER- } \\
\text { CENT } \\
\text { SATUR- } \\
\text { ATLON) }\end{array}$ \\
\hline
\end{tabular}

DATE

TIME LING ANCE

DEPTH (MICRO-

MHOS) (UNITS) (DEG C)

(NG/L) ATION)

JUL , 1977

28.1977

$\begin{array}{llll}1.0 & 58000 & 8.1 & 30.5\end{array}$

$28 \ldots . .1642$

1.0
7.5 58000

$8.0 \quad 30.5$

$\begin{array}{ll}4.9 & 86 \\ 4.1 & 72 \\ 4.3 & 76\end{array}$

58000

8.130 .5 
(FT . feet; $M G / L=$ milligramis per 1 iter)

284255095583500 LINE OBI SITE 02

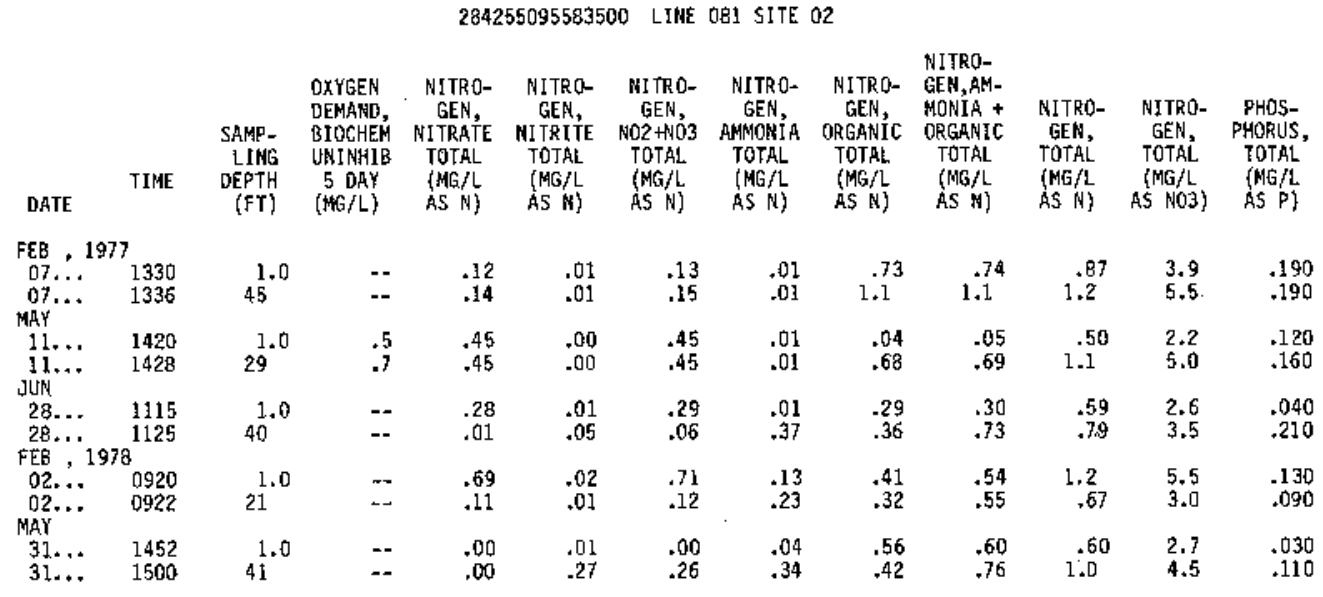

284005095575400 LINE 105 SITE O2

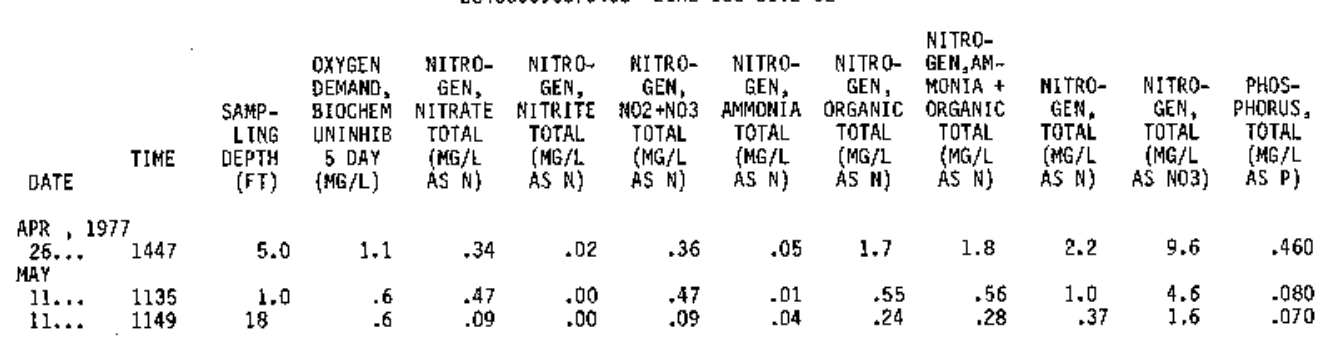

283807095580700 LINE 125 SITE 02

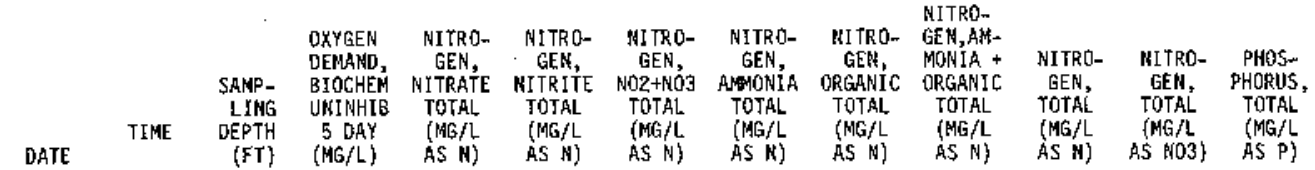

MAY, 1977

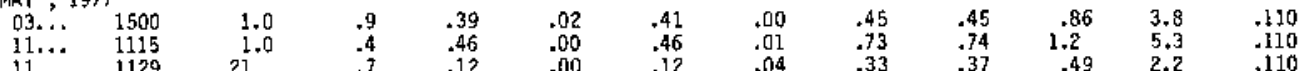

283706095582500 LINE 130 SITE 02

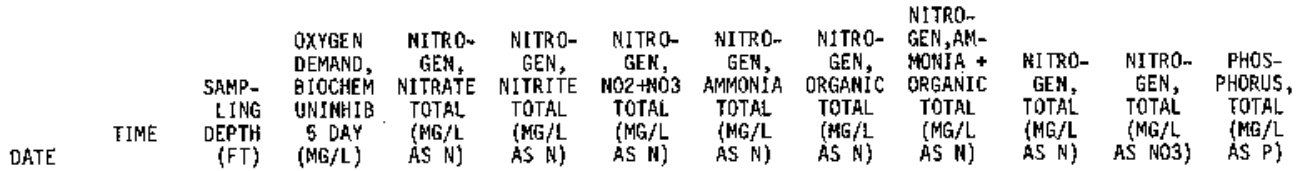

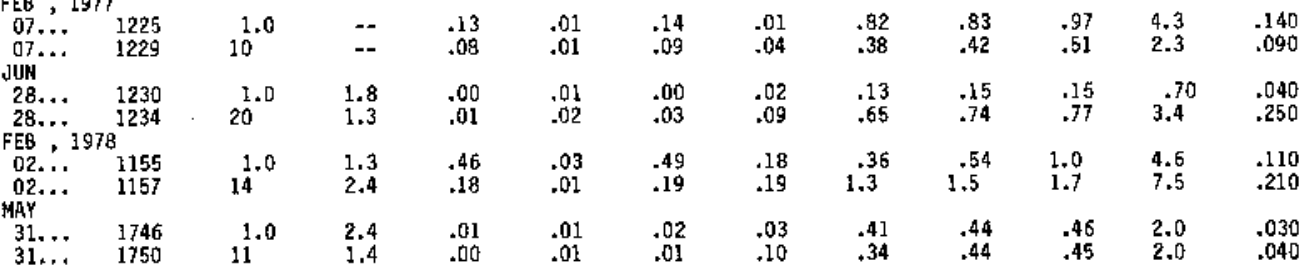


TabTe 5B.--Quality of water in the colorado estuary, water years 1977-78--Continued Nutrient Analyses--Continued

284149095563300 LINE 164 SITE Q2

NITRO- NITRO- WITRO- NITRO- NITRO- NITRO-

GEN

GEN, GEN, GEN, GEN, GEH, MONIA + NITRO- NITRO- PHOS-

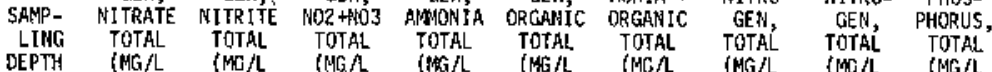

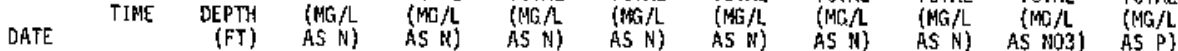

FEB , 1977

\begin{tabular}{|c|c|c|c|c|c|c|c|c|c|c|c|}
\hline $\begin{array}{c}07 \ldots \\
07 \ldots\end{array}$ & $\begin{array}{l}1425 \\
1429\end{array}$ & $1 \frac{1.0}{6}$ & $\begin{array}{l}.00 \\
.01\end{array}$ & $\begin{array}{l}.00 \\
.00\end{array}$ & $\begin{array}{l}.00 \\
.01\end{array}$ & $\begin{array}{l}.01 \\
.02\end{array}$ & $\begin{array}{l}.40 \\
.35\end{array}$ & $\begin{array}{r}.41 \\
.37\end{array}$ & $\begin{array}{r}.41 \\
.38\end{array}$ & $\begin{array}{l}1.8 \\
1.7\end{array}$ & $\begin{array}{r}.040 \\
.050\end{array}$ \\
\hline $\begin{array}{l}28 \ldots . \\
28 \ldots \\
F E B\end{array}$ & $\begin{array}{l}1045 \\
1049\end{array}$ & 13.0 & $\begin{array}{l}.16 \\
.13\end{array}$ & .01 & $\begin{array}{l}.17 \\
.15\end{array}$ & $\begin{array}{l}.03 \\
.09\end{array}$ & $\begin{array}{l}.10 \\
.19\end{array}$ & $\begin{array}{l}.13 \\
.28\end{array}$ & .30 & $\begin{array}{l}1.3 \\
1.9\end{array}$ & $\begin{array}{l}.050 \\
.080\end{array}$ \\
\hline $\begin{array}{cc}02 \ldots & 0 \\
02 \ldots & 0\end{array}$ & $\begin{array}{l}0950 \\
0952\end{array}$ & 18 & $\begin{array}{l}.05 \\
.01\end{array}$ & $\begin{array}{l}.00 \\
.02\end{array}$ & $\begin{array}{r}.05 \\
.03\end{array}$ & .01 & $\begin{array}{l}.44 \\
.70\end{array}$ & $\begin{array}{l}.45 \\
.73\end{array}$ & $\begin{array}{l}.50 \\
.76\end{array}$ & $\begin{array}{l}2.2 \\
3.4\end{array}$ & $\begin{array}{r}.080 \\
.080\end{array}$ \\
\hline $\begin{array}{l}31 \ldots \\
31 \ldots\end{array}$ & $\begin{array}{l}1430 \\
1434\end{array}$ & 19.0 & $\begin{array}{l}.01 \\
.00\end{array}$ & .01 & .02 & .06 & $\begin{array}{l}.45 \\
.45\end{array}$ & $\begin{array}{l}.51 \\
.51\end{array}$ & $\begin{array}{l}.53 \\
.52\end{array}$ & $\begin{array}{l}2.3 \\
2.3\end{array}$ & $\begin{array}{l}.04 \\
.06\end{array}$ \\
\hline
\end{tabular}


(FT - feet; MICROMHOS = micromhos per centimeter at $25^{\circ}$ Celsius; $\mathrm{HG} / L=$ milligrams per liter; AC-FT $=$ acre-feet $)$ 284255095583500 LIBE DBI SITE Q?

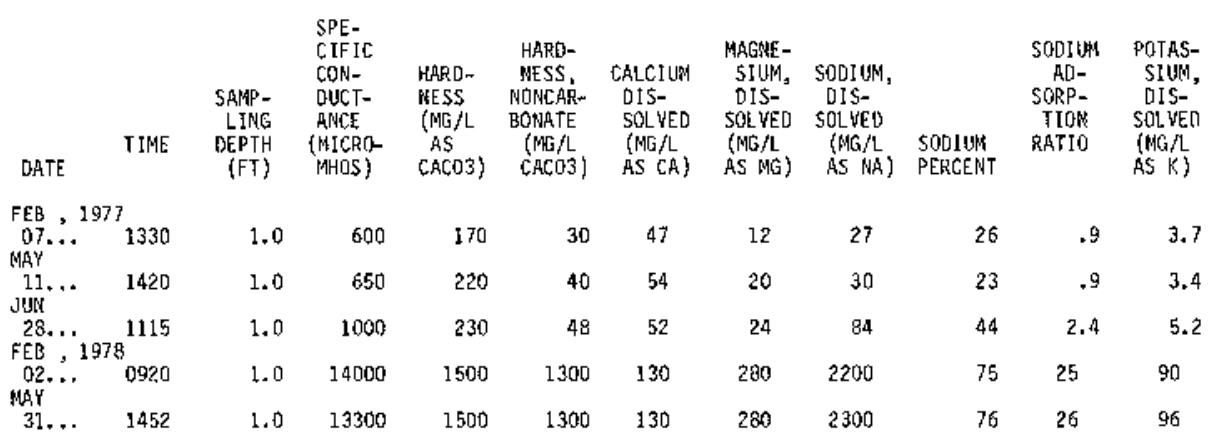

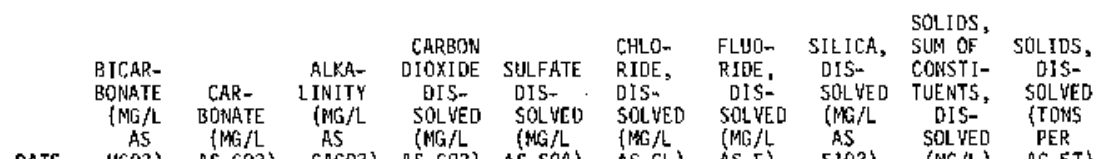

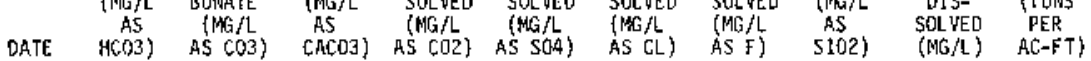

\begin{tabular}{|c|c|c|c|c|c|c|c|c|c|c|}
\hline $\begin{array}{l}\text { FEB }, 1977 \\
\text { O7... }\end{array}$ & 167 & -- & 137 & -- & 34 & 44 & .2 & 9.0 & 259 & .35 \\
\hline JuN & 216 & -- & 177 & - & 42 & 49 & .3 & 9.1 & 314 & .43 \\
\hline FEB 1978 & 220 & -- & 180 & -- & 50 & 140 & .3 & 10 & 474 & .64 \\
\hline MAY & 210 & 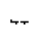 & 172 & - & 550 & 4000 & .4 & 8.8 & 7360 & 10.0 \\
\hline $31 \ldots$ & 200 & 0 & 164 & 1.6 & 580 & 4200 & .6 & 7.1 & 7690 & 10.5 \\
\hline
\end{tabular}

2B3807095580700 LINE 125 SITE 02

\begin{tabular}{|c|c|c|c|c|c|c|c|c|c|c|}
\hline DATE & TIME & $\begin{array}{c}\text { SAMP- } \\
\text { LING } \\
\text { DEPTH } \\
\text { (FT) }\end{array}$ & $\begin{array}{l}\text { SPE- } \\
\text { CIFIC } \\
\text { CON- } \\
\text { DUCT- } \\
\text { ANCE } \\
\text { (MICRD- } \\
\text { MHOS) }\end{array}$ & $\begin{array}{l}\text { HARD- } \\
\text { NESS } \\
\text { \{NALA } \\
\text { AS } \\
\text { CACO3) }\end{array}$ & $\begin{array}{l}\text { HARO- } \\
\text { MESS, } \\
\text { NDNCAR- } \\
\text { BONATE } \\
\text { (MG/L } \\
\text { CACO3) }\end{array}$ & $\begin{array}{l}\text { CALCIUM } \\
\text { DIS- } \\
\text { SOL VED } \\
\text { (IAG } f L \\
\text { AS CA) }\end{array}$ & $\begin{array}{l}\text { FAGNE- } \\
\text { SIUM, } \\
\text { DIS- } \\
\text { SOLVED } \\
\text { (MLG /E } \\
\text { AS MG) }\end{array}$ & $\begin{array}{l}\text { SDDFUM, } \\
\text { DIS- } \\
\text { SOLLED } \\
\text { (MG/L } \\
\text { AS NA) }\end{array}$ & $\begin{array}{l}\text { SODIUM } \\
\text { PERCENT }\end{array}$ & $\begin{array}{l}\text { SODIUA } \\
\text { AD- } \\
\text { SORP- } \\
\text { TIOH } \\
\text { RATIO }\end{array}$ \\
\hline $\begin{array}{l}\text { MAY, } \\
11 . . .\end{array}$ & 7129 & 21 & 36000 & 4000 & 3900 & 270 & 810 & 6900 & 78 & 47 \\
\hline DATE & $\begin{array}{l}\text { POTAS- } \\
\text { SIUM, } \\
\text { OIS- } \\
\text { SOLVED } \\
\text { (MG/L } \\
\text { AS KI) }\end{array}$ & $\begin{array}{c}\text { BICAR- } \\
\text { BONATE } \\
\text { (NA } / L \\
\text { AS } \\
\text { HCO3) }\end{array}$ & $\begin{array}{c}\text { CAR- } \\
\text { BONATE } \\
\text { (MG/L } \\
\text { AS COB })\end{array}$ & $\begin{array}{c}\text { ALKA- } \\
\text { LIKITY } \\
\text { (MGG } / L \\
\text { AS } \\
\text { CACO3) }\end{array}$ & $\begin{array}{l}\text { SULFATE } \\
\text { DIS } \\
\text { SOL VED } \\
\text { (MG/L } \\
\text { AS SO4) }\end{array}$ & $\begin{array}{l}\text { CHLO- } \\
\text { RIDE, } \\
\text { DIS- } \\
\text { SOLVEO } \\
\text { (MG/L } \\
\text { AS CL) }\end{array}$ & $\begin{array}{l}\text { FLUO- } \\
\text { RIDE, } \\
\text { DIS- } \\
\text { SOLWED } \\
\text { (MGA } \\
\text { AS F) }\end{array}$ & $\begin{array}{l}\text { SILICA. } \\
\text { DIS } \\
\text { SOL YED } \\
\text { (MG } / L \\
\text { AS } \\
\text { SI02) }\end{array}$ & $\begin{array}{l}\text { SOLIDS, } \\
\text { SEM OF } \\
\text { CONSTI- } \\
\text { TUENTS, } \\
\text { DIS- } \\
\text { SOLVED } \\
\text { (MG } / L \text { ) }\end{array}$ & $\begin{array}{c}\text { SOLIDS, } \\
\text { DIS- } \\
\text { SOL VED } \\
\text { (TONS } \\
\text { PER } \\
\text { AC-FT) }\end{array}$ \\
\hline
\end{tabular}

MAY, 1977

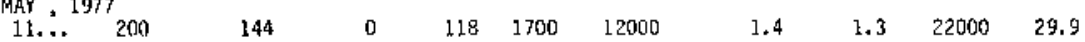




\section{Lavaca-Tres Palacios Estuary}

The Lavaca-Tres Palacios estuary, which has an area of about 350 square miles $\left(905 \mathrm{~km}^{2}\right)$, consists of the tidal parts of the Lavaca and Navidad Rivers, Tres Palacios Creek and other tributaries, Lavaca Bay, Cox Bay, Keller Bay, Carancahua Bay, Tres Palacios Bay, Matagorda Bay, Matagorda Bay Entrance Channel, Pass Cavallo, and part of the Intracoastal Waterway (Figure 7). Water depth at mean low water is 13 feet $(4.0 \mathrm{~m})$ or less in Matagorda Bay, except in the Matagorda Ship Channel, which is more than 40 feet $(12.2 \mathrm{~m})$ deep. Lavaca and Tres Palacios Bays are less than 8 feet $(2.4 \mathrm{~m})$ deep at mean low water, and Cox, Keller, and Carancahua Bays are less than 5 feet $(1.5 \mathrm{~m})$ deep. The rivers are generally less than 15 feet $(4.6 \mathrm{~m})$ deep.

Water-quality data (Table 6) were collected during February and April-June 1977 and February, June and September 1978.

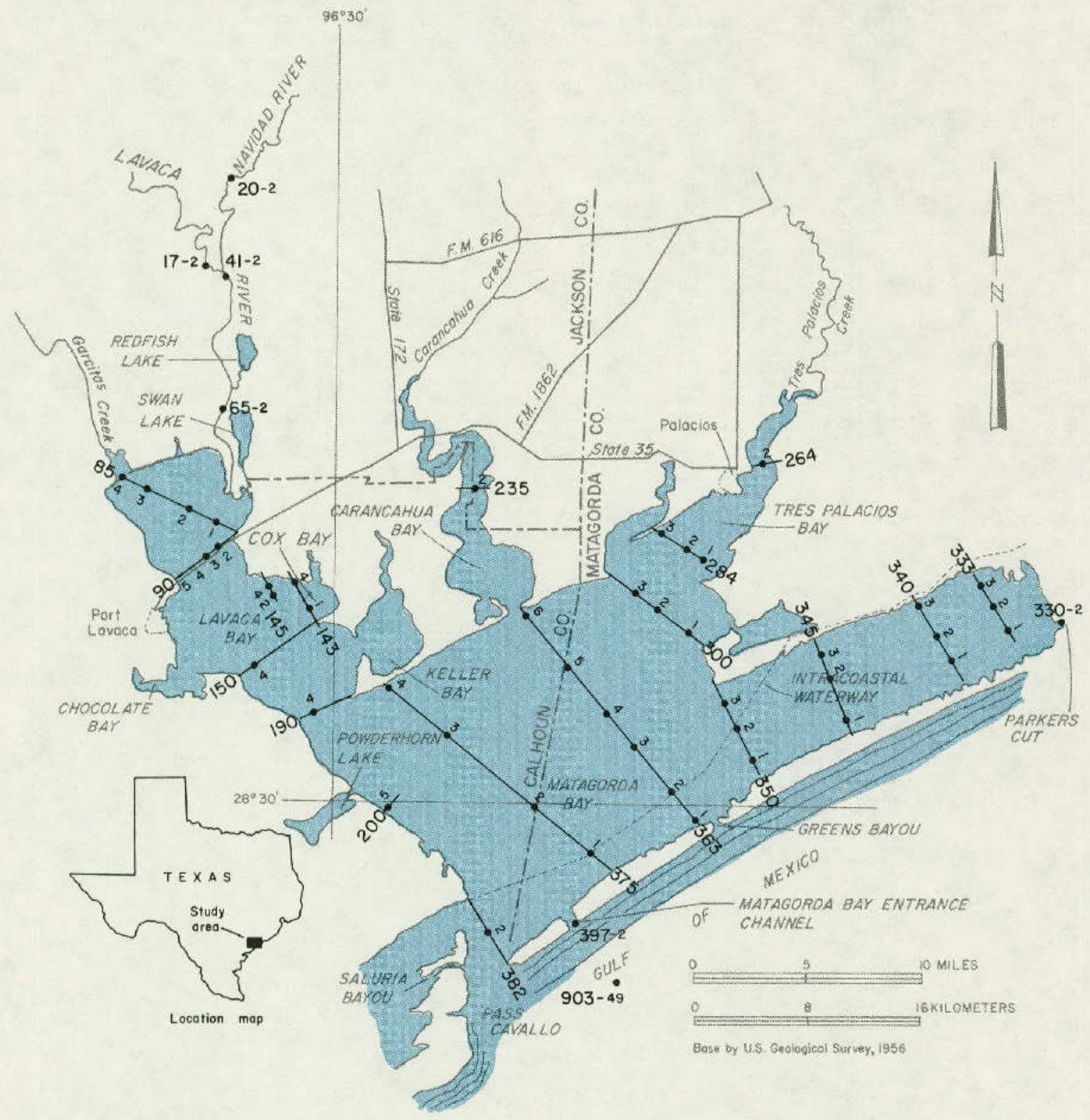

EXPLANATION

90-2 - DATA-COLLECTION LINE AND SITE NUMBER

Figure 7.-Data-Collection Sites in the Lavaca-Tres Palacios Estuary 

Table 6A.--Quality of water in the Lavaca-Tres Palacios estuary, water years 1977-78
Field Deterninations

$\left(F T=\right.$ feet; MICROMHOS $=$ micromhos per centimeter at $25^{\circ}$ Celsius; DEG $C=$ degrees Celsius; $M=$ meters: $M G / L=$ milligrams per 1iter)

285003096352400 LINE 017 SITE 02

\begin{tabular}{|c|c|c|c|c|c|c|c|c|}
\hline DATE & TIME & $\begin{array}{l}\text { SAMP- } \\
\text { LING } \\
\text { DEPTH } \\
\text { (FT) }\end{array}$ & $\begin{array}{l}\text { SPE- } \\
\text { CIFIC } \\
\text { CON- } \\
\text { DUCT- } \\
\text { ANCE } \\
\text { (MICRO- } \\
\text { MHOS) }\end{array}$ & $\begin{array}{c}\text { PH } \\
\text { (UNITS) }\end{array}$ & $\begin{array}{l}\text { TEMPER- } \\
\text { ATURE, } \\
\text { WATER } \\
\text { (DEG C) }\end{array}$ & $\begin{array}{l}\text { TRAMS- } \\
\text { PAR- } \\
\text { ENCY } \\
\text { (SECCHI } \\
\text { DISK) } \\
\text { (M) }\end{array}$ & $\begin{array}{c}\text { OXYGEN, } \\
\text { DIS- } \\
\text { SOLVED } \\
\text { (MG/L) }\end{array}$ & $\begin{array}{l}\text { OXYGEN, } \\
\text { DIS- } \\
\text { SOLVED } \\
\text { (PER- } \\
\text { CENT } \\
\text { SATUR- } \\
\text { ATION) }\end{array}$ \\
\hline \multicolumn{9}{|c|}{ FEB, 1977} \\
\hline $09 .$. & 1130 & 1.0 & 400 & 7.3 & 12.0 & -. & 9.2 & \\
\hline & & & -- & & 88 \\
\hline $27 \ldots$ & 1455 & 1.0 & 800 & 8.1 & 28.5 & .25 & 5.8 & 75 \\
\hline \multicolumn{9}{|c|}{ JUN, 1978} \\
\hline & 0957 & 1.0 & 418 & 8. & 30.5 & .41 & 6. & 87 \\
\hline & 09 & 10 & 451 & 8.1 & 30.5 & -- & 6.3 & 84 \\
\hline
\end{tabular}

285248096344400 LINE 020 SITE 02

\begin{tabular}{|c|c|c|c|c|c|c|c|}
\hline DATE & TIME & $\begin{array}{l}\text { SAMP- } \\
\text { LING } \\
\text { DEPTH } \\
\text { (FT) }\end{array}$ & $\begin{array}{l}\text { SPE- } \\
\text { CIFIC } \\
\text { CON- } \\
\text { DUCT- } \\
\text { ANCE } \\
\text { (MICRO- } \\
\text { MHOS) }\end{array}$ & $\begin{array}{c}\text { PH } \\
\text { (UNITS) }\end{array}$ & $\begin{array}{l}\text { TEMPER- } \\
\text { ATURE, } \\
\text { WATER } \\
\text { (OEG C) }\end{array}$ & $\begin{array}{c}\text { OXYGEN, } \\
\text { DIS- } \\
\text { SOLVED } \\
\text { (MG } / L)\end{array}$ & $\begin{array}{l}\text { OXYGEN, } \\
\text { DIS- } \\
\text { SOLVED } \\
\text { (PER- } \\
\text { CENT } \\
\text { SATUR- } \\
\text { ATION) }\end{array}$ \\
\hline
\end{tabular}

\begin{tabular}{|c|c|c|c|c|c|c|c|}
\hline \multicolumn{8}{|c|}{ SEP , 1978} \\
\hline $12 \ldots$ & 1130 & $\cdots$ & $=$ & $\cdots$ & 25.5 & $\cdots$ & $\cdots$ \\
\hline $12 \ldots$ & 1335 & 3.0 & 265 & 7.6 & 25.0 & - & - \\
\hline $12 . .$. & 1530 & $\cdots$ & $\cdots$ & $\cdots$ & 26.0 & - & $\cdots$ \\
\hline $12 \ldots$ & 1700 & 3.0 & 231 & 7.4 & 26.0 & -- & - \\
\hline $12 . .$. & 1930 & - & - & -. & 26.0 & - & -- \\
\hline $13 \ldots$ & 0845 & $\cdots$ & - & -- & 25.5 & - & - \\
\hline $13 . .$. & 0930 & 1.0 & 250 & 7.2 & 28.0 & 68.0 & 94 \\
\hline & 0945 & 3.0 & 169 & 7.1 & 25.5 & 7.6 & 95 \\
\hline 13. & 1100 & -. & -- & -- & 25.5 & -- & - \\
\hline 13. & 1630 & 3.0 & 176 & 7.3 & 25.0 & 4.4 & 54 \\
\hline 13. & 1900 & -- & -- & -. & 26.0 & -- & -- \\
\hline 19. & 0955 & $\cdots$ & 230 & -- & $\cdots$ & - & $\cdots$ \\
\hline & 0957 & -- & -- & - & 25.5 & - & \\
\hline
\end{tabular}

285223096343100 LINE 022 SITE 02

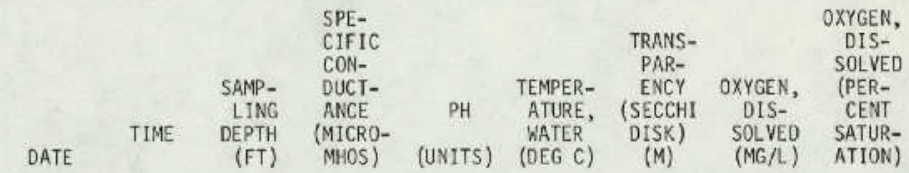

\begin{tabular}{|c|c|c|c|c|c|c|c|c|}
\hline \multicolumn{9}{|c|}{ FEB , 1977} \\
\hline $09 .$. & 1117 & 8.0 & 350 & 7.8 & 11.0 & $\ldots$ & 9.3 & $\begin{array}{l}88 \\
87\end{array}$ \\
\hline \multicolumn{9}{|l|}{ JUN } \\
\hline $27 \ldots$ & 1435 & 1.0 & 600 & 8.0 & 30.0 & .39 & 5.6 & \\
\hline \multicolumn{7}{|l|}{ JUN, 1} & 4.6 & \\
\hline $21 .$. & 0935 & 1.0 & 591 & 8.2 & 31.0 & .43 & 6.7 & \\
\hline $21 \ldots$ & 0937 & 5.0 & 201 & 8.2 & 31.0 & -. & 6.7 & \\
\hline
\end{tabular}


2B4921096342100 LINE 041 SITE 02

\begin{tabular}{|c|c|c|c|c|c|c|c|}
\hline DATE & TIME & $\begin{array}{c}\text { SAMP- } \\
\text { LING } \\
\text { DEPTH } \\
\text { (FT) }\end{array}$ & $\begin{array}{l}\text { SPE- } \\
\text { C1FIC } \\
\text { CON- } \\
\text { DUCT- } \\
\text { ANCE } \\
\text { (MICRO- } \\
\text { MHOS) }\end{array}$ & $\begin{array}{c}\mathrm{PH} \\
\text { (UNITS) }\end{array}$ & $\begin{array}{l}\text { TEMPER- } \\
\text { ATURE, } \\
\text { WATER } \\
\text { (DEG C) }\end{array}$ & $\begin{array}{c}\text { OXYGEN, } \\
\text { OIS- } \\
\text { SOLYED } \\
\text { (MG/L) }\end{array}$ & $\begin{array}{l}\text { OXYGEN, } \\
\text { DIS- } \\
\text { SOLVED } \\
\text { (PER- } \\
\text { CENT } \\
\text { SATUR- } \\
\text { ATION) }\end{array}$ \\
\hline \multicolumn{8}{|c|}{ SEP , 2978} \\
\hline $\begin{array}{l}12 \ldots \\
12 \ldots\end{array}$ & $\begin{array}{l}1206 \\
1208\end{array}$ & $20^{1.0}$ & $\begin{array}{l}264 \\
240\end{array}$ & $\begin{array}{l}7.7 \\
7.8\end{array}$ & $\begin{array}{l}25.5 \\
25.5\end{array}$ & $\begin{array}{l}4.4 \\
4.3\end{array}$ & $\begin{array}{l}55 \\
54\end{array}$ \\
\hline $12 \ldots$ & 1233 & - & - & $=$ & 25.5 & $=$ & $=$ \\
\hline $12 \ldots$ & 1238 & -- & 331 & - & - & -- & $=-$ \\
\hline $\begin{array}{l}12 \ldots \\
12 \ldots\end{array}$ & $\begin{array}{l}1530 \\
1532\end{array}$ & 1.0 & $\begin{array}{l}220 \\
310\end{array}$ & $\begin{array}{l}7.8 \\
7.8\end{array}$ & $\begin{array}{r}25.5 \\
.26 .0\end{array}$ & $\begin{array}{l}5.1 \\
5.0\end{array}$ & $\begin{array}{l}64 \\
62\end{array}$ \\
\hline $12, \ldots$ & 1622 & - & 252 & $=$ & -- &.- & - \\
\hline $12 .$. & 1810 & 1,0 & 220 & 7.7 & 25.5 & 5.2 & 65 \\
\hline 12. & 1812 & 20 & 270 & 7.7 & 25.5 & 5.1 & 64 \\
\hline $12 .$. & 1900 & -- & 290 & - & -- & -- & -- \\
\hline $12 \ldots$ & 2240 & 1.0 & 200 & 7.6 & 25.5 & 5.1 & 64 \\
\hline $12 \ldots$ & 2242 & 20 & 220 & 7.6 & 25.5 & 4.8 & 60 \\
\hline $12 .$. & 2315 & -- & 248 & $\because$ & 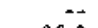 & $-\infty$ & $m$ \\
\hline 13. & 1020 & 1.0 & 170 & 6.1 & 26.0 & 5.0 & 62 \\
\hline $13 \ldots$ & 1050 & -- & -- & -- & 25.0 & $\cdots$ & $\rightarrow$ \\
\hline $13 .$. & 1325 & -- & -- & -- & 26.0 & - & $+\infty$ \\
\hline $14 . \ldots$ & 0815 & -- & -- & - & 25.0 & -- & $=$ \\
\hline $14 . .$. & 1425 & $=$ & -- & -- & 26.0 & -- & -- \\
\hline 14. & 1426 & 1.0 & 140 & 7.3 & 25.5 & 5.8 & 72 \\
\hline $14 .$. & 1430 & -- & 160 & -- & -- & -- & $\cdots$ \\
\hline $14 .$. & 1615 & -- & -- & -- & 26.0 & -- & $\cdots$ \\
\hline 14. & 1618 & 1.0 & 130 & 7.1 & 25.5 & 5,6 & 70 \\
\hline $14 .$. & 1822 & 1.0 & 150 & 7.4 & 25.5 & 5.7 & 71 \\
\hline 14. & 1824 & $\rightarrow$ & 155 & $-\infty$ & 26.0 & -- & $=$ \\
\hline 14. & 2030 & 1.0 & 150 & 7.4 & 25.5 & 5.8 & 72 \\
\hline 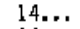 & 2228 & 1.0 & 150 & 7.1 & 25.0 & 5.8 & 72 \\
\hline 4. & 2230 & -- & 150 & - & 26.0 & -- & $\cdots$ \\
\hline 5, & 0715 & 1.0 & 140 & 7.7 & 25.0 & 6.9 & 85 \\
\hline & 0745 & -- & $=$ & -- & 25.0 & -- & $=$ \\
\hline 5. & 0755 & -- & 135 & -- & -- & -- & -- \\
\hline 5. & 0952 & 1.0 & 120 & 7.5 & 25.5 & 6.8 & B5 \\
\hline 1 & 0954 & + & 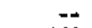 & - & 25.0 & -- & $=$ \\
\hline . & 1143 & 1.0 & 120 & 7.2 & 26.0 & 6.8 & 85 \\
\hline & 1348 & 1.0 & 120 & 7.7 & 25.5 & 6.9 & 86 \\
\hline 5. & 1350 & -- & $=$ & -- & 22.5 & $=$ & -- \\
\hline 15. & 1542 & 1.0 & 110 & 7.8 & 25.5 & 7.0 & 88 \\
\hline 15. & 1545 & $=$ & 113 & -- & 26.0 & - & -- \\
\hline 15. & 1747 & 1.0 & 110 & 7.6 & 25.5 & 7.0 & 88 \\
\hline & 1749 & $+\cdots$ & $\vec{H}$ & + & 26.0 & $=$ & $\because$ \\
\hline & 1940 & $\cdot 1.0$ & 110 & 7.6 & 25.5 & 6.8 & 85 \\
\hline & 1945 & - & -- & -- & 26.0 & $=$ & $\rightarrow$ \\
\hline & 1330 & 1.0 & 250 & 7.4 & 30.0 & 7.2 & 96 \\
\hline & 1335 & 19 & 250 & 7.4 & 29.5 & 7.2 & 95 \\
\hline 1. & 1358 & & -- & -- & 25.0 & -- & $=-$ \\
\hline
\end{tabular}

204516096343400 LINE 065 SITE 02

\begin{tabular}{|c|c|c|c|c|c|c|c|c|}
\hline DATE & TIME & $\begin{array}{r}\text { SAMP } \\
\text { LING } \\
\text { DEPTH } \\
\text { (FT) }\end{array}$ & $\begin{array}{l}\text { SPE- } \\
\text { CIFIC } \\
\text { CON- } \\
\text { DUCT- } \\
\text { ANCE } \\
\text { (MICRO- } \\
\text { MHOS) }\end{array}$ & $\begin{array}{c}\text { PH } \\
\text { (UNITS) }\end{array}$ & $\begin{array}{l}\text { FEMPER- } \\
\text { ATURE, } \\
\text { WATER } \\
\text { (DEG C ) }\end{array}$ & $\begin{array}{l}\text { TRANS- } \\
\text { PAR- } \\
\text { ERCY } \\
\text { (SECCHI } \\
\text { DISK) } \\
\text { (M) }\end{array}$ & $\begin{array}{c}\text { OXYGEN, } \\
\text { DIS- } \\
\text { SOLVED } \\
\text { (MG } / L)\end{array}$ & $\begin{array}{l}\text { OXYGEN, } \\
\text { DIS- } \\
\text { SOLVED } \\
\text { (PER- } \\
\text { CENT } \\
\text { SATUR- } \\
\text { ATYON) }\end{array}$ \\
\hline \multicolumn{9}{|c|}{ JUN , 1977} \\
\hline $\begin{array}{l}27 \ldots \\
27 \ldots \\
\text { JU木 }\end{array}$ & $8^{1410}$ & $13^{1.0}$ & $\begin{array}{l}800 \\
860\end{array}$ & $\begin{array}{l}8.7 \\
8.7\end{array}$ & $\begin{array}{l}29.0 \\
29.0\end{array}$ &. & $\begin{array}{l}7.2 \\
6.8\end{array}$ & $\begin{array}{l}95 \\
89\end{array}$ \\
\hline $\begin{array}{l}21 \ldots \\
21 \ldots \\
21 \ldots\end{array}$ & $\begin{array}{l}1020 \\
1022 \\
1024\end{array}$ & $\begin{array}{l}1.0 \\
7.5 \\
15\end{array}$ & $\begin{array}{r}5000 \\
12000 \\
12000\end{array}$ & $\begin{array}{l}8.0 \\
7.6 \\
7.6\end{array}$ & $\begin{array}{l}30.0 \\
30.0 \\
30.0\end{array}$ & $\begin{array}{l}.41 \\
-- \\
--\end{array}$ & $\begin{array}{l}6.6 \\
5.9 \\
6.0\end{array}$ & $\begin{array}{l}89 \\
81 \\
84\end{array}$ \\
\hline
\end{tabular}

284035096351200 LINE 085 SITE 01

\begin{tabular}{|c|c|c|c|c|c|c|c|c|}
\hline DATE & TIME & $\begin{array}{l}\text { SAMP- } \\
\text { LING } \\
\text { DEPTH } \\
\text { (FT) }\end{array}$ & $\begin{array}{l}\text { 5PE- } \\
\text { CIF IC } \\
\text { CON- } \\
\text { DUCT- } \\
\text { ARCE } \\
\text { (MICRO- } \\
\text { MROS) }\end{array}$ & $\begin{array}{c}\text { PH } \\
\text { (UNITS) }\end{array}$ & $\begin{array}{c}\text { TEMPER- } \\
\text { ATURE, } \\
\text { WATER } \\
\text { (DEG C) }\end{array}$ & $\begin{array}{l}\text { TRANS- } \\
\text { PAR- } \\
\text { ENCY } \\
\text { (SECCHI } \\
\text { DISK) } \\
\text { (M) }\end{array}$ & $\begin{array}{c}\text { OXYGEN, } \\
\text { DIS- } \\
\text { SOLVED } \\
\text { (MG/L) }\end{array}$ & $\begin{array}{c}\text { OXYGEN, } \\
\text { DIS- } \\
\text { SOLWED } \\
\text { (PER- } \\
\text { CENT } \\
\text { SATUR- } \\
\text { ATIOM) }\end{array}$ \\
\hline \multicolumn{9}{|c|}{ FEB, 1977} \\
\hline $\begin{array}{l}09 . \cdots 1 \\
09 . . . \\
\text { JUN }\end{array}$ & $\begin{array}{l}1045 \\
1047\end{array}$ & $\begin{array}{l}1.0 \\
5.0\end{array}$ & $\begin{array}{l}12000 \\
25000\end{array}$ & $\begin{array}{l}8.3 \\
8.2\end{array}$ & $\begin{array}{l}11.0 \\
11.0\end{array}$ & -- & $\begin{array}{l}9.5 \\
8.5\end{array}$ & $\begin{array}{l}93 \\
88\end{array}$ \\
\hline $\begin{array}{l}28 . \cdots \\
28, \cdots \\
\text { JUEN }\end{array}$ & ${ }^{0905}$ & $\begin{array}{l}1.0 \\
4.5\end{array}$ & $\begin{array}{l}1800 \\
1800\end{array}$ & $\begin{array}{l}8.4 \\
8.4\end{array}$ & $\begin{array}{l}27.5 \\
27.5\end{array}$ & $\begin{array}{r}.10 \\
--\end{array}$ & $\begin{array}{l}6.8 \\
6.7\end{array}$ & $\begin{array}{l}87 \\
86\end{array}$ \\
\hline $\begin{array}{l}21 . . . \\
21 \ldots\end{array}$ & $\begin{array}{l}0849 \\
0851\end{array}$ & $\begin{array}{l}1.0 \\
4.0\end{array}$ & $\begin{array}{l}19000 \\
19000\end{array}$ & $\begin{array}{l}8.0 \\
8.0\end{array}$ & $\begin{array}{l}29.0 \\
28.5\end{array}$ & .53 & $\begin{array}{l}6.3 \\
6.4\end{array}$ & $\begin{array}{l}88 \\
88\end{array}$ \\
\hline
\end{tabular}


Table 6A,--Quality of water in the Lavaca-Tres Palacios estuary, water years 1977-78-Continued

Field Determinations--Continued

2B4116096362700 LINE 085 SITE O2

\begin{tabular}{|c|c|c|c|c|c|c|c|c|}
\hline DATE & TIME & $\begin{array}{l}\text { SANP - } \\
\text { LIKG } \\
\text { DEPTH } \\
(\mathbf{F T})\end{array}$ & $\begin{array}{l}\text { SPE- } \\
\text { CIFIC } \\
\text { CON- } \\
\text { DUCT- } \\
\text { ANCE } \\
\text { (MICRO- } \\
\text { MHOS) }\end{array}$ & $\begin{array}{c}\text { PH } \\
\text { (UNITS) }\end{array}$ & $\begin{array}{l}\text { TEPPER- } \\
\text { ATURE, } \\
\text { WATER } \\
\text { (OEG C) }\end{array}$ & $\begin{array}{c}\text { TRABS- } \\
\text { PAR- } \\
\text { ENCY } \\
\text { (SECCHI } \\
\text { DISK) } \\
\text { (M) }\end{array}$ & $\begin{array}{c}\text { OXYGEN, } \\
\text { DIS- } \\
\text { SOL WED } \\
\text { (MG } / L)\end{array}$ & $\begin{array}{l}\text { OXYGEN, } \\
\text { DIS- } \\
\text { SOLVED } \\
\text { (PER- } \\
\text { CENT } \\
\text { SATUR- } \\
\text { ATION) }\end{array}$ \\
\hline \multicolumn{9}{|c|}{ JUM , 1977} \\
\hline $\begin{array}{l}28 \ldots \\
28 \ldots \\
\text { JUN }\end{array}$ & ${ }_{8}^{0915}$ & $\begin{array}{l}1.0 \\
5.0\end{array}$ & $\begin{array}{l}2500 \\
2400\end{array}$ & $\begin{array}{l}8.4 \\
8.4\end{array}$ & $\begin{array}{l}27.5 \\
27.5\end{array}$ & .10 & $\begin{array}{l}6.9 \\
6.9\end{array}$ & $\begin{array}{l}90 \\
90\end{array}$ \\
\hline $\begin{array}{l}21 \ldots \\
21 \ldots\end{array}$ & $\begin{array}{l}0838 \\
0840\end{array}$ & $\begin{array}{l}1.0 \\
5.0\end{array}$ & $\begin{array}{l}19000 \\
19000\end{array}$ & $\begin{array}{l}8.0 \\
8.0\end{array}$ & $\begin{array}{l}28.5 \\
28.5\end{array}$ & .40 & $\begin{array}{l}6.6 \\
6.5\end{array}$ & $\begin{array}{l}92 \\
90\end{array}$ \\
\hline
\end{tabular}

284200096374500 LINE 085 SITE 03

\begin{tabular}{|c|c|c|c|c|c|c|c|c|}
\hline ATE & TIME & $\begin{array}{l}\text { SAMP- } \\
\text { LING } \\
\text { DEPTH } \\
\text { (FT) }\end{array}$ & $\begin{array}{l}\text { \$PE- } \\
\text { CIFIC } \\
\text { CON- } \\
\text { DUCT- } \\
\text { ANCE } \\
\text { (MICRO- } \\
\text { MHOS) }\end{array}$ & $\begin{array}{c}\text { PH } \\
\text { (UNITS) }\end{array}$ & $\begin{array}{l}\text { TEMPER- } \\
\text { ATURE, } \\
\text { WATER } \\
\text { (DEG C) }\end{array}$ & $\begin{array}{l}\text { TRANS- } \\
\text { PAR- } \\
\text { ENCY } \\
\text { (SECCHI } \\
\text { DISK) } \\
\text { (II) }\end{array}$ & $\begin{array}{c}\text { DXYGEN, } \\
\text { DIS- } \\
\text { SOLVED } \\
\text { (HG } f \text { ) }\end{array}$ & $\begin{array}{c}\text { OXYGEN, } \\
\text { OIS- } \\
\text { SOLVED } \\
\text { (PER- } \\
\text { CENT } \\
\text { SATUR- } \\
\text { ATION) }\end{array}$ \\
\hline
\end{tabular}

\begin{tabular}{|c|c|c|c|c|c|c|c|c|}
\hline \multicolumn{9}{|c|}{ JUN ， 1977} \\
\hline $\begin{array}{r}28 \ldots \\
28 \ldots\end{array}$ & $\begin{array}{l}0920 \\
0922 \\
8^{-}\end{array}$ & $\begin{array}{l}1.0 \\
5.0\end{array}$ & $\begin{array}{l}2000 \\
2100\end{array}$ & $\begin{array}{l}8.3 \\
8.3\end{array}$ & $\begin{array}{l}27.5 \\
27.5\end{array}$ & .10 & $\begin{array}{l}6.7 \\
6.7\end{array}$ & $\begin{array}{l}86 \\
86\end{array}$ \\
\hline $\begin{array}{l}16 \ldots \\
16 \ldots \\
16 \ldots \\
16 \ldots \\
16 \ldots \\
16 \ldots \\
17 \ldots \\
17 \ldots\end{array}$ & $\begin{array}{l}0737 \\
0739 \\
1234 \\
1236 \\
1747 \\
1749 \\
0735 \\
0737\end{array}$ & $\begin{array}{l}1.0 \\
4.0 \\
1.0 \\
5.0 \\
1.0 \\
5.0 \\
1.0 \\
5.0\end{array}$ & $\begin{array}{l}21000 \\
23000 \\
19000 \\
20000 \\
19000 \\
20000 \\
21000 \\
21000\end{array}$ & $\begin{array}{l}8.5 \\
8.6 \\
8.6 \\
8.6 \\
8.8 \\
8.8 \\
8.9 \\
8.9\end{array}$ & $\begin{array}{r}8.0 \\
8.0 \\
9.5 \\
9.5 \\
10.5 \\
10.5 \\
10.0 \\
10.0\end{array}$ & $\begin{array}{l}-- \\
-- \\
-- \\
-- \\
-- \\
-- \\
--\end{array}$ & $\begin{array}{l}10.4 \\
10.2 \\
12.6 \\
12.8 \\
13.3 \\
13.5 \\
11.0 \\
11.2\end{array}$ & $\begin{array}{r}99 \\
97 \\
123 \\
124 \\
133 \\
135 \\
110 \\
112\end{array}$ \\
\hline $\begin{array}{l}20 \ldots \\
20 \ldots \\
21 \ldots \\
21 \ldots\end{array}$ & $\begin{array}{l}2012 \\
2014 \\
0815 \\
0817\end{array}$ & $\begin{array}{l}1.0 \\
4.0 \\
1.0 \\
5.0\end{array}$ & $\begin{array}{l}17000 \\
18000 \\
19100 \\
21000\end{array}$ & $\begin{array}{l}8.4 \\
8.4 \\
8.0 \\
8.0\end{array}$ & $\begin{array}{l}30.5 \\
30.0 \\
28.5 \\
28.5\end{array}$ & $\begin{array}{l}.26 \\
.35 \\
.-\end{array}$ & $\begin{array}{l}6.6 \\
6.8 \\
6.5 \\
6.7\end{array}$ & $\begin{array}{l}95 \\
96 \\
90 \\
95\end{array}$ \\
\hline
\end{tabular}

28423509638490 LINE OBS SITE O4

\begin{tabular}{|c|c|c|c|c|c|c|c|c|}
\hline DATE & TIME & $\begin{array}{l}\text { SANP - } \\
\text { LING } \\
\text { DEPTH } \\
\text { (FI) }\end{array}$ & $\begin{array}{l}\text { SPE- } \\
\text { CIF IC } \\
\text { CON- } \\
\text { DUCT- } \\
\text { ANCE } \\
\text { (MICRO- } \\
\text { MHOS) }\end{array}$ & $\begin{array}{c}\text { PH } \\
\text { (UNITS) }\end{array}$ & $\begin{array}{l}\text { TEMPER- } \\
\text { ATURE, } \\
\text { WATER } \\
\text { (DEG C) }\end{array}$ & $\begin{array}{l}\text { TRANS- } \\
\text { PAR- } \\
\text { ENCY } \\
\text { (SECCHI } \\
\text { DISK) } \\
\text { (M) }\end{array}$ & $\begin{array}{l}\text { OXYGEN, } \\
\text { DIS- } \\
\text { SOLVED } \\
\text { (NG/L) }\end{array}$ & $\begin{array}{c}\text { OXYGEN, } \\
\text { DIS- } \\
\text { SOLVED } \\
\text { (PER- } \\
\text { CENT } \\
\text { SATUR- } \\
\text { ATIOW) }\end{array}$ \\
\hline \multicolumn{9}{|c|}{ FEB, 1977} \\
\hline $\begin{array}{l}09 \ldots \\
09 . \ldots \\
\text { JUN }\end{array}$ & $\begin{array}{l}1030 \\
1032\end{array}$ & $\begin{array}{l}1.0 \\
3.0\end{array}$ & $\begin{array}{l}13000 \\
15000\end{array}$ & $\begin{array}{l}8.3 \\
8.3\end{array}$ & $\begin{array}{l}11.0 \\
11.0\end{array}$ & $=$ & $\begin{array}{l}9.3 \\
8.9\end{array}$ & $\begin{array}{l}92 \\
88\end{array}$ \\
\hline $\begin{array}{l}28 \ldots . \\
28 \ldots \\
\text { JUN , } 1\end{array}$ & $\begin{array}{l}0930 \\
0932 \\
8\end{array}$ & $\begin{array}{l}1.0 \\
3.5\end{array}$ & $\begin{array}{l}2400 \\
2300\end{array}$ & $\begin{array}{l}8.3 \\
8.4\end{array}$ & $\begin{array}{l}27.5 \\
28.0\end{array}$ & $\begin{array}{l}.10 \\
--\end{array}$ & $\begin{array}{l}7.0 \\
6.8\end{array}$ & $\begin{array}{l}90 \\
88\end{array}$ \\
\hline $\begin{array}{l}21 \ldots \\
21 \ldots\end{array}$ & $\begin{array}{l}0828 \\
0830\end{array}$ & $\begin{array}{l}1.0 \\
3.5\end{array}$ & $\begin{array}{l}16000 \\
19000\end{array}$ & $\begin{array}{l}8.0 \\
8.0\end{array}$ & $\begin{array}{l}28.5 \\
28.5\end{array}$ & $\begin{array}{r}.44 \\
--\end{array}$ & $\begin{array}{l}6.1 \\
6.3\end{array}$ & $\begin{array}{l}84 \\
87\end{array}$ \\
\hline
\end{tabular}

283924096352000 LINE 090 SITE O2

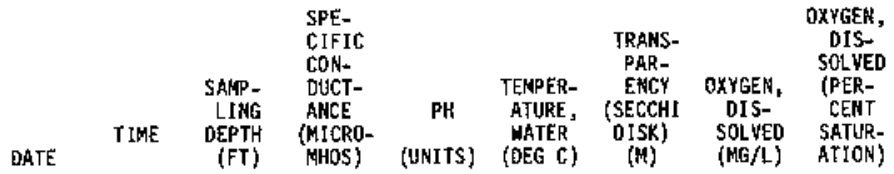

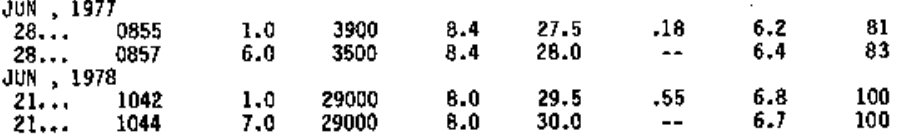


Table 6A,--Quality of water in the Lavaca-Tres Palacios estuary, water years 1977-78-Continued

Field Determinations-Cont inued

283912096354400 LINE 090 SITE 03

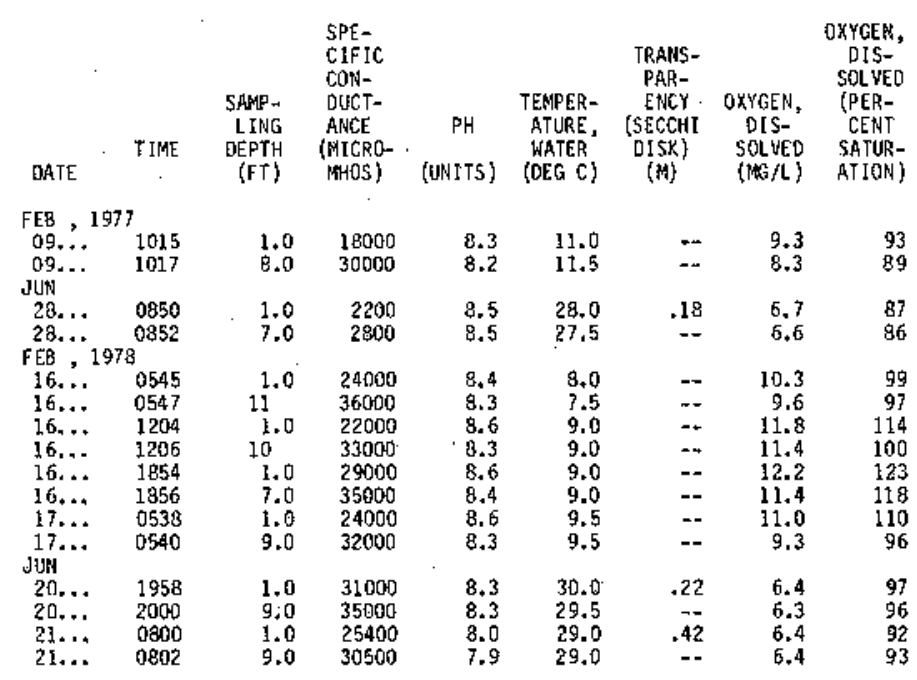

283843096361600 LINE O90 SITE O4

\begin{tabular}{|c|c|c|c|c|c|c|c|c|}
\hline OATE & TIME & $\begin{array}{l}\text { SAPF- } \\
\text { LING } \\
\text { DEPTH } \\
\text { (FT) }\end{array}$ & $\begin{array}{l}\text { SPE- } \\
\text { CIFIC } \\
\text { CON- } \\
\text { DUCT- } \\
\text { ANCE } \\
\text { (MICRO- } \\
\text { MHOS) }\end{array}$ & $\begin{array}{c}\text { PH } \\
\text { (UNITS) }\end{array}$ & $\begin{array}{l}\text { TEMPEA- } \\
\text { ATURE, } \\
\text { WATER } \\
\text { (DEG C) }\end{array}$ & $\begin{array}{c}\text { TRANS- } \\
\text { PAR- } \\
\text { ENCY } \\
\text { (SECCHI } \\
\text { DISK) } \\
\text { (N) }\end{array}$ & $\begin{array}{c}\text { OXYGEN, } \\
\text { DIS- } \\
\text { SDLVED } \\
\text { (IAG } / L \text { ) }\end{array}$ & $\begin{array}{c}\text { OXYGEN, } \\
\text { OIS- } \\
\text { SOLUEO } \\
\text { (PER- } \\
\text { CENT } \\
\text { SATUR- } \\
\text { ATION) }\end{array}$ \\
\hline \multicolumn{9}{|c|}{ JIN , 1977} \\
\hline $\begin{array}{l}28 \ldots . . \\
28 \ldots \\
\text { JUN }, 1\end{array}$ & $\begin{array}{l}0845 \\
0847 \\
8\end{array}$ & $\begin{array}{l}1.0 \\
3.5\end{array}$ & $\begin{array}{l}3800 \\
3800\end{array}$ & $\begin{array}{l}8.6 \\
8.6\end{array}$ & & .18 & $\begin{array}{l}6.6 \\
6.4\end{array}$ & $\begin{array}{l}86 \\
82\end{array}$ \\
\hline $\begin{array}{l}21 \ldots \\
21 \ldots\end{array}$ & $\begin{array}{l}1137 \\
1139\end{array}$ & $\begin{array}{l}1.0 \\
5.0\end{array}$ & $\begin{array}{l}26000 \\
29000\end{array}$ & $\begin{array}{l}8.0 \\
8.0\end{array}$ & $\begin{array}{l}30.0 \\
30.0\end{array}$ & $=-$ & $\begin{array}{l}6.7 \\
6.7\end{array}$ & $\begin{array}{r}99 \\
100\end{array}$ \\
\hline
\end{tabular}

283716096310400 LIINE 143 SITE 01

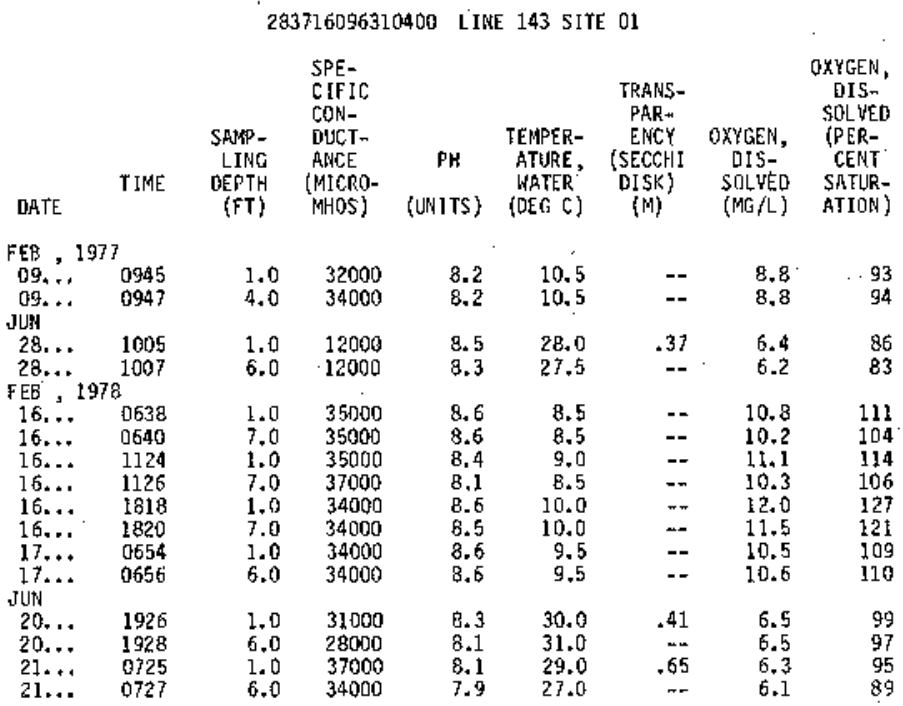

283819096321100 LINE 143 SITE O4

\begin{tabular}{|c|c|c|c|c|c|c|c|c|}
\hline DATE & TIME & $\begin{array}{l}\text { SAMP- } \\
\text { LING } \\
\text { DEPTH } \\
\text { (FT) }\end{array}$ & $\begin{array}{l}\text { SPE- } \\
\text { CIF IC } \\
\text { CON- } \\
\text { DUCT- } \\
\text { ANCE } \\
\text { (MICRO- } \\
\text { MHOS) }\end{array}$ & $\begin{array}{c}\text { PH } \\
\text { (UNITS) }\end{array}$ & $\begin{array}{c}\text { TEMPER- } \\
\text { ATURE, } \\
\text { WATER } \\
\text { (OEG C) }\end{array}$ & $\begin{array}{l}\text { TRANS- } \\
\text { PAR- } \\
\text { EMCY } \\
\text { (SECCHI } \\
\text { OISK) } \\
\text { (M) }\end{array}$ & $\begin{array}{c}\text { OXYGEN, } \\
\text { OIS- } \\
\text { SOLWED } \\
\text { (MG } / L)\end{array}$ & $\begin{array}{l}\text { OXYGEN, } \\
\text { DIS- } \\
\text { SOL HED } \\
\text { (PER- } \\
\text { CENT } \\
\text { SATUR- } \\
\text { ATION) }\end{array}$ \\
\hline \multicolumn{9}{|c|}{ JUN , 1977} \\
\hline $\begin{array}{l}28 . \cdots \\
28 \ldots \\
\text { JUN }\end{array}$ & $\begin{array}{l}1010 \\
1012 \\
8\end{array}$ & $\begin{array}{l}1.0 \\
5.0\end{array}$ & $\begin{array}{l}7300 \\
8400\end{array}$ & $\begin{array}{l}3.5 \\
8.3\end{array}$ & $\begin{array}{l}28.0 \\
28.5\end{array}$ & $\begin{array}{r}.26 \\
--\end{array}$ & $\begin{array}{l}7.0 \\
6.2\end{array}$ & $\begin{array}{l}92 \\
83\end{array}$ \\
\hline $\begin{array}{r}21 . \ldots \\
21 . \ldots\end{array}$ & $\begin{array}{l}1117 \\
1119\end{array}$ & $\begin{array}{l}1.0 \\
6.0\end{array}$ & $\begin{array}{l}35000 \\
35000\end{array}$ & $\begin{array}{l}8.0 \\
8.0\end{array}$ & $\begin{array}{l}30.0 \\
32.0\end{array}$ & $\begin{array}{r}.44 \\
-.\end{array}$ & $\begin{array}{l}6.6 \\
6.5\end{array}$ & $\begin{array}{l}100 \\
101\end{array}$ \\
\hline
\end{tabular}


Table 6A,--quality of water in the Lavaca-Tres Palacios estuary, water years 1977-78--Continued Field Determinations- - Continued

283902096330200 LINE 145 SITE 02

\begin{tabular}{|c|c|c|c|c|c|c|c|c|}
\hline DATE & TIME & $\begin{array}{l}\text { SAPP- } \\
\text { LIMG } \\
\text { DEPTH } \\
\text { (FT) }\end{array}$ & $\begin{array}{l}\text { SPE- } \\
\text { CIFIC } \\
\text { COHS- } \\
\text { OUCT- } \\
\text { ANCE } \\
\text { (MICRO- } \\
\text { MHOS) }\end{array}$ & $\begin{array}{c}\text { PH } \\
\text { (UNITS) }\end{array}$ & $\begin{array}{l}\text { TEROER- } \\
\text { ATURE, } \\
\text { WATER } \\
\text { (DEC C) }\end{array}$ & $\begin{array}{c}\text { TRAKS- } \\
\text { PAR- } \\
\text { EMCY } \\
\text { (SECCHY } \\
\text { OISK) } \\
\text { (M) }\end{array}$ & $\begin{array}{c}\text { OXYGEN, } \\
\text { DIS- } \\
\text { SOLYED } \\
\text { (YG/L) }\end{array}$ & $\begin{array}{c}\text { OXYGEN, } \\
\text { GIS- } \\
\text { SOLVED } \\
\text { (PER- } \\
\text { CENT } \\
\text { SATUR- } \\
\text { ATHON) }\end{array}$ \\
\hline \multicolumn{8}{|l|}{ FEB } & \multirow[b]{2}{*}{$\begin{array}{l}90 \\
85 \\
72 \\
75\end{array}$} \\
\hline $\begin{array}{l}09 . . . \\
09 . . . \\
09 . . . \\
09 . . .\end{array}$ & $\begin{array}{l}1000 \\
1002 \\
1004 \\
1006\end{array}$ & $\begin{array}{l}1.0 \\
10 \\
25 \\
35\end{array}$ & $\begin{array}{l}32000 \\
37000 \\
41000 \\
37000\end{array}$ & $\begin{array}{l}8.3 \\
8.1 \\
8.1 \\
8.1\end{array}$ & $\begin{array}{l}11.0 \\
11.0 \\
10.0 \\
10.0\end{array}$ & $\begin{array}{l}-- \\
=- \\
--\end{array}$ & $\begin{array}{l}8.4 \\
7.7 \\
6.5 \\
7.1\end{array}$ & \\
\hline $\begin{array}{l}26 . . . \\
28 . . .\end{array}$ & $\begin{array}{l}1025 \\
1027\end{array}$ & $\begin{array}{l}1.0 \\
4.0\end{array}$ & $\begin{array}{l}7600 \\
8300\end{array}$ & $\begin{array}{l}8.5 \\
8.4\end{array}$ & $\begin{array}{l}27.5 \\
28.5\end{array}$ & .25 & $\begin{array}{l}6.9 \\
6.5\end{array}$ & $\begin{array}{l}90 \\
87\end{array}$ \\
\hline $\begin{array}{l}\text { Jun , } 1 \\
21 \ldots . . \\
21 \ldots\end{array}$ & $\begin{array}{l}8 \\
1100 \\
1102\end{array}$ & $\begin{array}{l}1.0 \\
5.0\end{array}$ & $\begin{array}{l}35000 \\
35000\end{array}$ & $\begin{array}{l}8.0 \\
8.0\end{array}$ & $\begin{array}{l}30.0 \\
30.0\end{array}$ & $\begin{array}{r}.32 \\
--\end{array}$ & $\begin{array}{l}8.4 \\
6.6\end{array}$ & $\begin{array}{l}128 \\
100\end{array}$ \\
\hline
\end{tabular}

$293812096331200 \quad$ LIEE $\cdot 145$ SITE 04

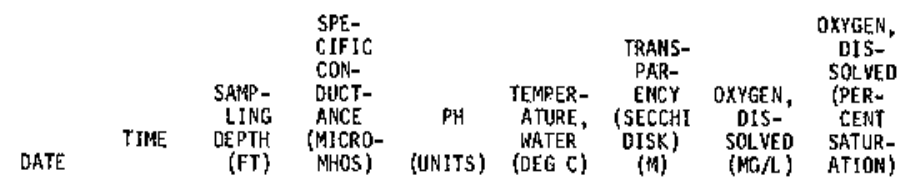

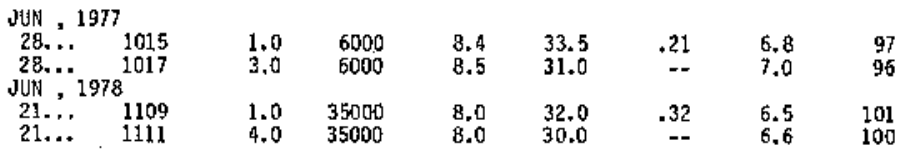

283522096332500 LINE 150 SITE O4

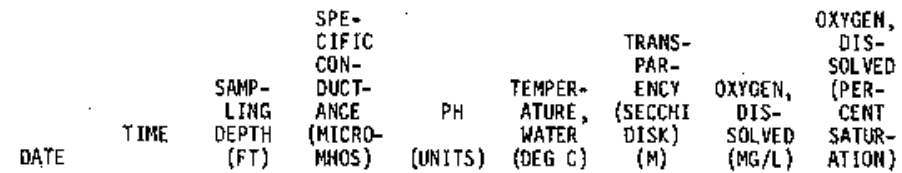

FEB , 1978

FEB. 19780623

$16 . . . \quad 0625 \quad 1.0 \quad 27000$

$16 . . .0627$

$16 . . .1145$

$16 . . . \quad 1147$

$16 \ldots \quad 1149$

$16 \ldots 1836$

$16 \ldots .1838$

$16 \ldots 21840$

$17 . . .0628$

17... 0632

JUWN 1945

$20 . . \quad 1945$

$20 \ldots \quad 1949$

$21 \ldots \quad 0742$

$21 \ldots .0744$

$\begin{array}{cc}1.0 & 27000 \\ 20 & 40000 \\ 42 & 43000 \\ 1.0 & 26000 \\ 20 & 36000 \\ 38 & 41000 \\ 1.0 & 32000 \\ 20 & 39000 \\ 40 & 40000 \\ 1.0 & 31000 \\ 20 & 35000 \\ 35 & 39000 \\ & \\ 1.0 & 37000 \\ 20 & 41000 \\ 40 & 39000 \\ 1.0 & 34100 \\ 20 & 36900 \\ 39 & 36900\end{array}$

$\begin{array}{ll}8.6 & 9.0 \\ 8.2 & 9.0 \\ 8.2 & 8.5 \\ 8.5 & 9.0 \\ 8.2 & 9.0 \\ 8.1 & 9.0 \\ 8.8 & 9.0 \\ 8.3 & 9.0 \\ 8.2 & 9.0 \\ 8.6 & 9.5 \\ 8.4 & 9.0 \\ 8.3 & 9.0 \\ & \\ 8.3 & 30.0 \\ 8.3 & 30.0 \\ 8.0 & 29.5 \\ 8.0 & 29.5 \\ 7.9 & 29.5 \\ 7.9 & 29.5\end{array}$

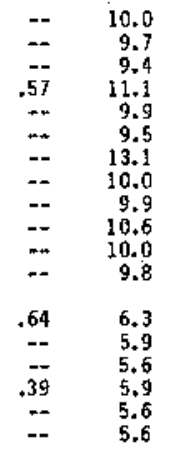

100
102
101
111
104
102
134
105
105
109
104
104
97
97
93
87
89
86
87 
283337096305000 LINE 190 SITE O4

\begin{tabular}{|c|c|c|c|c|c|c|c|c|}
\hline OATE & TIME & $\begin{array}{l}\text { SAYAP- } \\
\text { LING } \\
\text { DEPTH } \\
\text { (FT) }\end{array}$ & $\begin{array}{l}\text { SPE- } \\
\text { CIFIC } \\
\text { CON- } \\
\text { DUCT- } \\
\text { ARCE } \\
\text { (MICRO- } \\
\text { MHOS) }\end{array}$ & $\begin{array}{c}\text { PH } \\
\text { (UNITS) }\end{array}$ & $\begin{array}{l}\text { TEMPER* } \\
\text { ATURE, } \\
\text { WATER } \\
\text { (DEG C) }\end{array}$ & $\begin{array}{c}\text { TRANS- } \\
\text { PAR- } \\
\text { ENCY } \\
\text { (SECCHI } \\
\text { DISK) } \\
\text { (M) }\end{array}$ & $\begin{array}{c}\text { OXYGEW, } \\
\text { OIS- } \\
\text { SOLWED } \\
\text { (MG } f L)\end{array}$ & $\begin{array}{c}\text { OXYGEN, } \\
\text { OIS- } \\
\text { SOLWED } \\
\text { (PER- } \\
\text { CENT } \\
\text { SATUR- } \\
\text { ATION) }\end{array}$ \\
\hline $\begin{array}{l}\text { FEB . I } \\
09 . . . \\
09 . . . \\
09 . . \\
09 . . \\
\text { Jun }\end{array}$ & $\begin{array}{l}7 \\
0920 \\
0922 \\
0924 \\
0926\end{array}$ & $\begin{array}{l}1.0 \\
10 \\
25 \\
36\end{array}$ & $\begin{array}{l}34000 \\
34000 \\
43000 \\
43000\end{array}$ & $\begin{array}{l}8.2 \\
8.2 \\
8.1 \\
8.1\end{array}$ & $\begin{array}{l}10.5 \\
10.5 \\
10.0 \\
10.0\end{array}$ & $\begin{array}{l}-- \\
-- \\
--\end{array}$ & $\begin{array}{l}8.5 \\
8.5 \\
8.1 \\
8.1\end{array}$ & $\begin{array}{l}91 \\
91 \\
90 \\
90\end{array}$ \\
\hline $\begin{array}{l}27 \ldots \\
27 \ldots \\
27 \ldots \\
27 \ldots \\
27 \ldots \\
27 \ldots \\
27 \ldots \\
27 \ldots \\
28 \ldots \\
28 \ldots \\
28 \ldots \\
28 \ldots \\
28 \ldots \\
28 \ldots\end{array}$ & $\begin{array}{l}1510 \\
1512 \\
1514 \\
1516 \\
1518 \\
1520 \\
1522 \\
1524 \\
0920 \\
0922 \\
0924 \\
0926 \\
0928 \\
0930\end{array}$ & $\begin{array}{l}1.0 \\
5.0 \\
7.5 \\
10 \\
13 \\
15 \\
25 \\
32 \\
1.0 \\
5.0 \\
10 \\
15 \\
25 \\
32\end{array}$ & $\begin{array}{l}18000 \\
18000 \\
20000 \\
31000 \\
47000 \\
47000 \\
54000 \\
54000 \\
18000 \\
20000 \\
21000 \\
33000 \\
50000 \\
50000\end{array}$ & $\begin{array}{l}8.4 \\
8.4 \\
8.3 \\
8.1 \\
7.8 \\
7.9 \\
7.8 \\
7.8 \\
7.9 \\
7.9 \\
7.9 \\
7.9 \\
7.6 \\
7.6\end{array}$ & $\begin{array}{l}29.5 \\
29.5 \\
29.0 \\
28.5 \\
28.0 \\
28.0 \\
27.5 \\
27.5 \\
28.0 \\
28.0 \\
28.0 \\
28.0 \\
27.0 \\
27.0\end{array}$ & $\begin{array}{c}-21 \\
-- \\
-- \\
-- \\
-- \\
- \\
-5 \\
-- \\
-- \\
- \\
--\end{array}$ & $\begin{array}{l}7.8 \\
7.8 \\
7.3 \\
5.2 \\
3.7 \\
3.5 \\
3.4 \\
3.3 \\
6.6 \\
6.3 \\
5.8 \\
5.2 \\
3.5 \\
3.6\end{array}$ & $\begin{array}{r}111 \\
109 \\
103 \\
77 \\
58 \\
55 \\
55 \\
53 \\
90 \\
81 \\
82 \\
77 \\
54 \\
56\end{array}$ \\
\hline $\begin{array}{l}21 \ldots \\
21 \ldots \\
21 \ldots\end{array}$ & $\begin{array}{l}0725 \\
0727 \\
0729\end{array}$ & $\begin{array}{l}1.0 \\
20^{7}\end{array}$ & $\begin{array}{l}38000 \\
40000 \\
44000\end{array}$ & $\begin{array}{l}8.2 \\
8.2 \\
8.2\end{array}$ & $\begin{array}{r}29.0 \\
29.0 \\
29.0\end{array}$ & $\begin{array}{l}.58 \\
-- \\
--\end{array}$ & $\begin{array}{l}6.3 \\
6.3 \\
6.2\end{array}$ & $\begin{array}{l}97 \\
97 \\
97\end{array}$ \\
\hline
\end{tabular}

283018096275500 LINE 200 SITE 05

\begin{tabular}{|c|c|c|c|c|c|c|c|c|}
\hline GATE & TIME & $\begin{array}{l}\text { SAMP- } \\
\text { EING } \\
\text { DEPTH } \\
\text { (FT) }\end{array}$ & $\begin{array}{l}\text { SPE- } \\
\text { CIFIC } \\
\text { CON- } \\
\text { DUCT- } \\
\text { ANCE } \\
\text { (MICRO- } \\
\text { MHOS) }\end{array}$ & $\begin{array}{c}\text { PH } \\
\text { (UNITS) }\end{array}$ & $\begin{array}{l}\text { TEMPER- } \\
\text { ATURE, } \\
\text { WATER } \\
\text { (DEG C) }\end{array}$ & $\begin{array}{l}\text { TRANS- } \\
\text { PAR- } \\
\text { ENCY } \\
\text { (SECCHI } \\
\text { OISX) } \\
(M)\end{array}$ & $\begin{array}{c}\text { OXYGEN, } \\
\text { DIS- } \\
\text { SOL WED } \\
\text { (MGA) }\end{array}$ & $\begin{array}{l}\text { OXYGEN, } \\
\text { DIS } \\
\text { SOL WED } \\
\text { (PER- } \\
\text { CENT } \\
\text { SATUR- } \\
\text { ATION) }\end{array}$ \\
\hline \multicolumn{9}{|c|}{ FEB, 1977} \\
\hline $\begin{array}{l}08 . . . \\
08 . . \\
\text { JUA }\end{array}$ & $\begin{array}{l}1350 \\
1352\end{array}$ & $\begin{array}{l}1.0 \\
3.0\end{array}$ & $\begin{array}{l}41000 \\
41000\end{array}$ & $\begin{array}{l}8.0 \\
8.0\end{array}$ & $\begin{array}{l}11.5 \\
12.0\end{array}$ & $=-$ & $\begin{array}{l}9.8 \\
9.8\end{array}$ & $\begin{array}{l}109 \\
112\end{array}$ \\
\hline $\begin{array}{l}28 \ldots \\
28, \ldots \\
\text { FEB }\end{array}$ & $\begin{array}{l}0940 \\
8\end{array}$ & $\begin{array}{l}1.0 \\
3.0\end{array}$ & $\begin{array}{l}26000 \\
26000\end{array}$ & $\begin{array}{l}7.8 \\
7.8\end{array}$ & $\begin{array}{l}28.5 \\
28.5\end{array}$ & .38 & $\begin{array}{l}5.7 \\
5.7\end{array}$ & $\begin{array}{l}83 \\
83\end{array}$ \\
\hline $\begin{array}{l}16 \ldots \ldots \\
16 \ldots \\
16 . . \\
16 \ldots \\
16 . \ldots \\
16 \ldots \\
17 \ldots \\
17 \ldots\end{array}$ & $\begin{array}{l}0542 \\
0544 \\
1157 \\
1159 \\
1852 \\
1654 \\
0517 \\
0519\end{array}$ & $\begin{array}{l}1.0 \\
5.0 \\
1.0 \\
4.0 \\
1.0 \\
4.0 \\
1.5 \\
4.0\end{array}$ & $\begin{array}{l}24000 \\
26000 \\
31000 \\
31000 \\
32000 \\
33000 \\
32000 \\
32500\end{array}$ & $\begin{array}{l}8.5 \\
8.5 \\
8.7 \\
8.7 \\
8.5 \\
8.5 \\
8.7 \\
8.7\end{array}$ & $\begin{array}{r}8.0 \\
8.0 \\
11.0 \\
11.0 \\
9.5 \\
9.5 \\
9.5 \\
9.5\end{array}$ & $\begin{array}{l}-- \\
=- \\
-- \\
=- \\
-- \\
-- \\
--\end{array}$ & $\begin{array}{r}9.9 \\
9.9 \\
10.5 \\
10.5 \\
10.7 \\
10.5 \\
10.0 \\
9.8\end{array}$ & $\begin{array}{r}96 \\
96 \\
112 \\
112 \\
110 \\
109 \\
103 \\
101\end{array}$ \\
\hline $\begin{array}{l}20 \ldots \\
20 \ldots \\
21 \ldots \\
21 \ldots\end{array}$ & $\begin{array}{l}1904 \\
1906 \\
0658 \\
0900\end{array}$ & $\begin{array}{l}1.0 \\
4.0 \\
1.0 \\
4.5\end{array}$ & $\begin{array}{l}40000 \\
41000 \\
39000 \\
40000\end{array}$ & $\begin{array}{l}8.1 \\
8.1 \\
8.2 \\
8.2\end{array}$ & $\begin{array}{l}31.0 \\
31.0 \\
30.0 \\
30.0\end{array}$ & $\begin{array}{l}\ldots \\
.-2 \\
.83\end{array}$ & $\begin{array}{l}6.2 \\
7.3 \\
6.1 \\
6.0\end{array}$ & $\begin{array}{l}99 \\
99 \\
96 \\
93\end{array}$ \\
\hline
\end{tabular}

284153096240000 LINE 235 SITE 02

\begin{tabular}{|c|c|c|c|c|c|c|c|c|}
\hline DATE & TIME & $\begin{array}{c}\text { SAMP- } \\
\text { LING } \\
\text { DEPTH } \\
\text { (FT) }\end{array}$ & $\begin{array}{l}\text { SPE- } \\
\text { CIFIC } \\
\text { CON- } \\
\text { DULT- } \\
\text { ANCE } \\
\text { (MICRO- } \\
\text { MHOS) }\end{array}$ & $\begin{array}{c}\text { PH } \\
\text { (U:ATS) }\end{array}$ & $\begin{array}{l}\text { TEMPER- } \\
\text { ATURE, } \\
\text { WATER } \\
\text { (DEG C) }\end{array}$ & $\begin{array}{l}\text { TRANS- } \\
\text { PAR- } \\
\text { ENCY } \\
\text { (SECCHI } \\
\text { OISK) } \\
\text { (M) }\end{array}$ & $\begin{array}{c}\text { OXYGEN, } \\
\text { DIS- } \\
\text { SOLVED } \\
\text { (KG/L) }\end{array}$ & $\begin{array}{l}\text { OXYGEN, } \\
\text { OIS- } \\
\text { SOLWED } \\
\text { (PER- } \\
\text { CENT } \\
\text { SATUR- } \\
\text { ATIDN) }\end{array}$ \\
\hline \multicolumn{9}{|c|}{ FEB , 1977} \\
\hline 07... & $\begin{array}{l}1315 \\
1317\end{array}$ & $\begin{array}{l}1.0 \\
3.0\end{array}$ & $\begin{array}{l}24000 \\
24000\end{array}$ & $\begin{array}{l}8.5 \\
8.9\end{array}$ & $\begin{array}{l}12.0 \\
13.0\end{array}$ & $\begin{array}{c}.83 \\
--\end{array}$ & $\begin{array}{l}10.0 \\
10.3\end{array}$ & $\begin{array}{l}105 \\
111\end{array}$ \\
\hline $\begin{array}{l}27 \ldots \\
27 \ldots\end{array}$ & $\begin{array}{l}1245 \\
1247\end{array}$ & $\begin{array}{l}1.0 \\
5.5\end{array}$ & $\begin{array}{l}11000 \\
13000\end{array}$ & $\begin{array}{l}8.6 \\
8.6\end{array}$ & $\begin{array}{l}28.0 \\
28.5\end{array}$ & .23 & $\begin{array}{l}6.3 \\
5.8\end{array}$ & $\begin{array}{l}85 \\
78\end{array}$ \\
\hline \multicolumn{9}{|c|}{ JUN , 1978} \\
\hline $\begin{array}{l}20 \ldots \\
20 \ldots\end{array}$ & 09002 & $\begin{array}{l}1.0 \\
6.0\end{array}$ & $\begin{array}{l}27100 \\
33000\end{array}$ & $\begin{array}{l}8.2 \\
8.2\end{array}$ & $\begin{array}{l}29.0 \\
29.5\end{array}$ & .36 & $\begin{array}{l}6.5 \\
6.3\end{array}$ & $\begin{array}{l}95 \\
96\end{array}$ \\
\hline
\end{tabular}


284246096112800 LIAE 264 SITE 02

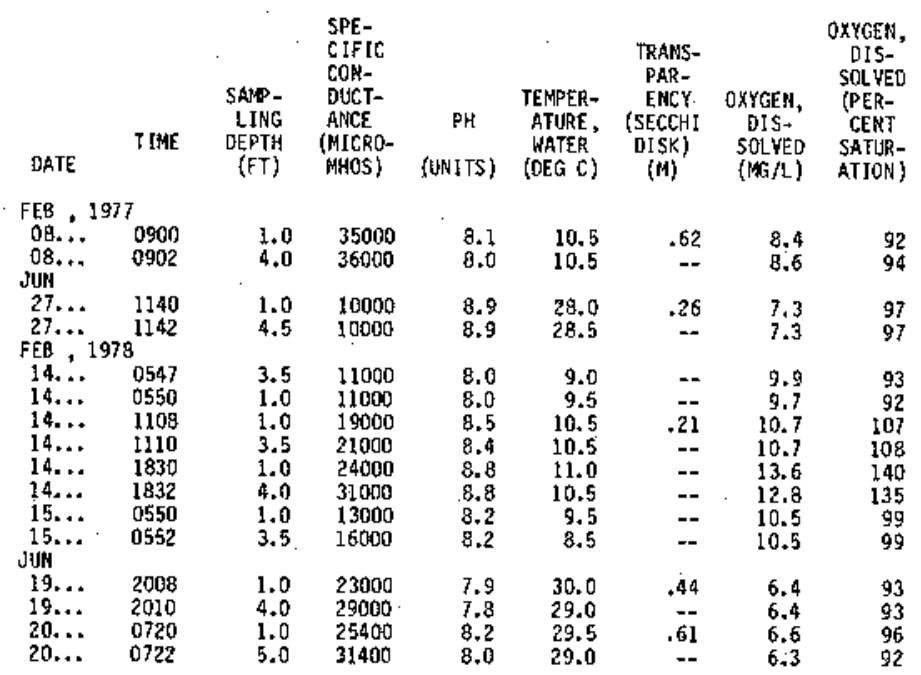

283914095140600 LINE 284 SITE 01

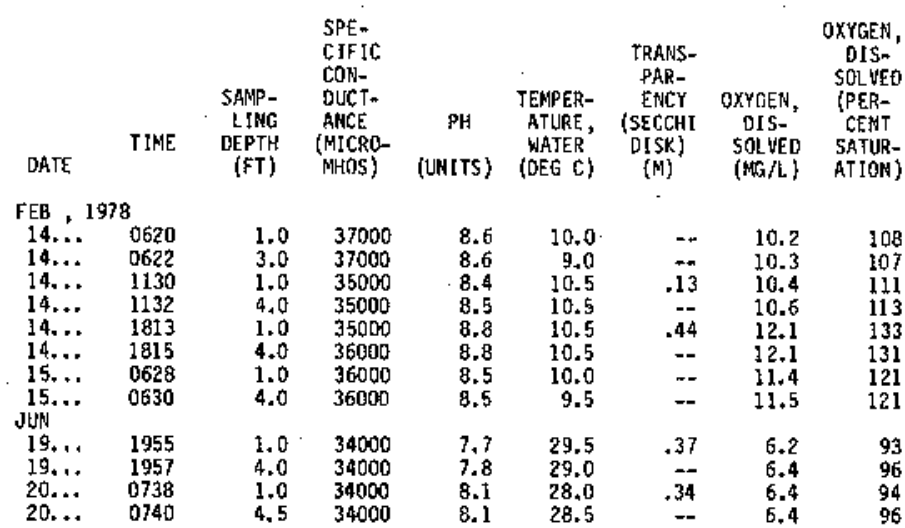

283634096144200 LINE 300 SITE OI

\begin{tabular}{|c|c|c|c|c|c|c|c|c|}
\hline DATE & TIME & $\begin{array}{c}\text { SAFAP- } \\
\text { LING } \\
\text { DEPTH } \\
\text { (FT) }\end{array}$ & $\begin{array}{l}\text { SPE- } \\
\text { CIFIC } \\
\text { CON- } \\
\text { DUCT- } \\
\text { ANCE } \\
\text { (MICRO- } \\
\text { MHDS) }\end{array}$ & $\begin{array}{c}\text { PH } \\
\text { (UNITS) }\end{array}$ & $\begin{array}{l}\text { TEMPER- } \\
\text { ATURE, } \\
\text { WATER } \\
\text { (DEG C) }\end{array}$ & $\begin{array}{l}\text { TRANS- } \\
\text { PAR- } \\
\text { ENEY } \\
\text { (SECCH1 } \\
\text { DISK) } \\
\text { (M) }\end{array}$ & $\begin{array}{c}\text { OXYGEN, } \\
\text { DIS- } \\
\text { SOL VED } \\
\text { (MG/L) }\end{array}$ & $\begin{array}{c}\text { OXYGEN, } \\
\text { DIS- } \\
\text { SOLVED } \\
\text { (PER- } \\
\text { CENT } \\
\text { SATUR- } \\
\text { ATION) }\end{array}$ \\
\hline EEB . & & & & & & & & \\
\hline $\begin{array}{l}08 \ldots . . \\
08 . . . \\
\text { JuN }\end{array}$ & $\begin{array}{l}0955 \\
0957\end{array}$ & $\begin{array}{l}1.0 \\
6.0\end{array}$ & $\begin{array}{l}41000 \\
41000\end{array}$ & $\begin{array}{l}8.2 \\
8.2\end{array}$ & $\begin{array}{l}10.5 \\
10.5\end{array}$ & $\begin{array}{r}1.65 \\
=-\end{array}$ & $\begin{array}{l}8.5 \\
8.7\end{array}$ & $\begin{array}{l}94 \\
96\end{array}$ \\
\hline $\begin{array}{l}27 \ldots \\
27 \ldots \\
\text { Jџพฺ . }\end{array}$ & $\begin{array}{l}1140 \\
1142 \\
8\end{array}$ & $\begin{array}{l}1.0 \\
5.0\end{array}$ & $\begin{array}{l}330000 \\
33000\end{array}$ & $\begin{array}{l}8.2 \\
8.2\end{array}$ & & .15 & $\begin{array}{l}5.8 \\
5.7\end{array}$ & $\begin{array}{l}87 \\
86\end{array}$ \\
\hline $\begin{array}{l}20 \ldots \\
20 \ldots\end{array}$ & $\begin{array}{l}0752 \\
0754\end{array}$ & 1.0 & $\begin{array}{l}37000 \\
35000\end{array}$ & $\begin{array}{l}8.1 \\
8.0\end{array}$ & $\begin{array}{l}28.5 \\
28.5\end{array}$ & .48 & $\begin{array}{l}6.4 \\
6.4\end{array}$ & $\begin{array}{l}97 \\
95\end{array}$ \\
\hline
\end{tabular}

283724096160100 LINE 300 SITE 02

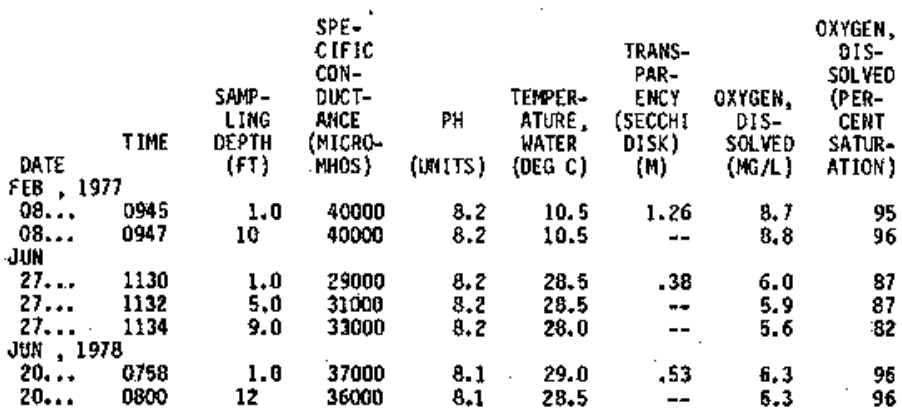


283816096170000 LINE 300 SITE 09

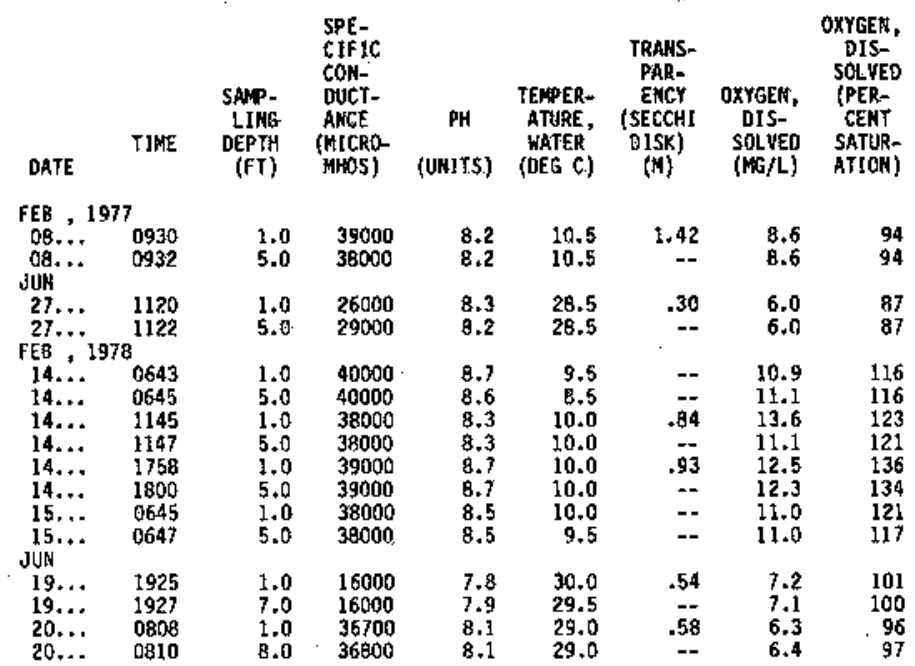

283702095583300 LINE 330 SITE 02

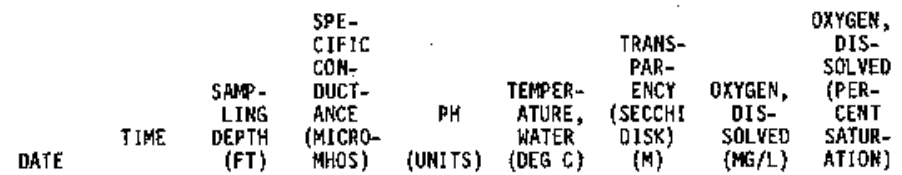

MAY, 1977

$25 . \cdots \quad 1250$

$25 . . \quad 1252$

25... 1254

25... 1535

$25 \ldots \quad 1537$

$\begin{array}{ll}25 . . . & 1539 \\ 25 \ldots & 1745\end{array}$

25... 1747

25,1749

$25 . . . \quad 2100$

25... 2102

25... 2104

26... $\quad 0335$

26... 0337

$26 . . . \quad 0600$

$26 . . .0602$

$26 . . .0604$

26... 0925

26... 0927

26... 0929

26... 1200

26... 1202

$27 \ldots \quad 0001$

$27 \ldots \quad 0002$

$27 \ldots .0004$

JUL

$1.0 \quad 4000$

$\begin{array}{rr}1.0 & 4000 \\ 5.0 & 8000 \\ 10 & 12000 \\ 1.0 & 6500 \\ 5.0 & 7500 \\ 10 & 9000\end{array}$

$\begin{array}{ll}1.0 & 4500 \\ 5.0 & 7000\end{array}$

$10 \quad 9500$

1.0
5.0

10

$1.0 \quad 5000$

$5.0 \quad 5500$

$10 \quad 5500$

$1.0 \quad 5500$

$3.5 \quad 6000$

$\begin{array}{rr}7.0 & 12000 \\ 1.0 & 3500\end{array}$

$\begin{array}{cc}5.0 & 3500 \\ 10 & 3500\end{array}$

$1.0 \quad 3000$

$5.0 \quad 3500$

10

4000
4000

$5.0 \quad 4500$

\subsection{6.}

$\begin{array}{ll}8.3 & 26.0 \\ 8.2 & 26.0\end{array}$

$8.2 \quad 26.0$

8.227 .0

$8.2 \quad 26.5$

$\begin{array}{ll}8.2 & 26.0 \\ 8.3 & 26.5\end{array}$

B.2 26.5

$\begin{array}{ll}8.1 & 26.0 \\ 8.2 & 25.0\end{array}$

$8.2 \quad 25.0$

$8.2 \quad 25.0$

$8.2 \quad 24.5$

$8.2 \quad 24.5$

$8.2 \quad 24.0$

$3.2 \quad 24.0$

$\begin{array}{ll}8.2 & 24.0 \\ 8.2 & 25.0 \\ 8.2 & 25.0\end{array}$

$\begin{array}{ll}8.2 & 25.0 \\ 8.2 & 25.0\end{array}$

$8.2 \quad 25.0$

$8.2 \quad 25.5$

8.2 25.0

$\begin{array}{ll}8.2 & 25.0 \\ 8.2 & 25.0\end{array}$

$\begin{array}{ll}8.2 & 25 . \\ 8.2 & \end{array}$

$27 \ldots 1600$

$27 \ldots . \quad 1602$

$27 \ldots \quad 1604$

$27 . . . \quad 1915$

27... 1917

27... $\quad 1919$

$27 \ldots \quad 2202$

$27 . . \quad 2204$

$28 \ldots .0100$

$28 . . .0102$

28... 0104

$28 . . .0430$

28... 0432

28... 0434

28... 0700

28... 0702

$28 . . .0704$

28... 1030

$28 \ldots \quad 1032$

$28 \ldots . .1315$

28... 1317

28... $\quad 1319$

$28 . . . \quad 1555$

$\begin{array}{ll}28 . . . & 1557 \\ 28 . . . & 1559\end{array}$

$\begin{array}{rr}1.0 & 35000 \\ 5.0 & 42000 \\ 9.0 & 42000 \\ 1.0 & 39000 \\ 5.0 & 43000 \\ 9.0 & 43000 \\ 1.0 & 23000 \\ 5.0 & 42000 \\ 9.5 & 43000 \\ 1.0 & 25000 \\ 5.0 & 25000 \\ 11 & 35000 \\ 1.0 & 55000 \\ 6.0 & 55000 \\ 12 & 55000 \\ 1.0 & 55000 \\ 6.0 & 55000 \\ 12 & 55000 \\ 1.0 & 55000 \\ 6.0 & 55000 \\ 11 & 55000 \\ 1.0 & 44000 \\ 5.0 & 51000 \\ 10 & 51000 \\ 1.0 & 40000 \\ 5.0 & 42000 \\ 10 & 42000\end{array}$

$\begin{array}{ll}8.4 & 31.5 \\ 8.4 & 31.5 \\ 8.4 & 31.0 \\ 8.0 & 30.5 \\ 8.0 & 30.5 \\ 8.0 & 30.5 \\ 7.8 & 30.5 \\ 7.9 & 30.0 \\ 7.9 & 30.0 \\ 7.7 & 29.0 \\ 7.7 & 29.0 \\ 7.6 & 30.0 \\ 7.7 & 29.0 \\ 7.5 & 29.0 \\ 7.6 & 29.0 \\ 7.7 & 29.0 \\ 7.6 & 29.0 \\ 7.7 & 29.0 \\ 7.7 & 29.0 \\ 7.7 & 29.0 \\ 7.7 & 29.0 \\ 8.0 & 29.5 \\ 8.1 & 29.5 \\ 8.0 & 29.5 \\ 8.2 & 32.0 \\ 8.3 & 32.0 \\ 8.3 & 32.0\end{array}$

$--\quad 7.7$

$\because \quad 7.2$

-4.8
-

$\begin{array}{ll}-- & 7.4 \\ - & 7.9\end{array}$

7.6
-2

$.33 \quad 7.5$

$\begin{array}{ll}-2 & 7.5 \\ 7- & 7.5\end{array}$

- 7.3

- 7.2

$=7.0$

- 6.9

$=7.6$

7.6

7.6

7.5
$-\quad 7.5$

$\begin{array}{ll}- & 7.1 \\ - & 7.1\end{array}$

$\begin{array}{lll}\because & 7.6 & 119 \\ - & 7.4 & 119\end{array}$

$\begin{array}{lll}-- & 7.4 & 119 \\ -- & 6.7 & 104\end{array}$

$\begin{array}{lll}- & 6.7 & 103 \\ = & 6.4 & 103\end{array}$

$\begin{array}{lll}- & 6.4 & 103 \\ - & 6.1 & 80\end{array}$

$\begin{array}{lll}- & 6.1 & \\ -- & 6.0 & 90 \\ - & 6.0 & 94\end{array}$

- 5.7

$\begin{array}{ll}-- & 5.2 \\ - & 5.3\end{array}$

$+4$

.. $\quad 5.3$

-- 5.1

$\begin{array}{ll}-- & 5.2 \\ - & 4.9\end{array}$

$\begin{array}{ll}- & 4.9 \\ - & 5.0\end{array}$

$\begin{array}{ll}-- & 4.8 \\ \cdots & 6.1\end{array}$

.- 5.9

$\begin{array}{ll}- & 5.9 \\ - & 7.8 \\ - & 7.2\end{array}$

$\overline{--}$ 
Table 6A.-2Quality of.water in the Layaca-Tres Palacios estuary, water years 1977-78--Continued Field Determinations-Continued

283608096011400 LINE 333 SITE 01

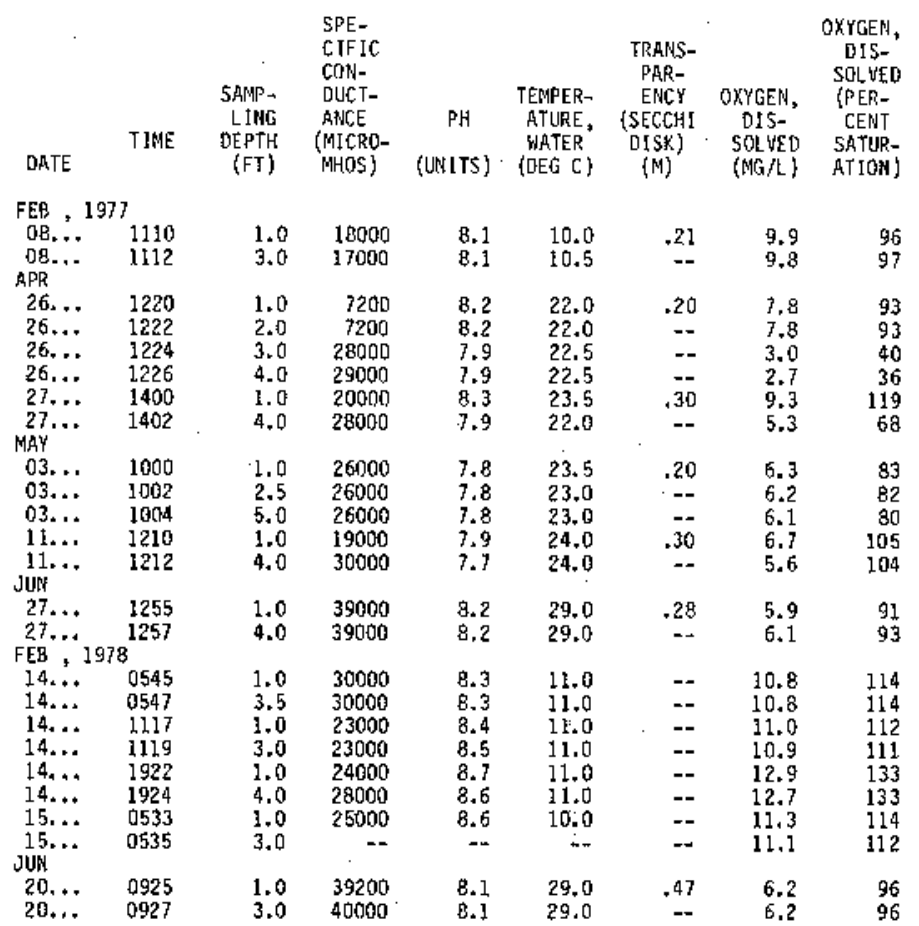

283700096013400 LINE 333 SITE O2

\begin{tabular}{|c|c|c|c|c|c|c|c|c|}
\hline DATE & TIME & $\begin{array}{r}\text { SAMP- } \\
\text { LING } \\
\text { DEPTH } \\
\text { (FT) }\end{array}$ & $\begin{array}{l}\text { SPE- } \\
\text { CIFIC } \\
\text { COHA- } \\
\text { DUCT- } \\
\text { AMCE } \\
\text { (MICRO- } \\
\text { MHOS) }\end{array}$ & $\begin{array}{c}\text { PH } \\
\text { (U:11TS) }\end{array}$ & $\begin{array}{c}\text { TEMPER- } \\
\text { ATURE, } \\
\text { WAJER } \\
\text { (OEG C) }\end{array}$ & $\begin{array}{c}\text { TRANSS- } \\
\text { PAR- } \\
\text { ENCY } \\
\text { (SECC.HI } \\
\text { DISX) } \\
\text { (M) }\end{array}$ & $\begin{array}{c}\text { OXYGEN, } \\
\text { DIS- } \\
\text { SGE VED } \\
\text { \{HG } A)\end{array}$ & $\begin{array}{c}\text { OXYGEN, } \\
\text { OIS- } \\
\text { SOL VED } \\
\text { (PER- } \\
\text { CENT } \\
\text { SATUR- } \\
\text { ATION) }\end{array}$ \\
\hline \multicolumn{9}{|c|}{$A P R, 1977$} \\
\hline $\begin{array}{l}26 . \ldots \\
26 \ldots \\
26 . \ldots \\
27 \ldots \\
27 \ldots \\
\text { TAY }\end{array}$ & $\begin{array}{l}1210 \\
1212 \\
1214 \\
1420 \\
1422\end{array}$ & $\begin{array}{l}1.0 \\
2.0 \\
4.0 \\
1.0 \\
5.0\end{array}$ & $\begin{array}{r}5500 \\
5500 \\
30000 \\
10000 \\
10000\end{array}$ & $\begin{array}{l}8.3 \\
8.2 \\
8.1 \\
8.6 \\
8.6\end{array}$ & $\begin{array}{l}21.0 \\
21.0 \\
23.0 \\
23.0 \\
23.5\end{array}$ & $\begin{array}{r}.20 \\
-- \\
-- \\
.46 \\
--\end{array}$ & $\begin{array}{r}7.5 \\
7.9 \\
5.4 \\
10.9 \\
10.9\end{array}$ & $\begin{array}{r}89 \\
93 \\
72 \\
135 \\
135\end{array}$ \\
\hline $\begin{array}{l}03 \ldots \\
03 \ldots \\
03 \ldots \\
11 \ldots \\
11 \ldots\end{array}$ & $\begin{array}{l}0945 \\
0947 \\
0949 \\
1225 \\
1227\end{array}$ & $\begin{array}{l}1.0 \\
3.0 \\
6.0 \\
1.0 \\
5.0\end{array}$ & $\begin{array}{l}12000 \\
14000 \\
18000 \\
26000 \\
27000\end{array}$ & $\begin{array}{l}7.9 \\
7.9 \\
7.8 \\
8.0 \\
7.9\end{array}$ & $\begin{array}{l}24.0 \\
24.0 \\
24.0 \\
24.0 \\
24.0\end{array}$ & $\begin{array}{l}.30 \\
-- \\
. \\
.30 \\
-.\end{array}$ & $\begin{array}{l}7.5 \\
7.0 \\
6.7 \\
6.9 \\
6.0\end{array}$ & $\begin{array}{l}95 \\
90 \\
85 \\
94 \\
82\end{array}$ \\
\hline
\end{tabular}

\section{LISE 333 SITE 03}

\begin{tabular}{|c|c|c|c|c|c|c|c|c|}
\hline DATE & TINE. & $\begin{array}{l}\text { SAHP- } \\
\text { LING } \\
\text { DEPTH } \\
\text { (FT) }\end{array}$ & $\begin{array}{l}\text { SPE- } \\
\text { CIFIC } \\
\text { COMW } \\
\text { DUCT- } \\
\text { ANCE } \\
\text { (MICRD- } \\
\text { MHOS) }\end{array}$ & $\begin{array}{c}\text { PH } \\
\text { (UNITS) }\end{array}$ & $\begin{array}{c}\text { TEMPER- } \\
\text { ATLRE, } \\
\text { WATER } \\
\text { (DEG C) }\end{array}$ & $\begin{array}{l}\text { TRANS- } \\
\text { PAR- } \\
\text { ENCY } \\
\text { (SECCHI } \\
\text { OISK) } \\
\text { (M) }\end{array}$ & $\begin{array}{c}\text { OXYGEN, } \\
\text { DIS- } \\
\text { SOLVED } \\
\text { (MGL) }\end{array}$ & $\begin{array}{l}\text { OXYGEN, } \\
\text { OIS- } \\
\text { SQLVED } \\
\text { (PER- } \\
\text { CERT } \\
\text { SATUR- } \\
\text { ATION) }\end{array}$ \\
\hline, 19 & & & & & & & & \\
\hline $\begin{array}{l}26 \ldots \\
26 \ldots \\
27 \ldots \\
27 \ldots\end{array}$ & $\begin{array}{l}1200 \\
1202 \\
1440 \\
1442\end{array}$ & $\begin{array}{l}1.0 \\
3.0 \\
1.0 \\
3.0\end{array}$ & $\begin{array}{r}10000 \\
12000 \\
7500 \\
7500\end{array}$ & $\begin{array}{l}8.1 \\
8.0 \\
8.1 \\
8.1\end{array}$ & $\begin{array}{l}21.0 \\
20.5 \\
23.5 \\
23.5\end{array}$ & $\begin{array}{r}.20 \\
.8 \\
.80 \\
-\end{array}$ & $\begin{array}{l}7.5 \\
6.5 \\
7.7 \\
7.7\end{array}$ & $\begin{array}{l}90 \\
77 \\
94 \\
94\end{array}$ \\
\hline $\begin{array}{l}03 \ldots . \\
03 \ldots \\
11 \ldots \\
11 \ldots\end{array}$ & $\begin{array}{l}0930 \\
0932 \\
1235 \\
1237\end{array}$ & $\begin{array}{l}1.0 \\
3.0 \\
1.0 \\
5.0\end{array}$ & $\begin{array}{r}6000 \\
6000 \\
26000 \\
29000\end{array}$ & $\begin{array}{l}7.7 \\
7.2 \\
8.0 \\
8.0\end{array}$ & $\begin{array}{l}24.0 \\
24.0 \\
24.0 \\
24.0\end{array}$ & $\begin{array}{r}.15 \\
.36 \\
.-\end{array}$ & $\begin{array}{l}7.3 \\
7.0 \\
6.6 \\
5.4\end{array}$ & $\begin{array}{r}90 \\
87 \\
106 \\
73\end{array}$ \\
\hline
\end{tabular}




\begin{tabular}{|c|c|c|c|c|c|c|c|c|}
\hline & & & 15180960 & 400 & E $340 \mathrm{~s}[\mathrm{~T}$ & E 01 & & \\
\hline DATE & T IME & $\begin{array}{l}\text { SAMP } \\
\text { LING } \\
\text { DEPTH } \\
\text { (FT) }\end{array}$ & $\begin{array}{l}\text { SPE- } \\
\text { CIFIC } \\
\text { COH- } \\
\text { OUCT- } \\
\text { ANCE } \\
\text { (MICRO- } \\
\text { MHOS) }\end{array}$ & $\begin{array}{c}\text { PH } \\
\text { (UNITS) }\end{array}$ & $\begin{array}{l}\text { TENPER- } \\
\text { ATURE, } \\
\text { WATER } \\
\text { (DEG C) }\end{array}$ & $\begin{array}{l}\text { TRANS- } \\
\text { PAR- } \\
\text { ENCY } \\
\text { (SECCHI } \\
\text { OTSK) } \\
\text { (M) }\end{array}$ & $\begin{array}{c}\text { OXYGEN, } \\
\text { DLS- } \\
\text { SOL VED } \\
\text { (KG /L) }\end{array}$ & $\begin{array}{l}\text { OXYGEN, } \\
\text { OIS- } \\
\text { SOLVED } \\
\text { (PER- } \\
\text { CENT } \\
\text { SATUR- } \\
\text { ATIOR) }\end{array}$ \\
\hline $\begin{array}{l}\text { APR .. } \\
26 . \ldots \\
26, \ldots \\
26 . . . \\
27 \ldots \\
27 \ldots\end{array}$ & $\begin{array}{l}7 \\
1410 \\
1412 \\
1414 \\
1215 \\
1217\end{array}$ & $\begin{array}{l}1.0 \\
3.0 \\
5.0 \\
1.0 \\
5.0\end{array}$ & $\begin{array}{l}14000 \\
30000 \\
30000 \\
24000 \\
30000\end{array}$ & $\begin{array}{l}8.5 \\
8.1 \\
8.1 \\
8.4 \\
8.0\end{array}$ & $\begin{array}{l}24.0 \\
23.0 \\
23.0 \\
23.0 \\
22.5\end{array}$ & $\begin{array}{r}.25 \\
. \\
.91 \\
. .\end{array}$ & $\begin{array}{l}8.1 \\
3.9 \\
3.8 \\
8.2 \\
5.5\end{array}$ & $\begin{array}{r}104 \\
52 \\
51 \\
108 \\
73\end{array}$ \\
\hline $\begin{array}{l}03 \ldots \\
03 . \ldots \\
03 . \ldots \\
03 . \ldots\end{array}$ & $\begin{array}{l}1100 \\
1102 \\
1104 \\
1106\end{array}$ & $\begin{array}{l}1.0 \\
2.5 \\
4.0 \\
5.0\end{array}$ & $\begin{array}{l}33000 \\
33000 \\
33000 \\
34000\end{array}$ & $\begin{array}{l}7.9 \\
7.9 \\
7.9 \\
7.8\end{array}$ & $\begin{array}{l}23.5 \\
23.5 \\
23.0 \\
23.0\end{array}$ & $\begin{array}{l}.56 \\
-- \\
-- \\
--\end{array}$ & $\begin{array}{l}6.7 \\
6.4 \\
5.7 \\
5.6\end{array}$ & $\begin{array}{l}92 \\
88 \\
79 \\
76\end{array}$ \\
\hline
\end{tabular}

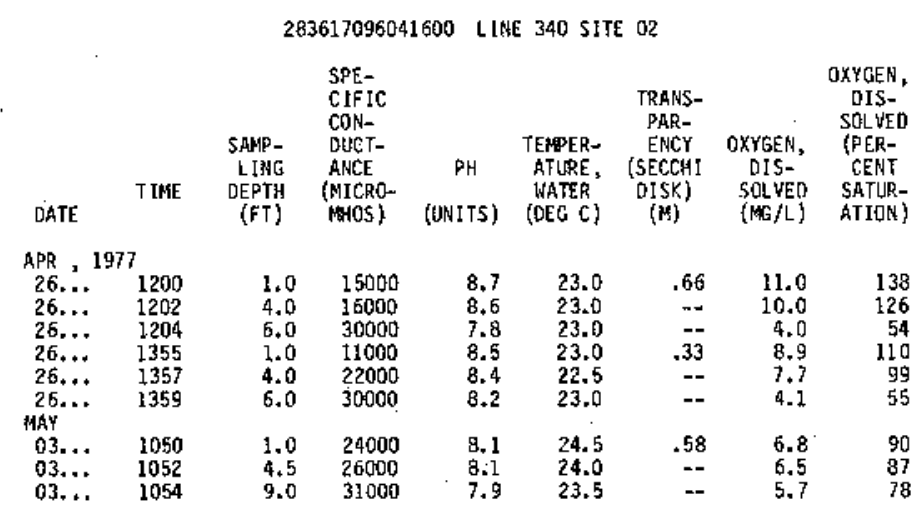

283704096044200 LINE 340 SITE 03

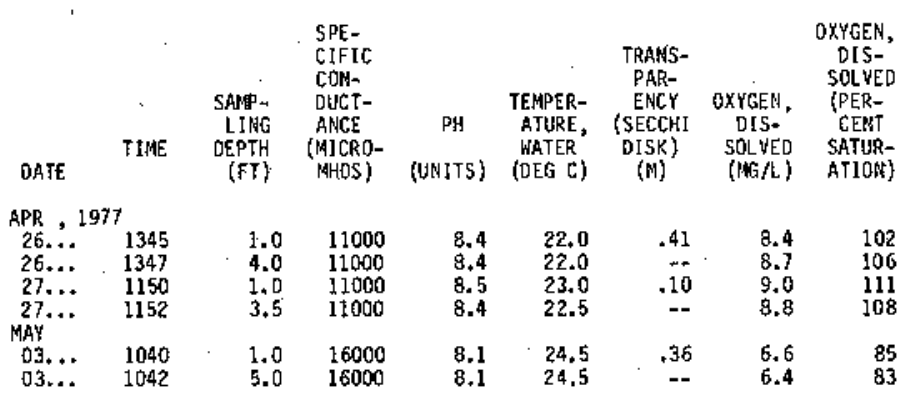

283327096080000 LINE 345 SITE O1

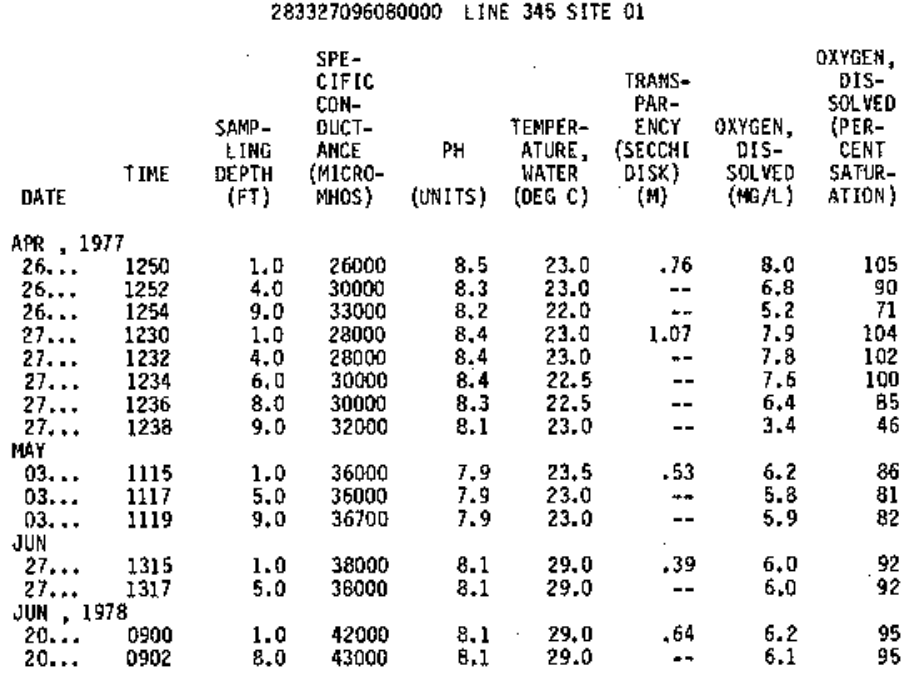


Table 6A.--nuality of water in the Lavaca-Tres Palacios estuary, water years 1977-78--Continued Field leterminations--Cont inued

283445096084200 LINE 345 SITE 02

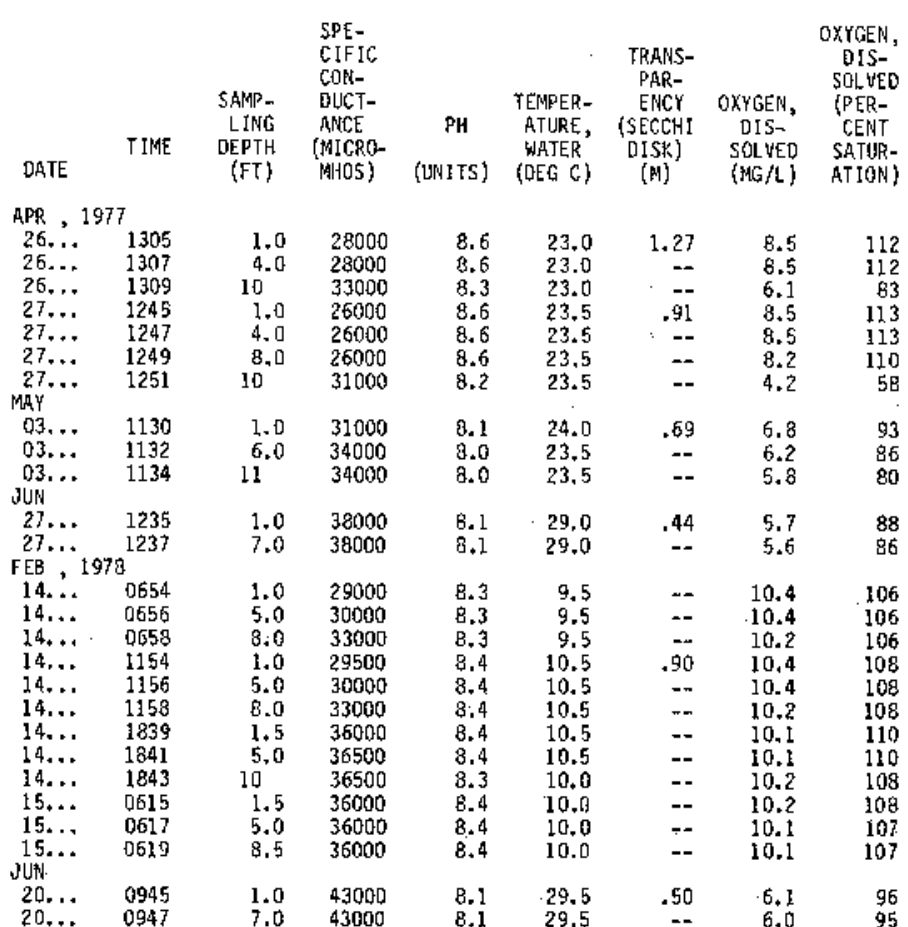

283557096092100 LINE 345 SITE 03

\begin{tabular}{|c|c|c|c|c|c|c|c|c|}
\hline DATE & TIME & $\begin{array}{l}\text { SAMP- } \\
\text { LING } \\
\text { BEPTH } \\
(F T)\end{array}$ & $\begin{array}{l}\text { 5PE- } \\
\text { CIFIC } \\
\text { CON- } \\
\text { DUCT- } \\
\text { AMCE } \\
\text { (MICRO- } \\
\text { MHOS) }\end{array}$ & $\begin{array}{c}\text { PH } \\
\text { (UNITS) }\end{array}$ & $\begin{array}{l}\text { TEMPER- } \\
\text { ATURE, } \\
\text { WATER } \\
\text { (DEG C). }\end{array}$ & $\begin{array}{c}\text { TRANS- } \\
\text { PAR- } \\
\text { ENCY } \\
\text { (SECCHI } \\
\text { OISK) } \\
\text { (M) }\end{array}$ & $\begin{array}{c}\text { OXYGEN, } \\
\text { OIS- } \\
\text { SOLYED } \\
\text { (MG } \Omega)\end{array}$ & $\begin{array}{c}\text { OXYGEN, } \\
\text { OIS- } \\
\text { SULYED } \\
\text { (PER- } \\
\text { CENT } \\
\text { SATUR- } \\
\text { ATION) }\end{array}$ \\
\hline \multicolumn{9}{|c|}{ FEB , 1977} \\
\hline $\begin{array}{l}08 \ldots \\
08 \ldots \\
A P R\end{array}$ & $\begin{array}{l}1130 \\
1132\end{array}$ & $\begin{array}{l}1.0 \\
4.0\end{array}$ & $\begin{array}{l}37000 \\
34000\end{array}$ & $\begin{array}{l}8.2 \\
8.2\end{array}$ & $\begin{array}{l}10.5 \\
11.5\end{array}$ & .50 & $\begin{array}{l}9.2 \\
9.4\end{array}$ & $\begin{array}{l}101 \\
103\end{array}$ \\
\hline $\begin{array}{l}26 \ldots \\
26 \ldots \\
27 \ldots \\
27 \ldots \\
27 \ldots \\
n_{4} . \ldots\end{array}$ & $\begin{array}{l}1315 \\
1317 \\
1300 \\
1302 \\
1304\end{array}$ & $\begin{array}{l}1.0 \\
6.0 \\
1.0 \\
4.0 \\
6.5\end{array}$ & $\begin{array}{l}24000 \\
24000 \\
25000 \\
25000 \\
25000\end{array}$ & $\begin{array}{l}8.7 \\
8.7 \\
8.6 \\
8.6 \\
8.6\end{array}$ & $\begin{array}{l}23.0 \\
22.5 \\
23.5 \\
23.5 \\
23.5\end{array}$ & $\begin{array}{r}1.22 \\
.46 \\
.- \\
--\end{array}$ & $\begin{array}{l}9.6 \\
9.8 \\
8.7 \\
8.6 \\
8.5\end{array}$ & $\begin{array}{l}126 \\
127 \\
114 \\
113 \\
112\end{array}$ \\
\hline $\begin{array}{l}03 \ldots \\
03 \ldots \\
03 \ldots \\
\text { Jin }\end{array}$ & $\begin{array}{l}1140 \\
1142 \\
1144\end{array}$ & $\begin{array}{l}1.0 \\
3.0 \\
6.0\end{array}$ & $\begin{array}{l}22000 \\
28000 \\
29000\end{array}$ & $\begin{array}{l}8.2 \\
8.0 \\
8.0\end{array}$ & $\begin{array}{l}24.5 \\
24.0 \\
24.0\end{array}$ & $\begin{array}{c}.46 \\
-. \\
-=\end{array}$ & $\begin{array}{l}5.8 \\
5.8 \\
5.5\end{array}$ & $\begin{array}{l}90 \\
78 \\
74\end{array}$ \\
\hline $\begin{array}{l}27 \ldots \\
27 \ldots \\
J \cup N, 1\end{array}$ & $\begin{array}{l}1220 \\
1222 \\
8\end{array}$ & $\begin{array}{l}1.0 \\
6.0\end{array}$ & $\begin{array}{l}37000 \\
37000\end{array}$ & $\begin{array}{l}8.2 \\
8.2\end{array}$ & $\begin{array}{r}28.5 \\
28.5\end{array}$ & .24 & $\begin{array}{l}5.7 \\
5.7\end{array}$ & $\begin{array}{l}87 \\
87\end{array}$ \\
\hline $\begin{array}{l}20 \ldots \\
20 \ldots\end{array}$ & $\begin{array}{l}0955 \\
0957\end{array}$ & $\begin{array}{l}1.0 \\
5.0\end{array}$ & $\begin{array}{l}37000 \\
41000\end{array}$ & $\begin{array}{l}8.1 \\
8.1\end{array}$ & $\begin{array}{l}29.0 \\
29.0\end{array}$ & .40 & $\begin{array}{l}6.2 \\
6.2\end{array}$ & $\begin{array}{l}96 \\
95\end{array}$ \\
\hline
\end{tabular}

223145096113700 LINE 350 SITE OI

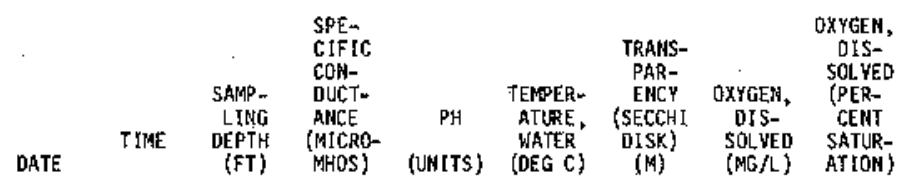

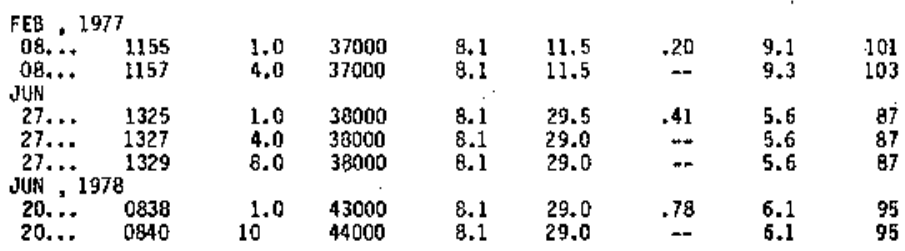


283315096123200 LINE 350 SITE 02

\begin{tabular}{|c|c|c|c|c|c|c|c|c|}
\hline DATE & TIME & $\begin{array}{l}\text { SAMP- } \\
\text { LING } \\
\text { DEPTH } \\
\text { (FT) }\end{array}$ & $\begin{array}{l}\text { SPE- } \\
\text { CIFIC } \\
\text { CON- } \\
\text { DUCT- } \\
\text { ANCE } \\
\text { (MICRO- } \\
\text { MHOS) }\end{array}$ & $\begin{array}{c}\text { PH } \\
\text { (UNITS) }\end{array}$ & $\begin{array}{l}\text { TEMPER- } \\
\text { ATURE, } \\
\text { WATER } \\
\text { (DEG C) }\end{array}$ & $\begin{array}{l}\text { TRAKS- } \\
\text { PAR- } \\
\text { ENCY } \\
\text { (SECCHI } \\
\text { DISK) } \\
\text { (M) }\end{array}$ & $\begin{array}{c}\text { OXYGEN, } \\
\text { DIS- } \\
\text { SOLVED } \\
\text { (MG } / \mathrm{L})\end{array}$ & $\begin{array}{c}\text { OXYGEN, } \\
\text { DIS-: } \\
\text { SOL YED } \\
\text { (PER- } \\
\text { CEMT } \\
\text { SATUR- } \\
\text { ATION) }\end{array}$ \\
\hline \multicolumn{9}{|c|}{ FEB , 1977} \\
\hline $\begin{array}{l}08 . . . \\
08 . . \\
08 . . .\end{array}$ & $\begin{array}{l}1030 \\
1032 \\
1034\end{array}$ & $\begin{array}{l}1.0 \\
10 \\
17\end{array}$ & $\begin{array}{l}39000 \\
39000 \\
39000\end{array}$ & $\begin{array}{l}8.2 \\
8.1 \\
8.2\end{array}$ & $\begin{array}{l}10.5 \\
11.0 \\
11.0\end{array}$ & $\begin{array}{l}.63 \\
-- \\
--\end{array}$ & $\begin{array}{l}8.8 \\
8.4 \\
8.2\end{array}$ & $\begin{array}{l}96 \\
93 \\
92\end{array}$ \\
\hline \multicolumn{9}{|l|}{ JUE⿱ } \\
\hline $\begin{array}{l}27 \ldots \\
27 \ldots \\
27 \ldots \\
27 \ldots\end{array}$ & $\begin{array}{l}1205 \\
1207 \\
1209 \\
1211\end{array}$ & $\begin{array}{l}1.0 \\
4.0 \\
8.0 \\
16\end{array}$ & $\begin{array}{l}37000 \\
370000 \\
37000 \\
37000\end{array}$ & $\begin{array}{l}8.2 \\
8.2 \\
8.2 \\
8.2\end{array}$ & $\begin{array}{l}29.0 \\
28.5 \\
28.5 \\
28.5\end{array}$ & $\begin{array}{l}.32 \\
-- \\
-- \\
--\end{array}$ & $\begin{array}{l}5.7 \\
5.7 \\
5.7 \\
5.7\end{array}$ & $\begin{array}{l}86 \\
86 \\
86 \\
86\end{array}$ \\
\hline \multicolumn{9}{|c|}{ JUN , 1978} \\
\hline $20 \ldots$ & 1018 & 1.0 & 40000 & 8.2 & & .46 & 6.2 & 96 \\
\hline 20. & 1020 & 11 & 3090 & 8.1 & 29 & חי & 5.9 & \\
\hline
\end{tabular}

283353096125600 LINE 350 SITE 03

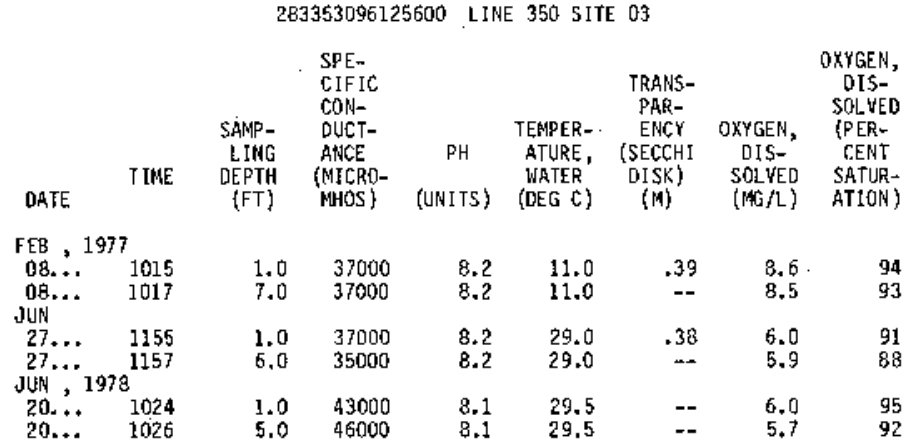

2B2928096144600 LINE 363 SITE 01

\begin{tabular}{|c|c|c|c|c|c|c|c|c|}
\hline DATE & TIMAE & $\begin{array}{c}\text { SAPP - } \\
\text { LING } \\
\text { DEPTH } \\
(\text { FT })\end{array}$ & $\begin{array}{l}\text { SPE- } \\
\text { C1FIC } \\
\text { CON- } \\
\text { OHET- } \\
\text { ANCE } \\
\text { (MICRO- } \\
\text { MHOS) }\end{array}$ & $\begin{array}{c}\text { PH } \\
\text { (UNITS) }\end{array}$ & $\begin{array}{l}\text { TEMPER- } \\
\text { ATURE, } \\
\text { WATER } \\
\text { (DEG C) }\end{array}$ & $\begin{array}{l}\text { TRANS- } \\
\text { PAR- } \\
\text { ENCY } \\
\text { (SECCHI } \\
\text { DISK) } \\
\text { (M) }\end{array}$ & $\begin{array}{l}\text { OXYGEM, } \\
\text { DISM } \\
\text { SOLYED } \\
\text { (MGA) }\end{array}$ & $\begin{array}{l}\text { OXYGEN, } \\
\text { DIS- } \\
\text { SOLVED } \\
\text { (PER- } \\
\text { CENT } \\
\text { SATUR- } \\
\text { ATION) }\end{array}$ \\
\hline \multicolumn{9}{|c|}{$F E B, 1977$} \\
\hline $\begin{array}{l}08 . . . \\
08 . \ldots \\
\text { JUN }\end{array}$ & $\begin{array}{l}1215 \\
1217\end{array}$ & $10^{1.0}$ & $\begin{array}{l}38000 \\
38000\end{array}$ & $\begin{array}{l}8.1 \\
8.0\end{array}$ & $\begin{array}{l}11.5 \\
11.5\end{array}$ & .42 & $\begin{array}{l}9.2 \\
9.2\end{array}$ & $\begin{array}{l}103 \\
103\end{array}$ \\
\hline $\begin{array}{l}27 \ldots \\
27 \ldots \\
27 \ldots\end{array}$ & $\begin{array}{l}1350 \\
1352 \\
1354\end{array}$ & $\begin{array}{l}1.0 \\
4.0 \\
8.0\end{array}$ & $\begin{array}{l}37000 \\
37000 \\
37000\end{array}$ & $\begin{array}{l}8.1 \\
8.1 \\
8.1\end{array}$ & $\begin{array}{l}29.0 \\
29.0 \\
29.0\end{array}$ & $\begin{array}{l}.58 \\
-- \\
--\end{array}$ & $\begin{array}{l}6.1 \\
6.0 \\
5.7\end{array}$ & $\begin{array}{l}93 \\
92 \\
88\end{array}$ \\
\hline \multicolumn{9}{|c|}{ FEB , 1978} \\
\hline & $\begin{array}{l}0734 \\
0736\end{array}$ & $\begin{array}{l}1.0 \\
5.0\end{array}$ & $\begin{array}{l}34000 \\
35000\end{array}$ & $\begin{array}{l}8.2 \\
8.2\end{array}$ & $\begin{array}{l}8.10 \\
8.5\end{array}$ & $\begin{array}{l}-- \\
--\end{array}$ & $\begin{array}{l}9.7 \\
9.7\end{array}$ & $\begin{array}{r}100 \\
99\end{array}$ \\
\hline $14 .$. & 0738 & 7.5 & 38000 & & 8. & $m-1$ & 9.7 & 101 \\
\hline 14. & 1234 & 1.5 & 31500 & 8. & 10. & .50 & 10.0 & 105 \\
\hline 14. & 1236 & 5.0 & 32500 & & 10. & 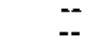 & 10.0 & 104 \\
\hline 14. & $\begin{array}{l}1238 \\
1809\end{array}$ & $\begin{array}{l}9.5 \\
1.0\end{array}$ & $\begin{array}{l}33500 \\
36000\end{array}$ & & $\begin{array}{l}10 . \\
10 .\end{array}$ & $=$ & $\begin{array}{l}9.8 \\
9.7\end{array}$ & $\begin{array}{l}103 \\
103\end{array}$ \\
\hline 14. & 1811 & 5. & 36000 & & 10 & - & 9.7 & 103 \\
\hline $14 .$. & 1813 & 9.0 & 36000 & 8. & 10. & -- & 9. & 103 \\
\hline & 0659 & 1.0 & & & & -- & 9. & 100 \\
\hline & 070 & 5. & 32000 & & 9. & -- & 9.6 & 99 \\
\hline H* & 0703 & 9. & 33000 & 8.2 & 10.0 & - & 9.6 & 101 \\
\hline & 1938 & 1.0 & 44000 & & & $=$ & & \\
\hline & 1940 & 8.0 & & & & $=$ & & \\
\hline & 0824 & 1.0 & & & & .94 & 6.1 & \\
\hline $20 \ldots$ & 0826 & 10 & 44000 & B.1 & 29.0 & -- & 6.1 & 96 \\
\hline
\end{tabular}

283015096154300 LINE 363 S1TE 02

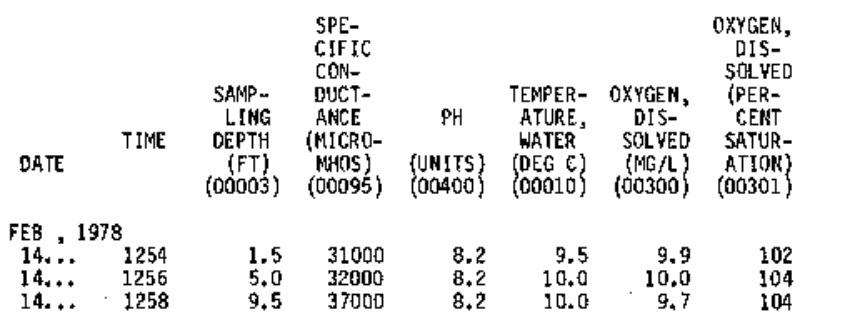


Table 6A.--Qual1ty of water in the Lavaca-Tres Palacios estuary, water years 1977-79--Continued

Field Determinations--continued

283135096170500 LISHE 363 SITE 03

\begin{tabular}{|c|c|c|c|c|c|c|c|c|}
\hline DATE & TIME & $\begin{array}{l}\text { SAMP- } \\
\text { LING } \\
\text { DEPTH } \\
(F T)\end{array}$ & $\begin{array}{l}\text { SPE- } \\
\text { CIFIC } \\
\text { CON- } \\
\text { DUCT- } \\
\text { ANCE } \\
\text { (MICRO- } \\
\text { MHOS) }\end{array}$ & $\begin{array}{c}\mathrm{PH} \\
\text { (UNITS) }\end{array}$ & $\begin{array}{l}\text { TEMPER- } \\
\text { ATURE, } \\
\text { WATER } \\
\text { (DEG C) }\end{array}$ & $\begin{array}{c}\text { TRANS- } \\
\text { PAR- } \\
\text { ENCY } \\
\text { (SECCHE } \\
\text { DISK) } \\
\text { (M) }\end{array}$ & $\begin{array}{c}\text { OXYGEN, } \\
\text { DIS- } \\
\text { SOLVED } \\
\text { (MG/L) }\end{array}$ & $\begin{array}{c}\text { OXYGEN, } \\
\text { DIS- } \\
\text { SOLLED } \\
\text { (PER- } \\
\text { CENT } \\
\text { SATUR- } \\
\text { ATION) }\end{array}$ \\
\hline \multicolumn{9}{|c|}{ FEB, 1977} \\
\hline $\begin{array}{l}08, \ldots \\
08 . . . \\
\text { JUN }\end{array}$ & $\begin{array}{l}1230 \\
1232\end{array}$ & $\begin{array}{l}1.0 \\
9.0\end{array}$ & $\begin{array}{l}41000 \\
41000\end{array}$ & $\begin{array}{l}\text { B. } 0 \\
\text { B.0 }\end{array}$ & $\begin{array}{l}11.0 \\
11.0\end{array}$ & $\begin{array}{r}.79 \\
\ldots\end{array}$ & $\begin{array}{l}9.5 \\
9.5\end{array}$ & $\begin{array}{l}107 \\
107\end{array}$ \\
\hline $\begin{array}{r}27 \ldots \\
27 \ldots \\
27 \ldots \\
\text { FEB },\end{array}$ & $\begin{array}{l}1400 \\
1402 \\
8^{1404}\end{array}$ & $\begin{array}{l}1.0 \\
4.0 \\
8.0\end{array}$ & $\begin{array}{l}33000 \\
33000 \\
33000\end{array}$ & $\begin{array}{l}\text { 8.1 } \\
\text { 8.1 } \\
\text { B.I }\end{array}$ & $\begin{array}{l}29.5 \\
29.5 \\
29.5\end{array}$ & $\begin{array}{r}.44 \\
-. \\
-.\end{array}$ & $\begin{array}{l}6.2 \\
6.2 \\
6.0\end{array}$ & $\begin{array}{l}93 \\
93 \\
91\end{array}$ \\
\hline $\begin{array}{l}14 \ldots \\
14 \ldots \\
14 \ldots \\
14 \ldots \\
14 \ldots \\
15 \ldots \\
15, \ldots \\
15 \ldots \\
\text { JUN }\end{array}$ & $\begin{array}{l}0718 \\
0720 \\
1749 \\
1751 \\
1753 \\
0724 \\
0726 \\
0728\end{array}$ & $\begin{array}{l}1.5 \\
8.0 \\
1.5 \\
5.0 \\
9.5 \\
1.5 \\
5.0 \\
9.0\end{array}$ & $\begin{array}{l}34000 \\
36000 \\
34000 \\
35000 \\
36000 \\
40000 \\
36000 \\
38500\end{array}$ & $\begin{array}{l}8.2 \\
8.2 \\
8.2 \\
8.2 \\
8.2 \\
8.2 \\
8.2 \\
8.2\end{array}$ & $\begin{array}{r}9.5 \\
9.0 \\
10.0 \\
10.0 \\
10.0 \\
10.0 \\
9.5 \\
9.5\end{array}$ & $\begin{array}{l}-- \\
=- \\
-- \\
=- \\
-- \\
=\end{array}$ & $\begin{array}{l}9.7 \\
9.6 \\
9.9 \\
9.8 \\
9.7 \\
9.4 \\
9.5 \\
9.4\end{array}$ & $\begin{array}{l}101 \\
100 \\
105 \\
104 \\
103 \\
102 \\
100 \\
100\end{array}$ \\
\hline $\begin{array}{l}19 \ldots \\
19 \ldots \\
20 \ldots \\
20 \ldots\end{array}$ & $\begin{array}{l}1924 \\
1926 \\
0805 \\
0806\end{array}$ & $\begin{array}{l}1.0 \\
9.0 \\
1.0 \\
8.0\end{array}$ & $\begin{array}{l}41000 \\
41000 \\
42000 \\
44000\end{array}$ & $\begin{array}{l}8.2 \\
8.2 \\
8.2 \\
8.2\end{array}$ & $\begin{array}{l}30.0 \\
29.5 \\
29.0 \\
29.0\end{array}$ & $\begin{array}{l}-- \\
.54 \\
-.\end{array}$ & $\begin{array}{l}6.1 \\
5.6 \\
6.4 \\
6.6\end{array}$ & $\begin{array}{r}97 \\
87 \\
99 \\
103\end{array}$ \\
\hline
\end{tabular}

283432096194600 LINE 363 SITE 05

\begin{tabular}{|c|c|c|c|c|c|c|c|c|}
\hline DATE & TIME & $\begin{array}{l}\text { SAMP- } \\
\text { LING } \\
\text { DEPTH } \\
(F T)\end{array}$ & $\begin{array}{l}\text { SPE- } \\
\text { CIF1C } \\
\text { CON- } \\
\text { DUCT- } \\
\text { ANCE } \\
\text { (MICRO- } \\
\text { MHOS) }\end{array}$ & $\begin{array}{c}\text { PH } \\
\text { (UNITS) }\end{array}$ & $\begin{array}{c}\text { TEMPER- } \\
\text { ATURE, } \\
\text { WATER } \\
\text { (DEG C) }\end{array}$ & $\begin{array}{c}\text { TRAKS- } \\
\text { PAR- } \\
\text { EHCY } \\
\text { (SECCHI } \\
\text { DISK) } \\
\text { (M) }\end{array}$ & $\begin{array}{c}\text { DXYGE } \\
\text { DIS- } \\
\text { SOLLVE) } \\
\text { (MG } / L)\end{array}$ & $\begin{array}{c}\text { DXYGEN, } \\
\text { DIS- } \\
\text { SOL YED } \\
\text { (PER- } \\
\text { CENT } \\
\text { SATUR- } \\
\text { ATION) }\end{array}$ \\
\hline \multicolumn{9}{|c|}{ FEB, 1977} \\
\hline $\begin{array}{l}08 . . . \\
08 . . \\
\text { JUN. }\end{array}$ & $\begin{array}{l}1255 \\
1257\end{array}$ & $10^{1.0}$ & $\begin{array}{l}41000 \\
41000\end{array}$ & $\begin{array}{l}8.0 \\
8.0\end{array}$ & $\begin{array}{l}11.0 \\
11.0\end{array}$ & .49 & $\begin{array}{l}9.3 \\
9.5\end{array}$ & $\begin{array}{l}105 \\
107\end{array}$ \\
\hline $\begin{array}{l}27 \ldots \\
27 \ldots \\
27 \ldots \\
\text { JUN, }\end{array}$ & $\begin{array}{l}1415 \\
1417 \\
1419 \\
8\end{array}$ & $\begin{array}{l}1.0 \\
5.0 \\
10^{\circ}\end{array}$ & $\begin{array}{l}32000 \\
32000 \\
32000\end{array}$ & $\begin{array}{l}8.1 \\
8.1 \\
8.1\end{array}$ & $\begin{array}{l}29.5 \\
29.5 \\
29.5\end{array}$ & $\begin{array}{l}.37 \\
-- \\
--\end{array}$ & $\begin{array}{l}6.3 \\
6.0 \\
5.9\end{array}$ & $\begin{array}{l}93 \\
89 \\
88\end{array}$ \\
\hline $\begin{array}{l}20 \ldots \\
20 \ldots\end{array}$ & $\begin{array}{l}0835 \\
0837\end{array}$ & 13.0 & $\begin{array}{l}39300 \\
39500\end{array}$ & $\begin{array}{l}8.1 \\
8.1\end{array}$ & $\begin{array}{l}29.5 \\
29.5\end{array}$ & .56 & $\begin{array}{l}6.3 \\
6.2\end{array}$ & $\begin{array}{l}97 \\
96\end{array}$ \\
\hline
\end{tabular}

283611096211900 LINE 363 SITE D6

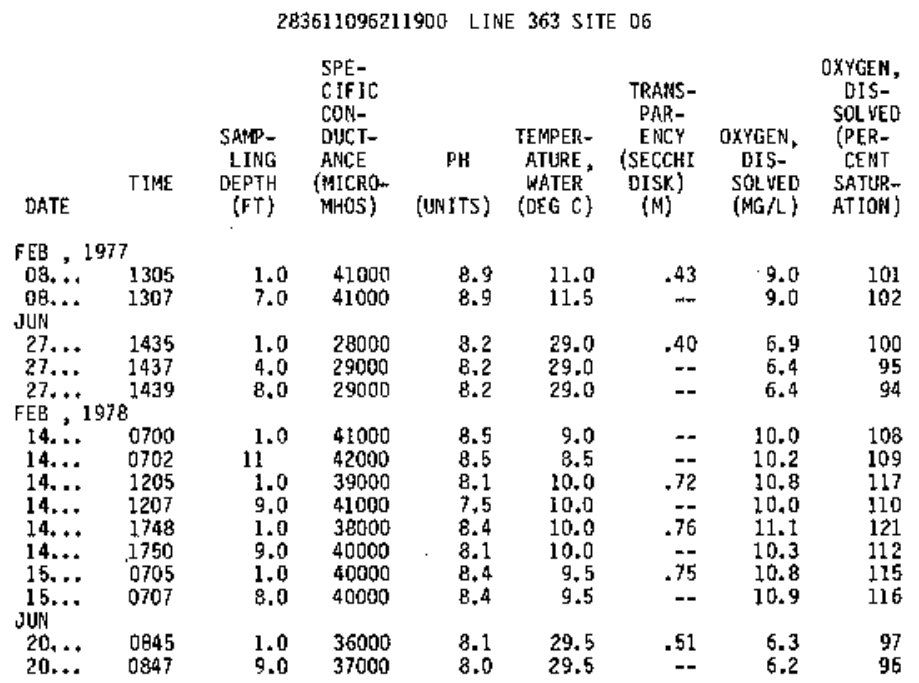


Table 6A.--Quality of water in the Lavaca-Tres Palacios estuary, water years 1977-78-Continued

Field Detenninations--Continued

282820096185100 LINE 375 SITE OI

\begin{tabular}{|c|c|c|c|c|c|c|c|c|}
\hline DATE & TIME & $\begin{array}{l}\text { SANP- } \\
\text { LING } \\
\text { DEPTH } \\
(\mathbf{F T})\end{array}$ & $\begin{array}{l}\text { SPE- } \\
\text { CIFIC } \\
\text { CON- } \\
\text { OUCT- } \\
\text { ANCE } \\
\text { (NICRO- } \\
\text { MANOS) }\end{array}$ & $\begin{array}{c}\mathrm{PH} \\
\text { (UNITS) }\end{array}$ & $\begin{array}{c}\text { TEMPER- } \\
\text { ATURE, } \\
\text { WATER } \\
\text { (DEG C) }\end{array}$ & $\begin{array}{l}\text { TRANS- } \\
\text { PAR- } \\
\text { ENCY } \\
\text { (SECCHI } \\
\text { DISK) } \\
\text { (M) }\end{array}$ & $\begin{array}{c}\text { OXYGEH, } \\
\text { OIS- } \\
\text { SOLYED } \\
\text { (MG } / L)\end{array}$ & $\begin{array}{c}\text { OXYGEN, } \\
\text { OIS- } \\
\text { SOLVED } \\
\text { (PER- } \\
\text { CENT } \\
\text { SATUR- } \\
\text { ATIOE) }\end{array}$ \\
\hline $\begin{array}{l}\text { FEB, } \\
08 . . . \\
08 . . . \\
08 . .\end{array}$ & $\begin{array}{l}1445 \\
1447 \\
1449\end{array}$ & $\begin{array}{r}1.0 \\
5.0 \\
12\end{array}$ & $\begin{array}{l}42000 \\
44000 \\
44000\end{array}$ & $\begin{array}{l}8.1 \\
8.0 \\
8.0\end{array}$ & $\begin{array}{l}11.5 \\
10.5 \\
11.0\end{array}$ & $\begin{array}{r}.43 \\
-- \\
--\end{array}$ & $\begin{array}{l}9.5 \\
9.5 \\
9.2\end{array}$ & $\begin{array}{l}108 \\
107 \\
105\end{array}$ \\
\hline $\begin{array}{l}\text { JUN } \\
28 \ldots . . \\
28 \ldots \\
28 . . . \\
\text { JUN , }\end{array}$ & $\begin{array}{l}1025 \\
1027 \\
1029 \\
8\end{array}$ & $\begin{array}{l}1.0 \\
6.0 \\
12\end{array}$ & $\begin{array}{l}52000 \\
54000 \\
50000\end{array}$ & $\begin{array}{l}7.9 \\
7.8 \\
7.8\end{array}$ & $\begin{array}{l}28.0 \\
27.5 \\
27.5\end{array}$ & $\begin{array}{l}-- \\
--\end{array}$ & $\begin{array}{l}5.7 \\
5.2 \\
5.2\end{array}$ & $\begin{array}{l}92 \\
83 \\
83\end{array}$ \\
\hline $\begin{array}{l}20 \ldots \\
20 \ldots \\
20 . \ldots\end{array}$ & $\begin{array}{l}1100 \\
1102 \\
1104\end{array}$ & $\begin{array}{r}1.0 \\
5.0 \\
13\end{array}$ & $\begin{array}{l}44000 \\
44000 \\
49000\end{array}$ & $\begin{array}{l}8.3 \\
8.2 \\
8.2\end{array}$ & $\begin{array}{l}30.0 \\
30.0 \\
30.0\end{array}$ & $\begin{array}{r}.80 \\
-7\end{array}$ & $\begin{array}{l}6.1 \\
6.1 \\
5.8\end{array}$ & $\begin{array}{l}97 \\
97 \\
95\end{array}$ \\
\hline
\end{tabular}

282943096204500 LINE 375 SITE 02

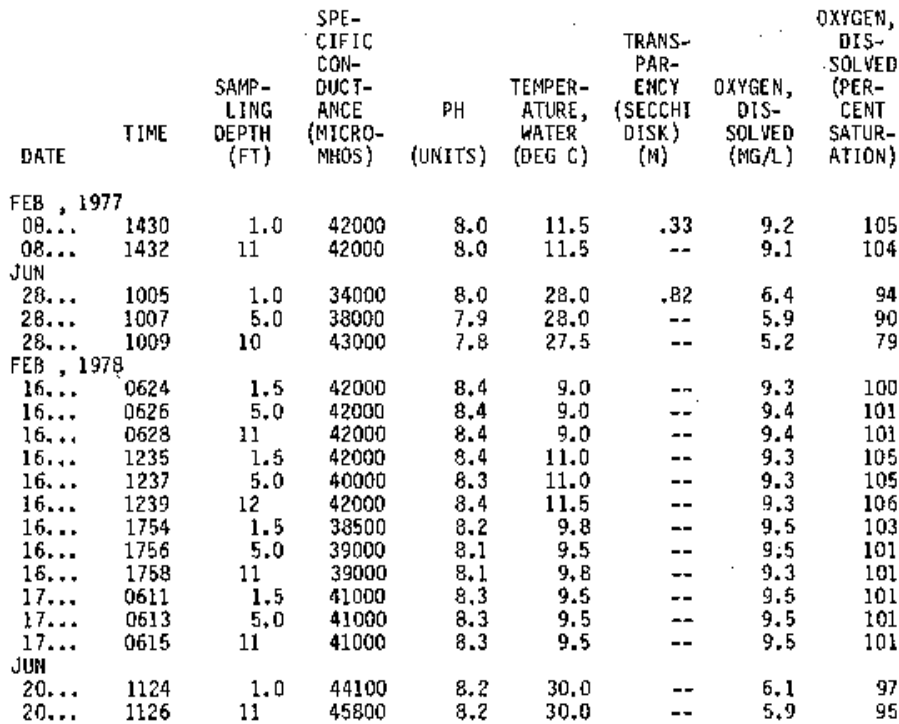

283233096244100 LINE 375 SITE 03

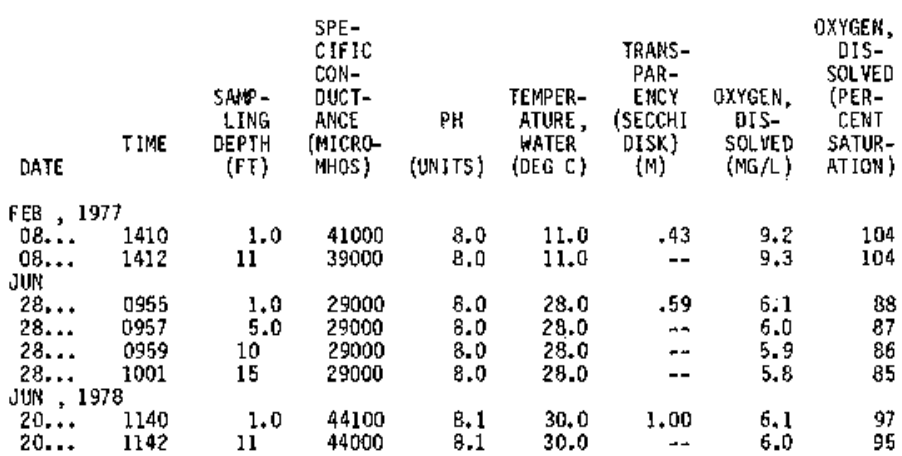


Table 6A.--Qualfty of water in the Lavaca-Tres Paldacios estuary, water years 1977-78--Continued Field Detenninations--Cont 1 nued

283430096272200 LINE 375 SITE 04

\begin{tabular}{|c|c|c|c|c|c|c|c|c|}
\hline DATE & TINE & $\begin{array}{l}\text { SAMG } \\
\text { LING } \\
\text { DEPTH } \\
\text { (FT) }\end{array}$ & $\begin{array}{l}\text { SPE- } \\
\text { C1FIC } \\
\text { CON- } \\
\text { DUCT- } \\
\text { ANCE } \\
\text { (MICRO- } \\
\text { MHOS) }\end{array}$ & $\begin{array}{c}\mathrm{PH} \\
\text { (UNITS) }\end{array}$ & $\begin{array}{l}\text { TEMPER- } \\
\text { ATURE, } \\
\text { WRTER } \\
\text { (DEG C) }\end{array}$ & $\begin{array}{l}\text { TRANSS- } \\
\text { PAR- } \\
\text { ENCY } \\
\text { (SECCHI } \\
\text { DISK) } \\
\text { (M) }\end{array}$ & $\begin{array}{c}\text { OXYGEN, } \\
\text { D15- } \\
\text { SOLUED } \\
\text { (MG } / L)\end{array}$ & $\begin{array}{l}\text { OXYGEN, } \\
\text { OIS- } \\
\text { SOLVED } \\
\text { (PER* } \\
\text { CENT } \\
\text { SASTUR- } \\
\text { ATION) }\end{array}$ \\
\hline \multicolumn{9}{|c|}{ FEB , 1977} \\
\hline $\begin{array}{l}08 \ldots \\
08 . . .\end{array}$ & $\begin{array}{l}1325 \\
1327\end{array}$ & $\begin{array}{l}1.0 \\
7.0\end{array}$ & $\begin{array}{l}39000 \\
39000\end{array}$ & $\begin{array}{l}8,0 \\
8.0\end{array}$ & $\begin{array}{l}11.5 \\
11.5\end{array}$ & .34 & $\begin{array}{l}9.1 \\
9.1\end{array}$ & $\begin{array}{l}102 \\
102\end{array}$ \\
\hline \multicolumn{9}{|l|}{ JUN } \\
\hline $\begin{array}{l}27 \ldots \\
27 \ldots\end{array}$ & $\begin{array}{l}1505 \\
150 \%\end{array}$ & $\begin{array}{l}1.0 \\
7.0\end{array}$ & $\begin{array}{l}29000 \\
28000\end{array}$ & $\begin{array}{l}8.2 \\
8.1\end{array}$ & $\begin{array}{l}28.5 \\
28.5\end{array}$ & $=-$ & $\begin{array}{l}6.2 \\
6.4\end{array}$ & $\begin{array}{l}90 \\
92\end{array}$ \\
\hline 16.. & 0659 & 1,0 & 39000 & 8.4 & 9.0 & -- & 9.5 & 100 \\
\hline $16 . .$. & 0701 & 5.0 & 39000 & 8.5 & 9. & $=$ & & \\
\hline $16 . .$. & 0703 & 8,0 & 40000 & 8. & 9. & -- & 9. & 98 \\
\hline $16 .$. & 1134 & 1.5 & 38500 & 8.2 & 11.0 & - & 9. & 105 \\
\hline $16 . \ldots$ & 1136 & 5.0 & 38500 & 8. & 11. & -- & & 106 \\
\hline 16. & 1138 & 8.0 & 38500 & 8, & 12. & $=$ & & 108 \\
\hline 16. & 1830 & 1.5 & 39000 & & 9. & -- & & 105 \\
\hline $16 .$. & 1832 & 5.0 & 38000 & 8. & 10. & -- & & 107 \\
\hline 17. & 0650 & 1.5 & 38000 & 8. & 9. & -- & & 102 \\
\hline 17 & 0652 & & 38500 & & 9. & -- & & 102 \\
\hline & 0654 & 8. & 385 & & 9. & -- & & 101 \\
\hline \multicolumn{9}{|l|}{ JUN } \\
\hline $20 . \ldots$ & 1918 & 1.0 & 38000 & 8.0 & 30.5 & -- & 6.1 & \\
\hline & 1920 & 9.0 & 39000 & 8.1 & 30.5 & $\overline{35}$ & 5. & 96 \\
\hline & 0737 & $\begin{array}{l}1.10 \\
8.0\end{array}$ & $\begin{array}{l}390000 \\
40000\end{array}$ & $\begin{array}{l}8.3 \\
8.3\end{array}$ & $\begin{array}{r}29.5 \\
29.5\end{array}$ & $=35$ & & $9 \sqrt{3}$ \\
\hline & & & & & & & & \\
\hline
\end{tabular}

282516096230000 LINE 382 5ITE 02

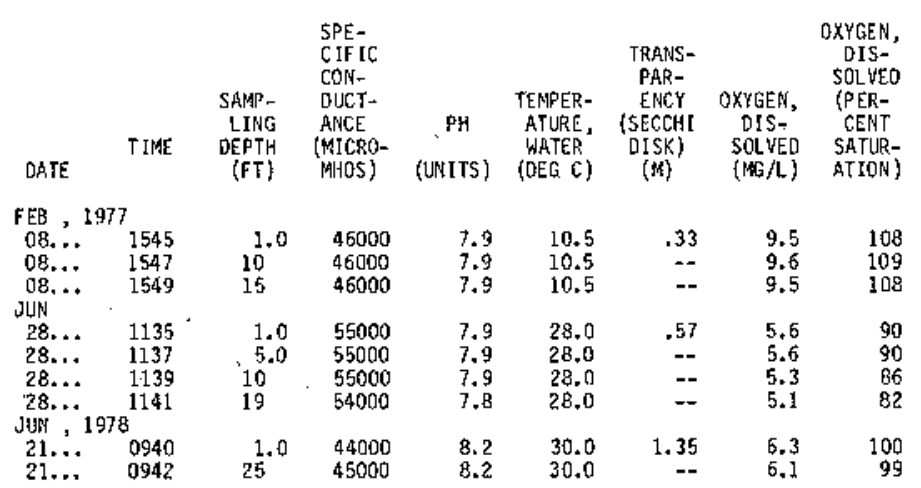

282516096192300 LINE 397 SITE QZ

\begin{tabular}{|c|c|c|c|c|c|c|c|c|}
\hline DATE & TIME & $\begin{array}{l}\text { SAMP- } \\
\text { LING } \\
\text { DEPTH } \\
\text { (FT) }\end{array}$ & $\begin{array}{l}\text { SPE- } \\
\text { CIFIC } \\
\text { CON- } \\
\text { DUCT- } \\
\text { ANCE } \\
\text { (MICRO- } \\
\text { MHOS) }\end{array}$ & $\begin{array}{c}\text { PH } \\
\text { (URITS) }\end{array}$ & $\begin{array}{l}\text { TEMPER- } \\
\text { ATURE, } \\
\text { WATER } \\
\text { (DEG C) }\end{array}$ & $\begin{array}{l}\text { TRAN5- } \\
\text { PAR- } \\
\text { ENCY } \\
\text { (5ECCHI } \\
\text { DISK) } \\
\text { (M) }\end{array}$ & $\begin{array}{c}\text { OXYCEN, } \\
\text { DIS- } \\
\text { SOLVED } \\
\text { (MG/L) }\end{array}$ & $\begin{array}{c}\text { OXYGEN, } \\
\text { OIS- } \\
\text { SOLVED } \\
\text { (PER- } \\
\text { CENT } \\
\text { SATUR- } \\
\text { ATION) }\end{array}$ \\
\hline $\begin{array}{l}\text { FEB , } \\
08 . \cdots \\
08 . . . \\
08 \ldots \\
08 \ldots . . \\
08 . .\end{array}$ & $\begin{array}{l}7 \\
1505 \\
1507 \\
1509 \\
1511 \\
1513\end{array}$ & $\begin{array}{l}1.0 \\
10 \\
20 \\
30 \\
47\end{array}$ & $\begin{array}{l}46000 \\
46000 \\
46000 \\
46000 \\
46000\end{array}$ & $\begin{array}{l}8.0 \\
8.0 \\
8.0 \\
8.0 \\
8.0\end{array}$ & $\begin{array}{l}10.5 \\
10.5 \\
10.5 \\
10.0 \\
10.0\end{array}$ & $\begin{array}{c}.43 \\
-- \\
-- \\
--\end{array}$ & $\begin{array}{l}9.5 \\
9.2 \\
9.2 \\
9.4 \\
9.4\end{array}$ & $\begin{array}{l}107 \\
105 \\
105 \\
106 \\
105\end{array}$ \\
\hline $\begin{array}{r}U A, \\
21 \ldots \\
21 \ldots \\
21 \ldots\end{array}$ & $\begin{array}{l}8 \\
0900 \\
0902 \\
0904\end{array}$ & $\begin{array}{l}1.0 \\
20 \\
42\end{array}$ & $\begin{array}{l}43000 \\
43000 \\
44000\end{array}$ & $\begin{array}{l}8.3 \\
8.3 \\
8.3\end{array}$ & $\begin{array}{l}29.5 \\
29.5 \\
29.5\end{array}$ & $\begin{array}{r}1,60 \\
\ldots \\
=\end{array}$ & $\begin{array}{l}6.2 \\
6.2 \\
6.1\end{array}$ & $\begin{array}{l}99 \\
99 \\
97\end{array}$ \\
\hline
\end{tabular}




\begin{tabular}{|c|c|c|c|c|c|c|c|c|}
\hline \multirow[b]{2}{*}{ DATE } & \multirow[b]{2}{*}{ TIME } & \multicolumn{3}{|c|}{282330096174000} & \multicolumn{2}{|c|}{ NE 903 SITE 49} & \multirow{2}{*}{\multicolumn{2}{|c|}{$\begin{array}{cc} & \text { OXYGEN, } \\
& \text { DIS- } \\
& \text { SOL VED } \\
\text { OXYGEN, } & \text { (PER- } \\
\text { DIS- } & \text { CFNT } \\
\text { SOLVED } & \text { SATUR- } \\
\text { (HAL) } & \text { ATION) }\end{array}$}} \\
\hline & & $\begin{array}{l}\text { SAMP- } \\
\text { LIMC } \\
\text { DEPTH } \\
\text { (FT) }\end{array}$ & $\begin{array}{l}\text { SPE- } \\
\text { CIFIC } \\
\text { CON- } \\
\text { DUCT- } \\
\text { AHCE } \\
\text { (MLCRO- } \\
\text { MHOS) }\end{array}$ & $\begin{array}{c}\mathrm{PH} \\
(\mathrm{UNITS})\end{array}$ & $\begin{array}{c}\text { TEMPER- } \\
\text { ATURE, } \\
\text { WATER } \\
\text { (OEG C) }\end{array}$ & $\begin{array}{c}\text { TRANS- } \\
\text { PAR- } \\
\text { ENCY } \\
\text { (SECCHI } \\
\text { DISK) } \\
\text { (M) }\end{array}$ & & \\
\hline $\begin{array}{l}\text { JUN , } \\
28, . \\
28 . . \\
28 . . \\
28 . . \\
28 . . \\
28 . .\end{array}$ & $\begin{array}{l}7 \\
1050 \\
1052 \\
1054 \\
1056 \\
1058 \\
1100\end{array}$ & $\begin{array}{l}1.0 \\
5.0 \\
10 \\
15 \\
25 \\
35\end{array}$ & $\begin{array}{l}54000 \\
57000 \\
57000 \\
57000 \\
57000 \\
57000\end{array}$ & $\begin{array}{l}7.9 \\
7.9 \\
7.9 \\
7.9 \\
7.8 \\
7.8\end{array}$ & $\begin{array}{l}28.0 \\
27.5 \\
27.0 \\
27.0 \\
26.5 \\
26.0\end{array}$ & $\begin{array}{c}1.15 \\
-- \\
= \\
= \\
- \\
+\end{array}$ & $\begin{array}{l}5.6 \\
5.2 \\
5.1 \\
4.8 \\
4.5 \\
4.1\end{array}$ & $\begin{array}{l}91 \\
85 \\
82 \\
77 \\
73 \\
65\end{array}$ \\
\hline $\begin{array}{l}\text { JUN, } \\
21, \ldots \\
21 \ldots \\
21 \ldots\end{array}$ & $\begin{array}{l}8 \\
0848 \\
0850 \\
0852\end{array}$ & $\begin{array}{l}2.0 \\
20 \\
33\end{array}$ & $\begin{array}{l}44000 \\
46000 \\
46000\end{array}$ & $\begin{array}{l}8.3 \\
8.3 \\
8.3\end{array}$ & $\begin{array}{l}29.5 \\
29.0 \\
29.5\end{array}$ & $\begin{array}{r}1.90 \\
-- \\
--\end{array}$ & $\begin{array}{l}6.2 \\
6.1 \\
6.0\end{array}$ & $\begin{array}{l}99 \\
96 \\
97\end{array}$ \\
\hline
\end{tabular}


Table 6B.--Quality of water in the Lavaca-Tres Palacios estuary, water years 1977-78

Nutrient AraTyses

$(F T=$ feet; $M G / L=$ milligrams per Titer)

2850030963524DO LINE 017 SITE 02

\begin{tabular}{|c|c|c|c|c|c|c|c|c|c|c|c|c|}
\hline DATE & TIME & $\begin{array}{l}\text { SAMP- } \\
\text { LING } \\
\text { DEPTH } \\
(F T)\end{array}$ & $\begin{array}{l}\text { OXYGEN } \\
\text { DEMAND, } \\
\text { BIOCHEM } \\
\text { UNIYHIB } \\
5 \text { DAY } \\
\text { (MAG/L) }\end{array}$ & $\begin{array}{l}\text { NITRO- } \\
\text { GEN, } \\
\text { NITRATE } \\
\text { TOTAL } \\
\text { (MG/L } \\
\text { AS N) }\end{array}$ & $\begin{array}{c}\text { NITRO- } \\
\text { GEN, } \\
\text { NITRITE } \\
\text { TOTAL } \\
\text { (MG/L } \\
\text { AS N) }\end{array}$ & $\begin{array}{c}\text { NITRO- } \\
\text { GEN, } \\
\text { MO2+NO3 } \\
\text { TOTAL } \\
\text { (MG/L } \\
\text { AS N) }\end{array}$ & $\begin{array}{l}\text { NITRO- } \\
\text { GEN, } \\
\text { AMMONIA } \\
\text { TOTAL } \\
\text { (MG/L } \\
\text { AS N) }\end{array}$ & $\begin{array}{l}\text { NITRO- } \\
\text { GEN, } \\
\text { ORGANIC } \\
\text { TOTAL } \\
\text { (MG/L } \\
\text { AS N) }\end{array}$ & $\begin{array}{l}\text { NITRO- } \\
\text { GEN, AN- } \\
\text { MONIA + } \\
\text { ORRGANIC } \\
\text { TOTAL } \\
\text { (MGG/L } \\
\text { AS N }\end{array}$ & $\begin{array}{l}\text { NITRO- } \\
\text { GEN, } \\
\text { TDTAL } \\
\text { (MG/L } \\
\text { AS N) }\end{array}$ & $\begin{array}{c}\text { NITRO- } \\
\text { GEM, } \\
\text { TOTAL } \\
\text { (MG } /( \\
\text { AS NO3) }\end{array}$ & $\begin{array}{l}\text { PHOS- } \\
\text { PHDRUS, } \\
\text { TOTAL } \\
\text { (MMG } / L \\
\text { AS P) }\end{array}$ \\
\hline \multicolumn{13}{|c|}{ FEB , 19Y7 } \\
\hline $\begin{array}{l}09 . . \\
\text { DUN }\end{array}$ & $\begin{array}{l}1130 \\
1132\end{array}$ & $10^{1.0}$ & $\begin{array}{r}1.1 \\
.9\end{array}$ & $\begin{array}{l}.36 \\
.37\end{array}$ & $\begin{array}{l}.00 \\
.00\end{array}$ & $\begin{array}{l}.36 \\
.37\end{array}$ & .03 & $\begin{array}{l}.50 \\
.47\end{array}$ & $\begin{array}{l}.53 \\
.51\end{array}$ & $\begin{array}{l}.89 \\
.88\end{array}$ & $\begin{array}{l}3.9 \\
3.9\end{array}$ & $\begin{array}{r}.030 \\
.080\end{array}$ \\
\hline $\begin{array}{l}27 \ldots \\
27 \ldots \\
\text { JUN, }\end{array}$ & $\begin{array}{l}1455 \\
1457 \\
8\end{array}$ & $\begin{array}{l}1.0 \\
8.5\end{array}$ & $\begin{array}{l}1.4 \\
1.3\end{array}$ & $\begin{array}{l}.38 \\
.38\end{array}$ & $\begin{array}{l}.01 \\
.01\end{array}$ & $\begin{array}{l}.39 \\
.39\end{array}$ & .09 & $\begin{array}{l}.76 \\
.48\end{array}$ & $\begin{array}{l}.85 \\
.57\end{array}$ & $\begin{array}{l}1.2 \\
.96\end{array}$ & $\begin{array}{l}5.5 \\
4.2\end{array}$ & $\begin{array}{l}.140 \\
.140\end{array}$ \\
\hline $\begin{array}{l}21 \ldots \\
21 \ldots\end{array}$ & $\begin{array}{l}0957 \\
0959\end{array}$ & 1.0 & $\begin{array}{l}2.8 \\
2.6\end{array}$ & $\begin{array}{l}.00 \\
.00\end{array}$ & $\begin{array}{l}.01 \\
.01\end{array}$ & $\begin{array}{l}.00 \\
.00\end{array}$ & .03 & $\begin{array}{l}.70 \\
.61\end{array}$ & $\begin{array}{l}.73 \\
.64\end{array}$ & $\begin{array}{l}.73 \\
.64\end{array}$ & $\begin{array}{l}3.2 \\
2.8\end{array}$ & $\begin{array}{r}.090 \\
.090\end{array}$ \\
\hline
\end{tabular}

285248096344400 LINE O2O SITE O?

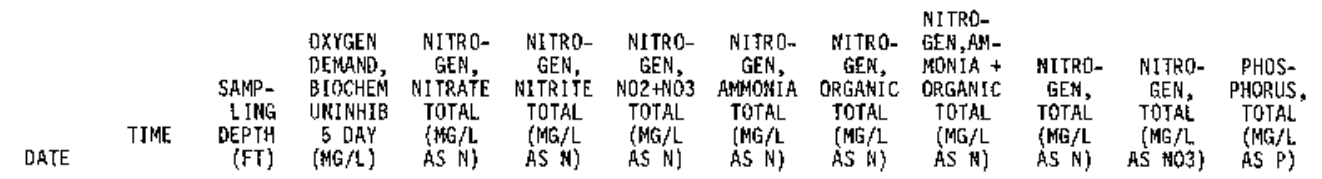

SEP , 1978

12... 1335

$12 \ldots .1700$

$13 \ldots 00945$

$19 \ldots .0955$

$\begin{array}{ll}3.0 & 4.2 \\ 3.0 & 4.1 \\ 3.0 & 2.9 \\ 3.0 & 2.3 \\ - & 3.6\end{array}$

$\begin{array}{ll}-17 & .09 \\ .19 & .02 \\ .18 & .01 \\ .01 & .01\end{array}$

$\begin{array}{ll}.26 & .74 \\ .21 & .17 \\ .19 & .08 \\ .02 & .05\end{array}$

$\begin{array}{ll}2.4 & 2.5 \\ 1.9 & 2.1 \\ 1.0 & 1.1 \\ 1.1 & 1.1\end{array}$

$\begin{array}{lll}2.8 & 12 & .250 \\ 2.3 & 10 & .220 \\ 1.3 & 5.7 & .220 \\ 1.1 & 5.0 & .340\end{array}$

285223096343100 LINE 022 SITE O2

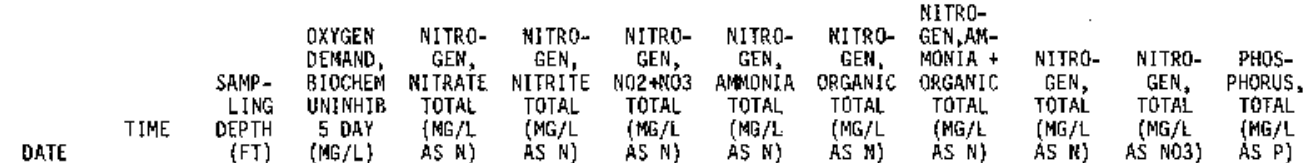

FEB , 1977

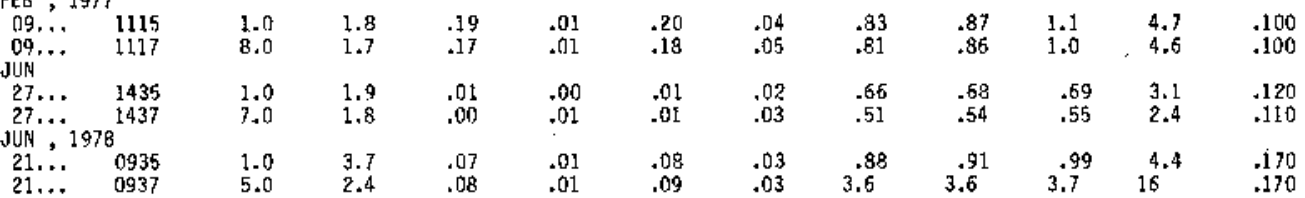

284921096342100 LINE 04I SITE 02

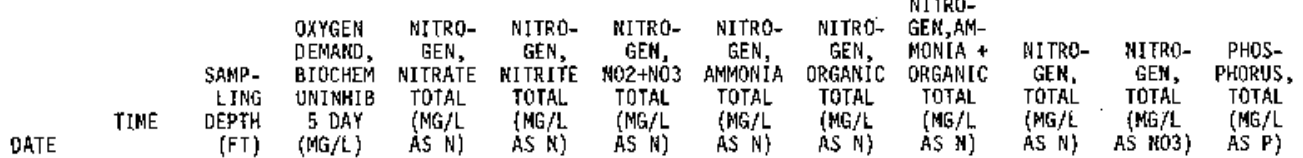

SEP , 1978

$12 \ldots{ }^{1978} 1238$

$12 \ldots .1238$

$12 \ldots \quad 1900$

$\begin{array}{ll}12 \ldots & 2315 \\ 13 \ldots . & 1052\end{array}$

$13 \ldots . \cdots \quad 1052$

$14 \ldots .1240$

$14 \ldots . .1430$

$14 \ldots .1824$

$14 . . .2032$

$14 . . .2230$

$15 \ldots .0945$

$15 \ldots \cdot 0954$

$15 . . . \quad 1350$

$15 . . . \quad 1545$

$15 \ldots .1749$

$\begin{array}{ll}15 \ldots . . & 1945 \\ 19 . . & 1410\end{array}$

$\begin{array}{ll}-. & 4.2 \\ -- & 4.9 \\ -- & 5.0 \\ -- & -- \\ -- & = \\ -- & -- \\ -- & = \\ -- & 2.8 \\ -- & 2.2 \\ -- & 2.3 \\ -- & 2.3 \\ -- & 2.5 \\ -- & 2.3 \\ -- & 2.2 \\ -- & -- \\ -- & 2.2 \\ -- & 2.1 \\ -- & 2.0 \\ - & 2.4 \\ -- & 2.6 \\ -- & 4.8\end{array}$

$\begin{array}{ll}.24 & .01 \\ .23 & .01 \\ .22 & .01 \\ .20 & .01 \\ .13 & .01 \\ .07 & .01 \\ .07 & .01 \\ .07 & .01 \\ .07 & .01 \\ .06 & .01 \\ .06 & .01 \\ .06 & .02 \\ .03 & .01 \\ . . & .- \\ .03 & .01 \\ .02 & .01 \\ .02 & .01 \\ .02 & .01 \\ .03 & .01 \\ .02 & .01 \\ .03 & .01\end{array}$

$\begin{array}{ll}.25 & .18 \\ .24 & .18 \\ .23 & .18 \\ .21 & .14 \\ .14 & .14 \\ .08 & .13 \\ .08 & .09 \\ .08 & .08 \\ .08 & .09 \\ .07 & .07 \\ .07 & .07 \\ .08 & .08 \\ .04 & .06 \\ .04 & .05 \\ .04 & .05 \\ .03 & .05 \\ .03 & .05 \\ .03 & .04 \\ .04 & .06 \\ .03 & .05 \\ .04 & .06\end{array}$

$\begin{array}{ll}.18 & 3.0 \\ .18 & 1.6 \\ .18 & 2.3 \\ .14 & 1.9 \\ .14 & 1.6 \\ .13 & 1.2 \\ .09 & 1.8 \\ .08 & 1.0 \\ .09 & .91 \\ .07 & .80 \\ .07 & 1.5 \\ .08 & .92 \\ .06 & 1.0 \\ .- & 1.3 \\ .05 & 1.3 \\ .05 & .77 \\ .05 & .75 \\ .04 & .73 \\ .06 & 1.0 \\ .05 & .79 \\ .06 & 1.3\end{array}$

$\begin{array}{cccc}3.2 & 3.5 & 15 & .320 \\ 1.8 & 2.0 & 9.0 & .540 \\ 2.5 & 2.7 & 12 & .290 \\ 2.0 & 2.2 & 9.8 & .300 \\ 1.7 & 1.8 & 8.1 & .310 \\ 1.3 & 1.4 & 6.1 & .250 \\ 1.9 & 2.0 & 8.8 & .190 \\ 1.1 & 1.2 & 5.2 & .180 \\ 1.0 & 1.1 & 4.8 & .180 \\ .87 & .94 & 4.2 & .180 \\ 1.6 & 1.7 & 7.4 & .220 \\ 1.0 & 1.1 & 4.8 & .190 \\ 1.1 & 1.1 & 5.0 & .180 \\ 1 .- & 1.3 & 5.9 & .150 \\ 1.3 & 1.3 & 5.9 & .150 \\ .82 & .85 & 3.8 & .150 \\ .80 & .83 & 3.7 & .150 \\ .77 & .80 & 3.5 & .130 \\ 1.1 & 1.1 & 5.0 & .140 \\ .84 & .87 & 3.9 & .140 \\ 1.4 & 1.4 & 6.4 & .320\end{array}$ 
284035096351200 LIHE 085 SITE 01

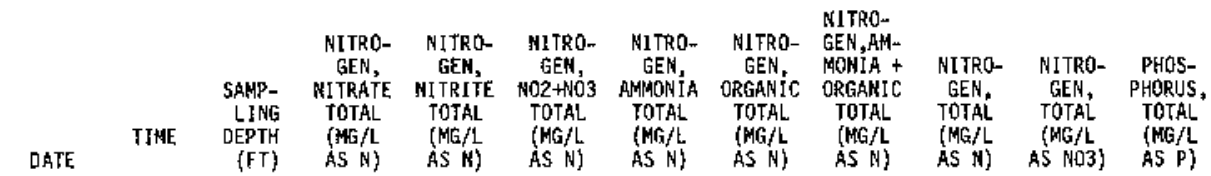

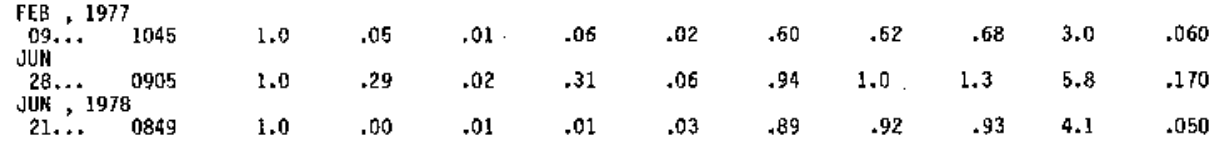

284200096374500 LINE OB5 SITE 03

\begin{tabular}{|c|c|c|c|c|c|c|c|c|c|c|c|}
\hline DATE & TIME & $\begin{array}{l}\text { SAMP } \\
\text { LING } \\
\text { DEPTH } \\
\text { (FT) }\end{array}$ & $\begin{array}{l}\text { NITRO- } \\
\text { GEN, } \\
\text { NITRATE } \\
\text { TOTAL } \\
\text { (WG/L } \\
\text { AS NA) }\end{array}$ & $\begin{array}{l}\text { NITRO- } \\
\text { GEN, } \\
\text { WITRITE } \\
\text { TOTAL } \\
\text { (MGG } / 1 \\
\text { AS N) }\end{array}$ & $\begin{array}{c}\text { NETRO- } \\
\text { GEN, } \\
\text { MO2+NO3 } \\
\text { TOTAL } \\
\text { (MG/L } \\
\text { AS H) }\end{array}$ & $\begin{array}{l}\text { MITRO- } \\
\text { GEM, } \\
\text { AMMONIA } \\
\text { TOTAL } \\
\text { (MG/L } \\
\text { ASS N) }\end{array}$ & $\begin{array}{l}\text { NITRO- } \\
\text { GEN, } \\
\text { ORGANIC } \\
\text { TOTAL } \\
\text { (MG/L } \\
\text { AS N) }\end{array}$ & $\begin{array}{l}\text { NITRO- } \\
\text { GEN,AM- } \\
\text { MONIA + } \\
\text { ORGANIC } \\
\text { TOTAL } \\
\text { (MGG } L \\
\text { AS N) }\end{array}$ & $\begin{array}{l}\text { NITRO- } \\
\text { GEN, } \\
\text { TOTAL } \\
\text { (MG/L } \\
\text { AS N) }\end{array}$ & 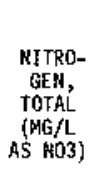 & $\begin{array}{l}\text { PHOS- } \\
\text { PHORUS, } \\
\text { JOTAL } \\
\text { (MA' } / L \\
\text { AS P) }\end{array}$ \\
\hline
\end{tabular}

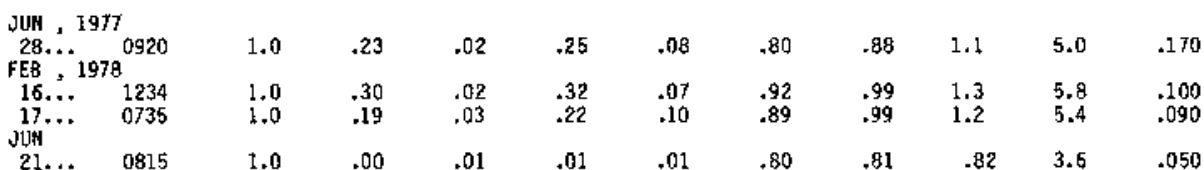

284235096324900 LINE 085 SITE Q4

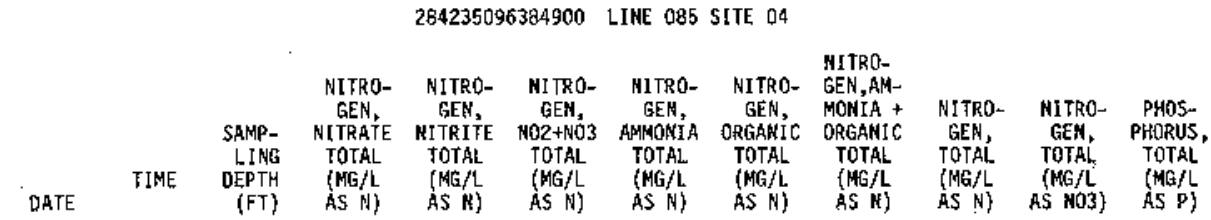

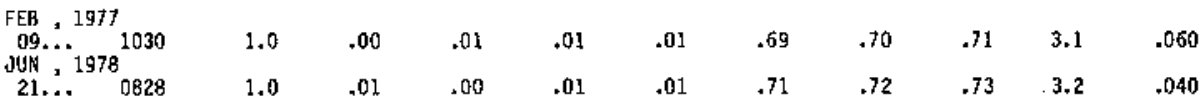

283912096354400 LJWE 090 SITE 03

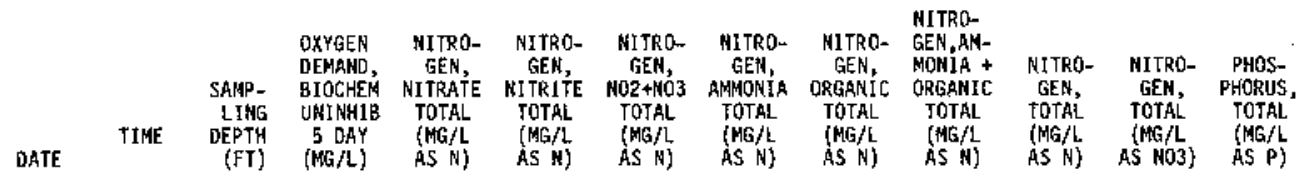

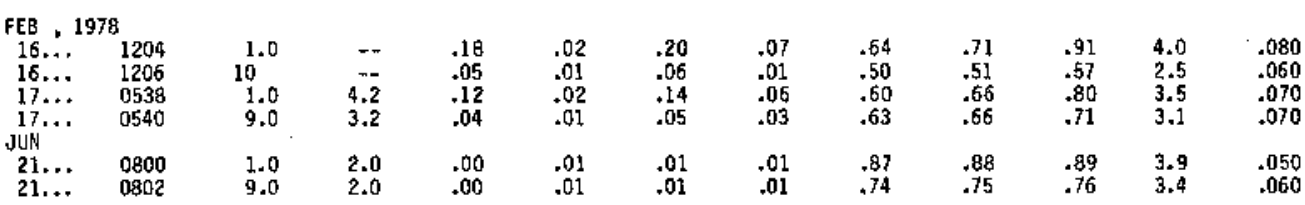

283716096310400 LINE 143 SITE 01

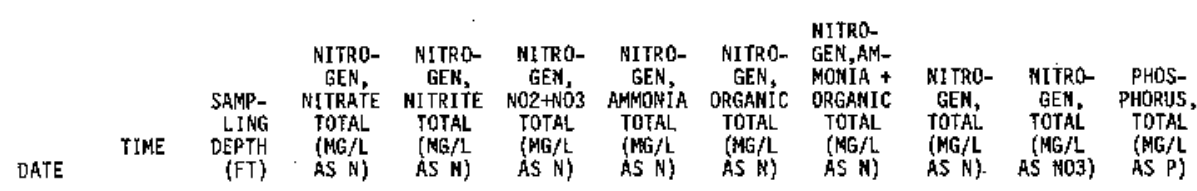

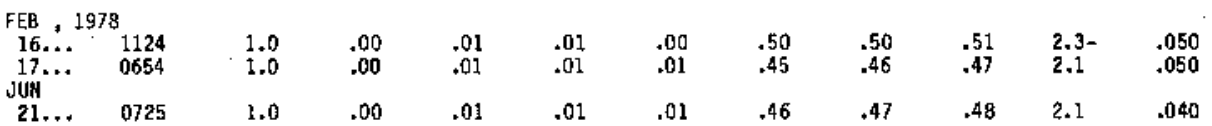


Table 6B.--Qual1ty of water in the Lavaca-Tres Palacios estuary, water years 1977-78--Continued

Mutríent Analyses-.-Cont inued

283902096330200 LINE 145 SITE 02

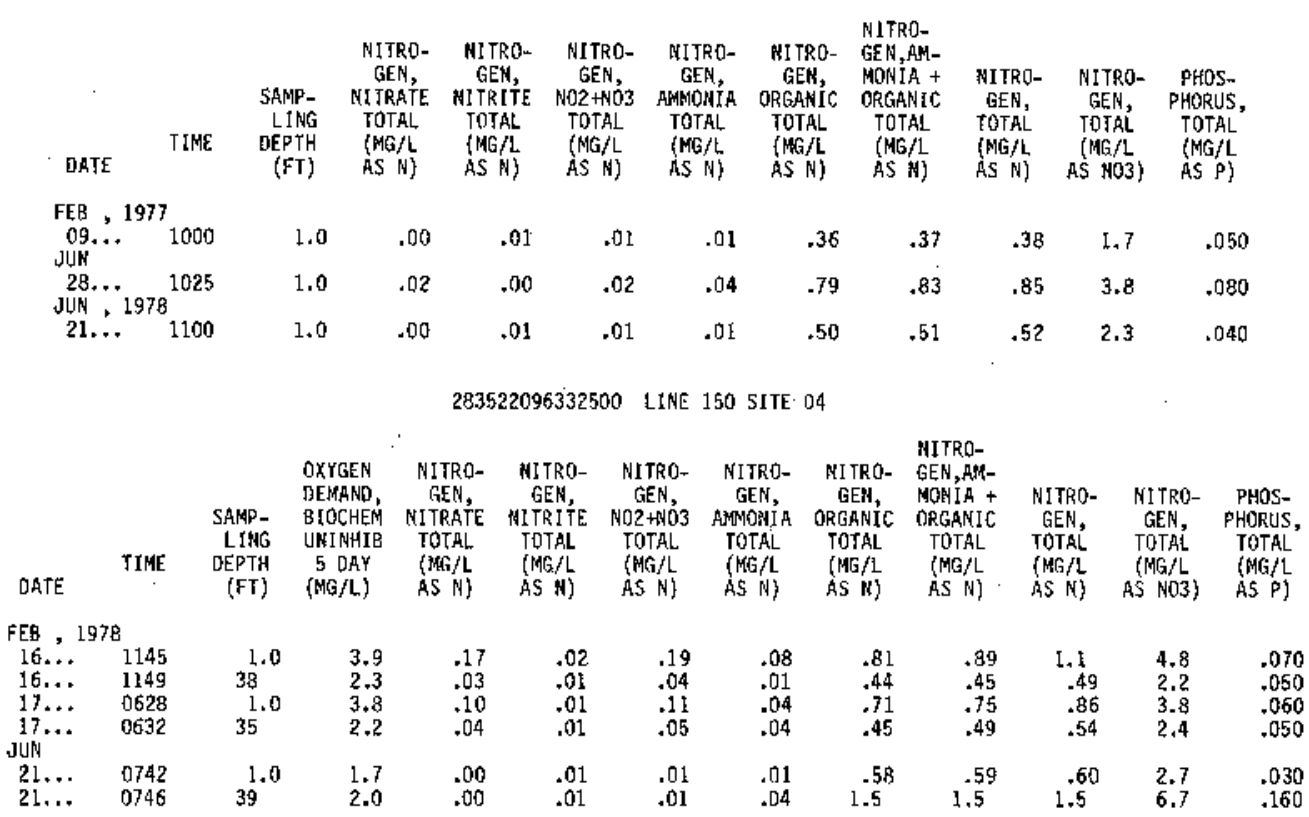

283337096305000 LIRE 190 SITE 04

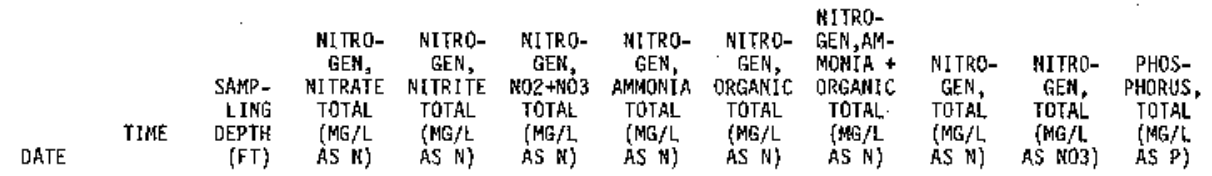

\begin{tabular}{|c|c|c|c|c|c|c|c|c|c|c|c|}
\hline \multicolumn{12}{|c|}{ FEB, 1977} \\
\hline $\begin{array}{c}09 . . . \\
09 . . . \\
\text { JUk }\end{array}$ & $\begin{array}{l}0920 \\
0926\end{array}$ & 36 & $\begin{array}{l}.00 \\
.00\end{array}$ & $\begin{array}{l}.01 \\
.01\end{array}$ & .01 & $\begin{array}{l}.04 \\
.11\end{array}$ & $\begin{array}{l}.26 \\
.27\end{array}$ & $\begin{array}{r}.30 \\
.38\end{array}$ & $\begin{array}{l}.31 \\
.39\end{array}$ & $\begin{array}{l}1.4 \\
1.7\end{array}$ & $\begin{array}{l}.050 \\
.000\end{array}$ \\
\hline $\begin{array}{r}27 \ldots \\
27 \ldots \\
\text { JUN, }\end{array}$ & $\begin{array}{r}1510 \\
1524 \\
8^{1524}\end{array}$ & $32^{1.0}$ & $\begin{array}{l}.00 \\
.02\end{array}$ & $\begin{array}{l}.01 \\
.04\end{array}$ & $\begin{array}{l}.01 \\
.06\end{array}$ & $\begin{array}{l}.03 \\
.10\end{array}$ & $\begin{array}{l}.58 \\
.32\end{array}$ & $\begin{array}{l}.51 \\
.42\end{array}$ & $\begin{array}{l}.62 \\
.48\end{array}$ & $\begin{array}{l}2.7 \\
2.1\end{array}$ & $\begin{array}{l}.050 \\
.050\end{array}$ \\
\hline $\begin{array}{l}21 \ldots \\
21 \ldots\end{array}$ & $\begin{array}{l}0725 \\
0729\end{array}$ & 1.0 & .01 & $\begin{array}{l}.00 \\
.01\end{array}$ & .01 & 101 & $\begin{array}{l}.51 \\
.51\end{array}$ & $\begin{array}{l}.52 \\
.52\end{array}$ & $\begin{array}{l}.53 \\
.53\end{array}$ & $\begin{array}{l}2.3 \\
2.3\end{array}$ & $\begin{array}{l}.040 \\
.040\end{array}$ \\
\hline
\end{tabular}

283018096275500 LINE $20051 T E \quad 05$

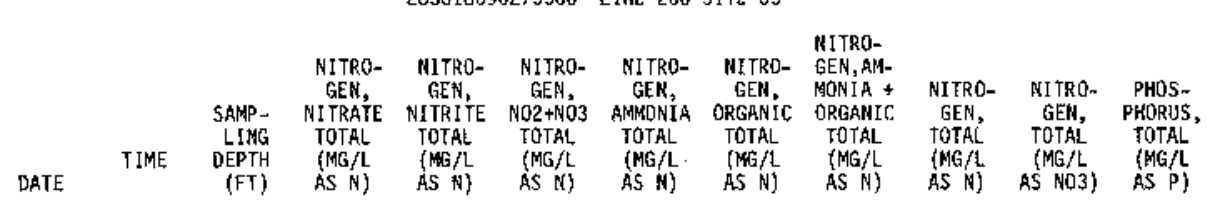

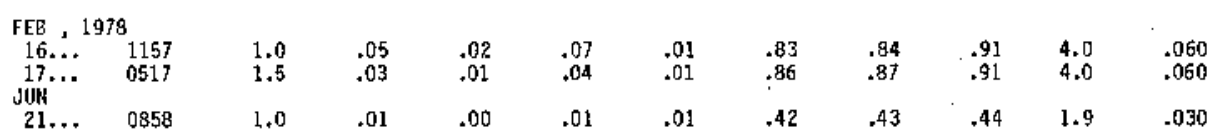

28415309624000 LINE 235 SITE 02

\begin{tabular}{|c|c|c|c|c|c|c|c|c|c|c|c|c|}
\hline DATE & TIME & $\begin{array}{l}\text { SAMP- } \\
\text { LIMG } \\
\text { OEPTH } \\
\text { (FT) }\end{array}$ & $\begin{array}{l}\text { OXYGEN } \\
\text { DEMAND, } \\
\text { BIOCHEN } \\
\text { UNINHIB } \\
5 \text { DAY } \\
\text { (MG } / L \text { ) }\end{array}$ & $\begin{array}{l}\text { NITRO- } \\
\text { GEN, } \\
\text { NITRATE } \\
\text { TOTAL } \\
\text { (MG } / \mathrm{L} \\
\text { AS N) }\end{array}$ & $\begin{array}{l}\text { NITRO- } \\
\text { GER, } \\
\text { NITRITE } \\
\text { TOJAL } \\
\text { (MG } / L \\
\text { AS N) }\end{array}$ & $\begin{array}{c}\text { NITRO- } \\
\text { GEN, } \\
\text { NO2+NO3 } \\
\text { TOTAL } \\
\text { (MG/L } \\
\text { AS N) }\end{array}$ & $\begin{array}{c}\text { NITRO- } \\
\text { GEN, } \\
\text { AMMONIA } \\
\text { TOTAL } \\
\text { (MG / } \\
\text { AS N) }\end{array}$ & $\begin{array}{l}\text { KITRO- } \\
\text { GEN, } \\
\text { ORGANIC } \\
\text { TOFAL } \\
\text { (MG } / \text { L } \\
\text { AS N) }\end{array}$ & $\begin{array}{l}\text { NITRO- } \\
\text { GEN,AM- } \\
\text { MONIA + } \\
\text { ORGANIC } \\
\text { TOTAL } \\
\text { (MGAL } \\
\text { AS N) }\end{array}$ & $\begin{array}{l}\text { NITRO- } \\
\text { GEN, } \\
\text { TDTAL } \\
\text { (MG/L } \\
\text { AS N) }\end{array}$ & $\begin{array}{l}\text { NITRO- } \\
\text { GEN, } \\
\text { TOTALL } \\
\text { (MG/L, } \\
\text { AS NO3) }\end{array}$ & $\begin{array}{c}\text { PHOS- } \\
\text { PHORUS, } \\
\text { TOTAL } \\
\text { (MG } / L \\
\text { AS P) }\end{array}$ \\
\hline $\begin{array}{l}\text { EB }, \\
07 . \\
U N\end{array}$ & 7315 & 1.0 & 1.7 & .00 & .01 & .01 & .01 & .39 & .40 & .41 & 1.8 & . 060 \\
\hline $27 \ldots$ & 1245 & 1.0 & 2.7 & , & 01 & .00 & .03 & .81 & .84 & .84 & 3.7 & .090 \\
\hline $20 .$. & 0900 & 1.0 & 2.6 & .02 & .01 & .03 & .03 & .84 & .87 & .90 & 4.0 & .040 \\
\hline
\end{tabular}


284246096112800 LINE 264 SITE 02

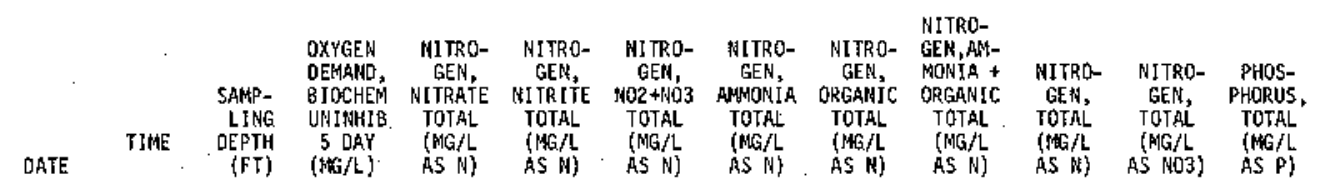

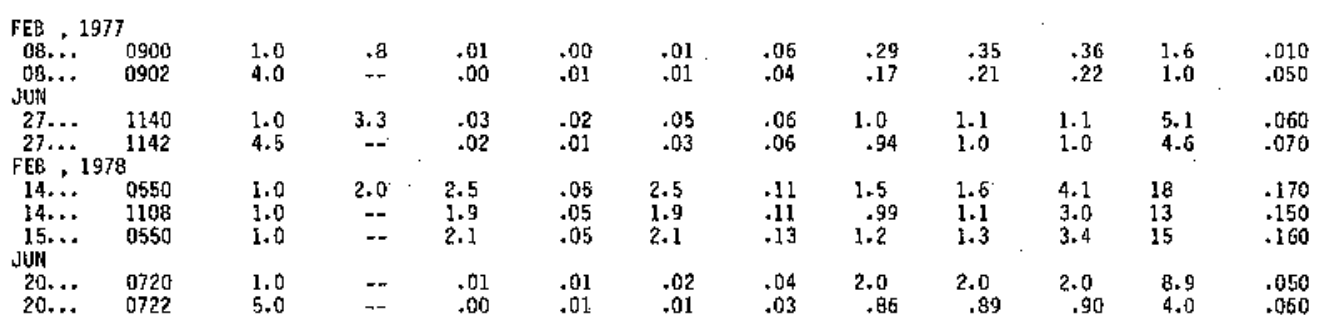

$283914096140600 \quad$ LINE 284 SITE 01

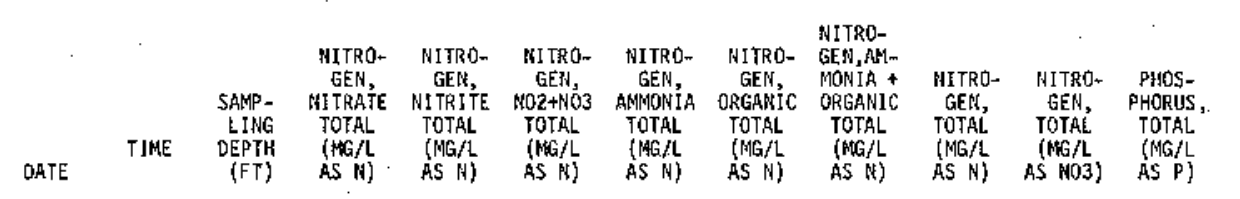

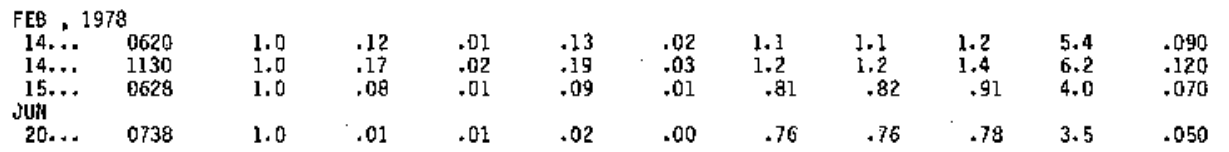

283816096170000 LINE 300 SITE 03

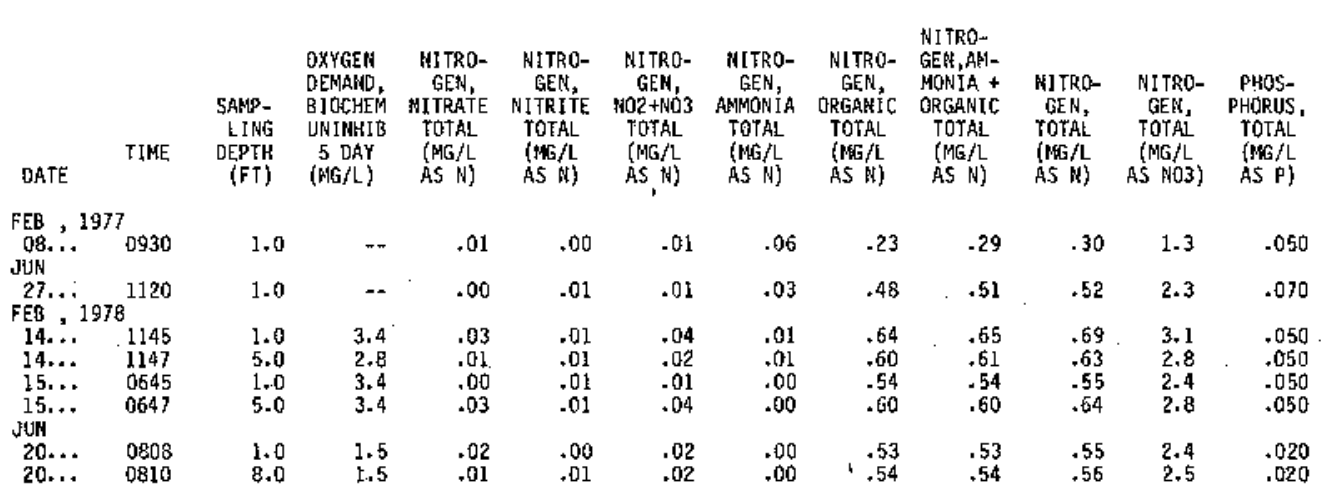


283608096011400 LINE 333 SITE 01

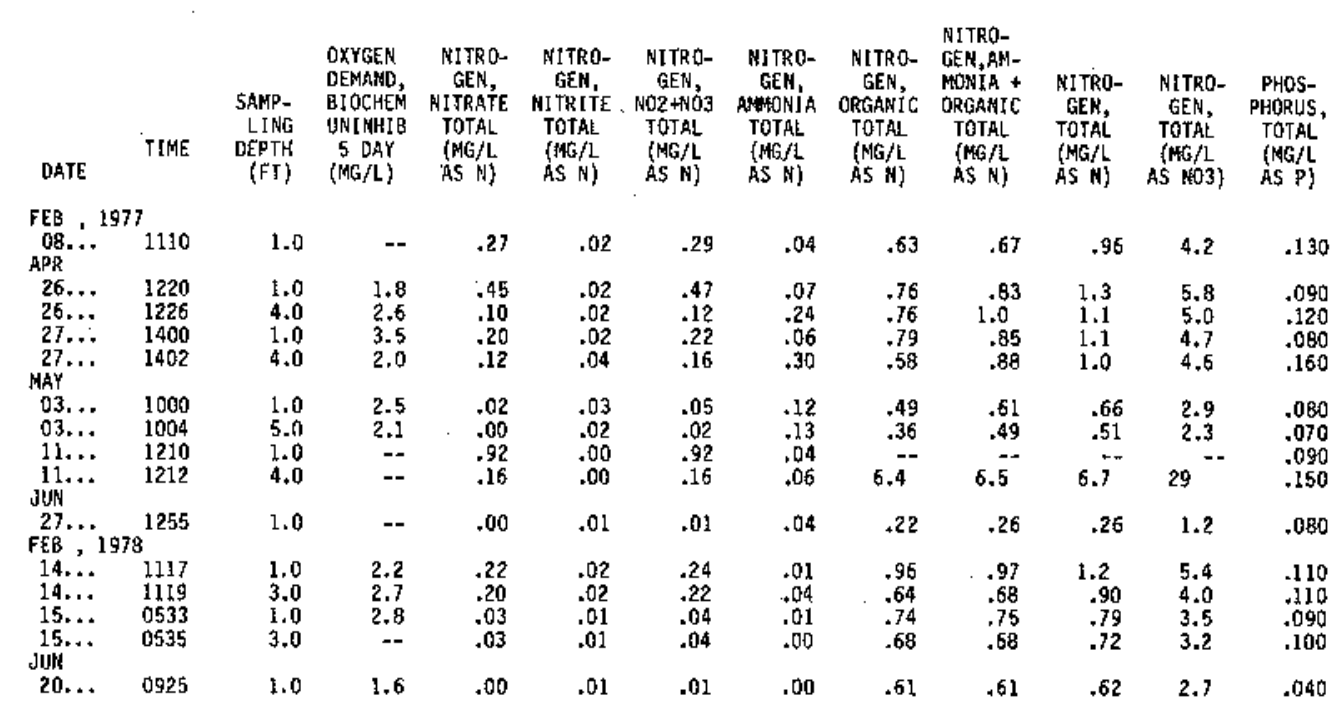

283749096015500 LINE 333 SITE 03

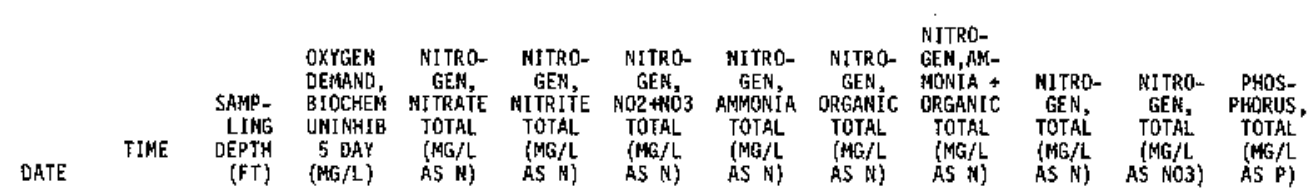

APR , 1977

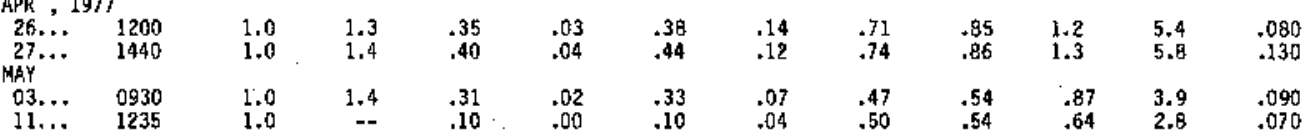

283518096034400 LINE 340 SITE OL

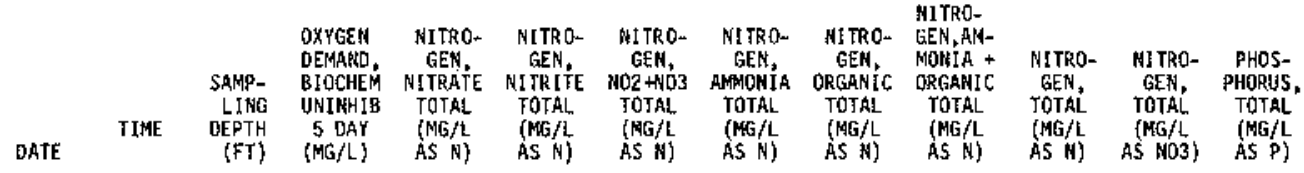

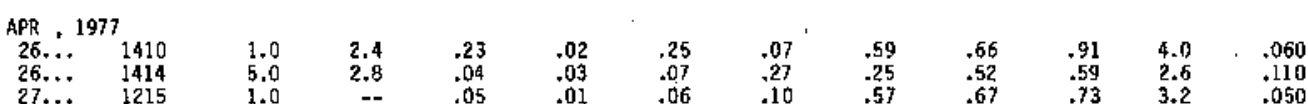

283704096044200 LINE 340 SITE 03

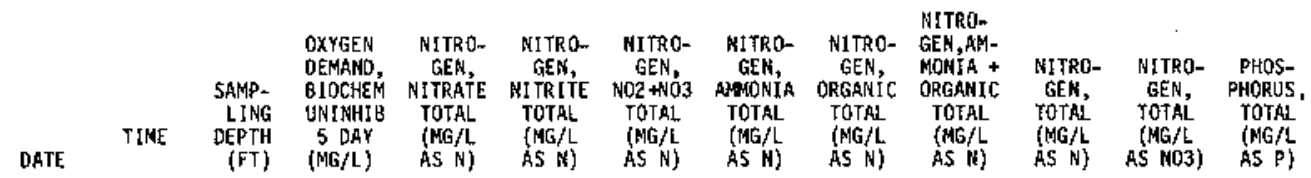

APR, 1977

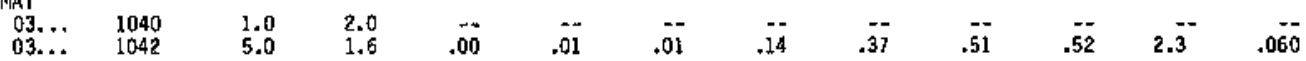


Table 6B.--Qual1ty of water in the Layaca-Tres Palacios estuary, water years 1977-78--Cont inued Mutrient Analyses--Cont inued

283327096080000 LINE 345 SITE OI

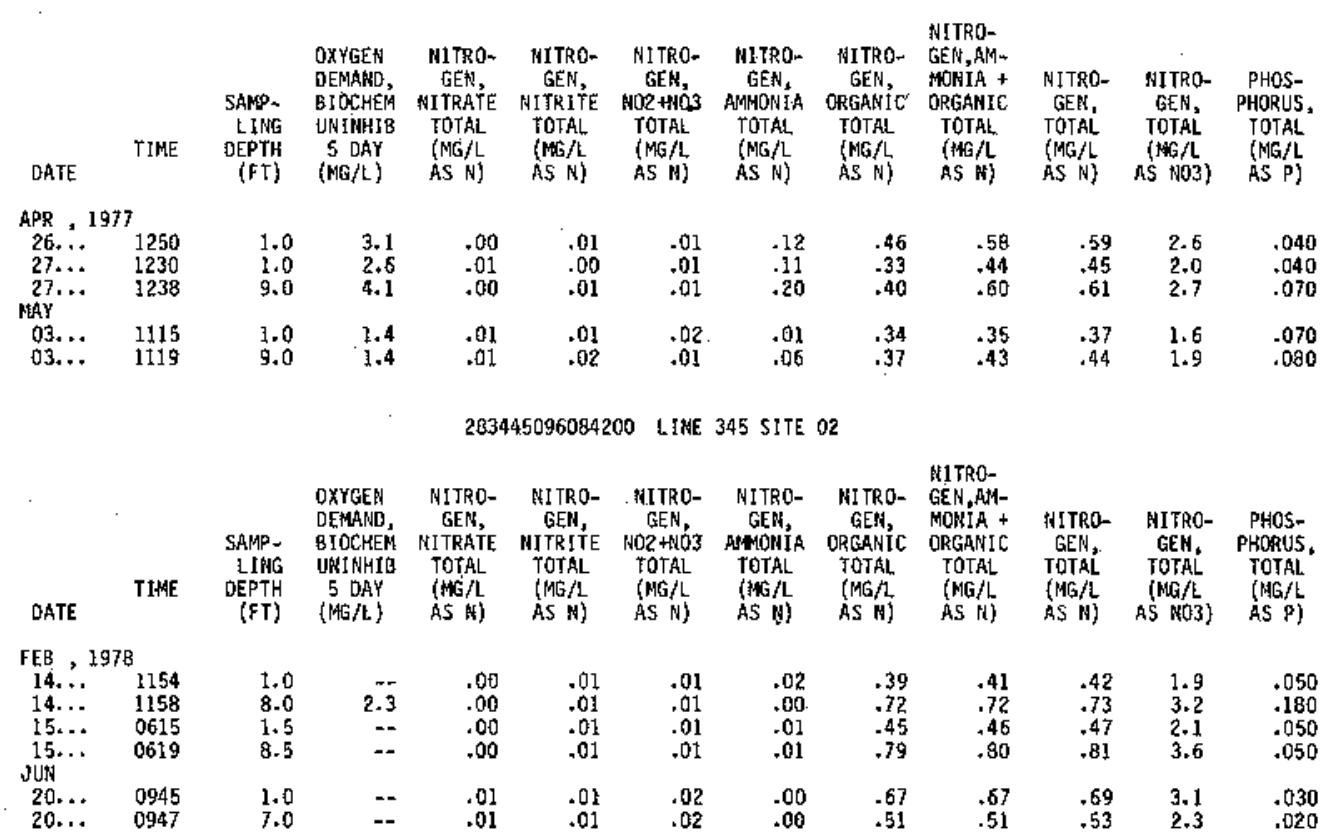

283557096092100 LINE 345 SITE 03

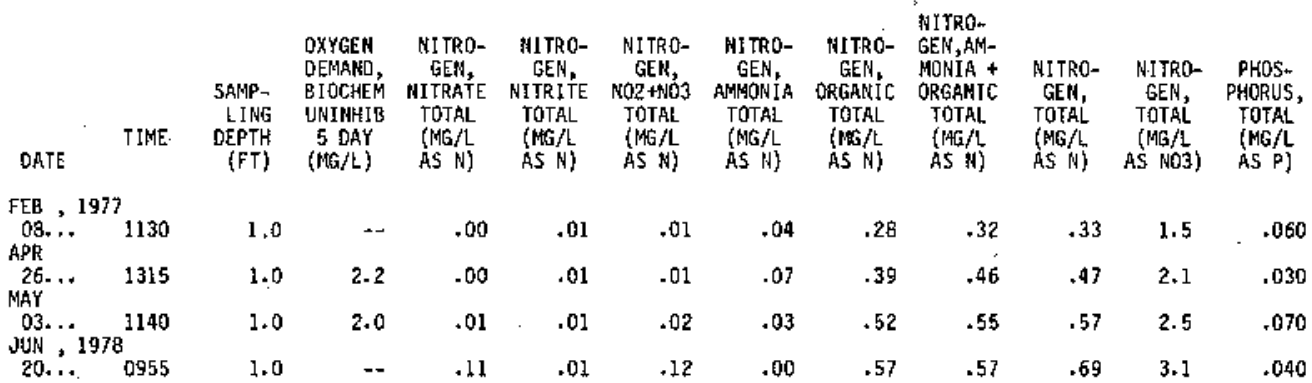

283353096125600 LINE 350 SITE 03

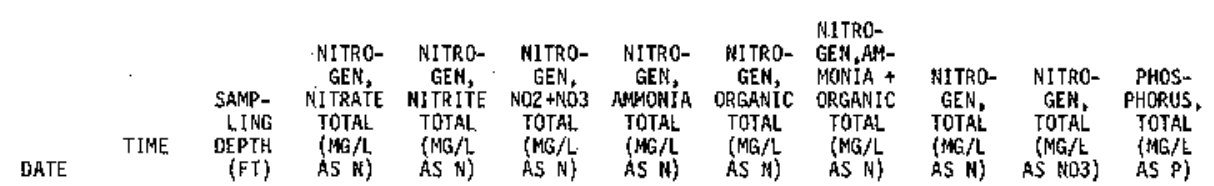

FEB , 1977

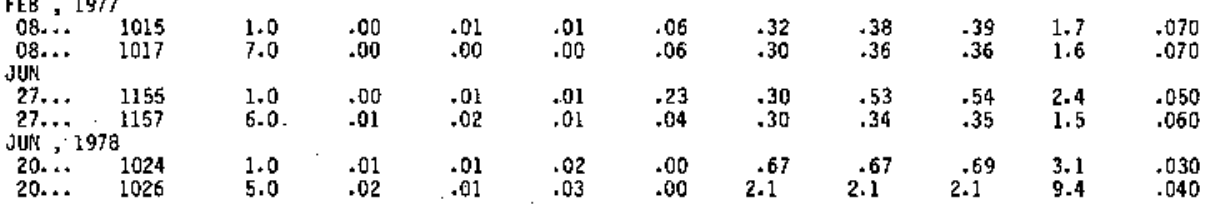

282928096144600 LINE 363 SITE 01

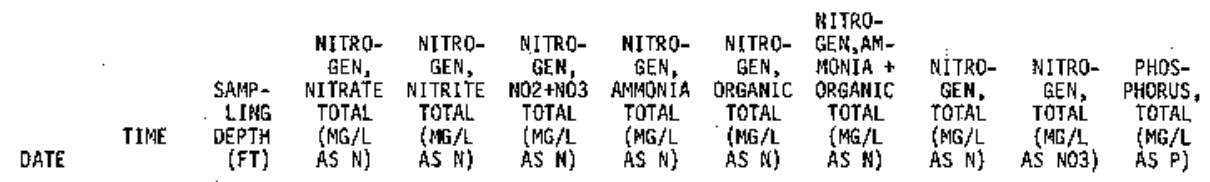

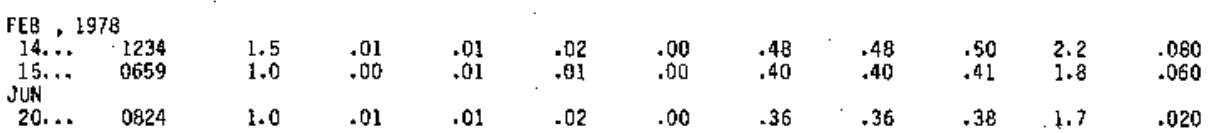


Table 6B.--Quality of water in the Lavaca-Tres Palacios estuary, water years 1977-78--Continued Nutrient Analyses*-Continued

283015096154300 LIRE 363 SITE 02

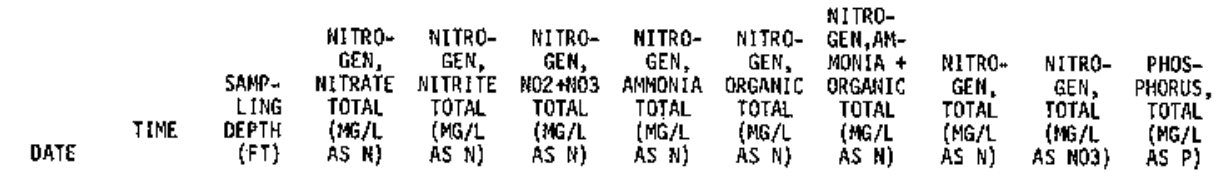

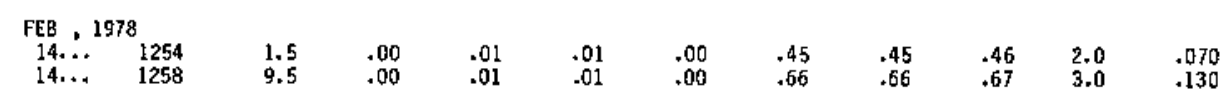

2B3135096170500 LINE 363 SITE 03

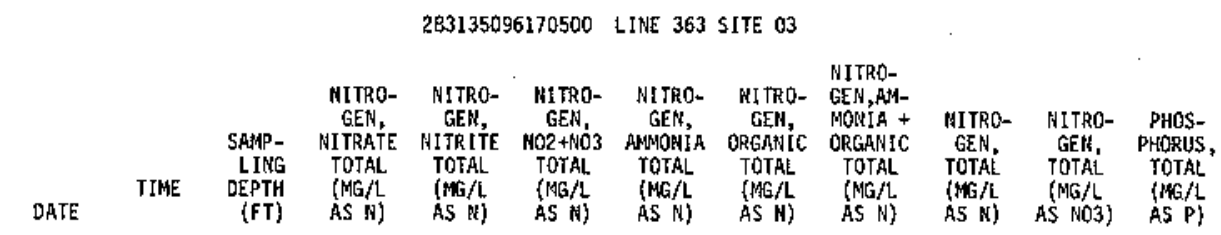

FEB, 1978

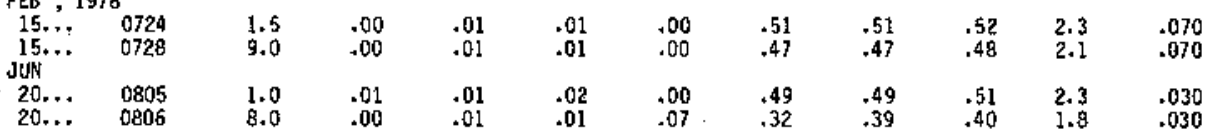

283432096194600 LINE 363 SITE 05

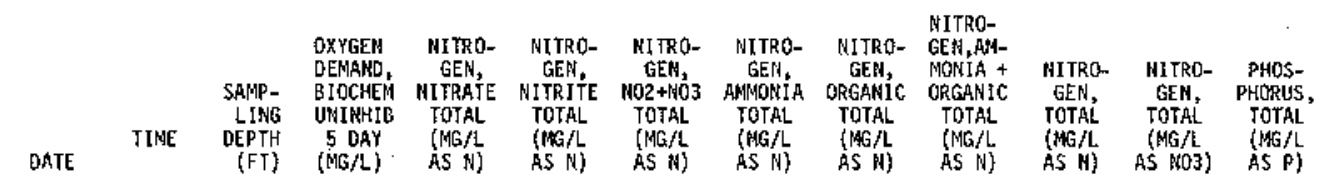

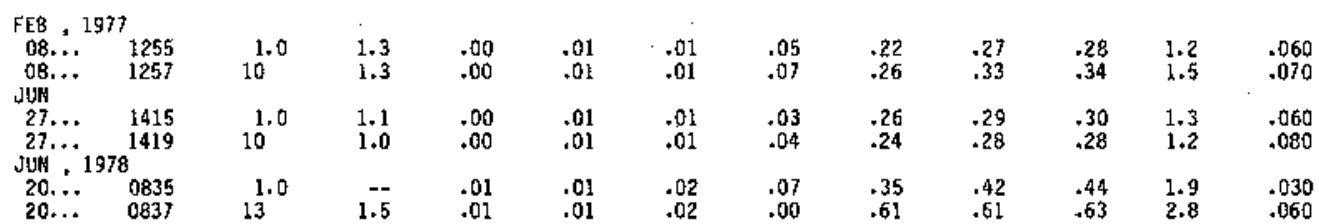

283611096211900 LINE 363 SITE 06

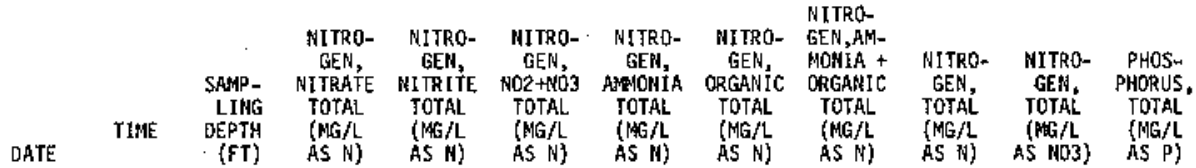

$\begin{array}{llllllllllll}\text { FEB }, 1978 & & & & & & & & & \\ 14 \ldots & 1205 & 1.0 & .05 & .01 & .06 & .01 & .53 & .54 & .60 & 2.7 & .050 \\ 14 \ldots & 1207 & 9.0 & .01 & .00 & .01 & .02 & .46 & .48 & .49 & 2.2 & .070 \\ 15 \ldots & 0705 & 1.0 & .01 & .00 & .01 & .01 & .49 & .50 & .51 & 2.3 & .050 \\ 15 \ldots & 0707 & 8.0 & .00 & .01 & .01 & .01 & .54 & .55 & .56 & 2.5 & .050\end{array}$

282943095204500 LJNE 375 SITE 02

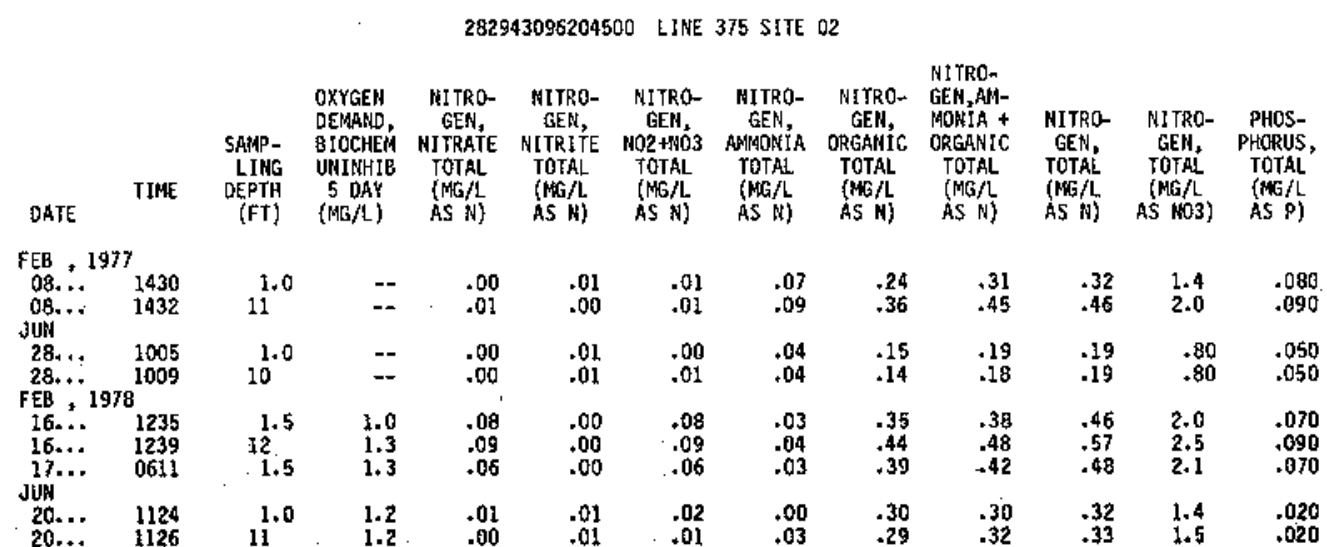


283430096272200 LINE 375 SITE 04

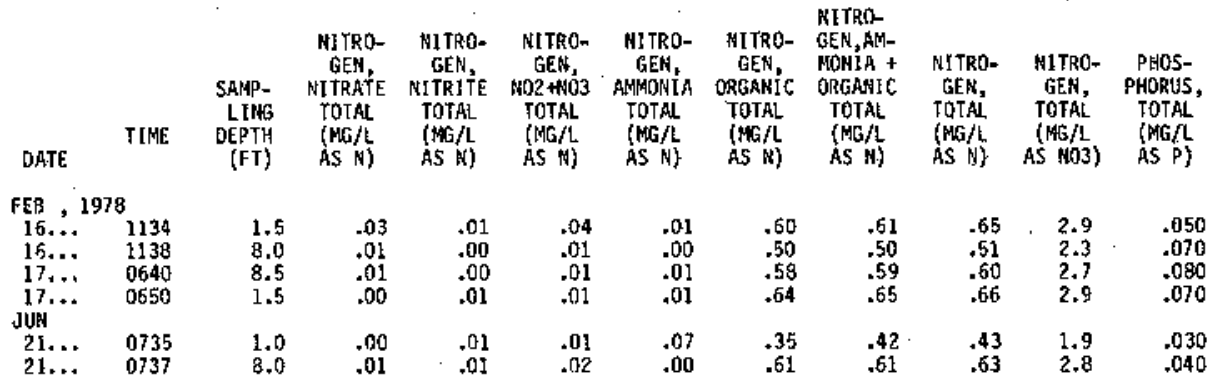

282516096230000 LINE 382 SITE 02

\begin{tabular}{|c|c|c|c|c|c|c|c|c|c|c|c|}
\hline DATE & TINAE & $\begin{array}{l}\text { SAMP- } \\
\text { LING } \\
\text { DEPTH } \\
\text { (FT) }\end{array}$ & $\begin{array}{l}\text { NITRO- } \\
\text { GEN, } \\
\text { NI TRATE } \\
\text { TOTAL } \\
\text { (MG/L } \\
\text { AS N) }\end{array}$ & $\begin{array}{l}\text { RITRO- } \\
\text { GEN, } \\
\text { N1TRITE } \\
\text { TOTAL } \\
\text { (MU/L } \\
\text { AS N) }\end{array}$ & $\begin{array}{c}\text { N1TRO- } \\
\text { GEN, } \\
\text { NO2+NO3 } \\
\text { TOTAL } \\
\text { (MG/L } \\
\text { AS N) }\end{array}$ & $\begin{array}{l}\text { MITRO- } \\
\text { GEN, } \\
\text { AIMWONIA } \\
\text { TOTAL } \\
\text { (MG/L } \\
\text { AS N). }\end{array}$ & $\begin{array}{c}\text { NITRO- } \\
\text { GEN, } \\
\text { ORGANIC } \\
\text { TOTAL } \\
\text { (MG/L } \\
\text { AS N) }\end{array}$ & $\begin{array}{l}\text { NITRO- } \\
\text { GEN,AHA- } \\
\text { MONIA + } \\
\text { ORGANIC } \\
\text { TOTAL } \\
\text { (WG/L } \\
\text { AS N) }\end{array}$ & $\begin{array}{l}\text { NITRO- } \\
\text { GEN, } \\
\text { TOTAL } \\
\text { (MG/L } \\
\text { AS N) }\end{array}$ & $\begin{array}{c}\text { NJTRO- } \\
\text { GEN, } \\
\text { TOTAL } \\
\text { (NG/L } \\
\text { AS N03) }\end{array}$ & $\begin{array}{c}\text { PHOS- } \\
\text { PHORUS, } \\
\text { TOTAL } \\
\text { (MG/L } \\
\text { AS P) }\end{array}$ \\
\hline \multicolumn{12}{|c|}{ FEB , 1977} \\
\hline $\begin{array}{c}08 . . \\
08 . .\end{array}$ & $\begin{array}{l}1545 \\
1549\end{array}$ & $15^{1.0}$ & .00 & $\begin{array}{l}.01 \\
.01\end{array}$ & .01 & $\begin{array}{l}.08 \\
.13\end{array}$ & $\begin{array}{r}.20 \\
.32\end{array}$ & $\begin{array}{r}.28 \\
.45\end{array}$ & $\begin{array}{l}.29 \\
.46\end{array}$ & $\begin{array}{l}1.3 \\
2.0\end{array}$ & $\begin{array}{l}.100 \\
.120\end{array}$ \\
\hline $\begin{array}{r}28 \ldots \\
28 \ldots \\
J \cup N\end{array}$ & ${ }_{8}^{1135}$ & $19^{1.0}$ & .00 & $\begin{array}{l}.01 \\
.01\end{array}$ & .01 & .03 & .08 & $\begin{array}{l}.00 \\
.14\end{array}$ & $\begin{array}{l}.01 \\
.15\end{array}$ & $\begin{array}{l}.00 \\
.70\end{array}$ & $\begin{array}{l}.030 \\
.050\end{array}$ \\
\hline $\begin{array}{r}21 \ldots \\
21 \ldots\end{array}$ & $\begin{array}{l}0940 \\
0942\end{array}$ & $\frac{1.0}{25}$ & $\begin{array}{l}.00 \\
.00\end{array}$ & .01 & $\begin{array}{l}.01 \\
.02\end{array}$ & $\begin{array}{l}.03 \\
.00\end{array}$ & $\begin{array}{l}.32 \\
.79\end{array}$ & $\begin{array}{l}.35 \\
.79\end{array}$ & $\begin{array}{l}.36 \\
.81\end{array}$ & $\begin{array}{l}1.6 \\
3.6\end{array}$ & $\begin{array}{l}.010 \\
.010\end{array}$ \\
\hline
\end{tabular}

282516096192300 LINE 397 SITE Q2

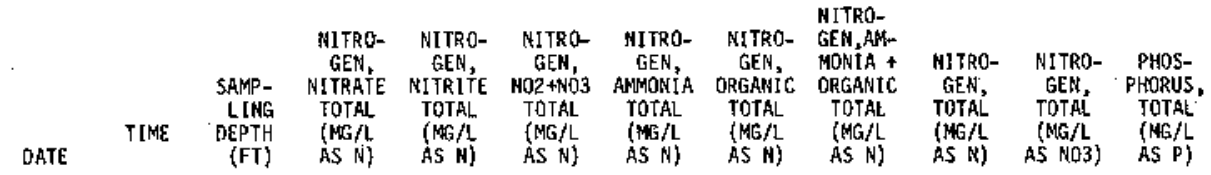

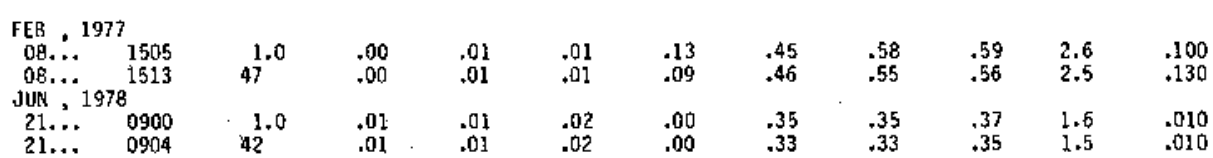

282400096243500 LINE 400 SITE 03

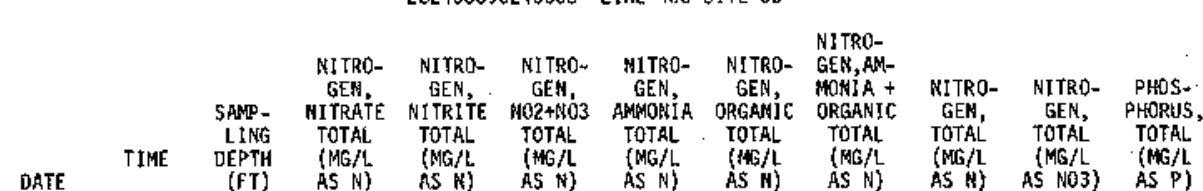

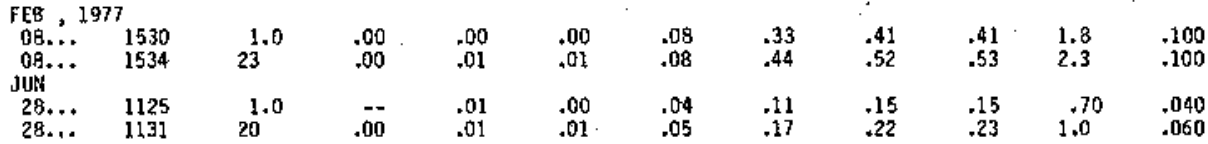

282330096174000 LINE 903 SITE 49

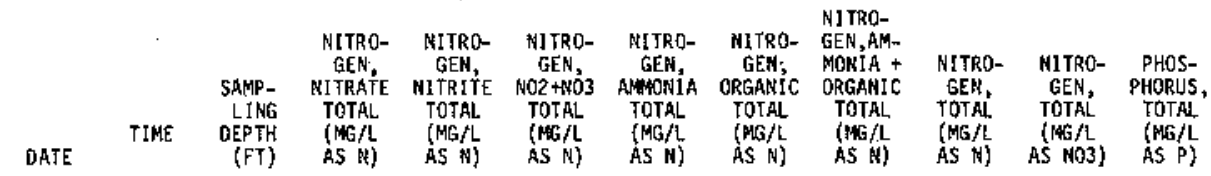

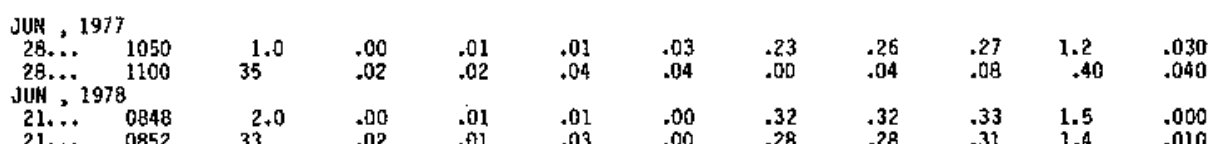


$285003096352400 \quad$ LINE 017 SIJE 02

\begin{tabular}{|c|c|c|c|c|c|c|c|c|c|c|}
\hline & TIME & $\begin{array}{l}\text { SAMP- } \\
\text { LING } \\
\text { DEPTH }\end{array}$ & $\begin{array}{l}\text { SPE- } \\
\text { CIFIC } \\
\text { CON- } \\
\text { OUCT- } \\
\text { ANCE } \\
\text { ONICRO- } \\
\text { MHOS }\end{array}$ & $\begin{array}{l}\text { MARD- } \\
\text { NESS } \\
\text { (1KG } / \mathrm{L} \\
\text { AS } \\
\text { A ACO3) }\end{array}$ & $\begin{array}{l}\text { HARD- } \\
\text { NESS, } \\
\text { NONCAR- } \\
\text { BONATE } \\
\text { (MG } f \text { L }\end{array}$ & $\begin{array}{l}\text { CALCIUM } \\
\text { OIS- } \\
\text { SOLVED } \\
\text { (MG/L }\end{array}$ & $\begin{array}{l}\text { MAGGE- } \\
\text { SIUM, } \\
\text { OIS- } \\
\text { SOL HED } \\
\text { (NGG } / \mathrm{L}\end{array}$ & $\begin{array}{l}\text { SODIUN, } \\
\text { DIS- } \\
\text { SOLVED } \\
\text { (MG/L }\end{array}$ & SODI IM & $\begin{array}{l}\text { SODIUS } \\
\text { AD- } \\
\text { SORP- } \\
\text { TION } \\
\text { RATIO }\end{array}$ \\
\hline DATE & & & & & & & AS MG) & RS NA) & PERCENT & \\
\hline
\end{tabular}
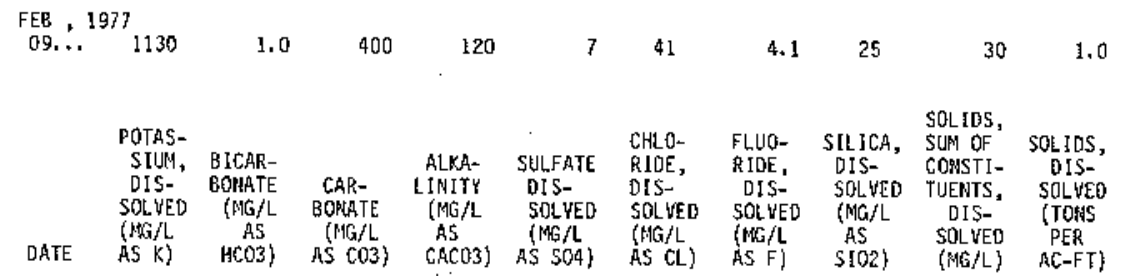
$\mathrm{FEB}, 1977$

$\begin{array}{lllllllllll}09 . . & 3.8 & 137 & 0 & 112 & 12 & 31 & .2 & 11 & 196 & .27\end{array}$

285248096344400 LINE 020 SITE 02

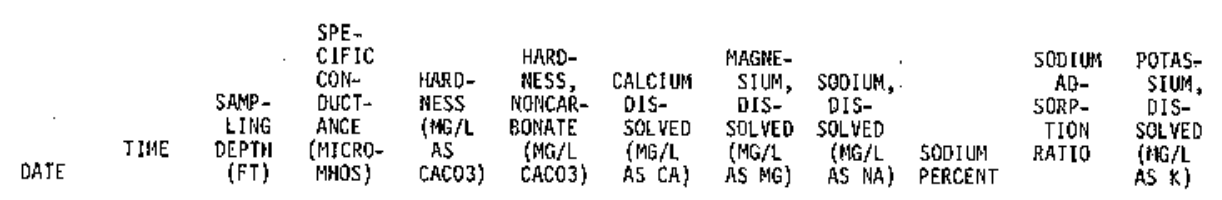

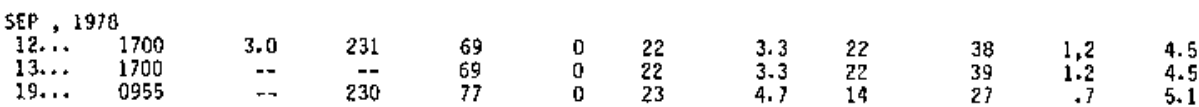

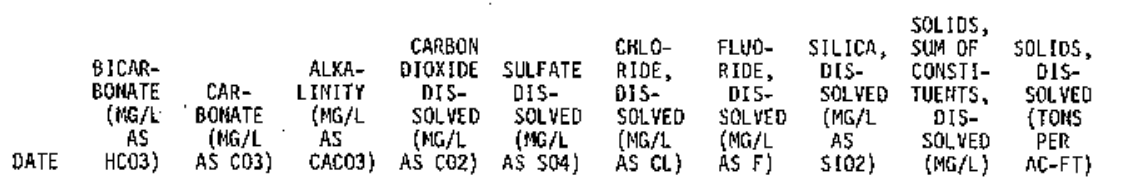

DATE HCO3) AS CO3) CACO3) AS CO2) AS SO4) AS CL) ASF) S(02) (MG (L) AC-FT)

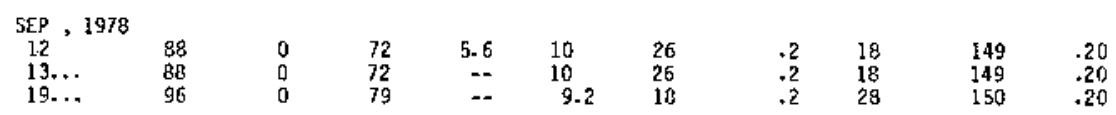

285223096343100 LJWE 022 SIJE Q2

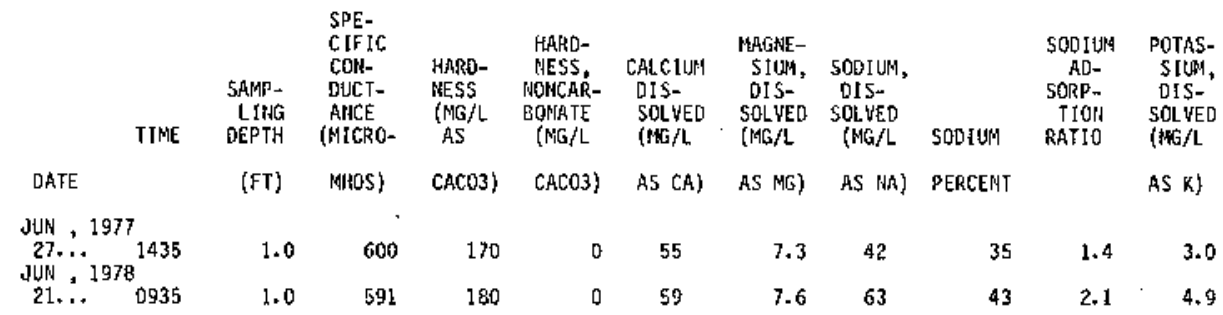

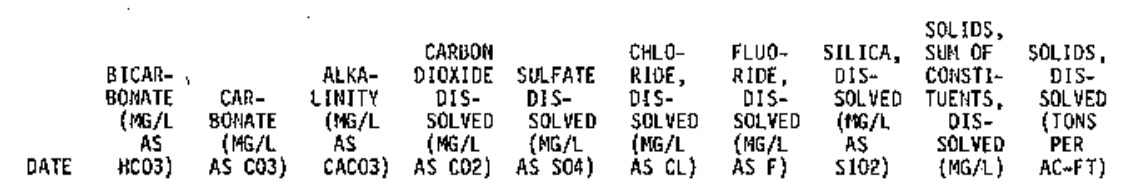
JUN, 1977

$\begin{array}{ccccccccccc}\text { JUN }, 1977 & & & & & & & & \\ 27 . . . & 220 & 0 & 180 & -- & 15 & 61 & .2 & 22 & 314 & .43 \\ \text { JUN }, 1979 & 220 & 0 & 180 & 2.2 & 17 & 74 & .3 & 15 & 349 & .47 \\ 21 . . . & 220 & 0 & \end{array}$


284921096342100 LIAE 041 SITE 02

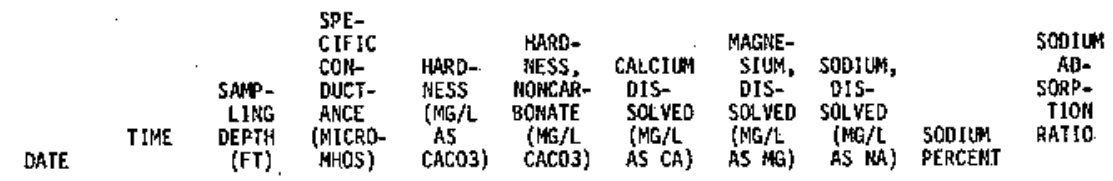

\begin{tabular}{ccccccccccc}
\multicolumn{2}{l}{ SEP , 1978} & & & & & & & \\
$12 . \ldots$ & 1238 & -- & 331 & 99 & 1 & 33 & 4.1 & 28 & 37 & 1.2 \\
$12 \ldots$ & 1622 & -- & 252 & 95 & 3 & 28 & 3.7 & 25 & 37 & 1.2 \\
$12 . .$. & 1900 & -- & 290 & 92 & 2 & 31 & 3.6 & 23 & 34 & 1.0 \\
$12 \ldots$ & 2315 &.- & 248 & 77 & 0 & 26 & 3.0 & 17 & 31 & .8 \\
$14 \ldots$ & 1430 & -- & 160 & 51 & 0 & 17 & 2.0 & 8.2 & 24 & .5 \\
$14 \ldots$ & 1824 & -- & 155 & 50 & 0 & 17 & 1.9 & 7.8 & 24 & .5 \\
$14 . \ldots$ & 2230 & -- & 150 & 49 & 0 & 16 & 2.1 & 9.2 & 27 & .6 \\
$15 \ldots$ & 0755 & -- & 135 & 49 & 0 & 16 & 2.1 & 7.1 & 23 & .4 \\
$15 \ldots$ & 1545 & -- & 113 & 42 & 0 & 14 & 1.7 & 5.8 & 22 & .4
\end{tabular}

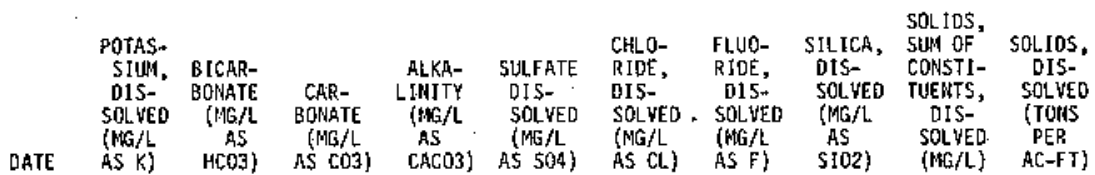

$\begin{array}{crr}\text { SEP . } 1978 & \\ 12 . . . & 4.6 & 120 \\ 12 \ldots & 4.5 & 100 \\ 12 \ldots & 4.5 & 110 \\ 12 \ldots & 4.3 & 96 \\ 14 \ldots & 3.6 & 64 \\ 14 \ldots & 3.6 & 64 \\ 14 \ldots . & 3.7 & 64 \\ 15 \ldots & 3.3 & 60 \\ 15 . . & 3.0 & 5\end{array}$

284200096374500 LINE 085 SITE 03

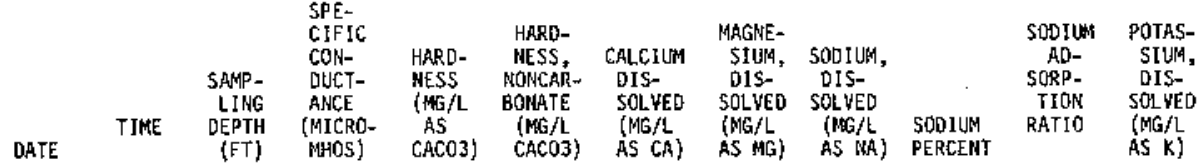

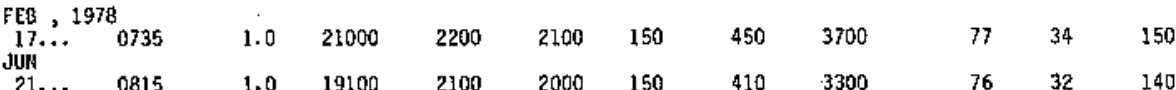

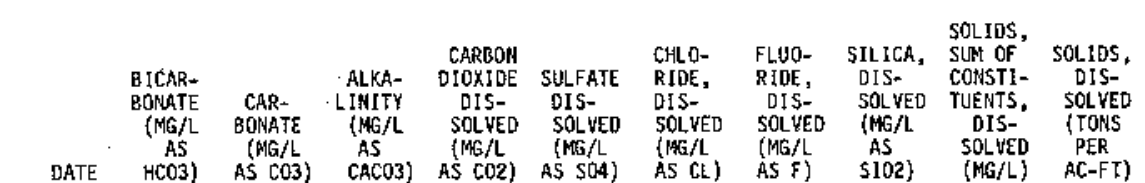

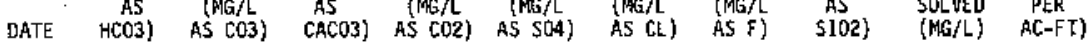

$$
\begin{array}{lrrrrrrrrrr}
\text { FEB } \\
\underset{\text { JUN }}{17 . .} & 100 & 0 & 92 & .2 & 890 & 6600 & .5 & .3 & 12000 & 16.3 \\
21 & 130 & 0 & 107 & 2.1 & 770 & 5900 & .5 & 8.3 & 10700 & 14.6
\end{array}
$$

284153096240000 LITAE 235 SITE 02

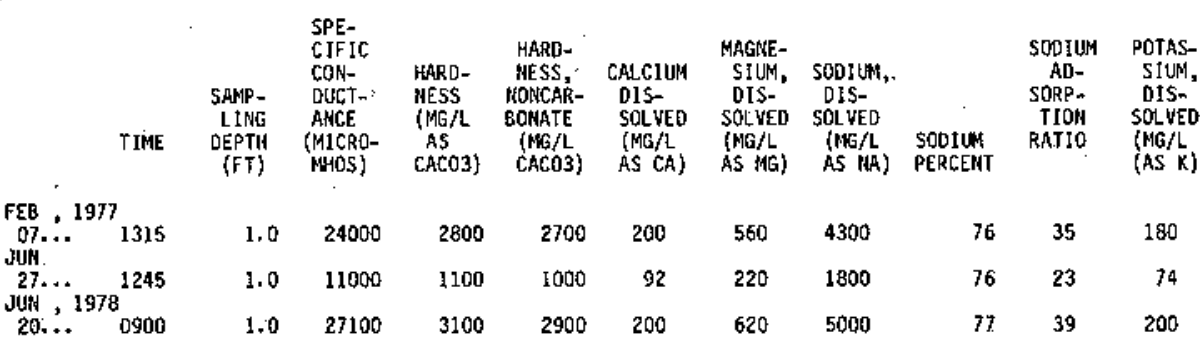


284153096240000 LINE 235 SIJE 02--Continued

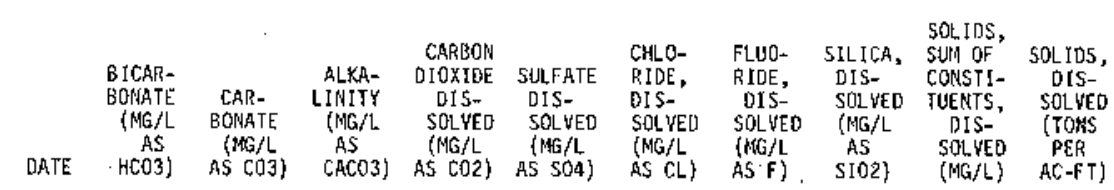

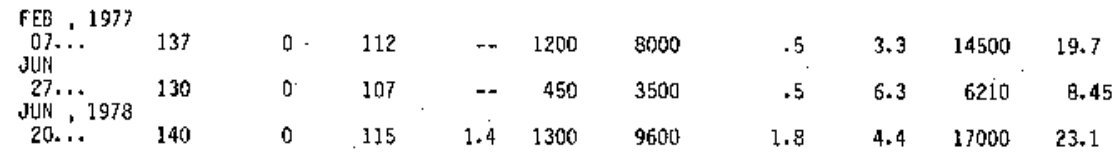

284246096112800 LINE 264 SLTE O2

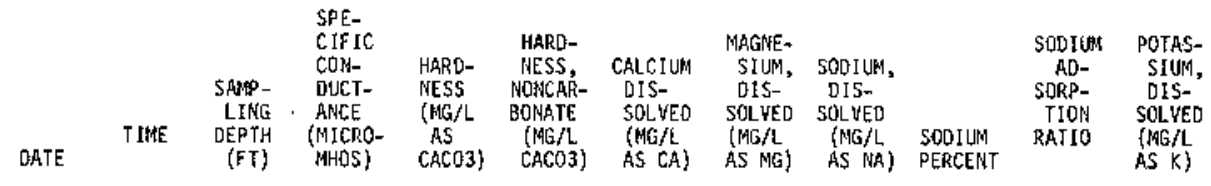

\begin{tabular}{|c|c|c|c|c|c|c|c|c|c|c|c|}
\hline \multicolumn{12}{|c|}{ FEE , 1978} \\
\hline $\begin{array}{l}14 \ldots \\
14 \ldots \\
15 \ldots\end{array}$ & $\begin{array}{l}0550 \\
1108 \\
0550\end{array}$ & $\begin{array}{l}1.0 \\
1.0 \\
1.0\end{array}$ & $\begin{array}{l}11000 \\
19000 \\
13000\end{array}$ & $\begin{array}{l}1100 \\
2000 \\
1400\end{array}$ & $\begin{array}{l}1100 \\
1900 \\
1300\end{array}$ & $\begin{array}{r}91 \\
140 \\
100\end{array}$ & $\begin{array}{l}220 \\
410 \\
270\end{array}$ & $\begin{array}{l}1800 \\
3300 \\
2200\end{array}$ & $\begin{array}{l}76 \\
77 \\
76\end{array}$ & $\begin{array}{l}23 \\
32 \\
26\end{array}$ & $\begin{array}{r}73 \\
130 \\
90\end{array}$ \\
\hline JUN & & & & & & & & & & & \\
\hline 20. & 0722 & 5. D & 31400 & 2500 & 2400 & 180 & 490 & 4500 & 18 & 39 & 190 \\
\hline
\end{tabular}

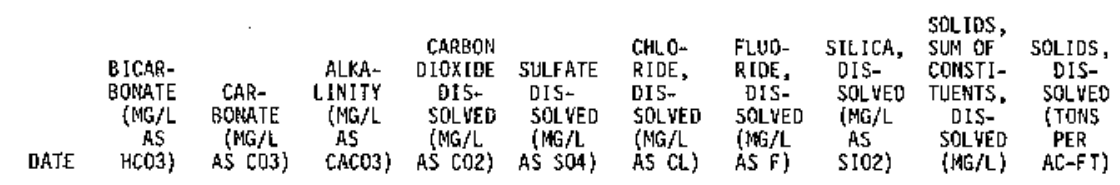

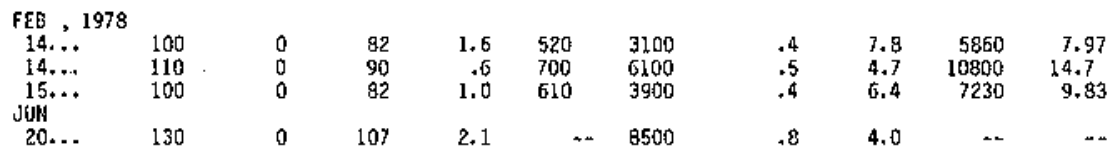

283608096011400 LIHE 333 SITE 01

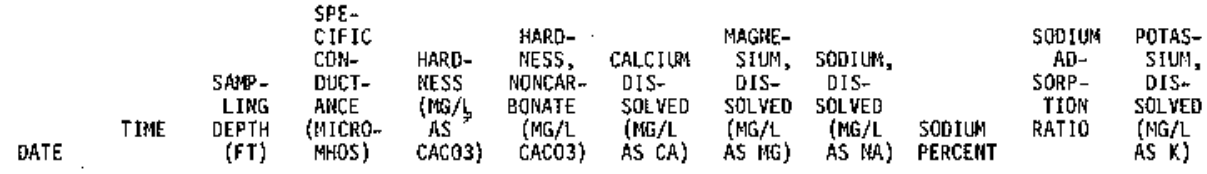

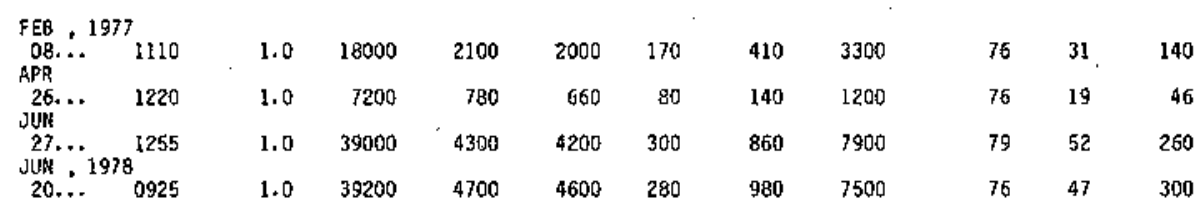

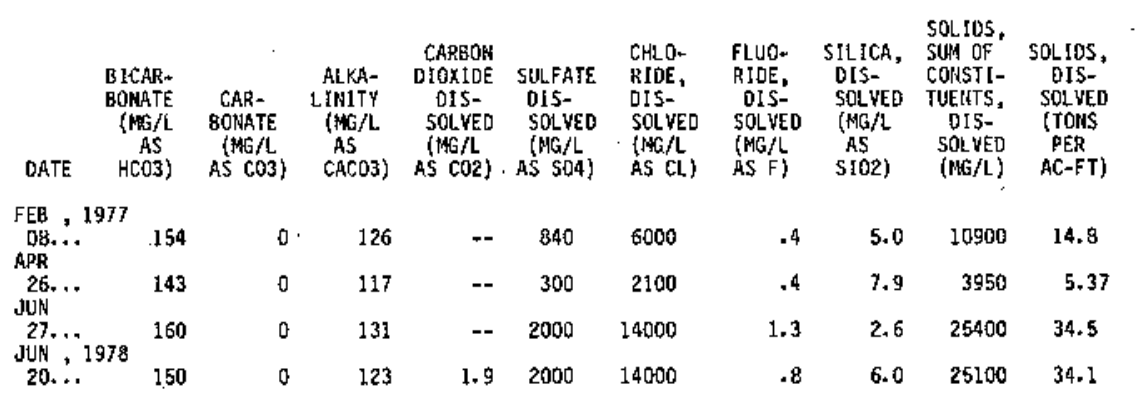


283432096194600 LINE 363 SITE 05

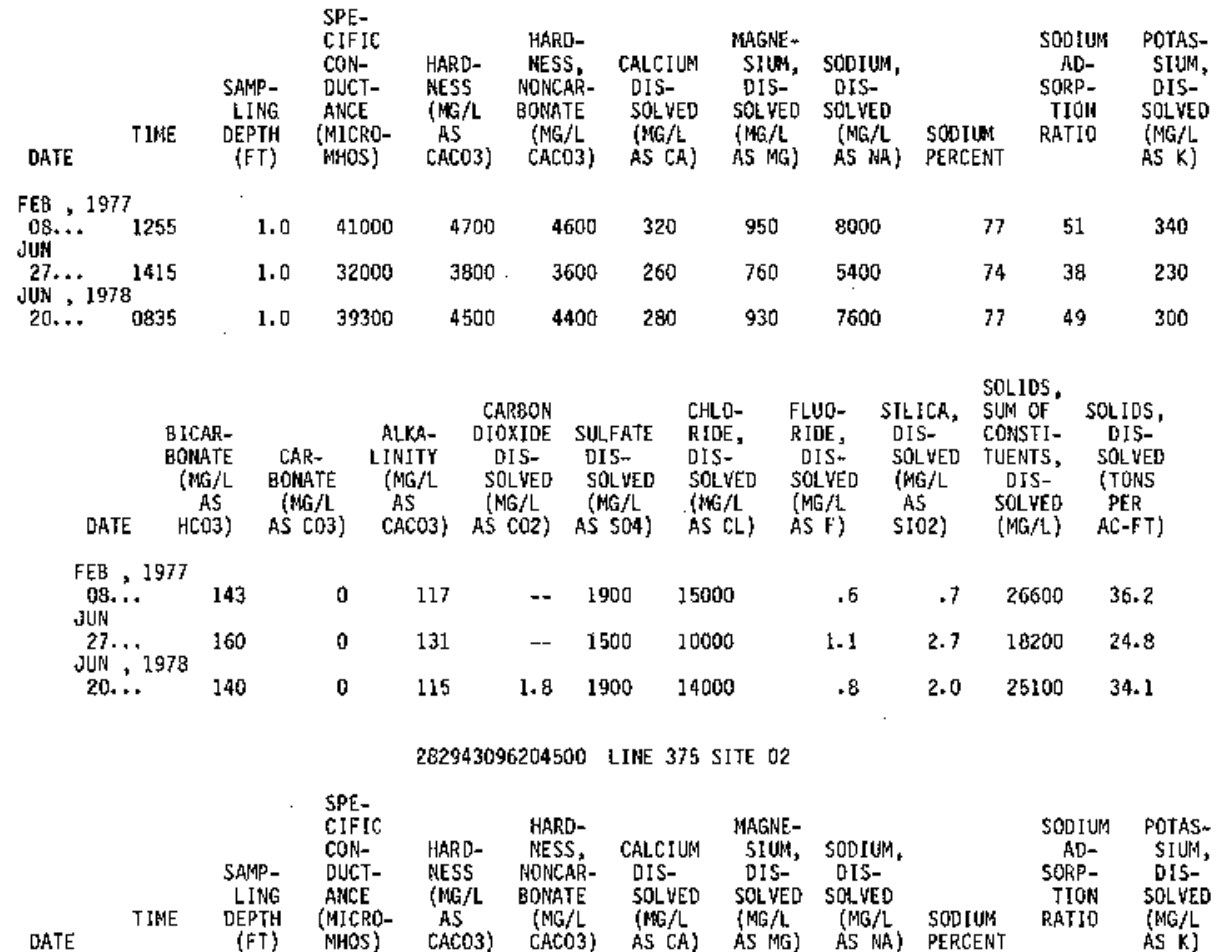

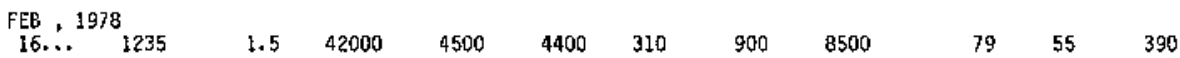

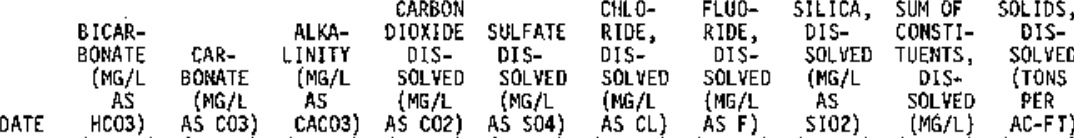

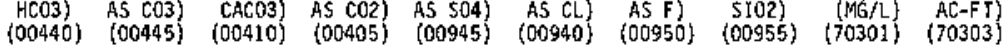

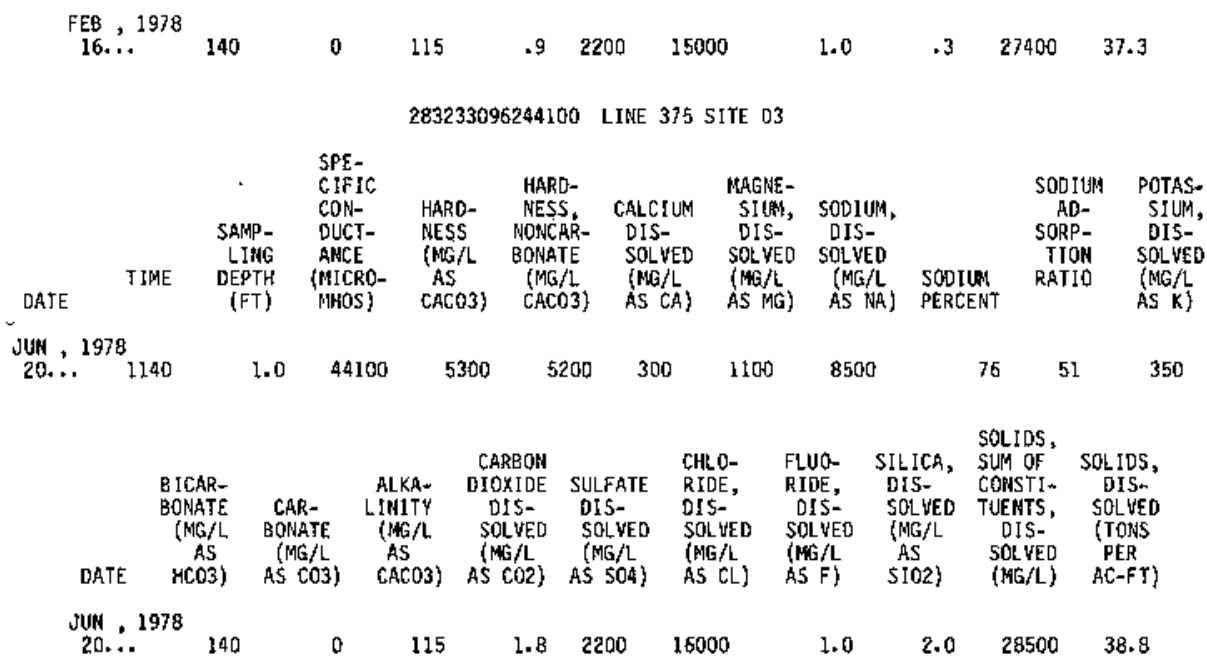




\section{Guadalupe Estuary}

The Guadalupe estuary, which has an area of about 210 square miles $\left(540 \mathrm{~km}^{2}\right)$, consists of the tidal parts of the Guadalupe River, Mission Lake, Guadalupe Bay, Hynes Bay, San Antonio Bay, Espiritu Santo Bay, Mesquite Bay, Victoria Channel, and part of the Intracoastal Waterway (Figure 8). At mean low water, the Guadalupe River is about 10 feet $(3.0 \mathrm{~m}$ ) deep; Mission Lake, Guadalupe Bay, and Hynes Bay are less than 3 feet $(1.0 \mathrm{~m})$ deep; San Antonio Bay is less than 6 feet $(1.8 \mathrm{~m})$ deep; Espiritu Santo Bay is about 8 feet $(2.4 \mathrm{~m})$ deep; Mesquite Bay is about 4 feet (1.2 $\mathrm{m})$ deep; Victoria Channel is more than 8 feet $(2.4 \mathrm{~m})$ deep; and the Intracoastal Waterway is about 15 feet $(4.6 \mathrm{~m})$ deep.

Water-quality data (Table 7) were collected during February and June 1977 and February and June 1978.

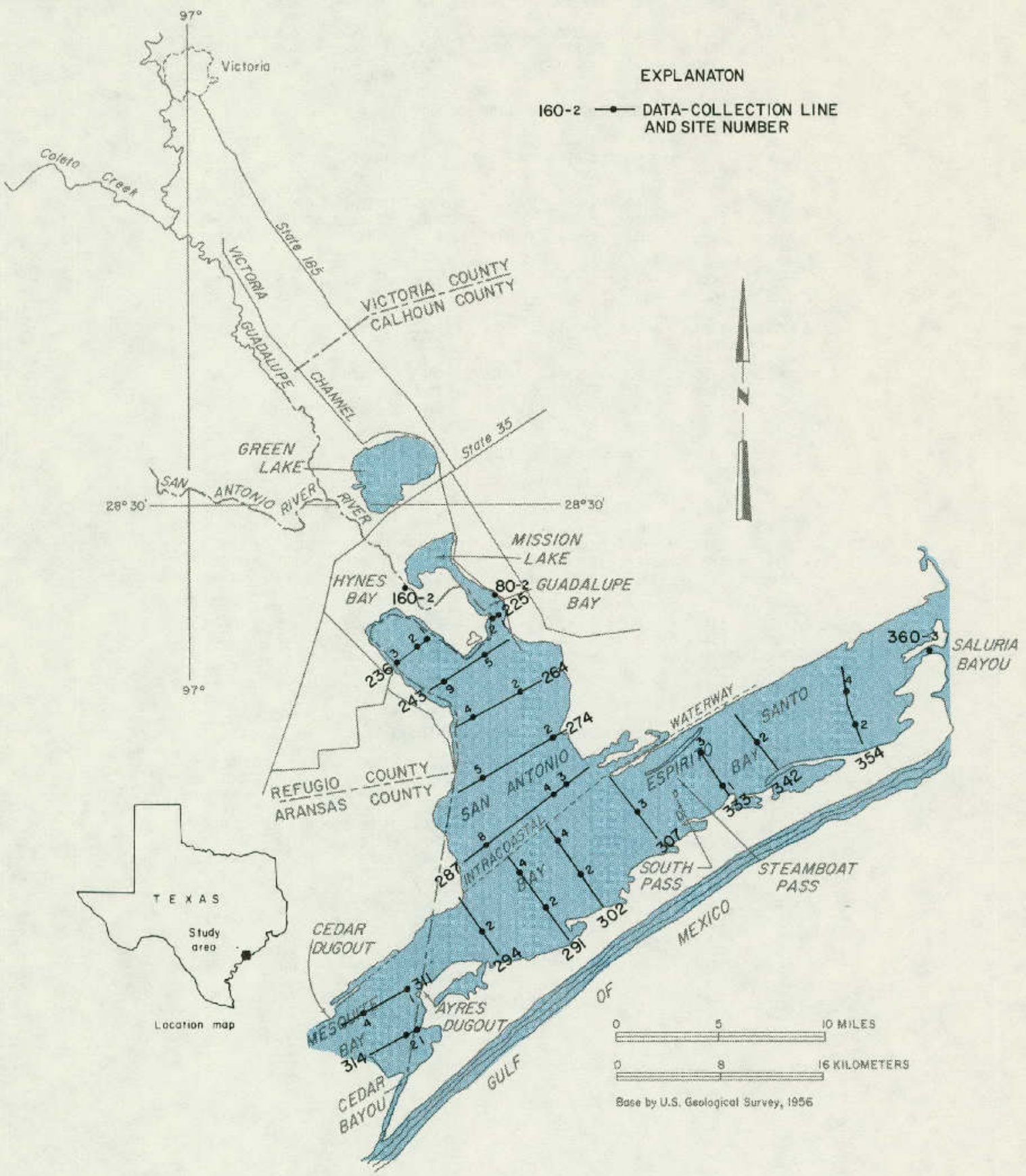

Figure 8.-Data-Collection Sites in the Guadalupe Estuary 
$\left(F T=\right.$ feet; MICROMHOS $=$ micromhos per centimeter at $25^{\circ}$ Celsius; $D E G C=$ degrees Celsius; $n=$ meters; $\mathrm{MG} / \mathrm{L}=$ milligrams per liter)

282644096455400 LINE 080 SITE 02

\begin{tabular}{|c|c|c|c|c|c|c|c|c|}
\hline DATE & TIME & $\begin{array}{c}\text { SAMP- } \\
\text { LING } \\
\text { DEPTH } \\
\text { (FT) }\end{array}$ & $\begin{array}{l}\text { SPE- } \\
\text { CIFIC } \\
\text { CON- } \\
\text { DUCT- } \\
\text { ANCE } \\
\text { (MICRO- } \\
\text { MHOS) }\end{array}$ & $\begin{array}{c}\text { PH } \\
\text { (UNITS) }\end{array}$ & $\begin{array}{l}\text { TEMPER- } \\
\text { ATURE } \\
\text { (DEG C) }\end{array}$ & $\begin{array}{l}\text { TRAIIS- } \\
\text { PAR- } \\
\text { ENCY } \\
\text { (SECCHI } \\
\text { DISK) } \\
\text { (M) }\end{array}$ & $\begin{array}{c}\text { OXYGEN, } \\
\text { DIS- } \\
\text { SOL VED } \\
\text { (MG/L) }\end{array}$ & $\begin{array}{l}\text { OXYGEN, } \\
\text { DIS- } \\
\text { SOLVED } \\
\text { (PER- } \\
\text { CENT } \\
\text { SATUR- } \\
\text { ATION) }\end{array}$ \\
\hline \multicolumn{9}{|c|}{ FEB , 1977} \\
\hline $09 . .$. & 1510 & 1.0 & 1400 & 8.2 & 11.0 & -. & 9.8 & \\
\hline & \\
\hline 28... & 1645 & 1.0 & 560 & 8.5 & 29.0 & .17 & 6.3 & 83 \\
\hline & 1647 & 11 & 630 & 8.3 & 29.0 & - & & \\
\hline \multicolumn{9}{|c|}{78} \\
\hline $22 \ldots$ & 1234 & 1.0 & 2400 & 8.0 & 30.0 & .15 & 6.9 & \\
\hline & & 1.0 & 2700 & & & $\cdots$ & & \\
\hline & & & & & & - & 6.7 & \\
\hline
\end{tabular}

\section{LINE 160 SITE 02}

\begin{tabular}{|c|c|c|c|c|c|c|c|c|}
\hline DATE & TIME & $\begin{array}{l}\text { SAMP- } \\
\text { LING } \\
\text { DEPTH } \\
(F T)\end{array}$ & $\begin{array}{l}\text { SPE- } \\
\text { CIFIC } \\
\text { CON- } \\
\text { DUCT- } \\
\text { ANCE } \\
\text { (MICRO- } \\
\text { MHOS) }\end{array}$ & $\begin{array}{c}\text { PH } \\
\text { (UNITS) }\end{array}$ & $\begin{array}{l}\text { TEMPER- } \\
\text { ATURE } \\
\text { (DEG C) }\end{array}$ & $\begin{array}{l}\text { TRANS- } \\
\text { PAR- } \\
\text { EIICY } \\
\text { (SECCHI } \\
\text { DISK) } \\
\text { (M) }\end{array}$ & $\begin{array}{c}\text { OXYGEN, } \\
\text { DIS- } \\
\text { SOL.VED } \\
\text { (MG/L) }\end{array}$ & $\begin{array}{c}\text { OXYGEN, } \\
\text { DIS-- } \\
\text { SOLVED } \\
\text { (PER- } \\
\text { CENT } \\
\text { SATUR- } \\
\text { ATION) }\end{array}$ \\
\hline \multicolumn{9}{|c|}{ FEB , 1977} \\
\hline $\begin{array}{l}09 . . \\
09 \ldots \\
\text { JUN }\end{array}$ & $\begin{array}{l}1545 \\
1547\end{array}$ & $10^{1.0}$ & $\begin{array}{l}800 \\
800\end{array}$ & $\begin{array}{l}7.9 \\
7.9\end{array}$ & $\begin{array}{l}12.0 \\
12.0\end{array}$ & -. & $\begin{array}{l}9.3 \\
9.5\end{array}$ & $\begin{array}{l}89 \\
91\end{array}$ \\
\hline $\begin{array}{l}28 \ldots \\
28 \ldots \\
\text { JUN , }\end{array}$ & $\begin{array}{l}1520 \\
1522 \\
8\end{array}$ & $14^{1.0}$ & $\begin{array}{l}650 \\
560\end{array}$ & $\begin{array}{l}8.1 \\
8.1\end{array}$ & $\begin{array}{l}30.0 \\
30.0\end{array}$ & $\begin{array}{l}.12 \\
--\end{array}$ & $\begin{array}{l}4.9 \\
4.5\end{array}$ & $\begin{array}{l}65 \\
60\end{array}$ \\
\hline $\begin{array}{l}22 \ldots \\
22 \ldots \\
22 \ldots\end{array}$ & $\begin{array}{l}1126 \\
1128 \\
1130\end{array}$ & $\begin{array}{l}1.0 \\
7.5 \\
15\end{array}$ & $\begin{array}{r}734 \\
734 \\
3410\end{array}$ & $\begin{array}{l}7.9 \\
7.9 \\
8.1\end{array}$ & $\begin{array}{l}30.5 \\
30.5 \\
31.0\end{array}$ & .20 & $\begin{array}{l}6.5 \\
6.6 \\
6.5\end{array}$ & $\begin{array}{l}87 \\
88 \\
89\end{array}$ \\
\hline
\end{tabular}

\section{LINE 225 SITE 01}

\begin{tabular}{|c|c|c|c|c|c|c|c|c|}
\hline DATE & TIME & $\begin{array}{l}\text { SAMP- } \\
\text { LING } \\
\text { DEPTH } \\
(F T)\end{array}$ & $\begin{array}{l}\text { SPE- } \\
\text { CIFIC } \\
\text { CON- } \\
\text { OUCT- } \\
\text { AHCE } \\
\text { (MICRO- } \\
\text { MHOS) }\end{array}$ & $\begin{array}{c}\text { PH } \\
\text { (UNITS) }\end{array}$ & $\begin{array}{l}\text { TEMPER- } \\
\text { ATURE } \\
\text { (DEG C) }\end{array}$ & $\begin{array}{l}\text { TRANS- } \\
\text { PAR- } \\
\text { ENCY } \\
\text { (SECCHI } \\
\text { DISK) } \\
\text { (M) }\end{array}$ & $\begin{array}{c}\text { OXYGEN, } \\
\text { OIS- } \\
\text { SOLVEO } \\
(M G / L)\end{array}$ & $\begin{array}{l}\text { OXYGEN, } \\
\text { DIS- } \\
\text { SCLVED } \\
\text { (PER- } \\
\text { CENT } \\
\text { SATUR- } \\
\text { ATION) }\end{array}$ \\
\hline \multicolumn{9}{|c|}{ JUN , 1977} \\
\hline $28 .$. & 1345 & 1.0 & 420 & 8.5 & 29.5 & .19 & 6.8 & \\
\hline & 1347 & 3.0 & 480 & 8.5 & 29.5 & - & 6.7 & 88 \\
\hline & 1038 & & 640 & & & .18 & 7.1 & \\
\hline & 1040 & 3.5 & 640 & 8.4 & 29.0 & $\ldots$ & 7.1 & 13 \\
\hline
\end{tabular}

282542096453400 LINE 225 SITE 02

\begin{tabular}{|c|c|c|c|c|c|c|c|c|}
\hline & TIME & $\begin{array}{r}\text { SAMP- } \\
\text { LING } \\
\text { DEPTH } \\
\text { (FT) }\end{array}$ & $\begin{array}{l}\text { SPE- } \\
\text { CIFIC } \\
\text { CON- } \\
\text { DUCT- } \\
\text { ARCE } \\
\text { (MICRO- } \\
\text { MHOS) }\end{array}$ & $\begin{array}{c}\text { PH } \\
\text { (UNITS) }\end{array}$ & $\begin{array}{l}\text { TEMPER- } \\
\text { ATURE } \\
\text { (DEG C) }\end{array}$ & $\begin{array}{l}\text { TRANS- } \\
\text { PAR- } \\
\text { ENCY } \\
\text { (SECCHI } \\
\text { DISK) } \\
\text { (M) }\end{array}$ & $\begin{array}{c}\text { OXYGEN, } \\
\text { DIS- } \\
\text { SOLVED } \\
\text { (MG/L) }\end{array}$ & $\begin{array}{l}\text { OXYGEN, } \\
\text { DIS- } \\
\text { SOLVED } \\
\text { (PER- } \\
\text { CENT } \\
\text { SATUR- } \\
\text { ATION) }\end{array}$ \\
\hline
\end{tabular}

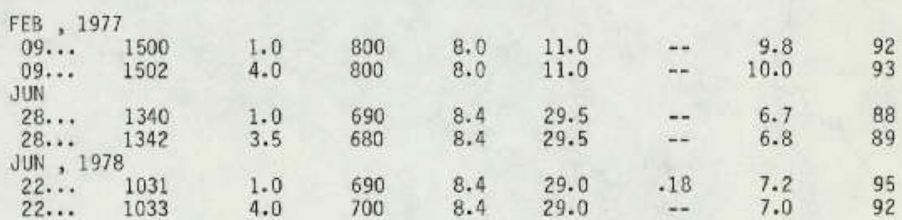


Table 7h.--Quality of water in the Guadalupe estuary, water years 1977-78--Continued Field Determinations $\rightarrow$ Cont inued

282423096493400 LINE 236 SITE 02

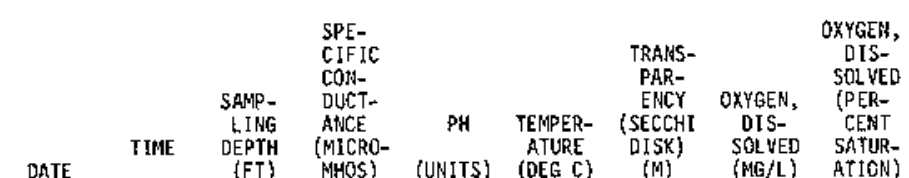

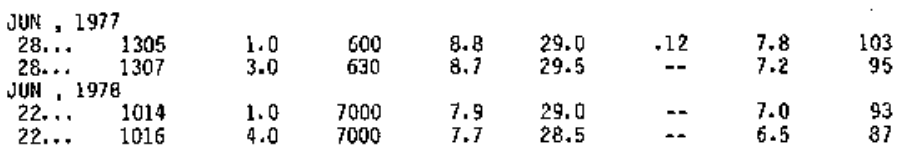

282404096452300 LINE 243 SITE OS

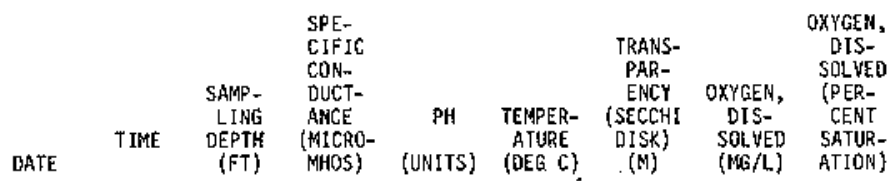

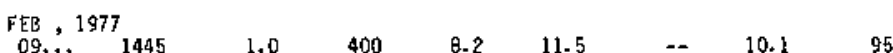

$\begin{array}{lllllllll}09 \ldots & 1445 & 1.0 & 400 & 8.2 & 11.5 & -- & 10.1 & 95 \\ 09 \ldots & 1447 & 4.0 & 400 & 8.2 & 11.5 & -- & 10.4 & 98\end{array}$

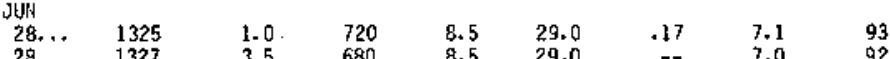

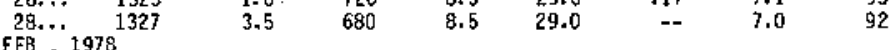

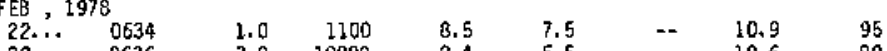

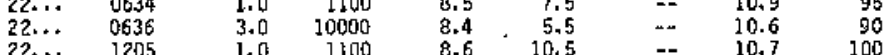

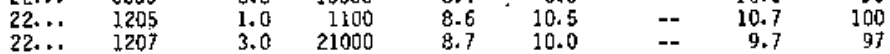

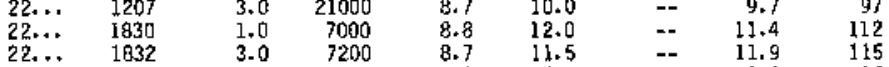

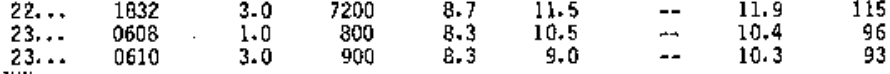

1904

21... 1904

$21 \ldots . . \quad 1906$

$22 \ldots . .0732$

$\begin{array}{ll}1.0 & 750 \\ 4.0 & 760 \\ 1.0 & 583\end{array}$

$\begin{array}{lllll}3.3 & .30 .0 & .12 & 7.4 & 99\end{array}$

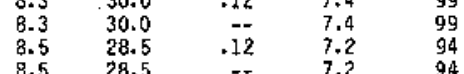

282254096474100 LINE 243 SITE 09

\begin{tabular}{|c|c|c|c|c|c|c|c|c|}
\hline DATE & T IME & $\begin{array}{l}\text { SAYP. } \\
\text { LING } \\
\text { DEPTH } \\
\text { (FT) }\end{array}$ & $\begin{array}{l}\text { SPE- } \\
\text { CIFIC } \\
\text { CON- } \\
\text { DUCT- } \\
\text { ANCE } \\
\text { (MICRO- } \\
\text { MHOS) }\end{array}$ & $\begin{array}{c}\mathrm{PH} \\
\text { (UNITS) }\end{array}$ & $\begin{array}{l}\text { TEHPER- } \\
\text { ATURE } \\
\text { (DEG C) }\end{array}$ & $\begin{array}{l}\text { TRANS- } \\
\text { PAR- } \\
\text { ENCY } \\
\text { (SECCHI } \\
\text { OISK) } \\
\text { (Y) }\end{array}$ & $\begin{array}{c}\text { OXYGEN, } \\
\text { DIS- } \\
\text { SOLWEO } \\
\text { (MG/L) }\end{array}$ & $\begin{array}{c}\text { OXYGEN, } \\
\text { DIS- } \\
\text { SOLVED } \\
\text { (PER- } \\
\text { CENT } \\
\text { SATUR- } \\
\text { ATION) }\end{array}$ \\
\hline
\end{tabular}

JU:, 1977

Ju․, 1977

$\begin{array}{lllllll}1.0 & 670 & 8.7 & 28.5 & .15 & 7.4 & 96\end{array}$

$\begin{array}{lllllllll}28 . & 1257 & 4.5 & 700 & 8.7 & 28.5 & - & 7.3 & 95\end{array}$

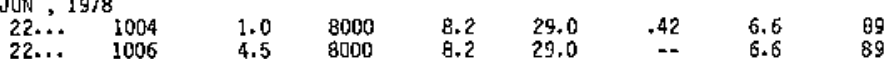

282256096442600 LINE 264 SITE 02

\begin{tabular}{|c|c|c|c|c|c|c|c|c|}
\hline DATE & TIME & $\begin{array}{l}\text { SAMP- } \\
\text { LING } \\
\text { DEPTH } \\
\text { (FT) }\end{array}$ & $\begin{array}{l}\text { SPE- } \\
\text { CIFIC } \\
\text { CON- } \\
\text { DUCT- } \\
\text { ANCE } \\
\text { (MICRD- } \\
\text { MHOS) }\end{array}$ & $\begin{array}{c}\mathrm{PH} \\
\text { (UNITS) }\end{array}$ & $\begin{array}{l}\text { TEMPER- } \\
\text { ATURE } \\
\text { (DEG C) }\end{array}$ & $\begin{array}{l}\text { TRANS- } \\
\text { PAR- } \\
\text { EACY } \\
\text { (SECCHI } \\
\text { DISK) } \\
\text { (M) }\end{array}$ & $\begin{array}{c}\text { OXYGEN, } \\
\text { DIS- } \\
\text { SOLVED } \\
\text { (IAG } / L)\end{array}$ & $\begin{array}{c}\text { OXYGEN, } \\
\text { DIS- } \\
\text { SOLVED } \\
\text { (PER- } \\
\text { CENT } \\
\text { SATUR- } \\
\text { ATION) }\end{array}$ \\
\hline \multicolumn{9}{|c|}{ Jun , 1977} \\
\hline $\begin{array}{l}28 \ldots \\
28 \ldots \\
\text { Juk . }\end{array}$ & $8^{1210}$ & $\begin{array}{l}1.0 \\
4.5\end{array}$ & $\begin{array}{l}1700 \\
1700\end{array}$ & $\begin{array}{l}8.7 \\
8.7\end{array}$ & $\begin{array}{l}28.0 \\
28.5\end{array}$ & $\begin{array}{r}.12 \\
-2\end{array}$ & $\begin{array}{l}7.2 \\
7.3\end{array}$ & $\begin{array}{l}94 \\
95\end{array}$ \\
\hline $\begin{array}{l}22 \ldots . \\
22 \ldots\end{array}$ & $\begin{array}{l}0947 \\
0949\end{array}$ & $\begin{array}{l}1.0 \\
5.0\end{array}$ & $\begin{array}{l}16000 \\
16000\end{array}$ & $\begin{array}{l}8.2 \\
8.2\end{array}$ & $\begin{array}{l}29.0 \\
29.0\end{array}$ & $\begin{array}{r}.55 \\
--\end{array}$ & $\begin{array}{l}6.7 \\
6.7\end{array}$ & $\begin{array}{l}93 \\
93\end{array}$ \\
\hline
\end{tabular}


Table 7A.--Quality of water in the Guadalupe estuary, water years 1977-78--Cont1nued Field Determinations-a Cont inued

282157096461700 LINE 264 SITE 04

\begin{tabular}{|c|c|c|c|c|c|c|c|c|}
\hline DATE & TIHE & $\begin{array}{c}\text { SAMP- } \\
\text { LING } \\
\text { DEPTH } \\
\text { (FT) }\end{array}$ & $\begin{array}{l}\text { SPE- } \\
\text { CIFIC } \\
\text { CON- } \\
\text { OUCT- } \\
\text { ANCE } \\
\text { (MICRO- } \\
\text { MHOS) }\end{array}$ & $\begin{array}{c}\mathrm{PH} \\
\text { (UNIJS) }\end{array}$ & $\begin{array}{l}\text { TEMPER- } \\
\text { ATURE } \\
\text { (DEG C) }\end{array}$ & $\begin{array}{c}\text { TRANS- } \\
\text { PAR- } \\
\text { EACY } \\
\text { (SECCHI } \\
\text { OISK) } \\
\text { (M) }\end{array}$ & $\begin{array}{c}\text { OXYGEA, } \\
\text { DIS- } \\
\text { SOLYED } \\
\text { (WG }\end{array}$ & $\begin{array}{c}\text { OXYGEN, } \\
\text { DIS- } \\
\text { SOLVED } \\
\text { (PER- } \\
\text { CENT } \\
\text { SATUR- } \\
\text { ATLON) }\end{array}$ \\
\hline
\end{tabular}

FEB , 1977

\begin{tabular}{|c|c|c|c|c|c|c|c|c|}
\hline $\begin{array}{c}09 . . \\
09 . . \\
\text { Jun }\end{array}$ & $\begin{array}{l}1410 \\
1412\end{array}$ & $\begin{array}{l}1.0 \\
5.0\end{array}$ & $\begin{array}{l}1100 \\
1100\end{array}$ & $\begin{array}{l}9.2 \\
8.2\end{array}$ & $\begin{array}{l}12.0 \\
11.0\end{array}$ & $\because$ & $\begin{array}{r}10.0 \\
9.9\end{array}$ & $\begin{array}{l}96 \\
93\end{array}$ \\
\hline $\begin{array}{r}28 . \\
28 . \\
\text { FEB }\end{array}$ & $\begin{array}{l}1245 \\
1247 \\
8\end{array}$ & $\begin{array}{l}1.0 \\
4.5\end{array}$ & $\begin{array}{l}2000 \\
1900\end{array}$ & $\begin{array}{l}8.6 \\
8.6\end{array}$ & $\begin{array}{l}28.5 \\
28.5\end{array}$ & .12 & $\begin{array}{l}7.3 \\
7.2\end{array}$ & $\begin{array}{l}95 \\
94\end{array}$ \\
\hline $\begin{array}{l}22 . \\
22 . \\
22 . \\
22 . \\
22 . \\
22 . \\
23 . \\
23 . .\end{array}$ & $\begin{array}{l}0650 \\
0652 \\
1150 \\
1152 \\
1814 \\
1916 \\
0630 \\
0632\end{array}$ & $\begin{array}{l}1.0 \\
4.0 \\
1.0 \\
4.0 \\
1.0 \\
4.0 \\
1.0 \\
4.0\end{array}$ & $\begin{array}{l}22000 \\
24000 \\
21000 \\
23000 \\
23000 \\
23000 \\
25000 \\
25000\end{array}$ & $\begin{array}{l}8.8 \\
8.7 \\
9.1 \\
9.0 \\
9.2 \\
9.2 \\
9.0 \\
8.9\end{array}$ & $\begin{array}{r}7.5 \\
8.0 \\
9.5 \\
8.5 \\
10.5 \\
10.5 \\
9.5 \\
9.5\end{array}$ & $\begin{array}{l}-- \\
- \\
.52 \\
-- \\
-- \\
-- \\
-- \\
--\end{array}$ & $\begin{array}{l}10.8 \\
10.7 \\
14.2 \\
14.9 \\
14.8 \\
15.6 \\
13.3 \\
13.2\end{array}$ & $\begin{array}{l}102 \\
103 \\
141 \\
143 \\
150 \\
157 \\
133 \\
132\end{array}$ \\
\hline $\begin{array}{l}\text { JUN } \\
21 . . \\
21 . . \\
22 . . \\
22 . .\end{array}$ & $\begin{array}{l}1923 \\
1925 \\
0745 \\
0747\end{array}$ & $\begin{array}{l}1.0 \\
5.0 \\
1.0 \\
4.0\end{array}$ & $\begin{array}{l}12000 \\
12000 \\
10000 \\
10000\end{array}$ & $\begin{array}{l}8.3 \\
8.3 \\
8.3 \\
8.3\end{array}$ & $\begin{array}{l}30.5 \\
30.0 \\
28.5 \\
28.5\end{array}$ & $\begin{array}{r}.83 \\
-. \\
.45 \\
--\end{array}$ & $\begin{array}{l}7.3 \\
7.3 \\
6.8 \\
6.7\end{array}$ & $\begin{array}{r}101 \\
101 \\
91 \\
90\end{array}$ \\
\hline
\end{tabular}

\begin{tabular}{|c|c|c|c|c|c|c|c|c|}
\hline & & \multicolumn{2}{|c|}{282029096431300} & \multicolumn{3}{|c|}{ LINE 274 SITE 02} & \multirow{2}{*}{\multicolumn{2}{|c|}{$\begin{array}{c}\text { OXYGEN, } \\
\text { DIS- } \\
\text { SOLVED } \\
\text { (PER- } \\
\text { CENT } \\
\text { SATUR- } \\
\text { ATION) }\end{array}$}} \\
\hline DATE & TIME & $\begin{array}{c}\text { SAMP- } \\
\text { LING } \\
\text { DEPTH } \\
\text { (FT) }\end{array}$ & $\begin{array}{l}\text { SPE- } \\
\text { CIFIC } \\
\text { CON- } \\
\text { DUCT- } \\
\text { ANCE } \\
\text { (MICRO- } \\
\text { TAHOS) }\end{array}$ & $\begin{array}{c}\text { PH } \\
\text { (UNAITS) }\end{array}$ & $\begin{array}{c}\text { TEMPER- } \\
\text { ATURE } \\
\text { (DEG C) }\end{array}$ & $\begin{array}{l}\text { TRANS- } \\
\text { PAR- } \\
\text { ENCY } \\
\text { (SECCHI } \\
\text { DISK) } \\
\text { (M) }\end{array}$ & & \\
\hline \multicolumn{9}{|c|}{ JUK , 1977} \\
\hline $\begin{array}{l}28 . . \\
28 \ldots \\
F E B,\end{array}$ & $8^{1222}$ & $\begin{array}{l}1.0 \\
3.5\end{array}$ & $\begin{array}{l}2100 \\
2000\end{array}$ & $\begin{array}{l}8.7 \\
6.7\end{array}$ & $\begin{array}{l}28.5 \\
29.0\end{array}$ & $\begin{array}{r}.14 \\
--\end{array}$ & $\begin{array}{l}7.0 \\
7.0\end{array}$ & $\begin{array}{l}91 \\
92\end{array}$ \\
\hline $\begin{array}{l}22 \ldots \\
22 \ldots \\
22 \ldots \\
22 \ldots \\
22 \ldots \\
22 \ldots \\
23 \ldots \\
23 \ldots\end{array}$ & $\begin{array}{l}0725 \\
0727 \\
1105 \\
1106 \\
1748 \\
1750 \\
0704 \\
0706\end{array}$ & $\begin{array}{l}1.0 \\
4.0 \\
1.0 \\
3.0 \\
1.0 \\
2.0 \\
1.0 \\
3.0\end{array}$ & $\begin{array}{l}30000 \\
32000 \\
29000 \\
30000 \\
29000 \\
29000 \\
31000 \\
30000\end{array}$ & $\begin{array}{l}8.8 \\
8.3 \\
8.9 \\
8.8 \\
9.1 \\
9.0 \\
8.9 \\
8.9\end{array}$ & $\begin{array}{r}9.0 \\
9.0 \\
9.5 \\
9.0 \\
13.0 \\
13.0 \\
9.5 \\
9.5\end{array}$ & $\begin{array}{c}-- \\
-59 \\
- \\
-- \\
- \\
-\end{array}$ & $\begin{array}{l}12.1 \\
10.8 \\
13.9 \\
14.1 \\
14.5 \\
14.5 \\
12.2 \\
12.5\end{array}$ & $\begin{array}{l}118 \\
108 \\
142 \\
143 \\
159 \\
159 \\
126 \\
128\end{array}$ \\
\hline 3UN & & & & & & & & \\
\hline $\begin{array}{l}21 \ldots \\
21 \ldots \\
22 \ldots \\
22 \ldots\end{array}$ & $\begin{array}{l}2000 \\
2002 \\
0820 \\
0822\end{array}$ & $\begin{array}{l}1.0 \\
3.0 \\
1.0 \\
3.0\end{array}$ & $\begin{array}{l}26000 \\
27000 \\
27000 \\
26000\end{array}$ & $\begin{array}{l}8.1 \\
8.1 \\
8.1 \\
8.1\end{array}$ & $\begin{array}{l}29.5 \\
28.5 \\
28.5 \\
28.5\end{array}$ & $\begin{array}{r}.45 \\
.41 \\
.4\end{array}$ & $\begin{array}{l}6.6 \\
6.5 \\
6.5 \\
6.5\end{array}$ & $\begin{array}{l}97 \\
94 \\
92 \\
92\end{array}$ \\
\hline
\end{tabular}

281923096460100 LIRE 274 SITE 05

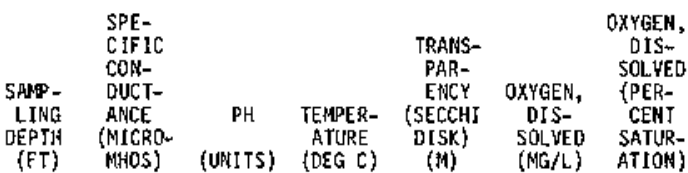

FEB, 1971

\begin{tabular}{|c|c|c|c|c|c|c|c|c|}
\hline $\begin{array}{ll}09 . . . & 1 \\
\text { JuN } & 1\end{array}$ & $\begin{array}{l}1425 \\
1427\end{array}$ & $\begin{array}{l}1.0 \\
3.0\end{array}$ & $\begin{array}{l}900 \\
900\end{array}$ & $\begin{array}{l}8.2 \\
8.2\end{array}$ & $\begin{array}{l}11.0 \\
11.5\end{array}$ & $=$ & $\begin{array}{l}9.8 \\
9.9\end{array}$ & $\begin{array}{l}92 \\
94\end{array}$ \\
\hline $\begin{array}{c}28 \ldots \ldots \\
28 \ldots, 1\end{array}$ & $\begin{array}{l}1235 \\
1237\end{array}$ & $\begin{array}{l}1.0 \\
4.5\end{array}$ & $\begin{array}{l}1700 \\
1800\end{array}$ & $\begin{array}{l}8.6 \\
8.6\end{array}$ & $\begin{array}{l}28.5 \\
28.5\end{array}$ & .16 & $\begin{array}{l}7.5 \\
7.4\end{array}$ & $\begin{array}{l}97 \\
96\end{array}$ \\
\hline $\begin{array}{cc}22 \ldots & 0 \\
22 \ldots & 0 \\
22 \ldots & 1 \\
22 \ldots & 1 \\
22 \ldots & 1 \\
22 \ldots & 1 \\
23 \ldots & 0 \\
23 \ldots & 0 \\
J \cup N & 0\end{array}$ & $\begin{array}{l}0705 \\
0707 \\
1130 \\
1132 \\
1800 \\
1802 \\
0648 \\
0650\end{array}$ & $\begin{array}{l}1.0 \\
6.0 \\
1.0 \\
7.0 \\
1.0 \\
6.5 \\
1.0 \\
6.0\end{array}$ & $\begin{array}{l}30000 \\
32000 \\
23000 \\
30000 \\
28000 \\
28000 \\
29000 \\
29000\end{array}$ & $\begin{array}{l}8.9 \\
8.9 \\
8.8 \\
8.4 \\
9.1 \\
9.0 \\
8.9 \\
8.9\end{array}$ & $\begin{array}{r}8.0 \\
7.5 \\
9.5 \\
9.0 \\
10.0 \\
10.5 \\
9.5 \\
9.5\end{array}$ & $\begin{array}{l}-- \\
.28 \\
-- \\
-- \\
-- \\
-\end{array}$ & $\begin{array}{l}11.4 \\
11.1 \\
12.7 \\
10.8 \\
12.0 \\
13.7 \\
12.2 \\
12.4\end{array}$ & $\begin{array}{l}111 \\
110 \\
126 \\
109 \\
123 \\
141 \\
124 \\
126\end{array}$ \\
\hline $\begin{array}{l}21 \ldots \\
21 \ldots \\
22 \ldots \\
22 \ldots\end{array}$ & $\begin{array}{l}1937 \\
1939 \\
0804 \\
0806\end{array}$ & $\begin{array}{l}1.0 \\
5.0 \\
1.0 \\
6.0\end{array}$ & $\begin{array}{l}17000 \\
17000 \\
16000 \\
16000\end{array}$ & $\begin{array}{l}8.1 \\
8.1 \\
8.1 \\
8.1\end{array}$ & $\begin{array}{r}30.5 \\
30.0 \\
29.0 \\
29.0\end{array}$ & $\begin{array}{r}.59 \\
.37 \\
.37\end{array}$ & $\begin{array}{l}6.9 \\
7.0 \\
6.3 \\
6.5\end{array}$ & $\begin{array}{l}99 \\
99 \\
88 \\
91\end{array}$ \\
\hline
\end{tabular}


$281833096421900 \quad$ LINE 287 SITE 03

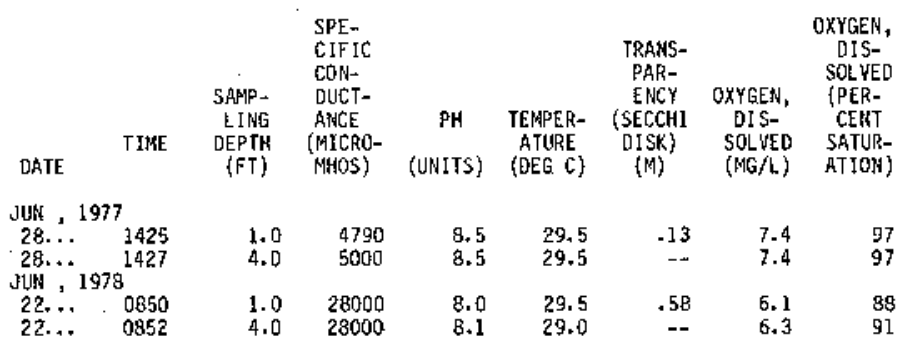

281801096431000 LINE 287 SITE 04

\begin{tabular}{|c|c|c|c|c|c|c|c|c|}
\hline DATE & T IME & $\begin{array}{l}\text { SAMP - } \\
\text { LING } \\
\text { DEPTH } \\
\text { (FT) }\end{array}$ & $\begin{array}{l}\text { SPE- } \\
\text { CIFIC } \\
\text { CON- } \\
\text { CUUCT- } \\
\text { ANCE } \\
\text { (MICRO- } \\
\text { MHOS) }\end{array}$ & $\begin{array}{c}\text { PH } \\
\text { (UNITS) }\end{array}$ & $\begin{array}{l}\text { TEMPER- } \\
\text { ATURE } \\
\text { (DEG C) }\end{array}$ & $\begin{array}{l}\text { TRANSS- } \\
\text { PAR- } \\
\text { ENCY } \\
\text { (SECCHI } \\
\text { DISK) } \\
\text { (M) }\end{array}$ & $\begin{array}{c}\text { OXYGEN, } \\
\text { DIS- } \\
\text { SOLVED } \\
\text { (MG/L) }\end{array}$ & $\begin{array}{c}\text { OXYGEN, } \\
\text { OIS- } \\
\text { SOLVED } \\
\text { (PER- } \\
\text { CEST } \\
\text { SATUR- } \\
\text { ATION) }\end{array}$ \\
\hline
\end{tabular}

$\begin{array}{ccccccccc}\text { FEB }, 1977 & & & & & & & \\ 09 . . . & 1050 & 1.0 & 10000 & 8.3 & 11.5 & .15 & 10.8 & 106 \\ 09 . . & 1052 & 4.0 & 10000 & 8.3 & 11.5 & -. & 10.7 & 105\end{array}$

281604096461300 LINE 287 SITE DB

\begin{tabular}{|c|c|c|c|c|c|c|c|c|}
\hline DATE & TIME & $\begin{array}{l}\text { SAMAP- } \\
\text { LING } \\
\text { DEPTH } \\
(\mathrm{FT})\end{array}$ & $\begin{array}{l}\text { SPE- } \\
\text { CIF IC } \\
\text { CDH- } \\
\text { DUCT- } \\
\text { ARCE } \\
\text { (RICRO- } \\
\text { MHOS) }\end{array}$ & $\begin{array}{c}\text { PH } \\
\text { (U\&1TS) }\end{array}$ & $\begin{array}{c}\text { TEMPER- } \\
\text { ATURE } \\
\text { (DEG C) }\end{array}$ & $\begin{array}{c}\text { TRAHS- } \\
\text { PAR- } \\
\text { EHCY } \\
\text { (SECCKI } \\
\text { DISK) } \\
\text { (A) }\end{array}$ & $\begin{array}{c}\text { OXYGEH, } \\
\text { OIS- } \\
\text { SOLWED } \\
\text { (MG } f L)\end{array}$ & $\begin{array}{c}\text { OXYGEN, } \\
\text { DIS- } \\
\text { SOLVED } \\
\text { (PER- } \\
\text { CENT } \\
\text { SATUR- } \\
\text { ATIOH) }\end{array}$ \\
\hline $\begin{array}{l}\text { FEB , } 1 \\
09 . . . \\
09 . . . \\
\text { JUN }\end{array}$ & $\begin{array}{l}1110 \\
1112\end{array}$ & $\begin{array}{l}1.0 \\
6.0\end{array}$ & $\begin{array}{l}15000 \\
16000\end{array}$ & $\begin{array}{l}8.2 \\
8.2\end{array}$ & $\begin{array}{l}11.0 \\
11.0\end{array}$ & .28 & $\begin{array}{l}10.5 \\
10.6\end{array}$ & $\begin{array}{l}105 \\
106\end{array}$ \\
\hline $\begin{array}{l}28 . . . \\
29 . \cdots \\
\text { JUN , } 1\end{array}$ & $\begin{array}{l}1445 \\
1447 \\
9\end{array}$ & $\begin{array}{l}1.0 \\
4.0\end{array}$ & $\begin{array}{l}4600 \\
5300\end{array}$ & & $\begin{array}{l}29.5 \\
29.0\end{array}$ & .13 & $\begin{array}{l}8.0 \\
7.7\end{array}$ & $\begin{array}{l}107 \\
103\end{array}$ \\
\hline $\begin{array}{l}22 \ldots \\
22 \ldots\end{array}$ & $\begin{array}{l}0908 \\
0910\end{array}$ & $\begin{array}{l}1.0 \\
7.0\end{array}$ & $\begin{array}{l}22000 \\
22900\end{array}$ & $\begin{array}{l}8.1 \\
8.0\end{array}$ & $\begin{array}{l}29.0 \\
29.0\end{array}$ & .44 & $\begin{array}{l}6.5 \\
6.6\end{array}$ & $\begin{array}{l}93 \\
95\end{array}$ \\
\hline
\end{tabular}

281327096431100 LINE 291 SITE 02

\begin{tabular}{|c|c|c|c|c|c|c|c|c|}
\hline DATE & TIME & $\begin{array}{c}\text { SAMP - } \\
\text { LIMG } \\
\text { DEPTH } \\
\text { (FT) }\end{array}$ & $\begin{array}{l}\text { SPE- } \\
\text { CIFIC } \\
\text { CON- } \\
\text { DUCT- } \\
\text { ARCE } \\
\text { (HICRO- } \\
\text { MHOS) }\end{array}$ & $\begin{array}{c}\text { PH } \\
\text { (UNITS) }\end{array}$ & $\begin{array}{l}\text { TEIAPER- } \\
\text { ATURE } \\
\text { (DEG C). }\end{array}$ & $\begin{array}{c}\text { TRARS- } \\
\text { PAR- } \\
\text { ENCY } \\
\text { (SECCHI } \\
\text { DISK) } \\
\text { (M) }\end{array}$ & $\begin{array}{c}\text { OXYGEN, } \\
\text { OIS- } \\
\text { SOLVED } \\
\text { (HG } / L)\end{array}$ & $\begin{array}{l}\text { OXYGEN, } \\
\text { DIS- } \\
\text { SOLYED } \\
\text { (PER- } \\
\text { CENT } \\
\text { SATUR- } \\
\text { ATION) }\end{array}$ \\
\hline
\end{tabular}

$\begin{array}{rrrrrrrrr}\text { FEB }, 1977 & & & & & & & & \\ 09 . . . & 1130 & 1.0 & 14000 & 8.4 & 11.0 & -27 & 10.8 & 107 \\ 09 . . . & 1132 & 6.0 & 14000 & 8.4 & 11.0 & -- & 10.9 & 108 \\ \text { JUN } & & & & & & & & \\ 28 . . . & 1500 & 1.0 & 8000 & 8.7 & 30.0 & -. & 9.8 & 135 \\ 28 . . & 1502 & 4.0 & 12000 & 8.7 & 30.0 & -- & 9.4 & 131\end{array}$

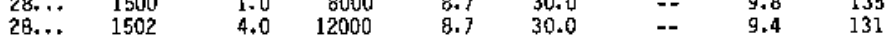

281453096441800 LIRE 291 5ITE O4

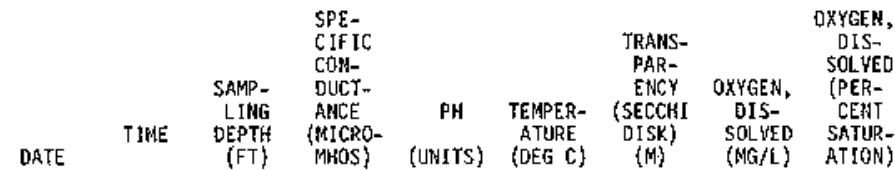

FEB , 1977

\begin{tabular}{|c|c|c|c|c|c|c|c|c|}
\hline $\begin{array}{l}09 . \ldots \\
09 . \ldots\end{array}$ & $\begin{array}{l}1120 \\
1122\end{array}$ & $\begin{array}{l}1.0 \\
6.0\end{array}$ & $\begin{array}{l}16000 \\
16000\end{array}$ & $\begin{array}{l}8.3 \\
8.3\end{array}$ & $\begin{array}{l}11.0 \\
11.0\end{array}$ & $\begin{array}{r}.48 \\
.-\end{array}$ & $\begin{array}{l}10.5 \\
10.6\end{array}$ & $\begin{array}{l}105 \\
106\end{array}$ \\
\hline $\begin{array}{l}28 \ldots \\
28 \ldots\end{array}$ & $\begin{array}{l}1445 \\
1447\end{array}$ & $\begin{array}{l}1.0 \\
4.0\end{array}$ & $\begin{array}{l}14000 \\
13000\end{array}$ & $\begin{array}{l}8.3 \\
8.3\end{array}$ & $\begin{array}{l}29.0 \\
29.0\end{array}$ & .15 & $\begin{array}{r}10.0 \\
9.4\end{array}$ & $\begin{array}{l}137 \\
129\end{array}$ \\
\hline JUK, 197 & & & & & & & & \\
\hline $\begin{array}{l}21 \ldots \\
21 \ldots\end{array}$ & $\begin{array}{l}1204 \\
1206\end{array}$ & $\begin{array}{l}1.0 \\
6.0\end{array}$ & $\begin{array}{l}27000 \\
32000\end{array}$ & $\begin{array}{l}8.0 \\
8.1\end{array}$ & $\begin{array}{l}30.0 \\
30.5\end{array}$ & $.3 B$ & $\begin{array}{l}6.2 \\
5.9\end{array}$ & $\begin{array}{l}91 \\
89\end{array}$ \\
\hline
\end{tabular}


281305096455500 LINE 294 SITE 02

\begin{tabular}{|c|c|c|c|c|c|c|c|c|}
\hline DATE & TIME & $\begin{array}{l}\text { SAHP- } \\
\text { LING } \\
\text { DEPTH } \\
\text { (FT) }\end{array}$ & $\begin{array}{l}\text { SPE- } \\
\text { CIFIC } \\
\text { CON- } \\
\text { DUCT- } \\
\text { ANCE } \\
\text { (MICRO- } \\
\text { MHOS) }\end{array}$ & $\begin{array}{c}\text { PH } \\
\text { (UNITS) }\end{array}$ & $\begin{array}{l}\text { TENPER- } \\
\text { ATURE } \\
\text { (DEG C) }\end{array}$ & $\begin{array}{c}\text { JRANS- } \\
\text { PAR- } \\
\text { ENCY } \\
\text { (SECCHI } \\
\text { DISK) } \\
\text { (M) }\end{array}$ & $\begin{array}{c}\text { OXYGEM, } \\
\text { DIS- } \\
\text { SOLVED } \\
\text { (MG/L) }\end{array}$ & $\begin{array}{c}\text { OXYGEN, } \\
\text { DIS- } \\
\text { SOLVEO } \\
\text { (PER- } \\
\text { CENT } \\
\text { SATUR- } \\
\text { ATYOH) }\end{array}$ \\
\hline \multicolumn{9}{|c|}{ FEY , 1977 } \\
\hline $\begin{array}{l}09 . . . \\
\text { JUA }\end{array}$ & $\begin{array}{l}1140 \\
1142\end{array}$ & $\begin{array}{l}1.0 \\
6.0\end{array}$ & $\begin{array}{l}15000 \\
15000\end{array}$ & $\begin{array}{l}8.4 \\
8.4\end{array}$ & $\begin{array}{l}11.0 \\
11.0\end{array}$ & $\begin{array}{r}.35 \\
--\end{array}$ & $\begin{array}{r}9.8 \\
10.1\end{array}$ & $\begin{array}{r}97 \\
100\end{array}$ \\
\hline $\begin{array}{r}28 . . \\
28 \ldots \\
\text { FEB },\end{array}$ & $\begin{array}{l}1510 \\
1512 \\
8\end{array}$ & $\begin{array}{l}1.0 \\
4.0\end{array}$ & $\begin{array}{l}14000 \\
14000\end{array}$ & $\begin{array}{l}8.5 \\
8.4\end{array}$ & $\begin{array}{l}29.5 \\
29.5\end{array}$ & $\begin{array}{l}.14 \\
--\end{array}$ & $\begin{array}{l}8.1 \\
7.2\end{array}$ & $\begin{array}{l}112 \\
100\end{array}$ \\
\hline $\begin{array}{l}22 \ldots \\
22 \ldots \\
22 \ldots \\
22 \ldots \\
22 . \ldots \\
22 \ldots \\
23 \ldots \\
23 \ldots \\
\text { JUN } \ldots\end{array}$ & $\begin{array}{l}0615 \\
0617 \\
1145 \\
1146 \\
1825 \\
1827 \\
0635 \\
0637 \\
8\end{array}$ & $\begin{array}{l}1.0 \\
5.0 \\
1.0 \\
4.0 \\
1.0 \\
4.0 \\
1.0 \\
5.0\end{array}$ & $\begin{array}{l}34000 \\
34000 \\
32000 \\
34000 \\
34000 \\
34000 \\
34000 \\
34000\end{array}$ & $\begin{array}{l}8.7 \\
8.7 \\
8.4 \\
8.3 \\
8.6 \\
8.7 \\
8.7 \\
8.7\end{array}$ & $\begin{array}{r}8.5 \\
8.5 \\
9.5 \\
9.0 \\
11.5 \\
11.5 \\
9.5 \\
9.5\end{array}$ & $\begin{array}{l}=- \\
m- \\
=- \\
=- \\
=- \\
=- \\
=\end{array}$ & $\begin{array}{r}10.2 \\
10.2 \\
10.1 \\
9.9 \\
10.0 \\
9.8 \\
10.0 \\
9.9\end{array}$ & $\begin{array}{l}104 \\
104 \\
104 \\
102 \\
109 \\
108 \\
104 \\
103\end{array}$ \\
\hline $\begin{array}{l}21 \ldots \\
21 \ldots\end{array}$ & $\begin{array}{l}1215 \\
1217\end{array}$ & $\begin{array}{l}1.0 \\
6.0\end{array}$ & $\begin{array}{l}31000 \\
31000\end{array}$ & $\begin{array}{l}8.1 \\
8.1\end{array}$ & $\begin{array}{l}30.0 \\
30.5\end{array}$ & $\begin{array}{r}.47 \\
--\end{array}$ & $\begin{array}{l}6.1 \\
6.1\end{array}$ & $\begin{array}{l}92 \\
92\end{array}$ \\
\hline
\end{tabular}

281438096413500 LINE 302 SITE D2

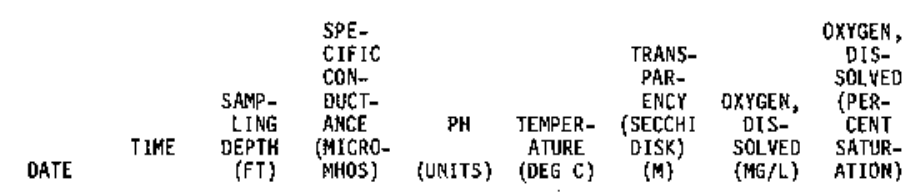

$\begin{array}{ccccccccr}\text { FEB }, 1977 & & & & & \\ 09 . . . & 1010 & 1.0 & 27000 & 8.2 & 11.0 & .41 & 9.9 & 103 \\ 09 . . . & 1012 & 5.0 & 31000 & 8.1 & 11.0 & -- & 9.7 & 103 \\ \text { J65 } & & & & & & & \\ 28 . . . & 1410 & 1.0 & 3800 & 8.7 & 29.5 & .13 & 7.3 & 97 \\ 28 . . & 1412 & 4.0 & 3200 & 8.7 & 29.5 & -. & 7.0 & 93\end{array}$

281605096424200 LINE 302 SITE O4

\begin{tabular}{|c|c|c|c|c|c|c|c|c|}
\hline DATE & TIME & $\begin{array}{l}\text { SAIP - } \\
\text { LING } \\
\text { DEPTH } \\
\text { (FT) }\end{array}$ & $\begin{array}{l}\text { SPE- } \\
\text { CIFIC } \\
\text { CON- } \\
\text { DUCT- } \\
\text { ANCE } \\
\text { (NICRO- } \\
\text { MHOS) }\end{array}$ & $\begin{array}{c}\mathrm{PH} \\
\text { (UAITS) }\end{array}$ & $\begin{array}{l}\text { TEMPER- } \\
\text { ATURE } \\
\text { (DEG C) }\end{array}$ & $\begin{array}{l}\text { TRANS- } \\
\text { PAR- } \\
\text { ENCY } \\
\text { (SECCHI } \\
\text { DISK) } \\
\text { (M) }\end{array}$ & $\begin{array}{c}\text { OXYGEN, } \\
\text { DIS- } \\
\text { SOLVED } \\
\text { (HG/L) }\end{array}$ & $\begin{array}{c}\text { OXYGEN, } \\
\text { DIS- } \\
\text { SOLYYEO } \\
\text { (PER- } \\
\text { CENT } \\
\text { SATUR- } \\
\text { ATION) }\end{array}$ \\
\hline \multicolumn{9}{|c|}{ FEB, 1971} \\
\hline $\begin{array}{l}09 . . . \\
09 . . .\end{array}$ & $\begin{array}{l}1025 \\
1027\end{array}$ & $\begin{array}{l}1.0 \\
5.0\end{array}$ & $\begin{array}{l}12000 \\
17000\end{array}$ & $\begin{array}{l}8.3 \\
8.2\end{array}$ & $\begin{array}{l}11.0 \\
11.0\end{array}$ & $\begin{array}{r}.30 \\
--\end{array}$ & $\begin{array}{l}10.8 \\
10.2\end{array}$ & $\begin{array}{l}106 \\
102\end{array}$ \\
\hline $\begin{array}{r}28 \ldots \\
28 \ldots\end{array}$ & $\begin{array}{r}1420 \\
1422\end{array}$ & $\begin{array}{l}1.0 \\
4.0\end{array}$ & $\begin{array}{l}2300 \\
3400\end{array}$ & $\begin{array}{l}8.5 \\
8.5\end{array}$ & $\begin{array}{l}29.5 \\
29.5\end{array}$ & .13 & $\begin{array}{l}7.8 \\
7.8\end{array}$ & $\begin{array}{l}104 \\
104\end{array}$ \\
\hline $\begin{array}{l}\text { JUN .. } \\
21 \ldots \\
21 \ldots .\end{array}$ & $\begin{array}{l}8 \\
1146 \\
1148\end{array}$ & $\begin{array}{l}1.0 \\
6.0\end{array}$ & $\begin{array}{l}34000 \\
34000\end{array}$ & $\begin{array}{l}8.3 \\
8.3\end{array}$ & $\begin{array}{l}30.0 \\
30.5\end{array}$ & .38 & $\begin{array}{l}6.1 \\
5.9\end{array}$ & $\begin{array}{l}92 \\
91\end{array}$ \\
\hline
\end{tabular}

281715096384500 LINE 307 SITE 03

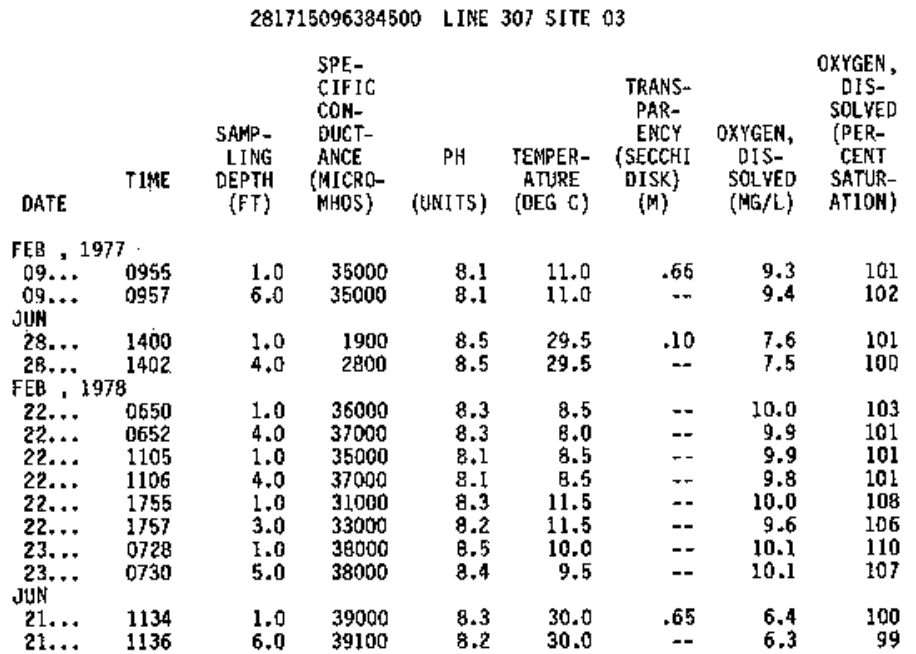


Table 7A.--Quality of water in the Guadal upe estuary, water years 1977-78--Continued Field Determinations--Cont f nued

281001096500000 L.JWE 311 SITE 01

\begin{tabular}{|c|c|c|c|c|c|c|c|c|}
\hline DATE & TIME & $\begin{array}{l}\text { SAMP- } \\
\text { LIHG } \\
\text { DEPTH } \\
\text { (FT) }\end{array}$ & $\begin{array}{l}\text { SPE- } \\
\text { CIFIC } \\
\text { CON- } \\
\text { DUCT- } \\
\text { ANCE } \\
\text { (\$ICRO- } \\
\text { MHOS) }\end{array}$ & $\begin{array}{c}\mathrm{PH} \\
\text { (UHATS) }\end{array}$ & $\begin{array}{c}\text { TEPAPER- } \\
\text { ATURE, } \\
\text { WATER } \\
\text { (DEG C) }\end{array}$ & $\begin{array}{c}\text { TRANS- } \\
\text { PAR- } \\
\text { ENCY } \\
\text { (SECCHI } \\
\text { DISK) } \\
\text { (M) }\end{array}$ & $\begin{array}{c}\text { OXYGER, } \\
\text { DIS- } \\
\text { SOLLWEL } \\
\text { (MG/L) }\end{array}$ & $\begin{array}{c}\text { OXYGEN } \\
\text { OIS- } \\
\text { SOLVED } \\
\text { (PER- } \\
\text { CENT } \\
\text { SATUR- } \\
\text { ATION) }\end{array}$ \\
\hline \multicolumn{9}{|c|}{ FEQ, 1977} \\
\hline $\begin{array}{c}09 . . . \\
09 . . \\
\text { JUN }\end{array}$ & $\begin{array}{l}1200 \\
1202\end{array}$ & $\begin{array}{l}1.0 \\
6.0\end{array}$ & $\begin{array}{l}15000 \\
15000\end{array}$ & $\begin{array}{l}8.5 \\
8.5\end{array}$ & $\begin{array}{l}11.5 \\
11.5\end{array}$ & .27 & $\begin{array}{l}11.0 \\
11.1\end{array}$ & $\begin{array}{l}110 \\
111\end{array}$ \\
\hline $\begin{array}{l}29 . . . \\
29 \ldots \\
\text { JuN }, 19\end{array}$ & $\begin{array}{l}1110 \\
1112 \\
8\end{array}$ & $\begin{array}{l}1.0 \\
3.0\end{array}$ & $\begin{array}{l}23000 \\
21000\end{array}$ & $\begin{array}{l}\text { B. } 4 \\
8.4\end{array}$ & $\begin{array}{l}28.5 \\
28.5\end{array}$ & .15 & $\begin{array}{l}6.7 \\
6.6\end{array}$ & $\begin{array}{l}95 \\
94\end{array}$ \\
\hline $\begin{array}{l}21 \ldots \\
21 \ldots\end{array}$ & $\begin{array}{l}1230 \\
1232\end{array}$ & $\begin{array}{l}1.0 \\
4.0\end{array}$ & $\begin{array}{l}39000 \\
39000\end{array}$ & $\begin{array}{l}8.1 \\
8.0\end{array}$ & $\begin{array}{l}30.0 \\
31.0\end{array}$ & .40 & $\begin{array}{l}6.0 \\
5.8\end{array}$ & $\begin{array}{l}93 \\
92\end{array}$ \\
\hline
\end{tabular}

280923096514100 LINE 311 SITE 04

\begin{tabular}{|c|c|c|c|c|c|c|c|c|}
\hline OATE & TIME & $\begin{array}{l}\text { SAMP- } \\
\text { LING } \\
\text { DEPTH } \\
\text { (FT) }\end{array}$ & $\begin{array}{l}\text { SPE- } \\
\text { CIFIC } \\
\text { CON- } \\
\text { DULT- } \\
\text { ANCE } \\
\text { (NICRO- } \\
\text { MHOS) }\end{array}$ & $\begin{array}{c}\text { PH } \\
\text { (UNITS) }\end{array}$ & $\begin{array}{l}\text { TEMPER } \\
\text { ATURE, } \\
\text { WATER } \\
\text { (DEG C) }\end{array}$ & $\begin{array}{c}\text { TRARS- } \\
\text { PAR- } \\
\text { ENCY } \\
\text { (SECCHI } \\
\text { DISK) } \\
\text { (M) }\end{array}$ & $\begin{array}{c}\text { OXYGEN, } \\
\text { DIS- } \\
\text { SOLUED } \\
\text { (HG/L) }\end{array}$ & $\begin{array}{c}\text { OXYGER, } \\
\text { DIS- } \\
\text { SOLVED } \\
\text { (PER- } \\
\text { CENT } \\
\text { SATUR- } \\
\text { ATION) }\end{array}$ \\
\hline
\end{tabular}

FEB 1977

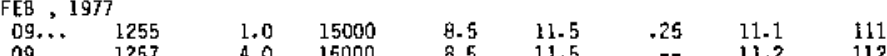

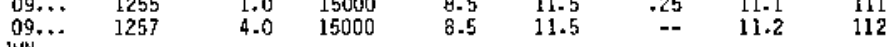

$\begin{array}{rrrrrrrrr}29 \ldots & 1100 & 1.0 & 20000 & 8.4 & 29.0 & .18 & 6.7 & 94 \\ 29 . . . & 1102 & 3.0 & 20000 & 8.4 & 29.0 & -- & 6.5 & 92\end{array}$

29... 1102

22... 0535

22... 0537

$22 . . \quad 1225$

1900

23... 0530

23... 0532

$21 . . \quad 1258$

1. 29000

3.D 32000

$\begin{array}{ll}4.0 & 29000 \\ 1.0 & 30000\end{array}$

$\begin{array}{ll}1.0 & 32000 \\ 4.0 & 33000\end{array}$

$\begin{array}{ll}4.0 & 33000 \\ 1.0 & 34000\end{array}$

$3.0 \quad 34000$

- 1.035000

$\begin{array}{llll}8.3 & 8.0 & -. & 9.4\end{array}$

$\begin{array}{lllll}8.3 & 8.0 & -- & 9.4 & 92 \\ 8.3 & 10.5 & -- & 9.0 & 90\end{array}$

$8.3 \quad 10.5$

$8.3 \quad 10.5$

8.511 .5

$8.5 \quad 11.5$

- $\quad 10.0$

- $\quad 9.7$

- $\quad 9.3 \quad 102$

$\begin{array}{lllll}8.3 & 10.5 & - & 9.2 & 98 \\ & 10.5 & -- & 9.2 & 98\end{array}$

21... 1300

280822096492800 LINE 314 SITE 01

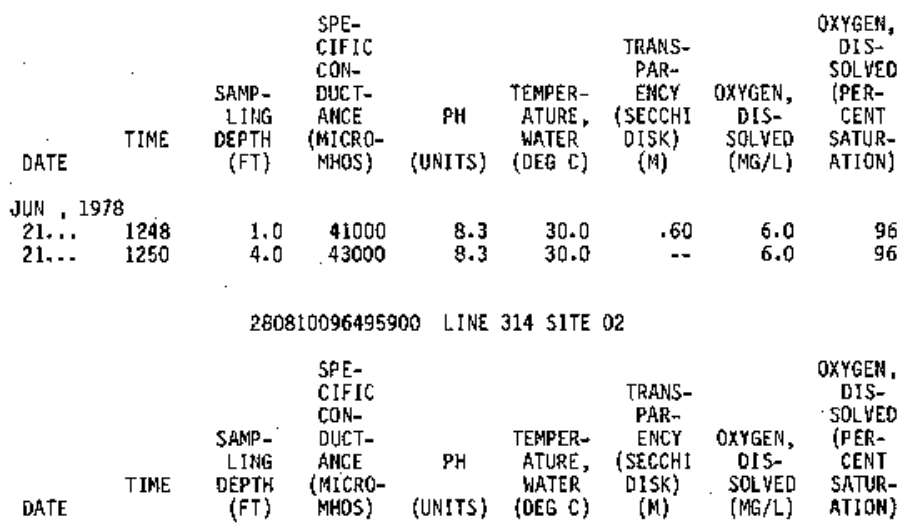

$\begin{array}{rrrrrrrrr}\text { FEB }, 1977 & & & & & \\ 09 . \ldots & 1245 & 1.0 & 13000 & 8.4 & 11.5 & .23 & 11.0 & 109 \\ 09 \ldots & 1247 & 4.0 & 14000 & 8.4 & 11.5 & -- & 10.9 & 109 \\ \text { JUN } & & & & & & & & \\ 29 . \ldots & 1120 & 1.0 & 23000 & 8.4 & 29.5 & .36 & 6.8 & 97\end{array}$



Table 7A.-Quality of water in the Guadalupe estuary, water years 1977-78--Continued
Ffeld Oeteminations--Continued

281834096345300 LIME 333 SITE 01

\begin{tabular}{|c|c|c|c|c|c|c|c|c|}
\hline DATE & TIME & $\begin{array}{c}\text { SAMP- } \\
\text { LING } \\
\text { DEPTH } \\
\text { (FT) }\end{array}$ & $\begin{array}{l}\text { SPE- } \\
\text { CIFIC } \\
\text { CON- } \\
\text { DUCT- } \\
\text { BRACE } \\
\text { (WICRO- } \\
\text { MHOS) }\end{array}$ & $\begin{array}{c}\text { PH } \\
\text { (UNITS) }\end{array}$ & $\begin{array}{l}\text { TEMPER- } \\
\text { ATURE } \\
\text { (DEG C) }\end{array}$ & $\begin{array}{c}\text { TRANS- } \\
\text { PAR- } \\
\text { ERCY } \\
\text { (SECCHI } \\
\text { DISK) } \\
\text { (M) }\end{array}$ & $\begin{array}{c}\text { OXYGEA, } \\
\text { DIS } \\
\text { SOLVED } \\
\text { (MG } f L)\end{array}$ & $\begin{array}{l}\text { OXYGER, } \\
\text { OIS- } \\
\text { SOLYEDD } \\
\text { (PER- } \\
\text { CERI } \\
\text { SATUR- } \\
\text { ATION) }\end{array}$ \\
\hline \multicolumn{9}{|c|}{ JUN , 1977} \\
\hline $\begin{array}{r}28 . . . \\
28 . . . \\
\text { JUN, }\end{array}$ & $\begin{array}{r}1335 \\
1337 \\
8\end{array}$ & $\begin{array}{l}1.0 \\
5.0\end{array}$ & $\begin{array}{l}2600 \\
2600\end{array}$ & $\begin{array}{l}8.6 \\
8.6\end{array}$ & $\begin{array}{l}29.0 \\
29.0\end{array}$ & $\begin{array}{r}.10 \\
--\end{array}$ & $\begin{array}{r}8.4 \\
8.2\end{array}$ & $\begin{array}{l}111 \\
108\end{array}$ \\
\hline $\begin{array}{l}21 \ldots \\
21 \ldots\end{array}$ & $\begin{array}{l}1120 \\
1122\end{array}$ & $\begin{array}{l}1.0 \\
7.0\end{array}$ & $\begin{array}{l}41000 \\
41000\end{array}$ & $\begin{array}{l}8.0 \\
8.0\end{array}$ & $\begin{array}{l}29.5 \\
30.0\end{array}$ & .64 & $\begin{array}{l}5.9 \\
5.9\end{array}$ & $\begin{array}{l}92 \\
93\end{array}$ \\
\hline
\end{tabular}

281937096354600 LINE 333 SITE 03

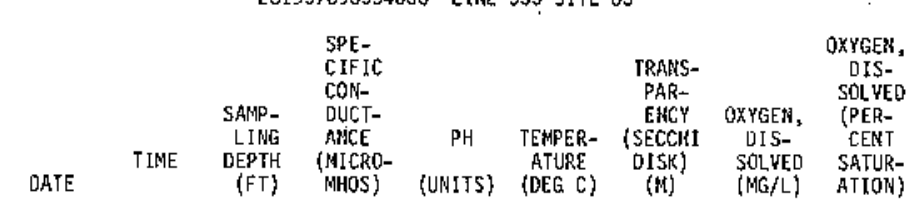

OATE (FT) MHOS) (UNITS) (DEG C) (M) (MG/L) ATION)

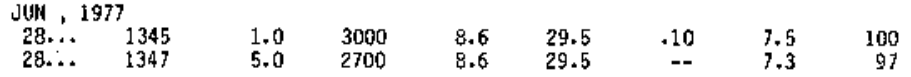

282021096331500 LINE 342 SITE 02

\begin{tabular}{|c|c|c|c|c|c|c|c|c|}
\hline DATE & TIME & $\begin{array}{l}\text { SAMP- } \\
\text { LIMG } \\
\text { DEPTH } \\
\text { (FT) }\end{array}$ & $\begin{array}{l}\text { SPE- } \\
\text { CIFIC } \\
\text { CON- } \\
\text { DUCT- } \\
\text { ANICE } \\
\text { (MICRO- } \\
\text { MHOS) }\end{array}$ & $\begin{array}{c}\text { PH } \\
\text { (UNITS) }\end{array}$ & $\begin{array}{l}\text { TEMPER- } \\
\text { ATURE } \\
\text { (DEG C) }\end{array}$ & $\begin{array}{c}\text { TRANS- } \\
\text { PAR- } \\
\text { ENCY } \\
\text { (SECCHI } \\
\text { OISK) } \\
(M)\end{array}$ & $\begin{array}{c}\text { OXYGEN, } \\
\text { DIS- } \\
\text { SOLVED } \\
\text { (MG/L) }\end{array}$ & $\begin{array}{l}\text { OXYGEN, } \\
\text { DIS- } \\
\text { SOLVED } \\
\text { (PER- } \\
\text { CENT } \\
\text { SATUR- } \\
\text { ATION) }\end{array}$ \\
\hline \multicolumn{9}{|c|}{$\mathrm{FEB}, 1977$} \\
\hline $\begin{array}{l}09 \ldots . . \\
\text { JUN }\end{array}$ & $\begin{array}{l}0935 \\
0937\end{array}$ & $\begin{array}{l}1.0 \\
7.0\end{array}$ & $\begin{array}{l}44000 \\
44000\end{array}$ & $\begin{array}{l}8.3 \\
8.3\end{array}$ & $\begin{array}{l}11.0 \\
11.0\end{array}$ & .82 & $\begin{array}{l}9.3 \\
9.4\end{array}$ & $\begin{array}{l}106 \\
107\end{array}$ \\
\hline $\begin{array}{l}28 \ldots \\
28 \ldots \\
\text { JuN, }\end{array}$ & $\begin{array}{l}1320 \\
1322 \\
8\end{array}$ & $\begin{array}{l}1.0 \\
6.0\end{array}$ & $\begin{array}{l}2800 \\
4000\end{array}$ & $\begin{array}{l}8.6 \\
8.6\end{array}$ & & $\begin{array}{r}-10 \\
-.\end{array}$ & $\begin{array}{l}8.0 \\
7.8\end{array}$ & $\begin{array}{l}105 \\
103\end{array}$ \\
\hline $\begin{array}{l}21 \ldots \\
21 \ldots\end{array}$ & $\begin{array}{l}1107 \\
1109\end{array}$ & $\begin{array}{l}1.0 \\
7.0\end{array}$ & $\begin{array}{l}45000 \\
45000\end{array}$ & $\begin{array}{l}8.1 \\
8.1\end{array}$ & $\begin{array}{l}30.0 \\
30.5\end{array}$ & $\begin{array}{r}.60 \\
--\end{array}$ & $\begin{array}{l}5.9 \\
5.7\end{array}$ & $\begin{array}{l}95 \\
93\end{array}$ \\
\hline
\end{tabular}

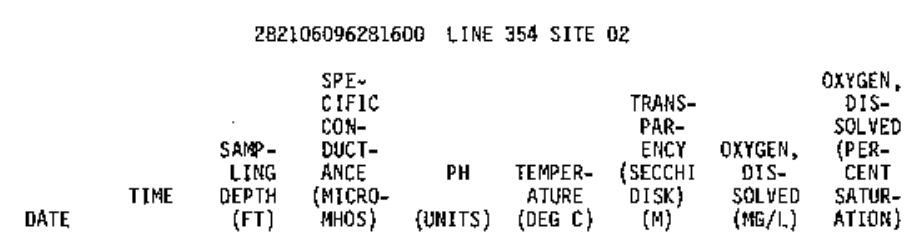

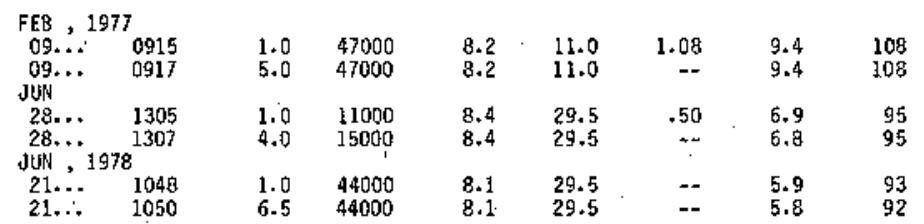

282246096294900 LINE 354 SITE 04

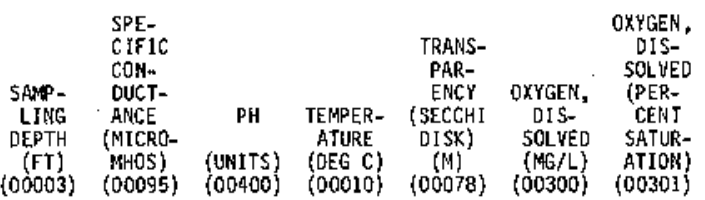

FEB , 1977

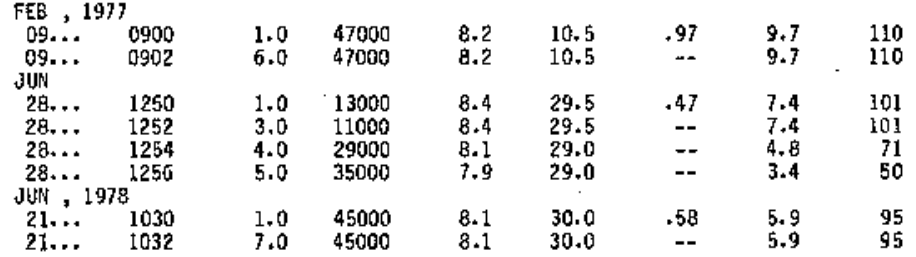


Table 7A.--QuaTtty of water in the Guadalupe estuary, water years 1977-78--Cont fnued Field Determinations--Contínued

282400096243500 LIHE 360 SITE 03

\begin{tabular}{|c|c|c|c|c|c|c|c|c|}
\hline DATE & TIME & $\begin{array}{l}\text { SAMP- } \\
\text { LIMG } \\
\text { DEPTH } \\
\text { (FT) }\end{array}$ & $\begin{array}{l}\text { SPE- } \\
\text { CIFIC } \\
\text { CON- } \\
\text { DUCT- } \\
\text { ANCE } \\
\text { (MICRO- } \\
\text { MHOS) }\end{array}$ & $\begin{array}{c}\text { pH } \\
\text { (UnlTS) }\end{array}$ & $\begin{array}{l}\text { TEMPER- } \\
\text { ATURE } \\
\text { (OEG C) }\end{array}$ & $\begin{array}{c}\text { TRANS- } \\
\text { PAR- } \\
\text { ENCY } \\
\text { (SECCHI } \\
\text { OISK) } \\
\text { (M) }\end{array}$ & $\begin{array}{c}\text { OXYGEN, } \\
\text { DIS- } \\
\text { SOLVED } \\
\text { (NG/L) }\end{array}$ & $\begin{array}{l}\text { OXYGEN, } \\
\text { OIS- } \\
\text { SOLYED } \\
\text { (PER- } \\
\text { CENT } \\
\text { SATUR- } \\
\text { ATION) }\end{array}$ \\
\hline \multicolumn{9}{|c|}{ FEB , 1977} \\
\hline $\begin{array}{l}08 . . \\
08 . . . \\
08 . .\end{array}$ & $\begin{array}{l}1530 \\
1532 \\
1534\end{array}$ & $\begin{array}{l}1.0 \\
10 \\
23\end{array}$ & $\begin{array}{l}46000 \\
46000 \\
46000\end{array}$ & $\begin{array}{l}7.9 \\
7.9 \\
7.9\end{array}$ & $\begin{array}{l}11.0 \\
11.0 \\
10.5\end{array}$ & $\begin{array}{l}.38 \\
-- \\
-\end{array}$ & $\begin{array}{l}9.4 \\
9.6 \\
9.5\end{array}$ & $\begin{array}{l}108 \\
110 \\
107\end{array}$ \\
\hline JUN & & & & & & & & \\
\hline $\begin{array}{l}28 \ldots \\
28 \ldots \\
28 \ldots \\
28 \ldots \\
\text { JUN }\end{array}$ & $\begin{array}{l}1125 \\
1127 \\
1129 \\
1131 \\
8\end{array}$ & $\begin{array}{l}1.0 \\
5.0 \\
10 \\
20\end{array}$ & $\begin{array}{l}54000 \\
55000 \\
55000 \\
54000\end{array}$ & $\begin{array}{l}7.9 \\
7.9 \\
7.9 \\
7.9\end{array}$ & $\begin{array}{l}28.5 \\
29.0 \\
28.5 \\
2 B .5\end{array}$ & $\begin{array}{l}.27 \\
-- \\
-- \\
--\end{array}$ & $\begin{array}{l}5.9 \\
5.9 \\
5.8 \\
5.6\end{array}$ & $\begin{array}{l}97 \\
97 \\
95 \\
92\end{array}$ \\
\hline $\begin{array}{l}21 \ldots \\
21 \ldots . . \\
21 . .\end{array}$ & $\begin{array}{l}0930 \\
0932 \\
0934\end{array}$ & $\begin{array}{l}1.0 \\
10 \\
25\end{array}$ & $\begin{array}{l}45000 \\
44000 \\
45000\end{array}$ & $\begin{array}{l}8.1 \\
8.1 \\
8.1\end{array}$ & $\begin{array}{l}30.0 \\
30.0 \\
30.0\end{array}$ & .76 & $\begin{array}{l}6.2 \\
6.3 \\
6.1\end{array}$ & $\begin{array}{r}100 \\
100 \\
99\end{array}$ \\
\hline
\end{tabular}


[FT = feet; $M G / L=$ milligrams per liter]

282644096455400 LINE 080 SITE 02

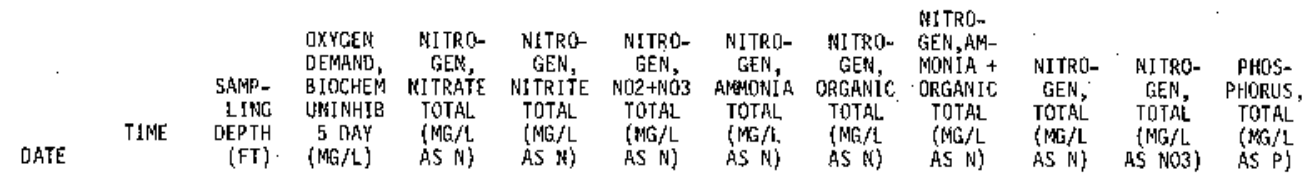

FER, 1977

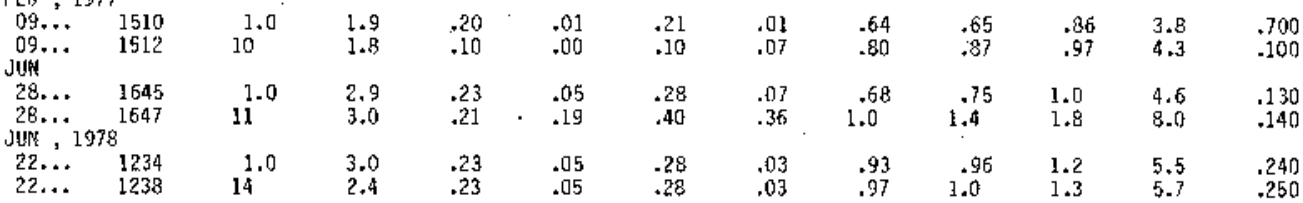

28264B096493700 LINE I60 SITE O2

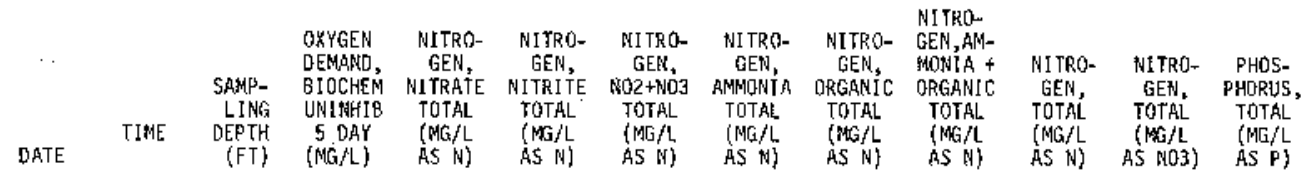

JUN, 1977

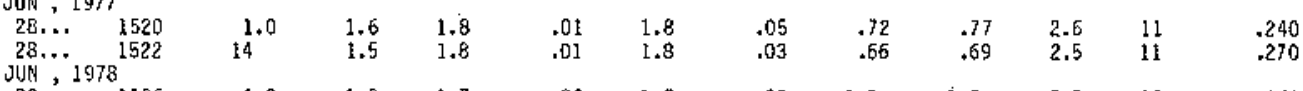

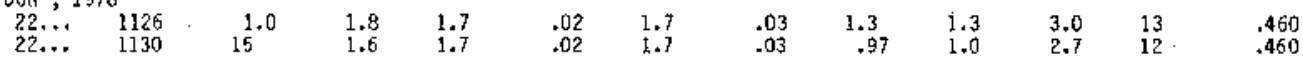

282404096452300 LINE 243 SITE 05

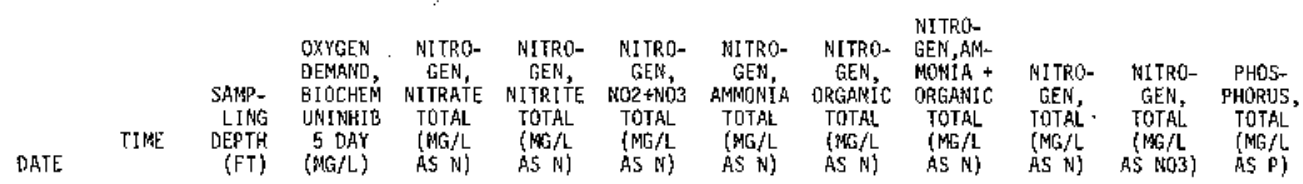

FEB 1973

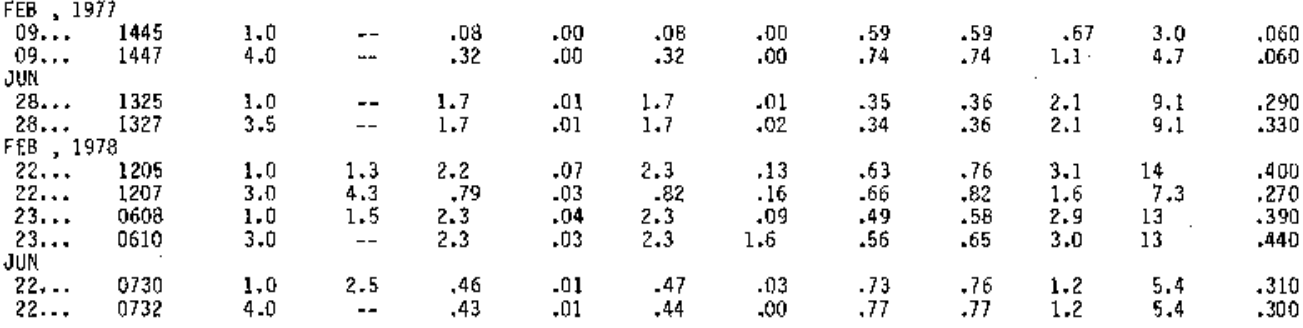

282157096461700 LINE 264 SITE OA

\begin{tabular}{|c|c|c|c|c|c|c|c|c|c|c|c|}
\hline DATE & TIME & $\begin{array}{l}\text { SAMP- } \\
\text { LIPGG } \\
\text { DEPTH } \\
(F T)\end{array}$ & $\begin{array}{c}\text { NITRO- } \\
\text { GEN, } \\
\text { NITRATE } \\
\text { TOTAL } \\
\text { (MG/L } \\
\text { AS N) }\end{array}$ & $\begin{array}{l}\text { NITRO- } \\
\text { GEN, } \\
\text { NITRITE } \\
\text { TOTAL } \\
\text { (MG } / L \\
\text { AS N }\end{array}$ & $\begin{array}{l}\text { NITRO- } \\
\text { GEN, } \\
\text { MOL+NDS } \\
\text { TOTAL } \\
\text { (MG/L } \\
\text { MS N }\end{array}$ & $\begin{array}{c}\text { UITRO- } \\
\text { GEN, } \\
\text { APMONIA } \\
\text { TDTAL } \\
\text { (MG/L } \\
\text { AS N) }\end{array}$ & $\begin{array}{l}\text { NITRO- } \\
\text { CEEN, } \\
\text { ORGANIC } \\
\text { TOTAL } \\
\text { (MG/L } \\
\text { RS N) }\end{array}$ & $\begin{array}{l}\text { NI TRO- } \\
\text { GEN, AM- } \\
\text { MONI } A+ \\
\text { ORGANIC } \\
\text { TOTAL } \\
\text { (MG/L } \\
\text { AS N) }\end{array}$ & $\begin{array}{l}\text { NITRO- } \\
\text { GEN, } \\
\text { TOTAL } \\
\text { (MG/L } \\
\text { AS } \$ \text { is) }\end{array}$ & $\begin{array}{l}\text { RITRO- } \\
\text { GEN, } \\
\text { TOTAL } \\
\text { (MG/L } \\
\text { AS NO3) }\end{array}$ & $\begin{array}{c}\text { PHOS- } \\
\text { PHORUS, } \\
\text { TOTAL } \\
\text { (MG/L } \\
\text { AS P) }\end{array}$ \\
\hline \multicolumn{12}{|c|}{ FEB , 1977} \\
\hline $\begin{array}{c}09 . . \\
09 . .\end{array}$ & $\begin{array}{l}1410 \\
1412\end{array}$ & $\begin{array}{l}1.0 \\
5.0\end{array}$ & $\begin{array}{l}.03 \\
.10\end{array}$ & $\begin{array}{l}.00 \\
.00\end{array}$ & $\begin{array}{l}.03 \\
.10\end{array}$ & .01 & $\begin{array}{l}1.5 \\
.68\end{array}$ & $\begin{array}{c}1.5 \\
.69\end{array}$ & $\begin{array}{c}1.5 \\
.79\end{array}$ & $\begin{array}{l}6.8 \\
3.5\end{array}$ & $\begin{array}{l}.240 \\
.130\end{array}$ \\
\hline $\begin{array}{r}28 \ldots \\
28 \ldots \\
F E B\end{array}$ & $\begin{array}{l}1245 \\
1247 \\
8\end{array}$ & $\begin{array}{l}1.0 \\
4.5\end{array}$ & $\begin{array}{l}.18 \\
.18\end{array}$ & 1 & $\begin{array}{l}.19 \\
.19\end{array}$ & $\begin{array}{l}.02 \\
.02\end{array}$ & .47 & $\begin{array}{l}.49 \\
.39\end{array}$ & $\begin{array}{r}.68 \\
.58\end{array}$ & $\begin{array}{l}3.0 \\
2.6\end{array}$ & $\begin{array}{l}.200 \\
.190\end{array}$ \\
\hline $\begin{array}{l}22 \ldots \\
22 \ldots \\
23 \ldots \\
23 \ldots\end{array}$ & $\begin{array}{l}1150 \\
1152 \\
0630 \\
0632\end{array}$ & $\begin{array}{l}1.0 \\
4.0 \\
1.0 \\
4.0\end{array}$ & $\begin{array}{l}.01 \\
.03 \\
.00 \\
.00\end{array}$ & $\begin{array}{l}.01 \\
.01 \\
.01 \\
.01\end{array}$ & $\begin{array}{l}.02 \\
.04 \\
.01 \\
.01\end{array}$ & $\begin{array}{l}.04 \\
.05 \\
.00 \\
.00\end{array}$ & $\begin{array}{l}.86 \\
1.3 \\
1.4 \\
1.4\end{array}$ & $\begin{array}{l}.90 \\
1.3 \\
1.4 \\
1.4\end{array}$ & $\begin{array}{l}.92 \\
1.3 \\
1.4 \\
1.4\end{array}$ & $\begin{array}{l}4.1 \\
5.9 \\
6.2 \\
6.2\end{array}$ & $\begin{array}{l}.130 \\
.180 \\
.190 \\
.200\end{array}$ \\
\hline JUN & & & & & & & & & & & \\
\hline $\begin{array}{l}22 \ldots \\
22 \ldots\end{array}$ & $\begin{array}{l}0745 \\
0747\end{array}$ & $\begin{array}{l}1.0 \\
4.0\end{array}$ & .00 & $\begin{array}{l}.01 \\
.01\end{array}$ & $\begin{array}{l}.01 \\
.05\end{array}$ & $\begin{array}{l}.00 \\
.00\end{array}$ & $\begin{array}{l}.67 \\
.82\end{array}$ & $\begin{array}{l}.67 \\
.82\end{array}$ & $\begin{array}{l}.68 \\
.87\end{array}$ & $\begin{array}{l}3.0 \\
3.9\end{array}$ & $\begin{array}{r}.190 \\
.190\end{array}$ \\
\hline
\end{tabular}




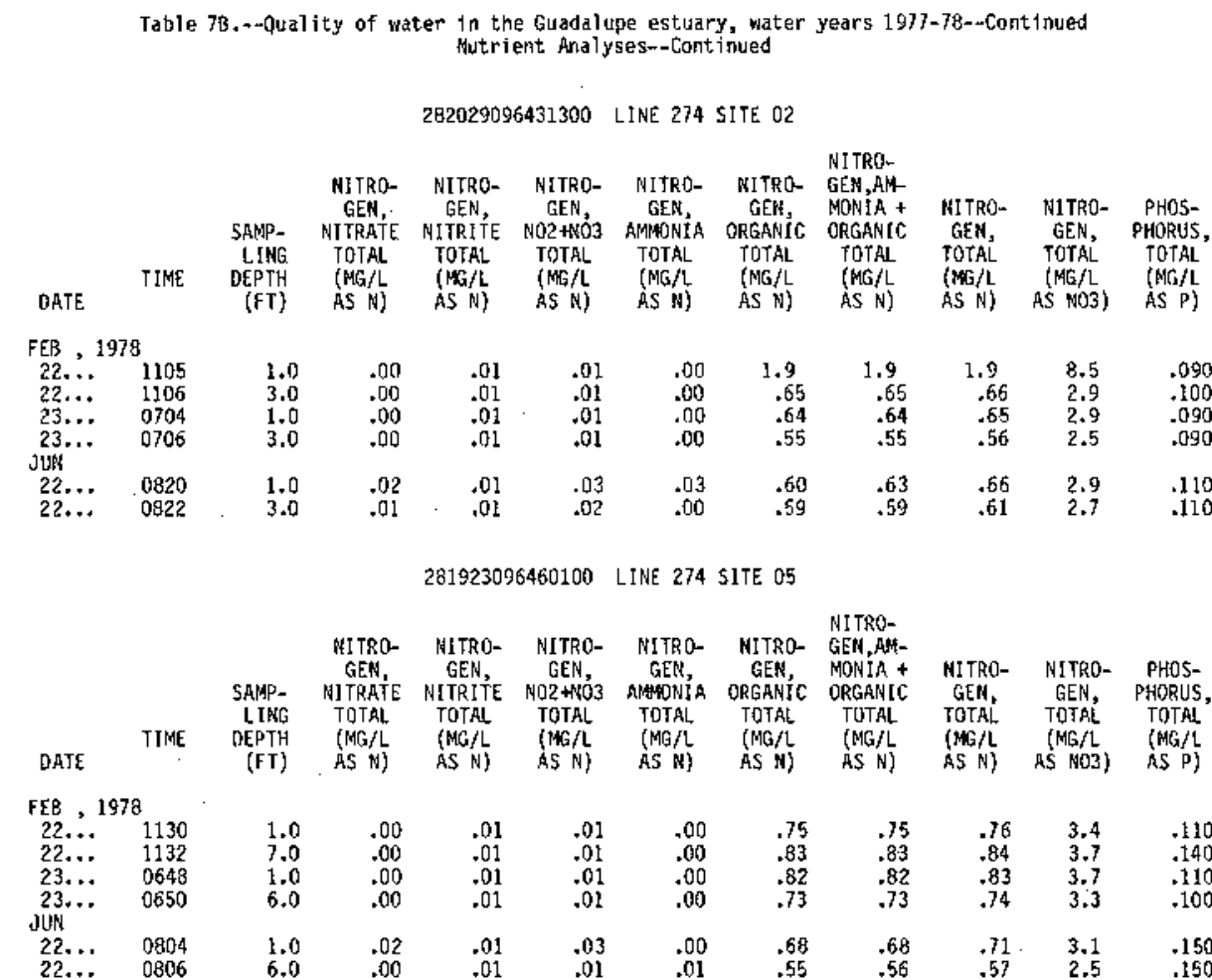

281833096421900 LIME 287 SITE 03

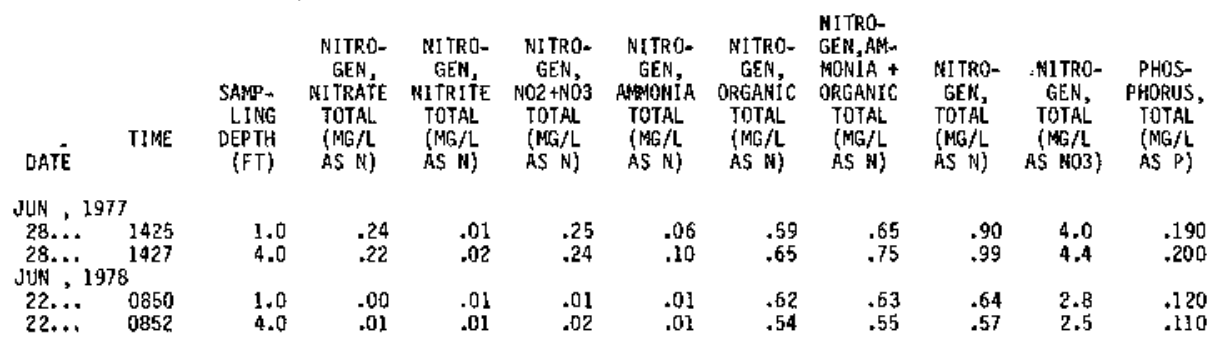

281801096431000 LINE 287 SITE O4

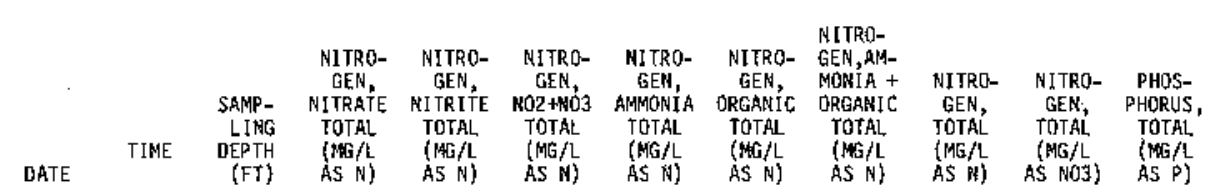

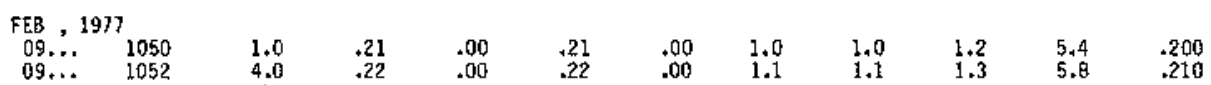

281604096461300 LINAF 287 SITE Q8

\begin{tabular}{|c|c|c|c|c|c|c|c|c|c|c|c|c|}
\hline DATE & TIME & $\begin{array}{c}\text { SAMP- } \\
\text { LING } \\
\text { DEPTH } \\
\text { (FT) }\end{array}$ & $\begin{array}{l}\text { OXYGEM } \\
\text { DEMANO } \\
\text { BIOCHEM } \\
\text { UNINHIB } \\
5 \text { DAY } \\
\text { (MG/L) }\end{array}$ & $\begin{array}{c}\text { NITRO- } \\
\text { GEN, } \\
\text { NITRATE } \\
\text { TOTRL } \\
\text { (MG/L } \\
\text { AS HI) }\end{array}$ & $\begin{array}{c}\text { RITROM } \\
\text { GER, } \\
\text { NITRITE } \\
\text { TOTAL } \\
\text { (MG GL } \\
\text { AS N) }\end{array}$ & $\begin{array}{c}\text { NITROH } \\
\text { GEN, } \\
\text { NO2+NO3 } \\
\text { TOTAL } \\
\text { (MG/L } \\
\text { ASS N) }\end{array}$ & $\begin{array}{c}\text { NITRO- } \\
\text { GEN, } \\
\text { AWNONIA } \\
\text { TOTAL } \\
(W G / L \\
\text { AS N) }\end{array}$ & 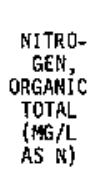 & $\begin{array}{l}\text { NI TRO- } \\
\text { GEN,AH- } \\
\text { MONIA + } \\
\text { ORGANIC } \\
\text { TDTAL } \\
\text { (MG/L } \\
\text { AS N) }\end{array}$ & $\begin{array}{l}\text { BITRO- } \\
\text { GEN, } \\
\text { TOTAL } \\
\text { (MG/L } \\
\text { AS N) }\end{array}$ & $\begin{array}{l}\text { NITRO- } \\
\text { GEN, } \\
\text { TOTAL } \\
\text { (MG/L } \\
\text { AS NO3) }\end{array}$ & $\begin{array}{l}\text { PHOS- } \\
\text { PHORUS, } \\
\text { TOTAL } \\
\text { (MG/L } \\
\text { AS P) }\end{array}$ \\
\hline $\mathrm{FEC}, 1$ & & & & & & & & & & & & \\
\hline $\begin{array}{l}09 . . . \\
09 . . . \\
\text { JUN }\end{array}$ & $\begin{array}{l}1110 \\
1112\end{array}$ & $\begin{array}{l}1.0 \\
6.0\end{array}$ & $\begin{array}{l}2.2 \\
2.2\end{array}$ & $\begin{array}{l}.13 \\
.13\end{array}$ & $\begin{array}{l}.00 \\
.00\end{array}$ & $\begin{array}{l}.13 \\
.13\end{array}$ & .01 & $\begin{array}{l}.80 \\
.97\end{array}$ & $\begin{array}{l}.81 \\
.98\end{array}$ & .94 & $\begin{array}{l}4.2 \\
4.9\end{array}$ & $\begin{array}{l}.150 \\
.170\end{array}$ \\
\hline $\begin{array}{l}28 . \ldots \\
28 \ldots \\
\text { JUK }, \ldots\end{array}$ & $\begin{array}{l}1445 \\
1447 \\
8\end{array}$ & $\begin{array}{l}1.0 \\
4.0\end{array}$ & $\begin{array}{l}1.5 \\
1.5\end{array}$ & $\begin{array}{r}.13 \\
.12\end{array}$ & $\begin{array}{l}.01 \\
.01\end{array}$ & $\begin{array}{l}.14 \\
.13\end{array}$ & $\begin{array}{l}.02 \\
.03\end{array}$ & $\begin{array}{l}.59 \\
.61\end{array}$ & $\begin{array}{l}.61 \\
.64\end{array}$ & $\begin{array}{l}.75 \\
.77\end{array}$ & $\begin{array}{l}3.3 \\
3.4\end{array}$ & $\begin{array}{l}.140 \\
.160\end{array}$ \\
\hline $22 \ldots$ & $\begin{array}{l}0908 \\
0910\end{array}$ & $\begin{array}{l}1.0 \\
7.0\end{array}$ & $\begin{array}{l}2.0 \\
1.8\end{array}$ & $\begin{array}{l}.00 \\
.00\end{array}$ & $\begin{array}{l}.01 \\
.01\end{array}$ & .01 & $\begin{array}{l}.01 \\
.03\end{array}$ & $\begin{array}{l}.53 \\
.50\end{array}$ & $\begin{array}{l}.54 \\
.53\end{array}$ & $\begin{array}{l}.55 \\
.54\end{array}$ & $\begin{array}{l}2.4 \\
2.4\end{array}$ & $\begin{array}{l}.130 \\
.130\end{array}$ \\
\hline
\end{tabular}


Table 7e.--quality of water in the Guadalupe estuary, water years 1977-78-Continued

Nutrient Analyses--Cont trued

281305096455500 LINE 294 SITE 02

\begin{tabular}{|c|c|c|c|c|c|c|c|c|c|c|c|}
\hline DATE & TIME & $\begin{array}{l}\text { SATAP- } \\
\text { LING } \\
\text { DEPTH } \\
\text { (FT) }\end{array}$ & $\begin{array}{l}\text { NITRO- } \\
\text { GEN, } \\
\text { NITRATE } \\
\text { TOTAL } \\
\text { (MG/L } \\
\text { A5 N) }\end{array}$ & $\begin{array}{l}\text { NITRD- } \\
\text { GEN, } \\
\text { NITRITE } \\
\text { TOTAL } \\
\text { (MG/L } \\
\text { AS N N) }\end{array}$ & $\begin{array}{c}\text { NITRO- } \\
\text { GEN, } \\
\text { NO2+NO3 } \\
\text { TOTAL } \\
\text { (MG } / L \\
\text { RS N) }\end{array}$ & $\begin{array}{l}\text { NITRO- } \\
\text { GEN, } \\
\text { AMMONIA } \\
\text { TOTAL } \\
\text { (MG } f \text { L } \\
\text { AS } B \text { ) }\end{array}$ & $\begin{array}{l}\text { NITRD- } \\
\text { GEN, } \\
\text { ORGARIC } \\
\text { TOTAL } \\
\text { (MG/L } \\
\text { AS N) }\end{array}$ & $\begin{array}{l}\text { NITRO- } \\
\text { GEN,AM- } \\
\text { MONIA + } \\
\text { ORGANIC } \\
\text { TOTRL } \\
\text { (MGG/L } \\
\text { ASN) }\end{array}$ & $\begin{array}{l}\text { NITRO- } \\
\text { GEN, } \\
\text { TOTAL } \\
(M G / L \\
\text { AS N } N)\end{array}$ & $\begin{array}{l}\text { NITRO- } \\
\text { GEN, } \\
\text { TOTAL } \\
\text { (WG/L } \\
\text { AS NO3) }\end{array}$ & $\begin{array}{l}\text { PHOS- } \\
\text { PHORUS, } \\
\text { TOTAL } \\
\text { (MG } / L \\
\text { AS P) }\end{array}$ \\
\hline
\end{tabular}

FEB , 1977

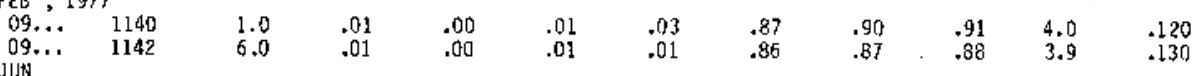

JUN

$28 \ldots .1510$

$\mathrm{FEB}, 1979$

$22 . . .1145$

$23 \ldots \quad 0635$

$23 \ldots .0637$

JUN, 1978

$21 . . \quad 1213$

$\begin{array}{lll}1.0 & .00 & .01 \\ 4.0 & .00 & .01\end{array}$

$\begin{array}{lll}1.0 & .00 & .0 \\ 4.0 & .00 & .00\end{array}$

$\begin{array}{lll}1.0 & .00 & .01 \\ 5.0 & .00 & .01\end{array}$

.0000

21... 1217

$\begin{array}{ll}6.0 & .01 \\ 1.0 & .0 \\ 6.0 & .0\end{array}$

.01

$\begin{array}{llllll}.02 & .44 & .46 & .46 & 2.0 & .110\end{array}$

.01

.01

.00
.00
.00

$\begin{array}{lllll}.60 & .60 & .61 & 2.7 & .060\end{array}$

$\begin{array}{ccccc}.2 & 1.2 & 1.2 & 5.4 & .070 \\ .45 & .44 & .45 & 2.0 & .050\end{array}$

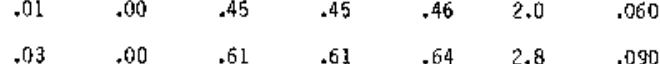

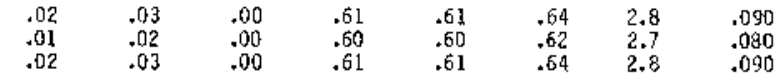

281715096384500 LINE 307 SITE 03

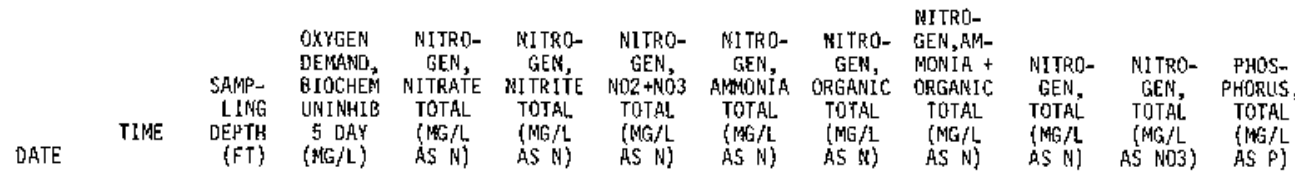

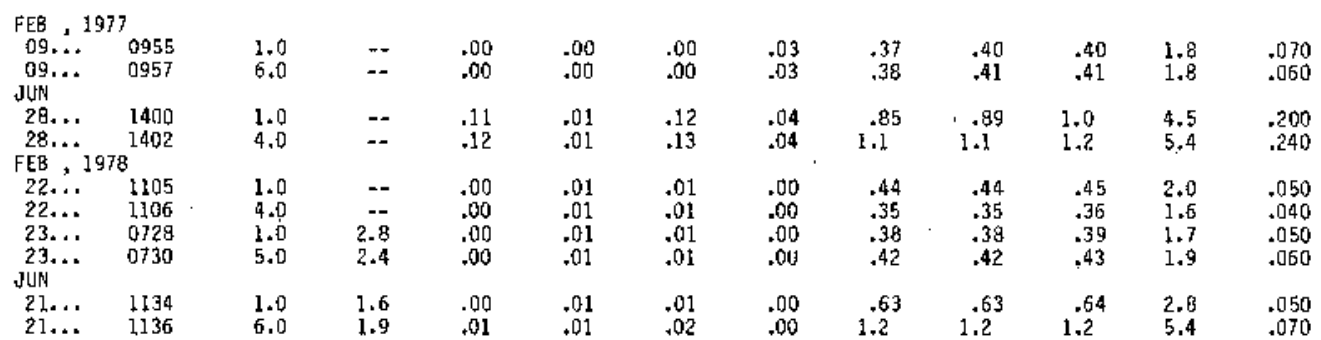

280923096514 I0D LIRE 311 SITE O4

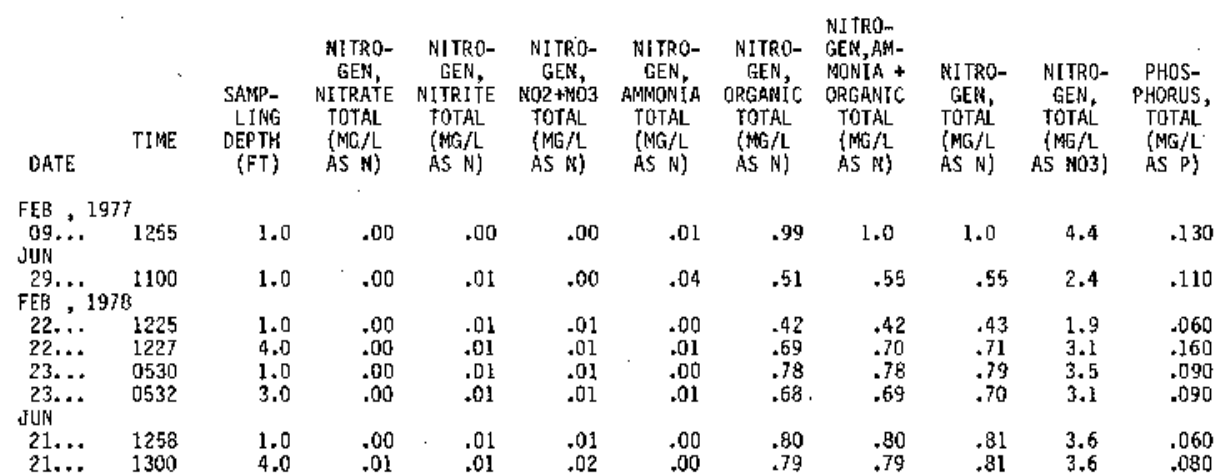

280810096495900 LINE 314 SITE O2

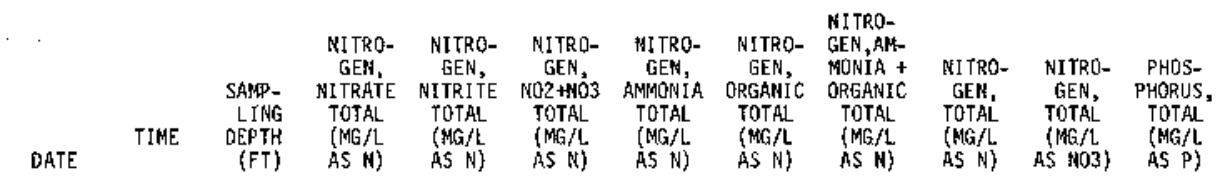

FEB , 1977

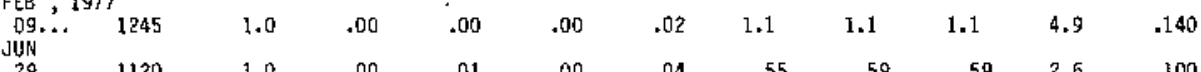




\begin{tabular}{|c|c|c|c|c|c|c|c|c|c|c|c|}
\hline \multirow[b]{2}{*}{ DATE } & \multirow[b]{2}{*}{ TIME } & \multirow[b]{2}{*}{$\begin{array}{c}\text { 5AMP- } \\
\text { LING } \\
\text { DEPTH } \\
\text { (FT) }\end{array}$} & \multirow[b]{2}{*}{$\begin{array}{l}\text { NITRO- } \\
\text { GEN, } \\
\text { NITRATE } \\
\text { TOTAL } \\
\text { (NSA } / L \\
\text { AS N) }\end{array}$} & \multicolumn{2}{|c|}{282021096331500} & \multicolumn{2}{|c|}{ LINE 342 SITE 02} & \multirow[b]{2}{*}{$\begin{array}{l}\text { NITRO- } \\
\text { GENAM- AM- } \\
\text { MONIA + } \\
\text { ORGANIC } \\
\text { TOTAL } \\
\text { (MG/L } \\
\text { AS N) }\end{array}$} & \multirow[b]{2}{*}{$\begin{array}{l}\text { NITRO- } \\
\text { GEPA, } \\
\text { TOTAL } \\
\text { (WAS } / L \\
\text { A5 N) }\end{array}$} & \multirow[b]{2}{*}{$\begin{array}{l}\text { N1TRO- } \\
\text { GEN, } \\
\text { TOTAL } \\
\text { (MGA } 2 \text {, } \\
\text { AS NO3) }\end{array}$} & \multirow[b]{2}{*}{$\begin{array}{l}\text { PHOS- } \\
\text { PHORUS, } \\
\text { TOTAL } \\
\text { (MG/L } \\
\text { AS P) }\end{array}$} \\
\hline & & & & $\begin{array}{l}\text { NITRO- } \\
\text { GEN, } \\
\text { NITRITE } \\
\text { TORAL } \\
(\text { ITAL } / L \\
\text { AS N } N)\end{array}$ & 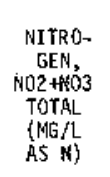 & $\begin{array}{c}\text { NITRO- } \\
\text { GEN, } \\
\text { AMMKNIA } \\
\text { TDTAL } \\
\text { (MGLL } \\
\text { AS N } N \text { ) }\end{array}$ & $\begin{array}{c}\text { NITRO- } \\
\text { GEE, } \\
\text { ORGANIC } \\
\text { TOTAL } \\
\text { (MGLL } \\
\text { RS N) }\end{array}$ & & & & \\
\hline $\begin{array}{l}\text { FEB }, 197 \\
09 . . \\
09 . \ldots\end{array}$ & $\begin{array}{l}7 \\
0935 \\
0937\end{array}$ & $\begin{array}{l}1.0 \\
7.0\end{array}$ & $\begin{array}{l}.00 \\
.00\end{array}$ & .00 & $\begin{array}{l}.00 \\
.01\end{array}$ & $\begin{array}{l}.06 \\
.07\end{array}$ & $\begin{array}{l}.24 \\
.17\end{array}$ & $\begin{array}{l}.30 \\
.24\end{array}$ & $\begin{array}{l}.30 \\
.25\end{array}$ & $\begin{array}{l}1.3 \\
1.1\end{array}$ & $\begin{array}{l}.050 \\
.060\end{array}$ \\
\hline 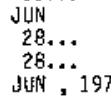 & $\begin{array}{l}1320 \\
1322\end{array}$ & $\begin{array}{l}1.0 \\
6.0\end{array}$ & $\begin{array}{l}.00 \\
.00\end{array}$ & .01 & $\begin{array}{l}.00 \\
.00\end{array}$ & $\begin{array}{l}.04 \\
.04\end{array}$ & $\begin{array}{l}.78 \\
.90\end{array}$ & $\begin{array}{l}.82 \\
.94\end{array}$ & $\begin{array}{l}.82 \\
.94\end{array}$ & $\begin{array}{l}3.6 \\
4.2\end{array}$ & $\begin{array}{r}.170 \\
. \quad .180 \\
\end{array}$ \\
\hline $\begin{array}{l}21 \ldots \\
21 \ldots\end{array}$ & $\begin{array}{l}1107 \\
1109\end{array}$ & $\begin{array}{l}1.0 \\
7.0\end{array}$ & .01 & $\begin{array}{l}.02 \\
.01\end{array}$ & $\begin{array}{l}.03 \\
.02\end{array}$ & .00 & $\begin{array}{l}.49 \\
.49\end{array}$ & $\begin{array}{l}.49 \\
.49\end{array}$ & $\begin{array}{r}.52 \\
.51\end{array}$ & $\begin{array}{l}2.3 \\
2.3\end{array}$ & $\begin{array}{r}.030 \\
.030\end{array}$ \\
\hline & & & & 28240009 & 96243500 & LINE 360 & SITE 03. & & & & \\
\hline DATE & TIME & $\begin{array}{c}\text { SAMP- } \\
\text { LIMG } \\
\text { DEPTH } \\
\text { (FT) }\end{array}$ & $\begin{array}{l}\text { HITRO- } \\
\text { GEN, } \\
\text { NITRRTE } \\
\text { TOTAL } \\
\text { (MG/L } \\
\text { AS N) }\end{array}$ & $\begin{array}{c}\text { NITRO- } \\
\text { GEN, } \\
\text { NITRITE } \\
\text { TOTAL } \\
\text { (MG } / \text { L } \\
\text { AS N }\end{array}$ & $\begin{array}{c}\text { NITRO- } \\
\text { GEN, } \\
\text { NO2+HO3 } \\
\text { TOTAL } \\
\text { (MG } / \mathrm{L} \\
\text { AS } \$ \text { ) }\end{array}$ & $\begin{array}{c}\text { AITRO- } \\
\text { GERS } \\
\text { AMMOHIA } \\
\text { TOTAL } \\
\text { (MG/L } \\
\text { AS N). }\end{array}$ & $\begin{array}{c}\text { MITRO- } \\
\text { GEN, } \\
\text { ORGANIC } \\
\text { TOTAL } \\
\text { (MGLL } \\
\text { AS N) }\end{array}$ & $\begin{array}{l}\text { NITRO- } \\
\text { GEN,AM- } \\
\text { MONIA + } \\
\text { ORGANIC } \\
\text { TOTAL } \\
\text { (NG/L } \\
\text { AS NS) }\end{array}$ & $\begin{array}{l}\text { NDITRD- } \\
\text { GEN, } \\
\text { TOTAL } \\
(M G / L \\
\text { RS K } \mathrm{K})\end{array}$ & $\begin{array}{l}\text { NITRO- } \\
\text { GEN, } \\
\text { TOTAL } \\
\text { (TMGL } \\
\text { AS NQ3) }\end{array}$ & $\begin{array}{l}\text { PHOS- } \\
\text { PHORUS, } \\
\text { TOTAL } \\
\text { (MG/L } \\
\text { AS P) }\end{array}$ \\
\hline JUN , 197 & & & & & & & & & & & \\
\hline $\begin{array}{l}21 \ldots \\
21 \ldots\end{array}$ & $\begin{array}{l}0930 \\
0934\end{array}$ & ${ }_{25}^{1.0}$ & $\begin{array}{l}.01 \\
.01\end{array}$ & $\begin{array}{l}.01 \\
.02\end{array}$ & $\begin{array}{l}.02 \\
.03\end{array}$ & .00 & $\begin{array}{l}.33 \\
.47\end{array}$ & $\begin{array}{l}.33 \\
.47\end{array}$ & $\begin{array}{l}.35 \\
.50\end{array}$ & $\begin{array}{l}1.5 \\
2.2\end{array}$ & $\begin{array}{l}.010 \\
.010\end{array}$ \\
\hline
\end{tabular}


(FT = feet; MICROMHOS = micromos per centimeter at, $25^{\circ}$ Celsius; MC/L = mi\}tigrals per 1 iter; AC,-FT = acre-feet $)$

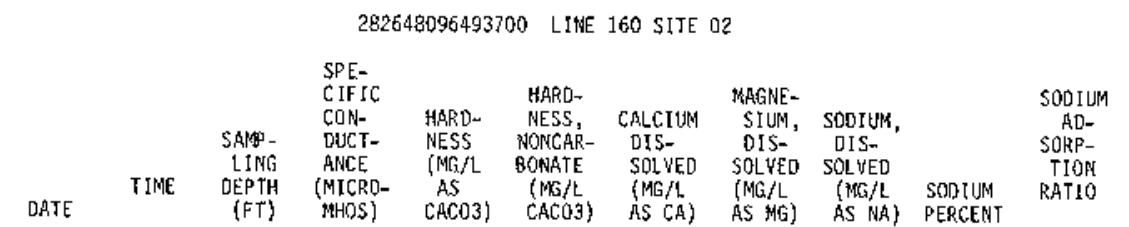

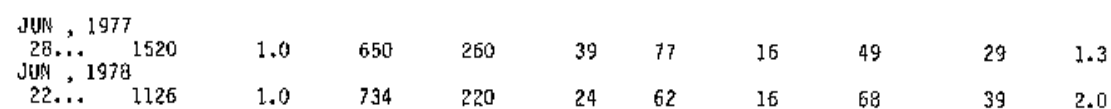

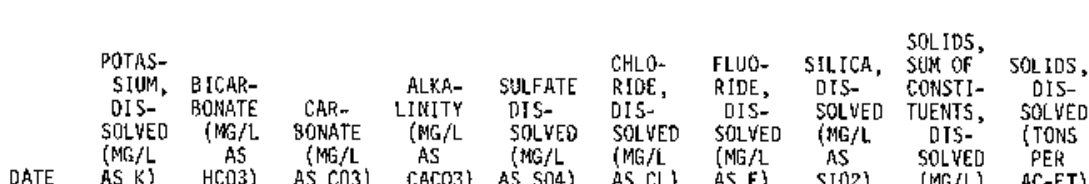

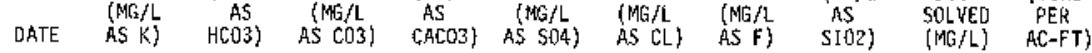

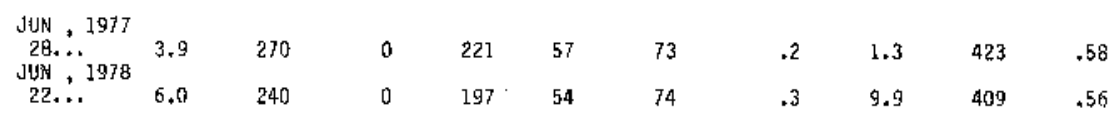

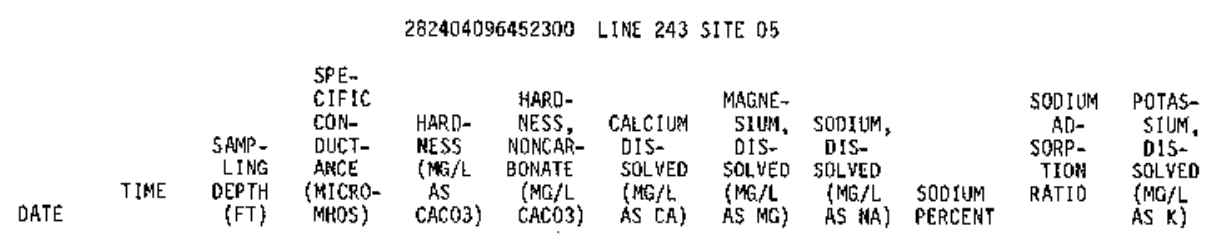

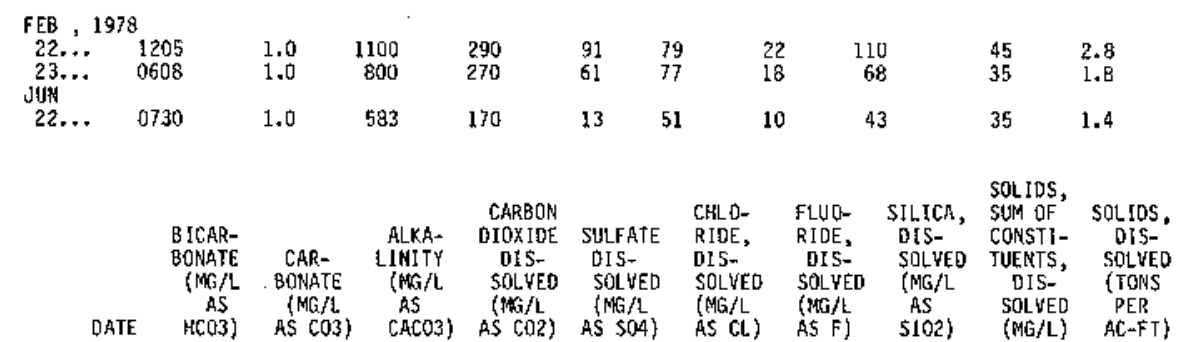

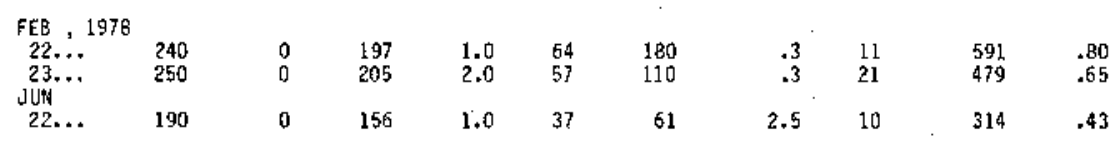

281933096421900 LINE 287 SITE 03

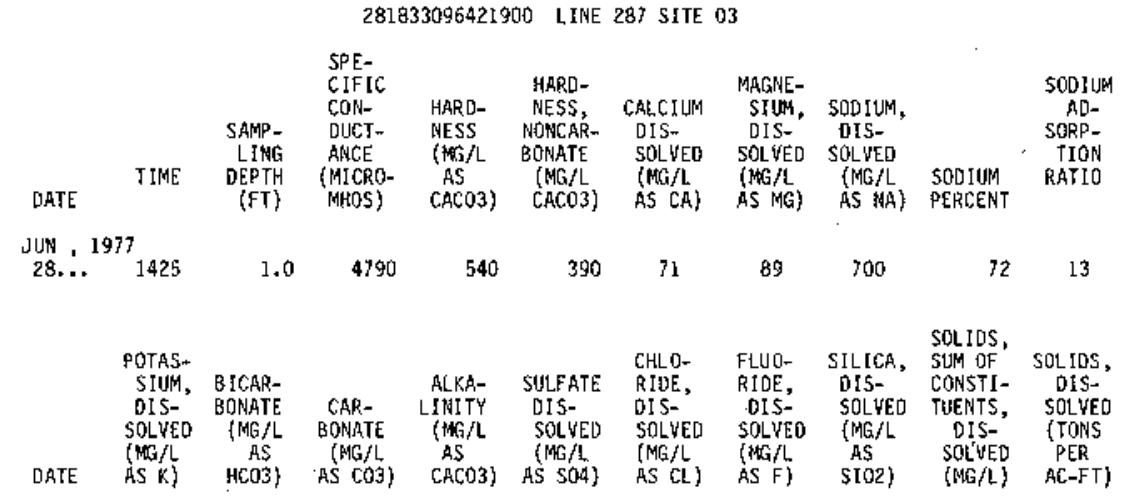

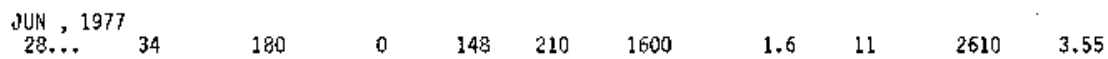


Table 7C.--Quality of water in the Guadalupe estuary, water years 1977-76--Cont inued Chemical Analyses--Cont inued

$281604096461300 \quad$ LINE 287 SITE 08

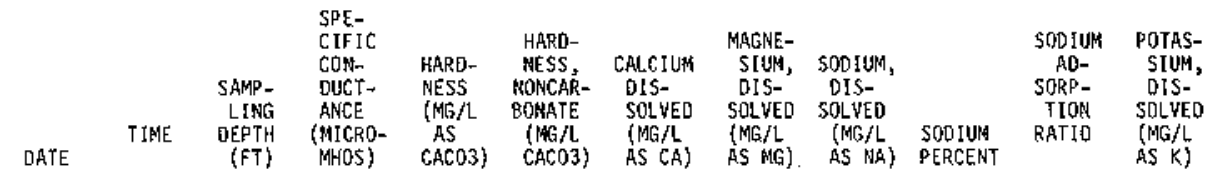

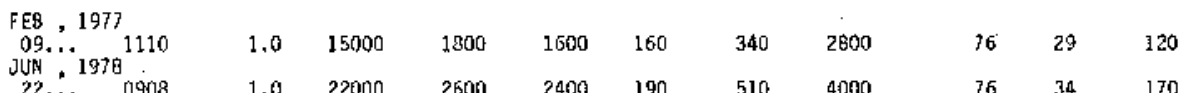

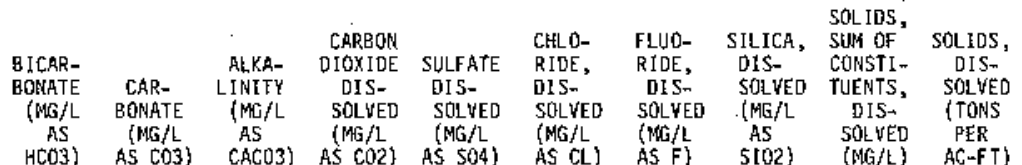

281715096384500 LINE 307 SITE 03

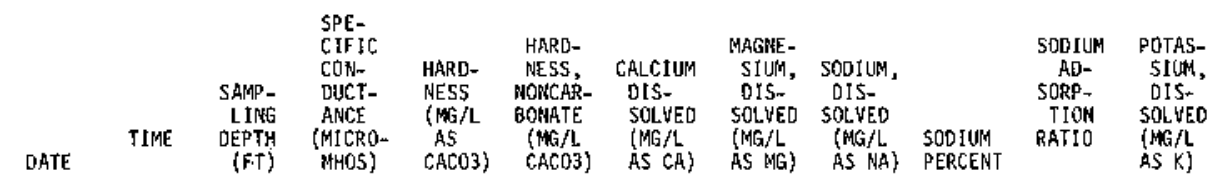

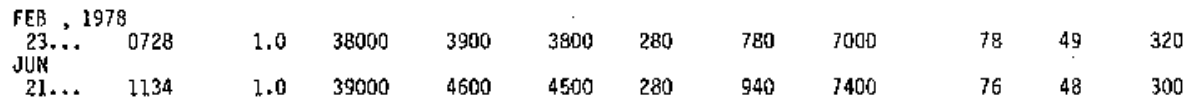

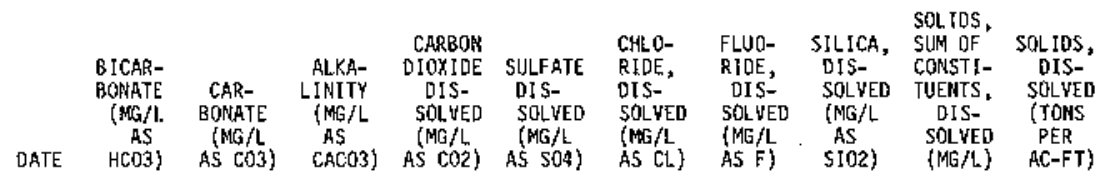

$\begin{array}{ccccccccccc}\text { FEB }, 1978 \\ 23 . . . & 160 & 0 & 131 & .8 & 1700 & 13000 & .8 & .1 & 23200 & 31.6 \\ \text { JUN } & & & & & & & & & & \\ 21 . . . & 140 & 0 & 115 & 1.1 & 1900 & 14000 & .8 & 4.2 & 24900 & 33.9\end{array}$

282021096331500 LINE 342 SITE 02

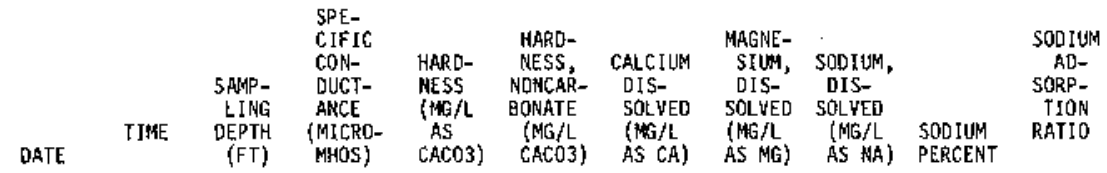

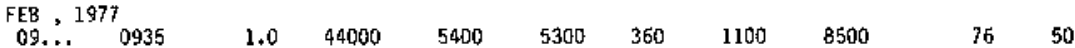

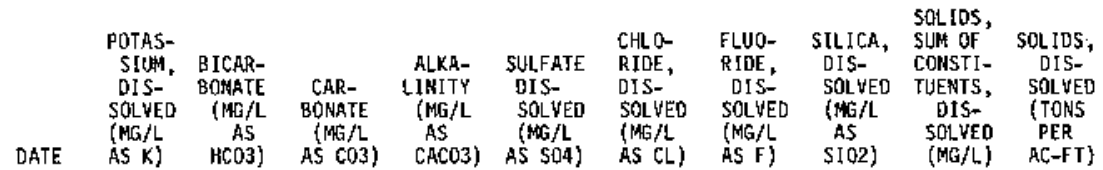
FEB, $197 \%$

$\begin{array}{llllllllll}09 . .{ }^{1977} 350 & 150 & 0 & 123 & 2300 & 15000 & .6 & .7 & 27700 & 37.7\end{array}$ 



\section{Mission-Aransas Estuary}

The Mission-Aransas estuary, which has an area of about 160 square miles $\left(415 \mathrm{~km}^{2}\right)$, consists of the tidal parts of Mission River, Aransas River, Copano Creek and other tributaries, Mission Bay, Copano Bay, Aransas Bay, St. Charles Bay, Carlos Bay, part of Redfish Bay, part of the Intracoastal Waterway, Lydia Ann Channel, and Aransas Pass (Figure 9). Water depth at mean low water is less than 2 feet $(0.6 \mathrm{~m})$ in Mission Bay; less than 8 feet $(2.4 \mathrm{~m})$ in Copano Bay; less than 13 feet $(4.0 \mathrm{~m})$ in Aransas Bay; less than 5 feet $(1.5 \mathrm{~m})$ in St. Charles Bay; 4 feet $(1.2 \mathrm{~m})$ or less in Carlos and Redfish Bays; about 15 feet $(4.6 \mathrm{~m})$ in the Intracoastal Waterway; about 20 feet ( 6.1 $\mathrm{m}$ ) in the Lydia Ann Channel; and more than 40 feet (12.2 m) in Aransas Pass.

Water-quality data (Table 8) were collected during February and June 1977 and January and June 1978.

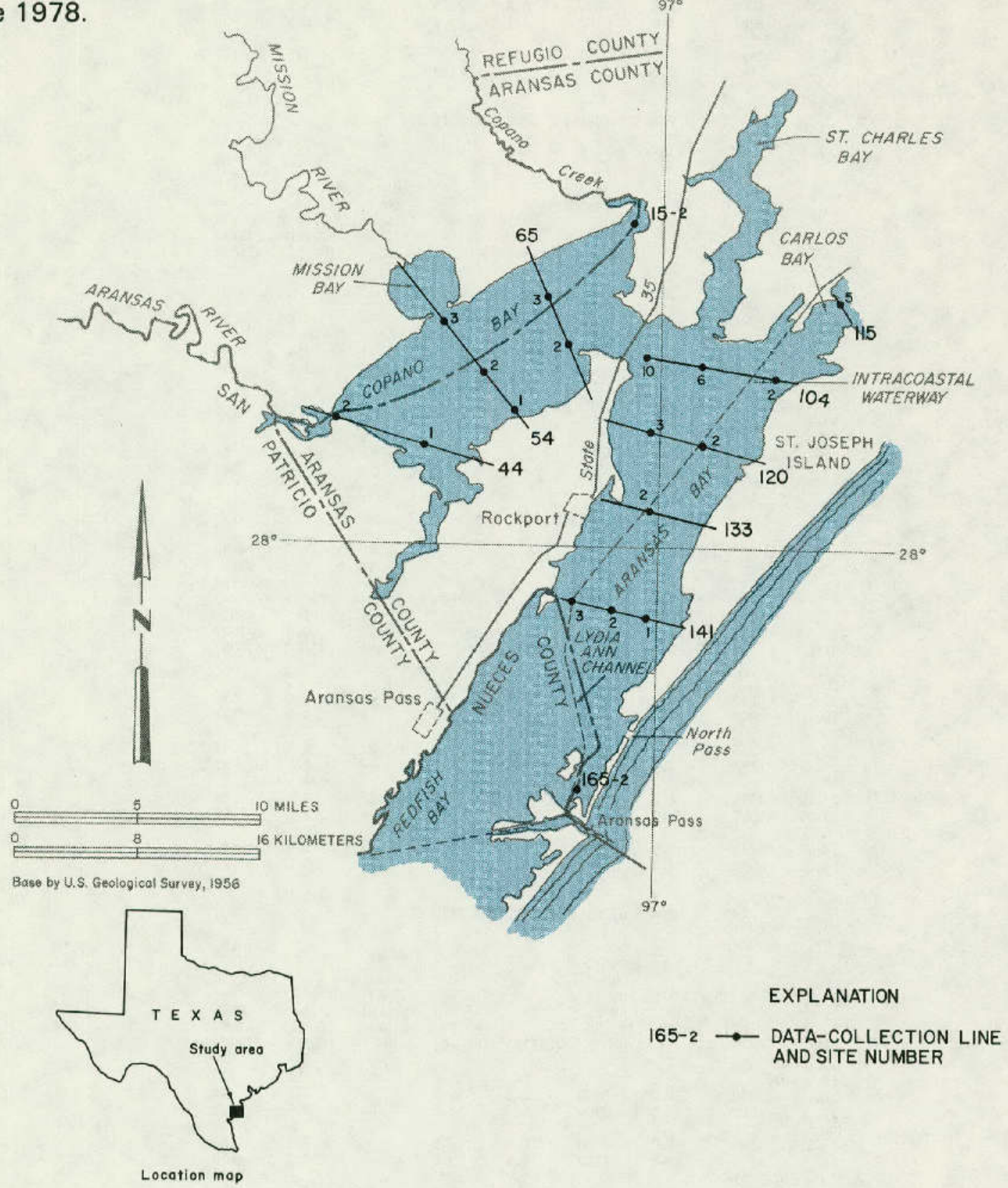

Figure 9.-Data-Collection Sites in the Mission-Aransas Estuary 
Table 8A.--Quality of water in the Mission-Aransas estuary, water years 1977-78 Field Determinations

(FT $=$ feet; MICROMHOS $=$ micromhos per cent ineter at $25^{\circ}$ Celsius; DEG $C=$ degrees Celsius; $M=$ meters; $\mathrm{MG} / \mathrm{L}=$ milligrans per liter)

281131097010500 LINE 015 SITE 02

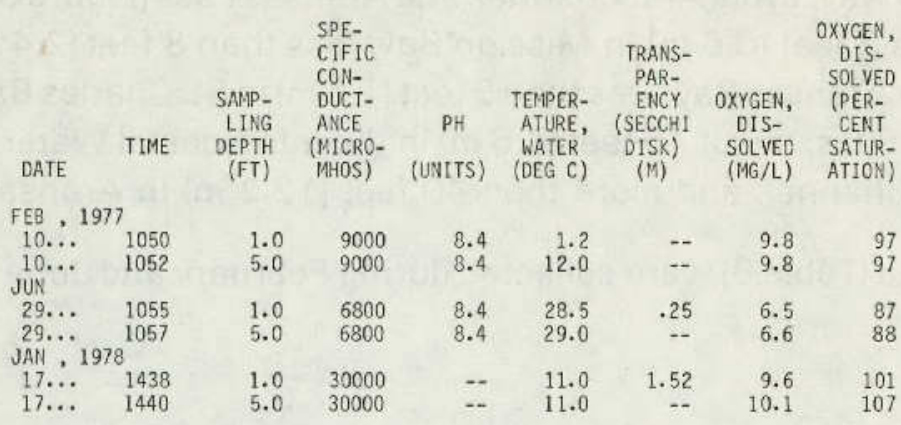

280331097092500 LINE 044 SITE 01

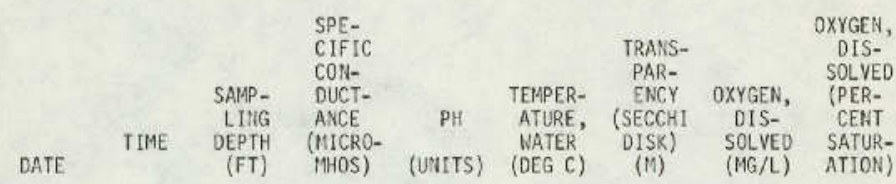

\begin{tabular}{|c|c|c|c|c|c|c|c|c|}
\hline & \multicolumn{8}{|c|}{ FEB , 1977} \\
\hline $10 \ldots$ & 1002 & 7.0 & 8000 & 8.2 & 11.5 & -. & 9.4 & 92 \\
\hline \multicolumn{9}{|l|}{ JUN } \\
\hline $29 . \ldots$ & 1240 & 1.0 & 6800 & 8.5 & 29.0 & .41 & 7.4 & \\
\hline $29 \ldots$ & 1242 & 7.5 & 6800 & 8.5 & 29.0 & -. & 7,4 & \\
\hline \multicolumn{8}{|c|}{ JAN , 1978} & \\
\hline $17 \ldots$ & 1302 & 1.0 & 26000 & -. & 10.5 & .40 & 9.5 & \\
\hline${ }_{\text {JUN }}^{17 . .}$ & 1304 & 7.0 & 26000 & -- & 10.5 & -- & 9.7 & \\
\hline \multicolumn{8}{|c|}{ JUN } & \\
\hline $14 \ldots$ & 0829 & 5.0 & 28000 & 8.2 & 30.0 & - & 5.8 & \\
\hline
\end{tabular}

280431097130300 LINE O44 SITE 02

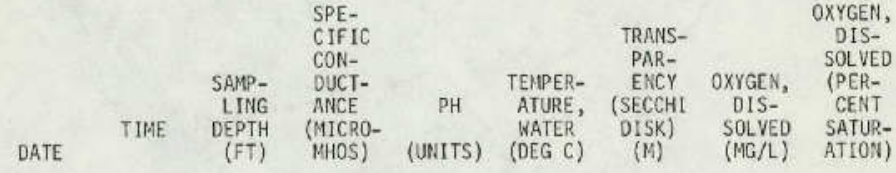

FEB, 19770940

$\begin{array}{lllllll}1.0 & 6000 & 8.3 & 11.5 & \ldots & 9.8 & 95 \\ 4.0 & 6000 & 8.2 & 11.5 & -- & 9.3 & 91 \\ 1.0 & 7000 & 8.6 & 29.0 & .22 & 6.9 & 92 \\ 4.5 & 6900 & 8.5 & 29.0 & -- & 6.8 & 91 \\ 1.0 & 26000 & \ldots & 10.5 & .19 & 9.5 & 98 \\ 4.0 & 27000 & -- & 10.0 & -- & 9.7 & 99 \\ 1.0 & 20000 & 8.2 & 31.5 & -- & 5.6 & 81 \\ 4.0 & 21000 & 8.2 & 31.5 & -- & 5.1 & 74\end{array}$

280452097054800 LINE 054 SITE 01

29... 1225

$29 \ldots 1227$

$\begin{array}{ll}17 \ldots & 1315 \\ 17 \ldots & 1317\end{array}$

JuII

$\begin{array}{ll}14 \ldots & 0838 \\ 14 \ldots & 0840\end{array}$

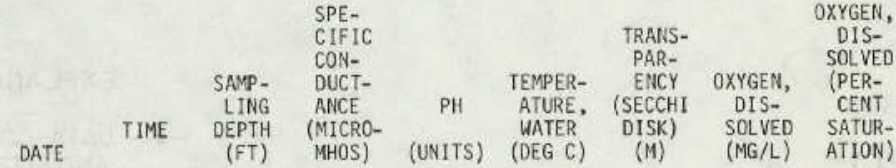

FEB , 1977

$1.0 \quad 9400$

$\begin{array}{lllll}8.3 & 11.5 & - & 9.6 & 94\end{array}$

$10 . . . \quad 1012$

$7.0 \quad 9000$

$8.3 \quad 11.5$

29... 1300

$29 \ldots \quad 1302$

JAN , $1978^{1302}$

$17 . . \quad 1232$

$17 \ldots \quad 1234$

$1.0 \quad 7400$

$7.5 \quad 7300$

$8.5 \quad 28.5$

1.030000

$\begin{array}{llll}29.0 & - & 7.5 & 100\end{array}$

$\begin{array}{ll}14 \ldots . & 0814 \\ 14 \ldots & 0816\end{array}$

7.029000

$=\quad 10.0 \quad .26$

$\begin{array}{ll}1.0 & 29300 \\ 5.0 & 30000\end{array}$

8.1
8.1

$\begin{array}{lll}10.5 & -- & 9.4\end{array}$

96

98

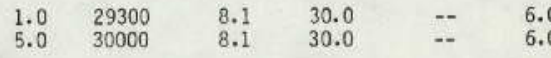


280632097070400 LIAdE 054 SITE 02

\begin{tabular}{|c|c|c|c|c|c|c|c|c|}
\hline DATE & TIME & $\begin{array}{l}\text { SAMP- } \\
\text { LING } \\
\text { DEPTH } \\
\text { (F.T) }\end{array}$ & $\begin{array}{l}\text { SPE- } \\
\text { CIFIC } \\
\text { CON- } \\
\text { OULT- } \\
\text { ANCE } \\
\text { (MICRO- } \\
\text { MHOS) }\end{array}$ & $\begin{array}{c}\text { PAI } \\
\text { (UNITS) }\end{array}$ & $\begin{array}{l}\text { TEMPER- } \\
\text { ATURE, } \\
\text { WATER } \\
\text { (OEG C) }\end{array}$ & $\begin{array}{c}\text { TRANS- } \\
\text { PAR- } \\
\text { ENCY } \\
\text { (SECCHI } \\
\text { OISK) } \\
\text { (M) }\end{array}$ & $\begin{array}{c}\text { OXYGEN, } \\
\text { O1S } \\
\text { SOLVED } \\
\text { (MG } / L)\end{array}$ & $\begin{array}{l}\text { OXYGER, } \\
\text { DIS- } \\
\text { SOLUED } \\
\text { (PER- } \\
\text { CEEST } \\
\text { SATUR- } \\
\text { AT ION) }\end{array}$ \\
\hline \multicolumn{9}{|c|}{ FEB, 1977} \\
\hline $10 . .$. & 1020 & 1.0 & 6000 & 8.2 & 11.5 & $=$ & 9.7 & \\
\hline $10 . .$. & 1022 & 7.0 & 8000 & & & - & 8.9 & \\
\hline $\begin{array}{l}29 \ldots \\
29 \ldots\end{array}$ & $\begin{array}{l}1150 \\
1152\end{array}$ & $\begin{array}{l}1.0 \\
7.5\end{array}$ & $\begin{array}{l}6100 \\
6100\end{array}$ & $\begin{array}{l}8.4 \\
8.4\end{array}$ & $\begin{array}{l}28.5 \\
28.5\end{array}$ & $\begin{array}{r}.20 \\
--\end{array}$ & $\begin{array}{l}7.1 \\
6.8\end{array}$ & \\
\hline \multicolumn{9}{|c|}{ JAN, $197 B$} \\
\hline $17 \ldots$ & 1355 & 1.0 & 23000 & -- & 10.5 & .36 & 9.5 & \\
\hline$\underset{\text { JUN }}{17 . . .}$ & 1357 & 7.0 & 28000 & $\cdots$ & 10.0 & -- & 9.7 & \\
\hline $\begin{array}{l}14 \ldots \\
14 \ldots\end{array}$ & $\begin{array}{l}0850 \\
0852\end{array}$ & $\begin{array}{l}1.0 \\
6.0\end{array}$ & $\begin{array}{l}25000 \\
26000\end{array}$ & $\begin{array}{l}8.2 \\
8.1\end{array}$ & $\begin{array}{l}29.5 \\
29.5\end{array}$ & $=$ & $\begin{array}{l}6.0 \\
6.0\end{array}$ & \\
\hline
\end{tabular}

280754097094000 LIIHE 054 SITE, 03

\begin{tabular}{|c|c|c|c|c|c|c|c|c|}
\hline DÁTE & TIHE & $\begin{array}{l}\text { SAMP- } \\
\text { LING } \\
\text { DEPTH } \\
(F T)\end{array}$ & $\begin{array}{l}\text { SPE- } \\
\text { CIFIC } \\
\text { CON- } \\
\text { OUCT- } \\
\text { ANCE } \\
\text { (MICRO- } \\
\text { MHOS) }\end{array}$ & $\begin{array}{c}\text { PH } \\
\text { (UNITS) }\end{array}$ & $\begin{array}{l}\text { TEMPER- } \\
\text { ATURE, } \\
\text { WRTER } \\
\text { (DEG C) }\end{array}$ & $\begin{array}{l}\text { TRAAS- } \\
\text { PAR- } \\
\text { ENCY } \\
\text { (SECCHI } \\
\text { DISK) } \\
\text { (M) }\end{array}$ & $\begin{array}{c}\text { OXYGEN, } \\
\text { DIS- } \\
\text { SOLVED } \\
\text { (MG } / L)\end{array}$ & $\begin{array}{c}\text { OXYGEN, } \\
\text { DIS- } \\
\text { SOLYED } \\
\text { (PER- } \\
\text { CENT } \\
\text { SATUR- } \\
\text { ATION) }\end{array}$ \\
\hline \multicolumn{9}{|c|}{ JEW , 1977} \\
\hline $\begin{array}{r}29 \ldots \\
29 \ldots \\
\text { JAN, }\end{array}$ & $\begin{array}{l}1200 \\
1202\end{array}$ & $\begin{array}{l}1.0 \\
6.0\end{array}$ & $\begin{array}{l}4800 \\
5100\end{array}$ & $\begin{array}{l}8.4 \\
8.4\end{array}$ & $\begin{array}{l}28.5 \\
28.5\end{array}$ & .15 & $\begin{array}{l}G .6 \\
6.5\end{array}$ & $\begin{array}{l}88 \\
87\end{array}$ \\
\hline $\begin{array}{l}17 \ldots \\
17 \ldots \\
\text { JUN }\end{array}$ & $\begin{array}{l}1345 \\
1347\end{array}$ & $\begin{array}{l}1.0 \\
6.0\end{array}$ & $\begin{array}{l}28000 \\
29000\end{array}$ & $=$ & $\begin{array}{l}10.5 \\
10.5\end{array}$ & $\begin{array}{r}.56 \\
--\end{array}$ & $\begin{array}{r}9.9 \\
10.0\end{array}$ & $\begin{array}{l}102 \\
104\end{array}$ \\
\hline $\begin{array}{l}14 \ldots \\
14 \ldots\end{array}$ & $\begin{array}{l}0857 \\
0859\end{array}$ & $\begin{array}{l}1.0 \\
4.0\end{array}$ & $\begin{array}{l}23000 \\
25000\end{array}$ & $\begin{array}{l}8.1 \\
8.1\end{array}$ & $\begin{array}{l}30.5 \\
30.5\end{array}$ & $m$ & $\begin{array}{l}5.4 \\
5.2\end{array}$ & $\begin{array}{l}79 \\
76\end{array}$ \\
\hline
\end{tabular}

280727097032700 LINE 065 SITE Q2

\begin{tabular}{|c|c|c|c|c|c|c|c|c|}
\hline DATE & TIME & $\begin{array}{c}\text { SAMP- } \\
\text { LING } \\
\text { DEPTH } \\
\text { (FT) }\end{array}$ & $\begin{array}{l}\text { SPE- } \\
\text { CIF IC } \\
\text { CON- } \\
\text { OUCT- } \\
\text { ANCE } \\
\text { (MICRO- } \\
\text { MHOS) }\end{array}$ & $\begin{array}{c}\mathrm{PH} \\
\text { (UNuts) }\end{array}$ & $\begin{array}{c}\text { TEMPER- } \\
\text { ATURE, } \\
\text { UATER } \\
\text { (DEG C) }\end{array}$ & $\begin{array}{l}\text { TRAAHS- } \\
\text { PAR- } \\
\text { EASCY } \\
\text { (SECCHI } \\
\text { DISK) } \\
\text { (M) }\end{array}$ & $\begin{array}{c}\text { OXYGEA, } \\
\text { OHIS- } \\
\text { SOLLWED } \\
\text { (MG/L) }\end{array}$ & $\begin{array}{l}\text { OXYGEN, } \\
\text { DIS- } \\
\text { SOLYED } \\
\text { (PER- } \\
\text { CENT } \\
\text { SATUR- } \\
\text { ATION) }\end{array}$ \\
\hline FEB , & & & & & & & & \\
\hline $\begin{array}{l}10 \ldots \\
10 \ldots \\
\text { JUN }\end{array}$ & $\begin{array}{l}1120 \\
1122\end{array}$ & $\begin{array}{l}1.0 \\
7.0\end{array}$ & $\begin{array}{l}14000 \\
13000\end{array}$ & $\begin{array}{l}8.3 \\
8.4\end{array}$ & $\begin{array}{l}12.0 \\
11.9\end{array}$ & $=$ & $\begin{array}{l}9.4 \\
9.2\end{array}$ & $\begin{array}{l}95 \\
92\end{array}$ \\
\hline $\begin{array}{l}29 . . \\
29 . \\
\text { JAN }\end{array}$ & $\begin{array}{l}1320 \\
1322 \\
8^{1322}\end{array}$ & $\begin{array}{l}1.0 \\
9.0\end{array}$ & $\begin{array}{l}8600 \\
8600\end{array}$ & $\begin{array}{l}8.4 \\
8.4\end{array}$ & $\begin{array}{l}28.5 \\
29.0\end{array}$ & $\begin{array}{r}-39 \\
--\end{array}$ & $\begin{array}{l}7.1 \\
7.0\end{array}$ & $\begin{array}{l}95 \\
95\end{array}$ \\
\hline $\begin{array}{l}17 . \cdots \\
17 \ldots\end{array}$ & $\begin{array}{l}1218 \\
1220\end{array}$ & $\begin{array}{l}1.0 \\
7.0\end{array}$ & $\begin{array}{l}30000 \\
30000\end{array}$ & $=$ & $\begin{array}{l}10.5 \\
10.0\end{array}$ & .26 & $\begin{array}{l}9.1 \\
9.4\end{array}$ & $\begin{array}{l}94 \\
97\end{array}$ \\
\hline $\begin{array}{l}14 \ldots \\
14 \ldots\end{array}$ & $\begin{array}{l}0920 \\
0922\end{array}$ & $\begin{array}{l}1.0 \\
8.0\end{array}$ & $\begin{array}{l}29000 \\
31000\end{array}$ & $\begin{array}{l}8.1 \\
8.0\end{array}$ & $\begin{array}{l}30.0 \\
30.0\end{array}$ & -- & $\begin{array}{l}5.7 \\
5.1\end{array}$ & $\begin{array}{l}85 \\
77\end{array}$ \\
\hline
\end{tabular}

280847097034700 LIRE 065 5ITE 03

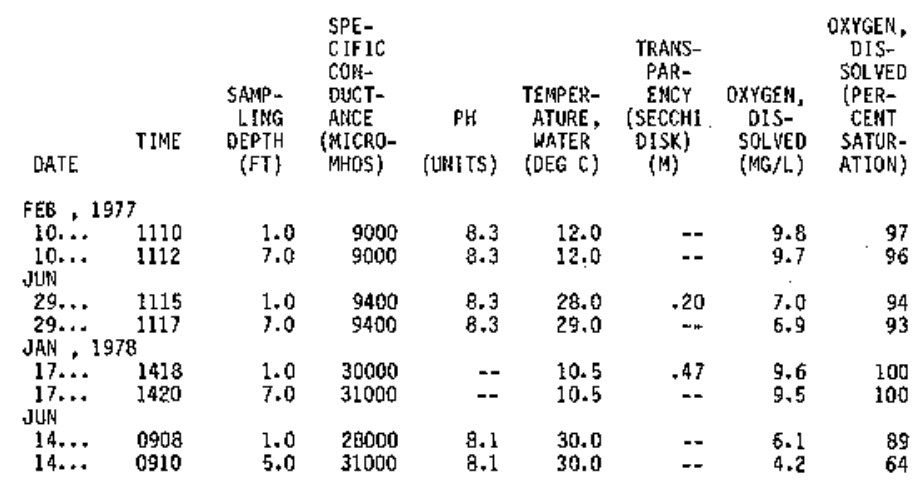


230608096551700 LINE 104 SITE Q2

\begin{tabular}{|c|c|c|c|c|c|c|c|c|}
\hline DATE & THE & $\begin{array}{l}\text { SAMP- } \\
\text { LING } \\
\text { DEPTH } \\
\text { (FT) }\end{array}$ & $\begin{array}{l}\text { SPE- } \\
\text { CIFIC } \\
\text { CON- } \\
\text { OUCT- } \\
\text { ANCE } \\
\text { (MICRO- } \\
\text { MHOS) }\end{array}$ & $\begin{array}{c}\mathrm{PH} \\
\text { (UNITS) }\end{array}$ & $\begin{array}{l}\text { TEMPER- } \\
\text { ATURE, } \\
\text { WATER } \\
\text { \{DEG C }\end{array}$ & $\begin{array}{c}\text { TRANS- } \\
\text { PAR- } \\
\text { ENCY } \\
\text { (SECCHI } \\
\text { OISK) } \\
\text { (M) }\end{array}$ & $\begin{array}{c}\text { OXYGEN, } \\
\text { DIS- } \\
\text { SOLYED } \\
\text { (NEG } / L)\end{array}$ & $\begin{array}{c}\text { OXYGEN, } \\
\text { DIS- } \\
\text { SOLUED } \\
\text { (PER- } \\
\text { CENT } \\
\text { SRTUR- } \\
\text { ATIONI) }\end{array}$ \\
\hline $\mathrm{FEB}, 197 \mathrm{~J}$ & & & & & & & & \\
\hline og... & $\begin{array}{l}1320 \\
1322\end{array}$ & $\begin{array}{l}1.0 \\
3.0\end{array}$ & $\begin{array}{l}12000 \\
12000\end{array}$ & $\begin{array}{l}8.4 \\
8.4\end{array}$ & $\begin{array}{l}11.0 \\
11.0\end{array}$ & $\begin{array}{r}.20 \\
--\end{array}$ & $\begin{array}{l}11+1 \\
11.1\end{array}$ & $\begin{array}{l}108 \\
108\end{array}$ \\
\hline $\begin{array}{l}29 \ldots \\
29 \ldots \\
\text { JAN, } 1970\end{array}$ & $\begin{array}{l}1040 \\
1042\end{array}$ & $\begin{array}{l}1.0 \\
4.0\end{array}$ & $\begin{array}{l}17000 \\
17000\end{array}$ & $\begin{array}{l}8.5 \\
8.5\end{array}$ & $\begin{array}{l}29.0 \\
29.0\end{array}$ & .39 & $\begin{array}{l}7.0 \\
6.9\end{array}$ & $\begin{array}{l}97 \\
96\end{array}$ \\
\hline $\begin{array}{l}17 \ldots \\
17 \ldots\end{array}$ & $\begin{array}{l}1242 \\
1244\end{array}$ & $\begin{array}{l}1.0 \\
4.0\end{array}$ & $\begin{array}{l}34000 \\
34000\end{array}$ & $\begin{array}{l}8.0 \\
8.1\end{array}$ & $\begin{array}{l}10.5 \\
10.5\end{array}$ & .87 & $\begin{array}{l}9.7 \\
9.7\end{array}$ & $\begin{array}{l}104 \\
104\end{array}$ \\
\hline $\begin{array}{l}14 \ldots \\
14 \ldots\end{array}$ & $\begin{array}{l}0830 \\
0832\end{array}$ & $\begin{array}{l}1.0 \\
5.0\end{array}$ & $\begin{array}{l}40000 \\
40000\end{array}$ & $\begin{array}{l}8.0 \\
8.0\end{array}$ & $\begin{array}{l}30.5 \\
30.5\end{array}$ & .37 & $\begin{array}{l}6.1 \\
6.1\end{array}$ & $\begin{array}{l}95 \\
95\end{array}$ \\
\hline
\end{tabular}

280633096581200 LINE 1045 ITE O6

\begin{tabular}{|c|c|c|c|c|c|c|c|c|}
\hline DATE & TIIAE & $\begin{array}{c}\text { SAHP- } \\
\text { LING } \\
\text { DEPTH } \\
\text { (FT) }\end{array}$ & $\begin{array}{l}\text { SPE- } \\
\text { CIFIC } \\
\text { CON- } \\
\text { DUCT- } \\
\text { ANCE } \\
\text { (MICRO- } \\
\text { MHOS) }\end{array}$ & $\begin{array}{c}\mathrm{PH} \\
\text { (UNITS) }\end{array}$ & $\begin{array}{l}\text { TEPAPER- } \\
\text { RTURE, } \\
\text { WATER } \\
\text { (DEG C) }\end{array}$ & $\begin{array}{l}\text { TRARS- } \\
\text { PAR- } \\
\text { EHCY } \\
\text { (SECCHI } \\
\text { DISK) } \\
\text { (M) }\end{array}$ & $\begin{array}{c}\text { OXYGEH, } \\
\text { DIS- } \\
\text { SOLWED } \\
\text { (MG } / L)\end{array}$ & $\begin{array}{c}\text { OXYGEN, } \\
\text { DIS- } \\
\text { SOL YED } \\
\text { (PER- } \\
\text { CENT } \\
\text { SATUR- } \\
\text { ATYOH') }\end{array}$ \\
\hline \multicolumn{9}{|c|}{$F E B ， 1977$} \\
\hline $\begin{array}{l}09 . . \\
09 . \\
\text { Jus }\end{array}$ & $\begin{array}{l}1345 \\
1347\end{array}$ & $\begin{array}{l}1.0 \\
6.0\end{array}$ & $\begin{array}{l}12000 \\
12000\end{array}$ & $\begin{array}{l}8.4 \\
8.4\end{array}$ & $\begin{array}{l}11.5 \\
11.5\end{array}$ & $\begin{array}{r}.30 \\
-.\end{array}$ & $\begin{array}{l}11.0 \\
10.8\end{array}$ & $\begin{array}{l}109 \\
107\end{array}$ \\
\hline $\begin{array}{r}29 . . \\
29 . \\
\text { JAN, }\end{array}$ & $\begin{array}{l}1030 \\
8^{1032}\end{array}$ & $\begin{array}{l}1.0 \\
\mathbf{5 . 0}\end{array}$ & $\begin{array}{l}15000 \\
14000\end{array}$ & $\begin{array}{l}8.4 \\
8.4\end{array}$ & $\begin{array}{l}28.5 \\
28.5\end{array}$ & .11 & $\begin{array}{l}7.3 \\
7.1\end{array}$ & $\begin{array}{r}100 \\
97\end{array}$ \\
\hline $\begin{array}{l}17 . . \\
17 . \\
\text { JUN }\end{array}$ & $\begin{array}{l}1232 \\
1234\end{array}$ & $\begin{array}{l}1.0 \\
6.0\end{array}$ & $\begin{array}{l}30000 \\
31000\end{array}$ & $\begin{array}{l}8.2 \\
8.2\end{array}$ & $\begin{array}{l}11.0 \\
11.0\end{array}$ & .86 & $\begin{array}{l}10.2 \\
10.1\end{array}$ & $\begin{array}{l}107 \\
107\end{array}$ \\
\hline $\begin{array}{l}14 . . \\
14 . .\end{array}$ & $\begin{array}{l}0818 \\
0820\end{array}$ & $\begin{array}{l}1.0 \\
6.0\end{array}$ & $\begin{array}{l}37000 \\
40000\end{array}$ & $\begin{array}{l}8.2 \\
8.2\end{array}$ & $\begin{array}{l}30.0 \\
29.5\end{array}$ & $\begin{array}{r}.81 \\
--\end{array}$ & $\begin{array}{l}5.9 \\
5.4\end{array}$ & $\begin{array}{l}91 \\
83\end{array}$ \\
\hline
\end{tabular}

280653097003300 LIRE 104 SITE 10

\begin{tabular}{|c|c|c|c|c|c|c|c|c|}
\hline DATE & TIME & $\begin{array}{c}\text { SAPMP- } \\
\text { LIPG } \\
\text { DEPTH } \\
\text { (FT) }\end{array}$ & $\begin{array}{l}\text { SPE- } \\
\text { CIFIC } \\
\text { CDH- } \\
\text { DUCT- } \\
\text { AMCE } \\
\text { (AICRO- } \\
\text { PIKOS) }\end{array}$ & $\begin{array}{c}P H \\
\text { (UNITS) }\end{array}$ & $\begin{array}{l}\text { TEMPER- } \\
\text { ATURE, } \\
\text { WRIER } \\
\text { (DEG C) }\end{array}$ & $\begin{array}{l}\text { TRANSS- } \\
\text { PAR- } \\
\text { ENCY } \\
\text { (SECCHI } \\
\text { DISX) } \\
\text { (M) }\end{array}$ & $\begin{array}{c}\text { OXYGEN, } \\
\text { DIS- } \\
\text { SOEVED } \\
\text { (HG/L) }\end{array}$ & $\begin{array}{l}\text { OXYGEN, } \\
\text { OIS- } \\
\text { SDLVED } \\
\text { (PER- } \\
\text { CENT } \\
\text { SATUR- } \\
\text { ATION) }\end{array}$ \\
\hline \multicolumn{9}{|c|}{ FEG , 1977} \\
\hline $\begin{array}{l}09 . . . \\
09 . . \\
\text { JUN }\end{array}$ & $\begin{array}{l}1355 \\
1357\end{array}$ & $\begin{array}{l}1.0 \\
7.0\end{array}$ & $\begin{array}{l}14000 \\
14000\end{array}$ & $\begin{array}{l}8.3 \\
8.3\end{array}$ & $\begin{array}{l}11.5 \\
11.5\end{array}$ & .35 & $\begin{array}{l}10.8 \\
10.6\end{array}$ & $\begin{array}{l}108 \\
106\end{array}$ \\
\hline $\begin{array}{l}29 \ldots . . \\
29 . \ldots \\
29 \ldots \\
\text { JAN . }\end{array}$ & $\begin{array}{l}0945 \\
0947 \\
0949\end{array}$ & $\begin{array}{l}1.0 \\
4.0 \\
7.0\end{array}$ & $\begin{array}{l}13000 \\
13000 \\
13000\end{array}$ & $\begin{array}{l}8.3 \\
8.3 \\
8.3\end{array}$ & $\begin{array}{l}28.5 \\
28.5 \\
28.5\end{array}$ & $\begin{array}{r}.29 \\
-- \\
--\end{array}$ & $\begin{array}{l}7.0 \\
7.0 \\
7.0\end{array}$ & $\begin{array}{l}95 \\
95 \\
95\end{array}$ \\
\hline $\begin{array}{l}17 \ldots \\
17 \ldots \\
170\end{array}$ & $\begin{array}{l}1224 \\
1226 \\
1228\end{array}$ & $\begin{array}{l}1.0 \\
5.0 \\
7.0\end{array}$ & $\begin{array}{l}29000 \\
29000 \\
28000\end{array}$ & $\begin{array}{l}8.0 \\
8.1 \\
8.1\end{array}$ & $\begin{array}{l}11.5 \\
11.5 \\
11.5\end{array}$ & $\begin{array}{r}.74 \\
-- \\
--\end{array}$ & $\begin{array}{l}9.6 \\
9.7 \\
9.8\end{array}$ & $\begin{array}{l}102 \\
103 \\
103\end{array}$ \\
\hline $\begin{array}{l}14 \ldots \\
14 \ldots \\
14 \ldots\end{array}$ & $\begin{array}{l}0806 \\
0808 \\
0810\end{array}$ & $\begin{array}{l}1.0 \\
5.0 \\
9.0\end{array}$ & $\begin{array}{l}24000 \\
23000 \\
25000\end{array}$ & $\begin{array}{l}8.2 \\
8.2 \\
8.2\end{array}$ & $\begin{array}{l}32.0 \\
29.5 \\
29.5\end{array}$ & $\begin{array}{l}.70 \\
--\end{array}$ & $\begin{array}{l}6.1 \\
6.1 \\
6.0\end{array}$ & $\begin{array}{l}88 \\
87 \\
87\end{array}$ \\
\hline
\end{tabular}

280755096533100 LIHE 115 SITE 05

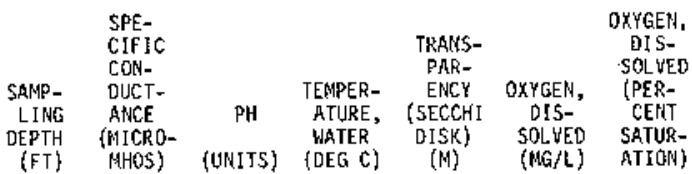

FEB , 1977

\begin{tabular}{|c|c|c|c|c|c|c|c|c|}
\hline $\begin{array}{l}09 . \ldots \\
09 \ldots\end{array}$ & $\begin{array}{l}1310 \\
1312\end{array}$ & $\begin{array}{l}1.0 \\
3.0\end{array}$ & $\begin{array}{l}14000 \\
14000\end{array}$ & $\begin{array}{l}8.4 \\
8.4\end{array}$ & $\begin{array}{l}11.5 \\
11.5\end{array}$ & .12 & $\begin{array}{l}10.8 \\
10.9\end{array}$ & $\begin{array}{l}108 \\
109\end{array}$ \\
\hline $\begin{array}{l}29 \ldots \\
29 \ldots \\
\text { JAN , } 1978\end{array}$ & $\begin{array}{l}1050 \\
1052\end{array}$ & $\begin{array}{l}1.0 \\
3.0\end{array}$ & $\begin{array}{l}19000 \\
19000\end{array}$ & $\begin{array}{l}8.3 \\
8.3\end{array}$ & $\begin{array}{l}28.5 \\
28.6\end{array}$ & $\begin{array}{r}.22 \\
--\end{array}$ & $\begin{array}{l}6.7 \\
6.6\end{array}$ & $\begin{array}{l}94 \\
92\end{array}$ \\
\hline $\begin{array}{l}17 \ldots \\
17 \ldots \\
\text { נun }\end{array}$ & $\begin{array}{l}1258 \\
1300\end{array}$ & $\begin{array}{l}1.0 \\
3.0\end{array}$ & $\begin{array}{l}33000 \\
33000\end{array}$ & $\begin{array}{l}8.1 \\
8.0\end{array}$ & $\begin{array}{l}11.5 \\
10.1\end{array}$ & .58 & $\begin{array}{l}9.4 \\
9.4\end{array}$ & $\begin{array}{l}104 \\
104\end{array}$ \\
\hline $\begin{array}{l}14 \ldots \\
14 \ldots\end{array}$ & $\begin{array}{l}0845 \\
0847\end{array}$ & $\begin{array}{l}1.0 \\
3.0\end{array}$ & $\begin{array}{l}38100 \\
39000\end{array}$ & $\begin{array}{l}8.1 \\
8.1\end{array}$ & $\begin{array}{l}30.5 \\
30.0\end{array}$ & $\begin{array}{r}.38 \\
-.\end{array}$ & $\begin{array}{l}5.8 \\
5.8\end{array}$ & $\begin{array}{l}91 \\
91\end{array}$ \\
\hline
\end{tabular}


280426096593500 LIRE 120 SITE 02

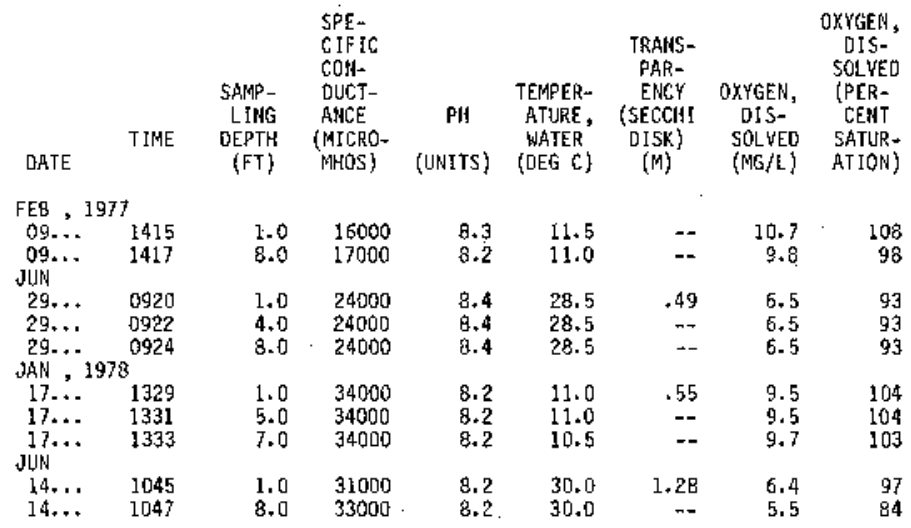

280458097004800 LINE 120 SITE 03

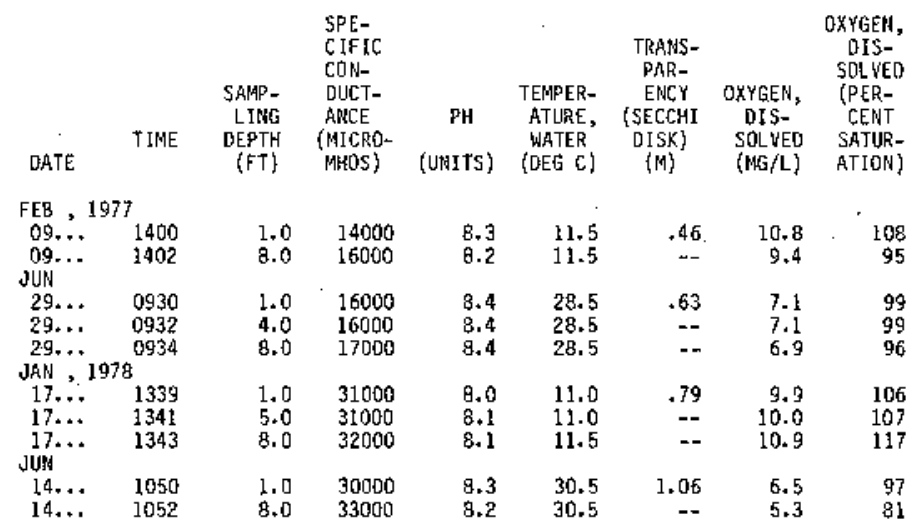

280132097002200 LIHE 133 SITE 02

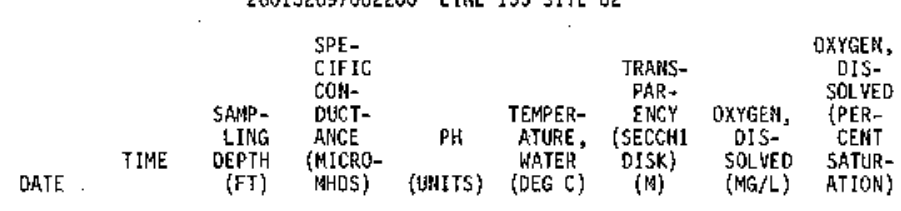

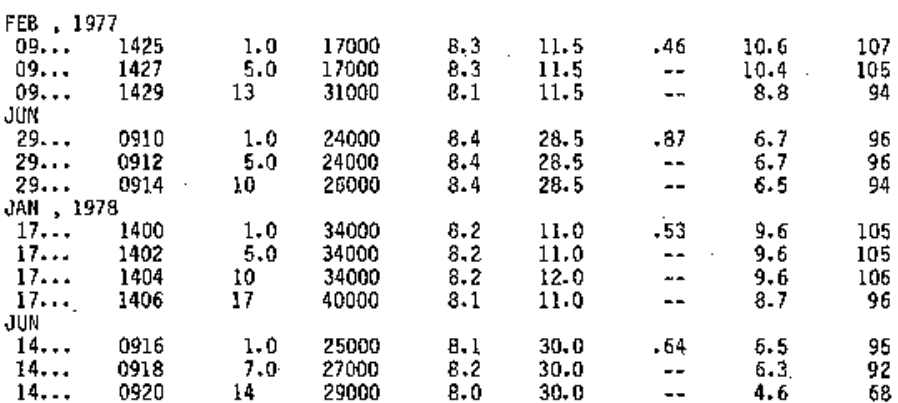


Table 8n.--Quality of water in the Mission-Aransas estuary, water years 1977-78--Continued Field Deterninations--Continued

27572309700130D LINE 141 SITE OH

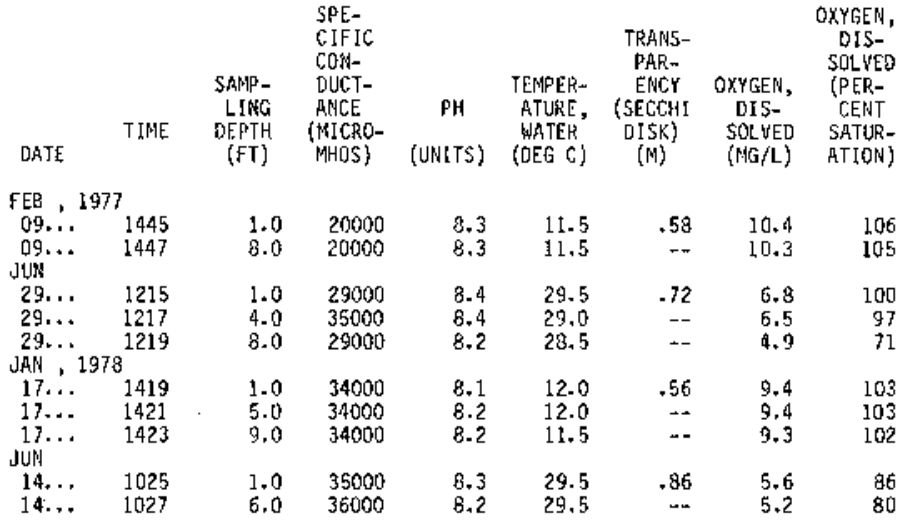

275750097015400 LINE 141 SITE 02

\begin{tabular}{|c|c|c|c|c|c|c|c|c|}
\hline DATE & TIME & $\begin{array}{c}\text { SAMP- } \\
\text { LING } \\
\text { DEPTH } \\
\text { (FT) }\end{array}$ & $\begin{array}{l}\text { SPE- } \\
\text { CIFIC } \\
\text { CON- } \\
\text { DUCT- } \\
\text { AUSCE } \\
\text { (AICRO- } \\
\text { MHOS) }\end{array}$ & $\begin{array}{c}\mathrm{PH} \\
\text { (UNITS) }\end{array}$ & $\begin{array}{l}\text { TEMPER- } \\
\text { ATURE, } \\
\text { WATER } \\
\text { (DEG C) }\end{array}$ & $\begin{array}{l}\text { TRANS- } \\
\text { PAR - } \\
\text { ENCY } \\
\text { (SECCHI } \\
\text { DISK) } \\
\text { (M) }\end{array}$ & $\begin{array}{c}\text { OXYGEK, } \\
\text { DIS- } \\
\text { SOLLED } \\
\text { (MG } / \mathrm{L})\end{array}$ & $\begin{array}{c}\text { OXYGEN, } \\
\text { DIS- } \\
\text { SOLUEO } \\
\text { (PER- } \\
\text { CENT } \\
\text { SATUR- } \\
\text { ATION) }\end{array}$ \\
\hline \multicolumn{9}{|c|}{ FEB , 1977} \\
\hline $\begin{array}{l}09 . . . \\
09 . . . \\
\text { JuN }\end{array}$ & $\begin{array}{l}1455 \\
1457\end{array}$ & $\begin{array}{l}1.0 \\
9.0\end{array}$ & $\begin{array}{l}21000 \\
24000\end{array}$ & $\begin{array}{l}8.3 \\
8.3\end{array}$ & $\begin{array}{l}11.0 \\
11.0\end{array}$ & $\begin{array}{l}-- \\
-\end{array}$ & $\begin{array}{l}10.3 \\
10.2\end{array}$ & $\begin{array}{l}105 \\
106\end{array}$ \\
\hline $\begin{array}{l}29 . \ldots \\
29 . \ldots \\
29 \ldots \\
\text { JAN , }\end{array}$ & $\begin{array}{l}1225 \\
1227 \\
1229 \\
8\end{array}$ & $\begin{array}{l}1.0 \\
5.0 \\
9.0\end{array}$ & $\begin{array}{r}29000 \\
31000 \\
* \quad 31000\end{array}$ & $\begin{array}{l}8.4 \\
8.4 \\
8.3\end{array}$ & $\begin{array}{l}29.0 \\
29.0 \\
29.0\end{array}$ & $\begin{array}{l}.65 \\
-- \\
--\end{array}$ & $\begin{array}{l}6.7 \\
6.4 \\
5.0\end{array}$ & $\begin{array}{l}99 \\
95 \\
74\end{array}$ \\
\hline $\begin{array}{l}17 \ldots \\
17 \ldots \\
17 \ldots \\
\text { JUN }\end{array}$ & $\begin{array}{l}1429 \\
1431 \\
1433\end{array}$ & $\begin{array}{l}1.0 \\
5.0 \\
9.0\end{array}$ & $\begin{array}{l}34000 \\
34000 \\
38000\end{array}$ & $\begin{array}{l}8.2 \\
8.2 \\
8.1\end{array}$ & $\begin{array}{l}11.5 \\
11.5 \\
11.0\end{array}$ & $\begin{array}{r}.51 \\
-- \\
--\end{array}$ & $\begin{array}{l}9.6 \\
9.5 \\
9.3\end{array}$ & $\begin{array}{l}106 \\
105 \\
103\end{array}$ \\
\hline $\begin{array}{l}14 \ldots \\
14 \ldots \\
14 \ldots\end{array}$ & $\begin{array}{l}1015 \\
1017 \\
1019\end{array}$ & $\begin{array}{r}1.0 \\
5.0 \\
10^{\circ}\end{array}$ & $\begin{array}{l}35000 \\
35000 \\
36000\end{array}$ & $\begin{array}{l}8.3 \\
8.2 \\
8.2\end{array}$ & $\begin{array}{l}29.5 \\
29.5 \\
29.5\end{array}$ & $\begin{array}{r}1.16 \\
= \\
2 .\end{array}$ & $\begin{array}{l}6.2 \\
6.1 \\
5.5\end{array}$ & $\begin{array}{l}93 \\
92 \\
84\end{array}$ \\
\hline
\end{tabular}

275815097031800 LINE 141 SITE 03

\begin{tabular}{|c|c|c|c|c|c|c|c|c|}
\hline DATE & TIME & $\begin{array}{l}\text { SAMP- } \\
\text { LING } \\
\text { DEPFH } \\
\text { (FT) }\end{array}$ & $\begin{array}{l}\text { SPE- } \\
\text { CIFIC } \\
\text { CON- } \\
\text { DUCT- } \\
\text { ANCE } \\
\text { ('AICRO- } \\
\text { MHOS) }\end{array}$ & $\begin{array}{c}\text { PH } \\
\text { (UNEITS) }\end{array}$ & $\begin{array}{l}\text { TEMPER- } \\
\text { ATURE, } \\
\text { WATER } \\
\text { (OEG C) }\end{array}$ & $\begin{array}{c}\text { TRANS- } \\
\text { PAR- } \\
\text { ENCY } \\
\text { (SECCHI } \\
\text { DISK) } \\
\text { (M) }\end{array}$ & $\begin{array}{c}\text { OXYGER, } \\
\text { DIS- } \\
\text { SOL.YED } \\
\text { (14G/L) }\end{array}$ & $\begin{array}{l}\text { OXYGEN, } \\
\text { OIS- } \\
\text { SOLYED } \\
\text { (PER- } \\
\text { CENT } \\
\text { SATUR- } \\
\text { ATIOH) }\end{array}$ \\
\hline$E B, 1$ & & & & & & & & \\
\hline $\begin{array}{l}09 \ldots \\
09 \ldots \\
09 \ldots \\
10 \ldots \\
10 \ldots \\
10 \ldots \\
\text { JuN }\end{array}$ & $\begin{array}{l}1510 \\
1512 \\
1514 \\
0810 \\
0812 \\
0814\end{array}$ & $\begin{array}{r}1.0 \\
5.0 \\
13 \\
1.0 \\
5.0 \\
13\end{array}$ & $\begin{array}{l}19000 \\
19000 \\
33000 \\
21000 \\
21000 \\
37000\end{array}$ & $\begin{array}{l}8.4 \\
8.4 \\
8.1 \\
8.3 \\
8.3 \\
8.0\end{array}$ & $\begin{array}{l}11.5 \\
11.5 \\
11.0 \\
11.5 \\
11.5 \\
11.0\end{array}$ & $\begin{array}{l}-- \\
-- \\
-82 \\
-- \\
--\end{array}$ & $\begin{array}{r}10.4 \\
10.2 \\
9.3 \\
9.5 \\
9.4 \\
8.7\end{array}$ & $\begin{array}{l}107 \\
104 \\
101 \\
98 \\
97 \\
95\end{array}$ \\
\hline $\begin{array}{l}29 \ldots \\
29 \ldots \\
29 . \ldots \\
\text { JAN , } 1\end{array}$ & $\begin{array}{r}1310 \\
1312 \\
1314 \\
8^{1312}\end{array}$ & $\begin{array}{l}1.0 \\
6.0 \\
12\end{array}$ & $\begin{array}{l}39000 \\
47000 \\
43000\end{array}$ & & & $\begin{array}{c}1.10 \\
\ldots \\
--\end{array}$ & $\begin{array}{l}6.8 \\
6.5 \\
5.8\end{array}$ & $\begin{array}{r}105 \\
103 \\
88\end{array}$ \\
\hline $\begin{array}{l}17 \ldots \\
17 \ldots \\
17 \ldots \\
\text { Juk }\end{array}$ & $\begin{array}{l}1444 \\
1446 \\
1448\end{array}$ & $\begin{array}{l}1.0 \\
5.0 \\
11\end{array}$ & $\begin{array}{l}39000 \\
40000 \\
40000\end{array}$ & $\begin{array}{l}8.1 \\
9.1 \\
8.1\end{array}$ & $\begin{array}{l}12.5 \\
12.5 \\
12.0\end{array}$ & $\begin{array}{l}.73 \\
-- \\
-\end{array}$ & $\begin{array}{l}9.5 \\
9.4 \\
9.4\end{array}$ & $\begin{array}{l}109 \\
108 \\
107\end{array}$ \\
\hline $\begin{array}{l}14 \ldots \\
14 \ldots \\
14 \ldots\end{array}$ & $\begin{array}{l}0935 \\
0937 \\
0939\end{array}$ & $\begin{array}{l}1.0 \\
6.0 \\
12\end{array}$ & $\begin{array}{l}29000 \\
32000 \\
34000\end{array}$ & $\begin{array}{l}8.1 \\
8.2 \\
8.2\end{array}$ & $\begin{array}{l}30.5 \\
30.0 \\
30.5\end{array}$ & $\begin{array}{l}.75 \\
-- \\
--\end{array}$ & $\begin{array}{l}6.2 \\
5.9 \\
5.8\end{array}$ & $\begin{array}{l}92 \\
89 \\
89\end{array}$ \\
\hline
\end{tabular}


Jable BA.--Quality of water in the Myssion-Aransas estuary, water years 197/-78--Cont1лwed Field Deteminations--Continued

275232097025000 LINE 165 SITE 02

\begin{tabular}{|c|c|c|c|c|c|c|c|c|}
\hline DATE & TIME & $\begin{array}{l}\text { SAMP- } \\
\text { LING } \\
\text { DEPTH } \\
\text { (FT) }\end{array}$ & $\begin{array}{l}\text { SPE- } \\
\text { CIF IC } \\
\text { COH- } \\
\text { DUCT- } \\
\text { ANCE } \\
\text { (MICRO- } \\
\text { MHOS ) }\end{array}$ & $\begin{array}{c}\text { PH } \\
\text { (UNITS) }\end{array}$ & $\begin{array}{l}\text { TEMPER- } \\
\text { ATURE, } \\
\text { WATER } \\
\text { (DEG C) }\end{array}$ & $\begin{array}{l}\text { TRANS- } \\
\text { PAR- } \\
\text { ENCY } \\
\text { (SECCHI } \\
\text { DISK) } \\
\text { (M) }\end{array}$ & $\begin{array}{c}\text { OXYGEN, } \\
\text { DIS- } \\
\text { SOL.VED } \\
\text { (MG/L) }\end{array}$ & $\begin{array}{l}\text { OXYGEN, } \\
\text { OIS- } \\
\text { SOLYED } \\
\text { (PER- } \\
\text { CENT } \\
\text { SATUR- } \\
\text { ATION) }\end{array}$ \\
\hline \multicolumn{9}{|c|}{$\mathrm{FEB}, 1977$} \\
\hline $10 .$. & 0835 & 1.0 & 28000 & 2. 3 & 11.5 & .93 & 9.3 & \\
\hline $10 \ldots$ & 0837 & 10 & 41000 & 8. & 11.0 & - & B.7 & 98 \\
\hline $10 .$. & 0839 & 20 & 42000 & 8.1 & 11.0 & $\sim$ & 8.7 & \\
\hline \multicolumn{9}{|l|}{ JUN } \\
\hline $29 \ldots$ & 1245 & 1.0 & 55000 & 8.0 & 25.5 & 74 & 5.8 & 90 \\
\hline $29 \ldots$ & 1247 & 5.0 & 55000 & 8. & 25.5 & -- & 5.8 & \\
\hline 29. & 1249 & 10 & 55000 & 8. & 25.5 & - & 5.7 & 8 \\
\hline $29 .$. & 1251 & 17 & 50000 & 8.0 & 25.5 & $=$ & 5.7 & 8 \\
\hline $30 .$. & .0840 & 1.0 & 540000 & 8. & 24.5 & .72 & 5.8 & \\
\hline $30 .$. & 0842 & 3.0 & 54000 & 8. & 24 & - & 5. & 9 \\
\hline $30 . .$. & 0844 & 18 & 54000 & 8.0 & & -- & 6.3 & \\
\hline \multicolumn{9}{|c|}{ JAN, 1978} \\
\hline $17 \ldots$ & 1507 & 1.0 & 39000 & R.t & 12.0 & .63 & 9.5 & 108 \\
\hline $17 \ldots$ & 1509 & 5.0 & 39000 & B.1 & 12.0 & $m$ & 9.3 & 10 \\
\hline 17. & 1511 & 10 & 40000 & 8.1 & 12.0 & -- & 9. & 10 \\
\hline $17 .$. & 1513 & 15 & 40000 & & 12 & $=$ & & 10 \\
\hline $17 .$. & 1515 & 19 & 41000 & & 12.0 & -- & 9.0 & 10 \\
\hline \multicolumn{9}{|l|}{ JUN } \\
\hline $14 \ldots$ & 0955 & 1.0 & 36000 & 8.1 & 30.0 & 1.21 & 5.8 & \\
\hline $14 \ldots$ & 0957 & 9.0 & 36000 & 8.1 & 30.0 & -- & 5.4 & 8 \\
\hline & 0959 & 18 & 42000 & 8.1 & 29.5 & -- & 4.5 & 71 \\
\hline
\end{tabular}


Table 8B.--Quality of water in the Mission-Arussas estuary, water years 1977-78 Nutrient Analyses.

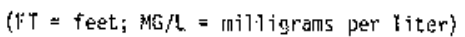

280452097054800 LIRE 054 SITE OI

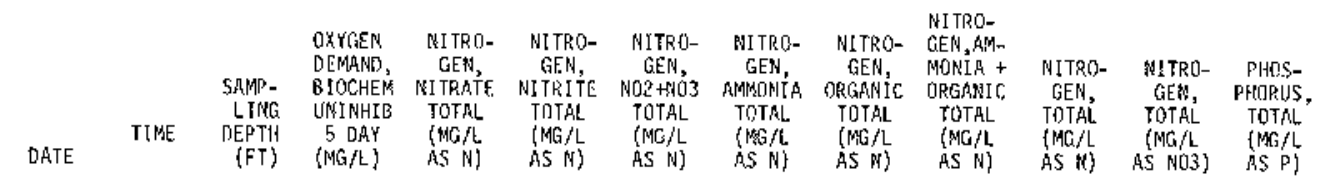
FEB , 1977

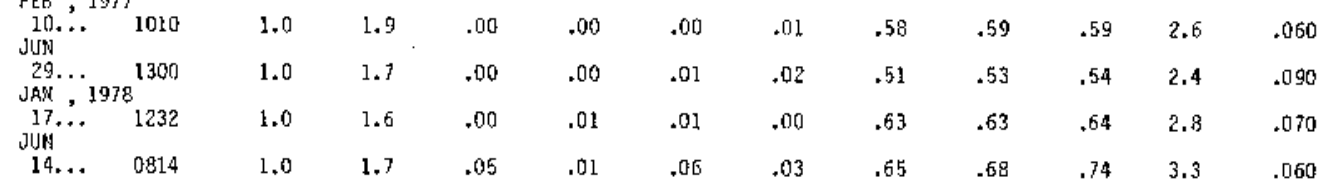

280847097034700 LINE 065 SITE 03

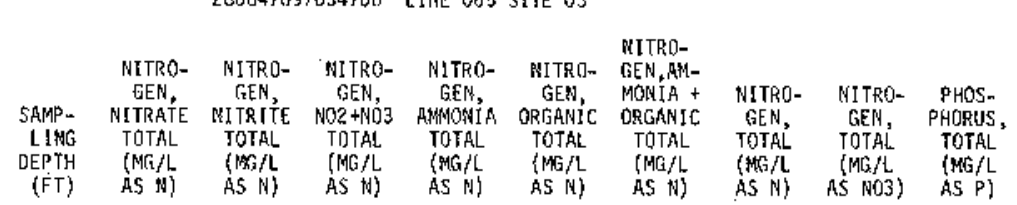

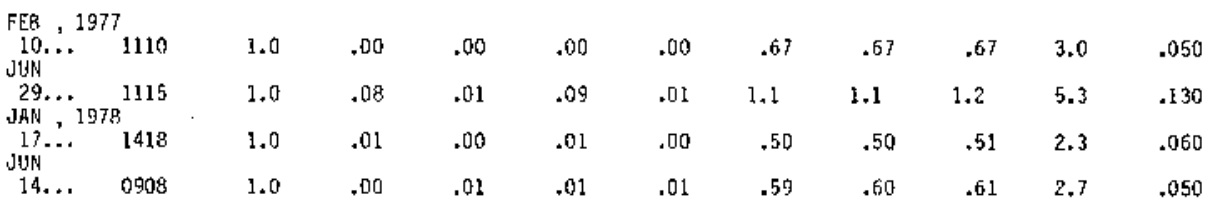

280608096551700 LINE 104 SITE 02

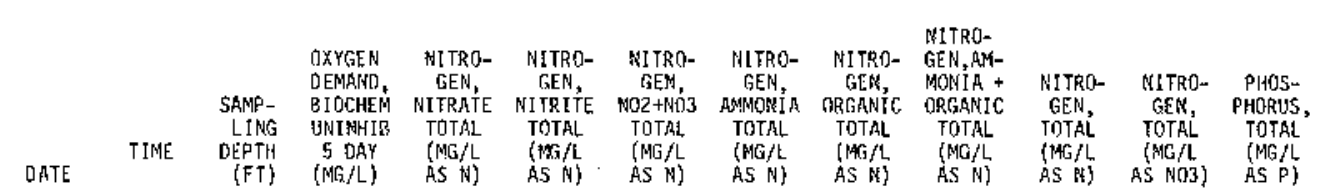

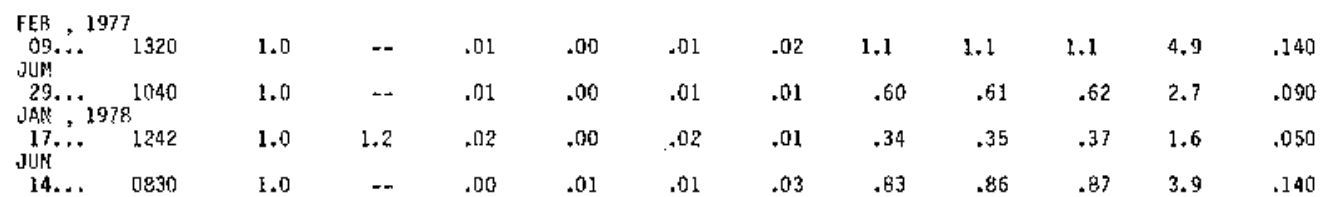

280755096533100 LINE 115 SITE 05

\begin{tabular}{|c|c|c|c|c|c|c|c|c|c|c|c|c|}
\hline DATE & TIME & $\begin{array}{l}\text { SAMP- } \\
\text { LING } \\
\text { DEPTH } \\
\text { (FT) }\end{array}$ & $\begin{array}{l}\text { OXYGEN } \\
\text { DEMAED, } \\
\text { BIOCHEM } \\
\text { UNINHI' } \\
5 \text { DAY } \\
\text { (MG/L) }\end{array}$ & $\begin{array}{l}\text { NITRO- } \\
\text { GEN, } \\
\text { NITRATE } \\
\text { TOTAL } \\
\text { (MG } / \text { L } \\
\text { AS N) }\end{array}$ & $\begin{array}{l}\text { NITRD- } \\
\text { GEN, } \\
\text { NITRITE } \\
\text { TOTAL } \\
\text { (MG/L } \\
\text { AS N) }\end{array}$ & $\begin{array}{c}\text { NITRO- } \\
\text { GEN, } \\
\text { ND2 +NÓ3 } \\
\text { TDTAL } \\
\text { (MG/L } \\
\text { AS N. }\end{array}$ & $\begin{array}{l}\text { NIJRO- } \\
\text { GER, } \\
\text { AMMONIA } \\
\text { TOTAL } \\
\text { (MG/L } \\
\text { AS N }\end{array}$ & $\begin{array}{l}\text { NITRO- } \\
\text { GEN, } \\
\text { ORGANIC } \\
\text { TDTAL } \\
\text { (MG/L } \\
\text { AS NS) }\end{array}$ & $\begin{array}{l}\text { OITRO- } \\
\text { GEN, AM- } \\
\text { MONIA + } \\
\text { ORGANIC } \\
\text { TOTAL } \\
\text { (NAS } / L \\
\text { AS N) }\end{array}$ & $\begin{array}{l}\text { NITRO- } \\
\text { GEN, } \\
\text { TOTAL } \\
\text { (MG } / 4 \\
\text { AS N) }\end{array}$ & $\begin{array}{l}\text { NITRO- } \\
\text { GEN, } \\
\text { TOTALL } \\
\text { (MG/L } \\
\text { AS NO3) }\end{array}$ & $\begin{array}{c}\text { PHOS- } \\
\text { PHORUS, } \\
\text { TOTAL } \\
\text { (MG/L } \\
\text { AS P) }\end{array}$ \\
\hline \multicolumn{13}{|c|}{ FEP $\quad 1977$} \\
\hline JuN & 1310 & 1.0 & 3.5 & ס0 & .00 & .00 & .01 & 1.4 & 1.4 & 1.4 & 6.2 & .150 \\
\hline JAN, & $8^{1050}$ & 1.0 & 1.7 & .03 & .01 & .04 & .03 & .45 & .48 & .52 & 2.3 & .100 \\
\hline $17 . \cdots$ & 1258 & 1.0 & -- & .01 & . 00 & .01 & .01 & .44 & .45 & .46 & 2.0 & .070 \\
\hline $14 \ldots$ & 0845 & 1.0 & 2.7 & .00 & .01 &, 01 & .03 & 1.5 & 1.5 & 1.5 & 6.7 & .260 \\
\hline
\end{tabular}


280458097004800 LINE 120 SITE 03

\begin{tabular}{|c|c|c|c|c|c|c|c|c|c|c|c|}
\hline DATE & TIME & $\begin{array}{l}\text { SAMP- } \\
\text { LING } \\
\text { DEPTH } \\
(F T)\end{array}$ & $\begin{array}{l}\text { RITRO- } \\
\text { GEN, } \\
\text { NITRATE } \\
\text { TOTAL } \\
\text { (MG/L } \\
\text { AS R) }\end{array}$ & $\begin{array}{l}\text { NITRO- } \\
\text { GEN, } \\
\text { NITRITE } \\
\text { TOTAL } \\
\text { (MG } / \text { L } \\
\text { AS N) }\end{array}$ & $\begin{array}{l}\text { NITRO- } \\
\text { GEN, } \\
\text { ND2+HO3 } \\
\text { TOTAL } \\
\text { (NA } / L \\
\text { AS N) }\end{array}$ & $\begin{array}{l}\text { NITRO- } \\
\text { GEN, } \\
\text { AMMONIA } \\
\text { TDTAL } \\
\text { (MG } f L \\
\text { AS R }\end{array}$ & $\begin{array}{l}\text { NITRO- } \\
\text { GEN, } \\
\text { ORGANIC } \\
\text { TOTAL } \\
\text { (MG/L } \\
\text { AS N) }\end{array}$ & $\begin{array}{l}\text { NITHRO- } \\
\text { GEN, AHT- } \\
\text { MONIA + } \\
\text { ORGANIC } \\
\text { TOTAL } \\
\text { (MG/L } \\
\text { AS N) }\end{array}$ & $\begin{array}{l}\text { NITRO- } \\
\text { GEN, } \\
\text { TDTAL, } \\
\text { (PAS/L } \\
\text { RSSN] }\end{array}$ & $\begin{array}{l}\text { NITRO- } \\
\text { GEN, } \\
\text { TOTAL } \\
\text { (MG/L } \\
\text { RS NO3) }\end{array}$ & $\begin{array}{c}\text { PHOS- } \\
\text { PHORUS, } \\
\text { TOTAL } \\
\text { (MG/L } \\
\text { AS P) }\end{array}$ \\
\hline
\end{tabular}

\begin{tabular}{|c|c|c|c|c|c|c|c|c|c|c|c|c|c|}
\hline \multicolumn{14}{|c|}{ FEB, 1977} \\
\hline $\begin{array}{c}09 . \\
\text { JUN }\end{array}$ & 1400 & & 1.0 & .01 & .00 & .01 & .00 & & .88 & .88 & .89 & 3.9 & .130 \\
\hline $\begin{array}{r}29, \cdots \\
\text { JAN, }\end{array}$ & $8^{0930}$ & , & 1.0 & .00 & .00 & .00 & .00 & • & .70 & .70 & .70 & 3.1 & .070 \\
\hline JUN & 1339 & & 1.0 & .02 & .00 & .02 & .02 & & .47 & .49 & .51 & 2.3 & .060 \\
\hline $14 \ldots$ & 1050 & & 1.0 & .00 & .01 & .01 & .01 & & .60 & .61 & .62 & 2.7 & .100 \\
\hline
\end{tabular}

275750097015400 LINE 14151 TE 02

\begin{tabular}{|c|c|c|c|c|c|c|c|c|c|c|c|}
\hline DATE & TIME. & $\begin{array}{l}\text { SAMP - } \\
\text { EING } \\
\text { DEPTH } \\
(F T)\end{array}$ & $\begin{array}{l}\text { SITRO- } \\
\text { GEN, } \\
\text { NITRATE } \\
\text { TOTAL } \\
\text { (MG } / L \\
\text { AS M }\end{array}$ & $\begin{array}{l}\text { NITRO- } \\
\text { GEN, } \\
\text { NITRI I E } \\
\text { TOTAL } \\
\text { (MG/L } \\
\text { AS N) }\end{array}$ & $\begin{array}{l}\text { NITRQ- } \\
\text { GEN, } \\
\text { NOZ+NOS } \\
\text { TOTAL } \\
\text { (MHA } / L \\
\text { RS N }\end{array}$ & $\begin{array}{l}\text { NITRO- } \\
\text { GEN, } \\
\text { AMMONIA } \\
\text { TOTAL } \\
\text { (MG/L } \\
\text { AS N) }\end{array}$ & $\begin{array}{c}\text { NIEPO- } \\
\text { GEN, } \\
\text { ORTANIC } \\
\text { TOTAL } \\
\text { (MO/L } \\
\text { AS N) }\end{array}$ & $\begin{array}{l}\text { NI TRO- } \\
\text { GEN,AM- } \\
\text { MONIA + } \\
\text { ORGANIC } \\
\text { TOTAL } \\
\text { (MGA } \\
\text { AS N) }\end{array}$ & $\begin{array}{l}\text { NITRO- } \\
\text { GEN, } \\
\text { TOTAL } \\
\text { (MG/L } \\
\text { ASN N) }\end{array}$ & $\begin{array}{l}\text { NITRO- } \\
\text { GEN, } \\
\text { TOTAL } \\
\text { (MSA } A \\
\text { AS NO3) }\end{array}$ & $\begin{array}{l}\text { PHOS- } \\
\text { PHORUS, } \\
\text { TOTAL } \\
\text { (MG/L } \\
\text { AS P) }\end{array}$ \\
\hline
\end{tabular}

FEF, 1977

\begin{tabular}{|c|c|c|c|c|c|c|c|c|c|c|c|}
\hline$\underset{\text { JuN }}{09} \cdot \cdots$ & 1455 & 1.0 & .00 & .00 & .00 & .01 & .64 & .65 & .65 & 2.9 & .070 \\
\hline JAN & $8^{1225}$ & 1.0 & .00 & .01 & .00 & .01 & .64 & .65 & .65 & 2.9 & .070 \\
\hline $\begin{array}{l}17 \ldots \\
17 \ldots\end{array}$ & $\begin{array}{l}1429 \\
1433\end{array}$ & $\begin{array}{l}1.0 \\
9.0\end{array}$ & $\begin{array}{l}.01 \\
.00\end{array}$ & $\begin{array}{l}.00 \\
.01\end{array}$ & $\begin{array}{l}.01 \\
.01\end{array}$ & .00 & $\begin{array}{l}.53 \\
.46\end{array}$ & $\begin{array}{l}.53 \\
.47\end{array}$ & $\begin{array}{l}.54 \\
.48\end{array}$ & $\begin{array}{l}2.4 \\
2.1\end{array}$ & $\begin{array}{l}.070 \\
.060\end{array}$ \\
\hline $14 \ldots$ & 1015 & 1.0 & .00 & .01 & .01 & .03 & .51 & .54 & .55 & 2.4 & .060 \\
\hline
\end{tabular}

275232097025000 LIHE 165 SITE O2

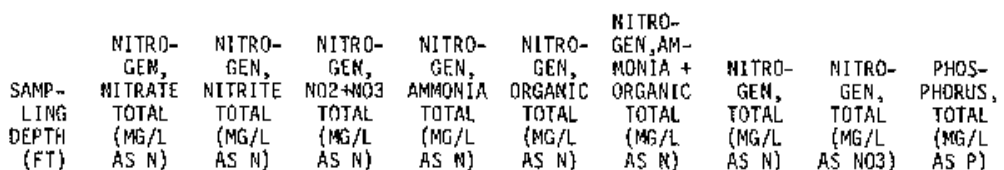

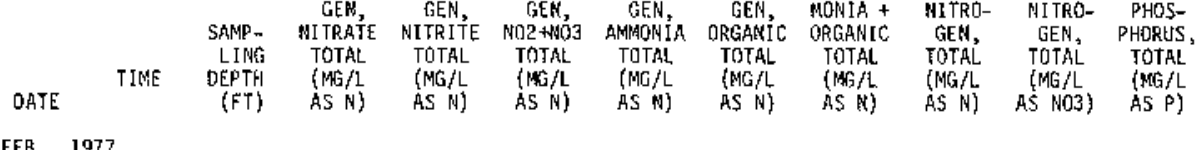

FEB, 19770835

$\begin{array}{lllllllllll}1.0 & .00 & .00 & .00 & .01 & .44 & .45 & .45 & . & 2.0 & .060\end{array}$

$29 . \ldots \quad 1245$

JAN, 1978,1507

$1.0 \quad .00 \quad .01$

$\begin{array}{llll}1.0 & .00 & .01 & .01\end{array}$

$\begin{array}{llll}.60 & .61 \quad .62 & 2.7 & .020\end{array}$

14... 0.955

$1.0 \quad .01 \quad .01$

.

.02 .03

$\begin{array}{lllll}.61 & .62 & .63 & 2.8 & .050\end{array}$

$\begin{array}{lllll}.72 & .75 & .77 & 3.4 & .070\end{array}$ 
Table 8C.--Quality of water in the His5ion-Aransas estuary, water yedrs 1977-78 Chemical Analyses

(FT = feet; MICROMHOS = micromhos per cent imeter at $25^{\circ}$ Celsius; (GG/L $=$ milligralis per 1 iter; $A C-f T=$ aCre-feet $)$

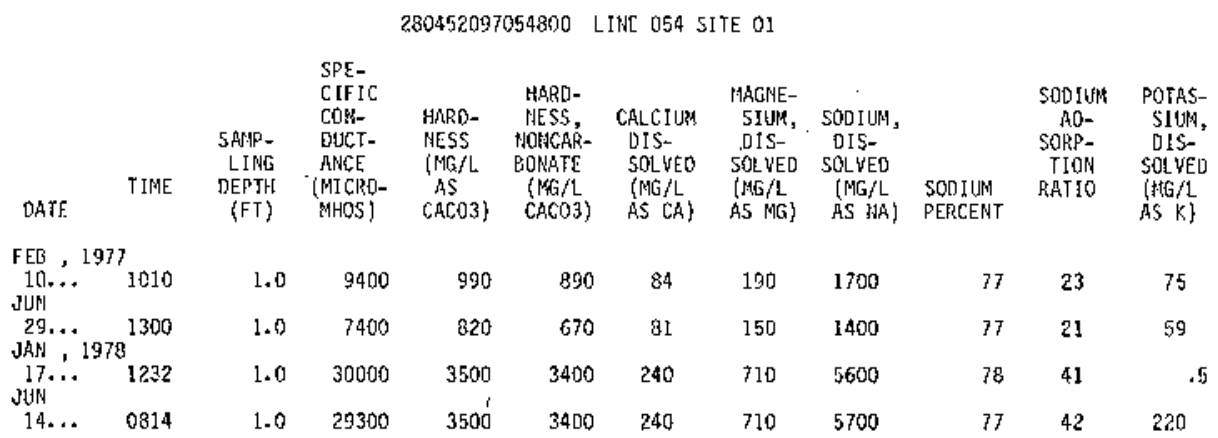

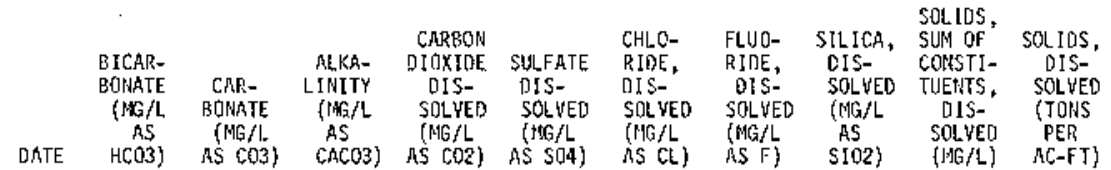

\begin{tabular}{|c|c|c|c|c|c|c|c|c|c|c|}
\hline $\begin{array}{l}\text { FEB , } 1977 \\
\text { J0... } \\
\text { JUN }\end{array}$ & 124 & 0 & 102 & -- & 410 & 2800 & .3 & 7.7 & 5330 & 7.25 \\
\hline $29, \ldots$ & 180 & -- & 148 & -- & 340 & 2500 & .4 & 14 & 4630 & 6.30 \\
\hline נIN & 160 & 0 & 131 & - & 1600 & 10000 & .7 & 5.7 & 18200 & 24.8 \\
\hline $14 \ldots$ & 150 & 0 & 123 & 1.9 & 1500 & 11000 & .7 & 10 & $\$ 9500$ & 26.5 \\
\hline
\end{tabular}




\section{Nueces Estuary}

The Nueces estuary, which has an area of about 200 square miles $\left(520 \mathrm{~km}^{2}\right)$, consists of the tidal parts of the Nueces River and other tributaries, Nueces Bay, Tule Lake Channel, Corpus Christi Bay, part of Redfish Bay, Corpus Christi Ship Channel, and Aransas Pass, and part of the Intracoastal Waterway (Figure 10). Water depth at mean low water is less than 13 feet $(4.0 \mathrm{~m})$ in Corpus Christi Bay; less than 3 feet $(1.0 \mathrm{~m})$ in Nueces Bay; more than 40 feet (12.2 $\mathrm{m}$ ) in Aransas Pass, Corpus Christi Ship Channel, and Tule Lake Channel; and about 15 feet $(4.6 \mathrm{~m})$ in the Intracoastal Waterway. A part of Redfish Bay is about 10 feet $(3.0 \mathrm{~m})$ deep, but about one-fourth of it is only 1 foot $(0.3 \mathrm{~m})$ deep at mean low water.

Water-quality data (Table 9) were collected during February and June 1977 and February, June, August, and September 1978.

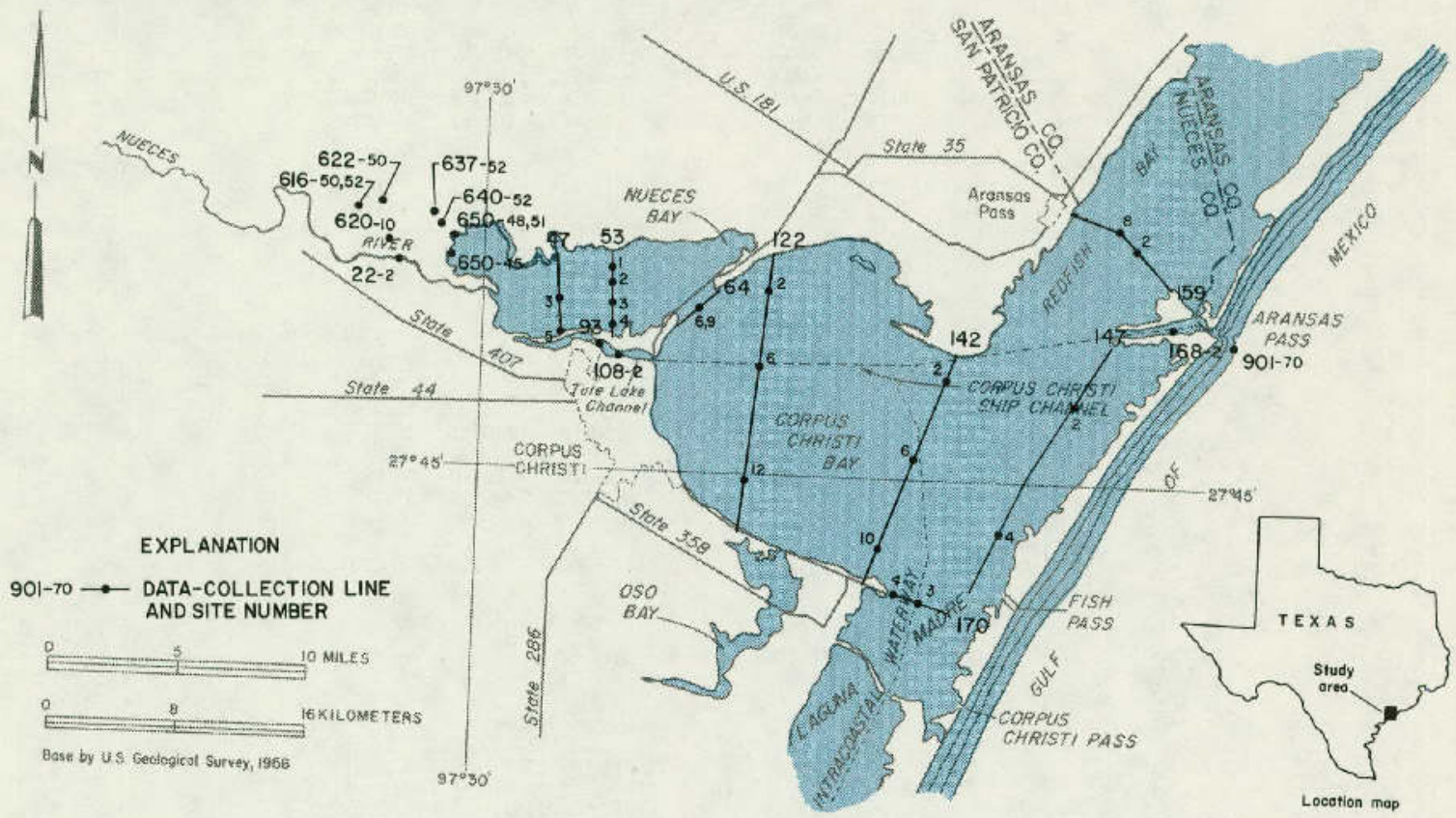

Figure 10.-Data-Collection Sites in the Nueces Estuary 
Table 9A.--Quality of water in the Nueces estuary, water years 1977-78 Field Detemninations

$\left(F T=\right.$ feet $;$ MICROMHOS $=$ micramhos per centineter at $25^{\circ}$ Celsius; $D E G C=$ degrees Celsius; $M=$ meters; $M G / L=$ milligrans per liter

275138097331900 LINE 022 SITE 02

\begin{tabular}{|c|c|c|c|c|c|c|c|c|}
\hline & TIME & $\begin{array}{l}\text { SAMP- } \\
\text { LING } \\
\text { DEPTH } \\
(F T)\end{array}$ & $\begin{array}{l}\text { SPE- } \\
\text { CIFIC } \\
\text { CON- } \\
\text { DUCT- } \\
\text { ANCE } \\
\text { (MICRO- } \\
\text { MHOS) }\end{array}$ & $\begin{array}{c}\text { PH } \\
\text { (UNITS) }\end{array}$ & $\begin{array}{l}\text { TEMPER- } \\
\text { ATURE, } \\
\text { HATER } \\
\text { (DEG C) }\end{array}$ & $\begin{array}{l}\text { TRARS- } \\
\text { PAR- } \\
\text { ENCY } \\
\text { (SECCHI } \\
\text { DISK) } \\
\text { (M) }\end{array}$ & $\begin{array}{c}\text { OXYGEN, } \\
\text { DIS- } \\
\text { SOLVED } \\
\text { (HG/L) }\end{array}$ & $\begin{array}{l}\text { OXYGEN, } \\
\text { DIS- } \\
\text { SOLVED } \\
\text { (PER- } \\
\text { CENT } \\
\text { SATUR- } \\
\text { ATION) }\end{array}$ \\
\hline
\end{tabular}

$\begin{array}{crlllllll}\text { JUN },{ }^{1977} & & & & & & & \\ 30 . . & 1130 & 1.0 & 1000 & 8.7 & 29.0 & .37 & 7.5 & 99 \\ 30 \ldots & 1132 & 8.5 & 1000 & 8.7 & 29.0 & \ldots & 7.4 & 97\end{array}$

275056097270500 LINE 047 SITE 03

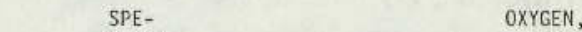

$\begin{array}{lr}\text { CIFIC } & \text { OIS- } \\ \text { CON- } & \text { SOLVED } \\ \text { SAMP- DUCT- } & \text { TEMPER- OXYGEN, } \\ \text { (PER- } & \text { OXIS- }\end{array}$

$\begin{array}{lll} & \\ \text { LING AEMPER- OXYGEN, } & \text { (PER- } \\ \text { DACTE- } & \text { PH ATURE, DIS- } & \text { CENT }\end{array}$

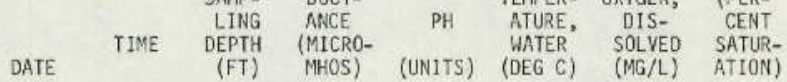

FEB , 1977

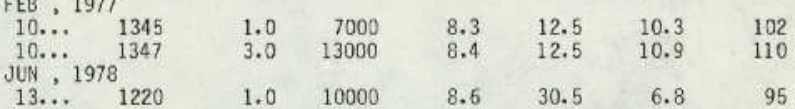

275143097252900 LINE 053 SITE 01

\begin{tabular}{|c|c|c|c|c|c|c|c|}
\hline ATE & TIME & $\begin{array}{r}\text { SAMP- } \\
\text { LING } \\
\text { DEPTH } \\
(F T)\end{array}$ & $\begin{array}{l}\text { SPE- } \\
\text { CIFIC } \\
\text { CON- } \\
\text { DUCT- } \\
\text { AHCE } \\
\text { (MICRO- } \\
\text { MHOS) }\end{array}$ & $\begin{array}{c}\text { PH } \\
\text { (UNITS) }\end{array}$ & $\begin{array}{l}\text { TEMPER- } \\
\text { ATURE, } \\
\text { WATER } \\
\text { (DEG C) }\end{array}$ & $\begin{array}{c}\text { OXYGEN, } \\
\text { DIS- } \\
\text { SOLVED } \\
\text { (MG/L) }\end{array}$ & $\begin{array}{l}\text { OXYGEN, } \\
\text { DIS- } \\
\text { SOLVED } \\
\text { (PER- } \\
\text { CENT } \\
\text { SATUR- } \\
\text { ATION) }\end{array}$ \\
\hline
\end{tabular}

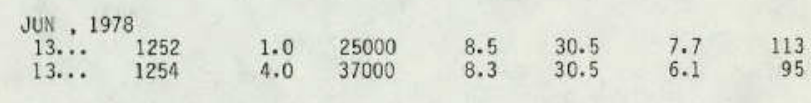

275125097252800 LINE 053 SITE 02

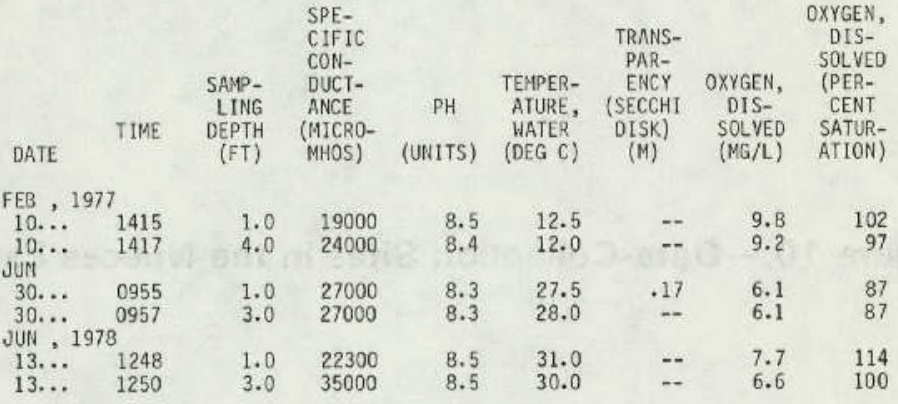

275056097252600 LINE 053 SITE 03

\begin{tabular}{|c|c|c|c|c|c|c|c|}
\hline ATE & TIME & $\begin{array}{l}\text { SAMP- } \\
\text { LING } \\
\text { DEPTH } \\
\text { (FT) }\end{array}$ & $\begin{array}{l}\text { SPE- } \\
\text { CIFIC } \\
\text { CON- } \\
\text { DUCT- } \\
\text { ANCE } \\
\text { (MICRO- } \\
\text { MHOS) }\end{array}$ & $\begin{array}{c}\text { PH } \\
\text { (UNITS) }\end{array}$ & $\begin{array}{c}\text { TEMPER- } \\
\text { ATURE, } \\
\text { UATER } \\
\text { (DEG C) }\end{array}$ & $\begin{array}{c}\text { OXYGEN, } \\
\text { DIS- } \\
\text { SOLVED } \\
\text { (MG/L) }\end{array}$ & $\begin{array}{l}\text { OXYGEN, } \\
\text { OIS- } \\
\text { SOLVED } \\
\text { (PER- } \\
\text { CENT } \\
\text { SATUR- } \\
\text { ATION) }\end{array}$ \\
\hline
\end{tabular}

JUN, 1978

$\begin{array}{rrrrrrrr}13 \ldots & 1245 & 1.0 & 22000 & 8.5 & 31.0 & 7.6 & 112 \\ 13 \ldots & 1247 & 4.0 & 42000 & 8.5 & 31.5 & 5.0 & 81\end{array}$ 
Table 9A.--Qual ity of water in the Nueces estuary, water years 1977-78--Continued Field Deteminations--Continued

275027097252300 LIHE O53 5ITE 04

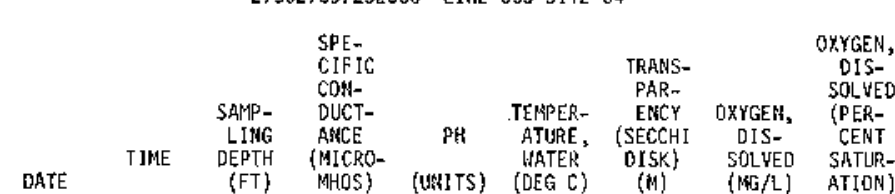

FEB , 1977

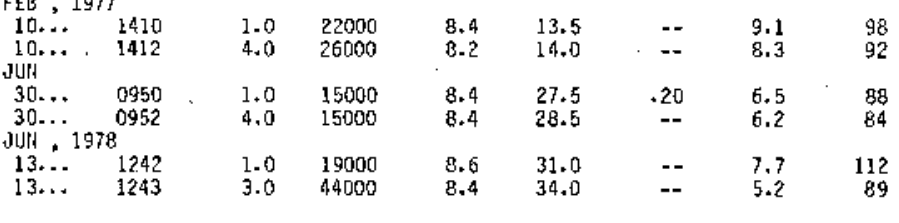

275043097220000 LIAHE O64 SITE 06

\begin{tabular}{|c|c|c|c|c|c|c|c|}
\hline DATE & TIME & $\begin{array}{c}\text { SAMP- } \\
\text { LI抵 } \\
\text { DEPTH } \\
(\mathrm{FT})\end{array}$ & $\begin{array}{l}\text { SPE- } \\
\text { CFFIC } \\
\text { CON- } \\
\text { DUCT- } \\
\text { ANCE } \\
\text { (NICRO- } \\
\text { MHOS) }\end{array}$ & $\begin{array}{c}\text { PH } \\
\text { (UNIIS) }\end{array}$ & $\begin{array}{c}\text { TEMPER- } \\
\text { ATURE, } \\
\text { HATER } \\
\text { (DEG C) }\end{array}$ & $\begin{array}{c}\text { OXYGEN, } \\
\text { DIS- } \\
\text { SOLYED } \\
\{\text { MUG } / L\}\end{array}$ & $\begin{array}{c}\text { OXYGEN, } \\
\text { DIS- } \\
\text { SOLYED } \\
\text { (PER- } \\
\text { CENT } \\
\text { SATUR- } \\
\text { ATION) }\end{array}$ \\
\hline \multicolumn{8}{|c|}{ JUN, 1978} \\
\hline $13 .$. & $\begin{array}{l}1146 \\
1148\end{array}$ & $\frac{1.0}{16}$ & $\begin{array}{l}44000 \\
46000\end{array}$ & $\begin{array}{l}8.3 \\
8.3\end{array}$ & $\begin{array}{l}30.0 \\
29.5\end{array}$ & $\begin{array}{l}5.7 \\
5.2\end{array}$ & 91 \\
\hline
\end{tabular}

275017097223200 LIHE 064 SITE 09

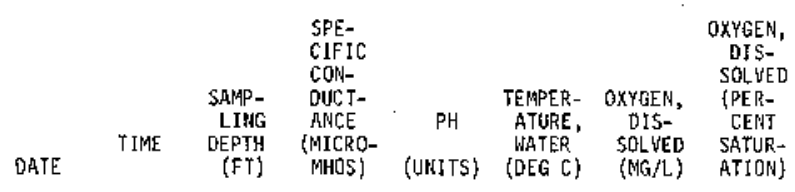

FER , 1978

$\begin{array}{rccccccc}24 \ldots & 1035 & 1.0 & 50000 & 8.1 & 12.5 & 9.2 & 112 \\ 24 \ldots & 1037 & 22 & 49000 & 8.1 & 12.5 & 9.3 & 112 \\ \text { JUN } & & & & & & & \\ 13 \ldots & 1151 & 1.0 & 44000 & 8.2 & 30.0 & 5.4 & 85 \\ 13 . \ldots & 1153 & 12 & 45000 & 8.2 & 30.0 & 5.2 & 84\end{array}$

274849097245600 LINE 108 SITE 02

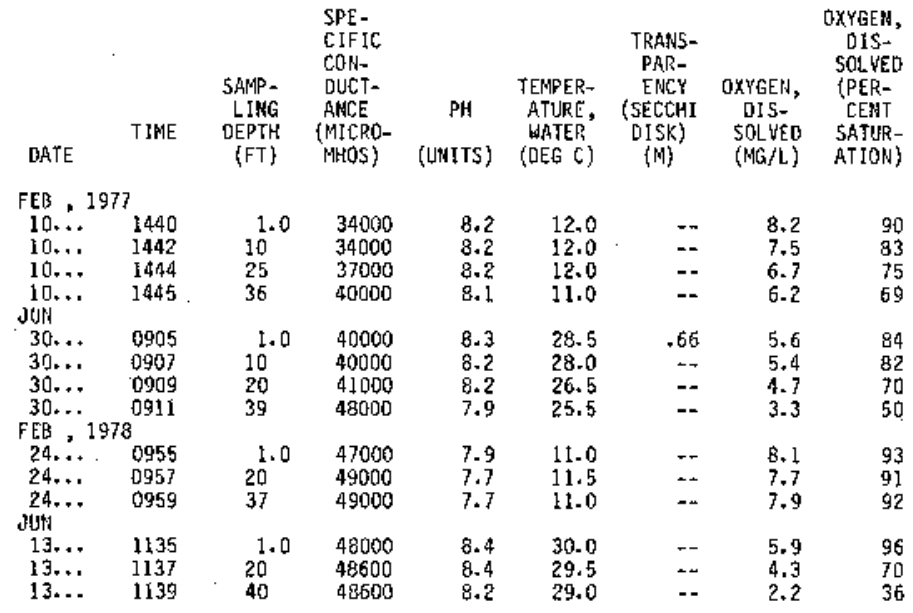


Table 9A.--Qqality of water in the Nueces estuary, water years 1977-78--Continued Field Determinations--Continued

275105097193100 LINE 122 SITE 02

\begin{tabular}{|c|c|c|c|c|c|c|c|c|}
\hline OATE & TIME & $\begin{array}{l}\text { SAMP- } \\
\text { LING } \\
\text { DEPTH } \\
\text { (FT) }\end{array}$ & $\begin{array}{l}\text { SPE- } \\
\text { CIFIL } \\
\text { CORS- } \\
\text { OUCT- } \\
\text { ANCE } \\
\text { (MICRO- } \\
\text { MHOS) }\end{array}$ & pHt & $\begin{array}{l}\text { JERAPER- } \\
\text { ATURE, } \\
\text { IATER } \\
\text { (DEG C) }\end{array}$ & $\begin{array}{l}\text { TRANS- } \\
\text { PAR- } \\
\text { ENCY } \\
\text { (SECCHI } \\
\text { DISK) } \\
\text { (M) }\end{array}$ & $\begin{array}{c}\text { OXYGEN, } \\
\text { DYS- } \\
\text { SOLVED } \\
\text { (HG/L) }\end{array}$ & $\begin{array}{c}\text { OXYGEN, } \\
\text { DIS- } \\
\text { SOLYED } \\
\text { (PER- } \\
\text { CENT } \\
\text { SATUR- } \\
\text { ATION) }\end{array}$ \\
\hline \multicolumn{9}{|c|}{$\mathrm{FEB}, 1977$} \\
\hline $\begin{array}{l}10 \ldots \\
10 \ldots\end{array}$ & $\begin{array}{l}1400 \\
1402\end{array}$ & $10^{1.0}$ & $\begin{array}{l}37000 \\
37000\end{array}$ & $\begin{array}{l}8.3 \\
8.3\end{array}$ & $\begin{array}{l}12.0 \\
12.0\end{array}$ & $\begin{array}{r}.80 \\
-.\end{array}$ & $\begin{array}{l}9.8 \\
9.2\end{array}$ & $\begin{array}{l}110 \\
104\end{array}$ \\
\hline $\begin{array}{l}30 \ldots \\
30 . \ldots \\
30 . .\end{array}$ & $\begin{array}{l}1225 \\
1227 \\
1229\end{array}$ & $\begin{array}{l}1.0 \\
5.0 \\
10\end{array}$ & $\begin{array}{l}37000 \\
37000 \\
38000\end{array}$ & $\begin{array}{l}8.2 \\
8.2 \\
8.2\end{array}$ & $\begin{array}{l}28.5 \\
28.5 \\
28.5\end{array}$ & $\begin{array}{l}.35 \\
-- \\
--\end{array}$ & $\begin{array}{l}6.1 \\
6.1 \\
6.1\end{array}$ & $\begin{array}{l}92 \\
92 \\
92\end{array}$ \\
\hline FEB, & & & & & & & & \\
\hline $\begin{array}{r}24 \ldots \\
24 \ldots\end{array}$ & $\begin{array}{l}0855 \\
0857\end{array}$ & $\begin{array}{l}1.0 \\
9.0\end{array}$ & $\begin{array}{l}50000 \\
50000\end{array}$ & $\begin{array}{l}7.4 \\
7.9\end{array}$ & $\begin{array}{l}11.5 \\
11.5\end{array}$ & -- & $\begin{array}{l}9.4 \\
9.4\end{array}$ & $\begin{array}{l}\text { 111 } \\
\text { III }\end{array}$ \\
\hline $\begin{array}{l}13 . . \\
13 . .\end{array}$ & $\begin{array}{l}1055 \\
1057\end{array}$ & $\begin{array}{l}1.0 \\
8.0\end{array}$ & $\begin{array}{l}47000 \\
47000\end{array}$ & $\begin{array}{l}8.3 \\
8.4\end{array}$ & $\begin{array}{l}29.5 \\
29.0\end{array}$ & $=$ & $\begin{array}{l}5.0 \\
5.1\end{array}$ & $\begin{array}{l}97 \\
82\end{array}$ \\
\hline
\end{tabular}

274838097201700 LINE 122 SITE 06

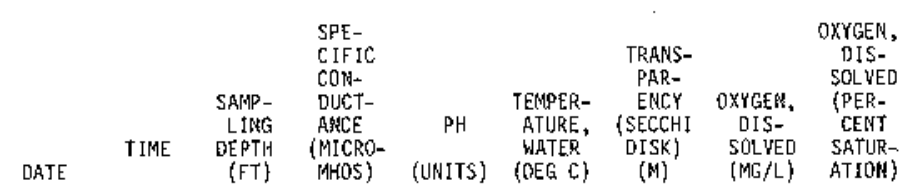

Jบห , 1977 ,

$30 . \cdots 1205$

$30 . \cdots \quad 1207$

$30 . .21219$

30..: 1213

30.. 1215

FEB , 1978

24. .. 0912

$\begin{array}{ll}24 \ldots & 0914 \\ 24 \ldots & 0916\end{array}$

24... 0916

$13 . . .1109$

$13 \ldots .111$

$13 . . .1113$

$\begin{array}{ccc}1.0 & 43000 \\ 5.0 & 44000 & 8 \\ 10 & 50000 & \\ 20 & 54000 & 7 \\ 30 & 54000 & 7 \\ 44 & 54000\end{array}$

$\begin{array}{rrrrr}8.1 & 28.5 & .44 & 6.1 & 95 \\ 8.1 & 28.5 & -- & 6.1 & 95 \\ 8.1 & 28.0 & -- & 5.6 & 91 \\ 7.9 & 26.0 & -- & 4.2 & 66 \\ 7.9 & 25.5 & -- & 4.1 & 64 \\ 7.7 & 25.5 & -- & .7 & 11 \\ & & & & \\ 7.1 & 11.0 & 1.30 & 9.5 & 110 \\ 8.1 & 10.5 & -- & 9.4 & 109 \\ 8.0 & 10.5 & -- & 9.1 & 106 \\ & & & & \\ 8.2 & 29.5 & -- & 6.5 & 101 \\ 8.3 & 29.0 & -. & 5.6 & 89 \\ 8.3 & 29.0 & -- & 4.0 & 64 \\ 7.3 & 27.5 & -- & .1 & 1\end{array}$

2745430972I1100 LINE 122 SITE 12

\begin{tabular}{|c|c|c|c|c|c|c|c|c|}
\hline DATE & TIME & $\begin{array}{c}\text { SAMP- } \\
\text { LING } \\
\text { DEPTH } \\
\text { (FT) }\end{array}$ & $\begin{array}{l}\text { SPE- } \\
\text { CIFIC } \\
\text { CON- } \\
\text { DUCT- } \\
\text { ANCE } \\
\text { (MICRO- } \\
\text { MHOS) }\end{array}$ & $\begin{array}{c}\text { PH } \\
\text { (UNITS) }\end{array}$ & $\begin{array}{l}\text { TEMPER- } \\
\text { ATURE, } \\
\text { WATER } \\
\text { (GEG C) }\end{array}$ & $\begin{array}{l}\text { TRAAS- } \\
\text { PAR- } \\
\text { ENCY } \\
\text { (SECCHI } \\
\text { DISK) } \\
\text { (M) }\end{array}$ & $\begin{array}{c}\text { OXYGEN, } \\
\text { DIS- } \\
\text { SOL VED } \\
\text { (MG } / L)\end{array}$ & $\begin{array}{c}\text { OXYGEN, } \\
\text { DIS- } \\
\text { SOLLED } \\
\text { (PER- } \\
\text { CENT } \\
\text { SATUR- } \\
\text { ATION) }\end{array}$ \\
\hline \multicolumn{9}{|c|}{ FEB, 1977} \\
\hline $\begin{array}{l}10 \ldots \\
10 \ldots \\
10 \ldots\end{array}$ & $\begin{array}{l}1325 \\
1327 \\
1329\end{array}$ & $\begin{array}{r}1.0 \\
5.0 \\
12\end{array}$ & $\begin{array}{l}35000 \\
35000 \\
36000\end{array}$ & $\begin{array}{l}8.3 \\
8.3 \\
8.2\end{array}$ & $\begin{array}{l}12.5 \\
12.0 \\
11.5\end{array}$ & $\begin{array}{l}-89 \\
-- \\
--\end{array}$ & $\begin{array}{r}10.0 \\
9.9 \\
B .7\end{array}$ & $\begin{array}{r}112 \\
109 \\
97\end{array}$ \\
\hline \multicolumn{9}{|l|}{ JUN } \\
\hline $\begin{array}{l}30 . \ldots \\
30 . \ldots \\
30 . \ldots\end{array}$ & $\begin{array}{l}1145 \\
1147 \\
1149\end{array}$ & $\begin{array}{l}1.0 \\
6.0 \\
12\end{array}$ & $\begin{array}{l}47000 \\
47000 \\
45000\end{array}$ & $\begin{array}{l}8.1 \\
8.1 \\
8.1\end{array}$ & $\begin{array}{l}28.5 \\
28.5 \\
28.5\end{array}$ & $\begin{array}{l}.63 \\
--\end{array}$ & $\begin{array}{l}5.9 \\
5.9 \\
5.7\end{array}$ & $\begin{array}{l}94 \\
94 \\
91\end{array}$ \\
\hline FEB , & \multicolumn{8}{|c|}{$\begin{array}{l}\mathrm{FEB}, 1978 \\
24 . .{ }_{0928}\end{array}$} \\
\hline $\begin{array}{l}24 . \ldots \\
24 \ldots \\
\text { JUश }\end{array}$ & $\begin{array}{l}0928 \\
0930\end{array}$ & $\begin{array}{l}1.0 \\
7.0\end{array}$ & $\begin{array}{l}47000 \\
48000\end{array}$ & $\begin{array}{l}8.2 \\
8.2\end{array}$ & $\begin{array}{l}11.0 \\
11.0\end{array}$ & $\begin{array}{r}1.53 \\
-.\end{array}$ & $\begin{array}{r}9.6 \\
10.0\end{array}$ & $\begin{array}{l}110 \\
116\end{array}$ \\
\hline $\begin{array}{l}13 . . \\
13 . \ldots\end{array}$ & $\begin{array}{l}1117 . \\
1119\end{array}$ & $10^{1.0}$ & $\begin{array}{l}46000 \\
46000\end{array}$ & $\begin{array}{l}8.3 \\
8.3\end{array}$ & $\begin{array}{l}30.0 \\
29.0\end{array}$ & $=$ & $\begin{array}{l}6.2 \\
4.9\end{array}$ & $\begin{array}{r}100 \\
78\end{array}$ \\
\hline
\end{tabular}


Table 9R.--quality of water fia the Nueces estuary, water years 1977-78--Continued Field Daterninations-Continued

274820097125900 LINE 142 SITE 02

\begin{tabular}{|c|c|c|c|c|c|c|c|c|}
\hline DATE & T IME & $\begin{array}{c}\text { SAMP- } \\
\text { LINǴ } \\
\text { DEPTH } \\
\text { (FT) }\end{array}$ & $\begin{array}{l}\text { SPE- } \\
\text { CIFIC } \\
\text { CON- } \\
\text { DULT- } \\
\text { ANCE } \\
\text { (MICRO- } \\
\text { MHOS) }\end{array}$ & $\begin{array}{c}\text { PH } \\
\text { (UNITS) }\end{array}$ & $\begin{array}{c}\text { ' } \\
\text { TEMPPER- } \\
\text { ATURE, } \\
\text { WNITER } \\
\text { (DEG C) }\end{array}$ & $\begin{array}{l}\text { TRANS- } \\
\text { PAR- } \\
\text { ENCY } \\
\text { (SECCHI } \\
\text { DISK) } \\
\text { (M) }\end{array}$ & $\begin{array}{c}\text { OXYGEN, } \\
\text { OIS- } \\
\text { SOLLWE } \\
\text { (KG } / L)\end{array}$ & $\begin{array}{c}\text { OXYGEN, } \\
\text { DIS- } \\
\text { SOLVED } \\
\text { (PER- } \\
\text { CENT } \\
\text { SATUR- } \\
\text { AJIDN) }\end{array}$ \\
\hline \multicolumn{9}{|l|}{ FEB } \\
\hline $\begin{array}{l}10 \ldots \\
10 \ldots \\
10 \ldots \\
\text { FEB. }\end{array}$ & $\begin{array}{l}1300 \\
1302 \\
1304 \\
8\end{array}$ & $\begin{array}{l}1.0 \\
5.0 \\
12\end{array}$ & $\begin{array}{l}37000 \\
37000 \\
37000\end{array}$ & $\begin{array}{l}8.3 \\
8.3 \\
8.2\end{array}$ & $\begin{array}{l}12.0 \\
12.0 \\
11.5\end{array}$ & $\begin{array}{l}: 88 \\
- \\
-\end{array}$ & $\begin{array}{l}9.6 \\
9.2 \\
8.7\end{array}$ & $\begin{array}{r}108 \\
104 \\
90\end{array}$ \\
\hline $\begin{array}{r}24 \ldots \\
24 \ldots\end{array}$ & $\begin{array}{l}1105 \\
1107\end{array}$ & $11^{1.0}$ & $\begin{array}{l}42000 \\
46000\end{array}$ & $\begin{array}{l}B .5 \\
8.4\end{array}$ & $\begin{array}{l}12.0 \\
12.5\end{array}$ & -- & $\begin{array}{l}9.2 \\
8.8\end{array}$ & $\begin{array}{l}106 \\
105\end{array}$ \\
\hline $\begin{array}{l}\text { JUN } \\
13 \ldots \\
13 \ldots \\
13 \ldots\end{array}$ & $\begin{array}{l}1110 \\
1111 \\
1112\end{array}$ & $\begin{array}{l}1.0 \\
6.0 \\
12\end{array}$ & $\begin{array}{l}24000 \\
24000 \\
24000\end{array}$ & $\begin{array}{l}3.1 \\
8.1 \\
8.0\end{array}$ & $\begin{array}{l}29.5 \\
29.5 \\
29.5\end{array}$ & $\begin{array}{l}.51 \\
=- \\
-\end{array}$ & $\begin{array}{l}6.1 \\
6.1 \\
5.0\end{array}$ & \\
\hline
\end{tabular}

274548097141000 LINE $14251 T E$ 06

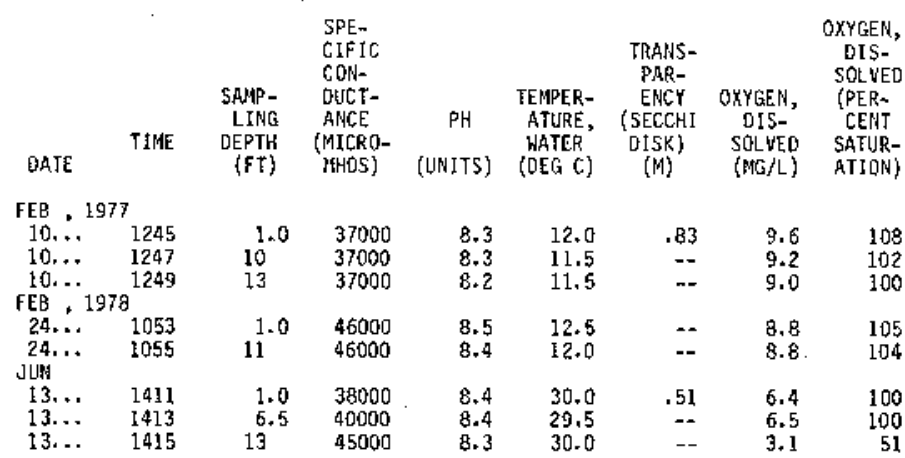

274315097152000 LINE 142 SITE 10

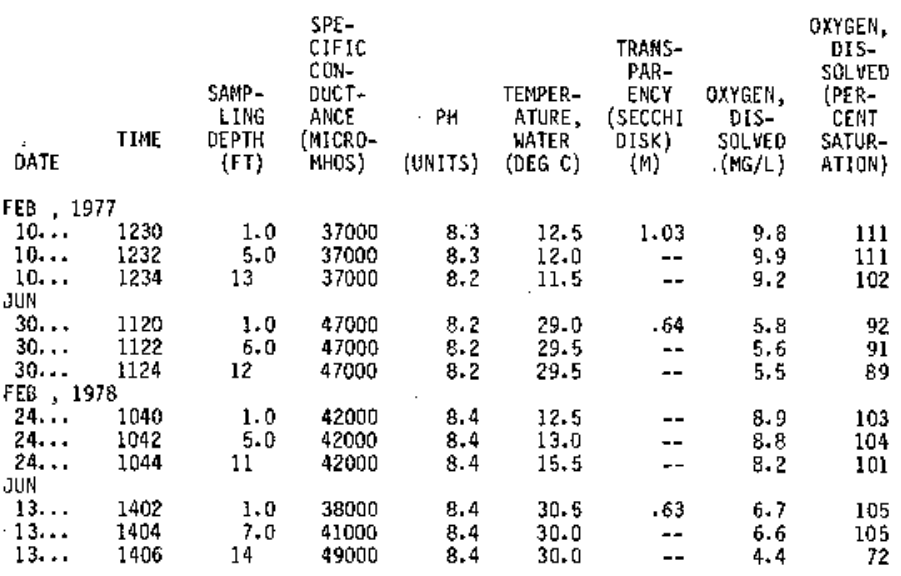

274720097092600 LINE 147 \$ITE 02

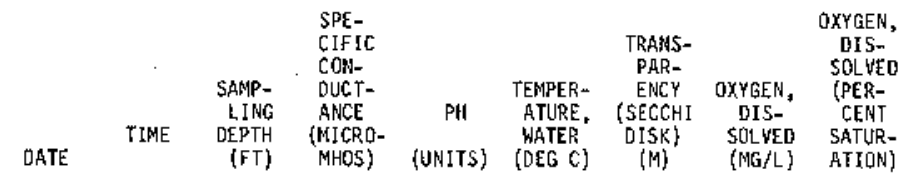

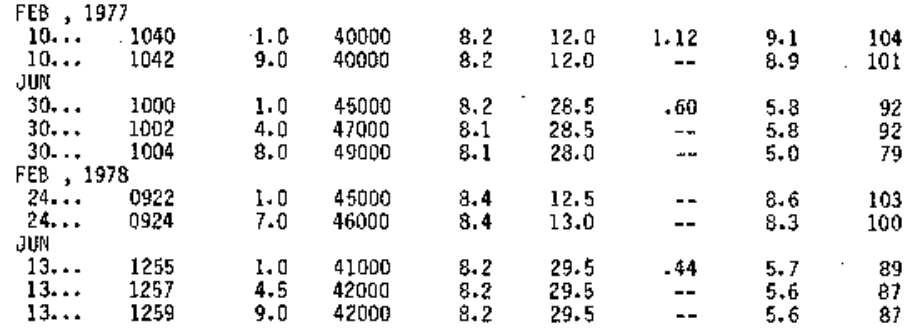


274335097111700 LINE 147 \$ITE 04

\begin{tabular}{|c|c|c|c|c|c|c|c|c|}
\hline DATE & TIME & $\begin{array}{c}\text { SAIM- } \\
\text { LING } \\
\text { DEPTH } \\
\text { (FT) }\end{array}$ & $\begin{array}{l}\text { SPE- } \\
\text { CIFIC } \\
\text { CON- } \\
\text { OUCT- } \\
\text { ARCE } \\
\text { (MICRO- } \\
\text { MHOS) }\end{array}$ & $\begin{array}{c}\text { Pli } \\
\text { (Utars) }\end{array}$ & $\begin{array}{l}\text { TEMPER- } \\
\text { ATURE, } \\
\text { WATER } \\
\text { \{DEG C) }\end{array}$ & $\begin{array}{c}\text { TRANS- } \\
\text { PAR- } \\
\text { ENCY } \\
\text { (SECCHI } \\
\text { UISK) } \\
\text { (M) }\end{array}$ & $\begin{array}{c}\text { OXYGEH, } \\
\text { DIS- } \\
\text { SOLYED } \\
\text { \{MG } / L\}\end{array}$ & $\begin{array}{c}\text { OXYGEN, } \\
\text { OIS- } \\
\text { SOLYEO } \\
\text { (PER- } \\
\text { CENT } \\
\text { SATUR- } \\
\text { ATION) }\end{array}$ \\
\hline \multicolumn{9}{|c|}{$F E B ， 1977$} \\
\hline $\begin{array}{l}10 . \ldots \\
10 . \ldots \\
10 . .\end{array}$ & $\begin{array}{l}1100 \\
1102 \\
1104\end{array}$ & $\begin{array}{r}1.0 \\
5.0 \\
12\end{array}$ & $\begin{array}{l}37000 \\
37000 \\
37000\end{array}$ & $\begin{array}{l}8.3 \\
8.3 \\
8.2\end{array}$ & $\begin{array}{l}12.0 \\
12.0 \\
12.0\end{array}$ & $\begin{array}{r}1.00 \\
- \\
--\end{array}$ & $\begin{array}{l}9.4 \\
9.2 \\
9.2\end{array}$ & $\begin{array}{l}106 \\
103 \\
103\end{array}$ \\
\hline \multicolumn{9}{|l|}{ JUN } \\
\hline $30 \ldots$ & 1020 & 1.0 & 47000 & 8.2 & 28.5 & .67 & 5.6 & 90 \\
\hline $\begin{array}{l}30 . . \\
30 . .\end{array}$ & 1022 & 5. & 45000 & & & $\cdots$ & & 90 \\
\hline \multicolumn{9}{|l|}{ FEB , 1} \\
\hline $\begin{array}{l}24 \ldots \\
24 \ldots\end{array}$ & $\begin{array}{l}0940 \\
0942\end{array}$ & $10^{1.0}$ & $\begin{array}{l}45000 \\
45000\end{array}$ & $\begin{array}{l}8.5 \\
8.4\end{array}$ & $\begin{array}{l}11.0 \\
11.5\end{array}$ & -- & $\begin{array}{l}8.9 \\
8.8\end{array}$ & $\begin{array}{l}103 \\
102\end{array}$ \\
\hline \multicolumn{9}{|l|}{ JUN } \\
\hline $13 . \ldots$ & 1315 & 1.0 & 43000 & 8.3 & 29. & .63 & 6. & 100 \\
\hline & 1317 & 6. & 43000 & 8.3 & & $=$ & 6. & \\
\hline & 1319 & 12 & 520 & 8.2 & 29.5 & - & 2.1 & 4 \\
\hline
\end{tabular}

275257097060000 LINE 159 SITE. 02

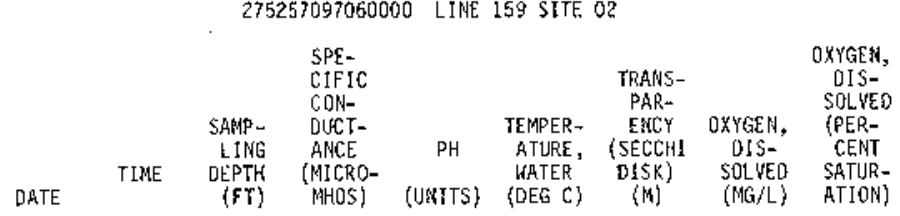

$\mathrm{FEB}, 1977$

10.. 0945

$\begin{array}{ll}10 . . & 0947 \\ 10 . \cdots & 0949\end{array}$

$\begin{array}{lllllll}1.0 & 32000 & 8.2 & 12.0 & .98 & 9.1 & 99 \\ 10 & 35000 & 8.2 & 11.5 & -- & 8.8 & 96 \\ 17 & 42000 & 8.1 & 11.5 & \cdots & 8.3 & 94\end{array}$

275326097063300 LINE 159 SITE OS

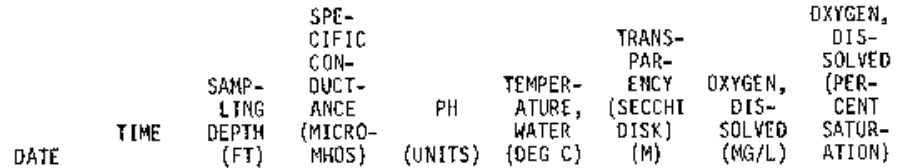

Juk , 1977

$\begin{array}{lllllllll}30 . .+ & 1415 & 1.0 & 50000 & 8.3 & 29.5 & .47 & 6.3 & 103\end{array}$

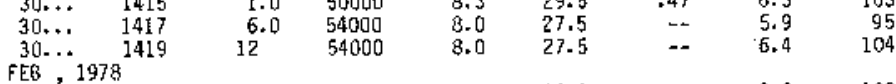

24... $\quad$ OBOO

$24 \ldots \quad 0802$

$24 . . . \quad 0604$

JUN

13... 1142

12.0

$\begin{array}{ll}1.0 & 40000 \\ 5.0 & 40000\end{array}$

$\begin{array}{lllll}8.6 & 12.0 & -- & 9.0 & 102 \\ 8.6 & 12.0 & - & 9.0 & 102\end{array}$

$\begin{array}{cc}5.0 & 40000 \\ 13 & 41000\end{array}$

$8.6 \quad 12.0$

$13 . . . \quad 1146$

$\begin{array}{cc}1.0 & 34000 \\ 7.5 & 34000 \\ 15 & 35000\end{array}$

$\begin{array}{rrrrr}8.3 & 29.5 & .79 & 5.2 & 79 \\ 8.3 & 30.0 & -- & 5.1\end{array}$

275023097045500 LIAJE 168 SITE 02

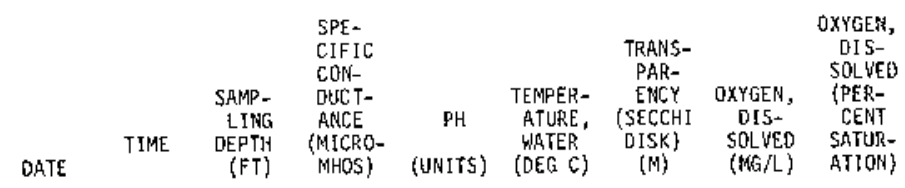

FEB, 1977

$\begin{array}{cc}10 . . & 1015 \\ 10 . . & 1017 \\ 10 . . & 1019\end{array}$

$10 \ldots .1021$

$10 \ldots .21023$

$10 . .$.

$30 . . .0940$

$\begin{array}{ll}30 . . . & 0940 \\ 30 . \ldots & 0942\end{array}$

$\begin{array}{ll}30 \ldots . & 0944 \\ 30 \ldots & 0946\end{array}$

$30 \ldots .0948$

FES , 1978

$24 . \cdots \quad 0850$

$\begin{array}{ll}24 \ldots . . & 0852 \\ 24 & 0854\end{array}$

JUN

JUN .. 1236

$\begin{array}{ll}13 . . & 1236 \\ 13 . . & 1238\end{array}$

$\begin{array}{ll}1.0 & 39000 \\ 10 & 42000 \\ 20 & 47000 \\ 30 & 47000 \\ 49 & 47000 \\ 1.0 & 55000 \\ 10 & 55000 \\ 20 & 55000 \\ 30 & 54000 \\ 46 & 54000 \\ 1.0 & 45000 \\ 25 & 46000 \\ 49 & 46000 \\ 1.0 & 40000 \\ 25 & 41000 \\ 50 & 42000\end{array}$

$\begin{array}{rrrrr}8.2 & 12.0 & .61 & 9.1 & 104 \\ 8.2 & 11.5 & -- & 8.9 & 101 \\ 8.2 & 11.5 & -- & 8.8 & 102 \\ 8.1 & 11.4 & -- & 8.8 & 102 \\ 8.1 & 11.5 & -- & 8.8 & 102 \\ 8.0 & 25.0 & 1.10 & 5.9 & 90 \\ 8.0 & 24.5 & -- & 5.7 & 88 \\ 8.0 & 24.5 & -- & 5.8 & 89 \\ 8.0 & 24.5 & -- & 5.7 & 88 \\ 8.0 & 25.0 & -- & 5.7 & 88 \\ 8.5 & 11.5 & -- & 8.6 & 100 \\ 8.4 & 11.5 & -. & 8.6 & 100 \\ 8.4 & 11.5 & -- & 8.6 & 100 \\ 8.1 & 28.5 & 1.15 & 5.5 & 83 \\ 8.0 & 27.5 & -- & 4.6 & 69 \\ 8.0 & 27.5 & -- & 3.9 & 59\end{array}$


Table gA.--Quality of water in the Nueces estuary, water years 1977-78--Continued F1eld deternifiations--Continued

274108097133200 LINE 170 \$1TE 03

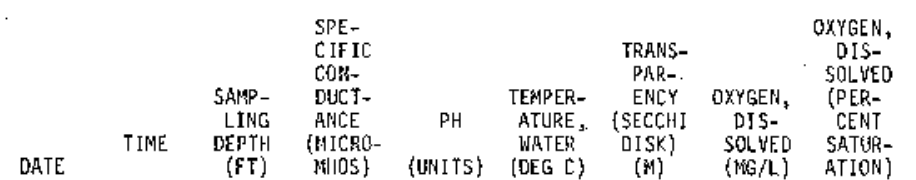

FEQ, 1977

G1... 1810

$01, \cdots \quad 1812$

\section{$1.0+3900$}

39000
39000
39000

$\begin{array}{lllll}8.2 & 9.0 & .57 & 9.4 & 99\end{array}$

Jute.

22... 1420

$22 \ldots \quad 1422$

$22 . . .1424$

MAR , 19781426

Ol... 1405

$01 \ldots 1407$

$\begin{array}{ll}1.0 & 39000 \\ 13 & 39000 \\ & 39000\end{array}$

$1.0 \quad 50000$

$5.0 \quad 52000$

$10 \quad 52000$

52000
52000

$\begin{array}{ll}8.2 & 9.0 \\ 8.2 & 9.0\end{array}$

$\begin{array}{rrr}.57 & 9.4 & 99 \\ -- & 9.5 & 100 \\ -- & 9.6 & 101\end{array}$

$8.3 \quad 29.0 \quad .7$

$\begin{array}{ll}8.3 & 29.0 \\ 8.3 & 29.0\end{array}$

$77 \quad 5.1$

$\begin{array}{ll}-\pi & 5.2 \\ - & 5.1\end{array}$

82

1352000

48000

15.5

$+$

01... 1409

$\begin{array}{cc}9.0 & 48000 \\ 16 & 48000\end{array}$

$\begin{array}{ll}8.4 & 15.5 \\ 8.4 & 15.5 \\ 8.4 & 17.0\end{array}$

274935097013500 LIME 901 \$ITE 70

\begin{tabular}{|c|c|c|c|c|c|c|c|c|}
\hline DATE & TIME & $\begin{array}{r}\text { SAMP- } \\
\text { LIMG } \\
\text { DEPTH } \\
\{F T\}\end{array}$ & $\begin{array}{l}\text { SPE- } \\
\text { CIFIC } \\
\text { CON- } \\
\text { DUCT- } \\
\text { AUCE } \\
\text { (MICRO- } \\
\text { MEOS }\end{array}$ & $\begin{array}{c}\text { PHE } \\
\text { (UNITS) }\end{array}$ & $\begin{array}{l}\text { TEMPER- } \\
\text { ATURE, } \\
\text { WATER } \\
\text { (DEG C) }\end{array}$ & $\begin{array}{c}\text { TRAYS- } \\
\text { PAR- } \\
\text { ENCY } \\
\text { (SECCHI } \\
\text { DISK) } \\
\text { (M) }\end{array}$ & $\begin{array}{c}\text { OXYGEN, } \\
\text { DIS- } \\
\text { SOLWEO } \\
\text { (MG } / L \text { ) }\end{array}$ & $\begin{array}{c}\text { OXYGEN; } \\
\text { DIS- } \\
\text { SOLYED } \\
\text { (PER- } \\
\text { CENT } \\
\text { SATUR- } \\
\text { ATIOH) }\end{array}$ \\
\hline \multicolumn{9}{|c|}{ JUN, 1977} \\
\hline $\begin{array}{l}30 . . \\
30 \ldots \\
30 . \ldots\end{array}$ & $\begin{array}{l}0900 \\
0902 \\
0904\end{array}$ & $25^{1.0}$ & $\begin{array}{l}57000 \\
57000 \\
54000\end{array}$ & $\begin{array}{l}8.0 \\
8.0 \\
8.0\end{array}$ & $\begin{array}{l}26.0 \\
24.5 \\
25.5\end{array}$ & $\begin{array}{r}.82 \\
--\end{array}$ & $\begin{array}{l}6.1 \\
5.9 \\
5.5\end{array}$ & $\begin{array}{l}96 \\
91 \\
86\end{array}$ \\
\hline \multicolumn{9}{|c|}{ FEB , 1978} \\
\hline $\begin{array}{l}24 \ldots \\
24 \ldots \\
24 \ldots \\
24 \ldots\end{array}$ & $\begin{array}{l}0830 \\
0832 \\
0834 \\
0830\end{array}$ & $\begin{array}{l}1.0 \\
10 \\
25 \\
50\end{array}$ & $\begin{array}{l}47000 \\
48000 \\
49000 \\
49000\end{array}$ & $\begin{array}{l}8.5 \\
8.4 \\
8.4 \\
8.4\end{array}$ & $\begin{array}{l}11.5 \\
11.5 \\
12.0 \\
12.0\end{array}$ & $\begin{array}{l}-- \\
-- \\
--\end{array}$ & $\begin{array}{l}8.6 \\
8.3 \\
8.4 \\
8.4\end{array}$ & $\begin{array}{r}100 \\
98 \\
100 \\
100\end{array}$ \\
\hline \multicolumn{9}{|l|}{ JUN } \\
\hline $\begin{array}{l}13 . \therefore \\
13 . \ldots \\
13 . \ldots\end{array}$ & $\begin{array}{l}1210 \\
1212 \\
1214\end{array}$ & $\begin{array}{l}1.0 \\
26 \\
52\end{array}$ & $\begin{array}{l}29500 \\
31000 \\
32000\end{array}$ & $\begin{array}{l}8.2 \\
8.1 \\
7.9\end{array}$ & $\begin{array}{l}29.0 \\
28.0 \\
25.5\end{array}$ & $\begin{array}{r}1.95 \\
-. \\
--\end{array}$ & $\begin{array}{l}5.7 \\
4.8 \\
4.9\end{array}$ & $\begin{array}{l}84 \\
71 \\
69\end{array}$ \\
\hline
\end{tabular}




$$
\begin{aligned}
& \text { (FT = fegt; } M G / L=\text { m\{11igrams per liter }) \\
& 275056097270500 \text { LIPYE } 047 \text { SITE } 03
\end{aligned}
$$

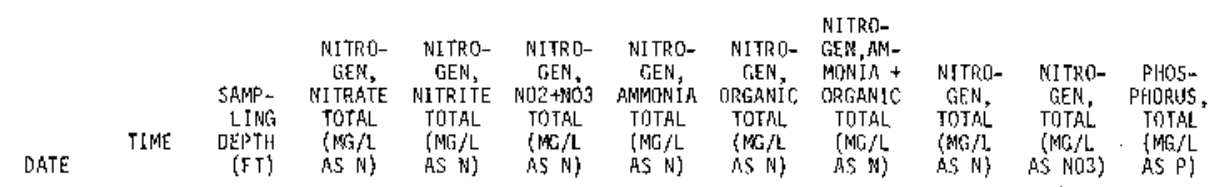

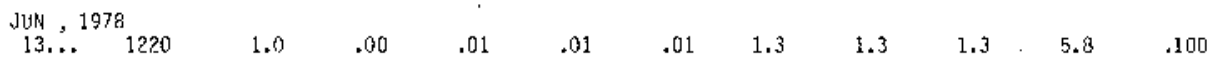

275125097252800 LIME 053 SITE 02

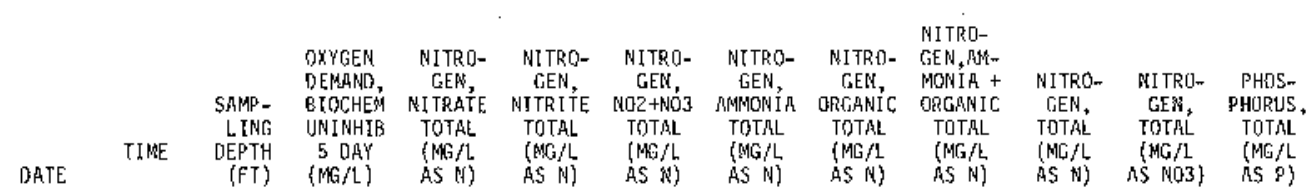

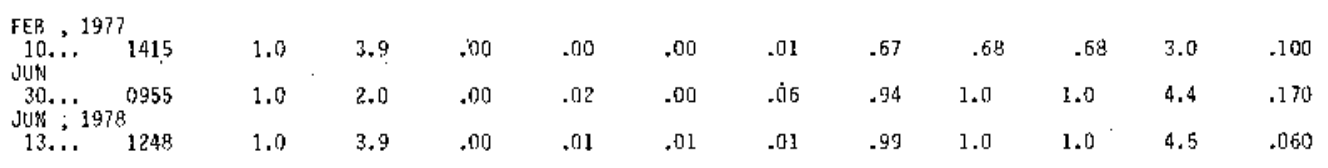

275017097223200 LINE 064 SITE O9

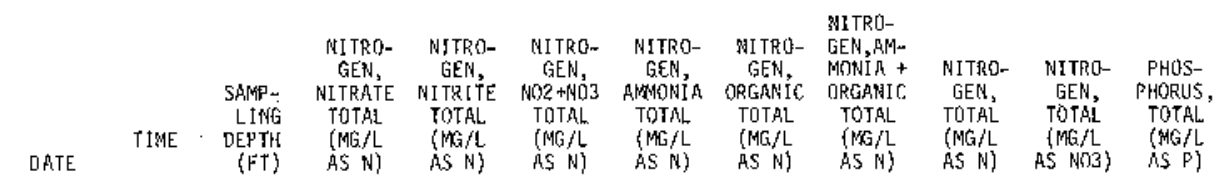

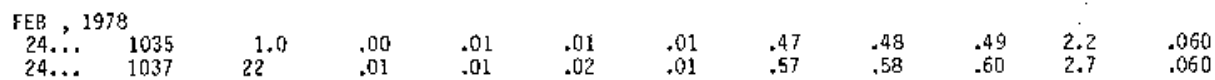

274849097245600 LINE 108 SITE 02

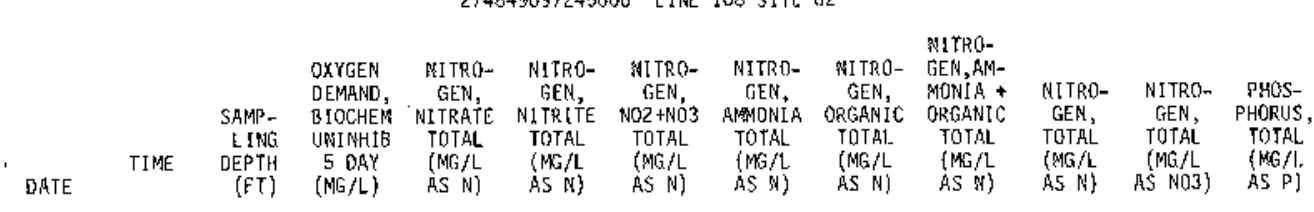

\begin{tabular}{|c|c|c|c|c|c|c|c|c|c|c|c|}
\hline DATE & TIME & $\begin{array}{c}\text { SAMP- } \\
\text { LING } \\
\text { DEPTH } \\
(F T)\end{array}$ & $\begin{array}{l}\text { NITRO- } \\
\text { GEN, } \\
\text { NITRATE } \\
\text { TOTAL } \\
\text { (MG } / \text { L } \\
\text { AS N) }\end{array}$ & $\begin{array}{l}\text { NITRO- } \\
\text { [AEN, } \\
\text { NITRLTE } \\
\text { TOTAL } \\
\text { (ME/L } \\
\text { AS N) }\end{array}$ & $\begin{array}{c}\text { MITRO- } \\
\text { GEN, } \\
\text { NO2 HNO3 } \\
\text { TOTAL } \\
\text { (MG/L } \\
\text { AS N) }\end{array}$ & $\begin{array}{l}\text { NIJRO- } \\
\text { GEN, } \\
\text { AMMDNIA } \\
\text { TOTALL } \\
\text { (MG } / 2 \\
\text { AS N) }\end{array}$ & $\begin{array}{l}\text { NI TRO- } \\
\text { GEN, } \\
\text { ORGANIC. } \\
\text { TOTAL } \\
(N G / L \\
\text { AS N) }\end{array}$ & $\begin{array}{l}\text { N1TRO- } \\
\text { GEN,AM- } \\
\text { MONLA + } \\
\text { ORGANIC } \\
\text { TOTAL } \\
(\text { MG } / L \\
\text { AS N) }\end{array}$ & $\begin{array}{l}\text { NITRO- } \\
\text { GEN, } \\
\text { TOTAL } \\
\text { (MG/ } / \mathrm{L} \\
\text { AS N })\end{array}$ & $\begin{array}{c}\text { NITRO- } \\
\text { GEN, } \\
\text { TOTAL } \\
\text { (MG/L } \\
\text { AS NO3) }\end{array}$ & $\begin{array}{c}\text { PHOS- } \\
\text { PHORUS, } \\
\text { TOTAL } \\
\text { (MG/L } \\
\text { AS P) }\end{array}$ \\
\hline FEB , & & & 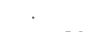 & & & & & & & & \\
\hline $\begin{array}{l}10 \ldots \\
10 \ldots\end{array}$ & $\begin{array}{l}1400 \\
1402\end{array}$ & 1.0 & חם & $\begin{array}{l}.00 \\
.00\end{array}$ & $\begin{array}{l}00 \\
.00\end{array}$ & .01 & $\begin{array}{l}.27 \\
.48\end{array}$ & $\begin{array}{l}.28 \\
.49\end{array}$ & $\begin{array}{l}.28 \\
.49\end{array}$ & $\begin{array}{l}1.2 \\
2.2\end{array}$ & $\begin{array}{l}.070 \\
.070\end{array}$ \\
\hline $\begin{array}{l}\text { JUN } \\
30, \ldots \\
30 . .\end{array}$ & $\begin{array}{l}1225 \\
1229\end{array}$ & $10^{1.0}$ & $\begin{array}{l}.00 \\
.00\end{array}$ & .01 & $\begin{array}{l}.00 \\
.01\end{array}$ & $\begin{array}{l}.04 \\
.04\end{array}$ & $\begin{array}{l}.49 \\
.39\end{array}$ & $\begin{array}{l}.53 \\
.43\end{array}$ & $\begin{array}{l}.53 \\
.44\end{array}$ & $\begin{array}{l}2.3 \\
1.9\end{array}$ & $\begin{array}{l}.060 \\
.060\end{array}$ \\
\hline $\begin{array}{l}F E B, \\
24 \ldots \\
24 \ldots\end{array}$ & $\begin{array}{l}8 \\
0855 \\
0857\end{array}$ & $\begin{array}{l}1.0 \\
9.0\end{array}$ & $\begin{array}{l}.00 \\
.00\end{array}$ & .01 & .01 & $\begin{array}{l}.00 \\
.00\end{array}$ & $\begin{array}{l}.54 \\
.52\end{array}$ & $\begin{array}{l}.54 \\
.52\end{array}$ & $\begin{array}{l}.55 \\
.53\end{array}$ & $\begin{array}{l}2.4 \\
2.3\end{array}$ & $\begin{array}{l}.050 \\
.050\end{array}$ \\
\hline $\begin{array}{l}\text { Jus } \\
13 \ldots \\
13 \ldots\end{array}$ & $\begin{array}{l}1055 \\
1057\end{array}$ & $\begin{array}{l}1.0 \\
8.0\end{array}$ & $\begin{array}{l}.00 \\
.00\end{array}$ & $\begin{array}{l}.01 \\
.01\end{array}$ & $\begin{array}{l}.01 \\
.01\end{array}$ & $\begin{array}{l}.03 \\
.03\end{array}$ & $\begin{array}{l}.93 \\
.97\end{array}$ & $\begin{array}{c}.96 \\
1.0\end{array}$ & 1.97 & $\begin{array}{l}4.3 \\
4.5\end{array}$ & $\begin{array}{l}.050 \\
.110\end{array}$ \\
\hline
\end{tabular}

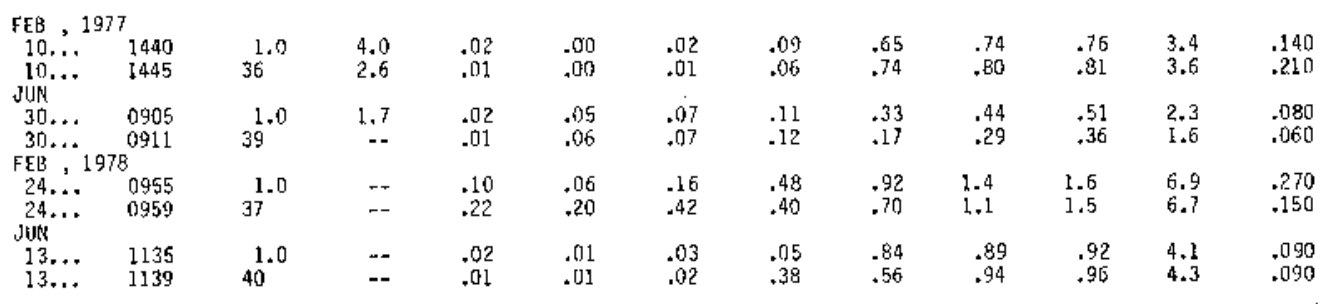

275105097193100 LINE 122 SITE O2 
274543097211100 LIRE 122 SITE 12

\begin{tabular}{|c|c|c|c|c|c|c|c|c|c|c|c|}
\hline ATE & TIME & $\begin{array}{l}\text { SAMP- } \\
\text { LING } \\
\text { DEPTH } \\
\text { (FI) }\end{array}$ & $\begin{array}{c}\text { NITRO- } \\
\text { GEN, } \\
\text { NITRATE } \\
\text { TOTAL } \\
\text { (MG/L } \\
\text { AS N) }\end{array}$ & $\begin{array}{l}\text { NIJRO- } \\
\text { GEM, } \\
\text { NITRITE } \\
\text { TOTAL } \\
\text { \{MG/L } \\
\text { AS NA }\end{array}$ & $\begin{array}{c}\text { MITRO- } \\
\text { GEN, } \\
\text { NOR+NOO3 } \\
\text { TOTAL } \\
\text { (MG/L } \\
\text { AS N) }\end{array}$ & $\begin{array}{l}\text { NITRO- } \\
\text { GEN, } \\
\text { AMMONIA } \\
\text { TOTRL } \\
\text { (MG/L } \\
\text { AS N) }\end{array}$ & $\begin{array}{c}\text { NITRO- } \\
\text { GEM, } \\
\text { ORGANIC } \\
\text { TOTAL } \\
(M G / L \\
\text { AS N) }\end{array}$ & $\begin{array}{l}\text { NIFRO- } \\
\text { GEN, AN- } \\
\text { MONIA + } \\
\text { ORGANIC } \\
\text { TOTAL } \\
\text { (MGG/L } \\
\text { AS N) }\end{array}$ & $\begin{array}{l}\text { N1TRO- } \\
\text { GEN, } \\
\text { TOTAL } \\
\text { (MG/L } \\
\text { AS N N) }\end{array}$ & $\begin{array}{c}\text { NITRO- } \\
\text { GEN, } \\
\text { TOTAL } \\
\text { (NGG/L } \\
\text { AS NO3) }\end{array}$ & $\begin{array}{c}\text { PHOS- } \\
\text { PHORUS, } \\
\text { TOTAL } \\
\text { (MG/L } \\
\text { AS P) }\end{array}$ \\
\hline
\end{tabular}

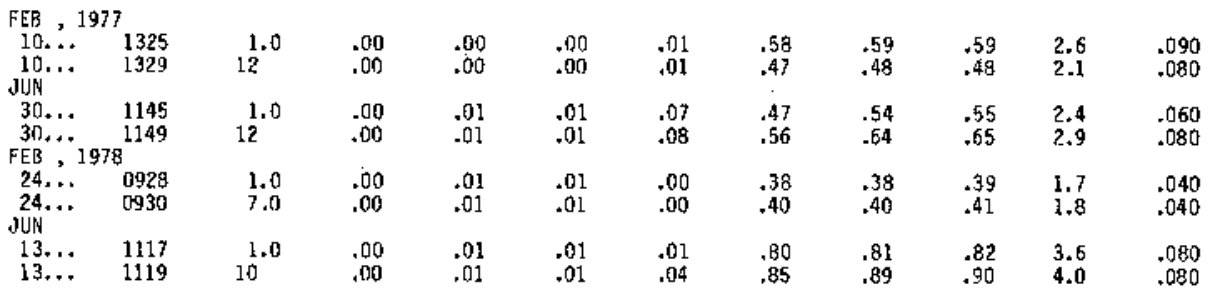

274548097141000 LINE 142 SITE 06

NITRO- NITRO- NITRO- NITRO- NITRO- GENRO-

GEN, GEN, GEN, GEN, GEN, MONIA + NITRO- NITRO- PHOS-

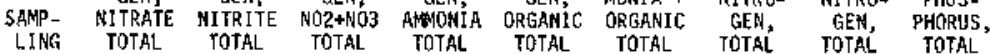

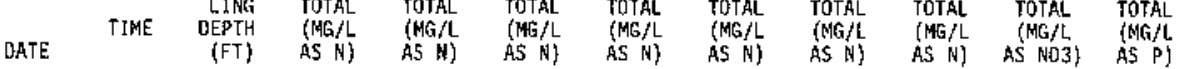

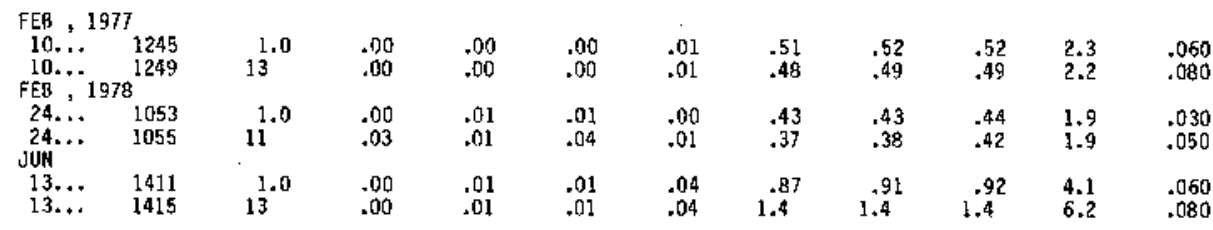

274335097111700 LINE 147 SITE 04

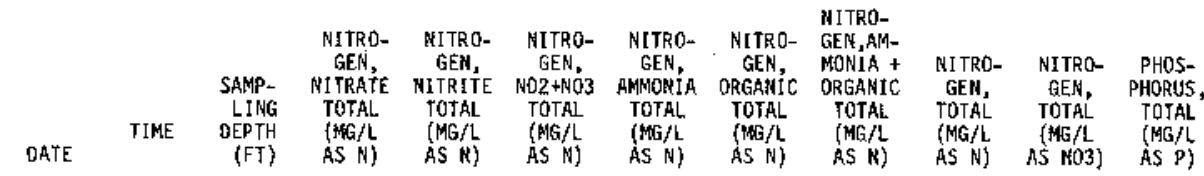

$\begin{array}{lllllllllllll}\text { FEB } & , 1977 \\ 10 . . . & 1100 & 1.0 & .00 & .00 & .00 & .01 & .44 & .45 & .45 & 2.0 & .060\end{array}$

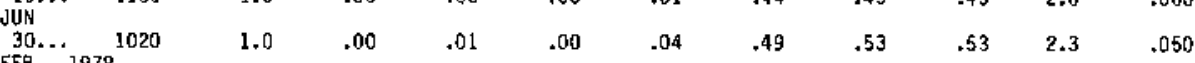

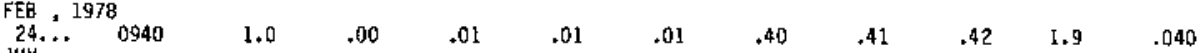

$\begin{array}{lllllllllll}13 \ldots & 1315 & 1.0 & .00 & .01 & .01 & .03 & .77 & .80 & .81 & 3.6\end{array}$

275257097060000 LINE 159 SITE 02

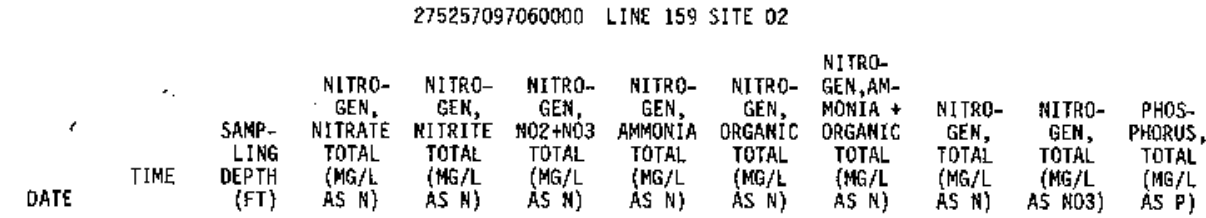

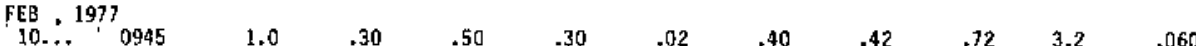

275326097063300 LINE 159 SITE 08

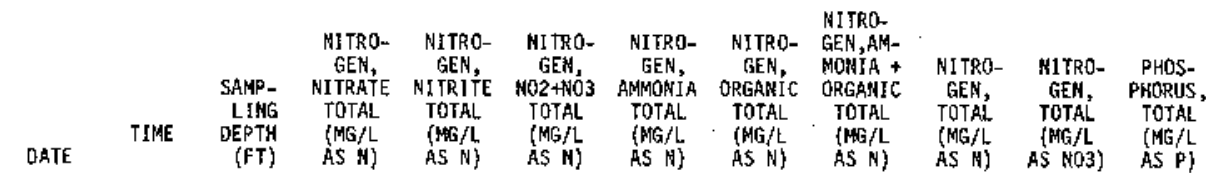

JUN , 1977

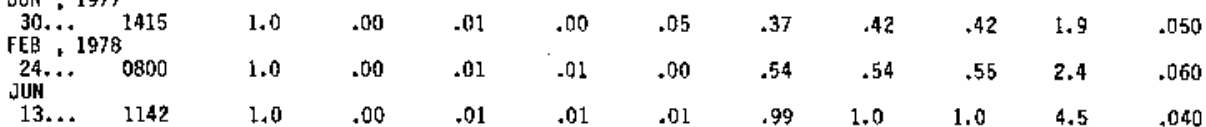




$$
\begin{aligned}
& \text { Tab1e 9R.--1? } \\
& \text { Nutrient Analyses--Continued } \\
& 275023097045500 \text { LINE } 168 \text { SITE } 02
\end{aligned}
$$

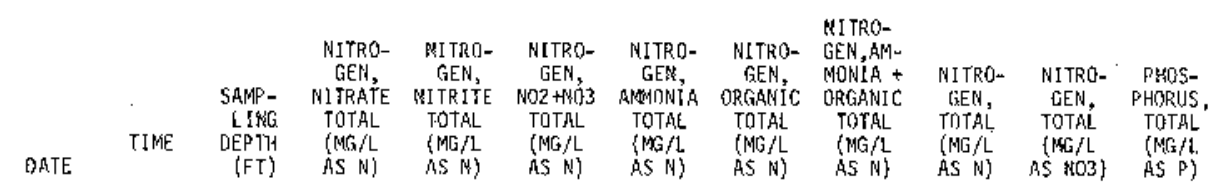

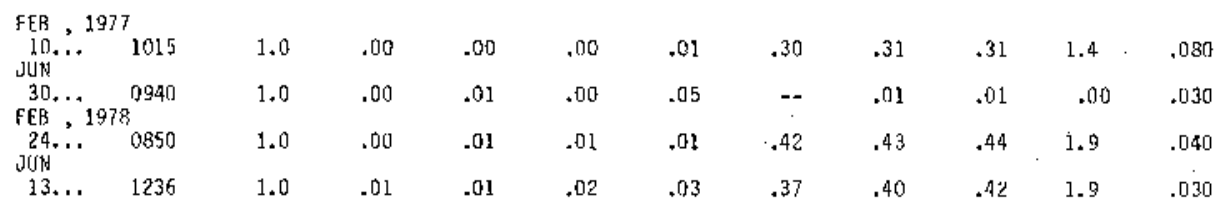

$$
\begin{aligned}
& 275310097345200 \text { LINE } 616 \text { SITE } 50
\end{aligned}
$$

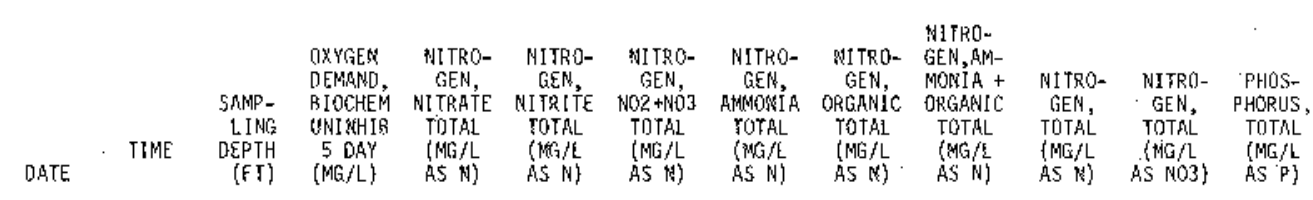

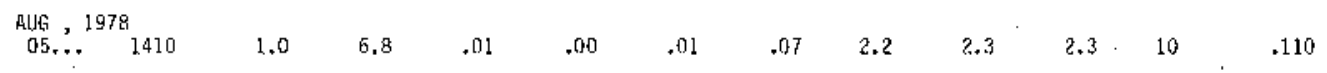

$$
\begin{aligned}
& 275314097344900 \text { LINE } 616 \text { SITE } 52
\end{aligned}
$$

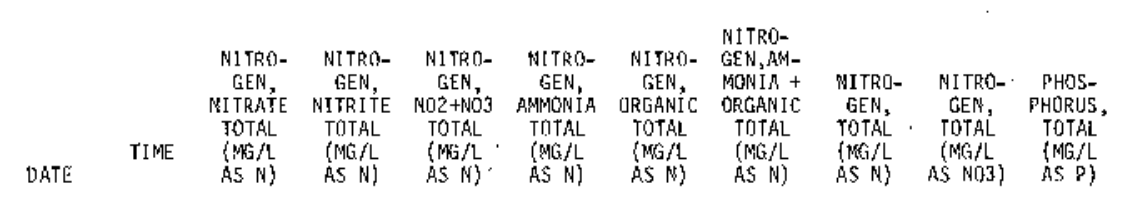

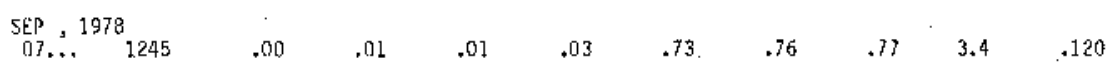

$$
\begin{aligned}
& 2752010973332000 \text { LINE } 620 \text { SITE } 10
\end{aligned}
$$

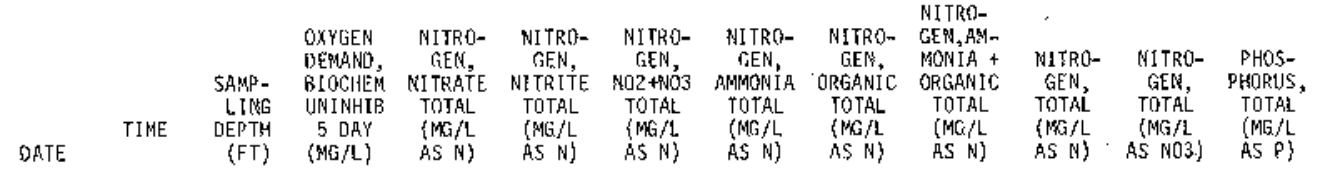

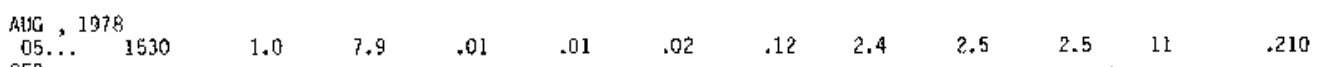

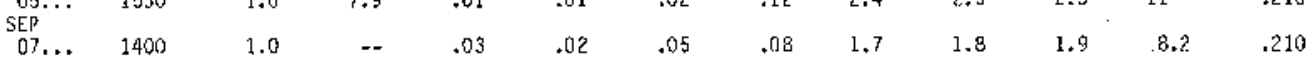

$$
\begin{aligned}
& 275326097335300 \text { LINE } 622 \text { SITE } 50
\end{aligned}
$$

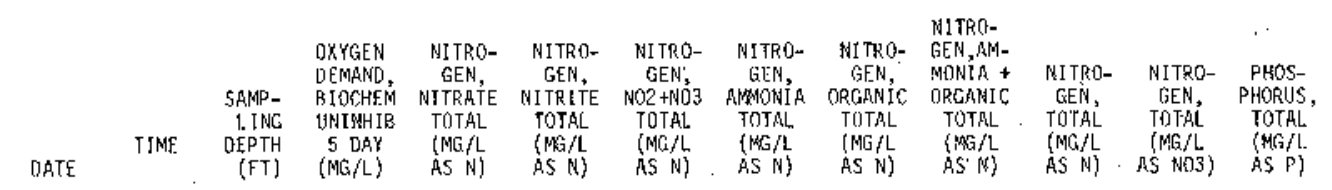

$$
\begin{aligned}
& \begin{array}{ccccccccccccccc}
\text { AUGG., } & 1978 \\
05 . . & 1400 & 1.0 & 8.0 & .01 & .01 & .02 & .10 & 1.9 & 2.0 & 2.0 & 8.9 & .130
\end{array} \\
& 275312097315500 \text { LINE } 637 \text { SITE } 52
\end{aligned}
$$

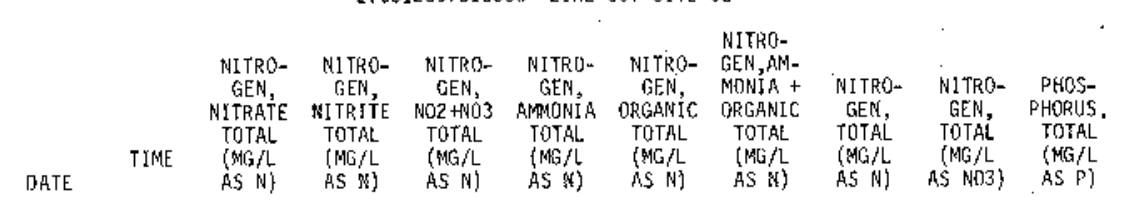

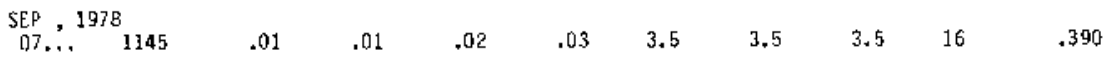




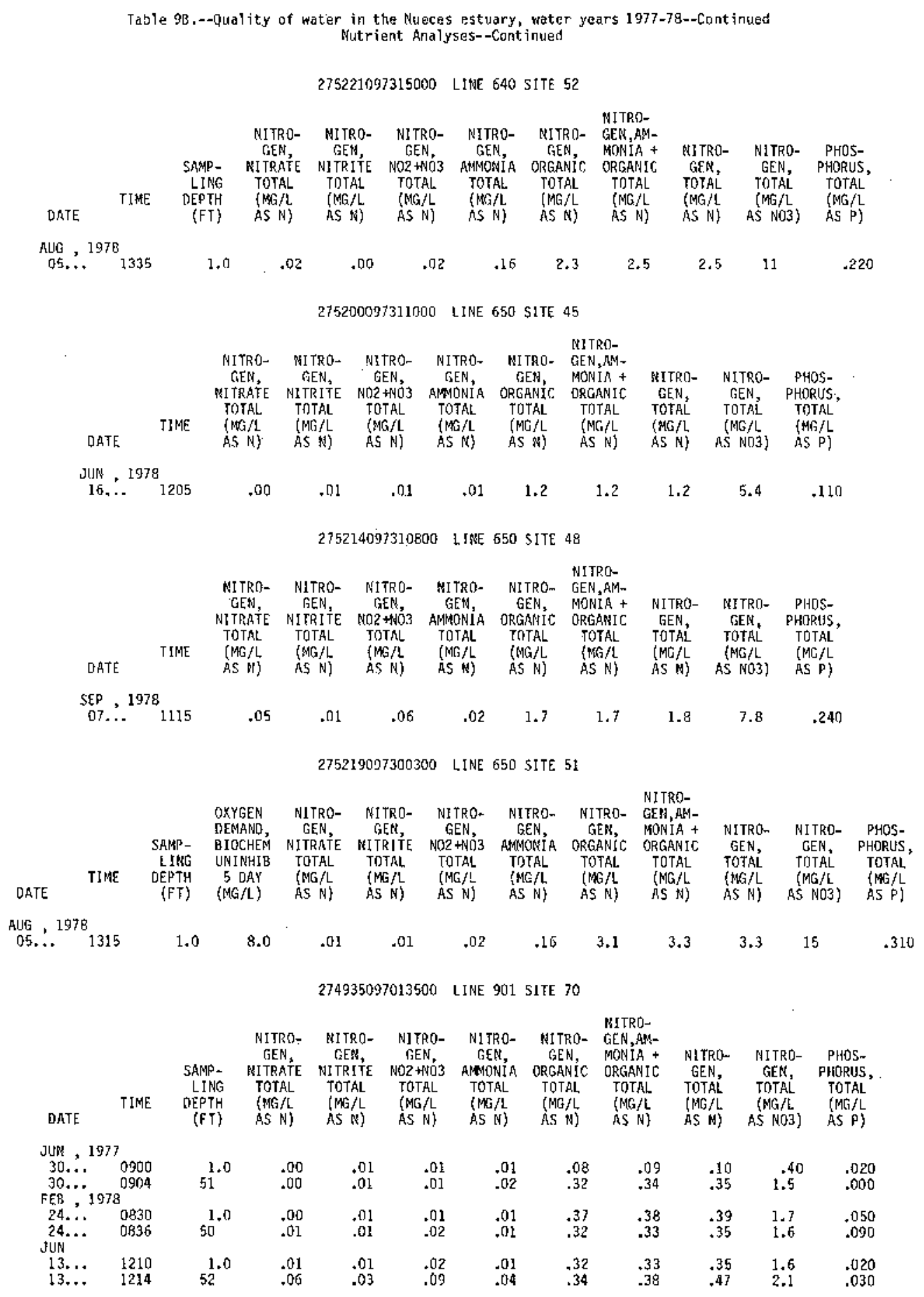


275125097252800 LINE, 053 SITE 02

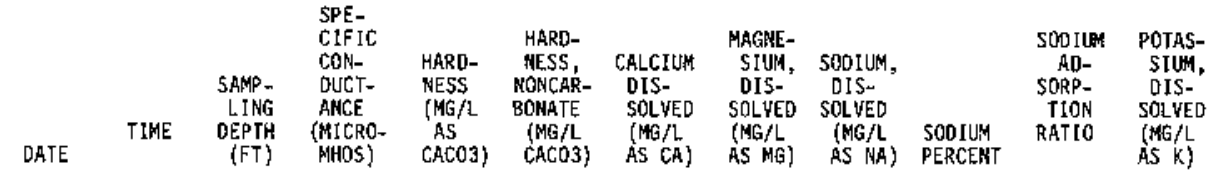

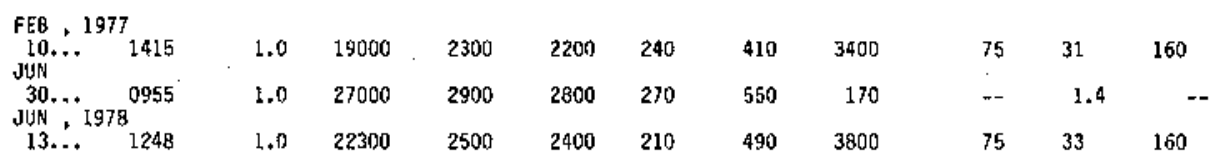

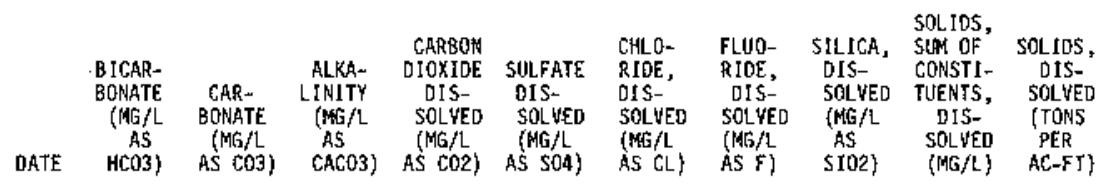

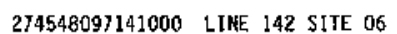

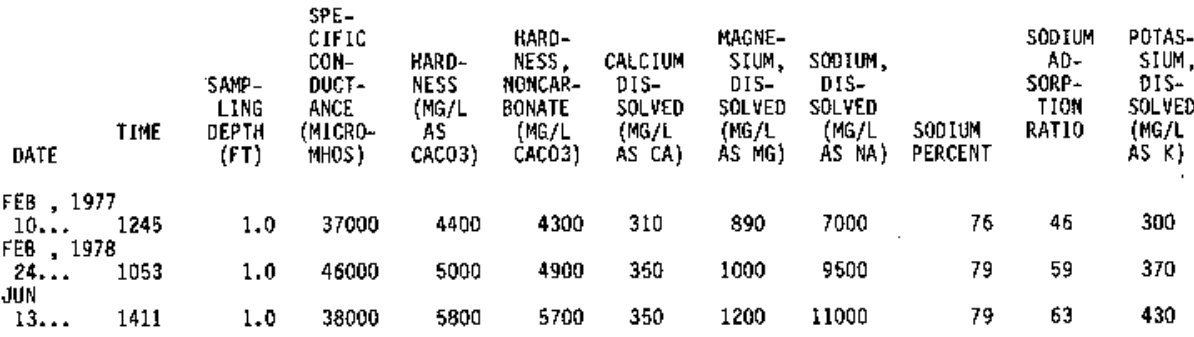

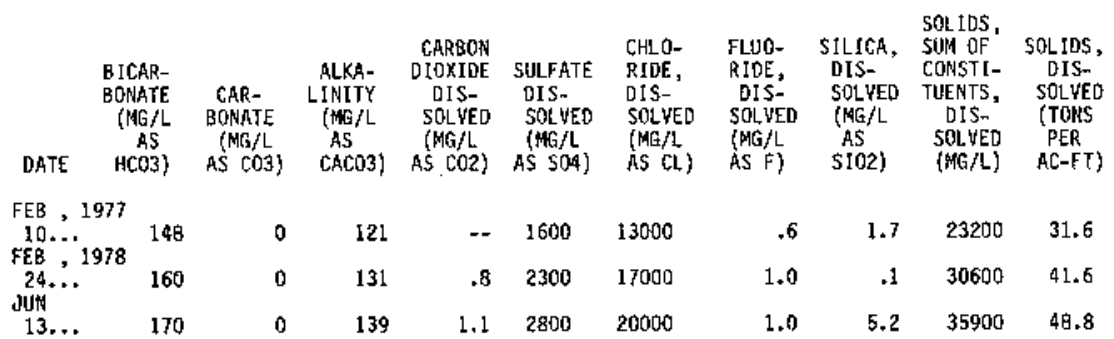

275310097345200 LINE 616 SITE 50

\begin{tabular}{|c|c|c|c|c|c|c|c|c|c|c|}
\hline & T1ME & $\begin{array}{l}\text { SAMP- } \\
\text { LING } \\
\text { DEPTH }\end{array}$ & $\begin{array}{l}\text { SPE- } \\
\text { CIFIC } \\
\text { CON- } \\
\text { DUCT- } \\
\text { ANCE } \\
\text { CMICRO- }\end{array}$ & $\begin{array}{l}\text { HARD- } \\
\text { NESS } \\
\text { (MG/L } \\
\text { AS }\end{array}$ & $\begin{array}{l}\text { MARD- } \\
\text { NESS, } \\
\text { NONCAR- } \\
\text { BOMATE } \\
\text { (HAG/L }\end{array}$ & $\begin{array}{l}\text { CALCIUH } \\
\text { DIS- } \\
\text { SOLVED } \\
\text { (AG/L }\end{array}$ & $\begin{array}{l}\text { MAGNE- } \\
\text { SILUN, } \\
\text { DIS- } \\
\text { SOLLED } \\
\text { (MG/L }\end{array}$ & $\begin{array}{l}\text { SODILA, } \\
\text { DIS- } \\
\text { SOLVED } \\
\text { (MG/L }\end{array}$ & SODIUM & $\begin{array}{l}\text { SODIUN } \\
\text { AD- } \\
\text { SORP- } \\
\text { TION } \\
\text { RATIO }\end{array}$ \\
\hline DTF & & & & & & & & & PERCENT & \\
\hline
\end{tabular}

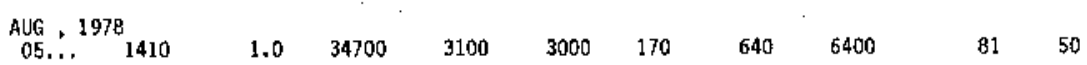

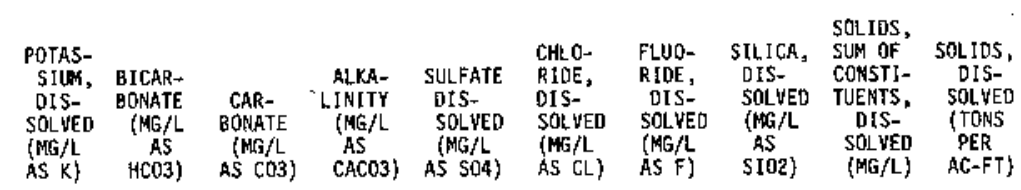

$\begin{array}{lllllllllll}\text { AUG }, & 1978 \\ 05 . . & 190 & 61 & 0 & 50 & 1800 & 11000 & .2 & 2.1 & 20200 & 27.5\end{array}$ 
Table 9C.--Quality of water in the Nukces estuary, water years 1977-78--Continud Cherical Aralyses--Continued

275314097344900 LINE 616 SITE 52

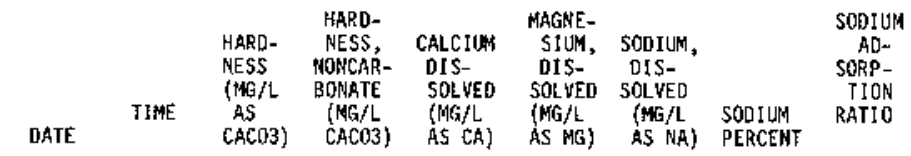
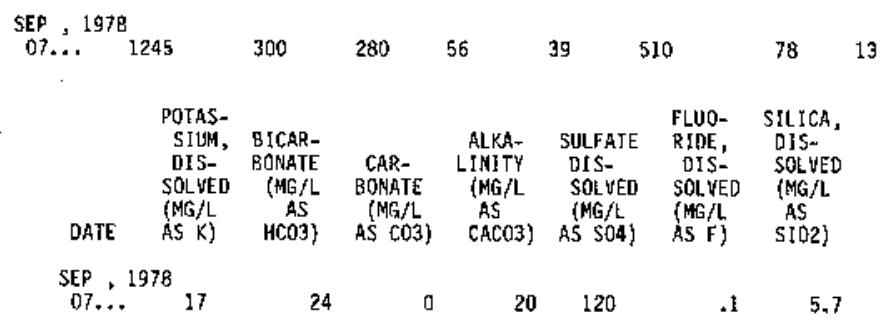

275201097333200 LIME 620 SITE 10

\begin{tabular}{|c|c|c|c|c|c|c|c|c|c|c|}
\hline DATE & TIME & $\begin{array}{l}\text { SAMP. } \\
\text { LING } \\
\text { OEPTH } \\
\text { (FT) }\end{array}$ & $\begin{array}{l}\text { SPE- } \\
\text { CIFIC } \\
\text { CON- } \\
\text { DUCT- } \\
\text { ANCE } \\
\text { (MICRO- } \\
\text { MHOS) }\end{array}$ & $\begin{array}{l}\text { HARD- } \\
\text { RESS } \\
\text { (MG/L } \\
\text { AS } \\
\text { CACO3) }\end{array}$ & $\begin{array}{l}\text { HARD- } \\
\text { NESS, } \\
\text { NONCAR- } \\
\text { BONATE } \\
\text { (NG/L } \\
\text { CACO3) }\end{array}$ & $\begin{array}{l}\text { CALCIUM } \\
\text { DIS- } \\
\text { SOLWEO } \\
\text { (NGG/L } \\
\text { AS CAS) }\end{array}$ & $\begin{array}{l}\text { MAGNE- } \\
\text { SIUM, } \\
\text { DIS- } \\
\text { SOL VED } \\
\text { (MG/L } \\
\text { AS MG) }\end{array}$ & $\begin{array}{l}\text { SODIUM, } \\
\text { DIS- } \\
\text { SOLVED } \\
\text { (MG } / L \\
\text { AS MA) }\end{array}$ & $\begin{array}{l}\text { SODIUHA } \\
\text { PERCENT }\end{array}$ & $\begin{array}{c}\text { SODIUM } \\
\text { AD- } \\
\text { SORP- } \\
\text { TIOH } \\
\text { RATIO }\end{array}$ \\
\hline \multicolumn{11}{|c|}{ AUG , 1978} \\
\hline DATE & $\begin{array}{l}\text { POTAS- } \\
\text { SIUA, } \\
\text { DIS- } \\
\text { SOLWED } \\
\text { (MIG/L } \\
\text { AS K) }\end{array}$ & $\begin{array}{c}\text { BICAR- } \\
\text { BONATE } \\
\text { (NAG/L } \\
\text { AS } \\
H C O 3)\end{array}$ & $\begin{array}{c}\text { CAR- } \\
\text { BONATE } \\
\text { (MG/L } \\
\text { AS C03) }\end{array}$ & $\begin{array}{c}\text { ALKA- } \\
\text { LINITY } \\
\{N G / L \\
\text { AS } \\
\text { CACO3 })\end{array}$ & $\begin{array}{l}\text { SULFATE } \\
\text { DIS- } \\
\text { SOLVED } \\
\text { (MGG/L } \\
\text { AS SO4) }\end{array}$ & $\begin{array}{l}\text { CHLO- } \\
\text { RIDE, } \\
\text { OIS- } \\
\text { SOLVED } \\
\text { (MG/L } \\
\text { AS CL) }\end{array}$ & $\begin{array}{l}\text { FLUD- } \\
\text { RIDE, } \\
\text { OIS- } \\
\text { SOLLED } \\
\text { (MG/L } \\
\text { AS F) }\end{array}$ & $\begin{array}{l}\text { SILICA, } \\
\text { DIS- } \\
\text { SOLVED } \\
\text { (NG/L } \\
A S \\
\text { SIO2) }\end{array}$ & $\begin{array}{l}\text { SOL IDS, } \\
\text { SUM OF } \\
\text { CONSTI- } \\
\text { TUENTS, } \\
\text { OIS- } \\
\text { SOLVEO } \\
\text { (MG/L) }\end{array}$ & $\begin{array}{c}\text { SOLIDS, } \\
\text { OIS- } \\
\text { 5OLVED } \\
\text { (TONS } \\
\text { PER } \\
\text { AC-FT) }\end{array}$ \\
\hline
\end{tabular}

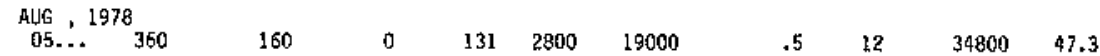

275221097315000 LINE 640 SITE 52

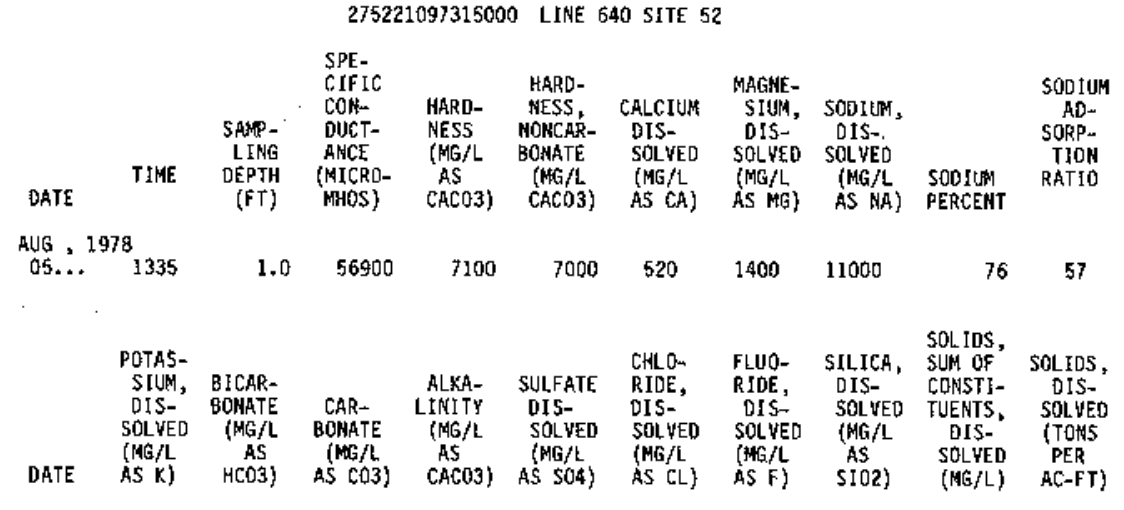

$\begin{array}{ccccccccccc}\text { AUG } & 1978 \\ 05 . . & 360 & 70 & 0 & 57 & 3200 & 21000 & .5 & .9 & 37500 & 51.0\end{array}$

275214097310800 LINE 650 SITE 48

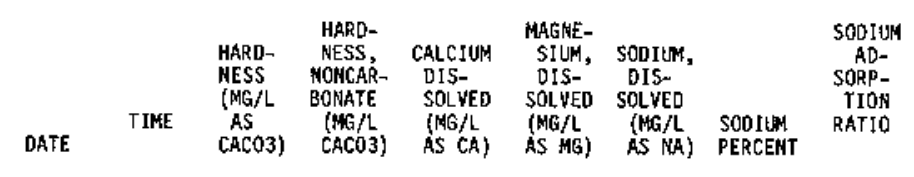

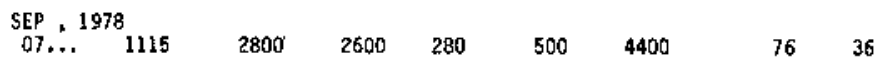

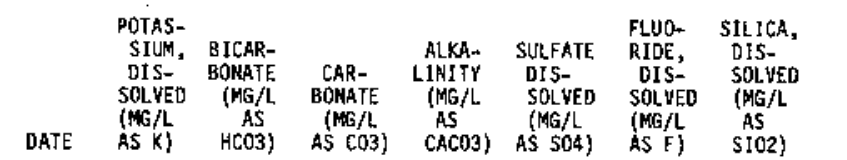

SEP 1978

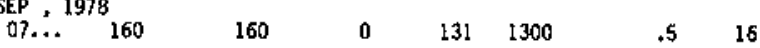





\section{Laguna Madre Estuary}

The Laguna Madre estuary, which has an area of about 640 square miles $\left(1,660 \mathrm{~km}^{2}\right)$, consists of the tidal parts of Arroyo Colorado and other tributaries, upper Laguna Madre, Baffin Bay, lower Laguna Madre, Brownsville Ship Channel, part of the Intracoastal Waterway, Port Mansfield Entrance Channel, and Brazos Santiago Pass (Figure 11). At mean low water, upper and lower Laguna Madre and Baffin Bay are generally less than 4 feet $(1.2 \mathrm{~m})$ deep, but in a few areas are as much as 10 feet $(3.0 \mathrm{~m})$ deep. The Intracoastal Waterway, Port Mansfield Entrance Channel, and Arroyo Colorado are about 15 feet $(4.6 \mathrm{~m}$ ) deep; and the Brownsville Ship Channel is about 40 feet $(12.2 \mathrm{~m})$ deep.

Water-quality data (Table 10) were collected in January, February, and June 1977 and February, March, and June 1978. 


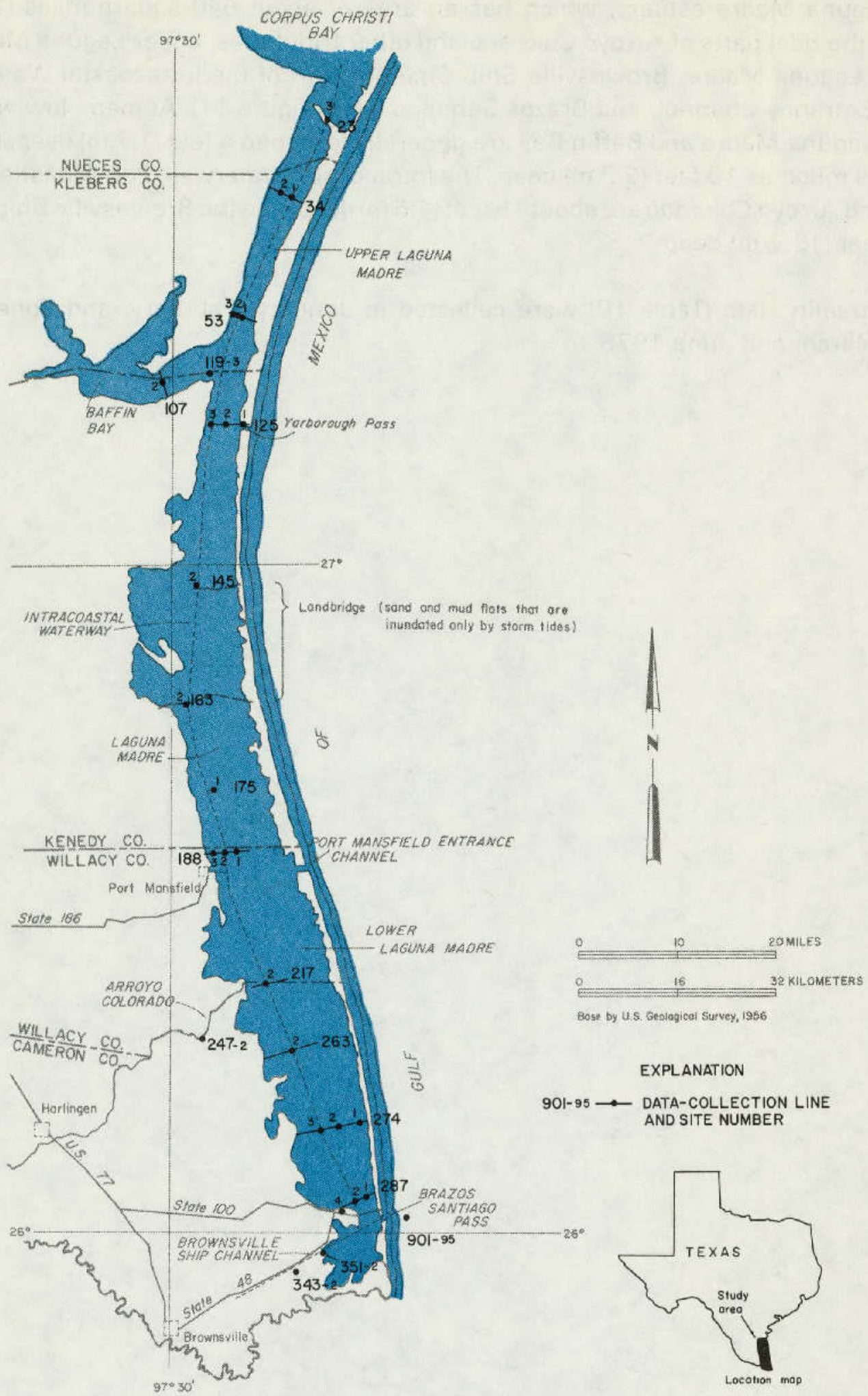

Figure 11

Data-Collection Sites in the Laguna Madre Estuary 
$\left\{F T=\right.$ feet; $M I C R O M H O S=$ micromhos per centineter at $25^{\circ}$ Celsius; $D E G C=$ degrees Celsilus; $M=$ bieters; 枯 $/ L=$ milligrams per 1 iter

273811097142100 LINE DR3 SITE 03

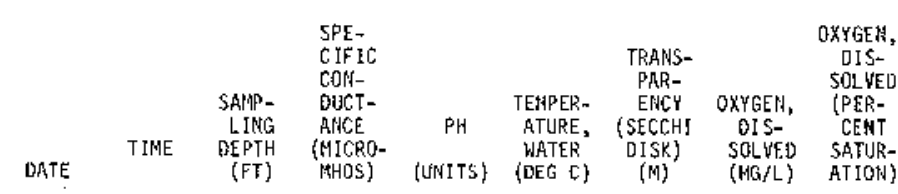

FEB , 1977

$\begin{array}{cccc}\text { FEB , } 1977 & & \\ 01 \ldots & 1755 & 1.0 & 38000 \\ 01 \ldots & 1757 & 8.0 & 37000 \\ 01 \ldots & 1759 & 16 & 37000 \\ 10 \ldots & 1125 & 1.0 & 37000 \\ 10 \ldots & 1127 & 5.0 & 37000 \\ 10 \ldots & 1129 & 10 & 37000\end{array}$

$10 . . . \quad 1129$

$10 \ldots .6131$

22... 1330

$22 \ldots \quad 1332$

$22 \ldots \quad 1334$

$22 \ldots .1336$

$30 \ldots 1050$

$\begin{array}{ll}30 \ldots . & 1052 \\ 30 \ldots & 1054\end{array}$

$30 \ldots 1056$

$30 . \cdots$

24... 1015

24... 1017

MAR .

01... 1345

$\begin{array}{ll}01 \ldots & 1347 \\ 01 . . . & 1349\end{array}$

JUR

$13 . . . \quad 1340$

$13 . . . \quad 1342$

$13 . . . \quad 1344$

$27 . . . \quad 1644$

$\begin{array}{ll}27 \ldots & 1646 \\ 27 \ldots & 1648\end{array}$

$10 \quad 37000$

$\begin{array}{ll}8.3 & 8.0 \\ 8.2 & 8.0 \\ 8.2 & 8 . \\ 8.2 & 13 . \\ 8.2 & 13 . \\ 8.2 & 12 . \\ 8.2 & 12 .\end{array}$

$\begin{array}{rrrr}8.0 & .81 & 10.2 & 105 \\ 8.0 & -- & 9.7 & 99\end{array}$

$1.0 .5 \% 000$

54000

$5.0 \quad 54000$

$\begin{array}{ll}15 & 54000 \\ 25 & 54000\end{array}$

$1.0 \quad 55000$

$5.0 \quad 55000$

$10 \quad 55000$

55000

$\begin{array}{lllll}8.3 & 29.5 & .93 & 4.4 & 74\end{array}$

2.1254000

$\begin{array}{cc}1.0 & 47000 \\ 13 & 48000\end{array}$

1.048000

$\begin{array}{ll}1.0 & 48000 \\ 12 & 48000 \\ 25 & 48000\end{array}$

$\begin{array}{llll}8.3 & 29.0 & - & 4.3 \\ 8.2 & 28.5 & -- & 3.4\end{array}$

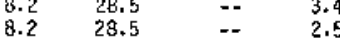

8.129 .5

8.129 .5

$\begin{array}{ll}1- & 4.1 \\ -0 & 3.9\end{array}$

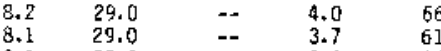

1.049000

1.0
7.5
15

$1.0 \quad 67000$

$15 \quad 67000$

8.129 .0

$8.4 \quad 14.0$

$=2.9$

27313209717AB0O LJME 034 SITE 01

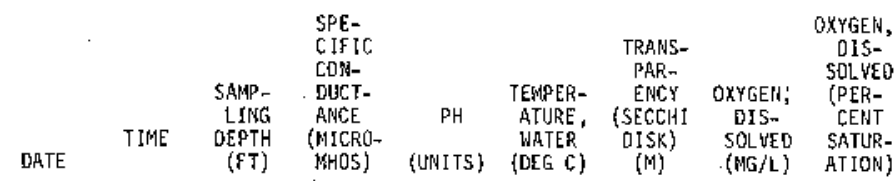

FEB , 1977

O1... 1700

$\begin{array}{ll}01 \ldots & 1702 \\ 01 \ldots . & 1704\end{array}$

JUพ $22 . . .1300$

$\begin{array}{ll}22 \ldots . . & 1300 \\ 22 . . & 1302\end{array}$

$22 \ldots .1304$

$\begin{array}{ccccccc}1.0 & 36000 & 8.1 & 3.0 & -- & 9.5 & 97 \\ 5.0 & 36000 & 8.1 & 8.0 & -- & 9.4 & 96 \\ 10 & 34000 & 8.1 & 7.0 & -- & 9.3 & 92 \\ 1.0 & 55000 & 8.2 & 29.0 & 1.02 & 4.7 & 76 \\ 5.0 & 55000 & 8.2 & 28.5 & -- & 4.5 & 74 \\ 13 & 55000 & 8.2 & 28.5 & -- & 4.3 & 70\end{array}$

273203097185100 LINE 034 SITE 02

\begin{tabular}{|c|c|c|c|c|c|c|c|c|}
\hline DATE & TIME & $\begin{array}{l}\text { SANP- } \\
\text { LING } \\
\text { DEPTH } \\
(F T)\end{array}$ & $\begin{array}{l}\text { SPE- } \\
\text { CIFIC } \\
\text { COR- } \\
\text { DUCT- } \\
\text { ANCE } \\
\text { (MICRO- } \\
\text { MHOS }\end{array}$ & $\begin{array}{c}\text { prᅡ } \\
\text { (UNITS) }\end{array}$ & $\begin{array}{l}\text { TEMPER- } \\
\text { ATURE, } \\
\text { WATER } \\
\text { (DEG C) }\end{array}$ & $\begin{array}{c}\text { TRANS- } \\
\text { PAR- } \\
\text { ENCY } \\
\text { (SECCH] } \\
\text { DISK) } \\
\text { (M) }\end{array}$ & $\begin{array}{c}\text { OXYGEN, } \\
\text { DIS- } \\
\text { SOLVED } \\
\text { (MG/L) }\end{array}$ & $\begin{array}{c}\text { OXYGEN, } \\
\text { DIS- } \\
\text { SOLVED } \\
\text { (PER- } \\
\text { CENT } \\
\text { SATUR- } \\
\text { ATHON) }\end{array}$ \\
\hline \multicolumn{9}{|c|}{ MAR , 1978 } \\
\hline $\begin{array}{l}01 \ldots \\
01 \ldots \\
01 \ldots \\
\text { Juk }\end{array}$ & $\begin{array}{l}1250 \\
1252 \\
1254\end{array}$ & $\begin{array}{l}1.0 \\
6.0 \\
12\end{array}$ & $\begin{array}{l}49000 \\
51000 \\
53000\end{array}$ & $\begin{array}{l}8.4 \\
8.3 \\
8.4\end{array}$ & $\begin{array}{l}17.5 \\
17.5 \\
18.5\end{array}$ & $\begin{array}{l}=- \\
=-\end{array}$ & $\begin{array}{l}7.6 \\
7.2 \\
7.1\end{array}$ & $\begin{array}{r}100 \\
96 \\
99\end{array}$ \\
\hline $\begin{array}{l}27 \ldots \\
27 \ldots\end{array}$ & $\begin{array}{l}1614 \\
1616\end{array}$ & $\begin{array}{l}1.0 \\
3.0\end{array}$ & $\begin{array}{l}66300 \\
66000\end{array}$ & $\begin{array}{l}7.9 \\
7.9\end{array}$ & $\begin{array}{l}32.0 \\
31.0\end{array}$ & $\begin{array}{r}.55 \\
-.\end{array}$ & $\begin{array}{l}5.3 \\
5.3\end{array}$ & $\begin{array}{l}96 \\
95\end{array}$ \\
\hline
\end{tabular}


Table 10A.--Quality of water in the Laguna Madre estuary, water yedrs 1977-78--Cont inued Field Deterninations--Cont f nued

272104097222800 LINE 053 SITE O1

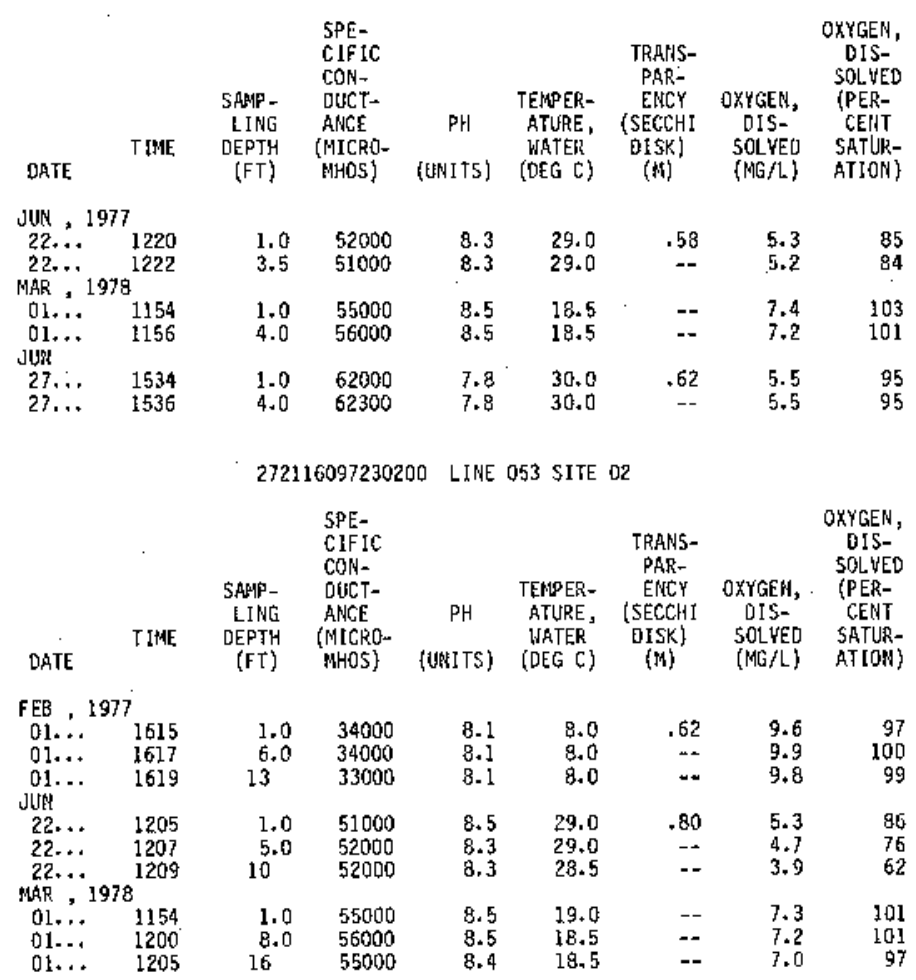

271543097301900 LIKE 107 SITE 02

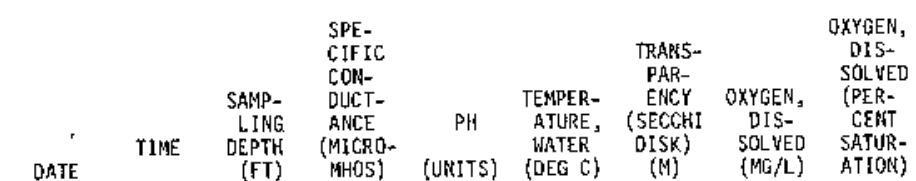

\begin{tabular}{|c|c|c|c|c|c|c|c|c|}
\hline \multicolumn{9}{|c|}{ FEB, 1977} \\
\hline $\begin{array}{l}01 \ldots \\
01 \ldots\end{array}$ & $\begin{array}{l}1530 \\
1532\end{array}$ & $\begin{array}{l}1.0 \\
7.0\end{array}$ & $\begin{array}{l}24000 \\
24000\end{array}$ & $\begin{array}{l}8.3 \\
8.3\end{array}$ & $\begin{array}{l}9.0 \\
9.0\end{array}$ & .19 & $\begin{array}{l}9.5 \\
9.7\end{array}$ & $\begin{array}{l}93 \\
96\end{array}$ \\
\hline \multicolumn{9}{|l|}{ JUN } \\
\hline $\begin{array}{l}22 \ldots \\
22 \ldots\end{array}$ & $\begin{array}{l}1130 \\
1132\end{array}$ & $\begin{array}{l}1.0 \\
6.0\end{array}$ & $\begin{array}{l}35000 \\
35000\end{array}$ & $\begin{array}{l}8.4 \\
8.4\end{array}$ & $\begin{array}{r}28.5 \\
28.5\end{array}$ & $\begin{array}{r}.78 \\
--\end{array}$ & $\begin{array}{l}5.9 \\
5.7\end{array}$ & $\begin{array}{l}8 \\
8\end{array}$ \\
\hline \multicolumn{9}{|c|}{$M M R, 1978$} \\
\hline $\begin{array}{l}01, \ldots \\
01, \ldots\end{array}$ & $\begin{array}{l}1113 \\
1115\end{array}$ & $\begin{array}{l}1.0 \\
6.0\end{array}$ & $\begin{array}{l}58000 \\
57000\end{array}$ & $\begin{array}{l}8.5 \\
0.6\end{array}$ & $\begin{array}{l}18.5 \\
18.5\end{array}$ & $=$ & $\begin{array}{l}7.3 \\
7.3\end{array}$ & $\begin{array}{l}103 \\
102\end{array}$ \\
\hline
\end{tabular}

271630097251900 LINE 119 SITE O3

\begin{tabular}{|c|c|c|c|c|c|c|c|c|}
\hline DATE & TIME & $\begin{array}{c}\text { 5AMP- } \\
\text { LING } \\
\text { DEPTH } \\
\text { (FT) }\end{array}$ & $\begin{array}{l}\text { SPE- } \\
\text { CIF IC } \\
\text { CON- } \\
\text { DUCT- } \\
\text { ANCE } \\
\text { (MICRO- } \\
\text { MHOS) }\end{array}$ & $\begin{array}{c}\mathrm{PH} \\
\text { (UNITS) }\end{array}$ & $\begin{array}{l}\text { TEMPER- } \\
\text { ATURE, } \\
\text { WATER } \\
\text { (DEG C) }\end{array}$ & $\begin{array}{l}\text { TRANS- } \\
\text { PAR- } \\
\text { ENCY } \\
\text { (SECCHI } \\
\text { DISK) } \\
\text { (M) }\end{array}$ & $\begin{array}{c}\text { OXYGEN, } \\
\text { DIS- } \\
\text { SOLVED } \\
\text { (MG } / L \text { ) }\end{array}$ & $\begin{array}{l}\text { OXYGEN, } \\
\text { DIS- } \\
\text { SOLVED } \\
\text { (PER- } \\
\text { CENT } \\
\text { SATUR- } \\
\text { RTION) }\end{array}$ \\
\hline $\begin{array}{r}\text { JUN, } \\
27 \ldots \\
27 \ldots\end{array}$ & $\begin{array}{l}8 \\
1510 \\
1512\end{array}$ & $\begin{array}{l}1.0 \\
6.0\end{array}$ & $\begin{array}{l}62300 \\
62300\end{array}$ & $\begin{array}{l}7.8 \\
7.8\end{array}$ & $\begin{array}{l}30.0 \\
30.0\end{array}$ & $\begin{array}{r}.57 \\
--\end{array}$ & $\begin{array}{l}5.5 \\
5.5\end{array}$ & $\begin{array}{l}95 \\
95\end{array}$ \\
\hline
\end{tabular}

271213097231100 LINE 125 SITE OI

\begin{tabular}{|c|c|c|c|c|c|c|c|}
\hline DATE & TIME & $\begin{array}{l}\text { SAMP- } \\
\text { LING } \\
\text { DEPTH } \\
\text { (FT) }\end{array}$ & $\begin{array}{l}\text { SPE- } \\
\text { CIFIC } \\
\text { CON- } \\
\text { DUCT- } \\
\text { ANCE } \\
\text { (MICRO- } \\
\text { MHOS) }\end{array}$ & $\begin{array}{c}\mathrm{PH} \\
\text { (UNITS) }\end{array}$ & $\begin{array}{l}\text { TEMPER- } \\
\text { ATURE, } \\
\text { WATER } \\
\text { (DEG C) }\end{array}$ & $\begin{array}{c}\text { OXYGEN, } \\
\text { OIS- } \\
\text { SOLVED } \\
\text { (PRG } / L)\end{array}$ & $\begin{array}{c}\text { OXYGEN, } \\
\text { DIS- } \\
\text { SOLVED } \\
\text { (PER- } \\
\text { CEAT } \\
\text { SATUR- } \\
\text { ATION) }\end{array}$ \\
\hline MAR , & & & & & & & \\
\hline $\begin{array}{l}01 . . \\
01 . .\end{array}$ & $\begin{array}{l}1017 \\
1019\end{array}$ & $\begin{array}{l}1.0 \\
7.0\end{array}$ & $\begin{array}{l}54000 \\
55000\end{array}$ & $\begin{array}{l}8.4 \\
8.4\end{array}$ & $\begin{array}{l}18.0 \\
18.0\end{array}$ & $\begin{array}{l}7.0 \\
7.0\end{array}$ & $\begin{array}{l}96 \\
96\end{array}$ \\
\hline
\end{tabular}



Table 10A.--Quality of water in the Laguna Madre estuary, water years 1977-78--Continued
Field Determinations.-Cont inued

271211097243300 LIJUE 125 SITE U2

\begin{tabular}{|c|c|c|c|c|c|c|c|c|}
\hline DATE & TIME & $\begin{array}{c}\text { SAMP- } \\
\text { LING } \\
\text { DEPTH } \\
\text { (FT) }\end{array}$ & $\begin{array}{l}\text { SPE- } \\
\text { CIFIC } \\
\text { CON- } \\
\text { DUCT- } \\
\text { ANCE } \\
\text { (MICRO- } \\
\text { MHOS) }\end{array}$ & $\begin{array}{c}P H \\
\text { (UNLTS) }\end{array}$ & $\begin{array}{l}\text { TEMPER- } \\
\text { ATURE, } \\
\text { WATER } \\
\text { (DEG C) }\end{array}$ & $\begin{array}{l}\text { TRANS- } \\
\text { PAR- } \\
\text { ENCY } \\
\text { (SECCHI } \\
\text { OISK) } \\
\text { (il) }\end{array}$ & $\begin{array}{c}\text { OXYGEN, } \\
\text { DIS- } \\
\text { SOLVED } \\
(\text { TAL } / L)\end{array}$ & $\begin{array}{c}\text { OXYGEN } \\
\text { OIS- } \\
\text { SOLVE } \\
\text { (PER- } \\
\text { CENT } \\
\text { SATUR } \\
\text { ATIDN }\end{array}$ \\
\hline $\begin{array}{l}\text { UUN, } \\
22 . . \\
22 \ldots\end{array}$ & $\begin{array}{l}1050 \\
1052\end{array}$ & $\begin{array}{l}1.0 \\
6.0\end{array}$ & $\begin{array}{l}54000 \\
54000\end{array}$ & $\begin{array}{l}8.4 \\
8.4\end{array}$ & $\begin{array}{l}28.0 \\
28.0\end{array}$ & $\begin{array}{r}.60 \\
--\end{array}$ & $\begin{array}{l}5.3 \\
5.4\end{array}$ & \\
\hline $\begin{array}{l}27 \ldots \\
27 \ldots\end{array}$ & $\begin{array}{l}1444 \\
1446\end{array}$ & $\begin{array}{r}1.0 \\
5.0\end{array}$ & $\begin{array}{l}60300 \\
60200\end{array}$ & $\begin{array}{l}3.0 \\
8.1\end{array}$ & $\begin{array}{l}30.0 \\
30.0\end{array}$ & $\begin{array}{l}.36 \\
--\end{array}$ & $\begin{array}{l}5.9 \\
5.8\end{array}$ & \\
\hline & & 271 & 13097253500 & LINE & 125 SITE & 03 & & \\
\hline DATE & TIME & $\begin{array}{c}\text { SAlPP - } \\
\text { LING } \\
\text { DEPTH } \\
(\mathrm{FT})\end{array}$ & $\begin{array}{l}\text { SPE- } \\
\text { CIFIC } \\
\text { CON- } \\
\text { DUCT- } \\
\text { ANCE } \\
\text { (MICRO- } \\
\text { PHOS) }\end{array}$ & $\begin{array}{c}\mathrm{PH} \\
\text { (UNITS) }\end{array}$ & $\begin{array}{l}\text { TEHPER- } \\
\text { ATURE, } \\
\text { WATER } \\
\text { (EEG C) }\end{array}$ & $\begin{array}{l}\text { TRANS- } \\
\text { PAR- } \\
\text { ENCY } \\
\text { (SECCHI } \\
\text { DISK) } \\
\text { (M) }\end{array}$ & $\begin{array}{c}\text { OXYGEN, } \\
\text { DIS- } \\
\text { SOLWED } \\
\text { (HG } / L)\end{array}$ & $\begin{array}{c}\text { OXYGE } \\
\text { DIS } \\
\text { SOL } \\
\text { (PEF } \\
\text { CEI } \\
\text { SATI } \\
\text { ATIC }\end{array}$ \\
\hline
\end{tabular}

FEB , 1977

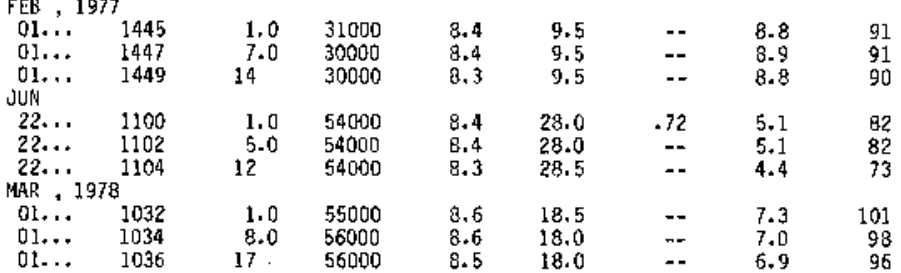

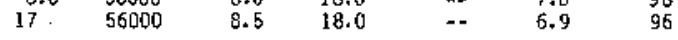

265857097270200 LIRE 145 SITE 02

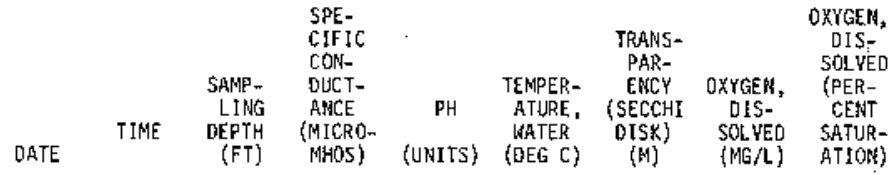

FEB, 1977

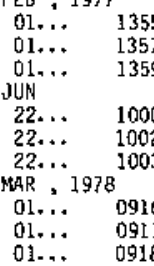

$\begin{array}{ccccccc}1.0 & 30000 & 8.2 & 8.5 & .25 & 9.0 & 90 \\ 10 & 30000 & 8.2 & 8.5 & -- & 9.0 & 90 \\ 19 & 30000 & 8.2 & 8.5 & -- & 8.9 & 89 \\ 1.0 & 50000 & 8.3 & 27.5 & 1.00 & 5.7 & 91 \\ 5.0 & 50000 & 8.3 & 27.5 & -- & 5.7 & 91 \\ 15 & 50000 & 8.3 & 27.5 & -- & 5.7 & 91 \\ 1.0 & 58000 & 8.4 & 19.5 & -- & 7.0 & 101 \\ 8.0 & 58000 & 8.4 & 19.5 & -- & 7.0 & 101 \\ 17 & 58000 & 8.4 & 18.0 & -- & 6.9 & 98\end{array}$

264822097281400 LINE 163 5ITE O2

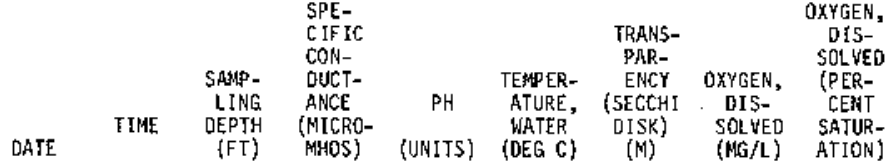

$\begin{array}{ll}\text { FEB , } 1977 \\ 01 \ldots & 1315 \\ 01 \ldots & 1317 \\ 01 \ldots & 1319 \\ \text { WAR, } 1978 \\ 01 \ldots & 0830 \\ 01 \ldots & 0832 \\ 01 \ldots & 0834 \\ \text { JUN } & \\ 27 \ldots & 1349 \\ 27 \ldots & 1351\end{array}$

$\begin{array}{cc}1.0 & 32000 \\ 7.0 & 32000 \\ 14 & 31000 \\ 1.0 & 57000 \\ 7.0 & 57000 \\ 15 & 57000 \\ 1.0 & 56000 \\ 17 & 55500\end{array}$

$\begin{array}{lrrrr}8.2 & 9.0 & .18 & 8.7 & 88 \\ 8.2 & 9.0 & -- & 8.7 & 88 \\ 8.3 & 9.0 & -- & 8.7 & 89 \\ & & - & & \\ 9.5 & 20.0 & -- & 6.7 & 98 \\ 8.4 & 20.0 & -- & 6.8 & 99 \\ 2.4 & 19.0 & -- & 6.9 & 98 \\ & & & & \\ 8.1 & 29.5 & .67 & 6.0 & 100 \\ 8.0 & 30.0 & -- & 5.9 & 100\end{array}$


Table 10A.--Quality of water in the Laguna Madre estuary, water years 1917-78--Continued Field Determinations--Cont inued

264136097245100 LIJUE 175 SITE 01

\begin{tabular}{|c|c|c|c|c|c|c|c|c|}
\hline DATE & TIME & $\begin{array}{l}\text { SAMP- } \\
\text { LING } \\
\text { DEPTH } \\
\text { (FT) }\end{array}$ & $\begin{array}{l}\text { SPE- } \\
\text { CIFIC } \\
\text { COH- } \\
\text { DUGT- } \\
\text { ANCE } \\
\text { (\$ICRO- } \\
\text { MHOS) }\end{array}$ & $\begin{array}{c}\text { PH } \\
\text { (UNITS) }\end{array}$ & $\begin{array}{c}\text { TEMPER- } \\
\text { ATURE, } \\
\text { HATER } \\
\text { (OEG C) }\end{array}$ & $\begin{array}{c}\text { TRANIS- } \\
\text { PAR- } \\
\text { ENCY } \\
\text { (SECCHI } \\
\text { DISK) } \\
\text { (M) }\end{array}$ & $\begin{array}{c}\text { OXYGEN, } \\
\text { DIS- } \\
\text { SOLVED } \\
\text { (MG/L) }\end{array}$ & $\begin{array}{l}\text { OXYGEK, } \\
\text { DIS- } \\
\text { SOLYVEO } \\
\text { (PER- } \\
\text { CENT } \\
\text { SATUR- } \\
\text { ATION) }\end{array}$ \\
\hline \multirow[t]{3}{*}{$\begin{array}{l}\text { JUNN , } \\
27, \ldots \\
27, \ldots\end{array}$} & $\begin{array}{l}8 \\
1320 \\
1322\end{array}$ & $\begin{array}{l}1.0 \\
5.0\end{array}$ & $\begin{array}{l}55000 \\
55000\end{array}$ & $\begin{array}{l}8.0 \\
8.0\end{array}$ & $\begin{array}{l}29.5 \\
29.5\end{array}$ & $\begin{array}{r}.67 \\
--\end{array}$ & $\begin{array}{l}6.0 \\
5.9\end{array}$ & \\
\hline & & 263 & 13097220 & LINE & I8B SITE & 01 & & \\
\hline & TIAE & $\begin{array}{l}\text { SANP- } \\
\text { LING } \\
\text { OEPTH } \\
\text { (FT) }\end{array}$ & $\begin{array}{l}\text { SPE- } \\
\text { CIFIC } \\
\text { CON- } \\
\text { DUCT- } \\
\text { ANCE } \\
\text { (MICRR- } \\
\text { MHDS) }\end{array}$ & $\begin{array}{c}\mathrm{PH} \\
\text { (UNITS) }\end{array}$ & $\begin{array}{c}\text { TEHOPER- } \\
\text { ATURE, } \\
\text { WATER } \\
\text { (OEG C) }\end{array}$ & $\begin{array}{c}\text { TRANS- } \\
\text { PAR- } \\
\text { ENCY } \\
\text { (SECCHI } \\
\text { DISK) } \\
(M)\end{array}$ & $\begin{array}{c}\text { OXYGEN, } \\
\text { OIS- } \\
\text { SOLYED } \\
\text { (MG/L) }\end{array}$ & $\begin{array}{c}\text { OXYGEN, } \\
\text { DIS- } \\
\text { SOLVED } \\
\text { (PER- } \\
\text { CENT } \\
\text { SATUR- } \\
\text { ATION) }\end{array}$ \\
\hline
\end{tabular}

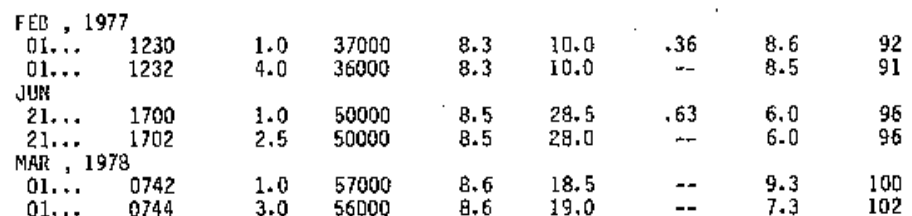

263532097242800 LINE 188 SITE 02

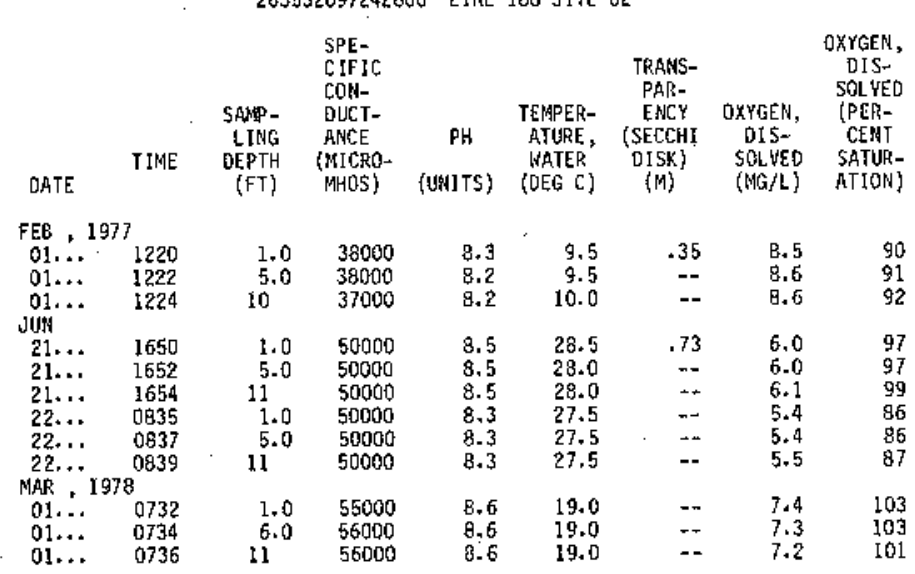

263516097252300 LITE 186 SITE 03

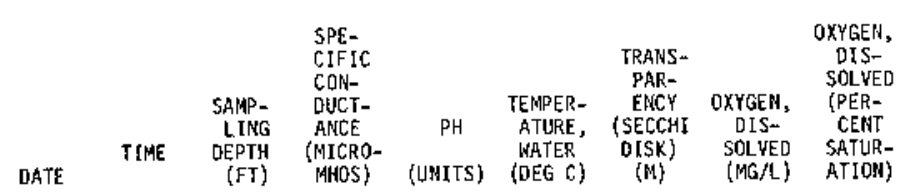

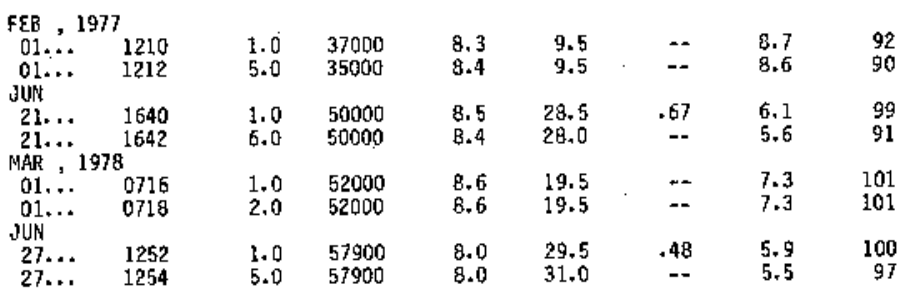


Table 10A.--Quality of water in the Lagund Madre estuary, water years 1977-78--Continued

Field leterminations--Cont1nued

262423097204400 LINE 217 SITE 02

\begin{tabular}{|c|c|c|c|c|c|c|c|c|}
\hline DATE & TIME & $\begin{array}{l}\text { SANT } \\
\text { LING } \\
\text { DEPTIA } \\
(F T)\end{array}$ & $\begin{array}{l}\text { SPE- } \\
\text { CIFIC } \\
\text { CON- } \\
\text { DUCT- } \\
\text { ANCE } \\
\text { (MICRO- } \\
\text { MHOS) }\end{array}$ & $\begin{array}{c}\text { PH } \\
\text { (UNITS) }\end{array}$ & $\begin{array}{c}\text { TEMPER- } \\
\text { ATURE, } \\
\text { HATER } \\
\text { \{DEG C) }\end{array}$ & $\begin{array}{l}\text { TRANS- } \\
\text { PAR- } \\
\text { ENCY } \\
\text { (SECCHI } \\
\text { DISK) } \\
\text { (M) }\end{array}$ & $\begin{array}{c}\text { OXYGEN, } \\
\text { DIS- } \\
\text { SOLWED } \\
\text { (MG } / L)\end{array}$ & $\begin{array}{c}\text { OXYGEN, } \\
\text { DIS- } \\
\text { SOLYEO } \\
\text { (PER- } \\
\text { CENT } \\
\text { SATUR- } \\
\text { ATION) }\end{array}$ \\
\hline \multicolumn{9}{|c|}{ FEB , 1977} \\
\hline $\begin{array}{l}01 \ldots \\
01 \ldots \\
01 \ldots \\
01 \ldots\end{array}$ & $\begin{array}{l}1030 \\
1032 \\
1034 \\
1036\end{array}$ & $\begin{array}{l}1.0 \\
5.0 \\
10 \\
15\end{array}$ & $\begin{array}{l}39000 \\
40000 \\
40000 \\
37000\end{array}$ & $\begin{array}{l}8.3 \\
8.3 \\
8.4 \\
8.4\end{array}$ & $\begin{array}{l}3.0 \\
8.0 \\
8.0 \\
8.0\end{array}$ & $\begin{array}{c}.42 \\
-- \\
-- \\
-\end{array}$ & $\begin{array}{l}9.2 \\
8.8 \\
8.8 \\
8.8\end{array}$ & $\begin{array}{l}95 \\
90 \\
90 \\
90\end{array}$ \\
\hline $\begin{array}{l}\text { JUN } \\
21, \ldots\end{array}$ & & 100 & & & & & & \\
\hline $\begin{array}{r}21 \ldots \\
21 \ldots \\
21 \ldots \\
\text { FEB... }\end{array}$ & $\begin{array}{l}1520 \\
1522 \\
1524 \\
8^{1524}\end{array}$ & $\begin{array}{l}1.0 \\
5.0 \\
15\end{array}$ & $\begin{array}{l}48000 \\
50000 \\
58000\end{array}$ & $\begin{array}{l}8.5 \\
8.4 \\
8.4\end{array}$ & $\begin{array}{l}28.0 \\
28.0 \\
28.5\end{array}$ & $\begin{array}{l}.40 \\
-. \\
-.\end{array}$ & $\begin{array}{l}6.0 \\
5.7 \\
4.5\end{array}$ & $\begin{array}{l}95 \\
92 \\
75\end{array}$ \\
\hline $\begin{array}{l}28 \ldots \\
28 \ldots \\
28 \ldots \\
\text { JUN }\end{array}$ & $\begin{array}{l}1600 \\
1602 \\
1604\end{array}$ & $\begin{array}{l}1.0 \\
6.0 \\
13\end{array}$ & $\begin{array}{l}50000 \\
50000 \\
48000\end{array}$ & $\begin{array}{l}8.4 \\
8.3 \\
8.3\end{array}$ & $\begin{array}{l}21.0 \\
20.5 \\
21.5\end{array}$ & $\begin{array}{l}- \\
--\end{array}$ & $\begin{array}{l}7.2 \\
6.9 \\
7.0\end{array}$ & $\begin{array}{r}103 \\
98 \\
100\end{array}$ \\
\hline $\begin{array}{l}27 \ldots \\
27 \ldots\end{array}$ & $\begin{array}{l}1108 \\
1110\end{array}$ & $13^{1.0}$ & $\begin{array}{l}53000 \\
59300\end{array}$ & $\begin{array}{l}8.3 \\
8.3\end{array}$ & $\begin{array}{l}29.0 \\
29.0\end{array}$ & $\begin{array}{r}.58 \\
-.\end{array}$ & $\begin{array}{l}6.1 \\
5.8\end{array}$ & $\begin{array}{r}100 \\
99\end{array}$ \\
\hline
\end{tabular}

261929097264500 LINE 247 S1TE 02

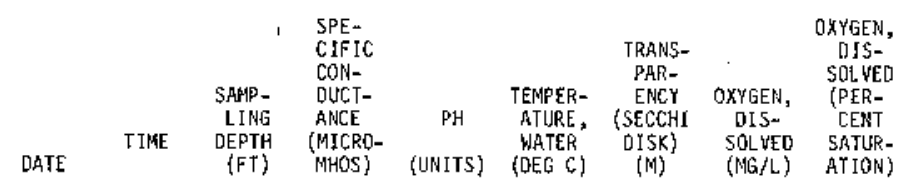

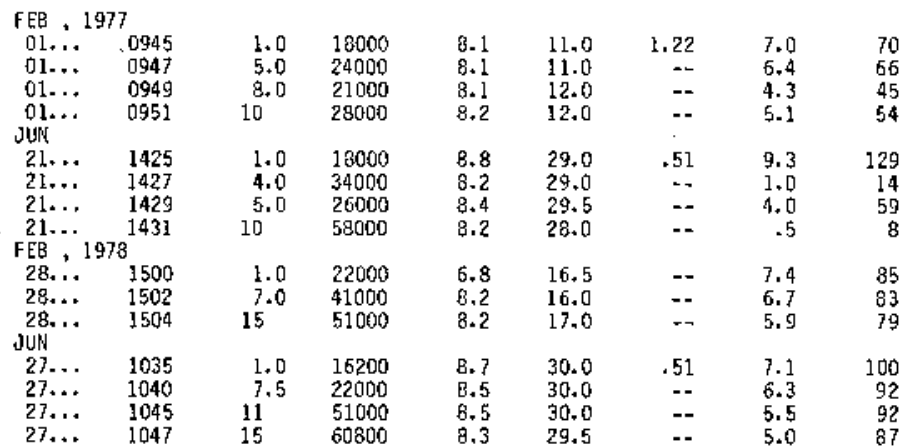

261922097182800 LINE 263 SITE 02

\begin{tabular}{|c|c|c|c|c|c|c|c|c|}
\hline DATE & T I TKE & $\begin{array}{l}\text { SAMP- } \\
\text { LING } \\
\text { DEPTH } \\
\text { (FT) }\end{array}$ & $\begin{array}{l}\text { SPE- } \\
\text { CIFIC } \\
\text { CON- } \\
\text { DUCT- } \\
\text { ANCE } \\
\text { (AICRO- } \\
\text { MHOS) }\end{array}$ & $\begin{array}{c}\text { PH } \\
\text { (UNITS\}) }\end{array}$ & $\begin{array}{l}\text { TEMPER- } \\
\text { ATURE, } \\
\text { WATER } \\
\text { (DEG C) }\end{array}$ & $\begin{array}{l}\text { TRANS- } \\
\text { PAR- } \\
\text { ENCY } \\
\text { (SECCHI } \\
\text { DISK) } \\
\text { (M) }\end{array}$ & $\begin{array}{c}\text { OXYGEN, } \\
\text { OIS+ } \\
\text { SOL VEO } \\
\text { (MG } / L)\end{array}$ & $\begin{array}{c}\text { DXYGEN, } \\
\text { DIS- } \\
\text { SOL YED } \\
\text { (PER- } \\
\text { CENT } \\
\text { SATUR- } \\
\text { ATIOAN) }\end{array}$ \\
\hline \multicolumn{9}{|c|}{ FEB, 1977} \\
\hline $01 .$. & 0855 & 1.0 & 38000 & 8.3 & 8.0 & .44 & 8.9 & 91 \\
\hline $01 \ldots$ & 0857 & 7.0 & 38000 & & & 2 & 9. & 92 \\
\hline & & \\
\hline $21 \ldots$ & 1340 & 1.0 & 58000 & B. 3 & 28.0 & .25 & 5.1 & \\
\hline $21 \ldots$ & 1342 & 5.0 & 53000 & & & $=$ & & \\
\hline & 1344 & 13 & 58000 & & & & & \\
\hline \multicolumn{9}{|c|}{ FEB, 1978} \\
\hline $28 . \cdots$ & 1404 & 1.0 & 52000 & 8. & 21. & -+ & 6.8 & \\
\hline 2 & 1406 & 6.0 & & 8. & 21 & -- & 6. & \\
\hline 2 & 1408 & 13 & 51000 & 8.3 & 21.5 & - & 6.9 & \\
\hline
\end{tabular}

261256097121800 LINE 274 SITE OI

\begin{tabular}{|c|c|c|c|c|c|c|c|c|}
\hline DATE & TIME & $\begin{array}{l}\text { SAMP- } \\
\text { LING } \\
\text { DEPTH } \\
(F T)\end{array}$ & $\begin{array}{l}\text { SPE- } \\
\text { CIFIC } \\
\text { CON- } \\
\text { DUCT- } \\
\text { ANCE } \\
\text { (MICRO- } \\
\text { MHOS) }\end{array}$ & $\begin{array}{c}\text { PH } \\
\text { (UNITS) }\end{array}$ & $\begin{array}{l}\text { TEMPER- } \\
\text { ATURE, } \\
\text { WATER } \\
\text { (DEG C) }\end{array}$ & $\begin{array}{l}\text { TRANS- } \\
\text { PAR- } \\
\text { ENCY } \\
\text { (SECCHI } \\
\text { DISK) } \\
\text { (M) }\end{array}$ & $\begin{array}{c}\text { OXYGEN, } \\
\text { DIS- } \\
\text { SOLVED } \\
\text { (MG/L) }\end{array}$ & $\begin{array}{c}\text { OXYGEN, } \\
\text { DIS- } \\
\text { SQLVED } \\
\text { (PER- } \\
\text { CENT. } \\
\text { SATUR- } \\
\text { ATION) }\end{array}$ \\
\hline \multicolumn{9}{|c|}{ Jos , 1978} \\
\hline $\begin{array}{l}27 \ldots \\
27 \ldots\end{array}$ & $\begin{array}{l}0950 \\
0952\end{array}$ & $\begin{array}{l}1.0 \\
3.0\end{array}$ & $\begin{array}{l}53900 \\
53900\end{array}$ & $\begin{array}{l}8.6 \\
8.6\end{array}$ & $\begin{array}{l}28.5 \\
28.5\end{array}$ & $\begin{array}{r}.91 \\
--\end{array}$ & $\begin{array}{l}5.8 \\
5.9\end{array}$ & $\begin{array}{l}95 \\
97\end{array}$ \\
\hline
\end{tabular}


Table 104.--Qual fty of water in the Laguna Pladre estuary, water years 1977-78--Continued Field Deterninations--Continued

261226097135700 LINE 274 SITE D2

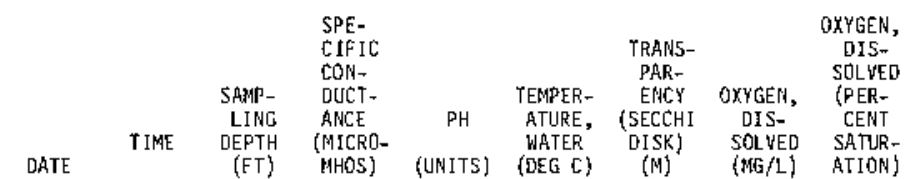

$F E B, 1977$

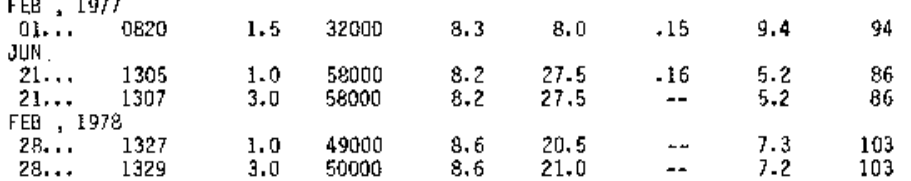

28.000

261153097153400 LINE 274 SITE 03

\begin{tabular}{|c|c|c|c|c|c|c|c|c|}
\hline DATE & TIME & $\begin{array}{c}\text { SAMP- } \\
\text { LING } \\
\text { DEPTH } \\
\text { (FT) }\end{array}$ & $\begin{array}{l}\text { SPE- } \\
\text { CIFIC } \\
\text { CON- } \\
\text { DUCT- } \\
\text { ANCE } \\
\text { (MICRO- } \\
\text { MHOS) }\end{array}$ & $\begin{array}{c}\mathrm{PH} \\
\text { (UNITS) }\end{array}$ & $\begin{array}{l}\text { TEMPER- } \\
\text { ATURE, } \\
\text { WATER } \\
\text { (DEG E) }\end{array}$ & $\begin{array}{l}\text { TRANS- } \\
\text { PAR- } \\
\text { ENCY } \\
\text { (SECCHI } \\
\text { DISK) } \\
\text { (M) }\end{array}$ & $\begin{array}{c}\text { OXYGEN, } \\
\text { OIS- } \\
\text { SOLWED } \\
\text { (MG } / \mathrm{L})\end{array}$ & $\begin{array}{c}\text { OXYGEN, } \\
\text { DIS- } \\
\text { SOL YED } \\
\text { (PER- } \\
\text { CENT } \\
\text { SATUR- } \\
\text { ATION) }\end{array}$ \\
\hline
\end{tabular}

$F E B, 1977$

FEB $=1977$

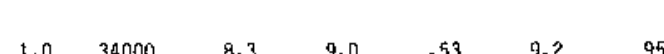

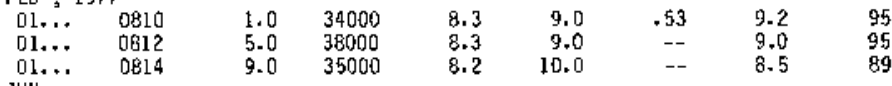

$01 . . .0814$

21... 1255

$21 \ldots \quad 1257$

$21 . \cdots 1978$

FEB, 1978

$28 . . . \quad 1307$

$28 .+1311$

$\begin{array}{ll}1.0 & 57000 \\ 5.0 & 57000\end{array}$

$\begin{array}{cc}5.0 & 57000 \\ 10 & 58000\end{array}$

$\begin{array}{lllll}8.2 & 27.5 & .16 & 5.3 & 87\end{array}$

$\begin{array}{lllllll}1.0 & 50000 & 8.5 & 21.0 & - & 7.5 & 107\end{array}$

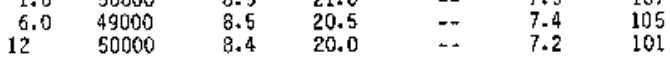

260553097111200 LIME 287 SITE 01

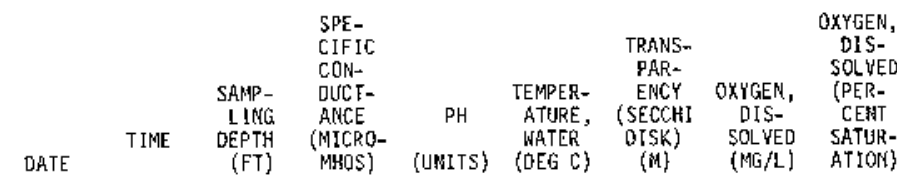

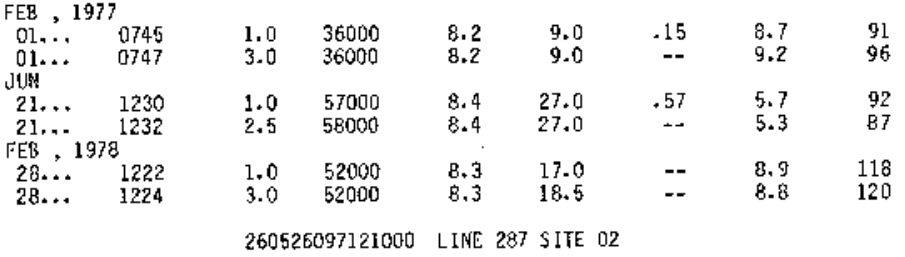

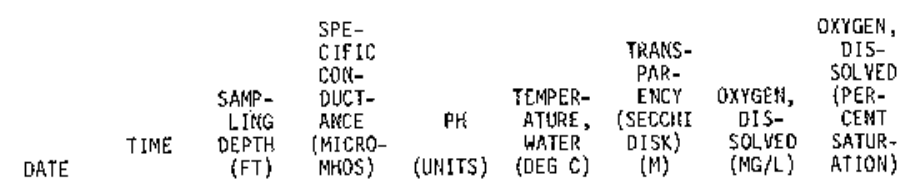

FEB, 1977

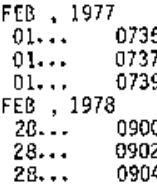

$\begin{array}{cc}1.0 & 39000 \\ 5.0 & 39000 \\ 11 & 39000 \\ 1.0 & 51000 \\ 8.0 & 51000 \\ 13 & 51000\end{array}$

$\begin{array}{lrrrr}8.2 & 9.0 & .22 & 8.7 & 91 \\ 8.2 & 9.0 & - & 9.2 & 96 \\ 8.2 & 9.0 & -- & 9.2 & 96 \\ 9.0 & 15.0 & -- & 8.1 & 104 \\ 9.0 & 15.0 & -- & 8.1 & 104 \\ 9.0 & 16.5 & -- & 8.0 & 105\end{array}$ 
Table 10A.--Quality of water in the Laguna Madre estuary, water years 1977-78--Continued field Determinat ions- Continued

260439097131000 LIPE 287 SITE 04

\begin{tabular}{|c|c|c|c|c|c|c|c|c|}
\hline DATE & TIME & $\begin{array}{c}\text { SANP - } \\
\text { LIRG } \\
\text { DEPTH } \\
(\mathrm{FT})\end{array}$ & $\begin{array}{l}\text { SPE- } \\
\text { CIFIC } \\
\text { CON- } \\
\text { DUCT- } \\
\text { ANCE } \\
\text { (MICRO- } \\
\text { BHOS) }\end{array}$ & $\begin{array}{c}\text { PH } \\
\text { \{UNITS\} }\end{array}$ & $\begin{array}{l}\text { TEMPER- } \\
\text { ATURE, } \\
\text { WATER } \\
\text { (DEG C) }\end{array}$ & $\begin{array}{l}\text { JRANS- } \\
\text { PAR- } \\
\text { ENCY } \\
\text { (SECCHI } \\
\text { DISK) } \\
\text { (AI) }\end{array}$ & $\begin{array}{l}\text { OXYGEN, } \\
\text { DIS- } \\
\text { SOLVED } \\
\text { (NG/L) }\end{array}$ & $\begin{array}{l}\text { OXYGEN, } \\
\text { DIS- } \\
\text { SOLLVED } \\
\text { (PER- } \\
\text { CENT } \\
\text { SATUR- } \\
\text { ATION) }\end{array}$ \\
\hline \multicolumn{9}{|c|}{ FEB , 1977} \\
\hline $\begin{array}{l}01 \ldots \\
01 \ldots \\
\text { jun }\end{array}$ & $\begin{array}{l}0720 \\
0722\end{array}$ & $\begin{array}{l}1.0 \\
6.0\end{array}$ & $\begin{array}{l}36000 \\
37000\end{array}$ & $\begin{array}{l}8.2 \\
8.1\end{array}$ & $\begin{array}{l}8.5 \\
8.5\end{array}$ & $\begin{array}{l}.14 \\
--\end{array}$ & $\begin{array}{l}9.3 \\
9.2\end{array}$ & \\
\hline $\begin{array}{l}21 \ldots \\
21 \ldots \\
\text { FEB, }\end{array}$ & $\begin{array}{l}1000 \\
1002\end{array}$ & $\begin{array}{l}1.0 \\
6.5\end{array}$ & $\begin{array}{l}53000 \\
50000\end{array}$ & $\begin{array}{l}8.3 \\
8.2\end{array}$ & $\begin{array}{l}28.0 \\
28.0\end{array}$ & $\begin{array}{r}.35 \\
--\end{array}$ & $\begin{array}{l}4.9 \\
5.0\end{array}$ & \\
\hline $\begin{array}{l}2 B \ldots \\
28 \ldots\end{array}$ & $\begin{array}{l}0846 \\
0848\end{array}$ & $\begin{array}{l}1.0 \\
9.0\end{array}$ & $\begin{array}{l}49000 \\
49000\end{array}$ & $\begin{array}{l}8.9 \\
8.8\end{array}$ & $\begin{array}{l}16.5 \\
17.0\end{array}$ & $\begin{array}{l}-- \\
--\end{array}$ & $\begin{array}{l}8.1 \\
7.8\end{array}$ & $\begin{array}{l}105 \\
102\end{array}$ \\
\hline
\end{tabular}

255952097172400 LINE 343 SITE 02

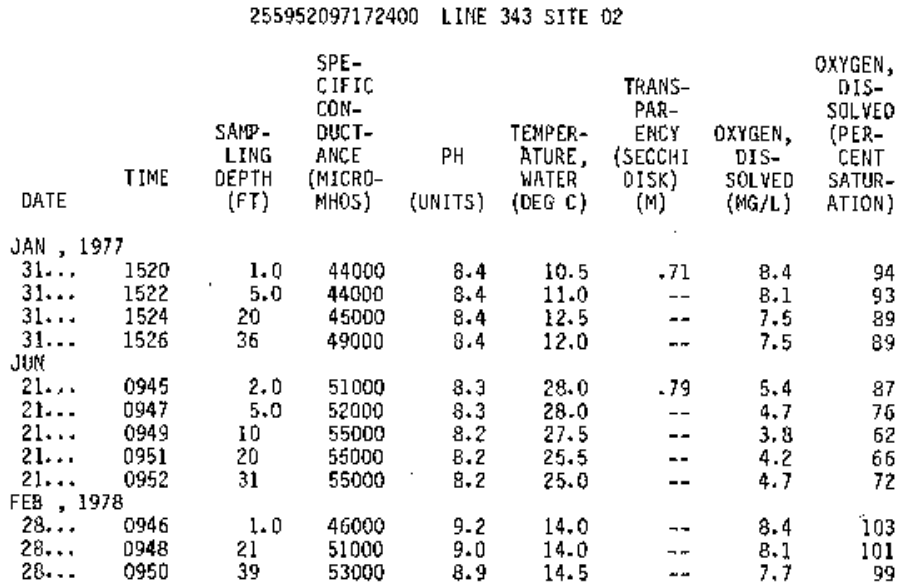

260106097151000 LINE 351 SITE 02

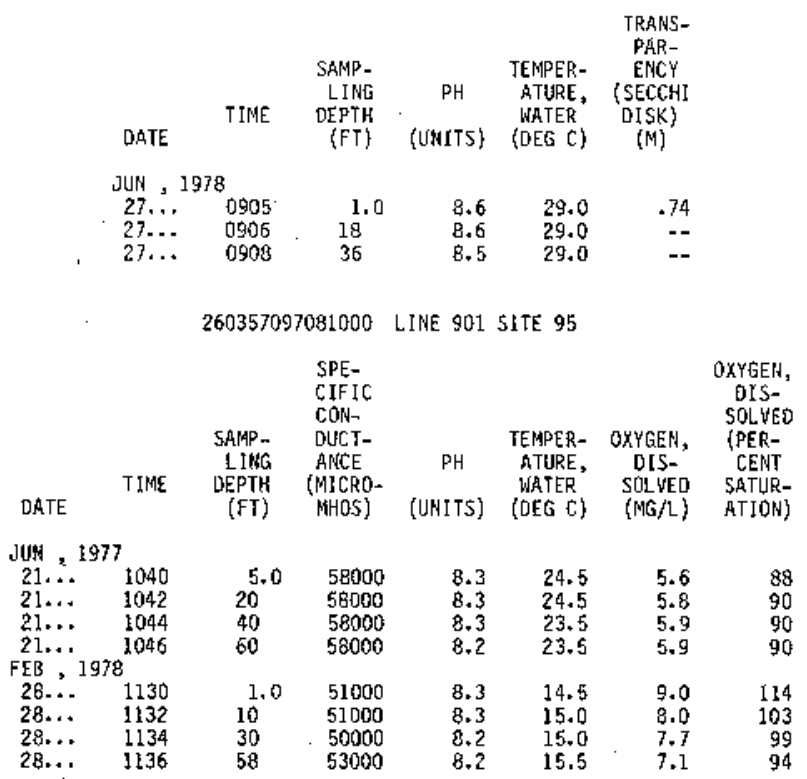


Table 1OB.--Quality of water in the Laguna Madre estuary, water years 1977-78 Nutrient Analyses

(FT = feet; MG $/ L=$ milliģrams per liter)

273811097142100 LINE 023 SITE 03

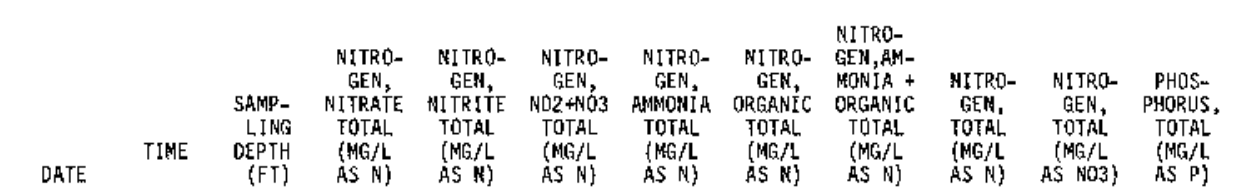

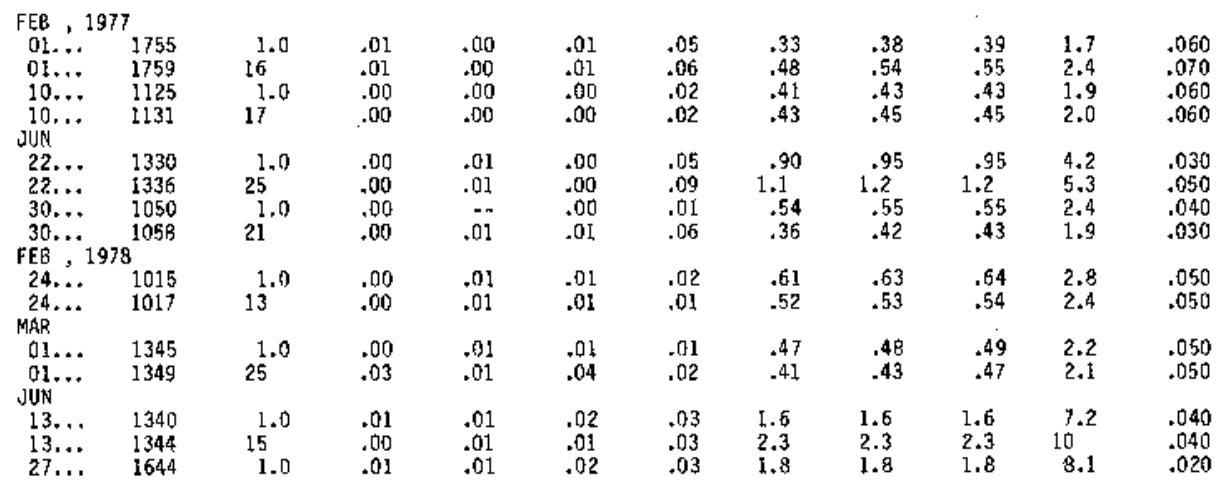

273203097185100 LINE 034 SITE 02

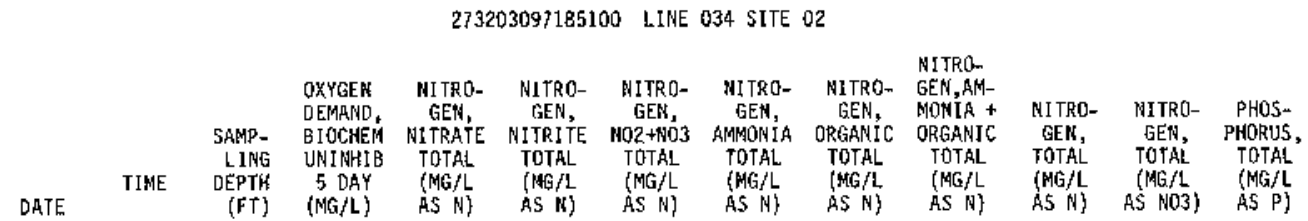

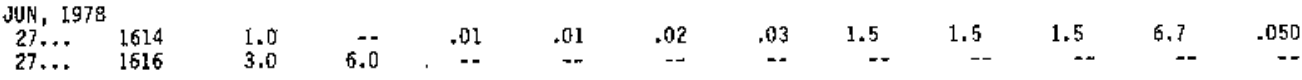

272104097222800. LINE 053 SITE DI

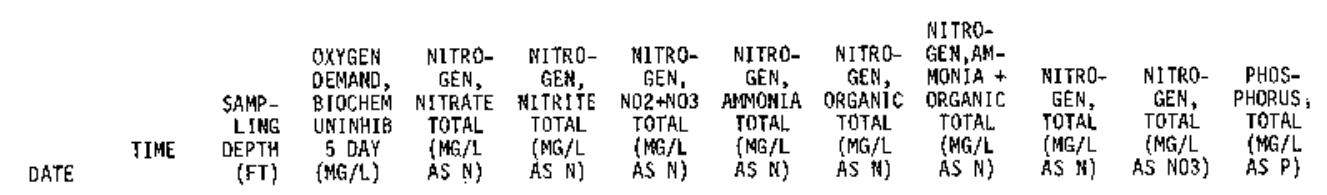

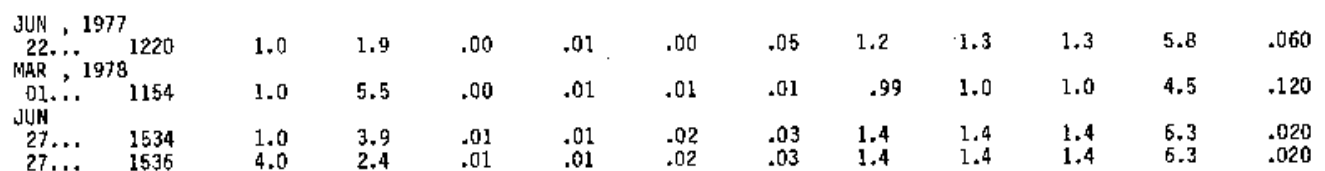

272116097230200 LINE 053 SITE 02

\begin{tabular}{|c|c|c|c|c|c|c|c|c|c|c|c|}
\hline DATE & TIME & $\begin{array}{l}\text { SAMP. } \\
\text { LING } \\
\text { DEPTH } \\
\text { (FT) }\end{array}$ & $\begin{array}{c}\text { MITRO- } \\
\text { GEN, } \\
\text { NITRATE } \\
\text { TOTAL } \\
\text { (MG/L } \\
\text { AS N) }\end{array}$ & $\begin{array}{l}\text { NITRO- } \\
\text { GEN, } \\
\text { NI TRITE } \\
\text { TOTAL } \\
\text { (MG/L } \\
\text { AS N) }\end{array}$ & $\begin{array}{l}\text { NITRD- } \\
\text { GEN, } \\
\text { NO2+NOO3 } \\
\text { TOTAL } \\
\text { (MG } / L \\
\text { AS N) }\end{array}$ & $\begin{array}{l}\text { NITRO- } \\
\text { GEN, } \\
\text { AMMONIA } \\
\text { TOTAL } \\
\text { (NGG } / \text { L } \\
\text { AS N) }\end{array}$ & $\begin{array}{l}\text { N1TRO- } \\
\text { GEN, } \\
\text { ORGANIC } \\
\text { TOTAL } \\
\text { (MG/L } \\
\text { AS RI) }\end{array}$ & $\begin{array}{l}\text { NITRO- } \\
\text { GEN,AM- } \\
\text { MONIA + } \\
\text { ORGANIC } \\
\text { TOTAL } \\
\text { (MG/L } \\
\text { AS NI) }\end{array}$ & $\begin{array}{l}\text { NITRO- } \\
\text { GEN, } \\
\text { TOTAL } \\
\text { (MG/L } \\
\text { RS KS) }\end{array}$ & $\begin{array}{c}\text { NITRO- } \\
\text { GEN, } \\
\text { TOTAL } \\
\text { (MG/L } \\
\text { AS NO3) }\end{array}$ & $\begin{array}{l}\text { PHOS- } \\
\text { PHORUS, } \\
\text { TOTAL } \\
\text { (MG/L } \\
\text { AS P) }\end{array}$ \\
\hline $\begin{array}{l}\text { FEB , } \\
01 . \cdots\end{array}$ & 1977 & 1.0 & .01 & .00 & .01 & .08 & .63 & .71 & .72 & 3.2 & .080 \\
\hline $\begin{array}{r}22 . \ldots \\
22 . \ldots\end{array}$ & $\begin{array}{l}1205 \\
1209\end{array}$ & $10^{1.0}$ & .00 & .01 & .01 & .05 & $\begin{array}{l}.93 \\
2.2\end{array}$ & 2.98 & $2.3^{.99}$ & $10^{4.4}$ & $\begin{array}{l}.070 \\
.040\end{array}$ \\
\hline
\end{tabular}


271543097301900 LINE 107 SITE 02

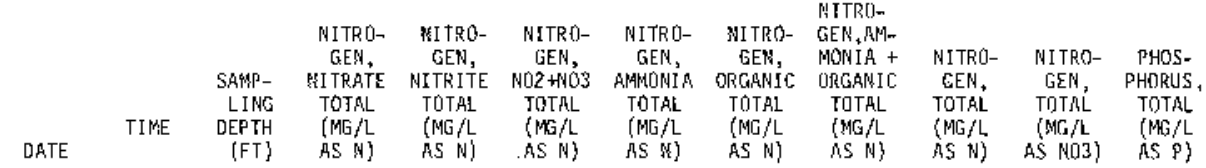

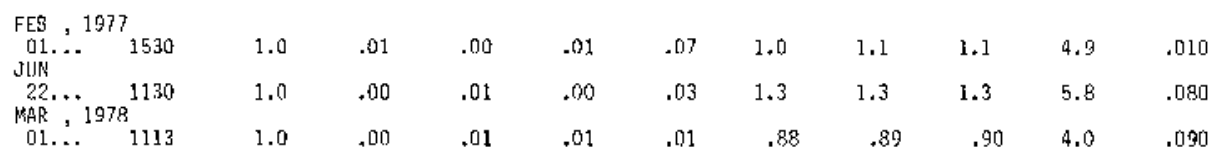

271630097251900 LIME 119 SITE 03

\begin{tabular}{|c|c|c|c|c|c|c|c|c|c|c|c|}
\hline DATE & TIME & $\begin{array}{l}\text { SAMP- } \\
\text { LYMG } \\
\text { DEPTH } \\
\text { (FT) }\end{array}$ & $\begin{array}{l}\text { RITRO- } \\
\text { GEN, } \\
\text { NITRATE } \\
\text { TOTAL } \\
\text { (MG /L } \\
\text { AS NI) }\end{array}$ & $\begin{array}{l}\text { NITRO- } \\
\text { GEN, } \\
\text { NITRITE } \\
\text { TOTAL } \\
\text { (WSIL } \\
\text { AS N) }\end{array}$ & $\begin{array}{c}\text { NITRO- } \\
\text { GEN, } \\
\text { WO2+NO3 } \\
\text { TDIALL } \\
\text { (MG/L } \\
\text { AS N) }\end{array}$ & $\begin{array}{l}\text { MITRO- } \\
\text { GEN, } \\
\text { AMMONIA } \\
\text { TOTAL } \\
\text { (NG/L } \\
\text { AS N) }\end{array}$ & 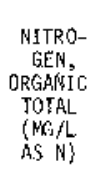 & $\begin{array}{l}\text { NITRO- } \\
\text { GEN,AM- } \\
\text { MONIA + } \\
\text { ORTAANIC } \\
\text { TOTAL } \\
\text { (MG/L } \\
\text { AS N })\end{array}$ & $\begin{array}{l}\text { NITRO- } \\
\text { GER, } \\
\text { TOTAL } \\
\text { (MG/L } \\
\text { A5 N) }\end{array}$ & $\begin{array}{c}\text { NITRO- } \\
\text { GEN, } \\
\text { TOTAL } \\
\text { (MG } / L \\
\text { AS NO3) }\end{array}$ & $\begin{array}{l}\text { PHOS- } \\
\text { PHORUS, } \\
\text { TOTAL } \\
\text { (MG/L } \\
\text { AS P) }\end{array}$ \\
\hline
\end{tabular}

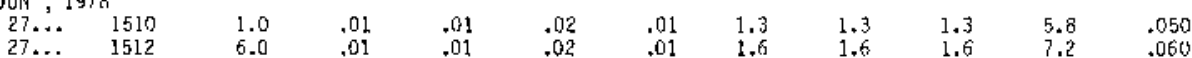

271211097243300 LINE 125 SITE 02

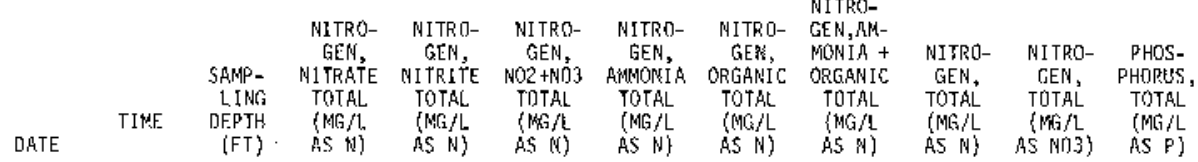

JIJN , 1978

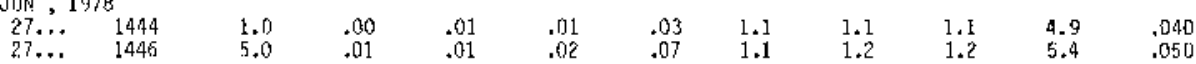

271213097253500 LINE 125 SITE 03

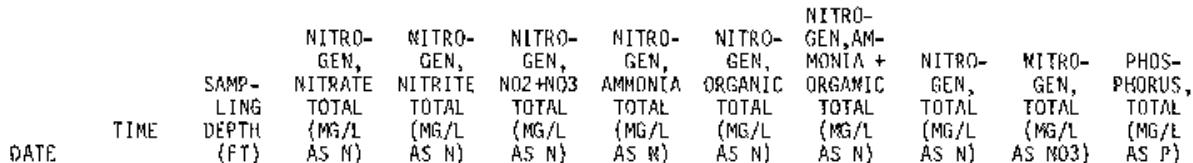
FEB, 1977

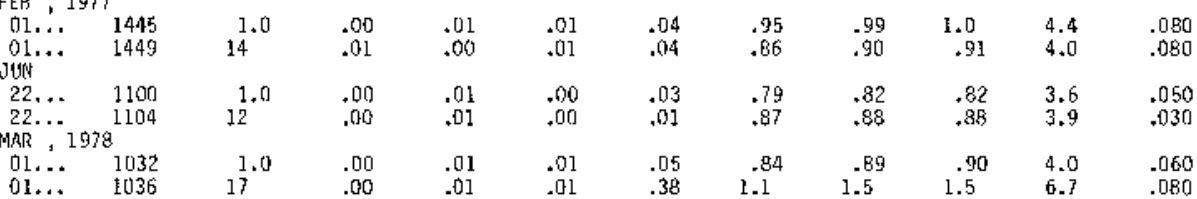$$
\text { 01... }
$$

$265857097270200 \quad$ LINE 145 SITE 02 i NITRO-

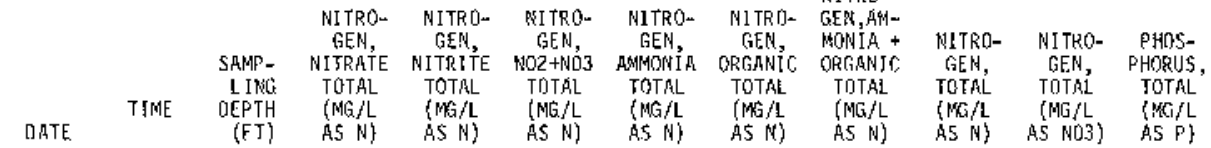

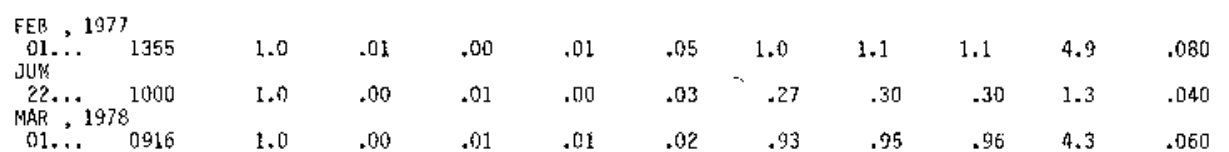


264822097281400 LINE 163 SITE 02

\begin{tabular}{|c|c|c|c|c|c|c|c|c|c|c|c|}
\hline ATE & TIME & $\begin{array}{l}\text { SAMP- } \\
\text { L IMG } \\
\text { DEPTH } \\
\text { (FT) }\end{array}$ & $\begin{array}{l}\text { NITRO- } \\
\text { GEN, } \\
\text { NITRATE } \\
\text { TRTAL } \\
\text { (MGAL } \\
\text { AS NI) }\end{array}$ & $\begin{array}{l}\text { NITRO- } \\
\text { GEN, } \\
\text { NITRITE } \\
\text { TOTAL } \\
\text { (HAL } / \\
\text { AS N N) }\end{array}$ & $\begin{array}{l}\text { NITRO- } \\
\text { GEN, } \\
\text { NOZ+NOB } \\
\text { TQTAL } \\
\text { (MC/L } \\
\text { AS N) }\end{array}$ & $\begin{array}{l}\text { NITRO- } \\
\text { GEN, } \\
\text { MMNOKIA } \\
\text { TOTAL } \\
\text { (NG/L } \\
\text { AS N) }\end{array}$ & $\begin{array}{c}\text { NITRO- } \\
\text { GEN, } \\
\text { ORGANLC } \\
\text { TOTAL } \\
\text { (NG/L } \\
\text { AS AA) }\end{array}$ & $\begin{array}{l}\text { NI TRD- } \\
\text { GEN, MN- } \\
\text { MONIR + } \\
\text { ORGANIC } \\
\text { TOTAL. } \\
\text { (MG/L } \\
\text { AS N) }\end{array}$ & $\begin{array}{l}\text { NITRQ- } \\
\text { GEN, } \\
\text { TOTAL } \\
\text { (MG/L } \\
\text { AS WS }\end{array}$ & $\begin{array}{c}\text { NITRO)- } \\
\text { CEN, } \\
\text { TOTAL } \\
\text { (MG/L } \\
\text { AS NO3) }\end{array}$ & $\begin{array}{l}\text { PHOS- } \\
\text { PHORUS, } \\
\text { TOTAL } \\
\text { (MGAL } \\
\text { AS P) }\end{array}$ \\
\hline
\end{tabular}

JUN 1978

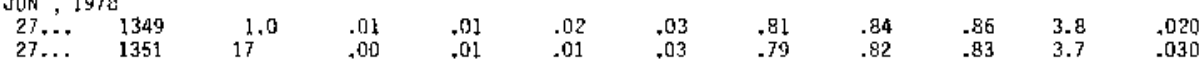

264136097245100 LINE I75 SITE OI

\begin{tabular}{|c|c|c|c|c|c|c|c|c|c|c|c|}
\hline ATE & TIMF, & $\begin{array}{r}\text { SAMP- } \\
\text { LING } \\
\text { DEPTH } \\
\text { (FT) }\end{array}$ & $\begin{array}{c}\text { NITRO)- } \\
\text { CEN, } \\
\text { NITRRTE } \\
\text { JOTRL } \\
\text { (MW } / L \\
\text { AS N) }\end{array}$ & $\begin{array}{c}\text { WITRO- } \\
\text { GEN, } \\
\text { AITRITE } \\
\text { TDTAL } \\
\text { (MG/L } \\
\text { AS N) }\end{array}$ & 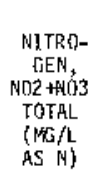 & $\begin{array}{l}\text { NITRO- } \\
\text { GEEN, } \\
\text { AMMONIA } \\
\text { TOTAL } \\
\text { (MG/L } \\
\text { AS N) }\end{array}$ & $\begin{array}{l}\text { NITRO- } \\
\text { CEN } \\
\text { ORGANIL } \\
\text { TOTAL } \\
\text { (MG/L } \\
\text { AS N) }\end{array}$ & $\begin{array}{l}\text { NITRO- } \\
\text { CEN , AM- } \\
\text { MONIA + } \\
\text { ORGANIC } \\
\text { TOTAL } \\
(A G / A \\
\text { AS A })\end{array}$ & $\begin{array}{l}\text { NITRO- } \\
\text { GEN, } \\
\text { TOTARL } \\
\text { (MG/L } \\
\text { AS N) }\end{array}$ & $\begin{array}{l}\text { NITR(O) } \\
\text { GEN, } \\
\text { TO†AL } \\
\{\mathrm{MG} / \mathrm{L} \\
\text { AS NO.3\}) }\end{array}$ & $\begin{array}{l}\text { PHOS- } \\
\text { PHORUS, } \\
\text { TOTAL } \\
\text { (MG/L } \\
\text { AS P) }\end{array}$ \\
\hline
\end{tabular}

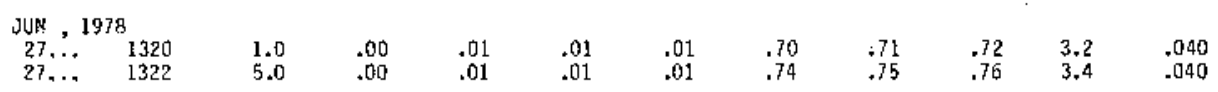

263532097242800 LIME 188 SITE 02

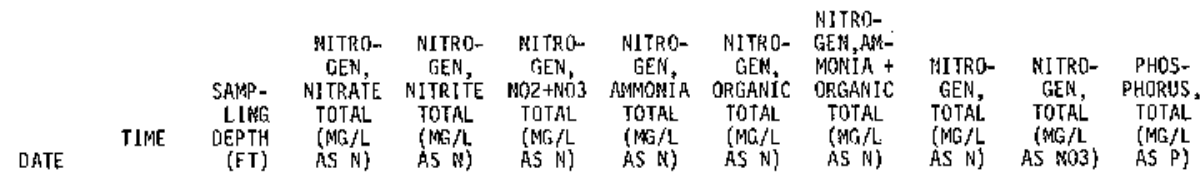

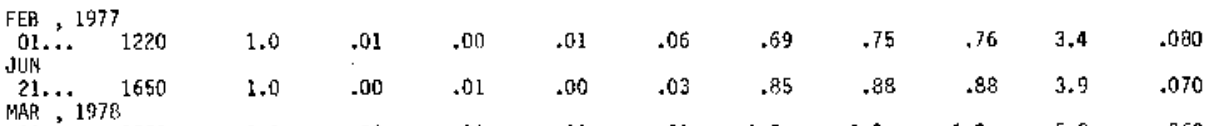

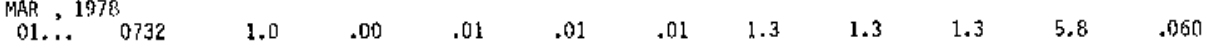

263516097252300 LINE. 188 SITE 03

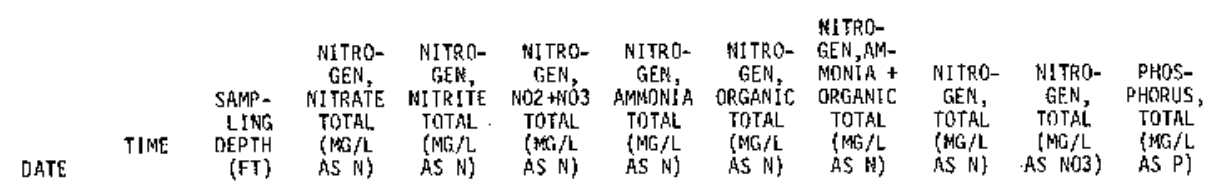

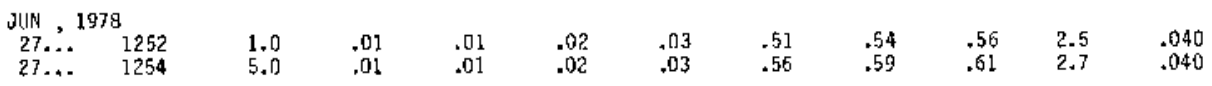

262423097204400 LINE 217 SITE 02

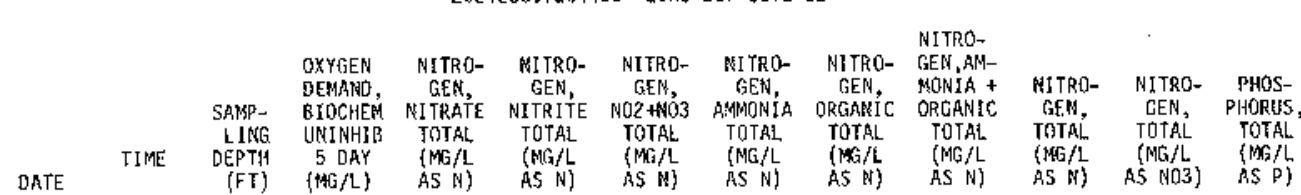

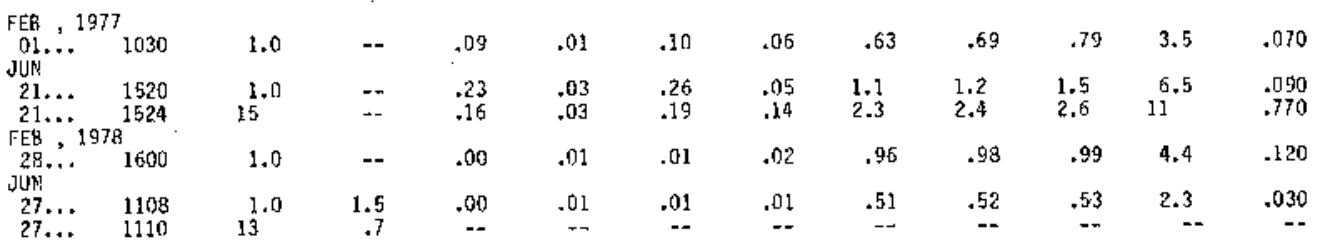


$261929097264500 \quad$ LISHE, $24751 \mathrm{TE} \quad 02$

\begin{tabular}{|c|c|c|c|c|c|c|c|c|c|c|c|c|}
\hline DATE & TIME & $\begin{array}{c}\text { SAMP - } \\
\text { LING } \\
\text { DEPTH } \\
(F T)\end{array}$ & $\begin{array}{l}\text { OXYGEN } \\
\text { DEMAND, } \\
\text { BIOCHEM } \\
\text { UNINHIB } \\
5 \text { DAY } \\
\text { (MCAL) }\end{array}$ & $\begin{array}{l}\text { NITRO- } \\
\text { CEN, } \\
\text { NITRATE } \\
\text { TOTAL } \\
\text { (MO/L } \\
\text { AS N) }\end{array}$ & $\begin{array}{l}\text { NITRO- } \\
\text { GEN, } \\
\text { NITRITE } \\
\text { TOJAL } \\
\text { (MGAL } \\
\text { AS N) }\end{array}$ & $\begin{array}{l}\text { NITRO- } \\
\text { GEN, } \\
\text { NO2+NO3 } \\
\text { TOTAL } \\
\text { (MG/L } \\
\text { AS N) }\end{array}$ & $\begin{array}{l}\text { NITRO- } \\
\text { GEN, } \\
\text { AMMONIA } \\
\text { TOTAL } \\
\text { (MG /L } \\
\text { AS N) }\end{array}$ & $\begin{array}{c}\text { NITRO- } \\
\text { GEN, } \\
\text { ORSGANIC } \\
\text { TOTAL } \\
\text { (MG } / L \\
\text { AS N) }\end{array}$ & $\begin{array}{l}\text { NITRO- } \\
\text { GEN, AM- } \\
\text { MONIA + } \\
\text { ORGANIC } \\
\text { TDTAL } \\
\text { (MG/L } \\
\text { AS NS }\end{array}$ & $\begin{array}{l}\text { NITRO- } \\
\text { GENA, } \\
\text { TOTAL } \\
\text { (MG } / L \\
\text { AS N) }\end{array}$ & $\begin{array}{l}\text { NITRO- } \\
\text { GEN, } \\
\text { TOTAL } \\
\text { (MO/L } \\
\text { AS NOO3) }\end{array}$ & $\begin{array}{l}\text { PHDS- } \\
\text { PHORUS, } \\
\text { TDTAL } \\
\text { (MG } / \text { L } \\
\text { AS P) }\end{array}$ \\
\hline \multicolumn{13}{|c|}{ FEB , 1917 } \\
\hline $01 \ldots$ & 0945 & 2.0 & 3.6 & 1.3 & .20 & 1.5 & .51 & .79 & 1.3 & 2.8 & 12 & .320 \\
\hline JUN & 0951 & 10 & 4.2 & .51 & & .58 & & .00 & .42 & & 4.4 & .200 \\
\hline $\begin{array}{r}21 \ldots \\
21 \ldots \\
\text { FEB }\end{array}$ & $\begin{array}{l}1425 \\
1431 \\
8\end{array}$ & 1.0 & $\begin{array}{l}8.2 \\
1.4\end{array}$ & .50 & $\begin{array}{r}.05 \\
.02\end{array}$ & .55 & .04 & $\begin{array}{c}3.4 \\
.88\end{array}$ & $\begin{array}{l}3.4 \\
1.2\end{array}$ & $\begin{array}{l}3.9 \\
1.3\end{array}$ & $\begin{array}{l}17 \\
5.6\end{array}$ & $\begin{array}{l}.200 \\
.160\end{array}$ \\
\hline $\begin{array}{l}28 . \\
28 .\end{array}$ & $\begin{array}{l}1500 \\
1504\end{array}$ & $15^{1.0}$ & $\begin{array}{l}8.4 \\
4.6\end{array}$ & $\begin{array}{l}.55 \\
.05\end{array}$ & $\begin{array}{l}.12 \\
.02\end{array}$ & $\begin{array}{l}.67 \\
.07\end{array}$ & $\begin{array}{r}.33 \\
.31\end{array}$ & $\begin{array}{l}2.5 \\
2.6\end{array}$ & $\begin{array}{l}2.8 \\
2.0\end{array}$ & $\begin{array}{l}3.5 \\
3.0\end{array}$ & $\begin{array}{l}15 \\
13\end{array}$ & $\begin{array}{r}.520 \\
.720\end{array}$ \\
\hline $\begin{array}{l}27 \ldots \\
27 \ldots\end{array}$ & $\begin{array}{l}1035 \\
1047\end{array}$ & $15^{1.0}$ & $\begin{array}{l}5.8 \\
2.4\end{array}$ & ס1.01 & $\begin{array}{l}.01 \\
.01\end{array}$ & $\begin{array}{l}.01 \\
.02\end{array}$ & $\begin{array}{l}.07 \\
.23\end{array}$ & $\begin{array}{c}7.5 \\
.87\end{array}$ & $\begin{array}{l}1.6 \\
1.1\end{array}$ & $\begin{array}{l}1.6 \\
1.1\end{array}$ & $\begin{array}{l}7.1 \\
5.0\end{array}$ & $\begin{array}{l}.150 \\
.080\end{array}$ \\
\hline
\end{tabular}

261256097121800 LINE 274 SITE O1

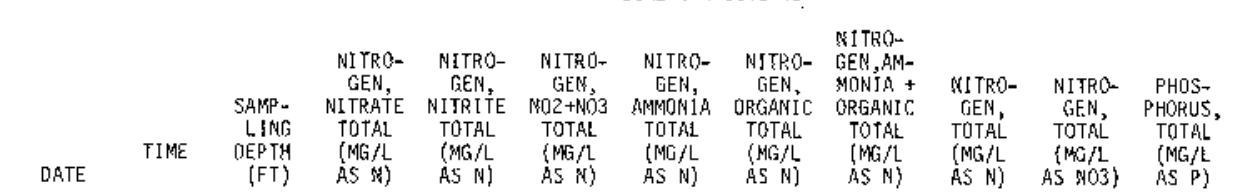
JUN, 1978

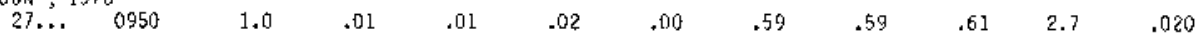

261153097153400 LIME 274 SITF, 03

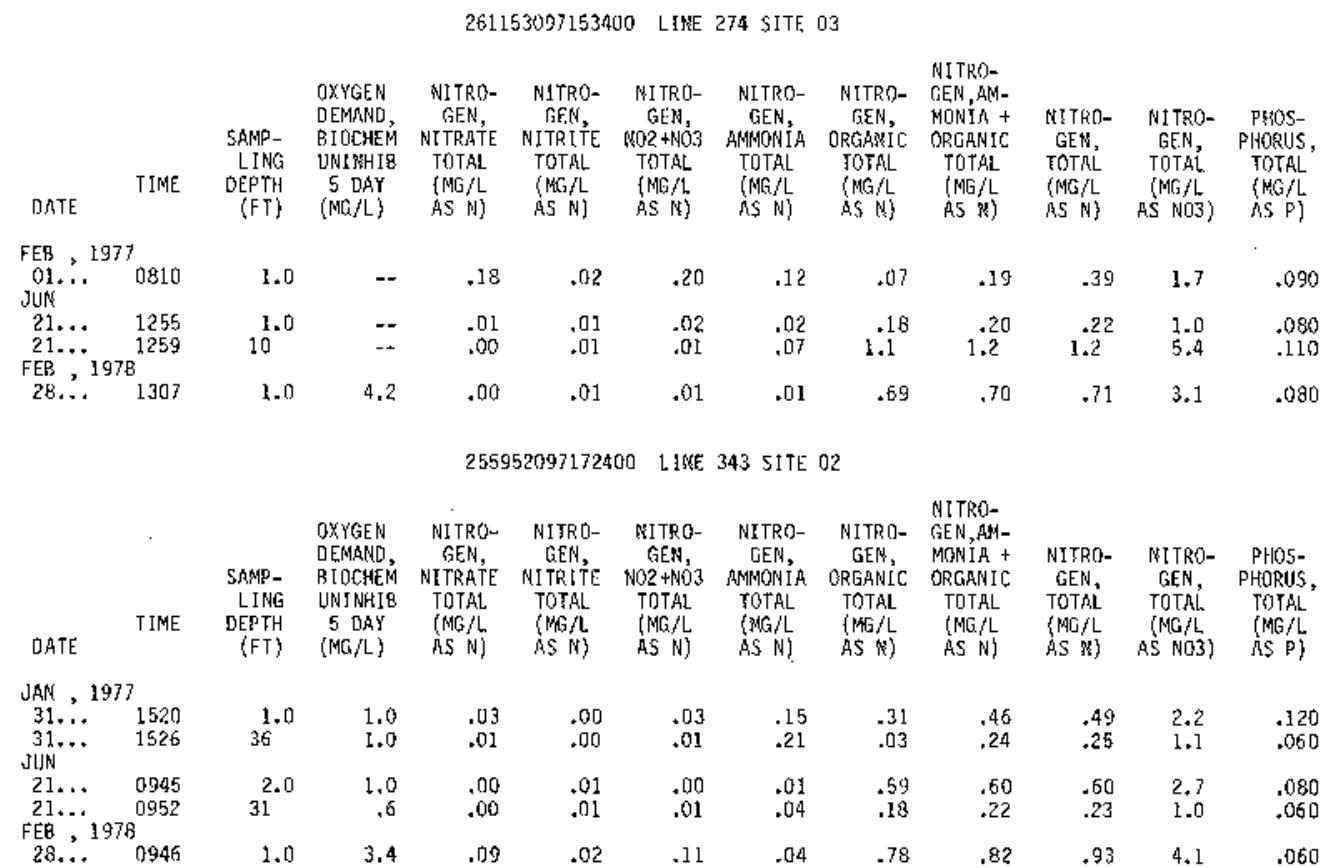


Table 100.--Quality of water in the Laguna Madre estuary, water years 1977-78--Continued Nutrient Analyses--Cont 1 nued

260106097151000 LINE 351 SITE 02

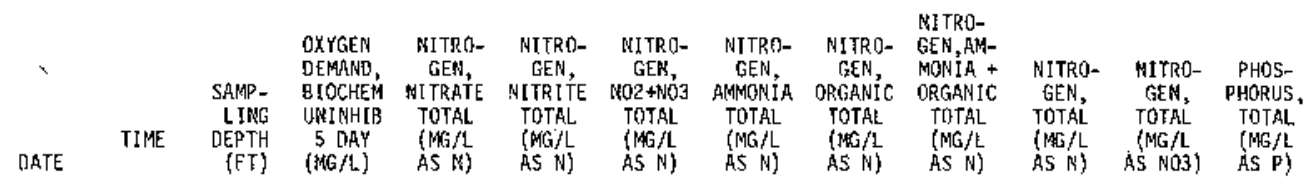

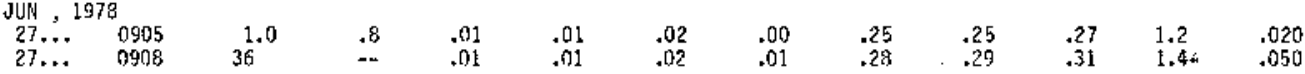

$260357097081000 \quad$ LIAE 901 SITE 95

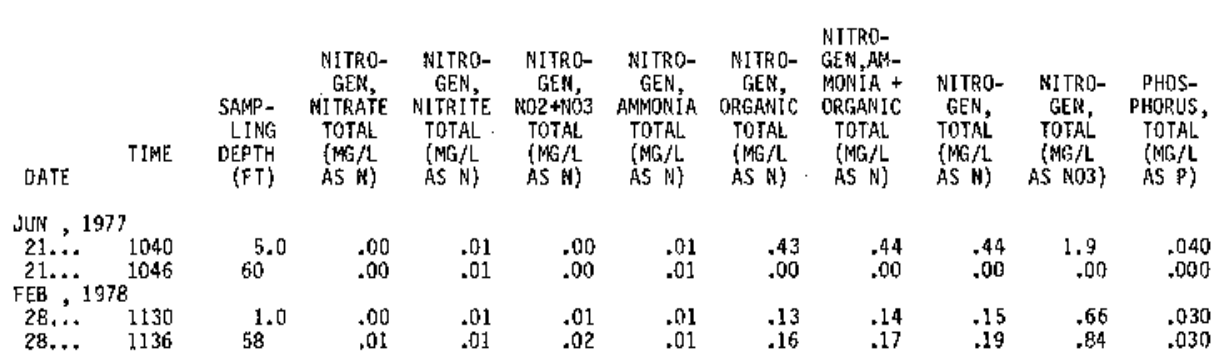


$\left\{F T=\right.$ feet: MJCROHHOS = micronhos per centimeter at $25^{\circ}$ Colsius; $K C / L=$ milligrams per Ijter; AC-FT = acre-feet

272104097222800 1IPEE. 053 SITE DI

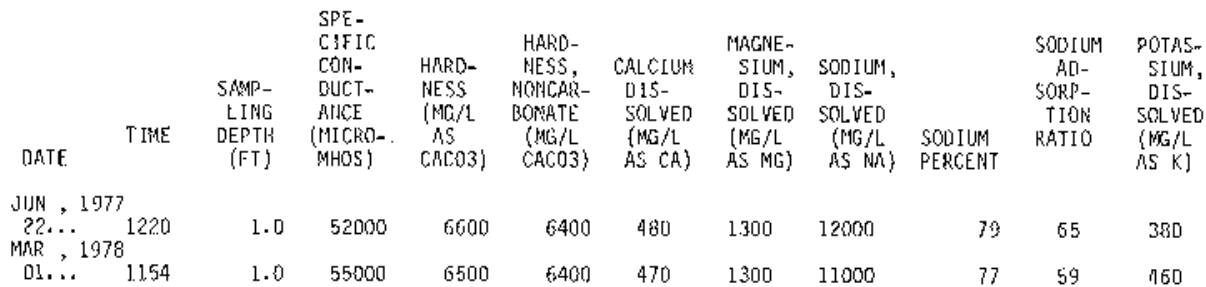

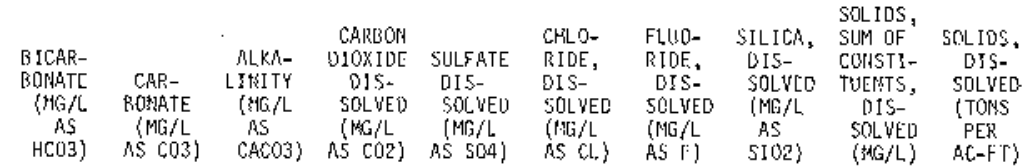

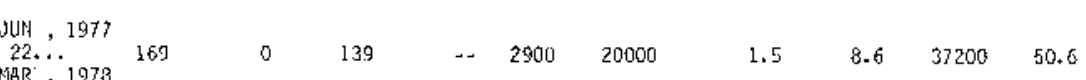

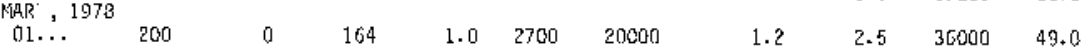

$$
\begin{aligned}
& 272116097230200 \text { LINE OS3 SITE 0? }
\end{aligned}
$$

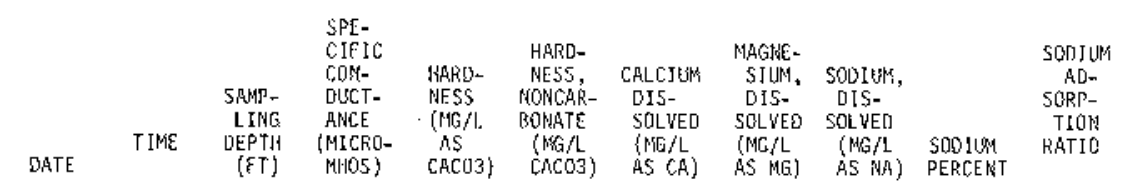

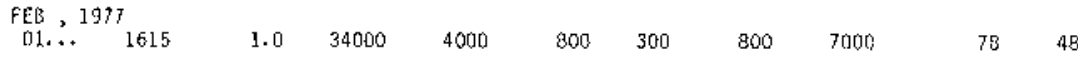

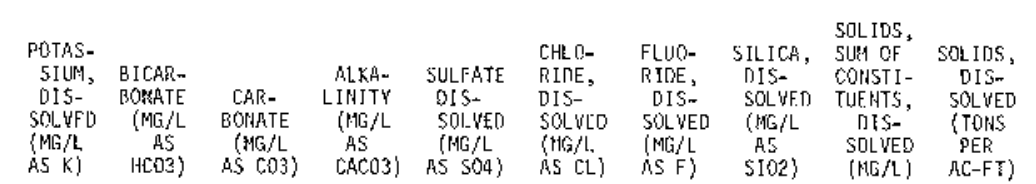

$$
\begin{aligned}
& \begin{array}{cccccccccc}
\text { FEF }, 1977 \\
01 . .+280 & 170 & 0 & 146 & 1600 & 12000 & 1.1 & 1.2 & 22100 & 30.1
\end{array} \\
& 271630097251900 \text { LINE } 119 \text { SITE } 03
\end{aligned}
$$

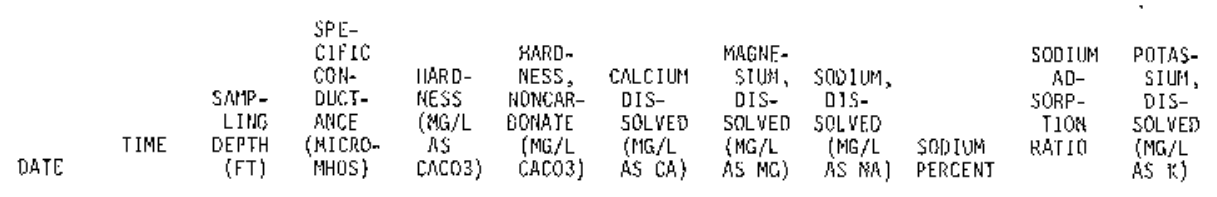

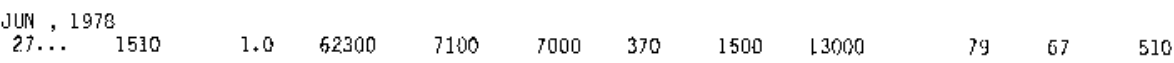

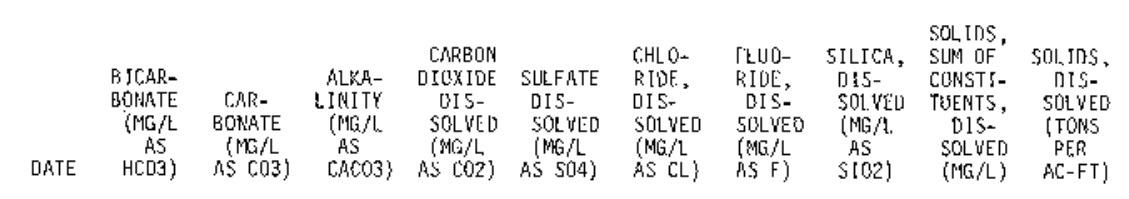

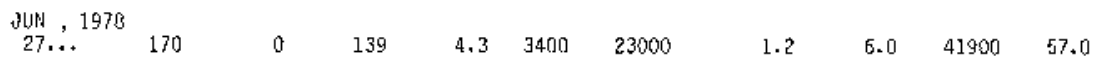


Table 10C.-mulatity of water ir the Laguna iladre estuary, water years 1977-78--Continued

Crenfoal Andyses--Continued

271213097253500 LINE 125 SITE. 03

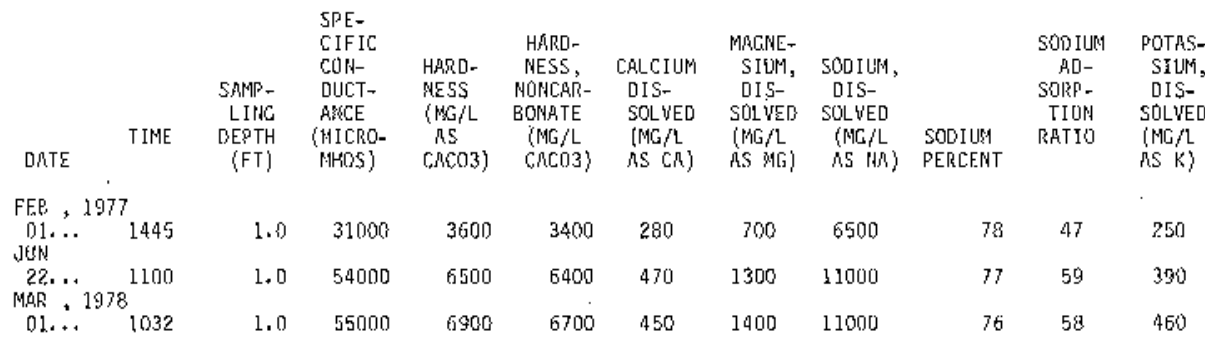

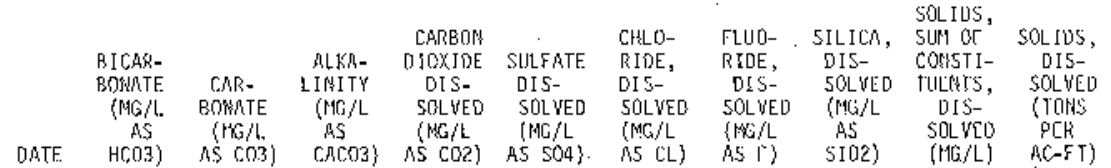

\begin{tabular}{|c|c|c|c|c|c|c|c|c|c|c|}
\hline $\begin{array}{l}\text { FEB , } 1977 \\
01 . . .\end{array}$ & 1,54 & 0 & 135 & 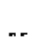 & 1500 & 13000 & 1.0 & .3 & 20300 & 27.6 \\
\hline MAR & 160 & 0 & 131 & -- & 2700 & 20000 & 3.8 & 8.0 & 36000 & 49. \\
\hline 01... & 200 & 0 & 164 & .8 & $2 \mathrm{and}$ & 20000 & 1.1 & 1.0 & 36200 & 49. \\
\hline
\end{tabular}

7,63532097242800 LINE 188 SITC 02

\begin{tabular}{|c|c|c|c|c|c|c|c|c|c|c|c|}
\hline OATE & TIME & $\begin{array}{l}\text { SAYtP. } \\
\text { LIMG } \\
\text { DEPTH } \\
\text { (FT) }\end{array}$ & $\begin{array}{l}\text { SPE- } \\
\text { CIFIC } \\
\text { COHS- } \\
\text { DSCT- } \\
\text { ANCF. } \\
\text { (MICRO- } \\
\text { MHUS) }\end{array}$ & $\begin{array}{l}\text { HARO- } \\
\text { NESS } \\
\text { (MG/L } \\
\text { AS } \\
\text { CACO3) }\end{array}$ & $\begin{array}{l}\text { IIARD- } \\
\text { NESS, } \\
\text { NOHCAR- } \\
\text { BONATE } \\
\text { (MGi/L } \\
\text { CACO3) }\end{array}$ & $\begin{array}{l}\text { CALCIUM } \\
\text { DIS- } \\
\text { SOLVCU } \\
\text { ( } \$ \text { MG/L. } \\
\text { AS CN) }\end{array}$ & $\begin{array}{l}\text { MRGNE- } \\
\text { SIUH, } \\
\text { OIS- } \\
\text { SOLVED } \\
\text { (KG/L } \\
\text { NS MG) }\end{array}$ & $\begin{array}{l}\text { SOUIUR:," } \\
\text { OIS- } \\
\text { SOLLED } \\
\text { (MG/L } \\
\text { A5 NA) }\end{array}$ & $\begin{array}{l}\text { SODIUM } \\
\text { PEREEIRT' }\end{array}$ & $\begin{array}{l}\text { SOIDSUM } \\
\text { AD- } \\
\text { SORP- } \\
\text { TION } \\
\text { RAT10 }\end{array}$ & $\begin{array}{l}\text { PORAS- } \\
\text { SIGH, } \\
\text { OIS- } \\
\text { SOLYED } \\
\text { (HAG/L } \\
\text { AS K) }\end{array}$ \\
\hline \multicolumn{12}{|c|}{$F E B, 1977$} \\
\hline 01.... & 1220 & 1.0 & 38000 & 4500 & 4400 & 340 & 890 & 8000 & 78 & 52 & 300 \\
\hline MRP, & 1650 & 1.0 & 50000 & 6000 & 5900 & 440 & 1200 & 9800 & 77 & 55 & 360 \\
\hline $01 .$. & 0732 & 1.0 & 55000 & 5700 & 5500 & 450 & 1100 & 11000 & 79 & 64 & 450 \\
\hline
\end{tabular}

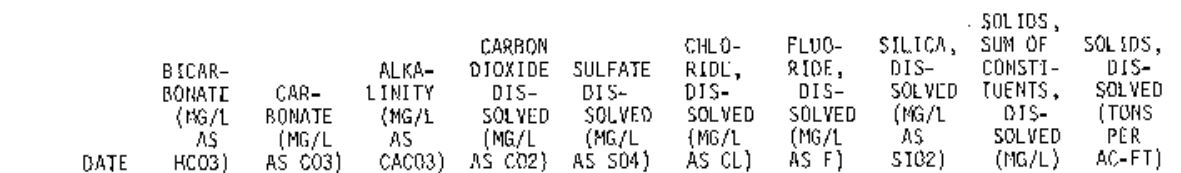
CATE HCO3) AS CO3) CACO3) ASCO2\} AS SO4) ASCL) ASF) SIO2) (HG/L) AC-FT)

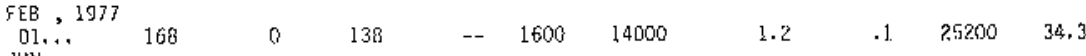

$$
\begin{aligned}
& \begin{array}{lllllllllll}
\text { JUN } & & & & & & & & & &
\end{array} \\
& \begin{array}{lllllllllll}
\text { MAR }, 1978 & 190 & 0 & 156 & .8 & 2500 & 20000 & 1.2 & 2.0 & 35900 & 48.8
\end{array}
\end{aligned}
$$

263516097252300 I TNF. 188 SITE O3

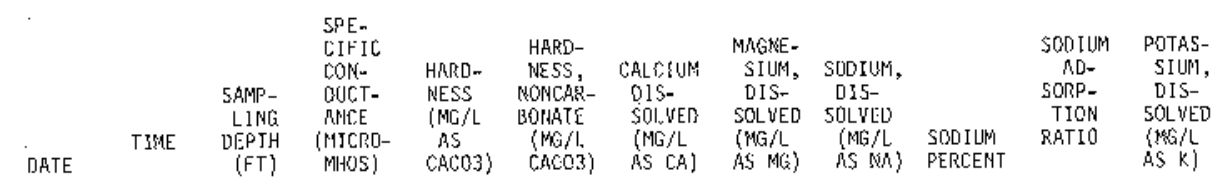

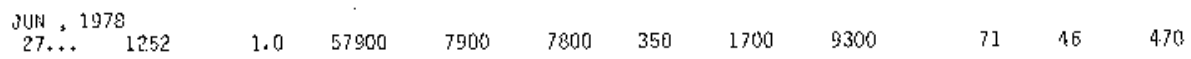
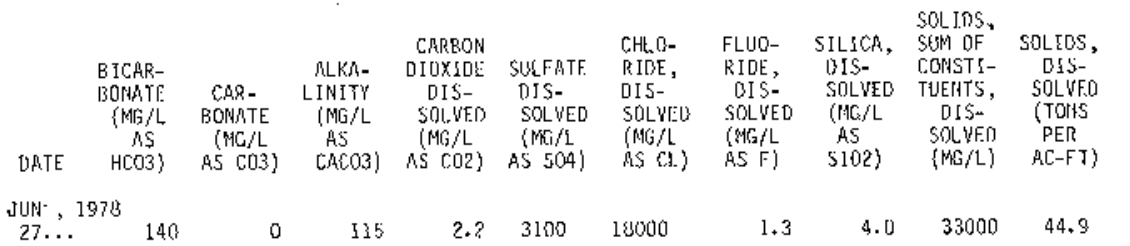
Table 10C. - Tuality of water in the Laguna Madre estuary, water years 1977-78--Continued Chenical Analyses--Continued

261929097264500 LINE 247 SITE 02

\begin{tabular}{|c|c|c|c|c|c|c|c|c|c|c|c|}
\hline DATE & TIME & $\begin{array}{c}\text { 5AMP- } \\
\text { LING } \\
\text { DEPTH } \\
\text { (FT) }\end{array}$ & $\begin{array}{l}\text { SPE- } \\
\text { CIFIC } \\
\text { CON- } \\
\text { DUCT- } \\
\text { ANCE } \\
\text { (SICRO- } \\
\text { MLOS) }\end{array}$ & $\begin{array}{l}\text { HARD'- } \\
\text { NESS } \\
\left(M_{1} / L\right. \\
\text { RS } \\
\text { CACOB) }\end{array}$ & $\begin{array}{l}\text { HARD- } \\
\text { NESS } \\
\text { HONCAR- } \\
\text { BONATE } \\
\text { (MG/L } \\
\text { CACO3) }\end{array}$ & $\begin{array}{l}\text { CALCIUNA } \\
\text { DIS- } \\
\text { SOLWEO } \\
\text { (MG/L } \\
\text { AS CA) }\end{array}$ & $\begin{array}{l}\text { MRGNE- } \\
\text { SIIDM, } \\
\text { DIS- } \\
\text { SOLYED } \\
\text { (WMG/L } \\
\text { AS MG) }\end{array}$ & $\begin{array}{l}\text { SODIUH, } \\
\text { O1S- } \\
\text { SOLUCD } \\
\text { (MG/L } \\
\text { AS NAS) }\end{array}$ & $\begin{array}{l}\text { 50DIUM } \\
\text { PERCENT }\end{array}$ & $\begin{array}{c}\text { SOUIUP: } \\
\text { AD- } \\
\text { SORP- } \\
\text { TION } \\
\text { RATIO }\end{array}$ & $\begin{array}{l}\text { PUTAS- } \\
\text { SIUN, } \\
\text { DIS- } \\
\text { SOLVED } \\
(M G / L \\
M S \quad K)\end{array}$ \\
\hline
\end{tabular}

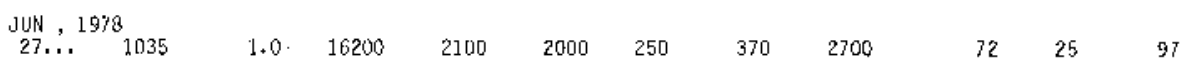

\begin{tabular}{|c|c|c|c|c|c|c|c|c|c|c|}
\hline & & & & CASRBON & & CHEDO- & FLU0- & SILICA, & $\begin{array}{l}\text { SOL LDS, } \\
\text { SUM Of }\end{array}$ & SOLIDS, \\
\hline & BICAR- & & ALKA- & DIOXIDE & SULГATE & RIDE, & RIDE, & DIS- & CONSTI- & DIS- \\
\hline & $\begin{array}{c}\text { BONATE } \\
\text { \{MG/L } \\
\text { AS }\end{array}$ & $\begin{array}{l}\text { CAR- } \\
\text { BONATE } \\
\text { (MG/L }\end{array}$ & $\begin{array}{l}\text { L.JNITY } \\
\text { (MG/L. } \\
\text { ASS }\end{array}$ & $\begin{array}{l}\text { DLS- } \\
\text { SOLVED } \\
\text { (NC/L }\end{array}$ & $\begin{array}{l}\text { DIS- } \\
\text { SOLVED } \\
\text { ING } / L\end{array}$ & $\begin{array}{l}\text { DIS- } \\
\text { SOLVED } \\
\text { (MG } / 2 / L\end{array}$ & $\begin{array}{l}\text { DIS- } \\
\text { SOLVED } \\
\text { SMG/2 }\end{array}$ & $\begin{array}{l}\text { SOLVET } \\
\text { CMG } / L \\
\text { AS }\end{array}$ & $\begin{array}{c}\text { TUENTS, } \\
\text { DIS- } \\
\text { SOLVED }\end{array}$ & $\begin{array}{l}\text { SOLVED } \\
\text { (TONSS } \\
\text { PER }\end{array}$ \\
\hline [ЗATE & HCn3) & A5 C031) & СACO3) & AS C02) & AS SO4] & SS [. & AS F & $5102)$ & (MG/L) & $\mathrm{RC}-\mathrm{FT}$ \\
\hline
\end{tabular}

$\begin{array}{ccccccccccc}\text { JUN }, 1978 & & & & & & & & & & \\ 27 . . & 190 & 0 & 156 & .6 & 1300 & 4800 & .9 & 14 & 9630 & 13.1\end{array}$

261153097153400 LINE 274 SITE 03

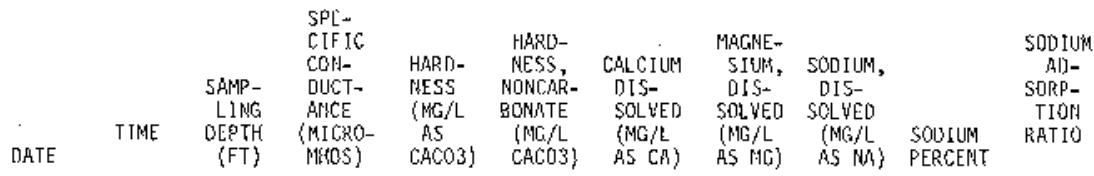

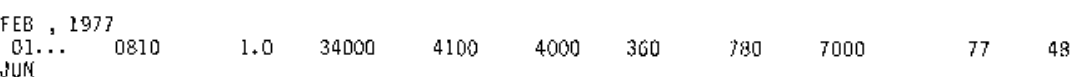

$\begin{array}{llllllllll}21 \ldots & 1255 & 1.0 & 57000 & 6500 & 6300 & 440 & 1300 & 11000 & 77\end{array}$

\begin{tabular}{|c|c|c|c|c|c|c|c|c|c|c|}
\hline Ies & $\begin{array}{l}\text { POTAS- } \\
\text { SIUM. } \\
\text { DIS- } \\
\text { SOLVED } \\
(M G / L \\
\text { AS K) }\end{array}$ & $\begin{array}{c}\text { B1CAR- } \\
\text { BONATE } \\
\text { (MG/L } \\
\text { AS } \\
\text { HCO3) }\end{array}$ & $\begin{array}{c}\text { CAR- } \\
\text { BONATF, } \\
\text { (MC/L } \\
\text { RS CO3) }\end{array}$ & $\begin{array}{l}\text { ALKA- } \\
\text { LIN1TY } \\
\text { (MGA } / \mathrm{L} \\
\text { AS } \\
\text { CACO3) }\end{array}$ & $\begin{array}{l}\text { SULFATE } \\
\text { DIS- } \\
\text { SOLYEO } \\
\text { ¿MG/L } \\
\text { nS SOL }\end{array}$ & $\begin{array}{l}\text { CHLD- } \\
\text { RIDE, } \\
\text { DIS- } \\
\text { 5OLVED } \\
\text { (MG/L } \\
\text { AS CL) }\end{array}$ & $\begin{array}{l}\text { FLUO- } \\
\text { RIOS: } \\
\text { DIS- } \\
\text { SOLVED } \\
(M G / L \\
\text { AS } F)\end{array}$ & $\begin{array}{l}\text { SILICA, } \\
\text { DIS- } \\
\text { SOLVED } \\
\text { (MG/L } \\
\text { AS } \\
\text { SIO2) }\end{array}$ & $\begin{array}{l}\text { SOL IDS, } \\
\text { SUM OF } \\
\text { CONSTI- } \\
\text { TUENSS, } \\
\text { DIS- } \\
\text { SOLVES } \\
\text { (MG/L) }\end{array}$ & $\begin{array}{c}\text { SOLIDS, } \\
\text { DIS- } \\
\text { SOLVED } \\
\text { (YONS } \\
\text { PER } \\
\text { AC-FT) }\end{array}$ \\
\hline
\end{tabular}

\begin{tabular}{|c|c|c|c|c|c|c|c|c|c|c|}
\hline FEB , & & & & & & & & & & \\
\hline O1." & 260 & 185 & 0 & 152 & 1900 & 12000 & 1.2 & 2.5 & 22400 & 30.5 \\
\hline $21 .$. & 410 & 150 & 0 & 1.23 & 2700 & 20000 & 3.6 & 3,9 & 35900 & 48.8 \\
\hline
\end{tabular}




\section{SELECTED HYDROLOGIC RECORDS}

\section{Climatological Records}

The climate of the region has a significant influence on the quality of the water in the estuaries. The types of climatological data available for an area about 60 miles $(95 \mathrm{~km})$ wide along the Texas Coast are shown in Figure 12.

Tabulations of daily precipitation, air temperature, and other data are published monthly; monthly summaries are published annually by the National Oceanic and Atmospheric Administration in the series titled "Climatological Data-Texas." For the period 1931-60, monthly and annual data are summarized in two publications by the National Weather Service (formerly U.S. Weather Bureau, 1958, 1965).

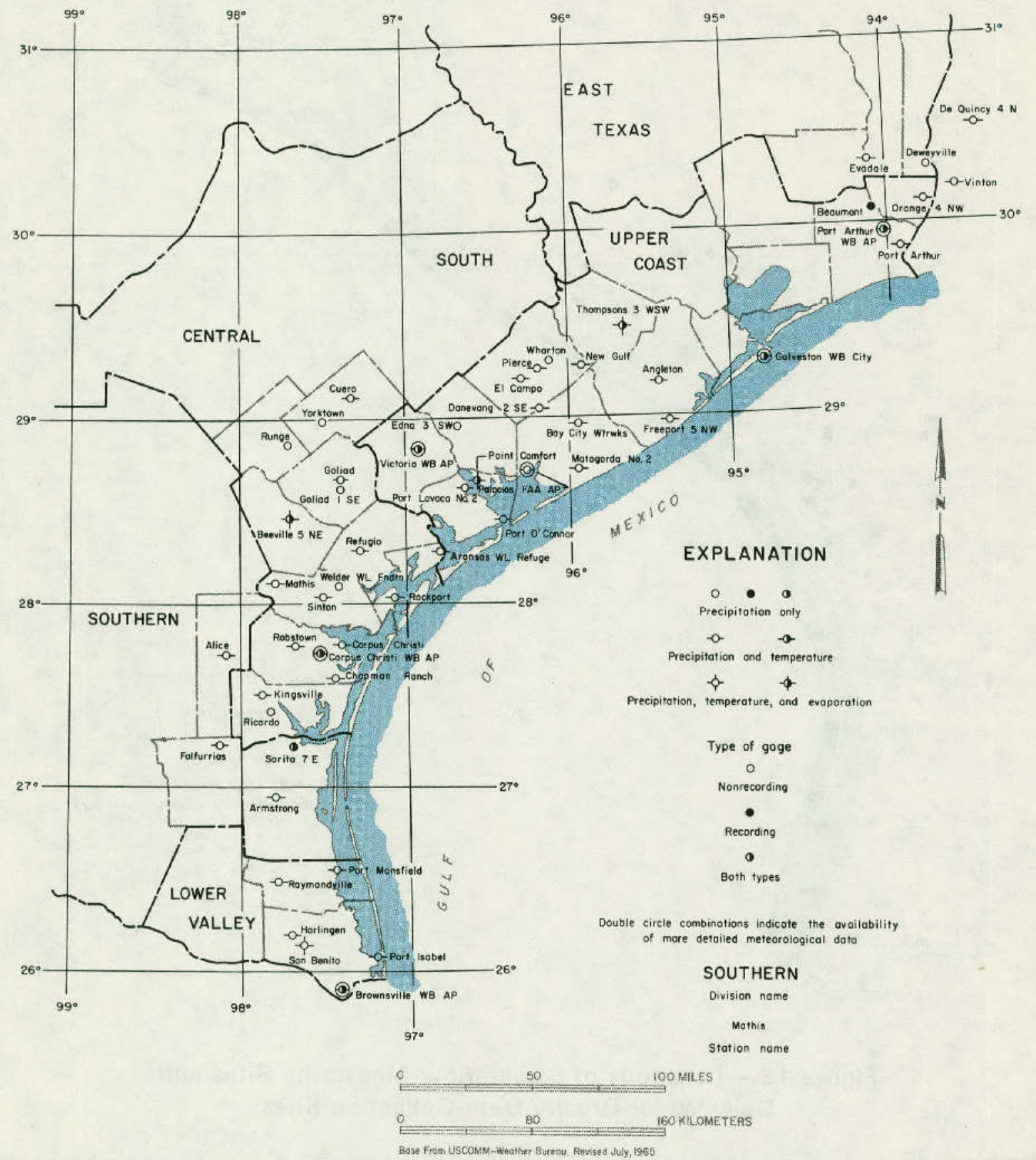

Figure 12.-Locations of Selected Climatological Stations 


\section{Streamflow and Water-Quality Records}

Streams along the Texas Coast flow across the flat coastal plain and are incised below sea level; therefore, changes in water stage within the bays are often reflected for many miles up the tributary streams. Consequently, the farthest downstream sites at which continuous streamflow data can be obtained are located many miles upstream from the principal estuarine embayments. The locations ${ }^{1}$ of the sites at which continuous streamflow and daily water-quality data are available are shown in Figure 13.

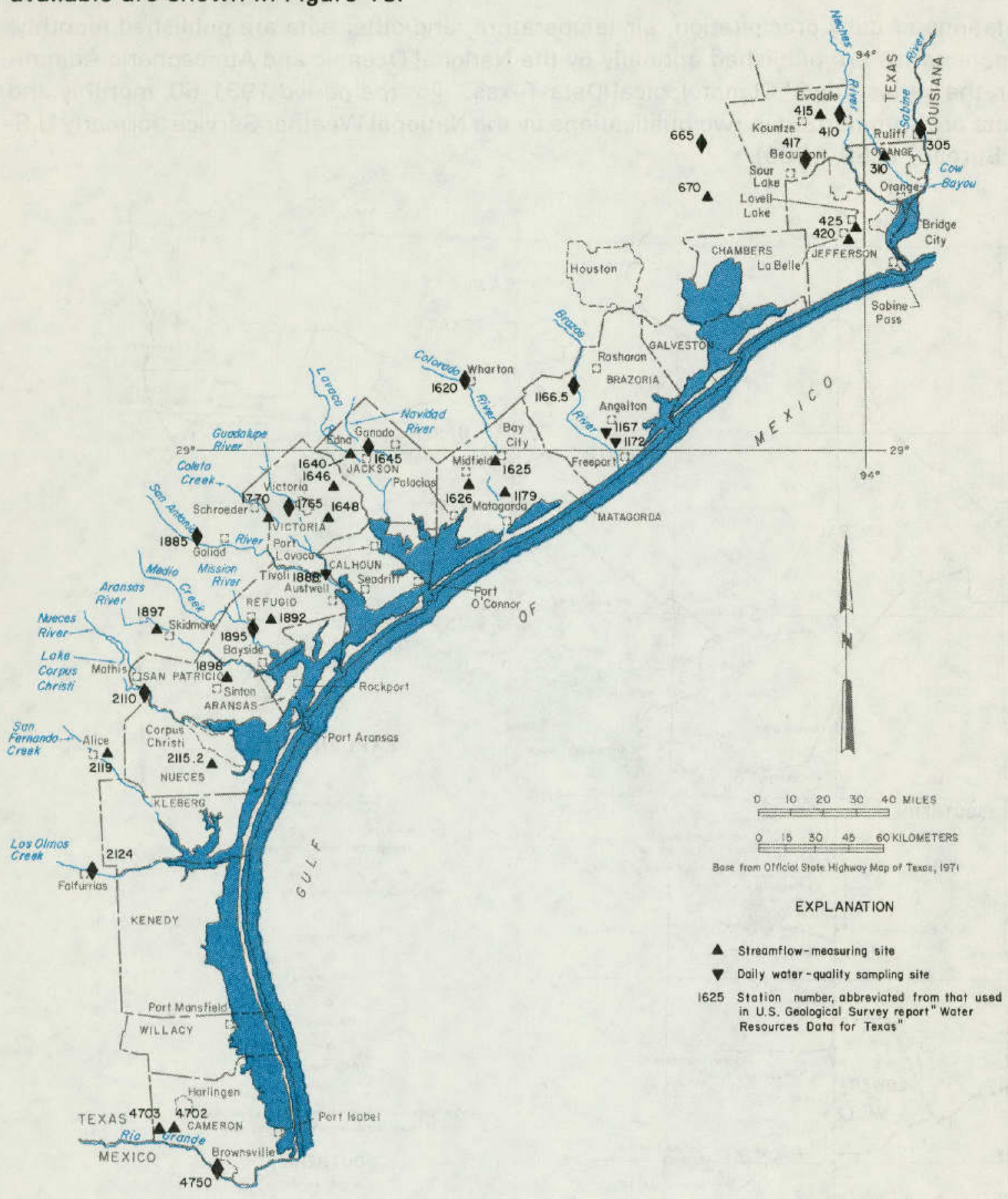

Figure 13.-Locations of Streamflow-Measuring Sites and Daily Water-Quality Data-Collection Sites 
The streamflow data for these sites represent runoff reaching the coastal area, but do not describe all of the flow from streams that enter the estuaries. Intervening drainage, diversion for irrigation, return flows, and evapotranspiration may influence streamflow between the measuring sites and the estuaries.

Analyses of water collected daily at streamflow-measuring sites show the effects of geology and cultural development on runoff from the drainage basins. At times, however, return flows from irrigation, evapotranspiration, and lack of significant runoff from areas upstream result in altered water quality between the data-collection site and the estuary.

The drainage areas from which unmeasured runoff enters the estuaries range from less than 100 square miles $\left(260 \mathrm{~km}^{2}\right)$ to more than 10,000 square miles $\left(25,900 \mathrm{~km}^{2}\right)$. Periodic measurements indicate that during some seasons, unmeasured runoff that reaches the estuaries exceeds measured flow from the major tributaries.

To completely describe the quality and quantity of runoff from the entire area between continuous streamflow-measuring stations and the estuaries is not feasible; however, representative data are collected periodically at the sites shown in Figure 14. Both continuous- and periodic-streamflow data and chemical-quality data are published by the U.S. Geological Survey (1978-79). 


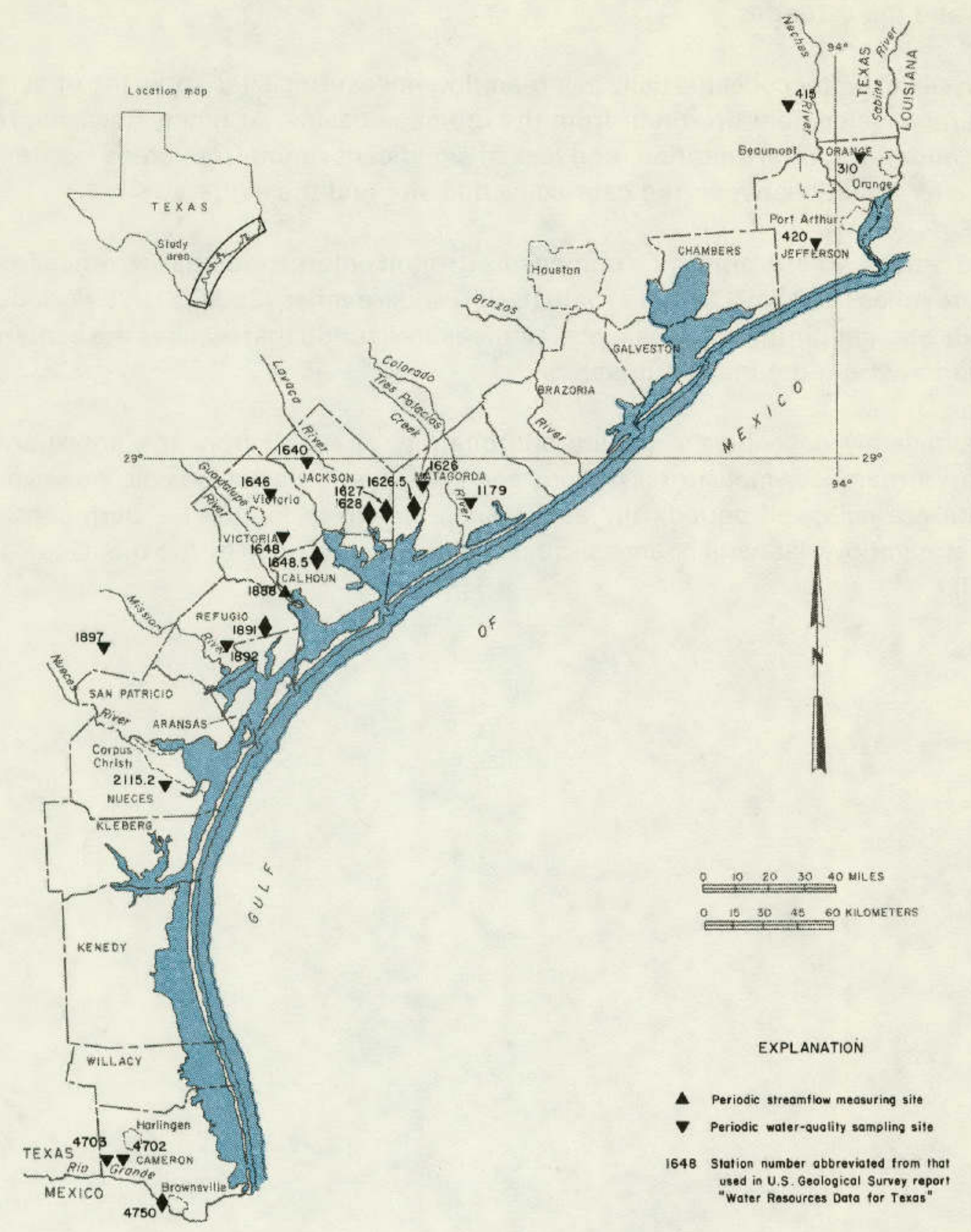

Figure 14

Location of Selected Water-Quality and Streamflow Data-Collection Sites 


\section{REFERENCES CITED}

American Public Health Association, American Water Works Association, Water Pollution Control Federation, 1971, Standard methods for the examination of water and wastewater: Am. Public Health Assoc., 13th ed., 874 p.

Grozier, R. U., and others, 1968, Floods from Hurricane Beulah in South Texas and Northeastern Mexico, September-October 1967: Texas Water Devel. Board Rept. 83, 195 p.

Hahl, D. C., and Ratzlaff, K. W., 1970, Chemical and physical characteristics of water in estuaries of Texas, September 1967-September 1968: Texas Water Devel. Board Rept. 117, 91 p.

1972. Chemical and physical characteristics of water in estuaries of Texas, October 1968-September 1969: Texas Water Devel. Board Rept. 144, 161 p.

1973. Chemical and physical characteristics of water in estuaries of Texas, October 1969-September 1970: Texas Water Devel. Board Rept. 171, 123 p.

1975, Chemical and physical characteristics of water in estuaries of Texas, October 1970-September 1971: Texas Water Devel. Board Rept. 191, 153 p.

Lauff, G. H., ed., 1967, Estuaries: Washington, D. C., Am. Assoc. Adv. Sci., 757 p.

Lind, W. B., 1980, Chemical and physical characteristics of water in estuaries of Texas, October 1974-September 1975: Texas Dept. Water Resources Rept. 245, 291 p.

1983, Chemical and physical characteristics of water in estuaries of Texas, October 1975-September 1976: Texas Dept. Water Resources Rept. 275, 203 p.

Lind, W. B., and Ratzlaff, K. W., 1979, Chemical and physical characteristics of water in estuaries of Texas, October 1973-September 1974: Texas Dept. Water Resources Rept. 231, 217 p.

Ratzlaff, K. W., 1976, Chemical and physical characteristics of water in estuaries of Texas, October 1971 -September 1973: Texas Water Devel. Board Rept. 208, 348 p.

Texas Water Development Board, 1968, The Texas water plan: Texas Water Devel. Board Planning rept., $215 \mathrm{p}$.

U.S. Geological Survey, 1978-79: Water resources data for Texas, water years 1977-78: U.S. Geol. Survey Water Data Repts. TX-77-1, 2, 3 and TX-78-1, 2, 3.

U.S. Weather Bureau, 1958, Climatic summary of the United States-supplement for 1931 through 1952, Texas: Climatography of the United States no. 11-36, U.S. Dept. of Commerce, $147 \mathrm{p}$.

1965. Climatic summary of the United States-supplement for 1951 through 1960 , Texas: Climatography of the United States no. 86-36, U.S. Dept. of Commerce, 198 p. 


